


\section{TO THE READER OF THIS VOLUME}

Kindly handle this book with the utmost care on account of its fragile condition. The binding has been done as well as possible under existing conditions and will wive reasonable wear with proper opening and handling.

Your thoughtfulness will be appreciated 





\title{
A CLASSIFIED
}

\section{INDEX AND SYNOPSIS}

\author{
OF THE
}

\section{ANIMAL K INGDOM}

ARRANGED IN CONFORMITY WITH ITS

ORGANIZATION,

\section{BY THE BARON CUVIER,}

MEMBER OF THE INSTITUTE OF FRANCE, \&c. \&c. \&c.

WITH

SUPPLEMENTARY ADDITIONS TO EACH ORDER,

BY

\section{EDWARD GRIFFITH, F.R.S. S.A. \& L.S.}

CORRESPONDING MEMBER OF THE ACADEMY OF NATURAL SCIENCES OF PHILADELPHIA, \&C.

AND OTHERS.

\section{LONDON:}

PRINTED FOR WHITTAKER AND CO. AVE-MARIA-LANE.

MDCCCXXXV. 
LONDON :

GILBERT \& RIVINGTON, PRINTERS,

ST. JOHN'S SQUARE.

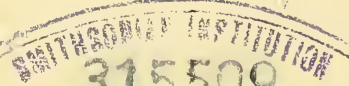

315509

MAR \&6 194

fect

c

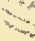

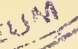




\section{A DVERTISEMENT.}

During the publication of the "Animal Kingdom," M. Guerin has produced in France a series of engravings, under the title "ICONOGRAPHIE DU REGNE Animal," illustrating nearly all the genera and subgenera of that work; including not only reduced copies of the most esteemed existing engravings of animals, but also a vast number of original figures of hitherto unpublished species, especially of insects and other invertebrata. M. Guerin has, moreover, added to these a most valuable series of anatomical figures, displaying, to the eye, much more intelligibly than words can do to the understanding, the peculiarities of structure on which Cuvier has founded his arrangement of the animal kingdom.

This work of M. Guerin has received the highest commendation, not merely from Cuvier himself, but also from most of the first zoologists in France, and is certainly of the greatest utility in the study of the work it is intended to illustrate, superseding, in a 
very great measure, constant references to Nature herself, otherwise necessary to those who would understand any natural system of arrangement.

The proprietors of the "Animal Kingdom" having now redeemed their pledges to the subscribers, by completing the work within the limits originally prescribed,-pledges which were made before Guerin's work was thought of,-have been strongly urged to publish the whole of these illustrations. Some of them have been already copied in the "Animal Kingdom;" and in order to render that work as useful as possible, it is proposed to publish the remainder as a Supplement to it, at as low a price as may be consistent with the proper execution of the plates.

The Iconography will moreover form a distinct work, or it may be applied to illustrate any general Zoology, as well as that of the Baron Cuvier. It will be published in ten or twelve Monthly Parts, to be completed contemporaneously with the original work in France, which will be early in 1836. A description of the figures will accompany the last Part. 


\section{N D E X}

TO THE

\section{ANIMAL KINGDOM.}

\section{MAMMALIA.}

Abdominales, vol. i. page lxvi.

Acanthion, v. 262

Daubentonii, v. 262

javanicum, v. 262

Accipitres, i. Ixii.

Acheus, v. 274

Acouchi, iii. 94

Acrobata pygmæa, v. 200

Acronotus, v. 361

Acronotine group, iv. 346

Acuti, v. 271

Addax, iv. 193 ; v. 328

Adimain, iv. 325

Adive, l', ii. 44 ; v. 146. 149

Agagre, iv. 23

Egagrus, iv. 303 ; v. 357

Aëllo, v. 83

Cuvieri, v. 83

Agouara, v. 114

Gouazou, ii. 42 ; v. 145

Agouarachy, v. 148

Agouti, v. 271

common, iii. 94

à crête, v. 272

des Patagons, iii. 243

Agoutis, iii. 94. 24]

Ahu, iv. 122; v. 313.332

Aï, iii. 253. 271 ; v. 274

ash-coloured, v. 275

collared, v. 274

spotless, v. 274

yellow-faced, v. 274

Aigocerine group, iv. 175

Cuv. Index.
Aigocerus, v. 324

Aigrette, v. 18

Akko, v. 357

Akoin, v. 336

Akouchy, v. 272

Alactaga, iii. 77. 143

Alb, the great, v. 321

Alce, v. 303

Alcine group, iv. 72

Alco, v. 140

Algazel, iv. 191 ; v. 327

Alliaire, l', v. 210

Allocamelus, v. 299

Alouatta, i. 349 ; v. 26

Beelzebut, i. 351

common, i. 222

howling, i. 351

Alouattas, i. 221

Amblotis, ii. 78 ; v. 206

Amphibia, i. Iviii. Ixiv. Ixxvii. ; v. 175

Anabula, v. 321

Anarnack of Gröenland, iv. 464

Andira, ii. 125 guaçu, ii. 9. 125 ; v. 71

Angingayer, v. 132

Angouan, l', v. 46

Angouya y bigoni, v. 218

Animal Anonyme, v. 152

body, active forces of the, i. 29

body, function and organs of the, i. 39

body, organic elements of the, i. 28 
Animal kingdom, general distribution of the, i. 60

kingdom, method of the, i. 58 moschiferum, v. 301

Animals, amphibious, i. Iviii.; ii. 55 first class of vertebrated, i. 72 intellectual function of, i. 49 pouched, ii. 61 vertebrated, i. 65 vertebrated into four classes, i. 69

Anisonys, v. 245 brachyura, v. 245 rufa, v. 245

Anoa, iv. 293 ; v. 355 compressicornis, v. 355 depressicornis, iv. 293 ; v. 355

Anoema, iii. 93. 234

Anoine group, iv. 292

Anoplotherium, iii. 334

Anseres, i. Ixii.

Anta, iii. 431 ; v. 294

Ant-eater, aculeated, iii. 313 ; v. 284 bear, v. 280

Cape, iii. 296 ; v. 279

great, v. 280

great American, iii. 299

little, iii. 306 ; v. 281

little bear, v. 280

maned, v. 280

porcupine, v. 284

two toed, iii. 261 ; v. 281

Ant-eaters, iii. 259

scaly, iii. 261

spiny, iii. 263

Antelope, awl-horned, iv. 253 ; v. 342

Barbary, iv. 212; v. 332

blue, iv. 20. 176; v. 324

broad-eared, iv. 260 ; v. 344

broad-hoofed, iv. 204 ; v. 330

Burchell's, iv. 262; v. 345

bush, iv. 258 ; v. 344

common, iv. 231 ; v. 336

cream-coloured, iv. 240 ; v. 338

des Buissons, v. 344

des Indes, v. 337

dodger, iv. 265 ; v. 347

Duvaucel's, v. 352

equine, iv. 20. 177

four-horned, iv. 256 ; v. 343

four-tufted,iv. 261 ; v. 346

Gambian, iv. 221 ; v. 334

great-horned, iv. 179

harnessed, iv. $274 ;$ v. 351

king, v. 349

long-horned, v. 325

Maxwell's, iv. 267; v. 347

nez taché, v. 330

niou, v. 368

of Honduras, v. 318
Antelope, of India, iv. 18

Ovine, iv. 289 ; v. 354

palmated, iv. 172 ; v. 323

Persian, iv. 210; v. 331

pourprée, v. $\mathbf{3 3 0}$

prong-horned, iv. 170 ; v. 323

red-necked, iv. 205

ribbed, iv. 275 ; v. 351

roan, iv. 177 ; v. 324

Salt's, iv. 271 ; v. 350

sharp-horned, iv. 252 ; v. 342

slate-coloured, iv. 268 ; v. 348

springer, iv. 208 ; v. 331

striped, v. 366

swift, iv. 206 ; v. 320

white-faced, iv. 202 ; v. 329

white-footed, iv. 363 ; v. 367

wool-bearing, iv. 286 ; v. 354

Antelopes, iv. 15

Antelopine group, iv. 218

Antes, v. 294

Antholops, v. 327

Antilocapra, iv. 167

Americana, v. 323

Antilope, iv. 15. 162 ; v. 322.334

acuticornis, iv. 252 ; v. 342

addax, iv. 193 ; v. 328

adenota, iv. 223 ; v. 335

algasel, v. 328

Americana, v. 354

arundinacea, v. 338

arundinium, v. 338

aurita, v. 325

barbata, iv. 180 ; v. 325

besoartria, v. 328

besoastica, v. 327

bezoastica, iv. 191

bubalis, iv. 18

Burchellii, iv. 162 ; v. 345

caama, iv. 18

capreolus, v. 339

cervicapra, iv. 18.231 ; v. 336

chickara, iv. 254 ; v. 343

cœrulea, iv. 268 ; v. 348

colus, iv. 226 ; v. 335

cora, iv. 216 ; v. 333

corinna, iv. 16.214 ; v. 333

dama, iv. 20.206 ; v. 330.341

diotragus, v. 342

dorcas, iv. 16. 212 ; v. 330.332

dorsata, v. 331

Duvaucelii, iv. 279 ; v. 352

eleotragus, iv. 237 ; v. 328.337

equina, iv. 20.177 ; v. 324

euchore, iv. 17. 208; v. 331

forfex, iv. 221; v. 334

fulvo rufula, iv. 239 ; v. 338

furcifer, iv. 170 ; v. 323

gnu, iv. 22 ; v. 368 
Antilope, goral, iv. 279 ; v. 352 grandicornis, iv. 179 ; v. 325 grimmia, iv. 266 ; v. 347 grisea, iv. 250 ; v 341 gutturosa, iv. 17. 229 ; v. 336 Ibex, v. 341

interscapularis, v. 352 isabellina, iv. 240 ; v. 338 kemas, iv. 196 ; v. 328 kevella, iv. 16. 213 ; v. 332 koba, v. 363

Lalandiana, v. 338

lanata, v. 339

lanigera, iv. 286 ; v. 354 leucophæa, iv. 20. 176; v. 324 leucoryx, iv. 188 ; v. 326 madoka, iv. 271 ; v. 350 marsupialis, v. 331 Maxwellii, iv. 267; v. 347 Mazama, iv. 289 ; v. 354 melampus, iv. 219; v. 334 melanotis, iv. 250 ; v. 341 mergens, iv. 264 ; v. 346 mytilopes, iv. 204 ; v. 330 naso maculata, v. 330 oreas, iv. 21 ; v. 365 oreotragus, iv. 245 ; v. 340 oryx, iv. $19.186 ;$ v. 325,326 ozanne, v. 325 palida, iv. 251 ; v. 342 pallah, v. 334 palmata, iv. 172 ; v. 323 perpusilla, iv. 269 ; v. 348 phalerata, iv. 275 ; v. 351 philamtomba, v. 349 picta, iv. 2 l : v. 367 platous, iv. 260 ; v. 344 ptoox, iv. 265 ; v. 347 pygarga, iv. 202; v. 329. 331 pygmea, iv. 270 ; v. 303.349 quadricornis, iv. 256 ; v. 343 quadriscopa, iv. 261 ; v. 345 redunca, iv. 238 ; v. 338 reversa, v. 338 rufescens, iv. 249 ; v. 341 ruficollis, iv. 205; v. 331 rupestris, iv. 248 ; v. 340 rupicapra, iv. 22.281 ; v. 353 saiga, iv. 17 ; v. 336

Saltatrix, v. 340 Saltiana, iv. 271 scoparia, iv. 244 ; v. 339 scripta, iv. 274 ; v. 351 Senegalensis, v. 363 silvicultrix, iv. 258 ; v. 344 strepsiceros, iv. 21 ; v. 366 subgutturosa, iv. 210 ; v. 331 subulata, iv. 253 ; v. 342 Sumatrensis, iv. 277 ; v. 352
Antilope, suturosa, iv. 363 sylvatica, iv. 272 ; v. 350 tao, iv. 189; v. 327 taurina, v. 369 temmamazama, iv. 291 ; v. 354 torticornis, v. 366 tragulus rupestris, v. 341 villosa, iv. 241 ; v. 339

Anzing Ager, v. 132

Aotus, v. 35 trivirgatus, v. 35

Apara, v. 275

Ape, Alpinus, v. 19 Barbary, i. 215. 280 ; v. 19 dog-faced, v. 21 great, v. 5 long-armed, v. 6 pygmy, v. 19 satyr, i. 350 tufted-tailed, v. 30

Aper Ethiopicus, v. 289 Apes, i. 207. 333. 349, 350

Aperea, v. 27]

Brasiliensibus, v. 270 wild, iii. 234

Aplocerine group, iv. 285

Aplocerus, v. 354

Apodes, i. lxvi.

Arabata, v. 27

Araguato, v. 27

Arctocephale, v. 182

Arctomys, iii. 80. 166 ; v. 243 Africana, v. 242 Alpina, v. 247 bobac, v. 244 brachyura, v. 245 citillus, v. 246 empetra, iii. 170 ; v. 245 Franklinii, v. 246

Hoodii, iii. 171

latrans, v. 248

Ludoviciana, v. 247

marmotta, v. 244

Missouriensis; v. 248

$\operatorname{monax}, \mathrm{v} .244$

Parryii, v. 247

Richardsonii, v. 246 rufa, v. 245

tridecimlineata, v. 247

Woodii, v. 247

Arctopithecus, i. 225

Argali, American, iv. 318 ; v. 359 Asiatic, iv. 316; v. 359 bearded, iv. 319 ; v. 359 of Siberia, iv. 25

Armadillo, iii. 255

Africanus, v. 277

Brasiliensis, v. 276

eight-banded, v. $276^{\circ}$ 
Armadillo, eighteen-banded, v. 278 giant, iii. 258. 294 ; v. 277 greatest, v, 277

Guyanensis, v. 276

hairy, iii. 292 ; v. 278

Indicus, v. 276

Mexicanus, v. 276

mule, iii. 294 ; v. 276

nine-banded, iii. 257 ; v. 276

Orientalis, v. 276

pig-headed, v. 276

seven-banded, v. 276

six-banded, iii. 257 ; v. 278

three-banded, iii. 256. 294 ; v. 275

twelve-banded, iii. 257 ; v. 277

Arnad, v. 372

weasel-headed, v. 278

Arnee, iv. 388 ; v. 372

Artak, v. 359

Artibeus, v. 84

Jamaicensis, v. 84

Arvicola, ii. 66. 109; v. 209

albicaudatus, v. 212

alliarius, v. 210

amphibia, v. 209

amphibia maculata, v. 209

amphibia nigra, v. 209

amphibia paludosa, v. 209

argentoretensis, v. 212

arvalis, v, 209

Astracan, v. 214

Astrachanensis, v. 214

Egyptian, v. 212

Floridanus, v. 214

fulvus, v. 212

gregalis, v. 211

hortensis, v. 213

musk, v. 208

Niloticus, v. 212

œconomus, v. 210

palustris, v. 213

Pennsylvanica, v. 214

pumilio, v. 211

riparius, v. 213

rutilus, v. 210

saxatilis, v. 210

socialis, v. 211

Strasbourg, v. 212

xanthognata, v. 213

yellow, v. 212

yellow-cheeked, v. 213

Ascagne, i. 213 ; v. 13

Ashaary, v. 298

Ashkokoo, iii. 431 ; v. 293

Asinus quagga, v. 295

Aspalax, v. 107. 241

Ass, iii. 343. 461 ; v. 295 common, v. 295
Assapan, v. 259

Atalapha, v. 98

Atele coaita de Cayenne, v. 24

Ateles, i. 222 ; v. 24 arachnoides, i. 223; v. 25

belzebeth, v. 24

hypoxanthus, v. 25

marginatus, i. 223 ; v. 25

melanochir, v. 25

niger, v. 24

paniscus, v. 24

pentadactylus, i. 222 ; v. 25

subpentadactylus, v. 25

Atok, v. 128

Attarsoak, v. 177

Attenk, v. 305

Atys, v. 16

Auchenia, iv. 5. 50 ; v. 298

araucana, v. 300

glama, v. 299

Huanaca, v. 299

Huemel, v. 300

paco, v. 299

vicugna, v. 300

Aurochs, iv. 29 ; v. 373

Aurox, true, v. 376

Aves, i. Iviii. Ixii.

Axine group, iv. 116

Axis, iv. 12; v. 312

brown porcine, iv. 119 ; v. 312

dwarf, iv. 120 ; v. 313

great, v. 310

spotted, iv. 117

Aye Aye, ii. 86. 196; v. 51.

BabI Alu, iii. 434

Babiroussa, iii. 332

Baboon, i. 349. 351 ; v. 21. 23

black, v. 26

brown, i. 290 ; v. 18

cinereous, v. 22

common, i. 218

dog-faced, i. 218 ; v. 21

dog-tailed, v. 14

great, v. 2 l

grey, v. 21

Guinea, v. 20

lion-tailed, v. 16

little, v. 20

long-legged, i. 289

Mandrill, i. 219. 289

of the woods, i. 219

Pennant's, v. 23

pig-faced, v. 20

pig-tailed, i. 288 ; v. 18

ribbed-nose, v. 22

thumbless, v. 23

variegated, v, 21 
Baboon, wood, v. 22 yellow, v. 22

Baboons, i. 214 Suppl. on the, i. 278

Babouin, v. 20 à museau de chien, v. 21 à longues jambes, v. 19

Babyroussa, iii. 408 ; v. 288

Badger, ii. 269; v. 116 American, v. 116 Bougainville's pouched, v. 195 Cape, v. 292 common, v. 116 dog, y. 274

fat-pouclied, v. 195

hog, ii. 274

Indian, v. 118 long-nosed pouched, v. 195 of Europe, ii. 31 rock, iii. 429

Badgers, ii. 30

Balæna, iv. 440 ; v. 389 boops, iv. 443 ; v. 391 cœrulescens, v. 391 gibbar, v. 390 gibbosa, iv. 498 ; v. 390 gibbosa, var., v. 390 glacialis, iv. 442 ; v. 390 Islandica, v. 390

Japonica, v. 390

lunulata, v. 390

maculata, v. 391

mœra, v. 390

major, v. 389

musculus, v. 391

mysticetus, iv. 441. 491 ; v. 389

nigra, v. 391

nodosa, iv. 498 ; v. 390

physalis, iv. 443 ; v. 391

punctata, v. 391

rostrata, iv. 478 ; v. 391

tripenni ventre lævi, v. 391

vulgaris Groenlandica, v. 389

Balænæ, iv. 471

Balænoptera, v. 390

Balantia, ii. 70

orientalis, v. 196

Baleine à bosses, v. 390

à museau pointu, v. 391

a six bosses, v. 390

bossue, v. 390

franche, v. 389

gibbar, v. 391

nord Caper, v. 390

noueuse, v. 390

tampon, v. 390

Baleinoptera gibbar, v. 391

with a smooth belly, iv. 413
Baleinoptera with a wrinkled belly, iv. 443

Baleinoptere Jubarte, v. 391

Bangsring, ii. 212 ; v. 52. 106

Barbaresque, iii. 83

Barbastel, v. 97

Barbastelle, ii. 14. 147

Barbet, ii. 41 ; v. 138

little, v. 138

grand, v. 138

Barensing'ha, v. 310

Baresque, v. 253

Basset à jambes droites, v. 139

Bat, Anglicè leaf-nosed, ii. 10 bearded, ii. 12.137

big-eared, v. 98

black-backed, v. 98

black-faced, v. 98

black-shouldered, v. 98

Blainville's mormoops, v. 74

blue-winged, v. 98

Bourbon, ii. 154

Brazil vespertilio, v. 91

broad-tailed molossus, v. 62

Brookes's celœno, v. 83

brown, ii. 126

brown-belly, v. 61

brown-striped, ii. 126

bull-dog, v. 60

chestnut, v. 62

chestnut-coloured molossus, v。 61

cinnamon, v. 62

common, ii. 142

common javelin, ii. 127

cordated, of Pennant, v. 74

Cuvier's Aëllo, v. 83

dark, v. 61

Daubenton's myopteris, v. 83

diadem rhinoloph, v. 77

dilated nyctinome, v. 65

dog, ii. 110

dog, of Java, v. 56

eighth, of Azara, v. 62

eleventh, of Azara, v. 96

flat-nosed phyllostome, v. 69

foliaceous megaderme, v. 75

fourth, of Azara, v. 71

great, v. 89

great-eared, ii. 14.152

great horse-shoe, ii. 11

javelin, ii. 126

great, of Madagascar, v. 55

great serotine, v. 9]

great-tailed molossus, v. 62

guyane molossus, v. 62

hairy, ii. 155

Hardwicke's, v. 92

hare-lipped, ii. 139 
Bat, heart-nosed, ii. 128

horse-shoe rhinolophus, v. 76

indented javelin, ii. 10

indented phyllostome, v. 68

Jamaica, v. 73

Jamaica artibeus, v. 84

javelin, ii. 9 ; v. 69

knobbed-tail glossophag. v. 73

lasiopter, ii. 155

leaf, of Pennant, v. 72

lesser horse-shoe, ii. 11 ; v. 76

lesser ternate, v. 75

Lewis's medateus, v. 74

Lily-leafed phyllostome, v. 71

long-eared, v. 96

long-leafed phyllostome, v. 68

lyre-leafed megaderme, v. 75

Madagascar, ii. 107

Monk, v. 98

netted, v. 98

New York, ii. 155

ninth, of Azara, v. 61

noctule, v. 89

nycteris, of Java, v. 80

nyctophilus, of Geoffroy, v. 86

obscure, ii. 128

of Carolina, ii. 143

ordinary, ii. 13

perforated taphozous, v. 82

Peruvian, v. 67

Peruvian, var. $\beta$. v. 67

pit-nosed, v. 77

pit-nosed rhinoloph, v. 77

Porto Rico, v. 97

pouch, v. 82

proboscis bull-dog, v. 63

pygmy vespertilio, v. 91

red bull-dog, v. 68

reddish, v. 67

reddish-brown, ii. 126

Redman's monophyllus, v. 85

rhinopome, of Carolina, v. 81

rough-haired nycteris, v. 79

rough-tailed, ii. 154 ; v. 90

round-leaved phyllostome, v. 70

scotophilus, of Kuhl. v. 84

second, of Azara, v. 70

Senegal, ii. 154 ; v. 90

serotine, v. 88.

seventh, of Azara, v. 96

short-eared English, v. 88

sixth, of Azara, v. 62

slender-tailed, v. 82

sloped-eared, ii. 153

small-leaved rhinopome, v. 80

smoky bull-dog, v. 63

sparred, v. 98

spear-leafed phyllostome, v. 69

spear-nosed, ii. 9
Bat, spectacle, ii. 127 ; v. 69

spectre, v. 71

striped, ii. 128 ; v. 90

streaked phyllostome, v. 70

tailed glossophag, v. 73

tailless diphylla, v. 85

tailless glossophag, v. 73

taphozous, of Mauritius, v. 82

taphozous, of Senegal, v. 81

Temminck's vespertilio, v. 92

tenth, of Azara, v. 62

Ternate, ii. 108

third, of Azara, v. 70

tiled, v. 93

Timor, ii. 154 ; v. 98

trident rhinoloph, v, 77

twelfth, of Azara, v. 96

ursine bull-dog, v. 63

vampyre, v. 71

veiled-eared, v. 98

vespertilio of Isid. St. Hiliere, v. 91

white-bellied bull-dog, v. 68

Bathyergus, iii. 79. 164 ; v. 241

Capensis, v. 242

Maritimus, v. 242

Cape, v. 242

coast, v. 242

Bats, ii. 3 ; v. 54 common, ii. 13

Bear, Arctic, v. 112

ash-coloured sea, v. 183

black, v. 112

black, of America, ii. 27. 224 ; v. 112

black, of Europe, ii. 27. 222 ; v. 111

brown, v. 111

brown, of Europe, ii. 26. 217

brown, of the Alps, ii. 217

brown, of Norway, ii. 217

cinnamon, ii. 229 ; v. 112

common, v. 111

common European, v. 111

common sea, v. 182

crowned sea, v. 182

European black, v. 111

gray, v. 113

grisly, ii. 229 ; v. 112

honey, ii. 269

Hudson's Bay, of Brisson, v. 117

long-lipped, v. 113

Malay, v. 113

New Holland, v. 197

Peron's sea, v. 182

polar, ii. 232; v. 112

sea, ii. 59. 509

Thibet, v. 113

thick-lipped, ii. 239 
Bear, yellow, v. 112

yellow, of Carolina, ii. 228

yellowish sea, v. 183 white, ii. 28

Bears, ii. 26

Beast, great-toothed, ii. 60

Beaver, iii. 101 ; v. 207

Canadian, iii. 105

musk, v. 209

of France, v. 208

white, v. 208

Beejoo, ii. 280

Beest, bastard wilde, v. 369 wilde, v. 368

Beings, of living, i. 11 organized into animals and ve-

Belette, v. 121 getables, i. 24

Belluo, i. lx. lxxvii. des niges, v. 121

Beluga, iv. 437. 457 ; v. 385

Belzebuth, Le, v. 24

Benawi, v. 146

Bentourong, v. 160

Berg-haas, iii. 165

Bhain, v. 372

Biche de Barallon, v. 31.8 des Bois, v. 318

Bievre, le, v. 208

Bimana, i. lxxvii. 82 ; v. 3

Binturong, ii. 417

Birds, i. lxii.

Bison, iv. 29.398 ; v. 373

American, iv. 401 ; v. 374 broad-headed fossil, iv. 410 of America, iv. 29

Bisontine group, iv. 395

Bisulca, i. lxxix.

Blaireau, v. 116 du Cap, v. 123 puant, v. 118

Blanc nez, Le, v. 13

Blauwbockje, v. 348

Bleekbock, iv. 251 ; v. 342

Boar, African, v. 289

Æthiopian, v. 289

masked, iii. 407 ; v. 288

Bobac, iii. 81.169 ; v. 244

Bobak, v. 244

Bock,

blauw, v. 324

bless, v. 330

bos, v. 351

bosch, v. 350, 351

ceinse, v. 326

duiker, iv. 264,265 ; v. 346

gemse, v. 353

grys, v. 341

kleene, v. 348
Bock, prouk, v. 331

riet, v. 337

riet $\mathrm{rhee}, \mathrm{v} .339$

spring, v. 331

steen, v. 340

stein, v. 356

vlackte steen, v. 341

Boeuf de haut cru, iv. 424

de nature, iv. 424

musque, v. 370

Boggo, i. 219 ; v. 21

Bokkol, iii. 187 ; v. 256

Bondar, v. 155

Bonnet-chinois, v. 17

Bos, iv. 28.376 ; v. 370

Americanus, iv. 29. 401 ; v. 374

arnee, v. 372

arni, iv. 388

bison, iv. 29 . 398 ; v. 373

bombifrons, iv. 410

bubalis, iv. 31.392 ; v. 372

caffer, iv. 31.384 ; v. 371

gaurus, iv. 399 ; v. 373

gavæus, iv. 406 ; v. 375

grunniens, iv. 30 ; v. 375

latifrons, iv. 410

moschatus, iv. 31 ; v. 370

pegasus, iv. 386 ; v. 371

poëphagus, iv. 404 ; v. 374

scoticus, v. 376

sylhetanus, v. 375

taurus, iv. 28.418 ; v. 376

urus, iv. 29.414 ; v. 376

urus scoticus, iv. 417

Boschbock, iv. 272; v. 350

Boselaphine group, iv. 354

Boselaphus, v. 364

Bouquetin, iv. 24 ; v. 356 de Nepal, v. 353

Bovidæ, v. 367

Bradypoda, i. lxxv.

Bradypus, iii. 252.266 ; v. 274 didactylus, iii. 254.275 ; v. 275 torquatus, iii. 277

tridactylus, iii. 258.271 ; v. 274

ursinus, iii. 254 ; v. 113

with a collar, iii. 277

Branchiostegi, i. lxvi.

Braque, v. 139

de Bengal, v. 139

Brocket, apara, iv. 141 ; v. 318 bira, iv. 142 ; v. 319 pita, iv. 140 ; v. 318

Brourong, ii. 239

Bruno, ii. 71 ; v. 197

Bruta, i. lx.

Bubale, v. 362

Bubaline group, iv. 378

Bubalis, iv. 18. 347 ; v. 362 
Bubalis of the ancients, iv, 18

Bubalus, v. 371

Bucher Abiad, El, v. 328

Buffalo, iv. 29 ; v. 374

Cape, iv. 30. 384; v. 371

domestic, iv. 392 ; v. 372

with horse's-tail, iv. 30

Buffle, v. 372

Buflus, v. 372

Buga, v. 308

Bukrus, v. 327

Bulan, v. 304

Bull, wild, v. 376

Busan, v. 372

Butskopf, iv. 459 ; v. 384

Buwol, v. 372

Canma, iv. 18.348 ; v. 362

Cabasson, iii. 257 deuxième, iii. 258

Cabaya, v. 271

Cabiai, iii. 93 ; v. 270

Cabiais, iii. 93

Cabree, v. 323

Cacajao, i. 318 ; v. 37

Cachalot, d'Anderson, v. 384 grand, v. 388 macrocephale, v. 388 microps, v. 388 svinewal, iv. 469 trumpo, iv. 468 cylindrical, iv. 469 furrowed, v. 389 great-headed, v. 388 small-eyed, v. 388

Cachalots, iv. 439.464

Cachicame, iii. 257 ; v. 276

Cœlogenus, iii. 95.247 ; v. 273 fulous, v. 273 subniger, v. 273

Cagui minor, v. 38

Caimiri, v. 33

Calf, sea, v. 176

Callithrix, i. 339. 348 ; v. 33 amictus, v. 33 capucinus, v. 32 incanescens, v. 34 infulatus, v. 34 lugens, v. 33 melanochir, v. 31 moloch, v. 34 personata, v. 33 sciureus, v. 33 torquatus, v. 34

Callitriche, v. 14 jeune, v. 14

Callitrix, i. 212. 263 collared, v. 34

Calocephale, v. 176
Calocephale discolor, v. 177

Cambing, Ootan, iv. 277 ; v. 352

Cambtan, v. 352

Camel, Arabian, iv. 49 ; v. 298 bactrian, iv. 48 ; v. 297 with one bunch, iv. 5 with two bunches, iv. 5

Camelidæ, v. 297

Camelopardalis, iv. 14 ; v. 321 giraffa, iv. 15.150 ; v. 321

Camels, iv. 3. 37

Camelus, iv. 3.37 ; v. 297 Arabia, v. 298 araucanus, v. 300 Bactriæ, v. 298

Bactrianus, iv. 5.48 ; v. 297

Dromedarius, iv. 5.49 ; v. 298

glama, v. 299

huanaco, v. 299

huanacus, iv. 55 ; v. 299

Indicus, v. 321

laniger, v. 300

Llama, iv. 6. 53

paco, iv. 157 ; v. 299

vicugna, iv. 6.58 ; v. 300

Campagnol, iii. 68. v.

doré, v. 211

economic, iii. 112

Florida, v. 214

garden, v. 213

marsh, v. 213

meadow, iii. 68

of Pennsylvania, v. 214

roux, v. 211

volant, v. 80

Campagnols, iii. 66. 109

Campagnon, v. 211

Caniche, v. 138

Canis, ii. 39. 317 ; v. 133

Ægyptiacus, v. 150

alopex, ii. 43.364 ; v. 147

Anglicus, ii. 339

antarcticus, v. 145

anthus, ii. 339 ; v. 147

aquaticus, ii. 334

aquaticus, aviarius, v. 138

argentatus, ii. 368 ; v. 148

aureus, ii. 42 ; v. 145

avicularius, ii. 335

barbarus, v. 146

brevipilis, ii. 333

cancrivorus, v. 145

cerdo, ii. 45 ; v. 152

cinereo argentatus, ii. 44.365 ;

v. 148

corsac, ii. 44 ; v. 149

crocuta, ii. 49 ; v. 162

crucigera, v. 147 
Canis culpæus, v. 145

decussatus, ii. 370 ; v. 149

domesticus, ii. $\mathbf{3 3 0}$

extrarius, ii. 332

familiaris, ii. 40 ; v. 133

Egyptius, v. 143

Americanus, v. 140

Andalusiæ, v. 143

Anglicus, v. 141

aquaticus, v. 138

Australasiæ, v. 134

avicularius, v. 139

Bengalensis, v. 139

Borealis, v. 140

Britannicus, v. 142

Danicus, v. 135

domesticus, v. 139

extrarius, v. 136

fricator, v. 142

Gallicus, v. 138

Grajus, v. 135

hybridus, v. 142

Islandicus, v. 142

laniarius, v. 134

leoninus, v. 137

minor, v. 138

Molossus, v. 141

Pomeranus, v. 140

Sibiricus, v. $\mathbf{1 4 0}$

Sumatrensis, v. 134

variegatus, v. 142

vertagus, v. 139

fulvus, v. 150

Graius, ii. 328

hyæna, ii. 49 ; v. 161

hyænomelas, v. 161

jubatus, v. 144

karagan, v. 149

lagopus, ii. 44.370 ; v. 148

laniarius, ii. 328

leoninus, ii. 334

lupus, ii. 41 ; v. 144

Lycaon, ii. 42 ; v. 144

Lycaon, var. v. 148

Americana, v. 144

megalotis, ii. 372 ; v. 152

mesomelas, ii. 45.371 ; v. 146

Mexicanus, ii. 42 ; v. 145

Molossus, ii. 336

Niloticus, ii. 365 ; v. 130

pantherinus, v. 139

pictus, v. 15]

pomeranus, ii. 331

sagax, ii. 334

Sibiricus, ii. 331

thous, v. 145

tricolor, v. 151

velong, v. 150

vertagus, ii. 336
Canis virginianus, v. 150

volans maxima aurito, v. 71

vulpes, ii. 43 ; v. 147

zerda, v. 152

Canna, iv. 21. 337 ; v. 365

Capistrate, iii. 178 ; v. 253

Capiygoua, iii. 93

Capparo, v. 26

Capra, iv. 23. 294 ; v. 355 ægagrus, iv. 23. 303 ; v. 357 Ammon, v. 359

Caucasica, iv. 25. 302 ; 357 depressa, iv. $\mathbf{3 1 0}$

hircus, iv. 24. 305 ; v. 357

Ibex, iv. 24. 300 ; v. 356

Jaela, iv. 301 ; 355

Jemlahica, iv. $\mathbf{3 0 8}$; v. 358

merga, v. 346

moschi, v. 301

rupicapra, v. 353

sylvestris Africana, v. 347

Caprea, v. 314

Capreoline group, iv. 121

Capreolus, v. 313 dorcas, v. 314

Capricerva paseng, v. 357

Capridæ, v. 322

Caprine group, iv. 155

Capuchin of the Orinoco, i. 316.

Capucin de l'Orinoque, v. 36

Capward, v. 270

Capybara, iii. 93. 232 ; v. 270 Brasiliensibus, v. 270

Capygona, v. 270

Cara, rayada, v. 35

Caracal, ii. 54. 490 ; v. 173

à longue queue, v. 173

of Algiers, v. 173

of Bengal, v. 173

of Nubia, v. 173

Caraya, i. 222.301 ; v. 28

Carcajou, ii. 274 ; v. 116

Cariacou, v. 317

Cariblanco, v. 32

Caribou des Bois, iv. 83 ; v. 305

great, v. 305

Labrador, v. 305

Polar, v. 305

Carigueia, ii, 63. v. 187

Cariquebein, v. 118

Carnassiers, ii. J. 53

first family of the, ii. 2 . suppl. to the, ii. 79

Carnivora, i. lxxvii. ; ii. 23 ; v. 111 Suppl. to the, ii. 217

Carpon, v. 223

Caruiri, v. 38

Castor, iii. 63.101 ; v. 207 fiber, iii. 64 ; v. 207

Cuv. Index. 
Castor moschatus, ii. 185 ; v. 105 of Canada, iii. 64 zibethicus, iii. 67. v. 208

Cat, Angora, ii. 489 ; v. 173

Bisaam, ii. 46

black, ii. 486

black-footed, v. 172

brinded, ii. 489

Cape, v. 170

Cape, of Forster, ii. 46 ; v. 170

chartreuse, ii. 489 ; v. 172

common, ii. 54. 487

common, wild, v. 172

Diard's Java, v. 171

domestic, ii. 489 ; v. 172

Javan, ii. 482

liver-coloured, v. 166

mountain, v. 175

nègre, ii. 486

New Spain, v. 173

Pageros, ii. 486 ; v. 170

Pampa, ii. 486 ; v. 170

pendant-eared, ii. 489

pennich, v. 299

Pensa, ii. 489

Persian, ii. 489

red, of Tobolsk, ii. 489

Spanish, v. 172

tiger, ii. 481

tiger, of Collinson, v. 175

tortoiseshell, ii. 487

waved, v. 171

wild, v. 172

Catin, v. 375

Catoblepas, iv. 366 ; v. 367

Brooksii, iv. 372 ; v. 369

gnu, iv. 367 ; v. 368

gorgon, iv. 371 ; v. 369

taurina, iv. 369 ; v. 368

Catodon, v. 388

macrocephalus, iv. 468

Cats, ii. 49

flying, ii. 14

Caudrieu, v. 382

Cavia, iii. 93

acuchi, iii. 94

acuschy, v. 272

aguti, iii. 94 ; v. 271

aperea, v. 271

capensis, v. 292

capybara, iii. 93 ; v. 270

cobai Brasiliensibus, v. 271

cobaia, v. 270

cristata, v. 272

Hudsonicus, v. 263

paca, iii. 95

Patachonica, v. 272

subniger, iii. 247

Cavicornian family, iv. 154
Cavy, bristly, v. 293

capybara, v. 270

long-nosed, v. 271

olive, v. 272

Patagonian, iii. 243 ; v. 272

restless, v. 271

rock, v. 271

variegated, v. 271

Cayopollin, ii. 65 ; iii. 32 ; v. 188

Cazoure, v. 280

Cebus, i. 221. 339.349 ; v. 28

albifrons, v. 31

albus, v. 29

apella, v. 28

apella, var. v. 30

barbatus, v. 29

capucinus, v. 32

cirrifer, v. 32

fatuellus, v. 31

flavus, v. 30

frontatus, v. 29

fulvus, v. 30

griseus, v. 29

hypoleucus, v. 32

lunatus, v. 31

moloch, v. 34

niger, v. 30

robustus, v. 28

satanus, v. 35

trepidus, v. 30

variegatus, v. 30

xanthosternos, v. 31

Celæno, v. 83

Brooksiana, v. 83

Centenes, ii. 21 ; v. 109

semispinosus, v. 109

setosus, v. 109

spinosus, v. 109

Cephalophus, v. 344

Cephalopine group, iv. 258

Cephalote, Pallas's, v. 59

Peron's, ii. 6 ; v. 59

Cephalotes, ii. 112 ; v. 59

Cercocebus, v. 14

æthiops, v. 16

atys, v. 16

cynocephalus, v. 18

cynomolgos, v. 18

cynosurus, v. 14

fuliginosus, v. 15

griseo viridis, v. 15

pygerythræus, v. 15

radiatus, v. 17

Sabæus, v. 14

Sinicus, v. 17

Cercoleptes, ii. 29 ; v. 115

Cercopithecus, i. 210.339 .348 ; v. 11. 20

王thiopicus, i. 261 
Cercopithecus, albocinereus, v. 14 auritus, i. 277 ; v. 11

barbatus, i. 212

cephus, v. 12

Diana, v. 13

entellus, v. 10

fuliginosus, i. 261

griseo viridis, v. 15

larvatus, v. 9

latibarbatus, i. 277 ; v. 11

maurus, v. 9

mona, v. 12

nasica, i. 350

nictitans, v. 12

niger, v. 13. 23

petaurista, v. 13

pileatus, v. 12

pygerythrœus, v. 15

ruber, v. 13

Sabæus, v. 14

talapoin, v. 11

Zeylonicus, v. 48

Cercou, iv. 446

Cerf, v. 308

blanc, v. 317

Bois de, trouvés à Etampes, v。 306

cochon, v. 313

couronné, v. 304

d'Abbeville, v. 307

d'Etampes, v. 306

de la Louissane, v. 315

de Virginie, v. $\mathbf{3 1 5}$

Cerigon, ii. $\mathbf{6 3}$

Cervula parvula Africana, v. 349

Cervidæ, v. $\mathbf{3 0 0}$

Cervo camelus, v. 299

Cervus, iv. 66 ; v. 303

alces, iv. $9.72 ;$ v. 303

Americanus, iv. 104 ; v. 309

Aristotle's, v. 310

aureus, iv. 148 ; v. 320

auritus, v. 308

axis, iv. 12.117 ; v. 312

bifurcatus, v. 323

campestris, iv. 136 ; v. 317

Canadensis, iv. 12.96 ; v. 308

capreolus, iv. 13.124 ; v. 314

clavatus, v. $\mathbf{3 1 5}$

coronatus, iv. 78 ; v. 304

dama, iv. 10. 84 ; v. 306

elaphus, iv. 11.90 ; v. 307

equinus, iv. 112 ; v. 311

giganteus, iv. 87 ; v. 306

Grænlandicus, v. 305

guetardi, v. 305

hamatus, iv. 172 ; v. 323

Hibernus, iv. 87 ; v. 306
Cervus hippelaphus, iv. 105 ; v. 309.311

leucogaster, v. $\mathbf{3 1 7}$

macrotis, iv. 133 ; v. 316

macrourus, iv. 134 ; v. 316

major, v. 308

Mariannus, iv. 115 ; v. 311

Mazath Chichiltic, v. 355

Mexicanus, v. 315

mirabilis, v. 305

moschatus, iv. 149 ; v. 320

muntjac, iv. 3.144 ; v. 319

nemoralis, iv. 137 ; v. 317

nemorivagus, iv. 142 ; v. 319

occidentalis, iv. 101 ; v. 308

palæodama, iv. 89 ; v. 307

palmatus, v. 305

paludosus, v. 316

Peronii, iv. 114 ; v. 311

Philippensis, iv. 147 ; v. 319

porcinus, iv. 119 ; v. 312

pumilio, iv. 120 ; v. 313

pygarga, iv. 13.122; v. 313

ramosicorris, v. 315

rangifer, v. 305

rufus, iv. 140 ; v. 318

simplicornis, iv. 141 ; v. 318

somonensis, iv. 89 ; v. 307

strongyloceros, iv. 12.96 ; v. 308

subcornutus, iv. 148; v. 320

tarandus, iv. 9. 79; v. 304

unicolor, iv. 108 ; v. 310

vaginalis, v. 319

Virginianus, iv. 12. 127; v. 314

Wallichii, iv. 103 ; v. 309

Cetacea, i. Ixxvii. ; iv. 429 ; v. 376

common, iv. 432 ; v. 380

lerbivorous, iv. 430 ; v. 377

large-headed, v. 387

Suppl. to the, iv. 445

Cete, i. Ix.; v. 380

Chacal, ii. 350 ; v. 145

of Senegal, ii. 354; v. 147

Chackal, Le, v. 146

Chacma, v. 20

Chacuro, v. 38

Chælopus, iii. 275 ; v. 275

Chameau, v. 298 du Perou, v. 299

Chameck, i. 222 ; v. 25

Chamois, iv. 22.281 ; v. 353

Cape, iv. 19

Persian, v. 353

Chat bizaam, v. 154

cervier, v. 174

marin, v. 182

pageros, v. 170

pampa, v. 170 
Chat pard, v. 167

sauvage, v. 172

sauvage à bandes noires des

Indes, v. 155

sauvage Iridien, v. 171

Chati, ii. 480 ; v. 169

Chauve souris, v。 88

souris, autre, v. 80

souris d'Egypte, v. 81

souris de la Vallée d'Ylo, v. 67

souris etrangère, v. 90

Cheirogaleus, i. 230 ; v. 50

large, v. 50

medius, v. 50

minor, v. 50

Cheiromales, v. 66

torquatus, v. 66

collared, v. 66

Cheiromys, iii. 86.196 ; v. 51

Daubentonii, v. 51

Madagascariensis, v. 51

Cheironectes, ii. 66 ; iii. 34 ; v. 191

Memina, v. 191

Yapock, iii. 35 ; v. 191

Cheiroptera, i. lxxvi. lxxvii.; ii. 2 ; v. 53 Suppl. to the, ii. 84

Cheloniscus, v. 276

Chetah, ii. 470 ; v. 166

Chèvre cossus, iv. 310

lmbrebe, iv. 310

Chevreuil, v. 314

de Tartarie, v. 313.

des Indes, v. 319

Chevrotain à peau marqué, de taches blanches, v. 302

de Guinea, v. 349

de Java, v. 302

Chibi-gouazou, v. 167

Chichiltic, iv. 291 ; v. 354

Chickara, iv. 254 ; v. 354

Chien, v. 133

Burgos, v. 139

canard, v. 138

courant, v. 138

crabier, v. 141

d'Alicante, v. 143

d'Artois, v. 143

de Berger, v. 140

de Cayenne, v. 143

d'Islande, v. 142

des Bois de Cayenne, v. 145

des Esquimaux, v. 140

dogue de forte race, v. 141

griffon, v. 138

Islois, v. 143

lillois, v. 143

lion, v. 137

loup, v. 140
Chien quatre vingts, v. 143

roquet, v. 142

turc, v. 143

turc à crinière, v. 144

volant, v. 55.57

Chlamyphorus, v. 283

truncatus, v. 283

Chloromys, iii. 94. 241 ; v. 271

Choak kama, v. 20

Chondropterygii, i. lxvi.

Choras, i. 219 ; v. 21

Choro, v. 27

Chorock, ii. 291 ; v. 122

Chrysochlore, ii. 192 Cape, ii. 192; v. 107 of the Cape, ii. 30

Chrysochloris, ii. 20 ; v. 107

Capensis, v. 107

Chuva, v. 25

Cirquineou, v. 275

Cirquirisa, iii. 257

Citillus, v. 246

Civet, ii. 46.379 ; v. 152

de Malacca, ii. 46

Civets, ii. 45

Civetta, v. 153

Civette, v. 153 de Java, petite, v. 155 de Malacca, v. 154

Chadobates ferruginea, v. 52. 160

Coagga, iii. 464

Coaita, i. 223 ; v. 24

à ventre blanc, v. 25

black, v. 24

black handed, v. 25

fawn-coloured, i. 223

white-bellied, i. 223

with bordered face, i. 223

Coala, v. 205

Coasse, v. 127

Coati, v. 115

brown, ii. 29 ; v. 115

Mondi, v. 115

red, ii. 29.253 ; v. 114

roux, v. 115

Coatis, ii. 29. 253

Cobaya, iii. 234 ; v. 270

Cobayas, iii. 93

Cochon d'Eau, v. 270

d'Inde, v. 271

de Terre, v. 279

Coëndou, v. 263

à longue queue, v. 264

Coesdoes, v. 366

Cogong, v. 369

Coleoptera, i. Ixvii.

Collier, Le, v. 217

Colobus, v. 7. 
Colobus ferruginosus, v. 8 polycomus, v. 7

Temminck's, v. 8

Colocolo, ii. 479 ; v. 169

Colour, varieties in, i. 155

Colugo, v. 99

flying, v. 99

Ternate, v. 99

varied, v. 99

Colus, v. 356 of Strabo, iv. 17

Condoma, v. 366

Condylura, ii. 23. 208 ; v. 110 cristata, v. 110 longicaudata, v. 110

Condylure, long-tailed, v. 110 radiated, v. 110

Conepate, v. 127, 128

Conepatl, v. 127, 128

Coney, v. 266

hedge, v. $266^{\circ}$ small Indian, v. 271

Coquallin, v. 253

Coquo, iv. 327

Cora, iv. 216 ; v. 333

Corine, v. 333

Corinne, iv. 16. 214 ; v. 333

Corsac, ii. 44 ; v. 149

Coscoes, v. 196

Cottie, v. 273

Cotting, iv. 340

Couagga, iii. 344 ; v. 295

Couassou, v. 318

Coudin, v. 382

Coudoma, iv. 21

Coudou, v. 365

Coudous, iv. 21

Couendou, iii. 208 ; v. 263

Cougoua, v. 163

Cougouar, v. 163

Couguar, ii. 52

Coui, v. 264

Couiy of Azara, v. 263

Couxio, i. 318 ; v. 35

Cow, Barbary, iv. 18

grunting, iv. 30

mountain, v. 294

sea, ii. 60 ; iv. 431 ; v. 378

Coypus, iii. 124; v. 222

Crab-eater, ii. 65

Crabier, v. 187

Cricetus, iii. 75. 134 ; v. 233

anomalus, v. 236

arenarius, v. 234

bursarius, v. 235

furunculus, v. 235

laniger, v. 235

migratorius, v. 233
Cricetus phæus, v. 234 songarus, v. 234 vulgaris, v. 233

Croque noix, v. 221

Cuaikare, v. 274

Cuchumbi, ii. 265

Cuendu, iii. 88

Cuetlachtle, v. 145

Cuguaçuarana, ii. 436

Culblanc, v. 359

Culpeu, v. 145

Cuniculus, v. 266

Americanus, v. 271

Bahamensis, v. 244

Brasiliensibus, v. 271

Indicus, v. 271

Norwegicus, v. 215

paca, v. 273

Cuscus Amboinensis, v. 196

Cynocephala, i. 217

Cynocephalus, i. 217. 348. 351 ; v. 19 , 20,21

Babouin, v. 20

Hamadryas, v. 21

leucophæus, v. 22

mormon, v. 21

papio, v. 20

porcarius, v. 20

Wurmbii, v. 5

Cynopterus marginatus, v. 58

\section{DAIM, v. 306}

d'une grande taille, v. 307

Dama, iv. 20 ; v. 306 fossil of Abbeville, v. 307

fossil of Ireland, v. 306

fossil of Scania, v. 307

vulgaris, v, 306

Damalis, iv. 343 ; v. 361

bubalis, iv. 347 ; v. 362

caama, iv. 348 ; v. 362

canna, iv. 357 ; v. 365

collared, iv. 350 ; v. 362

lunata, iv. 352 ; v. 363

oreas, iv. 355 ; v. 364

risia, iv. 363 ; v. 366

Senegalensis, iv. 351 ; v. 363

strepsiceros, iv. 359 ; v. 365

suturosa, iv. 351 ; v. 362

Daman, iii. 337 ; v. 236. 292

Israel, v, 293

Danois, v. 135

$$
\text { le petit, v. } 142
$$

Danta, v. 294. 327

Dasyprocta, iii. 94. 241 ; v. 271

acuschy, v. 272

acuti, v. 271 
Dasyprocta cristata, v. 272

Patagonica, v. 272

viscacha, v. 272

Dasypus, iii. 255. 283 ; v. 266. 275

apar, v. 275

dasycerus, v. 277

duodecimcinctus, v. 277

flavipes, v. 278

giganteus, v. 277

gigas, iii. 258 ; v. 277

hybridus, v. 276

maximus, v. 277

minutus, v. 279

novemcinctus, iii. 257 ; v. 276

octocinctus, iii. $259 ;$ v. 276

octodecimcinctus, iii. 257; v. 278

peba, v. 276

quadricinctus, v. 276

septemcinctus, iii. 257 ; v. 276

serratus, v. $276^{\circ}$

sexcinctus, iii. 257 ; v. 278

tatouay, v. 277

trachyurus, v. 276

tricinctus, iii. 256 ; v. 276

unicinctus, iii. 257 ; v. 277

villosus, v. 278

Dasyuri, ii. 66

Dasyure de White, v. 193

dwarf, v. 194

gutte, v. 193

nain, v. 194

of Maugé, v. 193

tacheté, v. 193

Dasyurus, ii. 66 ; iii. 36 ; v. 192

brush-tailed, ii. 68

cynocephalus, v. 195

dog-faced, iii. 36 ; v. 192

dog-headed, ii. 67

dwarf, ii. 68

long-tailed, ii. 67

macrourus, ii. 67; v. 193

Maugei, v. 193

minimus, v. 194

of Maugé, ii. 68

of White, ii. 68

pencilled, ii. 68

penicillatus, v. 194

rough, ii. 67

spotted, v. 193

tafa, v. 194

ursine, iii. 37 ; v. 192

ursinus, v. 192

viverrine, v. 193

zebrine, iii. 36

Dauphin à bec mince, v. 383

beluga, v. 386

butskopf, v. 386
Dauphin de Commerson, v. 385

de Geoffroy, v. 383

de Peron, v. 384

epaulard, v. 384

épée, de Mer, v, 384

gris, v. 385

leucoramphe, v. 384

marsouin, v. 383

Dauw, iii. 468 ; v. 296

Deab, v. 146

Deba, v. 321

Dedes, v. 153

Deer, iv. 66

black-tailed, v. 316

Cariacou, iv. 137 ; v. 317

common hog, v. 312

fallow, iv. 10.84 ; v. 306.315

fossil fallow, of Abbeville, iv. 89

fossil rein, iv. 84 ; v. 305

fossil Scanian fallow, iv. 89

great-eared, iv. 133 ; v. 316

Guazupuco, iv. 134 ; v. 316

Guazuti, iv. 136 ; v. 317

hog, v. 288

jumping, v. 317

long-tailed, iv. 134 ; v. 316

Mexican, iv. 130 ; v. 315

moose, iv. 9 ; v. 304

mule, v. 308.316

musk, of Nepaul, v. 321

North American rein, iv. 83

porcine, v. 313

red, v. 308

rein, iv. 9. 79 ; v. 304

rib-faced, v. 319

Virginian, iv. 127 ; v. 315

with a large tail, v. 316

Degu of Molina, v. 221

Delphinaptera, iv. 436

Delphinapteri, iv. 457

Delphinapterus, v. 385 beluga, v. 386

Delphinus, iv. 434 ; v. 380

albicans, v. 386

anarsak, v. 386

Bertini, v. 383

Boryi, v. 383

Butskopf, v. 386

Canadensis, v. 383

Chemnitzianus, v. 386

Commersonii, v. 384

Dalius, v. 383

Delphis, iv. 434.450 ; v. 381

edentulus, v. 386

epiodon, v. 386

feres, iv. 457 ; v. 383

frontatus, iv. 457 ; v. 383

gladiator, iv. 436.455 ; v. 384 
Delphinus globiceps, v. 385

grampus, v. 384

griseus, v. 385

Hunteri, v. 386

hyperoodon, v. 386

Leucas, iv. 437.457 ; v. 385

maximus, v. 384

Mongitori, v. 383

nesarnak, iv. 456 ; v. 382

niger, v. 382

orca, iv. 436.455 ; v. 383,384

Peronii, v. 384

phocæna, iv. 436 ; v. 383

pinna in dorso nulla, v. 386

Rissoanus, v. 385

rostratus, iv. 435 ; v. 382

Sinensis, v. 383

tursio, iv. 435 ; v. 382

ventricosus, v. 385

Delundung, ii. 419

Desman, ii. 185 ; v. 105

of Russia, ii. 18. 189

of the Pyrenees, ii. 190 ; v. 105

Desmans, ii. 18

Deva deve, v. 298

Devil of the Colonist, v. 193

Dhole, ii. 326

Diane, la, v. 13

Dib, v. 146

Dicotyles, iii. 333 ; v. 289 labiatus, iii. 334.413 ; v. 290 torquatus, iii. 334.412 ; v. 289

Dicranocerine group, iv. 167

Dicranocerus, v. 322

Didelphis, ii. 63 ; v. 185

Asiatica, v. 204

Azara, v. 186

brachyura, ii. 66 ; iii. 30 ; v. 190

Brunii, ii. 76 ; v. 204

cancrivora, ii. 65 ; iii. 30 ; v. 187

carcinophaga, v. 187

cayopollin, ii. 65 ; v. 190

cinerea, v. 188

crassicaudata, v. 189

cynocephala, ii. 67 ; v. 192

dorsigera, ii. 65 ; v. 189

gigantea, ii. 75 ; v. 202

lanigera, v. 190

lemurina, ii. 71 ; v. 197

macrotarsis, v. 50

macroura, ii. 72 ; iii. 45 ; v. 199

marina, ii. 66

marsupialis, ii. 65 ; iii. 30 ; v. 187

muneira, iii. 32

murina, v. 188

myosurus, v. 189

nudicaudata, v. 189

obesula, v. 195
Didelphis opossum, ii. 65; iii. 31; v. 187

orientalis, ii. 71 ; v. 198

palmata, ii. 66 ; iii. 35 ; v. 191

penicillata, ii. 68 ; v. 194

peregrinus, v. 197

petaurus, iii. 44 ; v. 199

philander, ii. 65 ; v. 188

pusilla, v. 191

pygmea, ii. 72 ; iii. 44 ; v. 200

Quica, v. 187

sciurea, v. 200

Sebæ, v. 190

tricolor, iii. 33 ; v. 190

ursina, ii. 67.78 ; v. 193

Virginiana, ii. 64 ; iii. 24 ; v. 186

viverrina, ii. 68 ; v. 193

vulpina, ii. 71 ; iii. 41 ; v. 197

Yapock, v. 191

Digitigrades, ii. 33.282 ; v. 119

Dikaja kosa, v. 313

Dilundung, v. 160

Dingo, ii. 326; v. 134

Diphylla, v. 84

ecaudatus, v. 85

Dipus, iii. 76 ; v. 236

Alagtaga, v. 237

brachyurus, iii. 149 ; v. 237

Cafer, iii. 80. 164 ; v. 243

Canadensis, v. 240

gerbillus, v. 240

gerboa, v. 236

jaculus, v. 237

jaculus var minor, v. 237

maximus, iii. 151 ; v. 238

minimus, v. 237

minutus, v. 237

pyramidum, iii. 158 ; v. 240

sagitta, v. 236

tamaricinus, v. 239

Dishon, v. 359

Ditylus, v. 298

Dog, v. 133

Albanian, ii. 327

Alicant, v. 143

Artois, ii. 338 ; v. 143

Australasian, v. 134

Barbary, v. 143

bull, ii. 41.336 ; v. 141

bull, black var. of Thibet, v. 141

Calabrian, v. 137

Ceylonese, of Pennant, v. 151

Dalmatian, v. 135

Danish, ii. 40 ; v. 135

domestic, ii. 40

drover's, v. 140

Egyptian, v. 143

Esquimaux, ii. 332; v. 140 
Dog, French shepherd's, v. 140

German, ii. 338

great Danish, ii. 328 ; v. 135

hyæna, ii. 376

Iceland, ii. 338 ; v. 142

king Charles', ii. 333

lion, ii. 334 ; v. 137

little Danish, ii. 338 ; v. 142

Maltese, ii. 334 ; v. 137

naked, ii. 338

Newfoundland, ii. 333

New Holland, ii. 326

North American, ii. 326

Peruvian, v. 141

Pomeranian, ii. 331

Prairie, iii. 198 ; v. 248

pug, ii. 337 ; v. 142

sea, v. 176

shepherd's, ii. 41.330 ; v. 139

shock, ii. 4 ] ; v. 142

Siberian, ji. 331 ; v. 140

spotted, v. 135

Surinam, v. 145

Turkish, ii. 338

turnspit, ii. 336

water, v. 138

wild, ii. 376

wild, of the East Indies, ii. 326

wolf, ii. 41.331 ; v. 140

Dogs, ii. 39.317

Dogue, le, v. 141

Doguin, le, v. 142

Dolichotes vischacha, v. 272

Dolichura, iv. 330

Dolphin, black, v. 382

Commerson's, v. 384

common, iv. 434.450 ; v. 381

fronted, v. 383

great, iv. 435 ; v. 382

of Commerson, iv. 457

of Honfleur, v. 386

of Peron, iv. 457 ; v. 384

round-headed, v. 385

slender-beaked, iv. 435 ; v. 382

Dolphins, iv. 434. 450

Dorcas, v. 332

Dormice, iii. 71 ; v. 21.9. 221

African, v. 221

common, iii. 122 ; v. 220

fat, iii. 118 ; v. 220

garden, iii. 122 ; v. 220

gilt-tailed, v. 218

greater, v. 220

murine, v. 221

Douc, i. 214. 276; v. 8,9

Douroucouli, i. 313 ; v. 35

Dril, v. 22

Dromédaire, v. 298

Dreron, iv. 17; v. 336
Dugong, iv. 432.446 ; v. 379

of the Indian Sea, v. 379

Duns, Suffolk, iv. 423

Dysopes, ii. 7 ; v. 86

mops, v. 86

Dzeren, iv. 17. 229 ; v. 336

Dziggetai, iji. 342. 459

Dziggtai, v. 295

ECHIDNA, iii. 263. 314; v. 283 bristly, v. 284

hystrix, iii. 264 ; v. 284

setosa, iii. 264 ; v. 284

spiny, v. 284

bristly, iii. 264

spiny, iii. 264

Echidnes, iii. 263

Echimys, iii. 70. 117 ; v. 217

bristly, v. 219

Cayenne, v. 219

chrysurus, v. 218

cristatus, v. 218

dactylinus, v. 218

d'Egypte, v. 233

didelphoides, v. 219

gilt-tailed, v. 218

hispidus, v. 219

long-toed, v. 218

perchal, v. 232

red, iii. 71.117 ; v. 218

rough-haired, v. 219

setosus, v. 219

spinosus, v. 218

with a golden tail, jii. 70

Ecureil à masque, v. 253

blanc de Siam, v. 252

de la côte de Malabar, grand, v. 250

de Madagascar, v. 250

éclatant, v. 260

rouge, v. 254

toupays, v. 257

volant de Sibérie, v. 259

Edentata, i. lxxvii ; iii. 251 ; v. 273

common, iii. 255

suppl. to the, iii. 266

Effodientia, i. lxxix.

Egin, v. 298

Egret, i. 216

Einhorn, v. 387

Eira, ii. 486

El Bucher el Achmer, v. 325 walrush, v. 327

Elan, iv. 9. 12 ; v. 303

Eland, bastard, v. 365

Gazelle, v. 365

Elant, v. 311

Elaphine group, iv. 90 
Elapliocamelas, v. 299

Elaphus, v. 307

Elch, v. 304

Elements, combination of chemical, i. 28

Elend, iv. 9 ; v. 304

Elephant, iii. 325

African, iii. 327.349 ; v. 286

Anson's sea, v. 180

Asiatic, iii. 346

Byron's sea, v. 181

Indian, iii. 326 ; v. 286

Patagonian sea, v. 180

sea, ii. 58 ; v. 180.184

Elephas, iii. 325 ; v. 285

Africanus, iii. 327 ; v. 286

Capensis, v. 286

Indicus, iii. $326 ; 286$

maximus, v. 286

Elk, iv. 9. 12. 72 ; v. 303. 308. 310

American, v. 308

Cape, iv. 12

crowned, iv. 78 ; v. 304

fossil, iv. 87 ; v. 309

fossil, of Ireland, v. 306

Irish, v. 306

round-horned, v. 308

Emmema, iv. 326

Emgalo, v. 289

Empabunga, v. 325

Empacasse, v. 372

Empaguessa, v. 372

Empalanga, v. 325

Empalunga, v. 325

Ena, v. 337

Encoubert, iii. 257 ; v. 278

Engalo, v. 289

Engry, v. 331

Enhydra, v. 132 marina, v. 132

Entelle, v. 10

Entellus, i. 210. 259 . v. 10

Epaulard, v. 384

Equus, iii. 340 ; v. 294 asinus, iii. 343.461 ; v 295

bisulus, iv. 53 ; v. 303

Brasiliensis, v. 296

caballus, iii. 341.439 ; v. 294

Hemionus, iii. 342.459 ; v. 295

Hemionus, Dziggtai dictus, v. 295

manius, v. 185

montanus, iii. 467 ; v. 296

quaccha, iii. 344. 464

quagga, iii. 464 ; v. 295

zebra, iii. 343.465 ; v. 295

Erethizon, v. 263

Buffonii, v. 263

dorsatum, v. 263

Cuv. Index.
Erinaceus, ii. 61.162; v. 100 auritus, ii. 16. 162; v. 101 ecanthurus, v. 109 ecaudatus, ii. 21 ; v. 109 Europæus, ii. 16. 168; v. 100 inauris, ii. 171 ; v. 101 Malaccensis, ii. 170 semispinosus, ii. 21 ; v. 109 setosus, ii. 21. 207 ; v. 109 Sibericus, ii. 171 ; v. 101 tanrec, v. 109

Ermine, ii. 35 ; v. 122

Eteocles semispinosus, v. 110

Eucritus, v. 263

Euryceros, v. 306

Exquima, i. 212; v. 13

Eyra, v. 171

Fegoule, le, v. 210

Felander, v. 204

Felis, ii. 49.421 ; v. 162

aurata, v. 206

borealis, v. 206

caligata, v. 206

Canadensis, ii. 53 ; v. 174

Capensis, v. 167. 170. 206 caracal, ii. 54.490 ; v. 173. 206

catenata, ii. 478 ; v. 168

catus, ii. 54 ; v. 172

catus Angorensis, v. 172

catus cæruleus, v. 172

catus ferus, v. 172

catus Hispanicus, v. 172

maculatus, v. 172

celidogaster, v. 206

cervaria, v. 206

chalybeata, ii. 473 ; v. 166 . 175

chati, v. 169

chaus, ii. 54.491 ; v. 173.206

chibi gouazou, v. 167

colocolo, v. 169

concolor, ii. 436 ; v. 163

Diardi, ii. 484 ; v. 171

discolor, ii. 52

eyra, v. 171

guttata, v. 166.175

Lybicus, v. 173

jaguar, v. 164

jaguarondi, ii. 54

jaquaramdi, v. 206

Javanensis, v. 170

jubata, ii. 52. 470 ; v. 166

leo, ii. 50 ; v. 162.206

leopardus, ii. 52 ; v. 165.206

lynx, ii. 53 ; v. 174

macrocelis, v. 206
D 
Felis macroura, ii. 478 ; v. 168.206 maniculata, v. 206

manul, v. 175

margay, v. 169

melas, ii. 53

microcelis, v. 164

mitis, v. 168.206

nebulosa, ii. 453 ; v. 164

nigra, v. 171

nigripes, ii. 489 ; v. 172

obscura, v. 171

ocelot, ii. 475 ; v. 168

onça, ii. 51 ; v. 164.206

pageros, v. 170

pardalis, ii. 53 ; v. 167.206

pardus, ii. 51 ; v. 165.206

pardus antiquorum, v. 165

(Priodonta) gracilis, v. 160

puma, v. 206

rufa, ii. 53 ; v. 174. 206

serval, ii. 54. 481 ; v. 167.206

Sumatrana, ii. 484 ; v. 171

tigrina, ii. 482 ; v. 206

tigris, ii. 50 ; v. 163.206

varia, v. 175

uncia, ii. 469 ; v. 166

undata, v. 171

venatica, v. 166

volans ternatea, v. 100

Wiedii, v. 169

Yagouaroundi, v. 170

Fennec, i. 332 ; ii. 45.372 ; v. 152

Bruce's, v. 152

Laland's, v. 15]

Fennecus Brucii, v. 152

Fer à cheval, grand, v. 76

Fer de lance, ii. 9 ; v. 69 grand, ii. 127 ; v. 70

Ferret, ii. 34. 287; v. 120

Java, v. 121

Javanese, ii. 288

Feuille, La, v. 73. 75

Fiber, iii. 66 ; v. 208 zibethicus, v. 208

Finnfisch, iv. 443

Fisch, fin, v. 391

Jupiter, v. 391

Knoten, v. 390

Swerd, v. 384

Fishtall, v. 360

Flacuatzin, v. 263

Fledermaus, blasse, v. 88 speck, v. 88

Foadh, v. 161

Foca a ventre bianco, v. 179

Fossane, ii. 390 ; v. 154 of Madagascar, ii. 47

Fouin, v. 123

Fouine, ii. 36 ; v. 124
Fouine de la Guyane, v. 117

Foutereau, ii. 37

Fox, v. 147

American cross, v. 149

Antarctic, v. 145

Arctic, ii. 370 ; v. 148

Bengal, v. 151

black, ii. 44

blue, ii. 44

Brant, v. 147

Cape, ii. 45. 371

Chili, v. 145

coal, ii. 43

common, ii. 43.361

Corsac, v. 149

cross, ii. 43.370 ; v. 149

Egyptian, v. 150

fulvous-necked, v. 148

grey, v. 148.150

Karagan, v. 149

red, v. 150

red, of Bartram, v. 151

silver, v. 148

silvery, ii. 44. 368

small yellow, ii. 44

sooty, v. 151

tri-coloured, ii. 44.365 ; v. 148

variable, ii. 371

Virginian, v. 150

Foxes, ii. 43. 361

Furet, v. 117. 120

Furunculus myoides, v. 235

GALAGo, v. 48.50

crassicaudatus, i. 230 ; v. 49

Demidoffii, v. 49

Demidoff's, i. 230 ; v. 49

Geoffroyii, v. 49

grand, v. 49

great, i. 230 ; v. 49

Guiniensis, v. 49

little, v. 48

Madagascariensis, v. 48

murinus, i. 332

of Madagascar, i. 332

of Senegal, i. $332 ;$ v. 49

Senegalensis, i. 230 ; v. 49.

Ternatensis, v. 99

variegatus, v. 99

with a tufted tail, i. 332

Galagos, i. 229. 330

Galeopitheci, ii. 14

Galeopithecus, ii. 14. 156 ; v. 99

red, ii. 158

rufus, v. 99

varied, ii. 158

variegatus, v. 99

Galeopithèque roux, v. 99 
Galera, ii. 278 ; v. 117. 126. 157

Gaour, v. 374

Garangan, ii. 398

Gasella, v. 329

Gaucohi, v. 310

Gaur, iv. 399 ; v. 373

Gauvera, v. 375

Gauzen, v. 310

Gaw dashti, v. 375

Gawzan, v. 367

Gayal, iv. 406 ; v. 375

Gazel, v. 332

Gazelle, iv. 16 ; v. 332 pouched, iv. 17

Gazelline group, iv. 200

Geldowesi, v. 298

Gemel gemel, v. 298

Genet, v. 154

var., v. 154

banded, v. 155

common, ii. 46

hyæna, ii. 390

Jndian, v. 155

of Barbary, ii. 387

of the Cape, ii. 46

Genets, ii. 46. 387

Genetta, ii. 46 ; v. 153

bondar, v. 155

fasciata, v. 155

fossa, v. 154

Geoffroyii, v. 154

Indica, v. 155

vulgaris, v. 154

Genette, v. 154

de France, v. 154. 159

du Cap, v. 154

Geoffroy's, v. 154

Geomys, v. 235 cinereus, v. 235

Georychus, iii. 68; v. 242

Gerbil, Canadian, v. 240

Egyptian, v. 240

Indian, v. 239

Labrador, v. 240

Tamarisk, v. 289

Torrid, v. 239

Gerbillus, iii. 153 ; v. 238

Egyptius, iii. 158 ; v. 240

Canadensis, v. 240

conurus, v. 241

Daviesii, v. 240

Hudsonius, v. 241

Indicus, iii. 156 ; v. 239

Labradorius, v. 240

megalops, v. 241

Meridianus, iii. 157 ; v. 239

of Canada, iii. 159

soricinus, iii. 159 ; v. 241

tamaricinus, v. 239
Gerbo, iii. 143 ; v. 236

Gerboa, iii. 77. 140 ; v. 236

African, iii. 157

brachyura, iii. 149

Canadian, iii. 159

great, iii. 151

little, iii. 149

major, v. 243

tamarisk, iii. 155

woolly, v. 50

Gerboise, v. 236

Gibbar, iv. 443 ; v. 390

Gibbon, i. 258 ; v. 5 active, i. 258 ; v. 7

agilis, i. 258

ash-coloured, i. 209. 254

black, i. 208

cendré, v. 6

little, v. 6.

ounko, v. 7

petit, v. 6

silvery, i. 209

Giraffa, iv. 14. 150; v. 32I camelopardalis, v. 321

Giraffe, v. 321

Giraffidæ, v. 321

Girafra, v. 321

Giudol, iii. 434

Glires, i. 1x. Ixxvi.

Glis, v. 220

œconomicus, v. 234

citillus, v. 246

cricetus, v. 233

lagurus, v. 216

marmotta, v. 244

marmotta argentoratensis, v. 233

monax, v. 244

moschiferus, ii. 186 ; v. 244

Polonica, v. 244

Tscherkensicus, v. 248

Zumui, v. 241

Glisorex, v. 106

Glossophaga, v. 72

amplexicaudata, v. 73

caudifer, v. 73

ecaudata, v. 73

soricina, v. 72

Gluton, v. 117 marpurito, v. 128

Gluttons, ii. 31

Glutton, v. 117

common, ii. 276

ferruginous, ii. 282 ; v. 119

Labrador, v. 118

masked, ii. 281 ; v. 119

Gnoo, iv. 367 ; v. 368 brindled, iv. 371 ; v. 369

Gnou, iv. 22

D 2 
Gnouroumy, v. 280

Goat, Angora, iv. 307 beardless, iv. 310

blue, iv. 20

bush, v. 344

Cachemire, iv. 307

cossus, iv. 310

domestic, iv. 315

dwarf, iv. 306

Egyptian, iv. 308

Jemlah, iv. 308 ; v. 358

Jueda long-haired, jv. 306

Nepaul, iv. 307

Persian, iv. 306

short-horned, iv. 306

Syrian long-haired, iv. 307

Tartar, iv. 307

Thibet, iv. 307

Welsh, iv. 306

Widah, iv. 306

wild, iv. 23

yellow, iv. 17 ; v. 336

Goats, iv. 23

Goazoupouco, v. 317

Goazouti, v. 317

Gona, v. 310

Gor, v. 374

Goral, iv. 279 ; v. 352

Gouazouara, v. 163

Gouazoubira, v. 319

Gouazu Pita, vo 318

Gozen, v. 310

Grampus, iv. 436.455 ; v. 384

Greber, le, v. 102

Gregari, le, v. 211

Greyhound, ii. 41 ; v. 135

common, ii. 328

Irish, ii. 328 ; v. 136

Italian, ii. 330 ; v. 136

Russian, ii. 330 ; v. 136

Scotch, ii. 330 ; v. 136

Turkish, ii. 330 ; v. 136

Griffon, v. 138

Grimee, v. 347

Grimm, iv. 266 ; v. 347

Gris, Petit, iii. 83 ; v. 254

Grisfuch, der, v. 148

Grison, ii. 32. 277; v. 117

Grisset, v. 47

Grivet, i. 265 ; v. 15

Grumm seler, v. 181

Grysbock, iv. 250

Guanaco, iv. 6. 55 ; v. 299

Guanque, v. 214

Guariba, i. 222.301 ; v. 27

Guazu Apara, v 318

Guazuara, ii. 436

Guenon, i. 348. 350 à camail, v, 8
Guenon à face alongée, v. 20

à face pourpré, v. 11

à long nez, v. 9

à long nez proéminent, v. 13

autre, v. 8

Chinese bonnet, i. 216

couronné, v. 12

crowned, i. 216

golden, i. 277 ; v. 11

long-nosed, i. 350

negre, v. 10

Guenons, i. 210. 259

Guerlinguet, le grand, v. 256

le petit, v. 256

little, v. 256

Guerlinguets, iii. 84

Guepard, ii. 52. 470

Guevei, iv. 270 ; v. 349

kaior, v. 349

Guib, le, v. 351

Gulo, ii. 31. 275 ; v. 116

barbatus, v. 117

castaneus, ii. 282

ferrugineus, v. 119

larvatus, ii. 281 ; v. 119

marpurito, v. 127

orientalis, ii. 280 ; v. 118

ratel, v. 118

Quitensis, v. 118. 127, 128

vittatus, v. 117

vulgaris, v. 117

wolverene, v. 117

Gyraffa, v. 321

HAGRI, Le, v. 233

Halicore, iv. 432 ; v. 379

Indicus, v. 379

Halmaturus, ii. 74

Brunii, v. 204

fasciatus, v. 203

gigantea, v. 202

Halychærus griseus, v. 178

Hamster, anomalous, iii. 139 ; v. 236

Astracan, v. 234

Baraba, v. 235

Canada, v. 235

Chinchilla, v. 235

common, iii. 75 ; v. 233

sand, v. 234

Songar, v. 234

Yaik, v. 233

Hamsters, iii. 75. 134

Hapales jaccus, v. 38 rosalia, v. 42

Hapales, i. 225 ; v. 38

Hardvark, v. 279

Hare, v. 265

African, iii. 91 
Hare, Alpine, iii. 222 ; v. 265. 269

American, v. 266

Baikal, v. 266

Brazilian, iii. 221 ; v. 267

calling, v. 269

Cape, v. 267

common, iii. 89.213 ; v. 265

leaping, iii. 165 ; v. 243

mountain, iii. 165

Ogotona, v. 269

Patagonian, v. 272

snowy, v. 265

spurious, v. 265

variable, iii. 90.219 ; v. 265

varying, v. 265

Virginian, v. 265

Hares, iii. 88

Harpygia, v. 59

Harrier, v. 138

Hartebeest, v. 362

Harts, king of the, v. 349

Head and brain, character of, i. 105

Hedgehog, ii. 162

American, v. 101

common, ii. 16 ; v. 100

dog, ii. 169

earless, ii. 171 ; v. 101

long-eared, ii. 16. 168; v. 101

Malacca, v. 101

of America, ii. 171

of Siberia, ii. 171

pendant-eared, v. 101

swine, ii. 169

with pendant ear, ii. 170

Hedgehogs, ii. 16

Madagascar, ii. 206

Helamys, iii. 80

Cafer, iii. 164 ; v. 243

marmot, v. 243

Heliodorus, v. 321

Hepoona koo, v. 199

Hermine, l', v. 122

Herisson, ii. 162 ; v. 101

d'Egypte, v. 10] pourceau, ii. 169

Herpestes, ii. 47 Edwardsii, v. 157

galera, v. 157

griseus, v. 157

Mungo, v. 156

Pharonis, v. 156

Heteromys, v. 236

Hippelaphus, v. 309. 310

Hippopotamus, iii. 329.397 ; v. 286

amphibious, v. 287

Capensis, v. 287

ecaudatus, v. 270

Senegalensis, v. 287

terrestris, v. 293
Hippotigre, v. 296

Hirsch, v. 308

Histrix chrysurus, v. 218 pilosus, v. 263

Hoang yang, iv. 17 ; v. 336

Hocheur, i. 213 ; v. 13

Hog, v. 287

black race with short limbs, iii. 405

English race, iii. 404

ground, v. 245. 279

race of France, iii. 405

race of Jutland, iii. 405

race of Poland, iii. 405

race of Russia, iii. $\mathbf{4 0 5}$

race of Zealand, iii. 405

race with a single toe, iii. 405

river, v. 270

stag, iii. 332

wild, iii. 331

Hoitzslaquatzin, iii. 88 ; v. 263

Homo, v. 3

lar, v. 6

nocturnus, v. 250

sapiens, v. 3

sylvestris, i. 238 ; v. 4

troglodytes, i. 250 ; v. 4

Horse, iii. 340 ; iv. 434 ; v. 294

Arabian, iii. 447

sea, ii. 60 ; iv. 431

Horses, Barbary, iii. 452

Dutch, iii. 455

English, iii. 456

of France, iii. 455

of Germany, iii. 454

of Italy, iii. 453

of Switzerland, iii. 455

Persian, iii. 451

Spanish, iii. 453

Tartar, iii. 450

Turkish, iii. 452

Hound, ii. 41. 334

Howler, brown, i. 222

red-handed, v. 28

Huemel, iv. 53 ; v. 300

Huitfisch, v. 386

Human face and form, var. of the, $i$. 163

species, causes of varieties of the, v. 174

species, general observations on the varieties of the, i. 183

Human species, var. of the, i. 96

Hyæna, ii. 48. 404 ; v. 161. 162

Abyssinian, v. 161

Capensis, v. 162

crocuta, v. 161

painted, ii. 376 ; v. 151

picta, v. 151 
Hyæna, spotted, ii. 49. 406; v. 161 striped, ii. 49.408 ; v. 161 vulgaris, v. 161

Hyænas, ii. 48

Hydrochærus, iii. 93. 232 ; v. 270 capybara, v. 270 tapir, v. 270

Hydromys, iii. 72.124 ; v. 222 chrysogaster, iii. 72. 124; v. 222

coypus, v. 222

leucogaster, iii. 72 ; v. 222

white-bellied, v. 222

yellow-bellied, v. 222

Hylobates, v. 5

agilis, v. 7

lar, v. 5

leuciscus, v. 6

syndactylus, v. 6

variegatus, v. 6

Hyperoodon, v. 386

Hyperoodontes, iv. 437. 459

Hypexanthus, mustache, v. 98

Hypexodon, v. 98

Hypsiprymnus, ii. 73 murinus, v. 201

Hypudæus, iii. 67

Hyrax, iii. 337; v. 292

Cape, iii. 430 ; v. 292

Capensis, iii. 338 ; v. 292

South African, iii. 430

Syriacus, iii. 338 ; v. 293

Syrian, iii. 431 ; v. 293

Hystrix, iii. 87.200 ; v. 261

aculeis apparentibus caudâ brevi, v. 263

Americanus major, v. 264

chrysuros, v. 70

couiy, v. 263

cristata, iii. 87 ; v. 261

cuandu, v. 263

dorsata, iii. 88.206 ; v. 262

fasciculata, iii. 88.207 ; v. 262

Hudsonicus, v. 263

longicauda, v. 262

macroura, v. 262

orientalis, v. 262

prehensilis, iii. 88. 208 ; v. 263

spinusa, v. 264

villosa, v. 264

IBEC, v. 314

Ibex, iv. 24. 300 ; v. 356

Abyssinian, iv. 301 ; v. 356

Alpium Sibiricarum, v. 356

Caucasian, iv. 25. 302 ; v. 357

Siberian, v. 356
Ichneumon, ii. 47.392 ; v. 156

Caffrarian, v. 157

Edward's, v. 157

Indian, v. 156

Javanese, v. 157

large, v. 158

major, v. 158

red, v. 158

Ictides, v. 159

albifrons, v. 159

white-fronted, v. 159

Ignavus arcthopithecus, v. 274

Impatoo, v. 321

Impoofo, iv. $\mathbf{3 5 5}$

Indri, v. 43

Macauco, v. 43

Indris, i. 228 ; v. 43

brevicaudatus, v. 43

laniger, v. 44

longicaudatus, v. 44

Insectivora, ii. 15; .v 100 suppl. to the, ii. 161

Irabubos, v. 270

Isates, v. 149

Isatis, ii. 44; v. 149

Isoodon, iii. 40 ; v. 195

Istiophorus, v. 71

Iyyol, v. $\mathbf{3 1 0}$

J A AL, v. 357

Jabartes, v. 391

Jacchus, v. 38

albifrons, v. 42

argentatus, v. 40

auritus, v. 39

black-tailed, v. 40

chrysomelas, v. 41

great-eared, v. 39

humeralifer, v. 39

labiatus, v. 41

leoninus, v. 42

leucocephalus, v. 39

melanurus, v. 40

penicillatus, v. 39

rosalia, v. 42

rufimanus, v. 40

tufted, v. 39

ursulus, v. 41

vulgaris, v. 38

white-headed, v. 39

white-shouldered, v. 39

Jachmur, v. 327

Jackal, ii. 42 ; v. 145

Barbary, v. 146

Cape, v. 146

Senegal, v. 147

Jaela, v. 357

Jaguar, ii. 51.452 ; v. 161 
Jaguar, of Buffon, v. 166

Jaguarondi, ii. 54

Jairou, v. 332

Jaquarete pope, v. 164

Jaqueparel, v. 146

Jaru, v. 314

Jelen, v. 308

Jeralang, iii. 179 ; v. 251

Jerboa, iii. 76. 140. 143 ; v. 236

Cape, iii. 164 ; v. 243

great, v. 238

Siberian, v. 237

striped, v. $\mathbf{2 3 7}$

Jerna, v. 314

Jevraschka, v. 246

J'hongnuaht, v. 375

Jirataka, v. 321

Jird, le, v. 239

Jocko, i. 238 ; v. 5

Jubarta, iv. 443 ; v. 391

Juscha, v. 305

KabarGA, v. 301

Kabasson, v. 277

Kadischi, iii. 448

Kahau, i. 213. 276

Kaidon, v. 295

Kalong, v. 54

Kalou, ii. 104

Kanchil, iv. 64 ; v. 302

Kanguroo, v. 202

banded, v. 203

elegant, ii. 76 ; v. 203

Eugene's, v. 203

giant, v. 202

gigantic, ii. 75 ; iii. 47

gray, v. 202

Labillardiere's, v. 203

large, v. 201

Le Brun's, v. 204

lesser, v. 201

mountain, v. 204

of Aroé, ii. 76

red, v. 202

red-necked, ii. 75 ; v. 203

smoked, ii. 75 .

sooty, v. 202

tufted-tailed, v. 204

with moustaches, ii. 75

woolly, iii. 49 ; v. 204

Kanguroos, ii. 74

Kangurus, v. 201

bicolor, v. 204

Brunii, v. 204

Eugenii, v. 203

fasciatus, v. 203

fuliginosus, v. 202

Gaimardi, v. 201
Kangurus griseus, v. 202

labiatus, v. 201

lanosus, v. 202

penicillatus, v. 204

ruficollis, v. 203

rufogriseus, v. 202

rufus, v. 202

Kechubu, iii. 194; v. 260

Kemas, v. 328

Kevel, iv. 16.213; v. 332

Kijang, iv. 144; v. 319

Kinkajou, ii. 262 ; v. 115

Kinkajous, ii. 29

Kiodote, ii. 108 ; v. 58

Kirivoula, ii. 153 ; v. 90

Kistu, v. 304

Klap, v. 179

migosen, v. 179

Kleenebock, iv. 269

Klipdaas, v. 292

Klipspringer, iv. 245 ; v. 340

Knouk, ii. 482

Koala, v. 205

Koalas, ii. 76 ; iii. 50

Kob, iv. 223; v. 335

Koba, iv. 351

Kochlani, iii. 449

Kokoon, v. 369

Koodoo, iv. 359 ; v. 365

Korin, v. 333

Korooko, v. 325

Korsaki, v. 149

Kosa Dikaja, v. 314

Koupara, v. 145

Kuwuk of Java, v. 170

Kyloe, iv. 423

LACERTus Indicus squamosus, v, 282 squamosus peregrinus, v. 282

Lagomys, iii. 91. 222 ; v. 268

Alpinus, v. 269

dwarf, iii. 92

grey, iii. 92

ogotona, v. 269

pica, iii. 92 ; v. 269

pusillus, v. 269

Lagothrix, v. 26

canus, v. 26

Humboldtii, v. 26

Lagure, le, v. 26

Lama, iv. 6. 53 ; v. 299

Lamantin, iv. 431 ; v. 377

American, v. 378

des Antilles, grand, v. 378

du Kamtschatka, grand, v. 380

du Sénégal, v. 379

of America, iv. 445

of Senegal, iv. 446 ; v. 378 
Lamantin of the North, iv. 448

Lamantins, iv. 445

Lamas, iv. 5.50

Landak, v. 262

Language, difference of, i. 170

Lapin de Sibérie, v. 267

Larog, v. 256

Lary, iii. 187

Lasiopyga, v. 8 nemæus, v. 9

Lawack, v. 159

Lemmar, v. 215

Lemming, iii. 69 ; v. 215

land, v. 217

Hudson's Bay, iii. 69

ringed, v. 217

talpine, v. 216

Zokor, v. 215

Lemmings, iii. 68. 114

Lemmus, v. 215

albicaudatus, v. 212

aspalax, v. 215

fulvus, v. 212

hare-tailed, v. 216

Hudsonius, v. 216

lagurus, v. 216

Niloticus, v. 212

novaboracenis, v. 215

talpinus, v. 216

talpoides, v. 215

terrestris, v. 217

torquatus, v. 217

vittatus, v. 215

Xanthognathus, v. 213

Lemur, v. 43

albifrons, i. 228.270 ; v. 46

ashy, v. 47

black, v. 45

black and red, v. 44

black-fronted, v. 46

catta, i. 228 ; v. 44

cinereus, v. 47

collared, v. 46

dwarf, i. 322

flocky, v. 44

fulvus, v. 45

galago, j. 332 ; v. 49

gracilis, i. 229

indri, v. 43

laniger, v. 44

little, v. 48

long-fingered, iii. 196 ; v. 5 ]

loris, v. 47

Macaco, i. 228 ; v. 45

minutus, v. 49

mongoz, i. 228 ; v. 45

murinus, i. 323.332 ; v. 48

niger, v. 45

nigrifrons, v. 47
Lemur potto, i. 230 ; v. 49 psilodactylus, iii. 196 ; v. 51

pusillus, v. 48

red, i. 228.324 ; v. 46

ring-tailed, v. 44

ruber, i. 228 ; v. 44

ruffed, v. 44

rufus, v. 46

slow, i. 330 ; v. 47

slow paced, v. 48

spectrum, i. 230 ; v. 50

tardigradus, i. 229.330 ; v. 47

tarsier, v. 50

volans, ii. 15. 156 ; v. 99

white-forehead, i. 325

white-fronted, i. 270 ; v. 46

white-handed, v. 45

whitish, v. 50

Lemurs, i. 227

suppl. to the, i. 321

Leo marinus, v. 184

Leontico, v. 42

Leopard, ii. 52. 459 ; v. 165

Cape, v. 175

hunting, ii. $470 ;$ v. 166

maned hunting, v. 166

maneless hunting, v. 166

Lepus, iii. 90.210 ; v. 264

Egyptius, v. 267

Africanus, v. 267

albus, v. 265

Alpinus, iii. 92 ; v. 269

Americanus, iii. 91 ; v. 267

Brasiliensis, v. 267

Capensis, iii. 91 ; v. 267

cuniculus, iii. 90 ; v. 266

Dauricus, v. 267

ecaudatus, iii. 221 ; v. 268

glacialis, v. 265

Hudsonius, v. 267

hybridus, iii. 221 ; v. 265

nanus, iii. 91 ; v. 267

nigricollis, v. 268

ogotona, v. 269

pusillus, iii. 92.227 ; v. 269

saxatilis, v. 268

tapeti, v. 268

timidus, iii. 89 ; v. 265

tolai, v. 266

variabilis, iii. 90 ; v. 265

Virginianus, v. 265

viscaccior, v. 268

Lepusculus, v. 266

Lerot, iii. 71. 122; v. 220

with golden tail, iii. 70 v. 218

Lerwee, v. 360

Lesang, v. 160

Leucoryx, v. 327

Lévrier, v. 135 
Lézard de Clusius, v. 282 écaillé, v. 282

Lichanotus, i. 228 ; v. 43 indri, v. 43

laniger, v. 44 niger, v. 43

Lièvre d'Afrique, v. 267 des roches, v. 268 du Grœnlandon, v. 265 pampa, v. 272 tapeti, v. 268

Lion, ii. 50. 428 ; v. 162 African, v. 163 American, ii. 52 ; v. 163 Asiatic, v. 163 crested sea, v. 179 sea, ii. 507 ; v. 180.184 sea, of Anson, ii. 58 sea, of Cox's Island, v. 181 sea, of Forster, ii. 509 sea, of Steller, ii. 59 tiger cubs, ii. 448

Lipurus cinereus, v. 205

Lizard, scaly, v. 282

Loir, iii. 7l. 118

Loncheres, iii. 70. 117 chrysurus, v. 218 myosurus, v. 218 paleacea, v. 219 rufa, v. 218

Loret volant, v. 81

Lori, i. 330

Loris, i. 229 ; v. 47

Ceylon, v. 48

Ceylonicus, v. 47

du Bengale, v. 48 gracilis, v. 47

Javanese, v. 48

paresseux, v. 47

slender, i. 229 ; v. 47

slow, i. 229

Loro of Java, v. 75

Lorvo assee, ii. 110

Los, v. 304

Loup, v. 144

cervier, v. 174

marin, v. 180

noir, v. 144

peint, v. 151

rouge, v, 145

Lout, v. 327

Loutre de Canada, v. 130 de Kamtschatka, v. 133 de la Guyane, petit, v. 192

Lowo assu, v. 56 churat of Java, v. 65 manir, v. 93

Lupus, v. 144 aureus, v. 146

Cuv. Index.
Lupus Indicus, v. 145

Lutra, ii. 38 ; v. 129

Barang, v. 132

Brasiliana, v. 130

Brasiliensis, ii. 314 ; v. 130

Canadensis, v. 130

enudris, v. 131

insularis, v. 131

inunguis, v. 131

lataxina, v. 131

leptonyx, ii. 315 ; v. 132

maculata, v. 130

marina, v. 132

memina, ii. 66 ; iii. 35 ; v. 191

minor, v. 122

nair, v. 131

vison, ii. 296

vittata, v. 117

vulgaris, v. 129

Loutre, le, v. 130

Lycaon, v. 151

Burchel's, v. 151

tricolor, v. 151

Lynx, v. 174

American, ii. 53

booted, ii. 54.491 ; v. 173

Canada, ii. 53 ; v. 174

cat, v. 174

common, ii. 53

du Canada, v. 174

fasciatus, v. 174

fox, v. 174

montanus, v. 175

of Barbary, ii. 490

of the ancients, v. 173

of the marshes, ii. 54. 491

red, v. 174

United States, ii. 494

wolf, v. 174

Lynxes, ii. 490

Lyre, ii. 1 l

MACACA, i. 351

Inuus, i. 351

Macacus, v. 16

cynomolgus, v. 17

Indicus, v. 19

radiatus, v. 17

silenus, v. 16

Sinicus, v. 17

Macaque, i. $348.350,351$; v. 18,19

à crinière, v. 16

à queue courte, v. 18

magot, i. 351

maimon, v. 18

maned, i. 216

of Buffon, i. 216

ordinaire, v. 18

Orion, v. 19 
Macaque, short-tailed, i. 289 toque, v. 172

Macaques, i. 215

Macauco, v. 45 black, v. 45

flying, v. 99

little, i. 332

loris, v. 47

ring-tailed, v. 45

woolly, v. 45

yellow, ii. 269

Machlis, v. 306

Macroglossus, v. 56

Macrorhina, v. 180

Macrorhine, v. 180

Macropus, ii. 74

elegans, ii. 76 ; iii. 49

giganteus, v. 202

lanigerus, iii. 49 ; v. 204

major, ii. 75

minor, ii. 74 ; iii. 46 ; v. 201

ruficollis, v. 203

rufogriseus, v. 202

Madoka, v. 350

Mafutiliqui, v. 127

Magot, i. 215. 348; v. 19

Magots, i. 215

Mahairy, v. 298

Maimon, i. 217.288 ; v. 22

Majot, i. 280

Maki, v. 47

à bourse, v. 44

aux pieds blancs, v. 46

aux pieds fauves, v. 46

d'Augouan, v. 46

fauve, v. 44

nain, i. 322

petit, v. 47

roux, v. 44.46

Makis, i. 227

Malanou bourou, ii. 104 ; v. 54

Málbrouc, i. 212 ; v. 14

Mammalia, i. lviii. 72.205 ; v. 2 division of the class into orders,

Man, i. 82

$$
\text { i. } 77
$$

American, var. v. 3

Caucasian, var. v. 3

differences between, and other animals, i. 205

food of, i. 130

Malay, var. v. 3

Negro, var. v. 3

of the woods, v. 5

peculiar conformation of, i. 82 suppl. history of, i. 103

Manati, v. 377

$$
\text { phocæ genus, v. } 378
$$

trichechus, v. 184
Manati, whale-tailed, v. 380

Manatis, iv. 431. 445

Manatus, iv. 431 ; v. 377.380

Americanus, iv. 445. 448 ; v. 378

Senegalensis, v. 378

Manaviri, ii. 265

Mandrill, i. 219 ; v. 21, 22

Mangabey, v. 15

à collier blanc, v. 16

collared, i. 261 ; v. 16

with collar, i. 211

without collar, i. 211

Mangouste, v. 156

of Egypt, ii. 47

of Java, ii. 398

of the Indies, ii. 47

of the Cape, ii. 47

vausire, v. 157

Mangoustes, ii. 47. 392

Mangusta, v. 156

Cafra, v. 157

Edwardsii, v. 157

galera, v. 157

Ichneumon, v. 156

Javanicus, v. 157

major, v. 158

mungos, v. 156

rubra, v. 158

Manicou, ii. 63

Manis, i. lx ; iii. 261. 306 ; v. 281

African, v. 282

brachyura, iii. 262 ; v. 282

broad-tailed, v. 282

crassicaudata, v. 282

Indian, v. 282

Javanica, v. 282

long-tailed, iii.262. 308; v. 282

longicaudata, v. 282

macroura, v. 282

pangolinus, v. 282

pentedactyla, iii. 262. 307 ; v. 282

phatagus, v. 282

short-tailed, iii. 262. 307 ; v。 282

tetradactyla, iii. 262. 308 ; v. 282

Mankind, general extension of, i. 123

Mans-gottung, v. 214

Mantegar, v. 21

Mapach, ii. 28 ; v. 114

Mapurita, v. 127

Mapurito, v. 118. 128

Margaia, ii. 482

Margay, ii. 482 ; v. 169

Margatsch, v. 336

Mariba, v. 294

Marikina, i. 226. 310 ; v. 42

Marimonda, i. 305 ; v. 24 
Marinose, ii. 66

Marmose, la, iii. 32 ; v. 188

Marmot, v. 244

Alpine, iii. 81.166 ; v. 244

barking, v. 248

Diana, iii. 170 ; v. 248

earless, v, 246

Franklin's, v. 246

Gundi, v. 248

hoary, v. 248

Hood's, v. 247

Maryland, v. 244

of Germany, iii. 75

of Poland, iii. 81. 169

Parry's, v. 247

Prairie, v. 247

Quebec, v. 245. 247

red, v. 245

Richardson's, v. 246

short-tailed, v. 245

striped American, iii. 171

tailless, v. 248

variegated, iii. 169

Marmots, iii. 166

Marmotte, v. 244

de Canada, v. 245

de Pologrie, v. 244

de Sibérie, v. 246

du cap, v. 292

volante, v. 90

Marmottes, iii. 80

Marsouin, iv. 453 ; v. 383

dolphin, v. 383

Marsupiata, ii. 61 ; v. 185

suppl. on the, iii. 1

Marte, v. 123

marron, v. 125

zorra, v. 125

Marten, v. 124

beech, ii. 293 ; v. 123

common, ii. 36. 294

Indian pine, v. 159

spotted, v. 193

Martens, ii. 33. 36. 292

Martes, v. 123

Canadensis, v. 124

foina, v. 123

leucotis, v. 126

Pennantii, v. 125

rufa, v. 125

sinuensis, v. 125

Vison, v. 124

vulgaris, v. 123

zibellina, v. 124

Martin, pine, v. 123

Massascus, v. 209

Mastiff, ii. 40. 337 ; v. 141

Englisb, v. 141

Mastodon, iii. 328
Mastodon, angustidens, iii. 329

giganteum, iii. 328

great, iii. 328

with narrow teeth, iii. 329

Mastodons, iii. 328

Mataco, iii. 256

Mâtin, v. 134

French, ii. 328

Maucauco, little, v. 48

murine, v. 48

Tarsier, v. 50

Maucoco, v. 115

Mazama, v. 314

dorsata, v. 354

sericea, v. 354

Mazame, iv. 12 ; v. 323.354

Mazamine group, iv. 127

Mbaracaya, ii. 482

Medateus, v. 74

Lewisii, v. 74

Meerkats, iii. 172

Megaderma, v. 74

frons, v. 75

lyra, v. 75

spasma, v. 74, 75

trifolium, v. 75

Megadermes, ii. 10. 128

Megalotis, ii. 45 ; v. 151

Brucei, v. 152

Megalotis, Lalandii, ii. 372 ; v. 151

Mejangan Banjoe, v. 311

Melas, ii. 53

Meles, ii. 30 ; v. 115,116

alba, v. 114

gulo, v. 117

Labradorica, v. 116

vulgaris, v. 116

Melursus, v. 113

Memina, iv. 63; v. 301

Men, wild, i. 207

Mephitis, ii. 37 ; v. 126

Americana, v. 127

Chilensis, v. 127

interrupta, v. 127

Javanensis, v. 129

meliceps, v. 129

Meriones, iii. 153

Mermaids, iv. 431

Merschswein, v. 383

Merycotherium Sibiricum, v. 322

Michaucanens, v. 141

Mico, i. 227 ; v. 40

Micouré, ii. 63

dwarf, v, 191

fifth, iii. 34

fourth, iii. 33

long-tailed, iii. 33

premier, v. 187

E 2 
Micouré, second, v. 190

sixth, v. 191

third, with a thick tail, v. 190

woolly, v. 190

Midas, v. 40

CEdipus, v. 42

albifrons, v. 41

chrysomelas, v. 41

labiatus, v. 41

leoninus, v. 42

rosalia, v. 42

rufimanus, v. 40

ursulus, i. 226 ; v. 41

Mimetes, v. 4

Mink, ii. 35. 37. 291 ; v. 122

of the Americans, v. 124

Mioroung, v. 180

Miriki, v. 25

Miriquouina, v. 36

Mirounga, v. 179

Ansonii, v. 180

Byronii, v. 181

Patagonica, v. 180

proboscidea, v. 180

Mistus, v. 370

Mitzli, ii. 436

Mococo, i. 228.329 ; v. 45

Mole, v. 108

albinose, v. 108

ash-coloured, ii. 201

blind rat, iii. 78

citron, ii. 201

common, ii. 23

fawn-coloured, ii. 201

golden, ii. 20

long-tailed, ii. 20. 211 ; v. 108. 110

maritime rat, iii. 79

of the Greeks, v. 241

pied, ii. 201

radiated, ii. 210 ; v. 110

rat, of the Cape, iii. 79

red, ii. 20. 193

red, of Seba, v. 108

sand, iii. 164

shrew, v. 107

small rat, iii. 79

spotted, v. 108

star muzzled, of Canada, ii. 23

water, v. 285

white, ii. 201 ; v. 108

yellow, v. 108

zand, iii. 164 ; v. 242

Moles, ii. 22. 194

rat, iii. 77

Molock, i. 209 ; v. 6.34

Molossus, ii. 7 ; v. 60

acuticaudatus, v. 63

amplexicaudatus, v. 62
Molossus, ater, v. 61

black, v. 61.

castaneus, v. 62

crassicaudatus, v, 62

fumarius, v. 63

fusciventer, ii. 7 ; v. 61 .

Guyanensis, ii. 7

longicaudatus, ii. 7 ; v. 62

nasutus, v. 63

obscurus, v. 61

rufus, v. 60

sharp-tailed, v. 63

ursinus, v. 65

Moluck, v. 304

Mona, i. 212. 268

Monardes paseng, v. 357

Monax, iii. 170 ; v. 245

Mone, la, v. 12

Mongooz, v. 45

Mongous, i. 228. 327 ; v. 45

grand, v. 45

with white forehead, i. 228

Mongsoo, v. 304

Mongul, iii. 143

Monk, ii. 291 ; v. 37

Monkey, i. 320

bay, v. 8

bonneted, i. 278

broad-bearded, v. 11

broad-tootlied, v. 11

callitrix, v. 14

capped, v. 7

capuchin, v. 32

Chinese, v. 17

Cochin China, i. 214. 276; v. 8

fair, v. 40

fearful, v. 29

four-fingered, v. 24

fox-tailed, v. 36

full bottom, v. 8

great-eared, v. 40

green, i. 212.263 ; v. 14

hare-lipped, i. 216. 286; v. 17

horned, v. 31

king, v. 8

lion, i. 226 ; v. 42

lion-tailed, i. 285

little black, v. 40

little lion, v. 43

long-nosed, i. 213

masked, v. 33

middle-size black, v. 9

mustache, i. 213.271 ; v. 12

Negro, i. 211.262 ; v. 9

night, i. 225

orange, v. 33

palatine, v. 13

perruque, v. 7

pig-tailed, v. 19 
Monkey, preacher, v. 27

proboscis, i. 213.276 ; v. 9

purple-faced, i. 277 ; v. 11

red, i. 211.261 ; v. 13

red howling, i. 222 ; v. 26

red-tailed, v. 43

red-vented, v. 15

royal, v. 26

silky, i. 310 ; v. 42

silver-haired, v. 26

spider, v. 25

spotted, i. 212 ; v. 13

squirrel, i. 307.312 ; v. 33

St. Jago's, v. 15

striated, i. $308 ;$.v. 38

stunted, v. 38

Talapoin, v. 1]

varied, i. 212.268 ; v. 12

vaulting, i. $213 ;$ v. 13

weeper, v. 28

white eye-lid, i. 211 ; v. 15

white-nosed, v. 12

widow, i. 320 ; v. 33

Monkeys, i. 206

American, v. 24

lazy, i. 229

long-tailed, i. 210

of America, i. 221

proper to the old world, i. 207

Mono, v. 25

capuchino, i. 316

colorado, v. 26

feo, i. 318

rabon, i. 318 ; v. 38

Monodon, i. lx ; iv. 437 ; v. 386

Andersonianus, v. 387

microcephalus, v. 387

monoceros, iv. 437 ; v. 387

narwhal, v. 387

spurius, iv. 464

Monophyllus, v. 85

Redmani, v. 85

Monotrema, v. 283

Moose, iv. 72; v. 304

Mopse, ii. 338

Morcassin, v. 288

Morfo, v. 185

Mormoops, v. 73

Mors, v. 185

Blainvillii, v. 74

Morse, ii. 511

Morses, ii. 59

Moschi capreolus, v. 301

Moschius, ii. 172

Moschus, i. lx ; iv. 6.59 ; v. 301 Javanicus, iv. 64 ; v. 302 memina, iv. 63 ; v. 301 moschiferus, iv. 6. $60 ; \mathrm{v} .301$ napu, iv. 63 ; v. 302
Moschus, pelandoc, iv. 66 ; v. 302 pygmæus, v. 303

Mouffette de Chili, v. 128

Mouffettes, ii. 37

Mouflon, v. 360

d'Afrique, v. 360

of Africa, iv. 26

of America, iv. 26

Mouse, v. 226

Astracan, v. 234

Baikal, v. 211

Barbary, v. 228

common domestic, iii. 131

field, iii. 74 ; v. 209.225

frugivorous, v. 229

garlic, v. 210

harvest, iii. 132 ; v. 226

Javanese, v. 224

lineated v. 211

meadow, iii. 110

minute, v. 227

of Cairo, iii. 74

red, v. 210

short-tailed field, v, 209

shrew, v. 102

soricine, v. 228

striated, v. 228

subtle, v. 227

white-footed, v. 232

white-tailed, v. 212

wood, iii. 132

Moussel, v. 268

Moustac, i. 213; v. 12

Muana conquo, iv. 327

Mufflon, iv. 26

Muffoli of Corsica, iv. 26

Mufione of Sardinia, iv. 26

Mule, wild, of Antiquity, v. 295

Mulot, iii. 74; v. 225

des champs, v. 226

petit, v. 226

volant, v. 60

volant, second, v. 61

Multungula, i. Ixxviii.

Mungo, v. 156

Mungutia, v. 156

Muntjak, iv. 144. 148

Blainville's, iv. 148 ; v. 320

Nepaul, iv. 149; v. 320

Philippine, iv. 147 ; v. 319

Ubi, iv. 148 ; v. 320

Mus, i. $1 x$; iii. 66 ; v. 208. 223

accedula, iii. 76 ; v. 233

Economus, iii. 68 ; v. 210

Egyptius, v. 236

Africanus cayopillin dictus, v. 188

agrarius, v. 227

agrestis, v. 290

agrestis major, v. 225 
Mus, Alexandrinus, v. 225

alliarius, v. 210

Alpinus, iii. 81 ; v. 234.244

amphibius, iii. 67 ; v. 209

amphibius terrestris, v. 217

angouya, v. 230

anomalus, iii. 139 ; v. 236

aquaticus, v. 209

exoticus, ii. 185 ; v. 105

aquatilis, ii. 185 ; v. 105.209

arctomys, v. 244

arenarius, iii. 76 ; v. 234

araneus, ii. 172

arvalis, iii. 68. 110

aspalax, iii. $69 ;$ v. 215

Astrachanensis, v. 214

auritus, v. 231

avellanarius, iii. 72. 122. v. 221

avellanarius minor, v. 221

avellanarius major, v. 220

Barabensis, v. 235

barbarus, v. 228

betulinus, v. 228

bobac, iii. 81

Braziliensis, v. 230

bursarius, iii. 138 ; v. 235

cafer, iii. 80 ; v. 243

cahirinus, iii. 74 ; v. 232

campestris, v. 226

campestris major, v. 225

campestris minor, v. 209

Canadensis, iii 159

Capensis, iii. 79 ; v. 242

caraco, iii. 74 ; v. 224

caugouya, v. 230

cephalotes, v. 231

citillus, iii. 81 ; v. 246

coypus, iii. 73 ; v. 223

cricetus, iii. 75 ; v. 233

cyanus, v. 214

decumanus, iii. 74.129 ; v. 224

dichrurus, v. 229

domesticus major, v. 225

domesticus medius, v. 225

domesticus vulgaris, v. 226

Donovani, v. 230

Egypticus, iii. 143

empetra, v. 245

fasciculatus, v. 262

Floridanus, v. 214

frugivorus, v. 229

furunculus, v. 235

giganteus, iii. 126 ; v. 223

glis, iii. 71 ; v. 220

gregalis, v. 211

gregarius, v. 211

griseus, v. 224

Hudsonius, iii, 69 ; v. 217

hydrochærus, v. 270
Mus, Icria, v. 229

Indicus, v. 224

Islandicus, v. 229

jaculus, iii. 77.143 ; v. 236

Javanicus, v. 224

Labradorius, v. 240

lagurus, v. 216

laniger, v. 235

laucha, v. 231

lemmus, iii. 69.114 ; v. 215

leucopus, v. 232

longipes, iii. 157 ; v. 240

macrourus, v. 263

Malabaricus, v. 223

maritimus, iii. 79.164 ; v. 242

marmotta, v. 244. 246

Maulinus, v. 248

Meridianus, v. 239

messorius, iii. 132 ; v. 226

microurus, v. 215

migratorius, v. 233

minutus, v. 227

monax, v. 244

muscardinus, iii. 122

musculus, iii. 73 ; v. 226

nigricans, v. 232

nigripes, v. 231

nitela, iii. 71

nitidula, v. 220

Noricus, v. 246

Norvegicus, v. 215. 224

Orientalis, v. 228

parvulus, v. 227

pendulinus, v. 227

Perchal, v. 232

phœus, iii. 76 ; v. 234

ponticus, v. 259

porcellus, v. 271

pumilio, v. 211

rattus, iii. 73 ; v. 225

rufus, v. 230

rutilus, v. 210

quercinus, v. 220

saccatus, v. 235

sagitta, iii. 77 ; v. 236

setifer, v. 229

seu Cuniculus Americanus et

Guineensis, v. 271

socialis, v. 211

songarus, iii. 76 ; v. 234

sorex, v. 226

soricinus, v. 228

striatus, v. 228

subtilis, v. 227

Sumatrensis, v. 224

suslica, v. 246

sylvaticus, iii. 74.132 ; v. 225

sylvestris, v. 224

sylvestris Americanus, v. 191.271 
Mus, tamaricinus, iii. 155 ; v. 239

talpinus, v. 216

terrestris, v. 209. 211

torquatus, v. 217

typhlus, iii. 78 ; v. 241

vagus, v. 227

zibethicus, iii. 67 ; v. 208

Musang, ii. 416

brilan, v. 159

Musaraigne, ii. 172 ; v. 102

à queue de rat, v. 104

d'eau, v. 102

dorée, v. 108

plaron, v. 103

taupe, v. 107

Musaraignes, ii. 172

Musc, v. 153

Muscardin, iii. 72 ; v. 221 volant, v. 90

Musculus dichrurus, v. 222. 229 frugivorus, v. 221.229

Muserain, ii. 172

Musette, ii. 17. 128

Musk, iv. 6

Indian, v. 302

Java, iv. 63

pygmeus, iv. 62 ; v. 303

Thibetian, iv. 60 ; v. 301

Musks, iv. 6. 59

Musmon, iv. 322 ; v. 360

Musquash, v. 208

Mustela, ii. 33 ; v. 119

abietinum, v. 123

abietum, ii. 294

Africana, v. 122

Alpina, v. 120

altaica, v. 120

Americana, v. 127

armelina, v. 122

barbara, ii. 32.278 ; v. 117

Canadensis, ii. 296 ; v. 124

candida, v. 122

Cuja, v. 126

erminea, ii. 35 ; v. 122

fagorum, ii. 293

flavigula, v. 126

foina, ii. 36 ; v. 124

furo, ii. 34 ; v. 120

glauca, v. 156

gulo, ii. 276 ; v. 117

Guyanensis, v. 126

Hudsonica, v. 130

leucotis, v. 126

lutra, ii. 38. 312; v. 130. 132

lutra Brasiliensis, ii. 38 ; v. 130

lutreola, ii. 35 ; v. 122

lutris, ii.39. 316 ; v. 132

martes, ii. 36 ; v. 123
Mustela, melanorhyncha, v. 125

nivalis, ii. 289 ; v. 121

Pennanti, ii. 297 ; v. 125

putorius, ii. 34 ; v. 120

quadricolor, v. 126

quiqui, v. 126

rufa, v. 125

Sarmatica, ii. 34

Sibirica, ii. 35 ; v. 122

sinuensis, ii. 297 ; v. 125

vison, v. 124

vulgaris, ii. 35 ; v. 121

zibellina, ii. 36.294 ; v. 124

Mutz, v. 179

Mycetes, i. 221 ; v. 26

flavicaudatus, v. 27

fuscus, v. 27

niger, v. 28

rufimanus, v. 28

seniculus, v. 26

stramineus, v. 27

ursinus, v. 27

Myadeus meliceps, ii. 305 ; v. 129

Mygale, ii. 18. 185 ; v. 105

Moscovitica, ii. 189 ; v. 105

Pyrenaïca, ii. 190 ; v. 105

Myonotes pratensis, v. 214

Myopotamus, v. 223

Canariensis, v. 223

Myopteris, v. 83

Daubentonii, v. 83

Myoxus, iii. 71. 118 ; v. 219

Africanus, v. 221

avellanarius, v. 220

avellanarius Lalandii, v. 221

chrysurus, v. 218

Compei, v. 221

dryas, v. 220

glis, iii. 118 ; v. 220

guerlinguet, v. 256

murinus, v. 221

muscardinus, v. 221

nitela, v. 220

tamaricinus, v. 239

Myrmecophaga, i. Ix ; iii. 259. 299 ; v. 280

aculeata, iii. 264 ; v. 284

Afra, v. 279

annulata, v. 281

Capensis, iii. 259 ; v. 279

didactyla, iii. 261 ; v. 28]

jubata, iii. 260.299 ; v. 280

minima, v. 281

nigra, v. 280

tamandua, iii. 260 ; v. 280

tetradactyla, iii. 260 ; v. 280

tridactyla, iii. 260 ; v. 280

unicolor, v. 281 
NABIS, v. 321

Næmorhedine group, iv. 277

Næmorhedus, v. 352

Nærs, v. 122

Nagor, iv. 238 ; v. 338

Naip, v. 321

Nanguer, le, iv. 20 ; v. 331

Napu, iv. 63 ; v. 302

Nari, v. 146

Narwhal, iv. 460 common, v. 387 microcephalus, iv. 463 vulgaire, v. 387

Narwhals, iv. 437

Nasalis larvatus, v. 9

Nasique, i. 276 ; v. 9

Nasua, ii. 29 ; v. 114 fusca, v. 115 rufa, v. 114

Natantia, i. Ixxix.

Neelghau, iv. 363 ; v. 366

Neitsek, v. 178

Neitsersoak, v, 179

Nems, ii. 47 ; v. 157

Neotoma, iii. 160 ; v. 214 Floridana, iii. 160 ; v. 214

Neotragine group, iv. 269

Neotragus, v. 349

Nesarnack, iv. 456 ; v. 382

Ngria, v. 331

Niborèbi, v. 294

Nil-bundar, v. 17

Niou, iv. 22

Noctilio, ii. 8 ; v. 67 à ventre blanc, v. 67 albiventer, v. 68 Americana, v. 67 dorsatus, v. 67 ferrum equinum, v. 76 hare-lipped, v. 67 leporinus, v. 67 Peruvian, v. 67 rufus, v. 68 unicolor, v. 67

Noctilions, ii. 8

Noctule, ii. 13. 144 ; v. 89

Noerz, ii. 35

Nord caper, iv. 442. 493 ; v. 390

Norek, ii. 35.291

Nots, Devonshire, iv. 340

Nougi-hari, ii. 355

Noumetje, v. 349

Nunel, v. 375

Nunni, v. 330

Nupu, v. 302

Nycteres, ii. 11

Nycteris, ii. 11 ; v. 79

Daubentonii, v. 80

Geoffroyii, v. 80
Nycteris, hispidus, v. 79 Javanica, v. 80 of Thebaïs, ii. 12. 137 Thebaïda, v. 80

Nycticebus, v. 47 Bengalensis, v. 47 Ceylonicus, v. 48 Javanicus, v. 48 potto, v. 49

Nycticeius, v. 98

Nyctinome, Bengal, v. 64 Brasilian, v. 65 d'Egypte, v. 64 Egyptian, ii. 8; v. 64 Murine, v. 61 Port Louis, v. 64

Nyctinomes, ii. 7

Nyctinomus, v. 64 acetabulosus, v. 64 Bengalensis, v. 64 Brasiliensis, v. 65 dilatus, v. 65 Mauritianus, v. 65 murine, v. 66 tenuis, v. 65

Nyctophilus, v. 86 Geoffroyi, v. 86

Nyentak, ii. 280 of Java, v. 118

Nylgau, iv. 21

OCELOT, ii. 53 ; v. 167 linked, v. 168

Ocelots, ii. 474

Octolicnus, i. 229 galago, v. 49

Odobenus, v. 185

Ogotone, iii. 226 ; v. 269

Oleck, ii. 158

Olen, v. 305. 308

Onager, v. 295

Once, v. 166

Ondatra, iii. 67.106 ; v. 208

Ondatras, iii. 66

Onza, ii. 452 ; v. 164

Oont, v. 298

Opeacha, v. 295

Ophion, v. 359

Opossum, ii. 63 ; v. 186.198 Azara's, v. 186 back-bearing, v. 189 Cayenne, v. 187 crab-eating, v. 187 dwarf, v. 191 four-eyed, v. 187 gray, v. 188 Javan, v. 204 lemurine, v. 197 Mexican, iii. 32 ; v. 188 
Opossum Molucca, v. 187

marine, ii. 66 ; iii. 32

murine, v. 188

naked-tailed, v. 189

New South Wales, v. 197

petaurine, v. 198

Philander, v. 188

Quica, v. 187

Seba's, v. 190

short-tailed, iii. 33 ; v. 190

spotted, v. 193

squirrel, v. 200

thick-tailed, v. 189

ursine, iii. 37.52

Virginian, iii. 24 ; v. 186

Viverrine, v. 194

vulpine, v. 197

white-tailed, v. 198

woolly, v. 190

Orang, i. 348

l', varié, i. 254

outang, i. 207.238 ; v. 5

Orangs, i. 207

Orc, iv. 455

Orca, v. 384

Orebi, v. 339

Oreillard, v. 97

Oreillards, ii. 14

Oreotragine group, iv. 245

Organization in general, i. 11

Orico, v. 264

Ornithorynchus, iii. 264. 316 ; v. 284

bristly, v. 284

brown, v. 285

crispatus, v. 285

fuscus, v. 285

histrix, iii. 264. 314; v. 284

histrix alter, v. 284

paradoxus, iii. 265 ; v. 284

porcupine, iii. 314 ; v. 284

red, v. 284

rufus, v. 284

setosus, iii. 264 ; v. 284

Orozo, l', v. 235

Orthula of Mexico, v. 128

Oryctère, iii. 79

Orycterope, iii. 258

of the Cape, iii. 259

Orycteropus, iii. 258.296 ; v. 279 Capensis, v. 279

Orygine group, iv. 182

Oryx, iv. 19 ; v. 325

Caffrarian, iv. 186 ; v. 326

Nubian, iv. 189 ; v. 327

white, iv. 188 ; v. 326

Otaria, v. 182

cinerea, v. 183

coronata, v. 182

Falklandica, v. 183

Cuv. Index.
Otaria flavescens, v. 183 jubata, v. 184

Peronii, v. 182

pusilla, v. 182

ursina, v. 182

Otaries, ii. 58

Otter, American, ii. 38. 314; v. 130

Canadian, v. 130

Carolina, v. 131

clawless, v. 131

common, ii. 38. 312 ; v. 129

Brasilian, ii. 314 ; v. 130

Javanese, v. 132

greater, v. 130

gryze, v. 132

little, of Guyane, iii. 35

Pondicherry, v. 131

sea, ii. 39. 316 ; v. 132

small, of Guyane, ii. 66

smaller, ii. 291

spotted, v. 130

Trinity, v. 131

white-headed sea, ii. 316

Otters, ii. 38.312

Ouanderou, i. 216. 284 ; v. 16

Ouarin, i. 222 ; v. 27

Ouavapavi, v. 31

Ouistiti, v. 38 common, is 226

Ouistitis, i. 225

Ounce, ii. 469

Ourebi, v. 340

Ours, v. 111

blanc, v. 112

d'Amérique, v. 112

gulaire, ii. 228

marin, v. 182

marin du Cap de Bonne Espérance, v. 182

Ovibos, iv. 372 ; v. 370 moschatus, iv. 373 ; v. 370

Pallantis, iv. 375 ; v. 370

Ovis, iv. 25. 311 ; v. 358

Ammon, iv. 25. 316 ; v. 359

argali, v. 359

aries, iv. 324 ; v. 361

montana, iv. 26 ; v. 354.359

musmon, iv. 322 ; v. 360

pudu, v. 355

pygargus, iv. 318 ; v. 359

Ox, iv. 28

tragelaphus, iv. 26.319 ; v. 359

Alderney, iv. 424

Bornou, iv. 426

Cape, v. 371

common, iv. 28

domestic, iv. 418

fossil musk, iv. 375 ; v. 370

Galla, iv. 426

F 
Ox, Guernsey, iv. 424

Highland, iv. 423

improved Leicester, iv. 422

Lancaster, iv. 422

long-horned, iv. 422

middle-horned, iv. 422

musk, iv. 31.373 ; v. 370

Orkney, iv. 423

polled, iv. 423

short-horned, iv. 422

Welsh, iv. 423

Zanga, iv. 426

Oxypterus, v. 383

PaART, berg, v. 296

mountain, v. 296

Paca Brasiliensibus, v. 273

brown, iii. 247 ; v. 273

femelle, v. 273

Pacas, iii. 95

ellow, v. 273

Paco, iv. 6. 57 ; v. 299

Pacos, v. 299

Pacasse, v. 372

Pachydermata, i. lxxvii ; iii. 323 ; v. 285 suppl. to the, iii. 345

Pag, v. 273

Pagasse, v. 371

Pague, v. 273

Palæotherium, iii. 338

Paletuveirs, v. 317

Pallah, iv. 219; v. 334

Palmiste, iii. 84; v. 253

Pangolin, v. 282 à queue courte, v. 282

Pangolins, iii. 261

Panther, ii. 51. 459 ; v. 165

black, ii. 53

of antiquity, v. 165

of exhibitors, v. 164

of the ancients, ii. 466

Panthère, v. 165

femelle, v. 164

mâle, v. 165

Papio, i. 339. 349; v. 274

apedia, v. 23

cinerea, v. 22

comatus, v. 20

cynocephalus, v. 20

leucophœus, v. 22

mormon, v. 21

niger, v. 23

Pennantii, v. 23

porcarius, v. 20

Silenus, v. 16

sublutea, v. 22

sus, v. 21

sylvicola, v. 22

Papion, i. 218 ; v. 20
Papion, à perruque, i. 218 ; v. 21

black, i. 218

noir, v. 20

petit, v. 20

short-tailed, i. 219

Paracyon, v. 192

Paradoxurus, ii. 412 ; v. 158

albifrons, v. 160

aureus, v. 159

common, v. 158

golden, v. 159

prehensile, v. 159

prehensilis, v. 159

typus, ii. 412 ; v. 158

Paræiasta, v. 121

Pardalis of the ancients, ii. 51

Paresseux pentadactyle du Bengale,v. 47

Parrah, v. 312

Passan, iv. 19; v. 326

Patas, i. 211.261 ; v. 13

à queue courte, i. 217 ; v. 18

Patira, iii. 334

Pay, v. 273

Peba, v. 276

Pecari, v. 290

Pecary, iii. 333

collared, iii. 412 ; v. 289

with collar, iii. 334

Pecora, i. Ix. lxxvi. ; iv. 1 ; v. 296

Pedetes, iii. 80. 164 ; v. 242

Capensis, v. 243

Pedimana, i. Ixxvii.

Pegasse, iv. 386

Pegasus, v. 372

Pekan, ii. 37; v. 124

Pelage, v. 179

Pelandok, iv. 66 ; v. 302

Perameles, ii. 68 ; iii. 38 ; v. 194

Bougainvillia, v. 195

nasuta, ii. 69 ; iii. 39 ; v. 195

obesula, iii. 39 ; v. 195

with pointed muzzle, ii. 69

Perouasca, ii. 34

Petaurista Cookii, v. 198

flaviventer, v. 199

large-tailed, v. 199

macroura, v. 199

pygmæa, v. 200

sciurea, v. 199

squirrel, v. 199

taguanoides, v. 198

yellow-bellied, v. 199

Petauristæ, iii. 43

Petaurus, ii. 71

flaviventer, v. 199

macrourus, v. 199

Peronii, v. 200

sciureus, v. 200

southern, v. 199 
Petaurus taguanoides, v. 199

Pfang, v. 372

Pflokfisch, v. 390

Phalanger, bear-like, v. 197

Cook's, ii. 71 ; iii. 42 ; v. 198

dwarf, v. 198

femelle, v. 196

flying dwarf, ii. 72 ; iii. 44

fox-like, ii. 71 ; v. 197

great-flying, ii. 72 ; iii. 44

long-tailed flying, ii. 72 ; iii. 45

male, v. 196

red, v. 196

spotted, v. 196

tauguanoides, v. 199

volant, grand, v. 199

volant à grande queue, v. 199

vulpine, iii. 41

yellow-tufted, v. 197

Phalangers, ii. 70 ; iii. 40 flying, ii. 71 ; iii. 43

Phalangista, ii. 70 ; v. 196

alba, v. 196

chrysorrhu, v. 197

Cookii, iii. 42 ; v. 198

maculata, v. 196

nana, v. 198

Papuensis, v. 196

Peronii, v. 200

petaurus, v. 199

pygmæa, v. 200

rufa, v. 196

sciurea, v، 200

ursina, v. 197

vulpina, v. 197

Phascochærus, iii. 333 ; v. 289

Africanus, v. 289

Phascogale, v. 194

minimus, v. 194

penicillata, v. 194

Phascolarctos, v. 204

fuscus, iii. $50 ;$ v. 205

koala, v. 205

Phascolomes, ii. 77

Phascolomys, ii. 77 ; iii. 52 ; v. 205

fusca, v. 206

wombat, v. 206

Phatagin, iii. 262 ; v. 282

Phattager, v. 282

Phé, le, v. 234

Philander, v. 187

Africanus, v. 188

maximus Orientalis, v. 187

Philandre de Surinam, v. 188

Philantomba, v. 349

Phoca, ii. 56 ; v. 175

Ansonii, v. 180, 181

barbata, v. 178

Byronii, ii. 509 ; v. 181
Phoca Coxii, v. 181

cristata, ii. 58. 507 ; v. 179

discolor, v. 177

fotida, v. 178

Falklandica, v, 183

fasciata, v. 181

flavescens, v. 183

Groënlandica, ii. 57

hispida, v. 178 ; v. 177.181

jubata, ii. 59. 508 ; v. 184

lagura, v. 177. 181

leonina, ii. 58 ; v. 179

leporina, v. 176. 181

leptonyx, ii. 507; v. 178. 181

lupina, v. 181

maculata, v. 176

major, v. 178 .

mitrata, v. 179

monachus, ii: 58 ; v. 178

oceanica, v. 177

Peronii, v. 182

proboscidea, v. 180

pusilla, ii. 59. 508 ; v. 182

semilunaris, v. 177

testudinea, v. 181

ursina, ii. 59. 509 ; v. 182

vitulina, ii. 57 ; v. 176.181

vitulina Bothionica, v. 176

Caspica, v. 176

Sibirica, v. 176

Phocæ, ii. 495

Phocæna, iv. 436 ; v. 383

Pholidolus longicaudatus, v. 282

Phoque à museau ridé, v. 181

à ventre blanc, v. 178

commune, v. 176

des Patagons, v. 180

grand, v. 178

nutsoak, v. 178

petit, v. 182

Phylacis, ii. 68

Phyllostoma, ii. 8 ; v. 68

crenulatum, ii. $\mathbf{~ 1 0}$; v. 68

elongatum, v. 69

hastatum, v. 69

lilium, v. 71

lineatum, v. 70

perspicillatum, ii. 127

planirostra, v. 69

rotundum, v. 70

soricinum, ii. $128 ;$ v. 73

spectrum, ii. 126 ; v. 71

Phyllostomes, ii. 8

Physalus, iv. 469 ; v. 388

Physeter, iv. 438 ; v. 327

catodon, iv. 469 ; v. 388

dorso pinnâ longâ, v. 388

macrocephalus, v. 388

microps, v. 388 
Physeter sulcatus, v. 389 trumpo, v. 388

Physeters, iv. 440, 469

Pichiy, iii. 293

Picka, v. 269

Pig, Guinea, iii. 93 ; v. 270

of Guinea, iii. 406

of New Guinea, v. 288

Siamese, iii. 406

Turkish, iii. 406

Pika, iii. 222 ; v. 269

calling, iii. 227

grey, iii. 226 ; v. 269

Pilosello, v. 154

Pinche, i. 226

Pinnipeda, i. lxxix.

Pipistrelle, ii. 13.151 ; v. 89

Pithecia, i. 224 ; v. 35

chiropotes, v. 36

leucocephala, v. 37

melanocephala, v. 37

miriquonina, v. 36

monachus, v. 37

ochrocephala, v. 37

rufibarba, v. 36

rufiventer, i. 225 ; v. 36

satanas, v. 35

Pithecus, i. 207. 348 ; v. $5.18,19$

agilis, v. 7

inuus, v. 19

lar, v. 6

leuciscus, v. 6

nemestrinus, v. 18

rhesus, v. 18

satyrus, v. 5

variegatus, v. 6

Wurmbii, v. 5

Pithèque, v. 19

Plantigrades, i. lxxvii.; ii. 25.217 ; v. 111

Platogna, v. 306

Platyceros, v. 306

Platycerine group, iv. 84

Platypus, iii. 264

anatinus, v. 284

duck-billed, v. 285

Platypyga, v. 271

Platyrhinque, v. 184

Plecotus, ii. 14 ; v. 96

auritus, v. 96

barbastellus, v. 97

Maugei, v. 97

Timoriensis, v. 98

velatus, v. 98

Poëphagus, v. 375

Pointer, ii. 41. 335 ; v. 139

Poisson à sabre, v. 384

Polatouche, iii. 191 ; v. 259 barbed, v. 260
Polatouche flêche, v. 260

Sapan, v. 259

Polatouches, iii. 84

Polecat, v. 120

Alpine, v. 120

Cape, ii. 35. 291

common, ii. 34. 285

of Catesby, v. 128

of Kalm, v. 127

of Poland, ii. 34

of Siberia, ii. 35

of the northern rivers, ii. 35

radiated, ii. 46

water, ii. 291

Polecats, ii. 34.285

Pollicata, i. Ixxviii.

Pone, le, v. 224

P'ongo, i. 295.351 ; v. 5

Pongos, i. 220

Poodle, v. 138

Pope, v. 114

Porcellus Indicus, v. 271

Porc-épic, v. 262 de Malacca, v. 262

Porcupine, Brazilian, v. 264 brush-tailed, iii. 207 ; v. 262

Canada, iii. 206 ; v. 263

crested, v. 261

common, iii. 87

hairy, iii. 88

iridescent, v. 263

long-tailed, v. 262

Malacca, iii. 207

maned, iii. 87

Marsden's, v. 262

Mexican, v. 263

pencillated tail, iii. 88 ; v. 262

prehensile-tailed, iii. 88. 208

rice-tailed, v. 262

Porcupines, iii. 87

Porcus, v. 288

aculeatus, v. 101

aculeatus sylvestris, v. 263

Porpoise, iv. 453

Porpus, v. 383 common, iv. 436

Porpusses, iv. 436

Portacine group, iv. 362

Portax, v. 366

Poto, ii. 262

Potoroo, ii. 74 ; iii. 46

Potorous, v. 200

Lesuerii, v. 201

murinus, v. 201

Peronii, v. 20I

Potot, v. 115

Potto, ii. 29 ; v. 49.115

Pottos caudivolvulus, v. 115

Prensiculantia, i. lxxviii. 
Presbytis, v. 7

Press, ii. 214 mitrula, v. 7

Primates, i. lx.

Priodontes, v. 277

Prionodon, ii. 412 ; v. 160 gracilis, ii. 412 ; v. 160

Proboscidea, v. 87

rivalis, v. 87

river, v. 87

rock, v. 87

saxatilis, v. 87

Prochilus ursinus, v. 113

Procyon, ii. 28. 246; v. 113 cancrivorus, ii. 252 ; v. 114 lotor, v. 114

Prosimia, v. 44

albifrons, v. 46

albimanus, v. 45

catta, v. 44

cinereus, v. 47

collaris, v. 46

fulvus, v. 45

macaco, v. 44

mongooz, v. 45

niger, v. 45

nigrifrons, v. 46

ruber, v. 44

rufus, v. 46

Proteles, v. 155

Lalandii, v. 156

Pseudotoma, v. 235

Pteromys, iii. 84. 190 ; v. 259

bright, v. 260

genibarbis, iii. 194 ; v. 260

lepidus, v. 261

nitidus, v. 260

petaurista, v. 260

sagitta, v. 260

Sibiricus, v. 259

volucella, v. 259

Pteropus, ii. 4.102 ; v. 54

Ægyptiacus, ii. 6 ; v. 57

amplexicaudatus, ii. 6. 108 ; v. 57

edulis, ii. 5.104 ; v. 54

Edwardsii, ii. 5 ; v. 55

fuscus, v. 55

griseus, ii. 6. 108 ; v. 55

Javanicus, ii. 105 ; v. 54

Leschenaultii, v. 56

marginatus, ii. 6 ; v. 57

minimus, ii. 6.108 ; v. 58

palliatus, ii. 110 ; v. 58

rostratus, v. 56

rubricollis, ii. 5 ; v. 55

stramineus, ii. 6.108 ; v. 57

vulgaris, ii. 5 ; v. 55

Puant of Lepage, v. 127
Pug, v. 142
bastard, ii. 338

Puma, ii. 52. 436 ; v. 163

Putois, v. 120

du Cap, v. 123 rayé des Indes, v. 155

Putorii, ii. 285

Putorius, ii. 34 ; v. 119

Alpinus, v. 120

Altaica, v. 120

erminea, v. 122

lutreola, v. 122

nudipes, v. 121

Sarmatica, v. 121

Sibirica, v. 122

vulgaris, v. 120

zorilla, v. 123

Putsche, v. 305

Pygargon, v. 359

Pygathrix nemæus, v. 9

Pyrame, v. 137

QUACCHA, v. 295

Quachi Valmont de Bomare, v. 115

Quadrumana, i. Ixxvii. 205 ; v. 4 addit. remarks on the, i. 332 suppl. on the, i. 231

Quantla mazame, v. 315

Qu'araho, v. 371

Quatreœil, v. 187

Quatto, v. 24

Quica, v. 187

Quickhatch, v. 117

Quocuya, v. 223

Quouiya d'Azara, iii. 73

RАввіт, iii. 90.216 ; v. 266

American, iii. 91

Angora, v. 266

collared, v. 268

long-nosed, v. 271

of Aroe, ii. 76

Russian, v. 266

Siberian, iii. 90

Racoon, ii. 246 ; v. 114

brown-throated, v. 114

common, ii. 246

crab, ii. 252 ; v. 114

crab-eater, ii. 29

of the Anglo-Americans, ii. 28 yellow, v. 114

Racoonda, v. 222

Racoons, ii. 28

Rangifer, v. 304

Rangiferine group, iv. 79

Raphicerine group, iv. 252

Raphicerus, v. 342 
Rasse, ii. 383 ; v. 153

Rat à bande noire, v. 227

Alexandrian, v. 225

Angouya, v. 230

Baikal, v. 211

bandicote, iii. 126

black, v. 225.232

black-footed, v. 231

blind, iii. 10I

bristle-bearing, v. 229

brown, iii. 74.128 ; v. 224

Canada, v. 235

Caraco, v. 224

common, iii. 73 ; v. 225

Daurian, v. 216

d'eau, v. 209

de Madagascar, v. 48

Donovan's, v. 230

Egyptian, v. 232

fauve, v. 227

field, iii. 132

fifth of Azara, v. 230

first of Azara, v. 218

Florida, iii. 160

fourth of Azara, v. 231

great-headed, v. 231

hamster, v. 233

hare-tailed, v. 216

Hudson's Bay, v. 217

Iceland, v. 229

Indian, v. 225

kanguroo, ii. 73,74 ; iii. 46 ; v. 201

Laucha, v. 231

long-eared, v. 231

long-legged, iii. 158

Malabar, v. 223

meadow, v. 209

moles, iii. 77

musk, v. 208

musk, of Canada, iii. 67

musqué de Canada, v. 208

nain, v. 211

Norway, v. 224

œconomic, v. 210

palmiste, v. 253

Perchal, iii. 74 ; v. 232

Pharaoh's, ii. 47 ; v. 156

Potooroo, v. 201

pouched, iii. 134

prickly, of Azara, iii. 71

red, of Azara, v. 230

ringed, v, 217

rock, v. 210

Russian musk, ii. 18

sand, v. 234

second, of Azara, v. 231

seventh, of Azara, v. 231

Sitnic, v. 227
Rat, sixth, of Azara, v. 231

sky-coloured, v. 214

small field, iii. 68

social, v. 211

Songar, v. 234

spiny, v. 218

Surinam, v. 196

tamarisk, v. 239

third, v. 230

two-coloured tailed, v. 229

water, iii. 67 ; v. 209

wood, v. 232

wood, of Cayenne, v. 256

wood, of Raffinesque, v. 232

volant, v. 83

Yaik, v. 233

zaryzin, v. 234

Ratel, ii. 33 ; v. 118

Ratellus, v. 118

Raton Crabier, v. 114

Rats, iii. 66. 126

American spineless, v. 230

kanguroo, ii. 73 ; iii. 46

spineless, of the old continent, v. 223

spiny, iii. 117 ; v. 232

Rattel, ii. 279 ; v. 118

Redunca, v. 337

Reduncine group, iv. 237

Rehe rehbock, v. 314

Remau-dahan, v. 164

Renard, v. 147 argenté, v. 148

gris, v. 148

noir, v. 148

Renne, v. 305

Renuthies, v. 305

Reptantia, i. lxxix.

Rheebock, red, iv. 239 riet, iv. 241

Rhesus, i. 217. 289 ; v. 18

Rhinoceros, iii. 336. 419 ; v. 290

Africarus, iii. 337 ; v. 291

bicornis, iii. 422 ; v. 291

bicornis Sumatrensis, v. 291

Camperis, v. 291

camus, v. 292

Indian, iii. 336.424 ; v. 290

Indicus, iij. 336 ; v. 290

simus, v. 292

sinusis, iii. 428

Sondaicus, v. 291

Sumatran, two-horned, v. 291

of Sumatra, iii. 336 ; v. 291

Sumatrensis, iii. 336; v. 291

two-horned, iii. 422

unicornis, v. 290

Rhinolophe cruminifera, v. 77

Rhinolophi, ii. 11 
Rhinolophus, ii. 11 ; v. 75

affinis, v. 78

bifer, ii. 11

bihastatus, v. 76

deformis, v. 79

diadema, v. 77

ferrum equinum, v. 76

ferrum equinum minor, v. 76

larvatus, v. 78

major, v. 76

marsupialis, v. 77

minor, v. 78

nobilis, v. 78

purse, ii. 119

speoros, ii. 119 ; v. 77

tridens, v. 77

unihastatus, v. 76

vulgaris, v. 78

Rhinopoma, v. 80

Carolinensis, v. 81

microphylla, v. 80

Rhinopome microphyle, ii. 12 ; v. 80

Rhinopomes, ii. 12

Rietbock, iv. 237

Rimau bulu, ii. 484 ; v. 171

Dahan, ii. 451

Rishya, v. 367

Rodentia, i. lxxvii.; iii. 61 ; v. 207 suppl. to the, iii. 96

Roe, Tartarian, iv. 122 ; v. 313

Roebuck, iv. 124 ; v. 314

American, v. 317

fossil, iv. 126

of Europe, iv. 13

of India, iv. 13

of Tartary, iv. 13

Roloway, i. 212; v. 13

Rona, le, v. 211

Rosmarus, v. 185

Indicus, v. $\mathbf{3 8 0}$

Rossomak, ii. 31

Rougette, v. 55

of Buffon, ii. 5. 107

Roussette, v. 55

à oreilles bordées, v. 58

amplexicaude, v. 57

black, ii. 5

bordered, v. 57

of Buffon, ii. 5

collared, ii. 5

common, ii. 107 ; v. 55

eatable, ii. 104

of Edwards, ii. 5.107 ; v. 55

Egyptian, v. 57

grey, ii. 108 ; v. 55

kiodote, v. 58

long-tailed, v. 57

mantled, ii. 110 ; v. 58

red-collared, v. 55
Roussette, red-necked, ii. 107 spotted, v. 56

Roussettes, ii. 4. 13. 102

Ruminantia, i. lxxvii ; iv. 1 ; v. 296 suppl. to the, iv. 33

Ruminants with hollow horns, iv. 15

Rupicapra, v. 353

Americana, v. 354

Rupicaprine group, iv. 281

Ruru, v. 312

Rusa, v. 309. 311

black, of Bengal, iv. 110

Etam, female of, v. 311

Gona, iv. 108 ; v. 310

great, iv. 105 ; v. 309

group, iv. 104

Malayan, iv. 112

of Malacca, iv. 115 ; v. 311

of the Mariannas, iv. 115 ; v. 311

of Timor, iv. 114; v. 311

Ubi, v. 320

Rytina, iv. 432

Ryzæna, ii. 48.404 ; v. 158

SABBEL, v. 124

Sable, ii. 36. 294 ; v. 124

le, v. 234

Saccopteryx lepturus, v. 82

Sægau, v. 305

Sagoin, i. 351 ; v. 33

black-handed, v. 34

Jacchus, i. 351

mitred, v. 34

ouistiti, i. 351

ruffed, v. 33

Saguinus ursulus, v. 41

Sai, i. 223 ; v. 32

à gorge blanche, v. 32

var., v. 29

Saiga, iv. 17. 226

Saija, v. 313

Saimiri, i. 224. 307 ; v. 33

Sajou, i. 223 ; v. 29

brun, v. 29

brun var., v. 30

cornu, v. 32

grey, v. 29

gris, v. 29

horned, i. 224. 307

Saki, v. 36, 37

black, i. 225

hand-drinking, v. 36

red-bearded, v. 36

red-bellied, i. 225

yellow-headed, v. 37

Saladang, iii. 434

Salientia, i. Ixxviii. 
Sanglier, iii. 331 ; v. 288

à masque, v. 288

d'Afrique, v. 289

de Madagascar, iii. 332; v. 288

du Cap, v. 289

masked, iii. 332

Sanglin, v. 38

Sapajou, bearded, v. 29

black, v. 30

crowned, v. 32

nègre, v. 30

ouavapavi, v. 31

paniscus, i. 351

spectacle, v. 31

varied, v. 30

yellow, v. 30

yellow-chested, v. 31

Sapajous, i. 221

common, i. 222

Sapan, iii. 192

Saricovienne, la, v. 118 de la Guyane, v. 130

Sarigue, ii. 63 ; v. 187

à long poil, v. 186

crab-eating, iii. 30

de Cayenne, v. 187

des Illinois, v. 186

four-eyed, ii. 65 ; iii. 31

great, of Cayenne, ii. 65

middle-sized, ii. 65

with bicoloured ears, ii. 64

Sarigues, ii. 63

Sarigues à oreilles bicolores, v. 186

Sariguoi, ii. 63

Sasi, v. 337

Sasin, v. 337

Sassasby, iv. 352. 363

Saumer, iv. 110 ; v. 310

Sauteur des rochers, v. 340

Scalope, ii. 190 of Canada, ii. 91. 191

Scalopes, ii. 19. 174

Scalops, ii. 191 ; v. 107 Canadensis, ii. 191 ; v. 107

Schakal, v. 146

$$
\text { Barbary, v. } 146
$$

Cape, v. 146

Schelch, v. 306

Schermaus, v. 212

Sciuropterus, v. 259

Sciurus, iii. 82.172 ; v. 248

Abyssinicus, v. 258

æstuans, v. 256

affinis, iii. 188 ; v. 251

albiceps, v. 251

albovittatus, v. 257

Alpinus, iii. 176 ; v. 249

annulatus, iii. 190 ; v. 257

anomalus, v. 258
Sciurus avellanarius, v. 221

bicolor, iii. 180 ; v. 251

bilineatus, v. 251

bivittatus, v. 257

Braziliensis, v. 257

capistratus, iii. 81 ; v. 253

Carolinensis, v. 254

Ceylonensis, v. 250

Ceylonicus, v. 250

cinereus, iii. 83.177 ; v. 254

Virginianus major, v. 254

Clarkii, v. 255

degus, v. 221

Dschinchicus, v. 257

epilepticus, v. 220

erythræus, v. 258

erythropus, v. 257

felenus, v. 259

Finlaysonii, v. 252

flavus, v. 258

Getulus, iii. 83. 185 ; v. 228. 253

Ginginianus, v. 256

glis, v. 220

grammurus, v. 255

Hudsonius, iii. 84

Indicus, v. 258

insignis, iii. 187 ; v. 256

Javanensis, v. 251

lateralis, v. 255.259

Leschenaultii, v. 251

Ludoviciana, v. 254

macrourus, iii. 83 ; v. 250.255

Madagascariensis, iii. 86 ; v. 51. 250

magnicaudatus, v. 255

maximus, iii. 83. 184 ; v. 249

melanotus, v. 259

nigrovittatus, v. 252

palmarum, iii. 84 ; v. 252

penicillata, v. 253

Persicus, v. 258

petaurista, v. 260

phaiopus, v. 259

platani, v. 251

Prevostii, v. 250

pusillus, v. 256

quadrivittatus, v. 255

quercinus, v. 220

ruber, v. 259

rubrilineatus, v. 251

rufiventer, v. 254

sagitta, iii. 85.194 ; v. 260

striatus, iii. 84

tamaricinus, v. 239

tenuis, iii. 188 ; v. 252

tridecim lineatus, iii. 171 ; v. 247

variegatus, v. 253 
Sciurus vittatus, v. 257

volans, iii. $85.191 ;$ v. 259

volucella, iii. 85.191 ; v. 259

vulgaris, iii. 82 ; v. 249

Zeylonicus, v. 250

Scotophilus, v. 84

Kuhlii, v. 84

Scythicus volans, v. 259

Seal, bearded, v. 178

black, v. 181

bottle-nosed, ii. 58; v. 181

brown-eared, ii. 509

common, ii. 57 ; v. 176.181

cowled, v. 179

crescent, ii. 57

eared, v. 183

Falkland Island, v. 183

fetid, v. 178

great, v. 178

Greek, v. 179

Greenland, v. 177

hare, v. 176

harp, v. 177

hooded, ii. 58. 507

leonine, v. 184

little, ii. 509

long-necked, v. 181

maned, ii. 59

Mediterranean, v. 179

Peron's, v. 180

proboscis, v. 180

red, v. 179

red-eared, ii. 509

ribbon, v. 181

small-clawed, v. 178

speckled, v. 181

spotted, v. 181

tiger, v. 181

tortoise-headed, v. 181

two-coloured, v. 177

white-bellied, ii. 58

white-tailed, v. 177

wolf, v. 181

yellow, ii. 59

Seals, ii. 56. 495

king of, v. 181

Segh, v. 306

Seloc, v. 375

Semnopithecus, v. 9

comatus, v. 10

entellus, v. 10

Maurus, v. 10

melalophus, v. 10

pruinosus, v. 10

Seraph, v. 321

Seraphah, v. 321

Serotine, ii. 150

de la Guyane, grande, v. 9 l great, v. 91

Cuv. Index.
Serval, ii. 481 ; v. 167

Setiger ecaudatus, v. 109

inauris, v. 109

variegatus, v. 109

Setter, ii. 333

Sewellel, v. 245

Sheep, the, iv. 25

Ardennoise, iv. 342

Astracan, iv. 329

Barbary, iv. 328

bearded, iv. 319

big-horned, iv. 318

black-faced heath, iv. 337

Cheviot, iv. 341

Circassian, iv. 330

Congo, iv. 326

Cretan, iv. 331

Dishley, iv. 340

domestic, iv. 324 ; v. 361

Dorset, iv. 337

dun-faced, iv. 338

Egyptian broad-tailed, iv. 328

fat-rumped, iv. $\mathbf{3 3 0}$

Flemish, iv. 341

goitered, iv. 327

Guinea, iv. 326

Hardwicke, iv. 341

Hebridean, iv. 339

Herefordshire, iv. 338. 340

Hottentot broad-tailed, iv. 327

long-legged, iv. 325

many-horned, iv. 331

Merino, iv. 332

Morocco, iv. 326

Mysore, iv. 330

New Leicester, iv. 340

Norfolk, iv. 337

Roussillonne, iv. 342

South Down, iv. 340

Solognot, iv. 341

Teeswater, iv. 340

Wallachian, iv. 332

wild, v. 359

Wiltshire, iv. 338

Zetland, iv. 339

Shial, v. 375

Shrew, Cape, ii. 183; v. 103

collared, v. 104

common, ii. 17 ; v. 102

Daubenton's, ii. 177

flat-tailed, v. 102

Indian, ii. 183; v. 103

musk, v. 105

oared, ii. 182 ; v. 103

rat-tailed, ii. 184; v. 104

square-tailed, v. 102

streaked, v. 103

striped, ii. 181

Tuscan, v. 104 
Shrew, water, ii. 18. 177; v. 102 white-toothed, ii. 181 ; v. 103

Shrews, ii. 17. 172

Si-nyn, v. 375

Siagous, ii. 490

Siamang, i. 255 ; v. 6

Sicista, v. 228

Sigmodon, v. 213

Sikistan, v. 227

Simia, i. 206. 339. 348. 350 ; v. 4.19 Æthiops, i. 211 ; v. 16 agrias, v. 5 amicta, v. 34 apedia, v. 23 apella, i. 223 ; v. 29 argentata, i. 227 ; v. 40 Atys, v. 16

auritus, v. 39 aygula, i. 216

Belzebuth, i. 222.305 ; v. 27 capileata, i. 278 capucina, i. 223 ; v. 32 cephus, i. 213 ; v. 12 chiropotes, v. 36 cinerea, v. 23 comosa, v. 8 cyanocephala, i. 216 ; v. 18.20 cynomolgus, i. 216 ; v. 18 cynosurus, i. 212 ; v. 14 dentata, v. 11

Diana, i. 212 ; v. 13

Diana faunus, v. 13 entellus, i. 210 ; v. 10 erythrea, i. 217.290 ; v. 18 fatuellus, i. 224 ; v. 31 faunus, i. 212 ; v. 14

ferox, v. 16

ferruginea, v. 8

flava, v. 30

fuliginosa, i. 211

fusca, v. 19

Geoffroyi, v. 39

Hamadryas, i. 218 ; v. 21

humeralifer, v. 39

hypoleuca, v. 32

inuus, i. 215 ; v. 19

Jacchus, i. 226 ; v. 38

lagothricha, v. 26

lar, i. 208 ; v. 6

leonina, i. 216 ; v. 16.42 leucisca, i. 209.258 ; v. 6 leucophœa, i. 219 ; v. 22

longimana, i. 253 ; v. 6

lugens, i. 320 ; v. 33

maimon, i. 219 ; v. 2]

Maura, i. 211

melalophus, v. 10

melanocephala, i. 318 ; v. 38

Midas, i. 226 ; v. 40
Simia, mona, i. 212; v. 12 monacha, i. 212 ; v. 12 mormon, i. 219 ; v. 21 nasalis, v. 9

nasica, i. 213 ; v. 9 nemæa, i. 214 ; v. 9 nemestrina, i. 217 ; v. 18 nictitans, i. 213 ; v. 12 nigra, v. 13

CEdipus, i. 226 ; v. 43 paniscus, i. 223 ; v. 24 patas, v. 13

personata, v. 33. 275 petaurista, i. 213 ; v. 13 pileata, v. 12

pithecia, i. 225 ; v. 36 pithecus, i. 215 ; v. 19 platypygos, i. 217 ; v. 19 polycomus, v. 7 porcaria, i. 218 ; v. 20 pygmæa, v. 4 roloway, v. 13 rosalia, i. 226 ; v. 42 rubra, i. 211

Sabæa, i. 212 ; v. 14 sangulata, v. 35 satanas, i. 225. 318 satyra, i. 207 satyrus, i. 350 ; v. 4 sciurea, i. 224 ; v. 33 seniculus, i. 222 ; v. 26 silenus, i. 216. 285 ; v. 16 silvanus, i. 215 ; v. 19 Sinica, i. 216 ; v. 17 sphyngiola, i. 218 ; v. 20 sphynx, i. $218 ;$ v. 21 sublutea, v. 22 syndactyla, i. 255 ; v. 6 . sylvestris, i. 219 sylvicola, v. 22 talapoin, v. 11 trivirgata, i. 313 trepida, v. 30

Troglodytes, i. 209 ; v. 4 ursina, i. $218 ;$ v. 20 veter, v. 11 villosa, v. 10

Simpai, v. 10

Simung, v. 132

Sinæthere, v. 264 prehensilis, v. 264

Singe à queue de Cochon, v. 18, 19 cheiropotes, i. 316 de Moco, i. 218; v. 21 de nuit, v. 36 de Wurmb. v. 5 noir, i. 218 ; v. 20 vert, v. 14

Sins ring, ii. 212 
Sirèn, iv. 432

Sirenia, v. 377

Skunk, v. 127

Sleeper, v. 221

Sloth, New Holland, v. 205

of Bengal, i. 229

three-toed, v. 274

two-toed, v. 275

ursiform, v. 113

ursine, ii. 238

Sloths, iii. 252. 266

Sobol, v. 124

Solidungula, i. Ixxvi. Ixxviii.

Solipeda, i. lxxvii.

Solipedes, iii. 340

Soora Goy, v. 375

Sorex, ii. 17. 172; v. 101

aquaticus, ii. 19. 174.191 ; v. 105. 107

araneus, ii. 17 ; v. 102

araneus maximus, v. 104

araneus maximus Capensis, ii. 184.

auratus, ii. 174

Brasiliensis, ii. 174 ; v. 105

cœcutiens, v. 104

Capensis, v. 103

carinatus, ii. 178; v. 102

collaris, v. 104

constrictus, ii. 179 ; v. 102

cristatus, ii. 23 . 174 . 210 ; v. 105. 110

cunicularis, v. 103

Daubentonii, ii. 173 ; v. 102

Etruscus, v. 104

exilis, v. 104

fodiens, ii. 18 ; v. 102

glis, v. 106

Javanica, ii. 211

Indicus, ii. 183; v. 103

leucodon, ii. 181 ; v. 103

lineatus, ii. 181 ; v. 103

minimus, v. 104

minutus, v. 104

moschatus, ii. 18.174 ; v. 105

Moschoviticus, v. 105

murinus, v. 104

myosurus, ii. 184 ; v. 104

pusillus, v. 105

pygmæus, v. 104

remifer, ii. 182 ; v. 103

Surinamensis, ii. 174

tetragonurus, ii. 178 ; v. 102

Souslik, iii. 81.169 ; v. 246

Spalax, iii. 77. 160

major, v. 241

microphthalmus, v. 241

minor, v. 216

vittata, v. 242
Spaniel, ii. 41.332 ; v. 136

Alpine, ii. 333 ; v. 137

great water, ii. $\mathbf{3 3 4}$

King Charles', v. 137

little, v. 137

Newfoundland, v. 137

smaller, ii. $\mathbf{3 3 3}$

Spasm of Ternate, ii. 11

Spatling, v. 88

Spermophilus, v. 246

Sphiggurus, v. 264

hairy, v. 264

spiny, v. 264

villosus, v. 264

Springbock, iv. 17

Squirrel, allied, v. 251

Alpine, iii. 176 ; v. 249

annulated, v. 257

aye aye, v. 51

Barbary, iii. 185 ; v. 253

barking, v. 248

black, v. 253

black banded, v. 252

Bokol, v. 256

burrowing, v. 245

Carolina, iii. 177 ; v. 254

Ceylon, v. 250

Chilian, v. 221.

Clarke's, v. 255.

common, iii. 82 . I72 ; v. 249

common flying, v. 259

European flying, v. 259

federation, v. 247

Finlayson's, v. 252

flying, iii. 85.194 ; v. 259

four banded, v. 255

Georgian, v. 258

gingi, iii. 189 ; v. 256

grey, iii. 83. 177 ; v. 254

great, iii. 184 ; v. 249

ground, iii. 84 ; v. 247

guerlinguet, v. 256

of Hudson's Bay, iii. 84

Javan, v. 251

large, iii. 83

large-tailed, v. 255

Leschenault's, iii. 179 ; v. $25 \mathrm{I}$

Lewis's, iii. 190 ; v. 257

line-tailed, v. 255

long tailed, v. 250

Madagascar, v. 250

masked, iii. 83. 178 ; v. 253

Mexican, v. 258

Norfolk Island, v. 200

palm, iii. 185 ; v. 252

plantain, v. 251. 258

Prevost's, v. 250

red-bellied, v. 254

red river, v. 254

G 2 
Squirrel, ruddy, v. 258 sailing, v. 260 Siberian flying, iii. 191 side marked, v. 255

$\triangle$ slender, v. 252 two banded, v. 257 two coloured, v. 251 two rayed, v. 251 white striped, v. 253

Squirrels, iii. 82

Stag, iv. 8.90 ; v. 307

of America, v. 308

Barbary, v. 307

Canadian, iv. 12.96

Cape, iv. 18

common, iv. 11

Corsican, v. 307

fossil of America, iv. 104; v. 309

great water, v. 311

Indian, iv. 12

Jamboe, v. 311

of Louisiana, iv. 12

Nepaul, iv. 103 ; v. 309

north-western, iv. 101 ; v. 308

of Virginia, iv. 12

Steatopyga, iv. 330

Steenbock, iv. 248

Vlackte, iv. 249

Stellere, v. $\mathbf{3 8 0}$ northern, v. 380

Stelleres, iv. 432.448 ; v. 380 Borealis, v. 380

Stematope, v. 179

Stenoderma, v. 66 rufa, v. 67

Stenoderme, v. 67 roux, v. 67

Stenodermes, ii. 8.

Stenops, i. 229 ; v. 47

Bengalensis, v. 47

gracilis, v. 47

Stentor flavicaudatus, v. 27

fuscus, v. 27.

niger, v. 28

seniculus, v. 26 .

stramineus, v. 27

ursinus, v. 27

Stepnie barani, v. 359

Stoat, v. 122

Strepsiceros, iv. 359 ; v. 328

Strepsicerotine group, iv. 359

Stylocerine group, iv. 143

Stylocerus, v. $\mathbf{3 1 9}$

Subr, v. 373

Subulo, v. 318

Subulonine group, iv. 139

Suhah, v. 336

Sukerkan le, v. 216
Sulok, v. 336

Sulgam, vi. 269

Suricata, v. 158

Capensis, v. 158

Suricate, ii. 399 ; v. 158

Surikate, ii. 48 ; v. 158

Surmulot, iii. 74.128 ; v. 224

Sus, iii. 330.401 ; v. 287

Africanus, iii. 332 ; v. 288

Angalla, v. 289

aper, v. 288

aquaticus multisulus, v. 293

babiroussa, iii. 332.408 ; v. 288

ferus, v. 288

hydrochœrus, v. 270

koiroptamus, v. 288

Tajassa, iii. 334

larvatus, iii. 332.407 ; v. 288

maximus palustris, v. 270

Papuensis, v. 288

scrofa, iii. $331.402 ;$ v. 287

tagassu, v. 290

Svartside, v. 177

Swartside, ii. 57

Swine, iii. 330. 401

Swinewal, v. 388

Tachyglossus, iii. 263

Taguan, iii. 82 ; v. 260

Taguicati, iii. 334 ; v. 290

Taira, ii. 32.278 ; v. 117

Taitetou, iii. 334

Tajassou, iii. 334. 413 ; v. 290

Takhaitze, v. 325

Talapoin, v. 11

Talpa, ii. 22.194 ; v. 108

Asiatica, ii. 20. 193 ; v. 107

cristata, ii. 23

Europæa, ii. 23 ; v. 108

fusca, ii. 191 ; v. 107

longicaudata, v. 110

maculata, v. 108

rubra, ii. 20.193

Sibirica aurea, v. 107

variegata, v. 108

vulgaris, v. 108

Tamandua, iii. 260 . 303 ; v. 280

Brasiliensibus, v. 280

ganga, v. 280

Tamanoir, iii. 260 ; v. 280

Tamarin, i. 218.226 ; v. 40

black, i. 226

leonine, v. 42

negro, v. 41

silky, v. 42

white fronted, v. 41

white lipped, v. 41

yellow fronted, v. 41 
Tamias, iii. 84

Tanrec, v. 109

$$
\text { jeune, v. } 110
$$

Tao, v. 327

Tapeti, iii. 221 Brasiliensis, v. 268

Taphien filet, ii. 12 perforated, ii. 12

Taphiens, ii. 12

Taphozous, ii. 12 lepturus, v. 82 Mauritianus, v. 82 perforatus, v. 82 Senegalensis, v. 81

Tapiluies, v. 294

Tapir, iii. 339 ; v. 293 American, iii. 431 ; v. 293 Americanus, iii. 340 ; v. 293 Indicus, v. 294

Malay, iii. 433 ; v. 294

Malayanus, v. 294

Mapouri, v. 293

Sumatrensis, v. 294

thick nosed, v. 270

Tapiti, v. 267

Tapoa tafa, ii. 68 ; v. 193.197

Tapoo tafa, iii. 38

Tapurete Brasiliensibus, v. 293

Tarandus, v. 305

Tardigrada, i. Ixxvii. Ixxix.

Tardigradus, v. 274

Ceylonicus catulus, v. 275

Tardigrades, iii. 251

Tarsier, v. 50

Bankanus, v. 5 l

fuscomanus, v. 51 yellow headed, v. 50

Tarsiers, i. 230

Tarsius, i. 230 ; v. 50

Bankanus, v. 51

Daubentonii, v. 50

Fischerii, v. 51

fuscomanus, v. 5]

Pallasii v. 50

spectrum, v. 50

Tartarin, i. 218 ; v. 21

Tatou apara, iii. 256 ; v. 276

black, iii. 257

eight-banded, v. 276

giant, iii. 258 ; v. 277

great, iii. 258 ; v. 277

mataco, v. 276

mulet, v. 277

mustelinus, v. 277. 282

nine banded, v. 276

noir, v. 276

peba, iii. 257 ; v. 276

pichiy, v. 279

poyou, v. 278
Tatou, six banded, iii. 257

tatouay, v. 277

three banded, iii. 256

twelve banded, iii. 257

velu, v. 279

Tatouay, iii. 257

Tatouhou, v. 276

Tatous, iii. 255 ; v. 278

Tatusies, v. 276

Taupe, v. 108 de Canada, v. 110 des Dunes, v. 242 grande, du cap, v. 242

Taurelephantes, v. 372

Taurine group, iv. 411

Taurus, v. 375

Taxus, v. 116 meles, ii. 275

Taytetou, v. 290

Tekus Urrok, v. 229

Teledu, ii. 305

Telagon, v. 129

Temary, v. 40

Temnamazame, v. 355

Tendrac, ii. 21. 207; v. 109

Tenrac, varied, ii. 208

Tenrec, ii. 21 ; v. 109 radiated, ii. 22 ; v. 109

Tenrecs, ii. 21. 206

Tepemaxtla, v. 128

Terrier, ii. 41. 331 black and $\tan$, v. 142 Scotch, v. 143

Tesbe, v. 331

Teschichi, v. 141

Tetracerine group, iv. 253

Tetracerus, v. 343 quadricornis, v. 344

Thlaquatrin, ii. 63

Thur, v. 376

Thybais nasuta, v. 195

Thylacinus Harrisii, v. 192

Thylaris obesula, v. 195

Thyroptera, v. $\mathbf{8 6}$ three coloured, v. 86 tricolor, v. 86

Tiger, v. 163 American, ai. 51

Bengal, ii. 440

black, ii. 473

clouded, ii. 449 ; v. 164

hunting of India, ii. 52

Iltis, v, 121

nebulose, ii. 449

royal, ii. 50.440

tortoiseshell, ii. 450 ; v. 164

white, ii. 444

Tigre, le, v. 163

Tigris Americana, v. 164 
Tigris fulva, v. 163

Titi, i. 226. 312 ; v. 33

de Carthagène, v. 43

Tjuja Tue, v. 298

Tolai, v. 267

Tolypeutes, v. 276

Tonatia, v. 71

Tong, v. 298

Touan, ii. 66 ; iii. 33 ; v. 190

Tragelaphine group, iv. 272

Tragelaphus, v. 350.360

Trago camelus, iv. 21

Traguline group, iv. 247

Tragulus, v. 340 memina, v. 302

Tragus moschiferus, v. 301

Trichecus, ii. 59. 511 ; v. 184. 377

Australis, v. 379

Borealis, v. 380

manatus, iv. 43]

rosmarus, ii. 61.512 ; v. 184

Troglodytes, v. 4 niger, $\mathrm{v}, 4$

Tseiran, iv. 20

Tucan, ii. 20

Tuhcuri, v. 122

Tupaia, ii. 211 ; v. 106 ferruginea, ii. 216 ; v. 106 Javanica, ii. 211 ; v. 106 press, ii. 211 tana, ii. 211 ; v. 106

Tupai, v. 257

Turnspit, ii. 336 ; v. 139 crooked-legged, v. 139

Tutucuri, ii. 291

Tzebi, v. 332

Tzeiran, iv. 20 ; v. 324. 332

Unau, iii. 254. 275 ; v. 275 little, v. 275

Unicorn, v. 329 sea, v. 387

Urchin, ii. 162

Urksuk, v. 178

Urson, iii. 88.206 ; v. 263

Ursus, ii. 26 ; v. 111

albus, v. 112

Americanus, ii. 27. 230 ; v. 112

Arctos, ii. 26. 217 ; v. 111

cancrivorus, ii. 29 ; v. 114

candescens, ii. 229 ; v. 112

cinereus, v. 112

ferox, v. 112

gulo, ii. 31. 276 ; v. 117

horribilis, v. 112

Indicus, v. 118

labiatus, ii. 238 ; v. 113

Labradoricus, เ. 116
Ursus longirostris, v. 113

lotor, ii. 28. 246 ; v. 114

luscus, ii. 32 ; v. 117

luteolus, ii. 228

Malayanus, ii. 236; v. 113

marinus, v. 182

maritimus, ii. 28. 232; v. 112

meles, ii. 31 ; v. 116

niger Americanus, v. 112

niger Europæus, v. 111

Thibetanus, ii. 243 ; v. 113

Urus, iv. 29 ; v. 376

fossil, iv. 414

white, iv. 417

Uyyol, v. 310

Vache brune, petite, v. 335

marine, v. 378

Vampyre, ii. 9

bearded of Sphix, v. 72

soricine of Sphix, v. 72

two-toothed, of Sphix, v. 72

Vampyrus, v. 71

bidens, v. 72

cirrhosus, v. 72

soricinus, v. 72

spectrum, v. 71

Vansire, v. 157

Vari, i. 228; v. 44

Varieties, intellectual, i. 170

Venegas, v. 359

Vespertilio, ii. 3. 14 ; v. 87 acetabulosus, ii. 8 ; v. 65 adversus, v. 92

albescens, ii. 156 ; v. 96

Americanus, ii. 126

Americanus vulgaris, v. 70

auritus, ii. 14. 152 ; v. 97

barbastellus, ii. 147; v. 97

Bechstein's, v. 95

black, v. 96

Borbonicus, ii. 154; v. 94

bordered-eared, v. 94

Bourbon, ii. 154 ; v. 94

Brasiliensis, v. 94

Carolinensis, ii. 143 ; v. 93

cephalotes, ii. 113 ; v. 59

cornutum, v. 7]

Daubentonii, v, 96

dingy, v. 94

discolor, v. 94

emarginatus, ii. 153 ; v. 94

ferrum equinum, ii. 11 ; v. 76

Hardwickii, v. 92

hastatus, ii. 9.126 ; v. 69

Hilarii, v. 91

hippocrepis, v. 76

hipposideros, ii. 11 ; v. 76 
Vespertilio, hispidus, ii. 12.137 ; v. 80 imbricatus, v. 93

indented, v. 91

ingens, ii. 107 ; v. 55

kirivoula, v. 90

Kuhlii, v. 95

levis, v. 92

lasiopterus, ii. 155 ; v. 89

lasiurus, ii. 154 ; v. 90

Leisler's, v. 95

leporinus, ii. 139 ; v. 67

lepturus, ii. 12 ; v. 82

major vulgaris, v. 88

marsupialis, v. 82

maugei, v. 97

maximus, ii. 155

microphyllus, ii. 12 ; v. 81

minor, ii. 152

minutus, v. 76

molossus, v. 60

murinus, ii. 13.142 ; v. 88

myotis, v. 88

mysticinus, v. 94

nasutus, v. 91

Nattererii, v. 95

nigrita, ii. 154 ; v. 90

noctula, ii. $13.144 ;$ v. 88

Novaboracensis, ii. 155 ; v, 98 perspicillatus, ii. 9.126 ; v. 69

pictus, ii. 153 ; v. 90

pipistrellus, v. 89

plicatus, ii. 8. v. 64

polythrix, v. 91

proterus, v. 89

pygmæus, v. 91

red, v. 96

red brown, v. 94

ruber, ii. 156 ; v. 96

Schreiber's, v. 95

serotinus, ii. 13.150 ; v. 88

shaggy, v. 96

silvery, v. 96

smooth, v. 92

soricinus, ii. 10; v. 73

spama, ii. 11 ; v. 75

spectrum, ii. 9 ; v. 7 ]

speoris, ii. 11; v. 77

Temminckii, v. 92

Ternatanus, v. 90

Timorensis, ii. 154 ; v. 98

tralatitius, v. 93

transposed, v. 93

vampyrus, ii. 102 ; v. 55

villosissimus, ii. 155 ; v. 96

Vespertilionidæ, v. 54

Vicognes, v. 300

Vicugna, v. 300

Vicunna, iv. 58 ; v. 300
Viduita, i. 320 ; v. 33

Vigogne, iv. 6

Viscaccia, v. 272

Viscache, iii. 230; v. 272

Vison, ii. 295 ; v. 124

Viverra, ii. 45 ; v. 152

bentourong, v. 160

bondar, v. 155 .

Cafra, ii. 47 ; v. 157

Capensis, v. 118

caudivolvula, ii. 30 ; v. 115

chinge, v. 127

civetta, ii. 46.381 ; vo 152

conepatl, v. 127

fasciata, ii. 46 ; v. 154

fossa, ii. 390 ; v. 154

galera, v. 157

genetta, ii. 46 ; v. 154

gracilis, v. 160

hyenoides, ii. 390 ; v. 156

ichneumon, ii. 47 ; v. 156

Indica, v. 155

Lesang, v. 160

maculata, v. 193

Malacensis, v. 154

mapurito, v. 128

marputio, v. 127

mellivora, ii. 33 ; v. 118

mephitis, v. 127

mongoz, v. 156

mungos, ii. 47

musanga, ii. 416 ; v. 159

narica, ii. 29

nasica, v. 115

nasua, ii. 29. 253

nigra, v. 159

prehensile, v. 159

putorius, v. 127

Quasje, v. 115

rase, ii. 383 ; v. 153

rufa, v. 115

striata, v. 127. 155

suricate, ii. 404

tetradactyla, ii. 48.404 ; v. 158

tigrina, v. 154

vittata, ii. 32.277 ; v. 117

zenick, v. 158

zibetha, ii. 46 ; v. 153

zorilla, ii. 35. 291 ; v. 123

Viverræ, ii. 377

Vohangshira, v. 157

Volitantia, i. Ixxix.

Vulpes, v. 147

alopex, v. 147

Americanus, v. 114

cœrulescens, v. 149

Vulpes, crucigera, v. 147

vulgaris, v. 147 
WA WAS KEESKO, v. 308

Wadan, v. 372

Walrus, Indian, v. 380

Wapiti, iv. 96 ; v. 308

Wargul, v. 132

Wasser maus, v. 209

Weasel, ii. 35 ; v. 121

Altaican, v. 120

American mephitic, v. 127

Cape, v. 118

chestnut, v. 125

common, ii. 289, v. 121

fasciated, v. 155

Fischer, ii. 297 ; v. 125

Fizzler, v. 118

fossane, v. 154.

great, of Azara, v. 118

grey-headed, v. 126

Guiana, v. 126

honey, v. 118

Madagascar, v. 157

mephitic, v. 129

musky, v. 126

Pekan, ii. 296 ; v. 124

Perouasca, v. 121

sable, v. 124

Sarmatian, ii. 288 ; v. 121

Siberian, ii. 291 ; v. 122

slender-toed, v. 126

South American, v. 126

striated, v. 127

white-cheek, v. 126

white-eared, ii. 297 ; v. 126 woolly, v. 126

Weasels, mephitic, ii. 297

Wha topoa voo, v. 197

Whale, bossed, v. 390

common, iv. 441.491 ; v. 389

knotted, v. 390

pike-headed, v. 478

Whales, iv. 440 proper, iv. 493

Whistler, iv. 435

Wilde paarde, iii. 468

Witfisch oder weissfisch, v. 386

Wizend, v. 373

Wolf, ii. 41.348 ; v. 144

Antarcticus, v. 145

black, ii. 42.348 ; v. 144

crab, v. 145

golden, ii. 42

Java, v. 144

Mexican, ii. 350

red, ii. 42 ; v. 144
Wolf, sea, ii. 58 ; v. 176

Wolverene, ii. 32 ; v. 117

Wombat, ii. 78 ; iii. 50 ; v. 206

Wombatus, v. 206

Woodchuck, v. 245

Wou wou, i. $209.254,258$; v. 6

Wow wow, v. 6.

$\mathrm{XE}, \mathrm{v} .301$

Xolsitzaintli, v. 145

Y ACK, iv. 30

Yagouare, v. 127

Yagouarete, v. 164

Yagouaroundi, ii. 486 ; v. 170

Yamus, v. 372

Yapock, v. 1.92

Yarke, i. 225 ; v. 36

Yarque, v. 37

Yazmur, v. 327

Yerbua Capensis, v. 243

Indica, v. 239

Y'Gann, v. 365

Yogouy, v. 280

Yzard, iv. 22; v. 353

Zamouse, v. 371

Zebra, iii. 343. 465 ; v. 295

femelle, v. 295

Indica, v. 296

Zebu, common, iv. 425

Zemni, iii. 161

Zenick, ii. 48

Zerda, i. 332; v. 152

Zibet, ii. 46.381 ; v. 153

Zikel, iii. 81

Zizel, v. 246

Zocor, iii. 69

Zokor, v. 216

Zomba, iv. 327

Zomer, v. 321

Zoology, rise and progress of, i. xiii.

Zoreille, v. 123

Zorille,.ii. 35. 291 ; v. 127

Zorillo, ii. 291 ; v. 123

Zorra, ii. 297 ; v. 125.128

Zubr, iv. 29

Zumni sleeper, iii. 78

Zunu, iv. 327

Zuraphate, v. 32]

Zurnapa, v. 321 


\section{A VE S.}

Abou Duch'n, vi. 192 hannes, viii. 359

Acahé, vii. 180

Acanthiza, vi. 470

Buchanani, vi. 471

frontalis, vi. 471

nana, vi. 470

pusilla, vi. 471

pyrrhopygia, vi. 471

reguloides, vi. 471

Accentor, vi. 465 ; vii. 7.35

Europæan, vi. 466

modularis, vii. 37

montanellus, vi. 466 ; vii. 39

Accipiter, vi. 53. 236

Delafonii, vi. 239

ruficaudus, vi. 62

Accipitres, vi. 14 suppl. on the, vi. 156

Acridotheres, vi. 422

Adjutant, viii. 505

Ægotheles, vii. 80

Erithina atricapilla, vi. 449

Agami, viii. 327. 469

Agelaius, vii. 168 ; viii. 688 badius, vii. 1.71

chrysopterus, vii. 167

cyanopus, vii. 171

frontalis, vii. 171

guirahuro, vii. 169

longirostris, vii. 171

pyrrhopterus, vii. 171

ruficapillus, vii. 171

textor, vii. 131

virescens, vii. 171

Aiaiai, viii. 508

Aigle autour huppé, vi. 43

de la Guiane, grand, vi. 42 petit, vi. 43

Ajaja, viii. 512

moyen de la Guyane, vi. 45

Akbobas, vi. 187

Alauda, vii. 116

Africana, vi. 479 ; vii. 119

Alpestris, vii. 118. 210

apiata, vii. 117. 210

arborea, vii. 117. 207

arvensis, vi. 116

Cuv. Index.

\author{
Alauda bifasciata, vii. 119 \\ bilopha, vii. 119. 212 \\ brachydactyla, vii. 117. 209 \\ calandra, vii. 118 \\ campestris spinoletta, vi. 478 \\ Capensis, vi. 479 \\ cinerea, vii. 120 \\ crassirostris, vii. 119 \\ cristata, vii. 116. 205 \\ crocea, vii. 119 \\ cunicularia, vii. 119 \\ Deserti, vii. 119 \\ flava, vii. 118 \\ fulva, vi. 479 \\ Gingica, vii. 120 \\ Italica, vii. 117 \\ Ludoviciana, vi. 479 \\ Lusitania, vi. 479 \\ magna, vii. 118. 173 \\ Malabarica, vii. 120 \\ melanocephala, vii. 119 \\ minor, vi. 477 \\ mirafa, vii. 119 \\ Mosellana, vi. 478 \\ mutabilis, vii. 118 \\ nemorosa, vii. 117 \\ Novæ Zealandiæ, vii. 120 \\ obscura, vi. 478 \\ pratensis, vi. 477 \\ pyrrhonotha, vii. 117 \\ rubra, vi. 479 \\ rufa, vi. 479 \\ rufescens, vi. 478 ; vii. 119 \\ rufipilea, vii. 118 \\ Senegalensis, vii. 120 \\ Sibirica, vii. 118 \\ spinoletta, vi. 479 \\ Tatarica, vii. 118 \\ trivialis, vi. 477 \\ undata, vii. 117 \\ Yeltoniensis, vii. 120 \\ Albatross, viii. 571 \\ brown, viii. 572 \\ chocolate, viii. 572 \\ eye-streaked, viii. 572 \\ short-tailed, viii. 572 \\ sooty, viii. 572. 573 \\ wandering, viii. 571


Albatross, yellow-nosed, viii. 573

Albatrosses, viii. 642

Alca, viii. 556. 558

antiqua, viii. 558

Arctica, viii. 556

Balthica, viii. 559

cirrhata, viii. 557

cristatella, viii. 557. 636

impennis, viii. 559. 637

Labradoria, viii. 556

minor, viii. 559

pica, viii. 559

psittacula, viii. 557. 635

pygmæa, viii. 557

sulcata, viii. 559

tetracula, viii. 557

torda, viii. 559. 636

Alcedo, vii. $407 ; 424$

Egyptia, vii. 413

Afra, vii. 408

halcyon, vii. 408

Amazonia, vii. 413

Americana, vii. 409

Asiatica, vii. 410

atricapilla, vii. 410

Bengalensis, vii. 409

bicolor, vii. 408

biru, vii. 410

corulea, vii. 413

cœruleocephala, vii. 409

cancrophaga, vii. 411

Capensis, vii. 410

Cayanensis, vii. 413

chlorocephala, vii. 411

cinereifrons, vii. 410

collaris, vii. 413

Coromandelica, vii. 411

cristata, vii. 409

crithaca, vii. 413

dea, vii. 411

diops, vii. 412

flavicans, vii. 413

fusca, vii. 413.426

galbula, vii. 436

gigantea, vii. 413.426

grandis, vii. 437

ispida, vii. 407

leuchoryncha, vii. 413

leucocephala, vii. 411

maculata, vii. 413.473

Madagascariensis, vii. 409

melanoptera, vii. 413

melanoryncha, vii. 412

meninting, vii. 414

Novæ Guineæ, vii. 413

omnicolor, vii. 412

purpurea, vii. 409

rudis, vii. 408

sacra, vii. 413. 428
Alcedo, semitorquata, vii. 410 Senegalensis, vii, 411

Smyrnensis, vii. 410

superciliosa, vii 409

Surinensis, vii. 413

torquata, vii. 408

tridactyla, vii. 414

tribrachys, vii. 414

tuta, vii. 413

venerata, vii. 413

Alcyon tridactyla, vii. 438

Alectelie de Durville, viii. 401

Alectors, viii. 2. 96

Alecturus, vi. 354

tricolor, vi. 354

Alouette à gros bec, vii. 119 sentinelle, vi. 479

Amblyramphus tricolor, vi. 173

Ammadovamus, vii. 149 bimaculatus, vii. 149

Ampelis, vi. 358. 503

Americana, vi. 365

astropurpurea, vi. 361

aureola, vi. 361

cœrulea, vi. 360

carnifex, vi. 359

carunculata, vi. 366. 505

Cayana, vi. 360

cinerascens, vi. 361

cinerea, vi. 359

cotinga, vi. 360

cristata, vi. 360

cucullata, vi. 366

cuprea, vi. 360.361

fusca, vi. 360

garrulus, vi. 364

garrulus,var. vi. 365

hypopyrra, vi. 361

lutea, vi. 355 ; viii. 683

Maynana, vi. 360

nudicollis, vi. 366

Pompadour, vi. 360

purpurea, vi. 361

tersa, vi. 360

umbellata, vi. 358

variegata, vi. 360.366

Anabates, vii. 346.388

amaurotis, vii. 346

atricapillus, vii. 347

cristatus, vii. 347

erythropthalmus, vii. 347

leucopthalmus, vii. 347

rufifrons, vii. 347

striolata, vii. 347

Anadorhynchus Maximiliani, vii. 482

Anas, viii. 597. 607. 616.657

acuta, viii. 618

adunca, viii. 619

Africana, viii. 613 
Anas albeola, viii. 611 albifrons, viii. 602 Alexandrini, viii. 625 anser, viii. 600. 601 Antarctica, viii. 605 Arabica, viii. 625 arborea, viii. 620 arcuata, viii. 623 atrata, viii. 599 autumnalis, viii. 620 Bahamensis, viii. 622 Beringii, viii. 603 bicolor, viii. 616. 624 Borealis, viii. 625 boschos, viii. 619 brachyptera, viii. 609 . 665 Brasiliensis, viii. 624 bucephala, viii. 611 cana, viii. 606. 622

Canadensis, viii. 599. 600 candidus, viii. 603 Capensis, viii. 624 caryophyllacea, viii. 614 casarea, viii. 622 cineraceus, viii. 608 cinerea, viii. 609 circia, viii. 623 clangula, viii. 610 clypeata, viii. 616. 669 Coromandelica, viii. $\mathbf{6 0 6}$ coscoroba, viii. 603 cristata, viii. 614. 624 curvirostra, viii. 619 cyanirostris, viii. 624 cyanoptera, viii. 624 cygnoides, viii. 600 cygnus, viii. 660 Damiatica, viii. 625 discors, viii. 671. 624 dispar, viii. 615 Dominica, viii. 614 Dominicana, viii. 625 Egyptica, viii. 604 epicuteri, viii. 624 ery thropos, viii. 604 fasciata, viii. 617 ferina, viii. 612.668 ferruginea, viii. 615 flavirostris, viii. 624 formosa, viii. 624 frænata, viii. 613 fuligula, viii. 614 fulva, viii. 615 fusca, viii. 608 fuscescens, viii. 624 galericulata,viii. 619 Gambensis, viii. 600 gattair, viii. 625 Georgica, viii. 624
Anas gerra, viii. 620 glacialis, viii. 609 glaucion, viii. 610 Gmelini, viii. 6 I6 grandis, viii. 603 Hina, viii. 624 histrionica, viii. 610 hybrida, viii. 603 hyperborea, viii. 608 Indica, viii. 603 Islandica, viii. 615 jaspedea, viii. 624 Javanica, viii. 623 jubata, viii. 621.670

Kalmani, viii. 624

Kekuschka, viii. 625

Labradora, viii. 615

leucomelas, viii. 621 leucophtalmos, viii. 613.669 leucoptera, viii. 605.608 lobata, viii. 609 maculirostris, viii. 620 Madagascariensis, viii. 606 Magellanica, viii. 605 Manillensis, viii. 624 marila, viii. 613 melanocephalus, viii. 599 melanoleuca, viii. 621 melanorhynchos, viii. 617 melanotis, viii. 601 melanura, viii. 624 membranacea, viii. 617 mersa, viii. 608 minuta, viii. 610 mollissima, viii. 611 monacha, viii. 625 montana, viii. 603 moschata, viii. 618 nigra, viii. 607.665 nigricollis, viii. 599

Nilotica, viii. 618

Novæ Hispaniæ, viii. 624

Novæ Hollandiæ, viii. 617 Nyroca, viii. 613 obscura, viii. 622 paturi, viii. 623

Penelope, viii. 621 perspicillata, viii. 608 picta, viii. 606 platalea, viii. 624 Plutonia, viii. 599 pœecilorhynchos, viii. 622 punctata, viii. 616 querquedula, viii. 623 radjah, viii. 621 regia, viii. 618 rhynchotis, viii. 617 rubida, viii. 615 rubrirostris, viii. 624 
Anas rufa, viii. 612

ruficollis, viii. 605

rufina, viii. 612

rutila, viii. 622

sarai, viii. 625

scandiaca, viii. 614

segetum, viii. 602

semipalmata, viii. 621

Sili, viii. 623

sisæir, viii. 625

spectabilis, viii. 611

spinicauda, viii. 624

spinosa, viii. 614

sponsa, viii. 620

stelleri, viii. 615

strepera, viii. 621

superciliosa, viii. 624

tadorna, viii. $\mathbf{6 1 7}$

torquata, viii. 605. 610. 624

typus, viii. 353

tzitzichoa, viii. 624

valisneria, viii. 615

variegata, viii. 606

versicolor, viii. 624

viduata, viii. 620. 671

virgata, viii. 616

Anastomus, viii. 353.510

albus, viii. 353

cinereus, viii. 353

Anhima, viii. 398

Anhinga, viii. 595

Levaillant, viii. 596

Anhingas, viii. 655

Ani, vii. 476.541

Animals, Oviparous vertebrated, vi. 1 second class of vertebrated vi. 3

Annumbi, vii. 354

Anser, viii. 601

bernicla, viii. 604

cœrulescens, viii. 603

cineraceus, viii. 602

cinereus, viii. 602

hyperboreus, viii. 603

jubatus, viii. 603

leucopsis, viii. 603

medius, viii. 602

rufescens, viii. 602

Anseranas, viii. 621

Ant-catcher, rocky mountain, vi. 409 red-bellied, vi. 545

Anteaters, vi. 399. 543 king of the, vi. 403

Anthochœra, vi. 413 Lewinii, vi. 413

Anthochœres, vi. 416

Anthropoides, viii. 329 Stanleyanus, viii. 330

Anthus, vi. 477 ; vii. 55 aquaticus, vi. 478
Anthus arboreus, vi. 477

Australis, vi. 479

breviunguis, vi. 477

campestris, vi. 478

Chi, vi. 480

fuliginosus, vi. 480

Ludovicianus, vi. 479

minimus, vi. 480

montanus, vi. 478

pallescens, vi. 480

pratensis, vi. 477

Richardi, vi. 479

rufescens, vi. 480

rupestris, vi. 478

sepiarius, vii. 56

spinoletta, vi. 479

Ant-thrush, black, vi. 284

long-legged, vi. 283

white--legged, vi. 283

white-shouldered, vi. 283

black-throated, vi. 284

white-spotted, vi. 284

Aptenodytes, viii. 559. 560. 636

Antarctica, viii. 562

Australis, viii. 563

catarrhactes, viii. 561

Chiloensis, viii. 562

chrysocoma, viii. 561. 638

demersa, viii. 563. 639

hirsutus, viii. 562

lanatus, viii. 562

Magellanica, viii. 562

minor, viii. 562

molinæ, viii. 562

Papua, viii. 561

Patagonica, viii. 638. 538

torquata, viii. 563

Apteryx, viii. 300. 562

Australis, viii. 563

southern, viii. 563

Apus, vii. 57

Aquape, viii. 395

Aquapeazo terutero, viii. 464

Aquapeccaca, viii. 395

Aquapuazos, viii. 395

Aquila, vi. 33. 43

Buson, vi. 67

chrysaëtos, vi. 225

fucosa, vi. 230

heliaca, vi. 34

picta, vi. 44

intermedia, vi. 35

Ara, vii. 481

gris à trompe, vii. 505

Ara, noir à trompe, vii. 505

Aracari, vii. 479 grooved-bill, vii. 480

Arachnothera inornata, vii. 367 longirostra, vii. 367 
Archnotheres, vii. 366. 393

Arada, vi. 544 ; vii. 45

Aramus, viii. 333.479 carau, viii. 333. 479

Araponga, vi. 366

Arara purpureo dorsalis, vii. 483

Ararauna, vii. 482

Aras, vii. 481. 574

Aratamus, vi. 287 cinereus, vi. 287

fuscus, vi. 288

lineatus, vi. 287

minor, vi. 287

Aratinga, Carolinæ, vii. 488 chrysocephalus, vii. 484 luteus, vii. 484

Ardea, viii. 335

affinis, viii. 341

agami, viii. 341

alba, viii. 338. 346.491

albicollis, viii. 340

Americana, viii. 331

Antigone, viii. 331. 332

Ardisiaca, viii. 342

argala, viii. 350.505

atricollis, viii. 337

Australis, viii. 347

badia, viii. 345

badra, viii. 345

bicolor, viii. 341

bilineata, viii. 347

botaurulus, viii. 340

botaurus, viii. 491

Brasiliensis, viii. 344

bubulculus, viii. 341

bubulcus, viii. $\mathbf{3 4 0}$

bullaranjaus, viii. 337

cœrulea, viii. 341, 342. 344

cœrulescens, viii. 337. 342

Caledonica, viii. 346

Canadensis, viii. 331

candida, viii. 339

candidissima, viii. 340

carunculata, viii. 332

Caspica, viii. 489

castanea, viii. 340

Cayennensis, viii. 346

chrysopelargus, viii. 349

ciconia, viii. 349 . 495

cinerea, viii. 336. 485

cinnamomea, viii. 347

cocoi, viii. 336

comata, viii. 339

cornuta, viii. 341

Coromandelica, viii. 341. 353

cracca, viii. 345

cyanocephala, viii. 346

cyanopus, viii. 342

cyanura, viii. 344
Ardea Danubialis, viii. 337. 347. 490

dubia, viii. 350.505

egretta, viii. 338.491

egret toides, viii. 339

equinoctialis, viii. 341

erythromelas, viii. 347

erythropus, viii. 340

exilis, viii. 347

fasciata, viii. 338

ferruginea, viii. 345

flava, viii. 338

flavicollis, viii. 342

flavirostris, viii. 339

fusca, viii. 341

fuscicollis, viii. 344

Gardeni, viii. 345

garzetta, viii. 338. 490

gigantea, viii. 331

grisea, viii. 345

grus, viii. 330

gularis, viii. 340

lielias, viii. 333. 480

Herodias, viii. 336

hoactli, viii. 345

Ibis, viii. 341

involucris, viii. 347

Jamaïcensis, viii. 345. 346

Javanica, viii. 344

jugularis, viii. 340

lamelliger, viii. 353

leuce, viii. 339

leucocephala, viii. 340

leucoceranus, viii. 331

leucogaster, viii. 341

leuconotus, viii. 344

leucops, viii. 342

leucoptera, viii. 343

lineata, viii. 338

Ludoviciana, viii. 337

Macquari, viii. 336

maculata, viii. 345. 348

Maguari, viii. 349. 507

major, viii. 336. 485

Malaccensis, viii. 340. 343

marmorata, viii. 338

Marsiglii, viii. 340

martook, viii. 340

melanocephala, viii. 337

melanoptera, viii. 348

minor, viii. 343

minuta, viii. 337. 347.490

misata, viii. 355

mokoho, viii 344

monticola, viii. 489

Nævia, viii. 338.345

nigra, viii. 342 . 349

Novæ Guineæ, viii. 337

Novæ Hollandiæ, viii. 342

nycticorax, viii. 345. 493 
Ardea obscura, viii. 345

Pacifica, viii. 337

palliata, viii. 337

pavonia, viii. 329

pavonina, viii. 473

Pealii, viii. 339

perciloptera, viii. 343

Philippensis, viii. 344

pileata, viii. 346

Pondiceriana, viii. 353

pumila, viii. 340

purpurata, viii. 489

purpurea, viii. 336. 489.491

pusilla, viii. 348

radiolata, viii. 344

ralloides, viii. 340

rufescens, viii. 341

ruficapilla, viii. 341

rugata, viii. 341

russata, viii. 340

scapularis, viii. 344

scolopacea, viii. 404

scolopax, viii. 333

segetacea, viii. 346

Senegalensis, viii. 340

sibilatrix, viii. 346

Sinensis, viii. 347

soco, viii. 338.344

Sologniensis, viii. 347

squaiotta, viii. 340

Sparrmanii, viii. 346

speciosa, viii. 342

stellaris, viii. 343. 491

Sturmii, viii. 347

Tayazu Guera, viii. 345

tigrina, viii. 338

torquata, viii. 345

'Tranquebarica, viii. 343

undulata, viii. 344

variegata, viii. 347

violacea, viii. 346

virescens, viii. 337

virgo, viii. 329,475

xanthodactyla, viii. 338

Ardeola, viii. 344. 347

Arenaria, viii. 378. 385

Argus, viii. 24. 235

pavoninus, viii. 24

Arremon, vi. 318 ; vii. 136 torquatus, vi. 318

Arrian, vi. 16

Astrapia, vi. 395

Astur, vi. 48. 236

approximans, vi. 49

fasciatus, vi. 49

Raii, vi. 49

Asturina, vi. 48

cinerea, vi. 48

Atites, viii. 391
Attagas, viii. 36

Attagen, viii. 36. 41

Auk, ancient, viii. 558

great, viii. 636. 637

perroquet, viii. 557. 635

puffin, viii. 634

razor-billed, viii. 636

snowy, viii. 557

tufted, viii. 557. 635

Autour de Cayenne, petit, vi. 47 huppé, vi. 45

Autours, vi. 48

Aves, vi. 1

division of the class into orders, vi. 11.

Avis tarda, viii. 451

Avoset, American, viii. 394 black-necked, viii. 393

long-legged, viii. 393

New Holland, viii. 394

Oriental, viii. 394

red-necked, viii. 394

white, viii. 375

Avosets, viii. 393.535

Ayam-alas, viii. 223

bankiva, viii. 219

Aythia, viii. 612

Azurin, vi. 400

Azurous, l'., vi. 331. 341

Bacha, vi. 62

Baglafecht, vii. 230

Baguari, viii. 507

Balbuzard, vi. 39

Balbuzzard, vi. 231

Baltimore, vii. 168

Barbacous, vii. 463. 538

Barbet à ventre rose, vii. 467 bearded, vii. 469

beautiful, vii. 469

black-breasted, vii. 470

black-throated, vii. 469

blue-semicollared, vii. 471

blue-throated, vii. 468.471

Cayenne, vii. 470

collared, vii. 472.539

elegant, vii. 469

great, vii. 468

green, vii. 468

Latham's, vii. 468

little, vii. 470

olive, vii. 471

party-coloured, vii. 471

Peruvian, vii. 470

red-collared, vii. 471

red-crowned, vii. 469

red-necked, vii. 469

red-rumped, vii. 470 
Barbet, rosy, vii. 469

yellow-cheeked, vii. 470 yellow-fronted, vii. 468 yellow-mustache, vii. 471 yellow-throated, vii. 468 wax-bill, vii. 463 white-breasted, vii. 470

Barbets, vii. 466.538

Barbican, Abyssinian, vii. 467 black-bellied, vii. 466 black-throated, vii. 467 Burchell's, vii. 467 groove-billed, vii. 466 masked, vii. 467 red, vii. 467 smooth-billed, vii. 467

Barbicans, vii. 466

Barge brune, viii. 386

Barita, vi. 288 destructor, vi. 290 anaphonensis, vi. 289

Barker, of Albin, viii. 387

Baryphonus, vii. 423

Basacaraguay, vii. 47

Batara, vi. 272

aconaledo, vi. 282 agallaspeladus, vi. $\mathbf{3 7 9}$ gola nigra, vi. 280 le grand, vi. 272 lisado, vi. 281 mordoré, vi. 273 negro y aplomado, vi. 279 noire et plombe, vi. 273 obscuro y negro, vi. 282 pardo dorado, vi. 280 rayé, vi. 272 roxa, vi. 282

Batteleur, le, vi. 64

Bec ouvert à lame, viii. $\mathbf{3 5 3}$

Becards, vi. 290. 487

Becafico, vi. 502

Bec figue, vi. 502

Becfins, vii. 3

Becquefigue, vi. 478

Bee-eater, Bullock's, vii. 404

Cayenne, vii. 402

chestnut, vii. 401

common, vii. 400. 422

Coromandel, vii. 403

Cuvier's, vii. 402

Indian, vii. 401

Lamarck's, vii. 402

Leschenault's, vii. 404

Malimbic, vii. 403

Philippine, vii. 402

pirik, vii. 404

red-throated, vii. 403

red-winged, vii. 402. 404

ruffed, vii. 403
Bee-eater, Sumatran, vii. 422

superb, vii. 401

supercilious, vii. 401

swallow-tailed, vii. 404

varied, vii. 403

variegated, vii. 401

wattle, vi. 413

yellow-tufted, vi. 416

Bee-eaters, vii. 400. 420

Beefeater, vii. 317

Beefeaters, vii. 164

Bequefigue, vi. 339

Bergeronnette à guimpe, vi. 475

de printemps, vi. 476 ; vii. 51 . 54

Bernacle, viii. 604

Bernacles, viii. 603. 663

Bernicla Canadensis, viii. 600 torquata, viii. 604

Bethyles, vi. 295. 488

Bethylus, vi. 295

Bibio, viii. 475

Bibombi, vii. 103

Bidens, vi. 31

Bigotillus, vi. 337

Bird, Brown's tailor, vi. 469

cat, vi. 385

cedar, vi. 365

crisped paradise, vii. 192

doubtful paradise, vii. 192

emerald-breasted paradise, vii. 192

frigate, viii. 592

frosted paradise, vii. 192

of God, vii. 339

gold-breasted paradise, vii. 190

golden paradise, vii. 191

hackled paradise, vii. 192

indigo, vii. 148

king paradise, vii. 190. 342

magnificent paradise, vii. 190

man of war, viii. 572

mocking, vi. 385

of paradise, great, vii. 189, 341

of paradise, Parkinson's, vi. 426

trumpet, viii. 328. 470

twelve-wired paradise, vii. 192

wattle, vii. 184

white-wing tailor, vi. 469

widow, vii. 228

of paradise, red, vii. 189

Pharaoh's, vi. 20

southern bristle, vi. 470

snow, vii. 148

summer red, vi. 315

superb paradise, vii. 191

Birds, first order of, vi. 14

gallinaceous, viii. 1

of Lybia, viii. 476 
Birds of paradise, vii. 188,338 of prey, vi. 14 of prey, diurnal, vi. 14 of prey, noble, vi. 24 of prey, nocturnal, vi. 69. 251 of Scythia, viii. 476 suppl. on, in general, vi. 87 tropic, viii. 656 widow, vii. 149. 228. 304

Bittern, common, viii. 491 lineated, viii. 338 little, viii. 347.490 of Europe, viii. 343 mokoho, viii. 343 rayed, viii. 490 tiger, viii. 338 zigzag, viii. 344

Bitterns, viii. 343

Biziura, viii. 609 Novæ Hollandiæ, viii. 609

Blac, vi. 57. 243

Blackbird, vi. 510. 530 common, vi. 374 rose-coloured, vi. 537

Blackbirds, vi. 374.530

Blackcap, vii. 30

Blanchot, le, vi. 266

Blevine, viii. 400

Blongosi, viii. 490

Boatbill, viii. 334. 480

Boatbills, viii. 480

Bombycilla, vi. 504 Canadensis, vi. 365 cedrorum, vi. 365

Bombyciphora, zanthocœlia, vi. 365

Bombycivore, vi. 364. 504

Bondrée huppée de Java, vi. 60

Boobies, viii. 593. 653

Booby, viii. 594

Boubou, vi. 265

Brachyptera, viii. 628

Brachypteræ, vii. 546

Brachypterix, vi. 453 montana, vi. 453 sepiaria, vi. 453

Brachypus, viii. 684 murarius, vii. 58

Bretonne, vii. 35

Breve des Philippines, vi. 400 blue-winged, vi. 402

Breviette, viii. 370

Brevipennes, viii. 294

Brubru, viii. 677

Brunoir, le, vi. 389

Bubo, vi. 76

Bucco, vii. 465. 467

Africanus, vii. 462 albifrons, vii. 463.471 armillaris, vii. 471
Bucco aureo-virens, vii. 471 barbiculus, vii. 469 calcaratus, vii. 471 Cayanensis, vii. 470 chacuru, vii. 472 chrysopogon, vii. 471 cinereus, vii. 471 collaris, vii. 472 cyanops, vii. 468 dubius, vii. 466 elegans, vii. 469 erythronotus, vii. 470 flavifrons, vii. 468 fuscus, vii. 463. 470 grandis, vii. 468 gularis, vii. 471 Lathami, vii. 468 leucops, vii. 463. 471 macrorhynchus, vii. 472 marginatus, vii. 471 Maynanensis, vii. 469 melanoleucos, vii. 472 mystacophanes, vii. 471 niger, vii. 460 nigrescens, vii. 471 nigrifrons, vii. 563. 471 nigrothorax, vii. 470 parvus, vii. 470

Peruvianus, vii. 470 Philippensis, vii. 468 roseus, vii. 469 rubescens, vii. 467 rubicauda, vii. 463 rubicula, vii. 471 rubricapillus, vii. 469 rubricollis, vii. 469 rufus, vii. 463.471 Saltii, vii. 467 somnolentus, vii. 473 striatus, vii, 463.471 strigillatus, vii. 472 tamatia, vii. 472 tenebrosus, vii. 471 torquatus, vii. 463.469 versicolor, vii. $\mathbf{4 7 1}$ viridis, vii. 468 Zeylanicus, vii. 470 Buceros, vii. 415. 430

Abyssinicus, vii. 417

Africanus, vii. 416 albirostris, vii. 416 albus, vii. 419 ambigenas, vii. 419 Bengalensis, vii. 418 bicornis, vii. 417 bucinator, vii. 416 cassidix, vii. 416. 434 cornutus, vii. 419 coronatus, vii. 418 
Buceros, enleirostris, vii. 418

erythrorynchos, vii. 418

exaratus, vii. 418

fasciatus, vii. 418

galeatus, vii. 419

gingianus, vii. 417

griseus, vii. 419

hastatus, vii. 418

hydrocorax, vii. 417

Javanicus, vii. 418

jubatus, vii. 419

leucocephalus, vii. 417

Malabaricus, vii. 416

Malayanus, vii. 419

Manillensis, vii. 418

melanoleucus, vii. 418

monoceros, vii. 416

niger, vii. 416

Orientalis, vii. 419

Panayensis, vii. 417

plicatus, vii. 419

Rhinoceros, vii. 416

ruber, vii. 419

sulcatus, vii. 417

undulatus, vii. 418

violaceus, vii. 417

viridis, vii. 419

Budytes, vi. 476

Bullfinch, vi. 297 ; vii. 158. 312

Bullfinches, vii. 158.312

Bundullock, vii. 579

Bunting, Amıazon, vii. 129

Angola, vii. 129

barred-tailed, vii. 129

black-crowned, vii. 129

black-headed, vii. 128

black-throated, vii. 129. 148

Chinese, vii. 129

cirl, vii. 125. 225

commanding, vii. 127.227; viii. 687

common, vii. 126. 225

crimson, vii. 131

Dominican, vii. 149

familiar, vii. 129

foolish, vii. 125. 225

gaur, vii. 130

grey, vii. 129

green, vii. 129

Lapland, vii. 129

Lesbian, vii. 126

long-tailed, vii. 150

Louisiana, vii. 129

Mexican, vii. 129

militaris, vii. 129

mustachio, vii. 125

olive, vii. 129

Oonalaschka, vii. 129

ortolan, vii. 226

Cuv. Index.
Bunting, painted, vii. 136

Panayan, vii. 150

passerine, vii. 125

pine, vii. 128

Plata, vii. 129

red-rumped, vii. $15 \mathrm{I}$

red-eyed, vii. 129

reed, vii. 126. 225

rusty, vii. 129

Sandwich, vii. 130

shaft-tailed, vii. 149

snow, vii. 128. 227

Surinam, vii. 130

Towhe, vii. 129

variegated, vii. 150

wlidah, vii. 150

white-crowned, vii. 148

yellow, vii. 125. 224

yellow-bellied, vii. 136

yellow-winged, vii. 129

Buntings, vii. 124. 223

Buphaga, vii. 164

Africana, vii. 165

Burhinus, viii. 322

Busards of Cuvier, vi. 65

Buseray, le, vi. 63

Bustard, viii. 449

Abyssinian, viii. 304

African, viii. 303

Arabian, viii. 303

black-headed, viii. 304

blue-necked, viii. 304

Cape, viii. 305

collared, viii, 303

great, viii. 301. 450

great-billed, viii. 307

Indian, viii. 303

little, viii. 301.453

marbled, viii. 305

New Holland, viii. 305

Nubian, viii. 303

Passarage, viii. 304

Rhoda, viii. 302

ruffed, viii. 302.454

thick-kneed, viii. 306. 456

white-ehinned, viii. 305

white-eared, viii. 302

Bustards, viii. 300

Buteo, vi. 60

cristatus, vi. 60

melanoleucus, vi. 61

vulgaris, vi. 246

Buzzard, vi. 65

black and white, vi. 61

common, vi. 61.246

common honey, vi. 59.247

crested, vi. 60

grey-cheeked, vi. 65

hobby, vi. 63 
Buzzard, Java honey, vi. 60 mantled, vi. 64 Montague, vi. 66 moor, vi. 66 naked-cheeked, vi. 68 Quoy's, vi. 68 spotted, vi. 64 whitish, vi. 64

Buzzards, vi. 60. 244 bald, vi. 39

honey, vi. 59

Buzzaret, vi. 63

Caïrina, viii. 618

Calandre, vii. 118. 211

Calandrelle, vii. 117

Calao, de waidjiou, vii. 418

Calidris, viii. 375. 529

Callæas, vii. 185

Calyptomena, vi. 428 viridis, vi. 428

Calyptorhynchus, vii. 494, 495

Camouche, viii. 398

Campephaga, vi. 362 ; viii. 683 ferruginea, vi. 363 flava, vi. 362 leucomela, vi. 363 niger, vi. 362

Campycorlumchus striotatus, vi. 408

Camy camy, viii. 470

Canard à bec tricolor, viii. 620

Canary, vii. 272

Cancroma, viii. 334.480 cochlearia, viii. 335.481

Canepetière, viii. 453

Canepetrace, viii. 453

Cangui, viii. 355

Cap, black, vi. 444

Cap-more, vii. 230

Capito, vii. 463 cyanocollis, vii. 468 elegans, vii. 469 maculatus, vii. 473 melanotis, vii. 472 rubrifrons, vii. 470

Capocier, le, vi. 467 Caprimulgi, vii. 104

Caprimulgus, vii. 70 acutus, vii. 77 Ægypticus, vii. 72 affinis, vii. 75 albicollis, vii. 74 albogularis, vii. 79 Americanus, vii. 75.78 Asiaticus, vii. 75.109 brachypterus, vii. 74 Brasilianus, vii. 76 campestris, vii. 76
Caprimulgus, Carolinensis, vii. $\mathbf{7 4}$

Cayennensis, vii. 72

cinerascens, vii. 76

climaturus, vii. 73

cornutris, vii. 78

cornutus, vii. 112

cyanus, vii. 72

diurnus, vii. 76

enicurus, vii. 77

Europæus, vii. 71. 106

forficatus, vii. 78

furcatus, vii. 78

furcifer, vii. 78

gracilis, vii. 73

grandis, vii. 71.78

griseus, vii. 73

Guianensis, vii. 76

guttatus, vii. 80

hirundinaceus, vii. 73

icteropus, vii. 72

Indicus, vii. 76

infuscatus, vii. 74

Isabellinus, vii. 72

Jamaicensis, vii. 77

leucopygus, vii. 77

leucurus, vii. 72

longicaudatus, vii. 73.77

longipennis, vii. 79. 108

macrodipterus, vii. 108

macropteropus, vii. 79

macrourus, vii. 75

manurus, vii. 79

megacephalus, vii. 81

nacunda, vii. 76

Nattereri, vii. 73

Novæ Hollandiæ, vii. 80

pectoralis, vii. 75.109

popetue, vii. 78

psalurus, vii. 78. 113

rubicus, vii. 72

rufitorquis, vii. 72

rufus, vii. 74.76

rupestris, vii. 78

semitorquatus, vii. 76

sphenurus, vii. 74

Strigoïdes, vii. 79

torquatus, vii. 73.74

variegatus, vii. 75

Virginianus, vii. 74. 78

vittatus, vii. 79

vociferus, vii. 71.74

Caracara, vi. 40 ; viii. 328.470

banded, vi. 41

negro, vi. 41

streaked, vi. 42

yellow-headed, vi. 41

Caraf, vii. 583

Carau, viii. 333. 479

Carbo, viii. 589 
Carbo Brasilianus, viii. 591

Desmaresti, viii. 592

Javanicus, viii. 592

Carburé, vi. 81

Carduelis, vii. 139

Caricaca, viii. 519 cucullata, vii. 145

Mexicanus, vii. 141

Cariama, viii. 326. 467

Brasilian, viii. 326

Carouge, true-black, vii. 134

Carouges, vii. 168. 319320

Caryocatactes, vii. 183

Casmarhynchos carunculatus, vi. 366 nudicollis, vi. 366

Cassicans, vi. 288. 487

Cassicus, vii. 165

angustifrons, vii. 166

ater, vii. 166

bifasciatus, vii. 165

hœmorrhous, vii. 166

nigerrimus, vii. 166

viridis; vii. 166

Cassique, vii. 165

black and mantled, vii. 133

noir, vii. 133

Cafriques, vii. 165. 317

Cassowary, viii. 297. 440 galeated, viii. 297

New Holland, viii. 298. 443

Cassuwaris, viii. 298

Cassyphus tristis, vi. 547

Casuarius, viii. 297

Novæ Hollandiæ, viii. 298

Catarrhactes, viii. 561

Catharista, vi. 20

Cathartes, vi. 18. 19. 20

aura, vi. 21

monachus, vi. 20

Catoptrophorus, viii. 389

Catracas, viii. 129

Cattaractes, viii. 638

Caurale, viii. 479

Ceblephyris, vi. 293. 361. 504; viii. 683

bicolor, vi. 362

cana, vi. 362

fimbriatus, vi. 363

Javanensis, vi. 293

Levaillantii, vi. 362

lineatus, vi. 364

lobatus, vi. 362

Madagascariensis, vi. 362

striga, vi. 294. 363

tricolor, vi. 364

Centropus, vii. 460

æthiops, vii. 461

ateralbus, vii. 461

gigas, vii. 461
Centropus membiki, vii. 461 nigro rufus, vii. 460 rufinus, vii. 461

Cephalopteres, vi. $\mathbf{3 5 8}$

Cephalopterus ornatus, vi. 388

Cephus, viii. 555. 633

Cereopsis, viii. 606. 664 cinereus, viii. 606 New Holland, viii. 606

Cerorhynca, viii. 558 Occidentalis, viii. $\mathbf{5 5 8}$

Certhia, vii. 348 amethystina, vii. 360

Antarctica, vii. 354. 384

Asiatica, vii. 366

atricapilla, vi. 418

auriculata, vi. 421 ; vii. 367

aurifrontalis, vii. 365

Australasiana, vi. 421 ; vii. 367

Borbonica, vii. 354

cœrulea, vi. 367. 420 ; vii. 353

cœrulescens, vi. 420

Caffra, vii. 360

cardinalis, vi. 420 ; vii. 354

Carolinensis, vi. 474

carunculata, vi. 413 ; vii. $36 \%$

Cayana, vii. 354

chalybea, vii. 360

chrysotis, vi. 416

cinnamomea, vii. 349

cocincinica, vii. 367

coccinea, vi. 421

cruentata, vii. 357

cucullata, vi. 420

cuprea, vii. 361

currucaria, vii. 362

cyanea, vii. 353

cyanocephala, vii. 361

dubia, vii. 361

erythropygia, vii. 357

erythronotos, vii. 357

familiaris, vii. 349

famosa, vii. 364

flaveola, vii. 355

fuliginosa, vii. 362

frontalis, vii. 366

fusca, vii. 352

goruck, vii. 367

graculina, vi. 421 ; vii. 367

ignobilis, vi. 418

lepida, vii. 362

Lotenia, vii. 360

lunata, vi. 415 ; vii. 367

maculata, vii. 356. 362

Madagascariensis, vii. $\mathbf{3 6 2}$

major, vii. 388

melanops, vi. 420

mellivora, vi. 420 ; vii. 367

Mexicana, vi. 421

I 2 
Certhia, muraria, vii. 352. 389

Novæ Hollandiæ, vi. 421 ; vii.367

obscura, vii. 358

omnicolor, vii. 361

palustris, vii. 49

pulchella, vii. 364

purpurata, vii. 361

rectirostris, vii. 365

rubra, vii. 357

rubrofusca, vii. 362

sanguinea, vi. 419 ; vii. 353

sannio, vii. 356

semitorquata, vii. 356

Śenegalensis, vii. 361 . 366

seniculus, vi. 420 ; vii. 367

sperata, vii. 361

Spiza, vi. 420 ; vii. 367

splendida, vii. 360

superba, vii. 360

tæniata, vii. 357

varia, vii. $356^{\circ}$

venusta, vii. 362

vestiaria, vii. $\mathbf{3 5 8}$

violacea, vii. 364

virens, vii. 356

xantholis, vi. 420 ; vii. 367

Zeilonica, vii. 361

Ceyx, vii. 414

Chacamel, viii. 7

Chacuru, vii. 472

Chacurus, vii. 472

Chœtura, vii. 69

Australis, vii. 70

Martinica, vii. 69

Sabini, vii. 70

Chaffinch, vii. 138. 246

Chaia, viii. 398.539

Chaima, viii. 398

Chamæza, vi. 410

memloides, vi. 410

Charadrius, viii. 305. 307

Agypticus, viii. 309. 316

albicapillus, viii. 32 」

albifrons, viii. 309

Alexandrias, viii. 316

annuligerus, viii. 317

apricarius, viii. 316

Asiaticus, viii. 311

atricapillus, viii. 316

atrogularis, viii. 321

autumnalis, viii. 392

Azarai, viii. 310

bilobus, viii. 317

bitorquatus, viii. 310

Brissonii, viii. 322

calidris, viii. 378

Cantianus, viii. 309

Caspius, viii. 311

Cayanus, viii. 316
Charadrius ceucurus, viii. 320

collaris, viii. $\mathbf{3 1 0}$

Coromandelicus, viii. 324

coronatus, viii. 316

crassirostris, viii. 307

cristatus, viii. $\mathbf{3 1 6}$

cucullatus, viii. 314

curonicus, viii. 309

curbepedesmos, viii. 313

Dudora, viii. 317

Duvaucelli, viii. 317

erythropus, viii. $\mathbf{3 0 9}$

Falklandicus, viii. 317

fluviatilis, viii. 309

fulvus, viii. 314

fuscus, viii. 315

Gallicus, viii. 324

gavia, viii. 320

Geoffroyi, viii. 313

griseus, viii. 314.315

gularis, viii. 311

heteroclitus, viii. 322

hiaticula, viii. 309.460

hiatula, viii. 312

himantopus, viii. 392

Indicus, viii. 310

Jamaicensis, viii. 309. 460

jugularis, viii. 311

keptuschka, viii. 311

larvatus, viii. 312

Leschenaultii, viii. 315

leucogaster, vii. $\mathbf{3 1 5}$

leucopolius, viii. 314

lugubris, viii. 312

magnirostris, viii. 310322

marginatus, viii. 313. 314

marmoratus, viii. 311

melanocephalus, viii. 316. 325

melanops, viii. $\mathbf{3 1 0}$

melodus, viii. 312

minor, viii. 309

modestus, viii. 320

morinellus, viii. 308.459

monachus, viii. 313

Mongolius, viii. 311

nebulosus, viii. $\mathbf{3 1 5}$

nigrifrons, viii. 310

Novæ Zealandiæ, viii. 317

obscurus, viii. 314

oedicnemus, viii. 306

Okenii, viii. 312

pastor, viii. 313

pectoralis, viii. 311

pecuarius, viii. 313

Philippinus, viii. 309

pileatus, viii. 317

pluvialis, viii. 308. 458

pusillus, viii. 312

pyrrocephalus, viii. 317 
Charadrius, rubidus, viii. 378.530 rubricollis, viii. 312 ruficapillus, viii. 313 sanguineus, viii. 314 semipalmatus, viii. 312 spinosus, viii. 316.320 Spixii, viii. 315 stolatus, viii. 317 Taitensis, viii. 314 Tartaricus, viii. 311 torquatus, viii. 309 tricollaris, viii. 310 tricolor, viii. 311 trifasciatus, viii. 310 varius, viii. 313 ventralis, viii. 317 vociferus, viii. 309 Wilsonii, viii. 310 xanthocheilus, viii. 314

Chardonneret, vii. 253

Charpentero nano, vii. 453

Cliasse fiente, le, vi. 15

Chat, wood, vi. 263

Chatterer, vi. 364 azure, vi. 365 black-headed, vi. 507 carunculated, vi. 505 European, vi. 364 umbelled, vi. 358 variegated, vi. 366

Chauche branche, vii. 106

Chenalopex, viii. 617 Ægyptica, viii. 605

Chenerotes, viii. 617

Chericote, viii. 405

Chevalier à longs pieds, viii. $\mathbf{3 8 7}$ varié, viii. 382.385 vert, viii. 372

Cheveche, vi. 259

Chevechette, vi. 79

Cheyarhis, vii. 507

Chicken, Pharaoh's, vi. 187

Chii, vi. 480

Chimachima, vi. 42

Chincou, vi. 16. 17

Cheuque, viii. 438

Chionis, viii. 416 alba, viii. 416

Forsteri, viii. 416 necrophaga, viii. 416 Novæ Hollandiæ, viii. 416 Chipui, vii. 130

Chirurgien de Luzon, viii. 397

Chloropsis, vi. 419 casmarh ynchos, vi. 391 hook-billed, vi. 391

Sonnerati, vi. 390

Alpine, vi. 396
Chocards, vi. 395

Chochi, vi. 523

Choucador, vi. 393

Choliba, vi. 85

Choucaris, vi. 292. 488

Choucas de Surinam, petit, vii. 133

Choucou; vi. 78

Chouette à terrier, vi. 258 petite, vi. 259 lapin, vi. 258

Chroni, viii. 438

Churi, viii. 296

Cichla Coraya, vi. 408

Ciconia, viii. 348. 494 Abdimii, viii. 350

Americana, viii. 350 argala, viii. 351 capillata, viii. 351 ephippirhynca, viii. 352 Guianensis, viii. 352 Javanica, viii. 351 jubura, viii. 349 leucocephala, viii. 350 maguari, viii. 350 marabou, viii. 350 mycteria, viii. 352 nigra, viii. 349. 504 umbellata, viii. 350

Cincinnurus, vii. 190

Cincle, vi. 545

Cincles, vi. 410

Cinclus, vi. 410 bicolor, vi. 410 aquaticus, vi. 410 Mexicanus, vi. 411 Pallasii, vi. 410 unicolor, vi. 410

Cinnyris, vii. 359. 390 æneus, vii. 365 affinis, vii. 365.367 Amboinensis, vii. 365 Asiaticus, vii. 366 aurantia, vii. 366 aurifrons, vii. 366 bombicinus, vii. 366 caudatus, vii. 366 chrysogenis, vii. 365 chrysoptera, vii. 366 cinereicolis;; vii. $\mathbf{3 6 6}$ cinereus, vii. 365 cirrhatus, vii. $\mathbf{3 6 5}$ Clementiæ, vii. 366 collaris, vii. 366 discolor, vii. 366 elegans, vii. 365 eques, vii. 363 erythrorhynchos, vii. 365 ery throthorax, vii. 366 
Cinnyris frontalis, vii. 366

fuscus, vii. 366

Indicus, vii. 365

Iodeus, vii. 366

leucogaster, vii. 366

lotenius, vii. 393

longirostris, vii. 366

Manillensis, vii. 365

melanurus, vii. 366

mornatus, vii. 365

nigralbus, vii. 365

nitens, vii. 366

Novæ Guineæ, vii. 366

ornatus, vii. 366

Phœnicotis, vii. 366

Perreini, vii. 366

politus, vii. 365

rubescens, vii. 366

rubrater, vii. 366

rubrocana, vii. 366

sericeus, vii. 366

Smaragdinus, vii. 366

sola, vii. 366

solaris, vii. 365

splendens, vii. 366

splendida, vii. 366

subflavus, vii. 366

sugninbindus, vii. 366

Circœtus, vi. 40

cinereus, vi. 40

Circus, vi. 59. 65

albicollis, vi. 68

axillaris, vi. 68

campestris, vi. 67

cinereus, vi. 68

Hudsonius, vi. 66

leucocephalus, vi. 68

melanopterus, vi. 68

rufulus, vi. 68

variegatus, vi. 68

Citrin, le, vi. 391. 469

Citta virescens, vi. 295

Clakis, viii. 604

Clangula, viii. 609. 665

Climacteris picumnus, vii. 355 scandens, vii. 355

Climbers, vii. 435

Clorios, vii. 519

Clypeata, viii. 616

Coccothraustes, vii. 151

Coccyzus, vii. 458. 536

Geoffroyii, vii. 459

Lathami, vii. 459

minutus, vii. 459

Cochevis, vii. 205

Cock, viii. 18. 19. 170

Bantam, viii. 20.217

bill, viii. 402-
Cock, crested, viii. 20. 216

crisped, viii. 19. 223

domestic, viii. 20. 180

Dorking, viii. 20

dwarf, viii. 20. 218

Friesland, viii. 223

frizzled, viii. 19

Jago, viii. 21. 174. 218

Knor, viii. 302

Malabar, viii. 21

negro, viii. 19. 221

of the plains, viii. 38

Paduan, viii. 21. 175. 218

Persian, viii. 21. 224

rumpless, viii. 21.224

silk, viii. 19. 222

Turkish, viii. 175. 217

wild, viii. 19

wild of Sonnerat, viii. 19. 20

Cockatoo, Banksian, vii. $\mathbf{4 9 5} .582$

black, vii. 505

Cook's, vii. 495. 582

funeral, vii. 495.582

giant, vii. 505

great sulphur-crested, vii. 494

great white, vii. 494

lesser sulphur-crested, vii. 494

Molucca, vii. 494

Nascian, vii. 494

Philippine, vii. 494

rose, vii. 495.584

Cockatoos, vii. 493

Cocoi, viii. 337

Cocorli, viii. 380

Coereba, vii. 354

Cola de Agudas, vi. 334

Colaris, vii. 187

Colibri, albogularis, vii. 379 crispus, vii. 379

helios, vii. 379

leucopygus, vii. $\mathbf{3 7 9}$

Colies, vii. 163. 316

Colin Sonnini, viii. 60

Colins, viii. 59. 267

Colius, vii. 163

Capensis, vii. 163

Coromandelensis, vii. 164

erythromelon, vii. 164

erythropus, vii. 164

gularis, vii. 164

Indicus, vii. 164

leuconotus, vii. 164

Panayensis, vii. 163

Senegalensis, vii. 164

striatus, vii. 163

viridis, vii. 164

Collier rouge, viii. 352

Colluricinca, vi. 296 
Colluricinca cinerea, vi. 296. 411

Colombe galline, viii. 72

Océanique, viii. 78

Colombihocco, viii. 72

Colon, le, vi. 336

Columba, viii. 71

Abyssinica, viii. 93. 291

ænea, viii. 78

Afra, viii. 83

alba, viii. 78.85

albicapilla, viii. 81

Amboinensis, viii. 91

Araucana, viii. 89

armillaris, viii. 78

aromatica, viii. 94

arquatrix, viii. 78

auricularis, viii. 90

aurita, viii. 81

Australis, viii. 93

Bantamensis, viii. 85

Barbarica, viii. 77

bitorquata, viii. 84

Brunnea, viii. 90

cœrulea, viii. 83

cœruleocephala, viii. 81

calva, viii. 94

Cambayensis, viii. 84

Capellei, viii. 95

Capensis, viii. 92

capistrata, viii. 86

Caribæa, viii. 79

Carolinensis, viii. 91

carunculata, viii. 72. 273

Cerensis, viii. 79

chalcoptera, viii. 78

cincta, viii. 80

cinerea, viii. 84

cobacola, viii. 75

coronata, viii. 72

cristat3, viii. 32. 77. 79

var. B., viii. 32

cruenta, viii. 74

cucullata, viii. 77

cyanocephala, viii. 73

curvirostra, viii. 95

cyanovirens, viii. 89

dasypus, viii. 77

dilopha, viii. 86

Dominicensis, viii. 92

Dussumieri, viii. 87

elegans, viii. 80

eques, viii. 77

erythroptera, viii. 82

erythrothorax, viii. 74

fasciata, viii. 90

Franciæ, viii. 80

frontalis, viii. 77

galeata, viii. 77
Columba Geoffroyi, viii. 84

griseola, viii. 75

Guineæ, viii. 79

gutturosa, viii. 77

gymnophthalmos, viii. 80

gyratrix, viii. 77

Hispanica, viii. 77

hispida, viii. 77

holocericea, viii. 82

Hottentotta, viii. 75

humeralis, viii. 91

humilis, viii. 88

hyogastra, viii. 88

Indica, viii. 81

Jamaicensis, viii. 74

jamboo, viii. 81

Javanica, viii. 81

jubata, viii. 77

lacernulata, viii. 86

laticauda, viii. 77

larvata, viii. 82

leucocephala, viii. 79

leucomela, viii. 87

leucoptera, viii. 81

leucotis, viii. 87

littoralis, viii. 78

Livia, viii. 76

locutrix, viii. 86

lophotes, viii. 91

loricator, viii. 89

luctuosa, viii. 88

Macquaria, viii. 92

maculosa, viii. 89

Madagascariensis, viii. 80

magnifica, viii. 86

Malabarica, viii. 85

Malaccensis, viii. 85

marginata, viii. 91

Martinica, viii. 74

Martinicana, viii. 81

Maugei, viii. 92

melanocephala, viii. 82

melanoleuca, viii. 78

melanoptera, viii. 93

migratoria, viii. 90

militaris, viii. 94

miniata, viii. 89

minor, viii. 555

minuta, viii. 75

monarcha, viii. 88

montana, viii. 73

mystacea, viii. 83

Nicobarina, viii. 73

Norwegica, viii. 77

oceanica, viii. 90

Enas, viii. 76

olax, viii. 94

oxyura, viii. 95 
Columba Pacifica, viii. 78 palumbus, viii. 76 Pampusan, viii. 88 passerina, viii. 75 percussor, viii. 77 perspicillata, viii. 88 phœenicoptera, viii. 95 phasianella, viii. 9l picazuro, viii. 89 picturata, viii. 88. 90 picui, viii. 75

Pinon, viii. 89 poïciloptera, viii. 89 Pompadora, viii. 95 porphyrea, viii. 85 psittacea, viii. 94 puella, viii. 86 purpurata, viii. 90 purpurea, viii. 95 Reinwartii, viii. 91 risoria, viii. 77. 290 rubri capillæ, viii. 80 ruffina, viii. 81 sanguinea, viii. 74

Sanctæ Thomæ, viii. 94 scripta, viii. 87

Senegalensis, viii. 85

Sinica, viii. 82 spadicea, viii. 77 speciosa, viii. 79 squamosa, viii. 85 strepitans, viii. 92 striata, viii. 85 superba, viii. 83 Suratensis, viii. 84 tabellaria, viii. 77 Talpacoti, viii. 74 tannensis, viii. 95 tigrina, viii. 84 turbita, viii. 77 Turcica, viii. 77 turtur, viii. 77 tympanistria, viii. 83 venusta, viii. 92 vernans, viii. 94 vinacea, viii. 77.84 violacea, viii. 81 viridis, viii. 82 Wallia, viii. 93 xanthonura, viii. 87 zenoida, viii. 90 zoæ, viii. 89

Coly African, vii. 164 black-throated, vii. 164 Cape, vii. 163 green, vii. 164 Panay, vii. 163 radiated, vii. 163
Coly, Senegal, vii. 164 white-backed, vii. 164 Colymbus, viii. 546. 551. 628 Arcticus, viii. 552. 631 auritus, viii. 548. 549 bicornis, viii. 549 Caspicus, viii. 548 Cayanus, viii. 550 cornutus, viii. 548 cristatus, viii. 547 Dominicus, viii. 550 glacialis, viii. 552.630 grylla, viii. 554

Hebridus, viii. 548 Immer, viii. 552 lacteolus, viii. 554 minor, viii. 548. 633 nigricans, viii. 548 obscurus, viii. 548 parotis, viii. 548 podiceps, viii. 549 Pyrenaïcus, viii. 549 rubricollis, viii. $\mathbf{5 4 8}$ Septentrionalis, viii. 553 stellatus, viii. 553 striatus, viii. 553 subcristatus, viii. 548 Thomensis, viii. 550 Troile, viii. 553. 632 urinator, viii. 547 vulgaris, viii. 548

Condor, vi. 19. 167

Conirostres, vii. 116 suppl. on the, vii. 193

Conophagus, vi. 409

Conurus, vii. 483.578

Coot, cinereous, viii. 415 grebes, viii. 550

Coq de roche, vii. 2 le petit, vi. 354

Coracias, vii. 185
Abyssinica, vii. 186
Afra, vii. 188
Bengalensis, vii. 186
cœrulea, vii. 187
Caffra, vii. 187
caudata, vii. 187
Cayana, vii. 187
Cayanensis, vi. 300
cyanea, vii. 187
cyanogaster, vii. 187
docilis, vi. 423 ; vii. 187
garrula, vii. 185
Indica, vii. $186^{\circ}$
Madagascariensis, vii. 188
Mexicana, vii. 187
militaris, vi. 359 ; vii. 187
nigra, vii. 187 
Coracias orientalis, vii. 188 pacifica, vi. 266 ; vii. 187 pilosa, vii. 187 puella, vi. 373 ; vii. 187 sagittatus, vi. 417 scutata, vi. 359 ; vii. 187 Sinensis, vii. 187 strepera, vi. 289 ; vii. 187 striata, vi. 266 ; vii. 187 Temminckii, vii. 186 tibicen, vi. 289 vagabunda, vii. 180 varia, vi. 289 ; vii. 187 viridis, vii. 186 ; vii. 187

Coracina, vi. 293 cephaloptera, vi. 358 cristata, vi. 424 fasciata, vi. 293 gymnocephala, vi. 358 gymnoderma, vi. 370 ornata, vi. 358 rubra, vi. 359

Corbi Calao, vi. 412 ; vii. 419

Cordon bleu, vi. 360

Coridonix, vii. 460

Cormorant, little, viii. 590 longap, viii. 591

Cormorants, viii. 589. 590

Corncrake, viii. 541

Corneille, à duvet blanc, vii. 177

Corvorant, common, viii. 651

Corvorants, viii. 651

Corvus, vii. 174 affinis, vii. 380

Africanus, vii. 180 albicollis, vii. 177 argyrophthalmus, vii. 183 Australis, vii. 178 azureus, vii. 180 balicassius, vi. 372 ; vii. 183 brachyurus, vi. 400 ; vii. 183 brachyurus $\beta$, vi. 401 brachyurus $\delta$, vi. 400 brachyurus $\varepsilon$, vi. 401 brachyurus $\eta$, vi. 401 Caledonicus, vii. 180 calvus, vi. 358 ; vii. 183

Canadensis, vii. 182 Caribæus, vii. 183 carunculatus, vii. 183 caryocatactes, vii. 183 Cayanus, vii. 179 clericus, vii. 178 collurio, vi. 295 Columbianus, vii. 178 corax, vii. 175 cornix, vii. 176. 332 corone, vii. 175 crinelus, vi, 396
Corvus crinitus, vi. 423 cristatellus, vii. 182 cristatus, vii. 181 crucirostra, vii. 329 cyaneus, vii. 179 cyanoleucus, vi. 290 ; vii. 182 cyanopogon, vii. 180 cyanurus, vi. 400 ; vii. 183 Dauricus, vii. 177 enca, vii. 178 eremita, vii. 183.380 erythrorhynchus, vii. $\mathbf{1 7 9}$ flavigaster, vi. 320 flaviventris, vii. 183 flavus, vi. 320 Floridanus, vii. 182 frugilegus, vii. 176. $33 \mathrm{I}$ garrulus, ví. 396 glandularius, vii. $18 \mathrm{l}$ gracula, vii. 380 graculinus, vi. 415 grallarius, vi. 403 ; vii. 183 gymnocephalus, vii. 183 Hottentottus, vii. 183 infaustus, vii. 182 Jamaicensis, vii. 177 leucognaphalus, vii. 177 leucophœus, vii. 175.178 melanoleucus, vi. 290 melanops, vi. 293.363 Mexicanus, vii. 178.183 monedula, vii. 176.332 montanus, vii. 178 nasicus, vii. 178

Novæ Guineæ, vi. 293. 363 ; vii. 183

nudus, vi. 370

olivaceus, vii. 180

ossifragus, vii. 178

Pacificus, vi. 290

Papuensis, vi. 293. 363 ; vii. 183

paradoxus, vi 413

Peruvianus, vii. 179

pica, vii. 179

pileatus, vii. 180

purpurascens, vii. 182

pyrrhocorax, vi. 396 ; vii. 183

rufipennis, vi. 393 ; vii. 183

rufus, vii. 180

Russicus, vii. 182

scapulatus, vii. 177

segetum, vii. 175.178

Senegalensis, vii. 179

sexsetaceus, vi. 396

Sibiricus, vii. 181

speciosus, vii. 183

spermologus, vii. 178

splendens, vii. 177

Cuv. Index. 
Corvus splendidus, vi. 393

squamulosus, vi. 295

Stelleri, vii. 181

Surinamensis, vii. 133

torquatus, vii. 182

tropicus, vi. 290

ultramarinus, vii. 182

varians, vii. 184.336

ventralis, vii. 179

zanöe, vii. 180

Corydalla, vi. 479

Corythaix, vii. 507. 584

Corythus, vii. 161

Cotingas, vi. 358.503 ; viii. 683 common, vi. 359. 503

Coturnix dactylisonans, viii. 56 torquata, viii. 58

Cotyle, vii. 61

Cou jaune, le, vi. 472

Couas, vii. 458.536

Coucals, vii. 460

Couhieh, vi. 243

Couigniop, vi. 393

Coupeur d' eau, viii. 586

Courlan, viii. 478

Coulicous, vii. 536

Courliri, viii. 478

Courlis à mèches étroites du Cap, viii. 364

Courol, African, vii. 461

Courols, vii. 461

Couroucoui, vii. 473

Courser, collared, viii. 325

Coromandel, viii. 324

double-collared, viii. 325

Coursers, viii. 324.467

Coz quauhtli, vi. 164

Crabeater of Mahon, viii. 339. 490

Crabeaters, viii. 337. 490

Craboli, viii. 535

Crake Australasian, viii. 332

Baillon, viii. 407

banded, viii. 542

black-bellied Indian, viii. 332

brown, viii. 331

common, viii. 330.476

gigantic, viii. 350.505

hooping, viii. 331

Indian, viii. 331, 332

Siberian, viii. 331

Stanley's, viii. 330

white-spotted, viii. 542

Cranes, viii. 468

crowned, viii. 329

Crapaud volant, vii. 105

Craticus, vi. 290

Crave, New Holland, vii. 380 of Europe, vii. 380

Craves, vii, 379. 398
Cravate frisée, le, vi. 416

Crax, viii. 3

alector, viii. 3

carunculata, viii. 5

Blumenbachii, viii. 5

faciolata, viii. 5

galeata, viii. 6

globicera, viii. 4.115

globulosa, viii. 5

Pauxi, viii. 6. 117

rubra, viii. 4. 108. 116

rubrirostris, viii. 5

tomentosa, viii. 6

tuberosa, viii. 7

uramutum, viii. 7

vociferans, viii. 7

Creadion, vi. 413

Creeper, beautiful, vii. $\mathbf{3 6 4}$

black and blue, vii. 353

black and yellow, vii. 355 ; viii. 689

blue-headed, vii. 361

Bourbon, vii. 354

Byron's, vii. 390

cardinal, vii. 354

Ceylonese, vii. 361

cinnamon, vii. 349

collared, vii. $\mathbf{3 6 0}$

copper, vii. 361

crimson, vii. 353

European, vii. 388

familiar, vii. 349

famous, vii. 364

great hook-billed, vii. $\mathbf{3 5 8}$

green-faced, vii. 362

grey, vii. 362

gold, vii. 361

hook-billed green, vii. 358

Loten's, vii. 360

orange-backed, vii. 357

picucule, vii. 350

pied, vii. 362

red-backed, vii. 357

red-breasted, vii. 361

red-brown, vii. 362

saccharine, vii. 364

scarlet, vii. 357

Senegal, vii. 361

shining, vii. 364

thorn-tailed, vii. 349

tufted-eared, vi. 421

violet-headed, vii. 364

wattled, vi. 413

yellow-rumped, vii. 354

Creepers, vii. 348. 388

of the wall, vii. 352

Cresserelle, vi. 26

Crex, viii. 406. 541

chloropus, viii. 411 
Crex galeata, viii. 412

lateralis, viii. 409

lugubris, viii. 410

Martinica, viii. 413

melampyga, viii. 409

mustelina, viii. 409

nigra, viii. 409

plumbea, viii. 410

pratensis, viii. 407

pulchra, viii. 410

rubiginosa, viii. 409

Criniger, vi. 296

Crombea, le, vii. 357

Crossbill, vii. 160 common, vii. 315 white-winged, vii. 161

Crossbills, vii. 160. 315

Crotophaga, vii. 457.476

major, vii. 476

minor, vii. $476^{\circ}$

Crow, carrion, vii. 175

chattering, vii. 177

cinereous, vii. 182

Clark's, vii. 178

downy, vii. 177

fish, vii. 178

hooded, vii. 176. 332

New Guinea, vi. 293

Papuan, vi. 293

rock, vi. 375

rufous, vii. 180

Senegal, vii. 179

Siberian, vii. 181

Steller's, vii. 181

Surinam, vii. 133. 233

white vi. 165. 189

white-breasted, vii. 177

Crows, vii. 174. 326

Crymophylus, viii. 383 rufus, viii. 384

Crypsirina, vii. 184

Cryptonix, viii. 31. 241

black, viii. 32

coronatus, viii. 31

ferrugineus, viii. 33

niger, viii. 32.33

rufus, viii. 33.55

Cryptura, fasciata, viii. 70 guagu, viii. 71 gubercola, viii. 67 magoua, viii. 67 solitaria, viii. 69

Crypturus, viii. 66 adspersus, viii. 68 cœrulescens, viii. 68 parvirostris, viii. 68

Cuckow, vii. 514 Ethiopian, vii. $46 \mathrm{I}$ black and white, vii. 461
Cuckow black-billed, vii. 455

blue, vii. 458

brazen, vii. 457

Cape, vii. 454

Carolina, vii. 455

Cayenne, vii. 459

chestnut, vii. 459

collared, vii. 455

common, vii. 454.516

cupreous, vii. 456

De Lalande's, vii. 458

edolio, vii. 455

Egyptian, vii. 460

Flinders'ś, vii. $\mathbf{5 3 6}$

giant, vii. 461

gilded, vii. 456

great Madagascar, vii. 458

Guira, vii. 457

honey, vii. 462

klaas, vii. 456

lark-heeled, vii. 460

lesser honey, vii. 462

long-billed, vii. 459

long-necked, vii. 460

Madagascar crested, vii. 458

mangrove, vii. 459

masked, vii. 463

Mindanao, vii. 457

noisy, vii. 455

Panayan, vii. 455

pheasant, vii. 459

Philippine, vii. 460

red-brown, vii. 460

rufous, vii. 461

rufous spotted, vii. 457

sacred, vii. 457.536

shining, vii. 456

society, vii. 457

solitary, vii. 454

spotted, vii. 459

white-billed, vii. 462

white rumped, vii. 463

white-rumped black, vii. 46.3

yellow-bellied, vii. 456

Cuckows, vii. 453

true, vii. 453

Cuculus, vii. 453. 514

Ethiops, vii. 461

Afer, vii. 461

albirostris, vii. 462

Americanus, vii. 455

ateralbus, vii. 461

auratus, vii. 451

Bengalensis, vii. 460

brachypterus, vii. 459

Brasiliensis, vii. 459

bubutus, vii. 460

cœruleus, vii. 458

canorus, vii. 454. 516

K 2 
Cuculus Capensis, vii. 454

Carolinensis, vii. 455

Cayanus, vii. 459

Chalcites, vii. 457

clamosus, vii. 455.464

Clasii, vii. 456

cornutus, vii. 459

Coromandus, vii. 455

cristatus, vii. 458

cupreus, vii. 456

Dominicus, vii. 464

edolius, vii. 455

Egyptius, vii. 460

erythrophthalmus, vii. 455

flavus, vi. 456

Flindersii, vii. 458

gigas, vii. 461

glandarius, vii. 454

Guira, vii. 457

honoratus, vii. 457.536

indicator, vii. 462

Indicus, vii. 458

Lalandii, vii. 458

lucidus, vii. 456

maculatus, vii. 457

Madagascariensis, vii. 458

melanoleucus, vii. 455

Mindanensis, vii. 457

minor, vii. 462

nævius, vii. 459

nigrorufus, vii. 460

Orientalis, vii. 458.464

Panayus, vii. 458

Paradiseus, vii. 463

Persa, vii. 507

Philippensis, vii. 460

Pisanus, vii. 454. 458

pluvialis, vii. 459

poliocephalus, vii. 458

punctulatus, vii. 459

punctatus, vii. 457

pyrrhocephalus, vii. 464

radiatus, vii. 455.458

regius, vii. 507

rubibandus, vii. 459

rufalbinus, vii. 463

rufinus, vii. 461

scolopaceus, vii. 457

Senegalensis, vii. 460

seniculus, vii. 459

serratus, vii. 455

Sinensis, vii. 464

solitarius, vii. 454

Sonneratii, vii. 458

Taitensis, vii. 457

tenebrosus, vii. 463

tolu, vii. 460

tranquillus, vii. 463

vetula, vii. 459
Cujelier, vii. 117

Culdor, le, vi. 389. 391

Culicivoræ, viii. 682

Cultirostres, viii. 327.468

Curassow blue, vii. 585 crested, viii. 3.109

Cumana, viii. 8. 9

cushew, viii. 6

globose, viii. 4. 115

piping, viii. 9. 123

razor-billed, viii. 6

red, viii. 4. 116

wattled, 5

Curicaca, viii. 361

Curlew, viii. 519

Cape, viii. 380. 531

Chili, viii. 366

common, viii. 364.520

Derhomai, viii. 366

Esquimaux, viii. 365

green, viii. 363

little, viii. 521

long-billed, viii. 365

Luzonian, viii. 366

Madagascar, viii. 364

Otaheite, viii. 366

pygmy, viii. 380

sea coast, viii. 365

sea side lesser, viii. 365

white-headed, viii. 366

Curlews, viii. 364

Curruca, vi. 440

garrula, vi. 445

Cursorius, viii. 324

Asiaticus, viii. 324

bicinctus, viii. 325

chalcopterus, viii. 325

grallator, viii. 325

Isabellinus, viii. 324

Temminckii, viii. 325

Curucau rase, viii. 361

Cyclaris, vi. 270 ; viii. 679

Cygnus, viii. 597

atratus, viii. 599

Canadensis, viii. 600

cucullatus, viii. 444

gelbus, viii. 598

melanocephalus, viii. 599

melanorhynchus, viii. 599

musicus, viii. 599

niger, viii. 661

nigricollis, viii. 599

olor, viii. 659

Sinensis, viii. 600

Cymbops, viii. 335

Cymindis, vi. 47. 62

Cyphos macrodactylus, vii. 473

Cypselus, vii. 57

albicollis, viii. 687 
Cypselus Alpinus, vii. 59

Alpinus Africanus, vii. 59

Balasiensis, vii. 60

Caffer, vii. 60

collaris, vii. 67. 70

comatus, vii. 59

giganteus, vii. 70 ; viii. 687

gutturalis, vii. 60

longipennis, vii. 59

inurarius, vii. 58

mystaceus, vii. 59 ; viii. 687

parvus, vii. 60

Dacelo, vii. 412. 426

cinnamoninus, vii. 412 ; viii. 689

concreta, vii. 412

cyanotis, vii. 413

Gaudichaud, vii. 413

pulchellus, vii. 413

Dacnis, vi. 465 ; vii. 171 ; viii. 688

Dædalion, vi. 48. 236

Dafila, viii. 618

Daptrius, vi. 40 ater, vi. 41

Darter, viii. 655

black-bellied, viii. 595

yellow-necked, viii. 596

Dasyornis, vi. 470

Australis, vi. 470

Dauphinois, vi. 490

Daw, vii. 332

Dedalion, vi. 52

Delophus, vi. 422. 425

Demoiselle de Numidie, viii. 475

Dendrocolaptes, vii. 350

bivittatus, vii. 351

crassirostris, vii. 352

decumanus, vii. 350

falcirostris, vii. $\mathbf{3 5 0}$

frontirostris, vii. 352

fuscus, vii. 351

guttatus, vii. 351

platyrostris, vii. $\mathbf{3 5 0}$

procurvus, vii. 351

rufus, vii. 351

sylviellus, vii. 348

tenuirostris, vii. 351

Wagleri, vii. 351

angustirostris, vii. 352

falcularius, vii. 352

guttatus, vii. 352

pyrrhosophius, vii. 352

rubricaudatus, vii. 352

Dendroplex, viii. 689

Dentirostres, vi. 262

Dicæum, vii. 356

choronotus, vii. 357

flavium, vii. 357
Dicæum rubescens, vii. 357 rufescens, vii. $\mathbf{3 5 7}$

Dicea, vi. 412 ; vii. 356

Diceum, vii. 390 ; viii. 685

Dicholophus, viii. 326

Dicrurus, vi. 371

æneus, vi. 372

cristatus, vi. 371

leucogaster, vi. 372

leucophœus, vi. 372

lophorinus, vi. 372

longus, vi. 371

macrocercus, vi. 372

musicus, vi. 373

mystaceus, vi. 373

platurus, vi. 371

Didus, viii. 299

ineptus, viii. 299. 443

Nazarinus, viii. 299. 300

solitarius, viii. 299

Dioch, vii. 137

rose, le, vii. 137

Diomedea, viii. 571

brachyura, viii. 572

Chinensis, viii. 572

chlororhynchos, viii. 573

exulans, viii. 571

fuliginosa, viii. 573

melanophris, viii. 572

spadicea, viii. 572

Diplectron, viii. 13

Dishwasher, vi. 369

Distingue roux à tête noire, vi. 292

Diver, black-throated, viii. 552

imber, viii. 552

Newcastle, viii. 552

northern, viii. 552

red-throated, viii. 553

striped, viii. 553

Divers, viii. 546. 551. 630

Greenland, viii. 555

Dod-aers, viii. 444

Dod-aersen, viii. 444

Dodo, vil. 299

hooded, viii. 299

Nazarene, viii. 299. 446

solitary, viii. 299. 446

Dolichonyx, viii. 688

Dotterel, viii. 308 sea, viii. 385

Double sourcil, le, vi. 469

Dove, ground, viii. 75 turtle, viii. 289

Doves, Portugal, viii. 77

Dragon, le, vii. 171

Dromaius, viii. 298. 443

Dromas, viii. 354

Ardeola, viii. 354

Drongear, vi. 373 
Drongi, vi. 372

Drongo à moustaches, vi. $\mathbf{3 7 3}$ bronzé, le, vi. 372

Drongolon, le, vi. 372

Drongos, vi. 371.510

Dronte, viii. 443

Drymoica, viii. 687

Drymophila, vi. 283

atra, vi. 284

leucopus, vi. 283

longipes, vi. 283

trifasciata, vi. 283

variegata, vi. 284

velata, vi. 284 ; viii. 679

Duck arched, viii. 623. 670

Arabic, viii. 625

American tufted, viii. 614

Alexandrine, viii. 625

Barbary, viii. 618

black, viii. 608

black-bellied whistling, viii. 620

black-tailed, viii. 624

brown, viii. 624

canvas-backed, viii. 615

castaneous, viii. 614

crested, viii. 624

curved-bill, viii. 619

Damietta, viii. 625

Dominican, viii. 625

dusky, viii. 622

European, viii. 614

falcated, viii. 624

ferrugineous, viii. 614,615

Gadwal, viii. 621

Garganey, viii. 623

Gattair, viii. 625

Georgian, viii. 624

Gmelin, viii. 616

golden-eye, viii. 610

Greenland, viii. 625

grey-headed, viii. 612

harlequin, viii. 610

Hawkesbury, viii. 621. 670

hook-billed, viii. 619

Iceland, viii. 615

Ilathera, viii. 622

Kekuschka, viii. 625

king, viii. 611

lobate, viii. 609

long-tailed, viii. 609

mallard, viii. 619

Mareca, viii. 624

membranaceous, viii. 617

Mexican, viii. 624

Muscovy, viii. 618

New Holland, viii. 617

New Zealand, viii. 616

olive-tufted, viii. 614

pied, viii. 615. 669
Duck pink-headed, viii. 614

pintail, viii. 618

pochard, viii. 612

radjah, viii. 621

red, viii. 615

red-billed whistling, viii. 620

red-breasted, viii. 616

red-crested, viii. 612

ruddy, viii. 615

scaup, viii. 613

scoter, viii. 607

shoveler, viii. 669

Sisæir, viii. 625

soft--bill, viii. 617

solitary, viii. 625

Spanish, viii. 620. 671

spirit, viii. 611

spotted-beaked, viii. 620

spotted-billed, viii. 622

summer, viii. 620

supercilious, viii. 624

tufted, viii. 614

Ural, viii. 608

velvet, viii. 608

western, viii. $\mathbf{6 1 5}$

white-faced, viii. 613. 624

wild, viii. 671

Ducks, viii. 597. 607. 664

Ducs, vii. 76

Dulang, vii. 579

Dulus, vi. 317

palmarum, vi. 318

Dumlin, viii. 370

Dysporus, viii. 593

Capensis, viii. 594

piscator, viii. 594

EAGLE bald, vi. 37

black and white, vi. 35

black-headed, vi. 46

black-tufted, of Africa, vi. 45

Blagre, vi. 39

Bonelli's, vi. 35

Chinese, vi. 46

common, vi. 33.228

crown, vi. 36

crowned, vi. 35.47

crying, vi. 37

fishing, vi. 38

golden, vi. 225

grey French, vi. 40

great, vi. 225

hook-billed, vi. 47

imperial, vi. 34

long-beaked, vi. 48

Mace's, vi. 38

marine, vi. 38

martial, vi. 35 . 229 
Eagle noisy, vi. 46 piscivorous, vi. 37

Pondicherry, vi. 38

Reinwardt's, vi. 35

ring-tailed, vi. 33

roc, vi. 175

rough-footed, vi. 34

royal, vi. 225

sea, vi. 36

small Cayenne, vi. 4

spotted, vi. 34. 46

tufted of Guiana, vi. 43

tyrant, vi. 46

vulture, vi. 22

vulturine, vi. 23

wedge-tailed, vi. 36. 230

white, vi. 49

white-bellied, vi. 38

Eagles, vi. 33. 220

fisher, vi. 36.42

properly so called, vi. 33

Eatua, vii. 428

Echassiers, viii. 293. 421

Echenilleurs, vi. 361. 503, 504

Eclatant, l'. vi. 393

Edolius, vi. 371

azureus, vi. 373

cineraceus, vi. 371

remifer, vi. 373

retifer, vi. 371

Effrayé, vi. 75

Egret, demi, viii. 34]

great, viii. 338, 339. 491

little, viii. 338,490

reddish, viii. 341

Egrets, viii. 338. 490

Eider, viii. 665

Eiders, viii. 611

Elanoides, vi. 58

Elanus, vi. 57 cæsius, vi. 58

Elgithina, vi. 448

Embaguari, viii. 507

Embergoose, viii. 631

Emberiza, vii. 124. 223

Amazona, vii. 129

Americana, vii. 129. 148

Angolensis, vii. 129. 150

Asiatica, vii. 130

atricapilla, vii. 129

Badensis, vii. $12 t^{\circ}$

Borbonica, vii. 127. 139

Brasiliensis, vii. 127. 139

brumalis, vii. 127

cæsia, vii. 127

Calfat, vii. 129

Capensis, vii. 127. 136

chlorocephala, vii. 126
Emberiza, chrysophrys, vii. 130

chrysoptera, vii. 129

cia, vii. 125

ciris, vii. 127. 136

cirlus, vii. 125

citrinella, vii. 125. 224

coccinea, vii. 129

cœrulea, vii. 127

cristata, viii. 687

cyanopis, vii. 127

erythropthalma, vii. 129

familiaris, vii. 129

ferruginea, vii. 129

flavifrons, vi. 298

fucata, vii. 130

fusca, vii. 129

grisea, vii. 129

gubernatrix, vii. 127. 227

hortulana, vii. 126. 226

jacarina, vi. 298

Lesbia, vii. 126

leucophrys, vii. 148

longicauda, vii. 150. 228

Lotharingica, vii. 125

luctuosa, vi. 340

Ludoviciana, vii. 129

melanocephala, vii. 128

melanodera, vii. 130

melbensis, vii. 126

Mexicana, vii. 129

miliaria, vii. 126. 225

militaris, vii. 129

mixta, vii. 129

montana, vii. 128

mustelina, vii. 128

nivalis, vii. 128

olivacea, vii. 129

Oonalashkensis, vii. 129

oryzivora, vii. 127. 148. 171; viii. 688

Panayensis, vii. 150

Paradisea, vii. 150

passerina, vii. 125

pecoris, vii. 168

pithyornis, vii. 128

Platensis, vii. 129

principalis, vii. 150

provincialis, vii. 125

psittacea, vii. $\mathbf{1 5 0}$

pusilla, vi. 298 ; vii. 129

quadricolor, vii. 127. 151

quelea, vii. 127. 137

regia, vii. 149

rubra, vii. 127. 131

rustica, vii. 129

rutila, vii. 129

Sandwichensis vii. 130

schœniclus, vi . 126. 225 
Emberiza serena, vii. 149

Sinensis, vii. 129

striolata, vii. 127

superciliosa, vii. 130

Surinamensis, vii. 130

textor, vii. 134

vidua, vii. 150

viridis, vii. 129

Emberizoides, vii. 127. 130 ; viii. 688 marginalis, vii. 130 melanotes, vii. 130

Eme, viii. 298

Emérillon, vi. 26

Emeu, viii. 298. 443

Emu, viii. 440

American, viii. 296

Entomyzon, vi. 415

Engoulevent, vii. 105

Enicurus, vi. 478 coronatus, vi. 478 velatus, vi. 478

Epervier patu, vi. 45

Epimachi, vii. 382.399

Epimachus, vii. 382

albus, vii. 383

magnificus, vii. 384

promefil, vii. 384

regius, vii. 384

superbus, viii. 689

with frizzled ornaments, vii. 382

Ereunetes, viii. 391

Erodia amphilensis, viii. 354

Erodies, viii. 353

Erody Abyssinian, viii. 354

Erolia, viii. 381 variegata, viii. 381

Espionneur, l', vi. 376

Etourneau à camail rouge, vii. 173

Eudynames, vii. 458

Eudytes, viii. 551

Euphone rufiventris, vi. 298

Eurinorhynchus, viii. 383. 531 griseus, viii. 383

Eurypyga, viii. 480

Eurystomus cyanocollis, vii. 188 gularis, vii. 188 purpurascens, vii. 188

Faisan de la Guiane, viii. 129

Falcinellus, vii. 381 ; viii. 381.531 cyanomelus, vii. 406 pygmæus, viii. 380

Falco, vi. 23, 24. 195

Acoli, vi. 67

Agyptius, vi. 59. 243

æruginosus, vi. 66
Falco aguia, vi. 35

albescens, vi. 46

albicans, vi. 65

albicaudus, vi. 36

albicella, vi. 36

albidus, vi. 62.64

albus, vi. 47. 49.66

Aldrovandi, vi. 27

ambustus, vi. 23

Angolensis, vi. 17

apivorus, vi. 59

aquiliuus, vi. 41 .

armiger, vi. 35

ater, vi. 58

aterrimus, vi. 41

atricapillus, vi. 46.49

aurantius, vi. 28

Austriacus, vi. 58

axillaris, vi. 37

bacha, vi. 62

badius, vi. 56

Banksia, vi. 54

barbarus, vi. 24,25

barbatus, vi. 22

bellicosa, vi. 35

Berigora, vi. 29

biarmicus, vi. 27

bidentatus, vi. 31

Blagrus, vi. 39

Bohemicus, vi. 66

Bonelli, vi. 35

Borealis, vi. 62

brachydactylus, vi. 40

brachypterus, vi. 50

Brasiliensis, vi. 40

Brissonianus, vi. 54

Brownii, vi. 56

busarellus, vi. 63

buteo, vi. 61.246

buzon, vi. 63

cachinnans, vi. 52

cœrulescens, vi. 30

Canadensis, vi. 33

candicans, vi. 32

Capensis, vi. 28

Carolinensis, vi. 39

Cayenensis, vi. 39. 47

cenchroides, vi. 29

cheriway, vi. 40

chicquera, vi. 28

chrysaëtos, vi. $\mathbf{3 3}$

cineraceus, vi. 66

cinereus, vi. 32

columbarius, vi. $\mathbf{3 0}$

communis, vi. 24. 61. 66

fuscus, vi. 62

concolor, vi. 27

connivens, vi. 61 . 
Falco coronatus, vi. 35, 36. 45.47

cristatellus, vi. 45

cristatus, vi. 42

crotophagus, vi. 42

cuculoides, vi. 55

cyaneus, vi. 65

cygneus, vi. 33

degener, vi. 42

deiroleucus, vi. 27

desertorum, vi. 63.65

destructor, vi. 42

diodon, vi. 31

dispar, vi. 58

dominicensis, vi. 30

Dussumieri, vi. 55

ecaudatus, vi. 64

femoralis, vi. 29

formosus, vi. 41

Forskahlii, vi. 59

frenatus, vi. 67

fringillarius, vi. 30

frontalis, vi. 28. 199

fucosa, vi. $\mathbf{3 6}$

fulvous, vi. 33

furcatus, vi. 58

fuscus, vi. 48

gabar, vi. 53

gallicus, vi. 40

gallinarius, vi. 48

gentilis, vi. 48

glaucopis, vi. 47

gracilis, vi. 50

griseus, vi. 66

Guianensis, vi. 43

gymnogenys, vi. 68

gyrfalco, vi. 48

haliætus, vi. 39

hamatus, vi. 48

harpyia, vi. 42

hemidactylus, vi. 50

herotinus, vi. 25

historionicus, vi. 68

hyemalis, vi. 67

ichthyætus, vi. 38

imperialis, vi. 34.42

Indicus, vi. 52

insectivorus, vi. 55

Islandicus, vi. 24

jackal, vi. 63

Jacquini, vi. 42

lagopus, vi. 60

laniarius, vi. 29

Lathami, vi. 30

latissimus, vi. 49

leuchauchen, vi. 51

leucocephalus, vi. 37. 61

leucogaster, vi. 38

leucomelas, vi. 67

leucopygus, vi, 47

Cuv. Index.
Falco leucorhynchus, vi. 52

Leverianus, vi. 62

limnæatus, vi. 45

lineatus, vi. 67

lithofalco, vi. 26

longipes, vi. 59

Macei, vi. 38

macrourus, vi. 56

maculatus, vi. 34

maculosa, vi. 46

Madagascariensis, vi. 18

magnirostris, vi. 51

magnus, vi. 22

Malayensis, vi. 35

melanaëtos, vi. 33

melanoleucus, vi. 68

melanops, vi. 52

melanopterus, vi. 57

milvus, vi. 58

minullus, vi. 53

Mississippiensis, vi. 57

minuta, vi. 54

mogilnik, vi. 33. 34

monogrammicus, vi. 27

montanus, vi. 66

musicus, vi. 54

nævius, vi. 34

niger, vi. 22. 33. 61

nisus, vi. 53

nitidus, vi. 50

niveus, vi. 44

Novæ Hollandiæ, vi. 49

Novæ Zelandiæ, vi. 41. 44.

nudicollis, vi. 41

occipitalis, vi. 45

œsalon, vi. 26

orientalis, vi. 52

ornatus, vi. 45

ossifragus, vi. 36

pœcilonotus, vi. 64

palliatus, vi. 64

palumbarius, vi. 48

palustris, vi. 67

parasiticus, vi. 59. 242

penriatus, vi. 34. 60

Pennsylvanicus, vi. 49. 54

peregrinus, vi. 24

pileatus, vi. 55

piscator, vi. 38

plancus, vi. 41

plumbea, vi. 57

poliogaster, vi. 51

poliogenys, vi. 65

polyosoma, vi. 65

Pondicerianus, vi. 38

pterocles, vi. 64

ptilorhynchos, vi. 60

punetatus, vi. 29

pygargus, vi. 65 
Falco radiatus, vi. 51 ranivorus, vi. 66 Riocourii, vi. 58 rufipes, vi. 26 rufus, vi. 56.66 rupicola, vi. 28 rupicolus, vi. 28. 202 rutilans, vi. 67 sacer, vi. 32

Sancti Johannis, vi. 61

Senegalensis, vi. 45 serpentarius, vi. 69 severus, vi. 27

Sinensis, vi. 46 soloensis, vi. 55 sparverius, vi. 30. 202 stellaris, vi. 25. 29 striolatus, vi. 50 subbuteo, vi. 26. 200 sufflator, vi. 53 superbus, vi. 45 Tachardus, vi. 62 Tachiro, vi. 63 thoracicus, vi. 29 tibialis, vi. 28. 199. 200 tinnunculoides, vi. 26 tinnunculus, vi. 26 torquatus, vi. 54 trivirgatus, vi. 51 tyrannus, vi. 46 uliginosus, vi. 66 uncinatus, vi. 47 unicinctus, vi. 44. 52 urubitinga, vi. 44 variegatus, vi. 62 velox, vi. 55 versicolor, vi. 62 vespertinus, vi. 26 virgatus, vi. 55 vocifer, vi. 37 vociferus, vi. 37 vulturinus, vi. 23.38 xanthothorax, vi. 50

Falcon, ashy, vi. 48 banded-throat, vi. 27

Barbary, vi. 25

Bengal, vi. 30

black and white, vi. 67

black and white Indian, vi. 68

black-thighed, vi. 28

booted, vi. 34

chaunting, vi. 54

Chicquera, vi. 28

chimachima, vi. 42

colvy, vi. 30

collared, vi. 54

common, vi. 24. 196

crested, vi. 45

crested Indian, vi. 28
Falcon, desert, vi. 63 double-bearded, vi. 27 dwarf, vi. 53 ger, vi. 31.32 golden red, vi. 67 jackal, vi. 63 kober, vi. 200 lake, vi. 45 laughing, vi. 52 long-legged, vi. 67 long-tailed, vi. 56 Madagascar, vi. 18 maritime, vi. 202 minute, vi. 54 mountain, vi. 202 New Zealand, vi. 41 notched, vi. 31 peregrine, vi. 24 radiated, vi. 51 red-legged, vi. 53 red-throated, vi. 41

Riocour's, vi. 58 rough-footed, vi. 60 Salvador, vi. 67 severe, vi. 27 short-tailed, vi. 64 short-toed, vi. 50 short-winged, vi. 50 snowy, vi. 44 spotted, vi. 29 streaked, vi. 52 Surinam, vi. 53 Tachard, vi. 62 two-toothed, vi. 31 uniform, vi. 27 white-necked, vi. 51 white-rumped, vi. 47 white-throated, vi. 27 winking, vi. $6 \mathrm{l}$ winter, vi. 67 yearling, vi. 25

Falconry, vi. 204

Falcons, vi. 23. 195 properly so called, vi. 24

Falcunculus, vi. 295 gutturalis, vi. 296

Fallow Sniech, vi. 435

Family, dentirostral, vi. 481

Father, white, vi. 187

Faucon à queue en ciseaux, vi. 243 chanteur, vi. 54

Faucons pélerins, vi. 25

Fauvette, vii. 7 des Alpes, vii. 36

Fauvettes, vii. 7 .

Ficedula, vi. 339. 439

Fieldfare, vi. 384. 524

Figeaters, vi. 471

Figuier à gorge jaune, vi. 472 
Figuier bleu, le, vi. 473 tacheté, le, vi. 472

Figulus, vii. 354 albogularis, vii. 354

Finch, Amaduvade, vii. 144 American yellow, vii. 143 Angola, vii. 145 Bahama, vii. 145 beautiful, vii. 136 black-collared, vii. 132. 232 black-faced, vi. 312 ; vii. 137 blue-bellied, vii. 144 Brazilian, vii. 144 Canary, vii. 143 citril, vii. 142. 271 common gold, vii. 139 crescent, vii. 135 crimson-crowned, vii. 162 glossy, vii. 144 gold, vii. 252 green gold, vii. 140 Lapland, vii. 119 lark, vii. 148 lazuli, vii. 148 lepid, vii. 143 mountain, vii. 138.251 orange, vi. 306 parrot, vii. 140 purple, vii. 157.162 red-headed, vii. 132 ring, vii. 154. 310 rose, vii. 162 savannah, vii. 148 scarlet, vii. 140 sea-side, vii. 149 Senegal, vii. 144 serin, vii. 142 sharp-tailed, vii. 149 snow, vii. 139 thistle, vii. 253 tree, vii. 135. 245

Finches, vii. 138. 246 gold, vii. 139. 252

Finfoot, African, viii. $55 \mathrm{I}$ American, viii. 551

Fissirostres, vii. 57 suppl. on the, vii. 82

Flamingo, viii. 543 little, viii. 420 pygmée, viii. 420 red, viii. 419

Flamingos, viii. 418

Flercher, viii. 304

Flûteur, le, vi. 392 ; vii. 348

Flycatcher, African, vi. $\mathbf{3 6 3}$ Australasian, vi. 346 azure, vi. 329 Banyumas, vi. 345 black, vi. 501
Flycatcher, black-collared, vi. 498 black of Europe, vi. 494 black-tipped, vi. 369 blue gray, vi. 459 Canada, vi. 455 chattering, vi. 355 ; viii. 683 coach whip, vi. 296 collared, vi. 329. 501 desert, vi. 329 dun, vi. 344 English, vi. 340. 501 fan-tailed, vi. 368 guava, vi. 388 hooded, vi. 455 indigo, vi. 345 Javan, vi. 346 mutable, vi. 330 obscure, vi. .345 Peruvian, vi. 336 pewit, vi. 350 pied, vi.339. 501 red-bellied, vi. 348 red-eyed, vi. 356 red-vented, vi. 344 round-crested, vi. 332 rufous-fronted, vi. 369 small-headed, vi. 459 solitary, vi. 355 spotted, vi. 339. 493 tyrant, vi. 324 warbling, vi. 356 white, vi. 326 white-eyed, vi. 356 wood pewit, vi. 350 yellow-necked, vi. 329 yellow-fronted, vi. $\mathbf{3 4 7}$ yellow-rumped, vi. 321.332 yellow-throated, vi. 355 Yetapa, vi. 355

Flycatchers, vi. 318. 328. 491 properly so called, vi. 339

Formicivora, vi. 284 maculata, vi. 284 nigricollis, vi. 284

Fourmillier, le, vi. 437

Fous, viii. 653

Fowl, Guinea, viii. 16. 163 Numidian, viii. 163 oat, vii. 227 pea, viii. 16 Wallichean, viii. 21

Francolin, viii. 261. 263 Le Vaillant's, viii. 49 long-beaked, viii. 50 lunated, viii. 48 thoracic, viii. 50

Francolins, viii. 45

Fratercula, viii. 556 arctica, viii, 634 
Fratercula cirrhata, viii. 635 psittacula, viii. 635

Fregillus, vii. 379

Frigates, viii. 652

Fringilla, vii. 130. 137. 138 Abyssinica, vii. 132 Ethiops, vii. 144 albicollis, vii. 148 Amandava, vii. 144 Americana, vii. 148 Anæna, vii. 148 Angolensis, vii. 145 arborea, vii. 148 arcuata, vii. 135 bella, vii. 310 Bengalus, vii. 144 bicolor, vii. 145 Brissonii, vii. 157 cœrulescens, vii. 145 calcarata, vii. 119. 129

Canadensis, vii. 148 Canaria, vii. 143 cannabina, vii. 142 capitalis, vii. 134 carduelis, vii. 139. 252 caudacuta, vii. 148.149 cinerea, vii. 139. 145

Cisalpina, vii. 135

citrinella, vii. 127. 142

coccinea, vii. 140

cœlebs, vii. 138.246

coracina, vi. 300

cristata, vi. 312 ; vii. 137

crocea, vii. 128

cruciger, vii. 138

cyanea, vii. 148

domestica, vii. 135

elegans, vii. 136

erythrocephala, vii. 127. 131. 132

erythronotos, vii. 146

erythropthalma, vii. 129.138

fasciata, vii. 148

flammea, vii. 148.162

flavicollis, vii. 148

frontalis, vii. 146

Georgica, vii. 149

gnatho, vii. 158

graminea, vii. 148

granatina, vii. 144

guttata, vii. 146

Hispaniolensis, vii. 135

hyemalis, vii. 148

ictera, vii. 143

ignicolor, vii. 134

illiaca, vii. 138

Italiæ, vii. 135

Lapponica, vii. 119. 129

laticauda, vii, 134
Fringilla lepida, vii. 143

linaria, vii. 141

leucocephala, vii. 140

lutea, vii. 147

luteola, vii. 134

macroura, vii. 130. 134

Magellanica, vii. 140

maritima, vii. 149

Martinicensis, vii. 158

melanotis, vii. 147

melba, vii. 140

melodia, vii. 148

melpoda, vii. 146

montana, vii. 135.245

monticola, vii. 148

monti $=$, vii. 138

montium, vii. 142

nitens, vii. 144

nivalis, vii. 139.148

noctis, vii. 158

ornata, vii. 147

oryx, vii. 134

otoleucus, vii. 147

palustris, vii. 148

passerina, vii. 148

Pennsylvanica, vii. 148

petronia, vii. 154. 310

plialerata, vii. 134

pinus, vii. 141.

polyzona, vii. 147

psaltria, vii. 141

psittacea, vii. 140

purpurea, vii. 157. 162

pusilla, vii. 148

quelea, vii. 137

quinticolor, vii. 146

rosea, vii. 162

rubra, vii. 131

rubriventris, vii. 146

rufa, vii. 138

rufobarbata, vii. 158

Sanguinolenta, vii. 147

savanna, vii. 148

savannarum, vii. 148

Senegala, vii. 144

serinus, vii. 142

simplex, vii. 147

socialis, vii. 148

spinus, vii. 142. 268

yar., vii. 140

superciliosa, vii. 150

textris, vii. 131

tricolor, vii. 145

tristis, vii. 141. 143; viii. 688

velata, vii. 134

vespertina, vii. 157

viridis, vii. 146

vitellina, vii. 134

zena, vi. 306 
Fringillæ, vii. 227

Fulica, viii. 411.542

Ethiops, viii. 415

Americana, viii. 415

armillata, viii. 415

atra, viii. 414

aterrima, viii. 415

Cayennensis, viii. 403.409

chloropus, viii. 411

cristata, viii. 415

fistulans, viii. 413

flavipes, viii. 413

flavirostris, viii. 413

Floridana, viii. 415

fusca, viii. 411

leucoptera, viii. 415

leucorix, viii. 415

maculata, viii. 413

Martinica, viii. 413

Mexicana, viii. 415

nævia, viii. 407. 411

Noveboracensis, viii. 408 porpliyrio, viii. 412.542 properly so called, viii. 414

Fuligula, viii. 607. 612. 668 cristata, viii. 614 nigra, viii. 608 oxyura, viii. 608 rufitorques, viii. 614

Furnarius, vii. 354

annumbi, vii. 354

Chilensis, vii. 354

fuliginosus, vii. 354

Lessonii, vii. 354

Fuscalbin, le, vi. 415

Gabiota circicienta, viii. 576

Galapiot, vii. 351 ; viii. 689

Galbula, vii. 435

albirostris, vii. 436

albiventris, vii. 437

grandis, vii. 437

macroura, vii. 436

paradisea, vii. 436

ruficauda, vii. 436

viridis, vii. 426.509

Galgulus, vii. 185

melanops, vii. 187

Gallina pratajuola, viii. 453

Gallinæ, viii. 1 suppl. to the, viii. 96

Gallinago media, viii, 368

minima, viii. 369

minor, viii. 369

saturata, viii. $\mathbf{3 7 0}$

Gallinula, viii. 411

Americana, viii. 412

cæsia, viii. $\mathbf{4 0 4}$
Gallinula Carolina, viii. 400

Cayennensis, viii. 404

flavirostris, viii. 413

fusca, viii. 412

galeata, viii. 412

gigas, viii. 404

gularis, viii. 410

Javanica, viii. 411

lugubris, viii. 410

obscura, viii. 412

porphyrio, viii. 413

rubiginosa, viii. 409

ruficeps, viii. 404

sarracura, viii. 404

stellaris, viii. 407

Gallinule, Abyssinian, viii. 410 ardoisée, viii. 411

black-blacked, viii. 413

black-pointed, viii. 414

Cayenne, viii. 403

Chericote, viii. 405

common, viii. 411

crake, viii. 406

crested, viii. 415

dwarf, viii. 407

favourite, viii. 413

Kookra, viii. 410

Jamaica, viii. 408

mangle, viii. 404

Martinico, viii. 413

minute, viii. 408

mournful, viii. 410

New Holland, viii. 414

purple, viii. 412 . 542

red-tailed, viii. 411

rufous-breasted, viii. 408

rusty, viii. 409

Soree, viii. 408

spotted, viii. 407

variegated, viii. 403

white, viii. 414

white-chinned, viii. 410.542

yellow-breasted, viii. 408

Gallinules, viii. 411. 542

Gallus, viii. 18. 170

Anstrutheri, viii. 21

Bankiva, viii. 20. 174. 219

crispus, viii. 19

cristatus, viii. 216

ecaudatus, viii. 21

furcatus, viii. 21. 223

gallinaceus peregrinus, viii. 444

giganteus, viii. 21. 174. 218

Javanicus, viii. 21

lanatus, viii. 19

Macartnyii, viii. 30

Morio, viii. 19

Mozambicus, viii. 19

Patavinus, viii. 21, 218 
Gallus Patuornus, viii. 21

pentedactylus, viii. 20

Persicus, viii. 21

pumilio, viii. 20

Sonneratii, viii. 19

Gampsonyx, vi. 56

Swainsonii, vi. 56

Gamulus Australis, vi. 355

Ganga, viii. 41 chata, viii. 257

Gangas, viii. 256

Gannet, viii. 594

lesser, viii. 594

Garrots, viii. 609. 665

Garrule torquéole, vii. 182

Garrulus, vii. 181 auritus, vii. 182

cœrulescens, vii. 182 galericulatus, vii. 182 gubernatrix, vii. 180 melanogaster, vii. 182 ultramarinus, vii. 182

Geai noir à collier blanc, vi. 268

Geese, viii. 601. 661

Gelinotte huppé, viii. 36

Gerfalcon, vi. 31. 202

Gerfalcons, vi. 31

Gilwut, viii. 21

Glareola, viii. 416

Australis, viii. 417

Austriaca, viii. 417

grallaria, viii. 417

Isabella, viii. 417

lactea, viii. 418

nævia, viii. 417

Orientalis, viii. 418

pratincola, viii. 417

torquata, viii. 417

Glaucopis, viii. 184. 336

cinerea, vii. 184

leucoptera, vii. 184

temnura, vii. 184

Glottis, viii. 375

Goatsucker, vii. 104

American, vii. 75

ash-coloured, vii. 80

banded, vii. 79

Bombay, vii. 75

Brasilian, vii. 76

Cayenne, vii. 109

Chuppa, vii. 75

Cold river, vii. 81

collared, vii. 109

European, vii. 106

fork-tailed, vii. 78. 110

gold-coloured, vii. 73

Guiana, vii. 76

Indian, vii. 76

Jamaica, vii. 77
Goatsucker, Javan, vii. 75

Leona, vii. 108

long-tailed, vii. 73

New Holland, vii. 80

rufous, vii. 76

scissor-tailed, vii. 78. 113

sharp-tailed, vii. 77

spotted, vii. 80

strigoïd, vii. 79

Trinidad, vii. 81. 114

varied, vii. 75

Virginian, vii. 108

white-collared, vii. 76

white-necked, vii. 109

white-throated, vii. 74. 79

Goatsuckers, vii. 70

Gobemouche à lunettes, vi. 329

étoilé, vi. 357

noir, vi. 498

Gobemouches, vi. 3 ] 8

Godwit, American, viii. 374

Barbary, viii. 373

black-tailed, viii. 374

Cambridge, viii. 387

cinereous, viii. 386

common, viii. 372

Hudsonian, viii. 374

lesser, viii. 373

marbled, viii. 374

red, viii. 373

Godwits, viii. 372.528

Goelands, viii. 574

Goldfinch, common, vii. 139 green, vii. 140

Goosander, viii. 674

Goose, Antarctic, viii. 605

bean, viii. 602

black and white, viii. 621

Brent, viii. 604

bustard, viii. 605

Canada, viii. 599

common, viii. 601

Egyptian, viii. 604

eider, viii. 611

Gambian, viii. 661

grey-headed, viii. 606. 622

loggerhead, viii. 609

Magellanic, viii. 605

painted, viii. 606

red-breasted, viii. 605

ruddy, viii. 622

semipalmated, viii. 621

short-legged, viii. 665

snow, viii. 603

swan, viii. 600

variegated, viii. 606

white-fronted, viii. 602

Gorto, viii. 44

Goruck, vi. 414 
Goshawk, vi. 239 common, vi. 48 crested, vi. 43.45

Goshawks, vi. 236

Goura, viii. 73. 274

Gracula, vi. 421 athis, vi. 381 barita, vii. 134. 168 calvus, vi. 414 calva, vi. 423 carunculatus, vi. 413.425 Cayennensis, vii. $\mathbf{3 5 0}$ cristatella, vi. 424. 548 fœtida, vii. 370 glauca, vi. 304 grisea, vi. 423 gryllivora, vi. 422 icterops, vi. 415 larvata, vi. 425 longirostris, vi. 379.416 melanocephala, vi. 418 melanopterus, vi. 423 nobilis, vi. 416 nudicollis, vi. 370 picoides, vii. 351 plicatus, vi. 418 quiscala, vii. 134. 168 saularis, vi. 294. 392 scandens, vii. 350 sturnina, vi. 425 ; vii. 174 tristis, vi. 422.547 viridis, vi. 399 boat-tailed, vii. 134 climbing, vii. 350 dial, vi. 294 green, vi. 399 Paradise, vi. 547 satin, vi. $\mathbf{5 4 8}$

Grakles, vi. $\mathbf{5 4 6}$

Grallæ, viii. 293 suppl, on the, viii. 421

Grallaria, vi. 403 ; viii. 685

Grallina, vi. 409. 545 melanoleuca, vi. 410

Graucalus, vi. 292 mentalis, vi. 293

Grebe, American, viii. 550 black-breasted, viii. 550 black-naped, viii. 550 Cayenne, viii. 550 Chili, viii. 550 crested, viii. 547 double-horned, viii. 549 eared, viii. 549 hoary, viii. 549 horned, viii. 548 little, viii. 555 Philippine, little, viii. 549 pied-bill, viii. 549
Grebe, red-necked, viii. 548 white-winged, viii. 550

Grebes, viii. 547. 628

Grenouillard, vi. $66^{\circ}$

Griffard, vi. 229

Griffins, vi. 21. 190

Griffon, le, vi. 161

Grimpart enfumé, vii. 350

flambré, vii. 350

grand, vii. 350

maillé, vii. 350

promerops, vii. 351

Grimpars, vii. 350

Griveron, le, vi. 386

Grives, vi. $\mathbf{5 1 0}$

Grivetin, le, vi. 391

Grosbeak, vii. 151

Abyssinian, vii. 230

azure, vii. 153

Bengal, vii. 134

black, vii. 154

black-headed, vii. 158

black-lined, vii. 153

blue, vii. 156

Brazilian, vii. 154

Brisson, vii. 157

brown, vii. 153

Caffrarian, vii. 150

Canada, vii. 157

Cape, vii. 137

cardinal, vii. 156

common, vii. 156. 310

crested Dominican, vii. 137

Dominican, vii. 137

evening, vii. 157

fasciated, vii. 155

Gambia, vii. 134

grey-necked, vii. 156

green, vii. 155. 309

grenadier, vii. 136

haw, vii. 156. 310

Java, vii. 153

lineated, vii. 159

Madagascar, vii. 155

Malacca, vii. 152

minute, vii. 159

Molucca, vii. 151

nitid, vii. 152

nun, vii. 159

pensile, vii. 132

Philippine, vii. 131. 230

pine, vii. 161. 316

Porto Rico, vii. 158 purple, vii. 312

red-billed, vii. 151. 314

red-breasted, vii. 154

Siberian, vii. 159

sociable, vii. 133. 232

striated, vii. 152 
Grosbeak, violaceous, vii. 311 warbling, vii. 153 wax-billed, vii. 153 white-headed, vii. 152 white-throated, vii. 157 Grosbeaks, vii. 308

Gros-bec bleu de ciel, vii. 157 longicone, vii. 151

Grous, viii. 34. 243

Bahtah, viii. 42 black, viii. 34. 247 crowned sand, viii. 44 double-banded, viii. 43 dusky, viii. 38 Franklin's, viii. 39 Gorto, viii. 44 hazel, viii. 36. 250 heteroclite, viii. 66.268 hybrid, viii. 35. 249

Indian, viii. 43

Louisiana, viii. 263

Lybian, viii. 42

Namaqua, viii. 42. 258

pinnated, viii. 37

pintado, viii. 251

pin-tailed, viii. 41. 257

ptarmigan, viii. 40. 253

red, viii. 41.256

Rehusak, viii. 40

Richardson's, viii. 39

ruffed, viii. 37. 25]

Sabine's, viii. 39

sand, viii. 43. 259

Senegal, viii. 42

sharp-tailed, viii. 44

singed, viii. 42.258

spotted, viii. 36. 251

Surmagur, viii. 44

willow, viii. 40.256

wood, viii. 34.243

Grus, viii. 327

Balearica, viii. 475

cinerea, viii. 330. 476

minor, viii. 475

Paradisea, viii. 330

poliocephœa, viii. 331

psophia, viii. 328

struthio, viii. 331

torquata, viii. 332

virgo, viii. 475

Grylle scapularis, viii. 554

Guacuguacu, viii. 585

Guan, viii. 8

courier, viii. 33

eyebrowed, viii. 10

obscure, viii. 9

supercilious, viii. 10

Guans, viii. 7

Guarauna, viii. 333
Gubernates, vi. 318

Cunninghami, vi. 319

Guépier Adanson, vii. 405

gris rose, vii. 405

rouge gorge, vii. 405

Sonnini, vii. 405

Guillemot, black, viii. 632

Brunnich's, viii. 554

foolish, viii. 553. 632

Mandt's, viii. 555

marbled, viii. 555

white, viii. 554

white-lined, viii. 554

Guillemots, viii. 553. 631

Guiraca melanocephala, vii. I57

Guirapita, viii. 512

Guitguits, vii. 389

Gull, Arctic, viii. 579

black-headed, viii. 574. 576

black-hooded, viii. 577

common', viii. 575

fish-eating, viii. 577

forked-tailed, viii. 576

glaucous, viii. 574

herring, viii. 574

ivory, viii. 575

Kittiwake, viii. 579

laughing, viii. 575

little, viii. 577

silvery, viii. 575

skua, viii. 579

white-eyed, viii. 576

Gulls, viii. 573. 644

Gur-Roo-Mul, viii. 563

Gymnocephalus, vi. 357. 503. 508

Gymnoderes, vi. 370.503

Gymnops, vi. 40

fasciatus, vi. 41

strigillatus, vi. 42

Gypaëte of the Alps, vi. 22

Gypaëtos, vi. 19. 21

barbatus, vi. 164

of Africa, vi. 192

Gypagus, vi. 18

Gypogeranidæ, vi. 68

Gypogeranus, vi. 68. 248

Gyps, vi. 16

Habia à bec orangé, vi. 300 cega blanca, vi. 301

cobigas pintadas, vi. 302

gola negra, vi. 303

jaune, vi. 317

Ponceau, vi. 317

purizo, vi. 302

robustana, vi. 302

roxisa, vi. 301

verde, vi. 301 
Hæmatopus, viii. 322

ater, viii. 324

Brasiliensis, viii. 323

Capensis, viii. 324

leucopus, viii. 324

luctuosus, viii. 323

niger, viii. 324

ostralegus, viii. 323.466

palliatus, viii. 323

Halcyon cinnamoninus, viii. 689

Haliætus, vi. 36

canorus, vi. 37

Calei, vi. 37

Halieus, viii. 589

Halicus Brasilianus, viii. 591

lucidus, viii. 592

Halodroma, viii. 570. 642

Hardbills, vii. 316

Harfang, vi. 79

Harpagus, vi. 31

Harpies, vi. 42

Harpy, vi. 66

booted, vi. 43

great, vi. 233

great, of America, vi. 42

Harpyia, vi. 42

braccata, vi. 43

ornata, vi. 43.45

Harrier, hen, vi. 65

Hatis à bec court, viii. 585 à tête noire, viii. 585

Hawk, American sparrow, vi. 30. 202 ash coloured, vi. 49

banded, vi. 49

banded-sided, vi. 64

black-capped, vi. 55

black sparrow, vi. 54

broad-winged, vi. 49

Brown's, vi. 56

Caley's, vi. 37

common, vi. 238

common sparrow, vi. 53

falcon like, vi. 56

fish of America, vi. 39

gos, vi. 49. 236. 239

grey-breasted, vi. 51

Indian sparrow, vi. 55

insectivorous sparrow, vi. 55

Javan sparrow, vi. 55

large-billed, vi. 51

marsh, vi. 66

Nankin, vi. 29

night, vii. 78

one banded, vi. 44. 52

orange speckled, vi. 29

painted, vi. 44

pigeon, vi. 30. 239

prince Maximilian's crested, vi. 236

Cuv. Index.
Hawk, Ray's, vi. 49

red femoral, vi. 29

red-tailed, vi. 62

sharp-shinned, vi. 55

shining, vi. 50

slate coloured, vi. 54

slender, vi. 50

sparrow, vi. 53

speckled sparrow, vi. 63

streaked, vi. 55

three-streaked, vi. 51

whistling, vi. 37

white-billed, vi. 52

yellow-throated, vi. 50

Hawks, vi. 48. 236

eagle, vi. 43

Helias, viii. 333. 480 phalanoides, viii. 334

Heliornis, viii. 550. 629 Senegalensis, viii. 551

Hemipalma, viii. 383

Hemipodius, viii. 61

Dussumierii, viii. 65

fasciatus, viii. 64

Hottentotus, viii. 64

lunatus, viii. 64

maculosus, viii. 64

Meiffrenii, viii. 63

nigrifrons, viii. 62

nivosus, viii. 63

pugnax, viii. 62

tachydromus, viii. 62

thoracicus, viii. 63

Heorotarii, vii. 357

Heron, Agami, viii. 341. 491

beautiful, viii. 342

black-crested white, viii. 346

blanc, viii. 491

blue, viii. $341,342.491$

Caledonian night, viii. 346

Cayenne, night, viii. 346

cinnamon, viii. 347

common, viii. 336.485

crested purple, viii. 489

crowned, viii. 473

demoiselle, viii. 329.475

gardenian night, viii. 345

great, viii. 336

laminated, viii. 510

Louisiana, viii. 337

Malacca, viii. 343

minute, viii. 347

New Guinea, viii. 337

night, viii. 345 . 493

Philippine, viii. 344

purple, viii. 489

red-necked, viii. 491

scolopaceous, viii. 333

snowy, viii. 340 
Heron, spotted, viii. 348 wattled, viii. 332 white-fronted, viii. 342 yellow-necked, viii. 342

Herons, viii. 335. 481 night, viii. 345

Herpethotheres, vi. 52

Heteroclite, l', viii. 66

Hians, viii. 353

Hierax, vi. 30

Hierofalco, vi. 31

Himantopus, viii. 392. 532 albicollis, viii. 392 atropurpureus, viii. 392 autumnalis, viii. 392 leucurus, viii. 393 melanopterus, viii. 392 melanurus, viii. 393 Mexicanus, viii. 393 nigricollis, viii. 393 rufipes, viii. 392

Hirundo, vii. 57. 60 acuta, vii. 69 albicollis, vii. 67.70 albiventer, vii. 67 Alpestris, vii. 64 ambrosiaca, vii. 64 Americana, vii. 62. 64. 68 apus, vii. 58

bicolor, vii. 65

Borbonica, vii. 68

Capensis, vii. 63

caudacuta, vii. 70

Cayanensis, vii. 60. 93

chalybea, vii. 63

cinerea, vii. 61.69

corhinca, vii. 61

cristata, vii. 64

cyanoleuca, vii. 65

cyanoptyrha, vii. 68

Daurica, vii. 64

domestica, vii. 65.94

Dominicensis, vii. 67

erythrocephala, vii. 69

esculenta, vii. 62

fasciata, vii. 63

filifera, vii. 66

flavigaster, vii. 69

Francica, vii. 67

fuciphaga, vii. 62

fulva, vii. 62.68 ; viii. 687

fusca, vii. 69.93

fuscata, vii. 64

Hortensis, vii. 69

Indica, vii. 63

Javanica, vii. 68. 95

jugularis, vii. 65

klecho, vii. 59

leucoptera, vii. 67
Hirundo leucorhæa, vii. 69. 93

leucothea, vii. 59

Ludoviciana, vii. 61

lunifrons, vii. 68

Martinicana, vii. 69

melampiga, vii. 65

melanogaster, vii. 62 ; viii. 687

melanoleuca, vii. 65

melba, vii. 58

minuta, vii. 65

montana, vii. 61. 94

nigra, vii. 64

nigricans, vii. 66

Oonalaschkensis, vii. 66

oxyura, vii. 70. 103

Pacifica, vii. 70

paludicola, vii. 64

Panayensis, vii. 63

Pelasgia, vii. 70. 104

Peruviana, vii. 66

purpurea, vii. 63. 92

pyrrhonota, vii. 65.68

riparia, vii. 61.91

rufa, vii. 62

ruficeps, vii. 66

rufifrons, vii. 64

rupestris, vii. 61.94

rupicollis, vii. 68

rustica, vii. 61

rutila, vii. 67

Senegalensis, vii. 63

Sinensis, vii. 59

Smithii, vii. 61

subis, vii. 63

Tahitica, vii. 66

tapera, vii. 64

thalassinus, vii. 66

torquata, vii. 67

urbica, vii. 60. 89

velox, vii. 59

violacea, vii. 63

virescens, vii. 68

viridis, vi. 365 ; vii. 65

Histrionicus, viii. 609

Hoazin, viii. 4. 132

Hobby, vi. 26. 200

orange-breasted, vi. 28

orange-legged, vi. 26

spotted-tailed, vi. 57

Hoccos, viii. 3.98

Holopodius, viii. 392

Honey-eater, cowled, vi. 412 graculine, vi. 415

knob-fronted, vi. 412

Honey-suckers, vi. 412

Hoopoe, Cape, vii. 381

Hoopoes, vii. 379. 398 proper, vii. 380

Hornbill, Angola, vii. 418 
Hornbill, bifronted, vii. 417

Bontian, vii. 417

channelled, vii. 417

crested, vii. 434

crowned, vii. 418

furrowed, vii. 417

gingi, vii. 417

great-billed, vii. 416

Indian, vii. 417

Malabar, vii. 416

Panayan, vii. 417

plowed, vii. 418

psittaceous, vii. 465

rhinoceros, vii. 416

striped-tailed, vii. 418

trumpet, vii. 416

unicorn, vii. 416

violaceous, vii. 417

Hornbills, vii. 415

Horsefoot, viii. 385

race, viii. 609

Houbara, viii. 302

Houppifère, Macartney, viii. 240

Houppifères, viii. 30. 240

Howler, great grey, of Sweden, vi. 74

Howler, vi. 74

Humming-bird, broad-shafted, vii. 374 crested brown, vii. 373

crested green, vii. 372

giant, vii. 398

harlequin, vii. 372

long-tailed, vii. 369

racket-tailed, vii. 376

red-throated, vii. 375

sapphire, vii. $\mathbf{3 7 5}$

smallest, vii. 376

supercilious, vii. 368

Tobago, vii. 375

topaz, vii. 368

tufted-necked, vii. 373

white-bellied, vii. 375

Humming-birds, vii. 367. 393

Huppart, vi. 45

Hute du soleil, viii. 346

Hybarra, viii. 455

Hydrobates, viii. 607. 609

Hydrocorax dilophus, viii. 591

Hylophilus, vi. 464

poicilotis, vi. 464

thoracicus, vi. 464

Ibagau, vii. 113

Ibis, viii. 358. 512

albicollis, viii. 519

bald, viii. 361

bare-necked, viii. 360

Bengala, viii. 360

black, viii. 363
Ibis, black-faced, viii. 362,518

calva, viii. 361

candida, viii. 355

Cayenne, viii. 361

chalcoptera, viii. 362

crested, viii. 363

Egyptian, viii. 355

infuscata, viii. 361

green, viii. 363. 518

leucopygus, viii. 363

macca, viii. 360

melanocephalus, viii. 360

Molucca, viii. 360

nandapoa, viii. 355

nudifrons, viii. 361

oxycercus, viii. 361

papillosa, viii. 360

plumbeus, viii. 362

religiosa, viii. 359.518

sacred, viii. 359

scarlet, viii. 362.518

Theban, viii. 360

white, viii. 363

white-headed, viii. 356.363

white-necked, viii. 361

wood, viii. 355

1bycter, vi. 40

leucogaster, vi. 41

Icteria dumicola, vi. $\mathbf{3 5 5}$

Icterus, vii. 167 ; viii. 688

anticus, vii. 171

pecoris, vii. 168

sulcirostris, vii. 168

Tanagrinus, vii. 171

lctinia, vi. 56

Ilha do Cirne, viii. 443

1 mber, lesser, viii. 552

Imbrim, viii. 63]

Indicator, great, vii. 537

Levaillantii, vii. 462

major, vii. 462

Indicators, vii. 462. 537

löra scapularis, vi. 448 ; viii. 686

Ipecati apoa, viii. 601

Irena, viii. 683 puella, vi. 373

Iriburubicha, vi. 164

lrupero, vi. 326

Ispida Carolinensis, vii. 408

1 xos, vi. 388. 390 ; viii. 684

virescens, vi. 389

chalcocephalus, vi. 390

Jabiru, viii. 349

American, viii. 351.508

Brasiliensis, viii. 507

guacu, viii. 508

Senegal, viii. 352

\section{2}


Jabiru, white-bellied, viii. 349 Jabirus, viii. 351. 507

Jacamaciri, vịi. 437

Jacamar, alcyon, vii. 437 great, vii. 437 green, vii. 436 Paradise, vii. 436 rufous-tailed, vii. 436 three-toed, vii. 438 Vaillant's great, vii. 437 white-billed, vii. 436 white-bellied, vii. 437

Jacamars, vii. 435

Jacamerops, vii. 437

Jacana, viii. 395 chestnut, viii. 396 Chinese, viii. 397 faithful, viii. 398 . 539 Indian, viii. 396 variable, viii. 396

Jacanas, viii. 395.537

Jacbird, vii. 387

Jackdaw, vii. 176. 332 little, vii. 134

Jaco, vii. 496

Jaculot, cinereus, vii. 408

Jacuruti, vi. 72

Jahana, viii. 395

Janfredic, le, vi. 391. 438

Jaseurs, vi. 503. 508

Jay, vii. 181

blue, vii. 179. 181

Cayenne, vii. 179 of Europe, vii. 181 Paraguan, vii. 180 Peruvian, vii. 179 red-billed, vii. 179. 335

Jays, vii. 335

Jean le Blanc, vi. 40

Jensan, viii. 622

Julang, vii. 579

Kakelick, viii. 52

Kamichi, viii. 397. 538

Kestrill, vi. 26. 201 lesser, vi. 26 rufous-backed, vi. 28. 202

Kingfisher, Asiatic, vii. 410 azure, vii. 414 belted, vii. 408 black and white, vii. 408 black-billed, vii. 412 black-capped, vii. 410 Biru, vii. 410 blue-eared, vii. 413 blue-headed, vii. 409 Cape, vii. 410 cinereous, vii. 408
Kingfisher, common, vii. 426 Coromandel, vii. 411 crab-eating, vii. 411 crested, vii. 409 double-eyed, vii. 412 Gaudichaud's, vii. 428 great African, vii. 408 great brown, vii. 413 green-headed, vii. 411 grey-fronted, vii. 410 half-collared, vii. 410

Indian, vii. 409

Leach's, vii. 429

little, vii. 413

Meninting, vii. 414

purple, vii. 409 rufous, vii. 409 rufous and green, vii. 40 sacred, vii. 428 Senegal, vii. 411 Smyrna, vii. 410 spotted, vii. 408 stubbed, vii. 112 supercilious, vii. 409

Ternate, vii. 411 three-toed, vii. 414 varied, vii. 112 white and green, vii. 408 white-headed, vii. 411

Kingfishers, vii. 407. 424

King, little, vii. 246 of the couroumous, iv. 164

Kinki, viii. 235

Kite, black, vi. 58

Brasilian, vi. 40. 235

Carolina, vi. 58

common, vi. 58. 241

Etolian, vi. 243

Mississippi, vi. 243

swallow-tailed, vi. 58

Kites, vi. 57. 241

Klake, viii. 603

Knot, viii. 376

Krai, white, vi. 189

Labbes, viii. 579

Lagopedes, viii. 39

Lamellirostres, viii. 597. 657

Lamprotornis, vi. 393 erythrophiis, vi. 395 metallicus, vi. 394

Langrayen, vi. 286. 487

Laniarius, vi. 270

Lanio, vi. 270. 315

Lanius, vi. 262

Afer, vi. 267

albus, vi. 269

Americanus, vi. 266 
Lanius Antiguanus, vi. 271

Ardosiaceus, vi. 270

atricapilius, vi. 272

atricilla, vi. 291

atrococcineus, vi. 272

backakiri, vi. 271

barbarus, vi. 270

bicolor, vi. 266

Borealis, vi. 269. 485

boubou, vi. 265 . 272

boubo, viii. 678

brubru, vi. 265

cæsius, vi. 273

Canadensis, vi. 273

Capensis, vi. 265

carbo, vi. 317

Carolinensis, vi. 270

Cayanus, vi. 290

cærulescens, vi. 372

collaris, vi. 265

collurio, vi. 264

collurio rufus, vi. 263

corvinus, vi. 267

cristatus, vi. 268

Cuba, vi. 267 ; viii. 678

curvirostris, vi. 285

doliatus, vi. 272

Dominicanus, vi. 288

domicilla, vi. 275

drongo, vi. 371

emeria, vi. 486

erythropterus, vi. 267. 271 ; viii. 678

excubitor, vi. 263. 269. 485

excubitor minor, vi. 263

ferrugineus, vi. 267. 272

frontalis, vi. 295

forficatus, vi. 371

Gambensis, vi. 267

guttatus, vi. 273

gutturalis, vi. 271

infaustus, vi. 375

inquisitor, vi. 290 ; viii. 680

karu, vi. 269 ; viii. 677

kirkocephalus, vi. 269

leucocephalus, vi. 287; viii. 679

leucogaster, vi. 287

leucorhynchus, vi. 288

Leverianus, vi. 295

luctuosus, vi. 275

Ludovicianus, vi. 270. 485

macrourus, vii. 164

Madagascariensis, vi. 266

Malabaricus, vi. 371

melanocephalus, vi. 269. 389

meleager, vi. 276

Mellivorus, vi. 267

Manillensis, vi. 288

Meridionalis, vi. 264; viii. 677
Lanius mitratus, vi. 292

mystaceus, vi. 268

nævius, vi. 273

Naotka, vi. 270

Natka, vi. 270

Nengeta, vi. 322

Nubicus, vi. 265

oleagineus, vi. 270

olivaceus, vi. 270

ornatus, vi. 271

Pacificus, vi. 268

palliatus, vi. 275

Panayensis, vi. 269

pendens, vi. 268

personatus, vi. 265

picatus, vi. 295

pileatus, vi. 292

pitungua, vi. 320

plumatus, vi. 286

poliocephalus, vi. 266

Pomeranus, vi. 264

punctatus, vi. 273

rufescens, vi. 265

ruficeps, vi. 265

ruficollis, vi. 264

rufus, vi. 264. 294

rutilus, vi. 264

rutilus $\gamma$, vi. 271

scapulatus, vi. 268

scapularis, viii. 677

schah, vi. 268

Senegalensis, vi. 267

Septentrionalis, vi. 269

severus, vi. 275

signatus, vi. 267

silens, vi. 268

spinitorquens, vi. 264

stagurus, vi. 272

sulphuratus, vi. 320

superciliosus, vi. 265

Tabuensis, vi. 269

tyrannus var., vi. 323

validus, vi. 292

viridis, vi. 288

Lanner, vi. 29

Lanting, viii. 50

Lapwing, viii. 460. 462

Lapwings, viii. 318, 319

Lark, vii. 116

African, vi. 479

Alpine, vii. 210

Azara's, vii. 119

black, vii. 118. 196;

cinereous, vii. 120

clapper, vii. 117. 210

common, vii. 194

crested, vii. 116. 205

desert, vii. 119

double-banded, vii. 119 
Lark, double-crested, vii. 119. 212

dusky, vi. 478

field, vii. 194. 477

gingi, vii. 120

great-billed, vii. 119

Isabella, vii. 196

Italian, vii. 117

Malabar, vii. 120

meadow, vi. 477

mutable, vii. 118

New Zealand, vii. 120

red, vi. 479

red-backed, vii. 117. 210

red-crowned, vii. 118

rufous, vi. 479

Senegal, vii. 120

short-toed, vii. 117. 209

sky, vii. 116. 194

undated, vii. 117

white, vii. 196

white-banded, vii. 119

willow, vi. 478

wood, vii. 117. 207

Larks, vii. 193

field, vii. 55 ; vi. 477

sea, viii. 530

Larus, viii. 573. 644

Andouinii, viii. 578

Arcticus, viii. 576

argentatoides, viii. 577

argentatus, viii. 574.576

atricella, viii. 576

canus, viii. 575

cataractes, viii. 579

cirrhocephalus, viii. 576

crepidatus, viii. 579

cyanorhynchus, viii. 575

Dominicanus, viii. 578

eburneus, viii. 575

erythropus, viii. 575

flavipes, viii. 575

fuscus, viii. 575

glaucoides, viii. 576

glaucus, viii. 574

hæmatorhynchus, viii. 578

Hybernus, viii. 575

ichthyætus, viii. 577

leucomela, viii. 574

leucopthalmus, viii. 576

leucopterus, viii. 576

maculipennis, viii. 578

marinus, viii. 574

melanocephalus, viii. 577

melanurus, viii. 577

minutus, viii. 577

nævius, viii. 574

Pacificus, viii. 574

parasiticus, viii. 579. 580

poliocephalus, viii. 576
Larus ridibundus, viii. 575

rissa, viii. 579

roseus, viii. 578

Sabini, viii. 576

tridactylus, viii. 579

Lavandières, vii. 53

Leptopteryx, vi. $\mathbf{2 8 7}$

leucorhynchos, vi. 287

Leptosomus, vii. 461

Lestris, viii. 579

catarractes, viii. 580

pomarinus, viii. 580

Limacula glottis, viii. 386 marmorata, viii. 374

Limosa, viii. 372. 528 ægocephala, viii. 374

candida, viii. 375

Meyeri, viii. 373

rufa, viii. 373

Linaria, vii. 141

Lindo azul, vi. 299

azul cabeza blanca, vi. 306

bello, vi. 305

celeste oro y negro, vi. 306

pardo carpo amarillo, vi. 333

Linnet, vii. 262

great red-headed, vii. 142

lesser red-headed, vii. 141

Linnets, vii. 139. 141. 252

Liponix, viii. 31

Lobipes, viii. 391. 532

Lobopesineanus, viii. 392

Loggerhead, vii. 387

Lohong, viii. 304

Longipennes, viii. 563. 639

Longirostres, viii. 358. 512

Lophophores, viii. 13

Lophophorus, viii. 13. 153

Cuvieri, viii. 14

refulgens, viii. 14

Wallichii, viii. 15

Lophorina, vii. 191

Lophyrus, viii. 73

Loriots, vi. 540

Lory, black-capped, vii. 500

blue-tailed, vii. 501

Bornean, vii. 492

Ceram, vii. 501

collared, vii. 500

grand, vii. 491

king's, vii. 580

unicolor, vii. 500

Lorys, vii. 500

Loxia, vii. 160

Abyssinica, vii. 132. 134

Angolensis, vii. 160

Astrild, vii. 153

atricapilla, vii. 154

bella, vii. 153 
Loxia Brasiliana, vii. 154

Brissonii, vii. 157

Canadensis, vii. 157

cantans, vii. 153

Capensis, vii. 137

cardinalis, vii. 156

chloris, vii. 155. 309

coccothraustes, vii. 156. 309

cœrulea, vii. 157

collaria, viii. 159

cuvirostra, vii. 160. 161

cyanea, vii. 153

Dominicana, vii. 137

enucleator, vii. 161

erythrina, vii. 162

erythromelas, vii. 158

falcirostris, vi. 161

fasciata, vii. 155

flamengo, vii. 162

frontalis, vii. 146

fusca, vii. 153

fuscata, vii. 153

grossa, vii. 157

guttata, vii. 155

hæmatina, vii. 155

Hamburgia, vii. 135

ignicolor, vii. 137

leucoptera, vii. 161. 316

lineola, vii. 159

Ludoviciana, vii 154

macroura, vii. 134. 151

Madagascariensis, vi. 266 ; vii. 155

Maia, vii. 152

Malacca, vii. 152

melanura, vii. 156

melanocephala, vii. 134

melanoleuca, vi. 288

Mexicana, vi. 315

minuta, vii. 159

Molucca, vii. 151

nigra, vii. 154

nitida, vii. 152

noctis, vii. 160

ostrina, vii. 156

oryx, vii. 136

oryzivora, vii. 153

pensilis, vii. 132

Philippina, vii. 131

Portoricensis, vii. 158

psittacea, vii. 162

punctularia, vii. 152

pyrrhula, vii. 158

pytiopsittacus, vii. 161. 316

quadricolor, vii. 151

quinticolor, vii. 155

rosea, vii. 157. 162

sanguinirostris, vii. 151

Siberian, vii. 159
Loxia socia, vii. 133

striata, vii. 152

torrida, vii. 160

variegata, vii. 152

violacea, vii. 158

Virginica, vi. 315

Loyca, vii. 326

Lulu, vii. 117. 207

Lypornix, vii. 463 unicolor, vii. 463

Lyres, vi. 426 ; vii. 1

Macao à bec crochu, viii. 549 cornu, viii. 549 petit, viii. 550

Macareux, viii. 633

Maccaw, vii. 481 blue and yellow, vii. 482 Brazilian green, vii. 483 hyacinthine, vii. 482.577 military, vii. 482 parrakeet, vii. 483 parrot, vii. 483 purple-back ed, vii. 483 red and blue, vii. 482.577 red, yellow, and blue, vii. 482 varied, vii. 482

Maccaws, vii. 576

Machetes, viii. 381

Macreuse, viii. 607. 665

Macrodactyli, viii. 394

Macrodactylus, viii. 537

Macroptera, viii. 687

Macropteryx, viii. 687

Macropus, vii. 458

caixana, vii. 459

phasianellus, vii. 459

Macroramphus, viii. 371. 608

Macrotarsus, viii. 532

Macucagua, viii. 328

Mænura, vi. 426 ; vii. 1

Magpie, vii. 179

Bernet's, vii. 334

common, vii. 333

Malaconotus, vi. 271 ; viii. 678

Malcoha à bec peint, vii. 465 red and green, vii. 464 red-headed, vii. 464 rouverdin, vii. 464

Malcohas, vii. 464. 538

Maleyka, viii. 349

Malkoha Java, vii. 465

Malurus, vi. 466 ; vii. 39

Africanus, vii. 348

Brownii, vi. 469

clamans, vi. 467

exilis, vi. 469

galactoides, vi. 467 
Malurus gracilis, vi. 467

hirundinaceus, vi. 469 ; viii. 686

Lambertii, vi. 468

leucopterus, vi. 469 ; vii. 164

palustris, vi. 468

superciliosus, vi. 469

textilis, vii. 164

Manakin, Papuan, vi. 345

Peruvian, vi. 427

rock, vi. 427 ; vii. 2

striped-headed, vi. 431

tuneful, vi. 298

Manakins, vi. 427 ; vii. 1 true, vi. 428

Manchots, viii. 637

Mandurria, viii. 519

Mangoipe, viii. 400

Mankerro, viii. 400

Manloague, viii. 400

Mantelé, le, vi. 329

Manucode, vii. 339

Marabous, viii. 350

Marcia, viii. 622 fistularis, viii. 622

Marten, vii. 89

Martens, vii. 57

Martin, common, vi. 547 greatest, vii. 59

Martins, vi. 421.546

Martins-chasseurs, vii. 413

Mauve, viii. 574

Mbiyui, vii. 94

Mbiyiumbopi, vii. 103

Megalurus, vi. 467 palustris, vi. 480

Megapode à pieds rouges, viii. 401

Megapodius, viii. 71. 399. 540

Duperrey, viii. 400

Freycinet, viii. 400. 540

La Perouse, viii. 400

mænura, vi. 426

Reinwardtii, viii. 540

Meleagris, viii. 15. 154

gallopavo, viii. 16

ocellata, viii. 16

satyra, viii. 31

Meliphaga, vi. 412, 413. 416

chrysocephala, vi. 398

cyanops, vi. 415

Javanica, vi. 390

maculata, vi. 419

mystacalis, vi. 419

reticulata, vi. 419

Melithreptus, vi. 420 ; vii. 357.390

agilis, vii. 359

albicapillus, vii. 359

albicollis, vii. 359

ater, vii. 359

atricapillus, vii. 358
Melithreptus canescens, vii. 358

cardinalis, vii. 359

cœrulescens, vii. 358

collaris, vii. 358

cucullatus, vii. 359

dibaphus, vii. 359

erythropigius, vii. 359

flavicans, vii. 359

flavicollis, vii. 359

fuscus, vii. 358

guttatus, vii. 359

melanoleucus, vii. 359

melanops, vii. 359

Novæ Hollandiæ, vii. 359

obscurus, vii. 358

Pacificus, vii. 358

pyrrhopterus, vii. 358

sanguinolentus, vii. 359

sannio, vii. 359

tenuirostris, vii. 358

vestiarius, vii. 358

virescens, vii. 359

Mellisuga, vii. 360

Merganser, viii. 673

Brasilian, viii. 627

goosander, viii. 625

hooded, viii. 627

red-breasted, viii. 626

smew, viii. 626

Mergulus alle, viii. 555

Mergus, viii. 551. 625. 673

albellus, viii. 626

Brasiliensis, viii. 627

castor, viii. 626

cucullatus, viii. 627

merganser, viii. 625. 674

minutus mustelinus, viii. 626

serrator, viii. 626

Merion leucoptère, vii. 164 natté, vii. 164

Meriones maculatus, vi. 470

Merla bicolor, vi. 397

Merle curouje, vi. 388

de la nouvelle Guinée, vi. 395

écaillé, le, vi. 414

fluteur, le, vi. 470

Merles, vi. 374. 510

Merlin, vi. 26

common, vi. 200

rock, vi. 200

Merops, vii. 400

albifrons, vi. 417 ; vii. 405

amictus, vii. 403

apiaster, vii. 400

auritus, vi. 418

badius, vii. 401

Bengalensis, vii. 401

Brasiliensis, vii. 405

Bullock, vii. 404 
Merops cærulescens, vii. 405

Cafer, vii. 365. 405

carunculatus, vi. 413 ; vii. 405. 413

Cayanensis, vii. 402

chrysocephalus, vii. 405

chrysopterus, vi. 417

cinereus, vii. 405

Cincinnatus, vi. 416 ; vii. 405

congener, vii. 405

corniculatus, vi. 412 ; vii. 405

Coromandus, vii. 403

cucullatus, vi. 417 ; vii. 405

Cuvieri, vii. 402

cyanops, vi. 415 ; vii. 405

Daudin, vii. 403

Egyptius, vii. 401

erythropterus, vii. 402

fasciculatus, vi. 416 ; vii. 405

garrulus, vi. 417 ; vii. 405

gularis, vii. 403

Javanicus, vii. 405

Lamarckii, vii. 402

Leschenault, vii. 404

Malimbicus, vii. 403

melanurus, vii. 405

minutus, vii. 404

Molluccensis, vi. 414

monachus, vi. 412 ; vii. 405

niger, vi. 416

Novæ Zealandiæ, vi. 416

olivaceus, vi. 418 ; vii. 356

Orientalis, vii. 405

ornatus, vi. 417 ; vii. 401

Philippinus, vii. 402

Phrygius, vi. 414 ; vii. $40 J$

puella, vii. 422

quinticolor, vii. 403

rufus, vii. 354 ; viii. 689

Savignii, vii. 402

Senegalensis, vii. 401

Spiza, vi. 420

superbus, vii. 401

superciliosus, vii. 401

torquatus, vii. 401

urica, vii. 404

viridis, vii. 401.402

Merula, vi. 510. 530

candida, vi. 374

flavirostris, vi. 380

leucocephala, vi. 374

tristis, vi. 380

varia, vi. 374

Messenger, vi. 251

Microdactylus, viii. 326 cristatus, viii. 326

Micropterus, viii. 609. 665

Micropus Alpinus, vii. 59 murarius, vii. 58

Cuv. Index.
Millouins, viii. 612. 668

Milvago, vi. 40

ochrocephalus, vi. 41

Milvina, vi. 57

Milvus, vi. 57 cenchris, vi. 57 sphenura, vi. 36.59

Mimeta, vi. 399 ; viii. 684 flavocinctus, vi. 399 meruloides, vi. 399 viridis, viii. 684

Mira mata, viii. 28

Mirafa Javanica, vii. 119

Missel, vi. 524

Mitou poranga, viii. 3

Mitous, viii. 3

Mitu, viii. 6

Mniotella, vii. 356 ; viii. 686 varié, vii. 356

Monarcha, vi. 328

Monasa, vii. 463 personata, vii. 463

Monaulus Impeyanus, viii. 14

Mocking-birds, vi. 510

Moqueurs, vi. 510

Moineau du Brésil, vii. 150 ignicolor, vii. 137

Molenar, le, vi. 329

Molinar, vi. 494

Momotus; vii. 423

Montain, grand, vii. 129

Mormon, viii. 556 fratercula, viii. 556 glacialis, viii. 557

Morinellus marinus, viii. 385

Morphnus, vi. 43

Morus parvus, viii. 595

Moschatus, viii. 618

Motacilla, vi. $434,435.474$; vii. 3.7 . 50

Motacilla Enanthe, vi. 435 ; vii. 4 æstiva, vi, 460. 472

Afra, vi. 476

Africana, vi. 470

aguimp, vi. 475

alba, vi. 475 ; vii. 51

albicapilla, vi. 448

albicollis, vi. 460.462

Alpina, vi. 465 ; vii. 37

aquatica, vi. 443

arcola, vi. 447

arundinacea, vi. 442 ; vii. 30

atrata, vi. 440

atricapilla, vi. 340. 369. 444. 475

aurocapilla, vi. 454

A watcha, vi. 464

bananivora, vi. 463

Blackburniæ, vi. 460 
Motacilla, boarula,vi. 476

Bonariensis, vi. 450

Borealis, vi. 461

cærulea, vi. 459

cærulescens, vi. 475

Cafra, vi. 447

calidris, vi. 463

Calliope, vi. 440

campestris, vi. 463

Canadensis, vi. 454. 457

Capensis, vi. 475. 476.477

caprata, vi. $436^{\circ}$

caryophyllacea, vi. 448

Cayana, vii. 172

chloroleuca, vi. 462

chrysocephala, vi. 462

chrysogastra, vi. 476

chrysoptera, vii. 172

cincta, vi. $\mathbf{4 5 4}$

cinerea, vi. 475

Cingalensis, vi. 448

citrina, vi. 452

citreola, vi. 476

coronata, vi. 454

cristata, vi. 333

curruca, vi. 445 ; vii. 34

cyanea, vi. 450.468

cyanocephala, vii. 172

cyanura, vi. 447

Dominica, vi. 462

dorsalis, vi. 464

dumetorum, vi. 445

erithacus, vi. 440

erythrogaster, vi. 447

familiaris, vii. 129

ficedula, vi. 340

flava, vi. 476 ; vii. 54

flavescens, vi. 449

flavicauda, vi. 333

flavifrons, vii. 172

fulicata, vi. 436

fulva, vi. 472

fuscata, vi. 450

fuscescens, vi. 462

fuscicollis, vi. 461

garrula, vi. 445

Gibraltariensis, vi. 440

Guira, vi. 463

gularis, vi. 402.463

hirundinacea, vii. 357

hortensis, vi. 446

Hudsonica, vi. 475

hypolaïs, vi. 472

icterocephala, vi. 456

Indica, vi. 476

ischecantschia, vi. 435.450

Kamtschatkensis, vi. 463

leucomela, vi. 436

leucorhoa, vi. 437
Motacilla lineata, vii. 172

littorea, vi. 450

livida, vi. 449

longipes, vi. 452

longirostra, vi. 450

Ludoviciana, vi. 460. 472

lugubris, vi. 475

luscinia, vi. 441

lutescens, vi. 447

macroura, vi. 391

maculosa, vi. 455

Madagascariensis, vi. 463

Maderaspatana, vi. 453. 475

Mauritiana, vi. 449. 473

melanocephala, vi. 476

melanops, vi. 476

modularis, vi. 465

multicolor, vi. 462

murina, vi. 447

nævia, vi. 443

nigrirostris, vi. 447

nisoria, vi. 445

ochirura, vi. 450

olivacea, vi. 448

palmarum, vi. 454

Patagonica, vi. 461

passerina, vi. 445

Pennsylvanica, vi. 456

pensilis, vi. 460

petechia, v1. 460

phænicurus, vi. 440

Philippensis, vi. 436

Philomela, vi. 441

pinguis, vi. 454

protonotharius, vii. 172

provincialis, vi. 446

pusilla, vi. 471

regulus, vii. 39

rubecula, vi. 439

rubicola, vì. 435

ruficauda, vi. 461

ruticilla, vii. 122

salicaria, vi. 443

scialis, vi. 440

schænobanus, vi. 443

Scheltobriusk, vi. 476

silvia, vi. 444

Sinensis, vi. 449

solitaria, vi. 438

Southern, vi. 370

speciosa, vi. 478

spinicauda, vii. 349

Stapazina, vi. 435

striata, vi. 456

subflava, vi. 391.469

Suecica, vi. 439

sulphurea, vi. 476

sunamisica, vi. 450

sutoria, vi. 450 
Motacilla tigrina, vi. 457 titys, vi. 440 typhia, vi. 448 trochilus, vi. 472 ; vii. 41 Troglodytes, vi. 473 Tschutschensis, vi. 476 umbria, vi. 454 undata, vi. 449 velia, vi. 305 varia, vi. 464 ; vii. 356 variegata, vi. 475 vermivora, vii. 172 virens, vi. 456 viridis, vi. 475 Zeylonica, vi. 448

Motmot, vii. 423

Brasilian, vii. 406

Dombey, vii. 407 red-headed, vii. 407

Motmots, vii. 406

Mouette cendrée, viii. 578 grande, viii. 578

Mouettes, viii. 574

Mud-hen, fresh-water, viii. 402

Murier, vi. 502

Muscicapa, vi. 318. 328.339

Aëdon, vi. 344. 494

Afra, vi. 330

agilis, vi. 352

albicapilla, vi. 333

albifrons, vi. 342

albiventer, vi. 327

alector, vi. $\mathbf{3 5 4}$

alectura, vi. 354

ampelina, vi. 354

animosa, vi. 323

Arcadica, vii. 337

armillata, vi. 333

atra, vi. 324.350

atricapilla, vi. $339,340.343$. 501

audax, vi. 320

aurantia, vi. 292. 337. 339

auricomis, vi. 417

Australis, vi. 347. 370

azurea, vi. 341

azurou, vi. 493

Banyumas, vi. 345

barbata, vi. 324. 332. 347 ; viii. 681

bicolor, vi. 337. 350, 351. 362

biloba, vi. 372

Borbonica, vi. 330

cæruleocapilla, vi. 342

cærulea, vi. 329. 341. 459 ; viii. 683

cæsia, vi. 273. 353

Caledonica, vi. 367

Cambaiensis, vi. 347
Muscicapa Canadensis, vi. 351 cana, vi. $\mathbf{3 6 2}$

cantatrix, vi. 345.356

Capensis, vi. 341

carinata, vi. 328.331

Carolinensis, vi. 385

caudacuta, vi. 334

Cayanensis, vi. 349

cinerascens, vi. 325

cinerea, vi. 32 l. 323

Cingalensis, vi. $\mathbf{3 3 1}$

cinnamomea, vi. 332

coccinigastra, vi. 348

Cochinsinensis, vi. 341

collaris, vi. 329.340

colonus, vi. 336

comata, vi. 341

coronata, vi. 332. 338.349 ; viii. 682

crepitans, vi. 296

cristata, vi. 328 . 330. 352

cucullata, vi. 347

cyanea, vi. 329

cyanocephala, vi. 342

cyanomelas, vi. $\mathbf{3 4 4}$

cyanorostris, vi. 333

Deserti, vi. 329

despotes, vi. 319

diope, vi. 277

diops, vi. 353

divaricata, vi. 373

Dominicana, vi. 327

emarginata, vi. 373

erincta, vi. 320

erythrogaster, vi. $\mathbf{3 4 8}$

erythropis, vi. 341

eximia, vi. 353

ferox, vi. 324

ferruginea, vi. 35]

flabellifera, vi. 368

flammea, vi. 346

flammiceps, vi. 354

flava, vi. 327

flavicauda, vi. 326.350

flavicollis, vi. 329

flavifrons, vi. 347

flavigaster, vi. $\mathbf{3 6 8}$

flaviventris, vi. 334

forficata, vi. 319

fucata, vi. 319

fuliginosa, vi. 351 ; vii. 122

fusca, vi. 324. 334. 350

fuscescens, vi. 330

galeata, vi. $326^{\circ}$

Goodenovii, vi. 348

grisea, vi. $\mathbf{3 4 3}$

griseo capilla, vi. 343

grisola, vi. 339. 493

gularis, vi. 353 
Muscicapa hœmorhousa, vi. 344. 381. 388

hirundinea, vi. 345 ; viii. 682

hyacinthina, vi. 346

icteropterys, vi. 334

indigo, vi. 345

Joazeiro, vi. 322

Javanica, vi. 344. 346

labrosa, vi. 331 ; viii. 681

Lathami, vi. 348

legatus, vi. 321

leucura, vi. 342

longicauda, vi. 319

longipes, vi. 356 ; viii. 683

Ludoviciana, vi. 320

lutea, vi. 367

Luzoniensis, vi. 330

maculata, vi. 348

Madagascariensis, vi. 330

mœsta, vi. 326

malachura, vi. 468

Manillensis, vi. 342

mastacalis, vi. 354

melanictera, vi. 345

melanoleuca, vi. 329

melanocephala, vi. 347. 469

melanopis, vi. 367

melanops, vi. 333

melanoptera, vi. 329. 351

melodia, vi. 356

meloxantha, vi. 341

miniata, vi. 346

minuta, vi. 351.459

mitrata, vi. 455

monacha, vi. 336

multicolor, vi, 348

muscipeta, vi. 340

mutata, vi. 330

mystacea, vi. 327.367

nævia, vi. 367

nigerrima, vi. 333

nigricans, vi. 334

nigriceps, vi. 326

nigrifrons, vi. 343

nitens, vi. 345

nitida, vi. 341

nivea, vi. 326

Novæ Hollandiæ, vi. 367

Noveboracensis, vi. 356

nunciola, vi. 324.350

obscura, vi. 332. 345.347 ; viii. 682

obsoleta, vi. 352

ochracea, vi. 363

ochrocephala, vi. 367

ochroleuca, vi. 252

oleaginea, vi. 325

ulivacea, vi. 356

pagana, vi. 325
Muscicapa Paradisii, vi. 330

parva, vi. 340)

passerina, vi. 348

pectoralis, vi. 367.434

petechia, vi. 336

Phœebe, vi. 324. 350

phœnoleuca, vi. 333

Philippensis, vi. 330

plumbea, vi. 325

polyglotta, vi. 322

Pondicheria, vi. 342

pririt, vi. 340

pristinaria, vi. 344. 494

psalura, vi. 355

Psidii, vi. 342.388

punctata, vi. 334

pygmæa, vi. 352

querula, vi. 349. 350

rapax, vi. 350

rhodogastra, vi. 348

rhodoptera, vi. 346

rivularis, vi. 354

rosea, vi. 342

rubra, vi. 315.334

rubricollis, vi. 314. 359

rufescens, vi. 338

ruficapilla, vi. 334

rufifrons, vi. 351.369

rufina, vi. 327

rufiventris, vi. 344

rupestris, vi. 354

ruticilla, vi. 350 ; viii. 682

ruticolla, vi. 333

Sandwichensis, vi. 347

Saya, vi. 350 ; viii. 682

scita, vi. 344

sebrica, vi. 344

Senegalensis, vi. 331. 340

sibilans, vi. 326

sibilator, vi. 334

silva, vi. 356

similis, vi. 322

Sinensis, vi. 343

solitaria, vi. 355

spadicea, vi. 332

stellata, vi. 357 ; viii. 683

stenura, vi. 354 ; viii. 682

straminea, vi. 354

strangulata, vi. 325

streptophora, vi. $\mathbf{3 4 0}$

subflava, vi. 346

Suiriri, vi. 352

sulfurea, vi. 326

superciliosa, vi. 343

Surinama, vi. 352

sylvicola, vi. 355

tectec, vi. 343

thamnophiloides, vi. 322

torquata, vi, 341 
Muscicapa tyrannus, vi. 319 undulata, vi. 344 varia, vi. 327 variegata, vi. 343 velata, vi. 325 ven tralis, vi. 353 vetula, vi. 319 virens, vi. 350 virescens, vi. 353 virgata, vi. 352 viridis, vi. 355 vittigera, vi. 325 vociferans, vi. 354 Yetapa, vi. 320 Yiperu, vi. 319

Musophaga, vii. 508. 584 gigantea, vii. 507 variegata, vii. 507 violacea, vii. 508

Muthurau, viii. 27

Mycteria, viii. 351. 507 Americana, viii. 351. 508 Senegalensis, viii. 352 macropterus, vi. 368

Myiagra, vi. 368 plumbea, vi. 368 rubecoloides, vi. 368

Myiothera, vi. 399 ; viii. 685 affinis, vi. 400 brachyura, vi. 400 campanisona, vi. 404 capistrata, vi. 405 Coraya, vi. 408 ferruginea, vi. 408 fuliginosa, vi. 405 leuconota, vi. 409 loricata, vi. 406 malura, vi. 407 melanothorax, vi. 405 mentalis, vi. 405 nematura, vi. 406 perspicillata, vi. 406 pileata, vi. 406 ruficeps, vi. 409 rufimarginata, vi. 408 squamata, vi. 406 strictothorax, vi. 405 superciliaris, vi. 278. 405 tetema, vi. 404 tinnicus, vi. 403 umbretta, vi. 404 velata, vi. 400

Myioturdus, vi. 403

Myophonus, vi. 402 metallicus, vi. 403

Myrmothera, vi. 403 ; viii. 685 axillaris, vi. 407 cœrulescens, vi. 409 Colma, vi. 404
Myrmothera fuscicapilla, vi. 407 gutta, vi. 409 longipes, vi. 407 melanoleucos, vi. 407 tetema, vi. 404 tinnica, vi. 544

Mystacinus, vii. 123

Nabirop, vi. 393

Nacagua, vi. 52

Nacunda, vii. 76

Nacurutu, vi. 72 sans aigrettes, vi. 83

Nandou, viii. 296

Nandu, viii. 438

Nauclerus, vi. 58

Nasican le, vii. 351

Nectarinia, vi. 412. 418 ; vii. 353.389 aurifrons, vii. 365 Cafer, vii. 365 coccinogaster, vii. 363 eximia, vii. 363 Hasselti, vii. 363 Kuhlii, vii. 365 lepida, vii. 363 metallica, vii. 364 melanopogon, vii. 365 melanorhyncus, vii. 365 mysticalis, vii. 364 pectoralis, vii. 363 rubricosa, vii. $\mathbf{3 5 7}$ solaris, vii. 363.365

Nectris fuliginosa, viii. 570 munda, viii. 570

Nelicourvi, vii. 132. 231

Nemosia, vi. 310 flavicollis, vi. 310 ruficapilla, vi. 310

Neophron, vi. 19

Neops, ruficauda, vii. 346

Nightingale, vi. 441 ; vii. 12 river, vi. 441 ; vii. 29

Nisser, vi. 22

Nisus, vi. 53

Noctua, vi. 77

Noddies, viii. 585

Notherodius Guarauna, viii. 333

Nothura, viii. 70

Noumenois, viii. 519

Numenius, viii. 364 Africanus, viii. 380.531 arcuata, viii. 365 arquatus, viii. 519 atricapillus, viii. 366 brevirostris, viii. 366 cyanopus, viii. 365 Hudsonicus, viii. 365 leucocephalus, viii. 366 
Numenius longirostris, viii. 365

Luzoniensis, viii. 366

Madagascariensis, viii. 364

pusillus, viii. $\mathbf{3 8 0}$

pygmæus, viii. 366.380

rufus, viii. 364 . 366

Tahitiensis, viii. 366

tenuirostris, viii. 365

variabilis, viii. 379

virgatus, viii. 364

Numida, viii. $] 6$

Ægyptica, viii. 18

cristata, viii. 17. 168

Meleagris, viii. 17. 163

mitrata, viii. 18

ptylorhyncha, viii. 18

Nutcracker, vii. 183. 336. 387

Nutjobber, vii. 387

Nuthatch, vii. 344.385

common, vii. 385

spotted, vi. 408

Nyroca, viii. 612

Ocypterus, vi. 286. 487

albovittatus, vi. 287

fuscatus, vi. 287

rufiventer, vi. 288

Odontophorus, viii. 268 rufus, viii. 59

Edicnemus, viii. 306. 322. 455 crepitans, viii. 306 Europæus, viii. 456 grallarius, viii. 307 longipes, viii. 307 maculosus, viii. 306 magnirostris, viii. 307 recurvirostris, viii. 307

Enanthe, vi. 440 ; vii. 3

Enas cata, viii. 42 bicinctus, viii. 44

Oidemia, viii. 607 leucoptera, viii. 608

Oiseau de Paradis noir, vii. 191 de Saint Martin, vi. 65

Onocratalus, viii. 587 Phœnix, viii. 589

Onores, viii. 337

Onychorlıynchus, vi. 332

Opæthus, vii. 507 Buffonia, vii. 507 erythrolophus, vii. 507

Opetiorhynchus, vii. 354

Ophie, vii. 354

Ophiotheres, vi. 68

Ophistolophus, viii. 399 fidelis, viii. 399

Opisthocomus, viii. 11. 133

Orambleu, l', vi. 394
Oranga, vii. 474

Oranor, vii. 1.22

Oricou, l', vi. 163

Oriole, black, vii. 318

black and yellow, vii, 166

Bonana, vii. 170

chestnut, vii. 167

Coudougan, vi. 397

crested, vii. 166.317

golden, vi. 540

of Europe, vi. 397

olive, vii. 167

Paradise, vi. 398

red, vii. 173

red-breasted, vii. 169

red-rumped, vii. 166

rice, vii. 133

St. Domingo, vii. 167

weaver, vii. 131

white-winged, vi. 311 ; vii. 170

yellow, vii. 318

yellow-winged, vii. 167

Orioles, vi. 540

true, vi. 396

Oriolus, vi. 396

Americanus, vii. 169

annulatus, vi. 397

arundinarius, vi. 398

auratus, vi. 397

aureus, vii. 191. 398

Baltimore, vii. 170

bicolor, vi. 397 ; vii. 403

Bonana, vii. 170

Capensis, vi. 398 ; vii. 167

castaneus, vii. 167

caudacutus, vii. 149

Cayanus, vii. 167

chrysocephalus, vii. 167

citrinus, vii. 169

Cochinchinensis, vi. 397

cristatus, vii. 17l. 166

Dominicensis, vii. 167

furcatus, vi. 398

galbula, vi. 397.540

galbula $\beta$, vi. 397

galbula $\gamma$, vi. 398

Guyanensis, vii. 171

hippocrepis, vi. 398

icterocephalus, vii. 170

icterus, vii. 169

Jamaicaii, vii. 171

larvatus, vi. 397

leucogaster, vi. 398

leucopterus, vi. 311 . 398 ; vii. 170

Ludovicianus, vii. 133

maculatus, vi. 398

melaleucos, vi. 311

melancholicus, vii. 171 
Oriolus melanocephalus, vi. 397 Mexicanus, vii. 168.170 minor, vi. 134. 169 monachus, vi. 397 mutatus, vii. 167 niger, vii. 133 Novæ Hispaniæ, vi. 397 olivaceus, vii. 168 oryzivorus, vii. 133.134 Persicus, vii. 166 Phœnicus, vii. 169 picus, vii. 351.398 radiatus, vi. 397 regens, vi. 398 ruber, vii. 173

Sinensis, vi. 397. 424 spurius, vii. 170 textor, vi. 398 ; vii. 131.134 variegatus, vi. 399 varius, vii. 167 viridis, vi. 399 xanthocephalus, vii. 170 xanthonotus, vi. 398 xanthornus, vii. 170

Orphæa, vii. 34

Orpheus cærulescens, vi. 387 curvirostris, vi. $\mathbf{3 8 7}$

Ortalida, viii. 10

Orthonyx, vii. 347 spinicaudatus, vii. 347

Orthorhyncus, vii. 372 amazilia, vii. 379 Cora, vii. 376 frontalis, vii. 345 stephanoides, vii. 373

Ortolan, vii. 126

Ortygis, viii. 62

Ortygonometra, viii. 406 crex, viii. 407 marmorata, viii. 407

Ortyx Douglasii, viii. $6 \mathrm{l}$ picta, viii. 61

Osprey, vi. 36. 39. 230 Carolina, vi. 39 Cayenne, vi. 39

Ossifrage, vi. 230

Ostralega Europæa, viii. 323 leucopus, viii. 324

Ostrich, viii. 422 black, viii. 295 of Magellan, viii. 438

Ostriches, viii. 295

Otis, viii. 300

Abyssinica, viii. 304

Afra, viii. 302

Arabs, viii. 303

atriceps, viii. 304

aurita, viii. 304

Australis, viii. 305
Otis Barrowii, viii. 304

Bengalensis, viii. 303

Caffra, viii. 305

Denhami, viii. 303. 455

Houbara, viii. 302. 454

Indica, viii. 305

Nubia, viii. 303

Rhoda, viii. 302

tarda, viii. 301

tetrax, viii. 301. 453

torquata, viii. $\mathbf{3 0 3}$

Otus, vi. 71

Ouira-ouassa, vi. 181

Ourax, viii. 5 mitu, viii. 6. 119

Ourigourap, vi. 20.189

Owzel, brook, viii. 402 ring, vi. 375.534 white-tailed, vi. 375

Owl, Arcadian, vi. 81 bare-legged, vi. 83 barn, vi. 75 bay, vi. 75 black-headed, vi. $84 ?$ Brasilian, vi. 76 brown, vi. 71 Cayenne, vi, 82 chestnut-winged, vi. 81 common long-eared, vi. 71 common passerine, vi. 79 common white, vi. 75. 257 Coquimbo, vi. 79.258 cross-bearing, vi. 86 crossed, vi. 85 downy, vi. 82 falconine, vi. 78 fasciated, vi. 82 ferruginous, vi. 81 great American horned, vi. 72 great-horned, vi. 76

hairy, vi. 85

Hard wicke's naked-legged, vi. 77 hawk, vi. 78

howling, of Canada, vi. 74

hutum, vi. 77

Indian, vi. 84. 85

Lempyi, vi. 84

large-billed, vi. 73

little, vi. 80. 259

little-eared, vi. 255

long-billed, vi. 73

masked, vi. 83

Mauge's, vi. 85

mouse, vi. 75

noisy, vi. 73

occipital, vi. 82

Oriental eared, vi. 73

pagoda, vi. 85

pearl, vi. 81 
Owl, red and mottled, vi. 83 red-eared, vi. $256^{\circ}$ red-passerine, vi. 80 short-crested, vi. 71 short-eared, vi. 71 snowy, vi. 79. 256 Sonnerat's, vi. 85 sparrow-like, vi. 82 spectacle, vi. 83 spotted-eared, vi. 72 supercilious, vi. 77

Tenglmalm's, vi. 80 tuidara, vi. 75

Ural, vi. 78 variegated, vi. 78 white, vi. 79 white-eared, vi. 84 white-edged, vi. 86 white-horned, vi. 73 wood of England, vi. 76

Owls, vi. 25]

falconine, vi. 77

horned, vi. 71

Oxtyxelus, viii. 63

Oxyglossus, viii. 686

Oxyrhinci, vii. 321. 171

Oxyrhincus, vii. 171 cristatus, vii. 347 flammiceps, vii. 171. 347

Oxyura, viii. 615

Oyster-catcher, brown-backed, viii. $\mathbf{3 2 3}$ New Holland, viii. 323 pied, viii. 323. 466

Oyster-catchers, viii. 322. 466

Pachycephala, vi. 369 ; viii. 685 fuliginosa, vi. 370

fusca, vi. 369

olivacea, vi. 370

striata, vi. 434

Pachyptila, viii. 570. 642

Pachyrhynchus, viii. 679. 680

Cayanus, vi. 290

cinerascens, vi. 291

Cuvierii, vi. 291

niger, vi. 291

rufescens, vi. 291

semifasciatus, vi. 290

variegatus, vi. 292

Palæornis, vii. 484. 558

Palamedea, viii. 397.538 cornuta, viii. 398 cristata, viii. 326

Palikours, vi. 544

Palmipedes, viii. 545. 628

Pandion, vi. 39 caracara, vi. 235
Paons, viii. 12

Paonzello, viii. 462

Paradisea, vii. 188 alba, vii. 191. 383

apoda, vii. 189.341

aurea, vii. 191 ; viii. 684

chalybea, vi. 289 ; vii. 191

cirrhatus, vii. 191

furcata, vii. 191

gularis, vi. 395 ; vii. 191

leucoptera, vii. 191

magnifica, vii. 190

minor Papuana, vii. 191

nigra, vi. 395 ; vii. 191

regia, vii. 190 . 342

rubra, vii. 189

sexsetacea, vii. 190

superba, vii. 191

tristis, vi. 422.547

viridis, vi. 289

Pardalotus, vi. 431

cristatus, vi. 430

Parkinsonius mirabilis, vi. 426

Parotia, vii. 190

Parra, viii. 395

Enea, viii. 396

Africana, viii. 396.397

Brasiliensis, viii. 396

Cayennensis, viii. 321

chavaria, viii. 396.398

Chinensis, viii. 397

Dominica, viii. 322

gallinacea, viii. 397

Goensis, viii. 321

Indica, viii. 396

Jacana, viii. 396. 537

Ludoviciana, viii. $\mathbf{3 2 1}$

Luzoniensis, viii. 397

melanchloris, viii. 396

mutica, viii. 397

nigra, viii. 396

Senegalla, viii. 32I

superciliosa, viii. 396

variabilis, viii 396

viridis, viii. 396

Parrakeet, vii. 483

Alexandrine, vii. 485

Amboina red, vii. 488

Angola yellow, vii. 484

banded, vii. 484

Bengal, vii. 485

black, vii. 486 . 492

blossom-headed, vii. 485

blue-banded, vii. 578

Brazil yellow, vii. 488

bridled, vii. 491

brown-throated, vii. 489

Carolina, vii. 489

Cayenne, vii. 490 
Parrakeet, collared, vii. 484 crimson-fronted, vii. 492 crimson-winged, vii. 487 golden, vii. 492 golden-crowned, vii. 489 grey-breasted, vii. 490 grey-cheeked, vii. 490 Illinois, vii. 489 lace-winged, vii. 491 lory, vii. 491

Malacca, vii. 485 mustache, vii. 490 nonpareil, vii. 487 Papuan, vii. 486 Pavouane, vii. 483 Pennant's, vii. 487 racket-tailed, vii. 493 red and blue-headed, vii. 489 red-breasted, vii. $486^{\circ}$ red-shouldered, vii. 492 red-winged, vii. 491 scaly-breasted, vii. 484 Sincialo, vii. 486 Tabuan, vii. 488 Turcosine, vii. 493 undulated, vii. 492 wave-breasted, vii. 484 yellow-collared, vii. 485 yellow-winged, vii. 490 zoned, vii. 493

Parraqua, viii. 128

Parraquas, viii. 10

Parrot, Amazon, vii. 497. 584

Amboina, vii. 499

autumnal, vii. 498

Barnard's, vii. 581

Bauer's, vii. 580

black-headed, vii. 502

black-winged, vii. 501

blue-crested, vii. 503

blue-faced, vii. 497.579

blue-fronted, vii. 498

blue-headed, vii. 496.503

bonneted, vii. 500

Brazil, vii. 497

Brown's, vii. 487. 580

collared, vii. 502

common green, vii. 497

diadema, vii. 500

Dufresne's, vii. 497

dusky, vii. 497

dusty green, vii. 498

festive, vii. 498

Geoffroy's, vii. 499

gold-headed, vii. 501

great-bellied, vii. 491

green, vii. 584

green and red Chinese, vii. 499 gregarious, vii. 504

Cuv. Index.
Parrot, grey, vii. 496

grey-headed, vii. 502

ground, vii. 505

Guinea, vii. 503

Havanna, vii. 498

hawk-headed, vii. 499

Indian green, vii. 502

King's, vii. 580

large black, vii. 487

Le Vaillant's, vii. 499

marked, vii. 496

masked, vii. 487.579

New Zealand, vii. 506

nonpareil, vii. 579

Otaheite, vii. 503

passerine, vii. 501

Pavouane, vii. 489. 578

Pennant's, vii. 579

Philippine, vii. 502

pileated, vii. 501

plain, vii. 503

purple, vii. 496

racket-tailed, vii. 582

red-banded, vii. 492.497

red-crowned, vii. 495

red-masked, viii. 499

red-sided, vii. 499

Rosehill, vii. 579

sapphire-crowned, vii. 502

Senegal, vii. 499

short-winged, vii. 503

small, vii. 492

Sparman's, vii. 503

spotted, vii. 579

Swindernian, vii. 502

Tabuan, vii. 488. 581

Ternate blue-headed, vii. 490

Tui, vii. 501

varied, vii. 581

vernal, vii. 502

violet, vii. 503

white-breasted, vii. 496

white-fronted, vii. 498

white-headed, vii. 498

yellow-faced, vii. 499

yellow-green, vii. 504

Parrots, vii. 481.547

filamentous tongued, vii. 579

nimble, vii. 505

with a trunk, vii. 504

Partridge, viii. 37.45

African, viii. 50

Asiatic, viii. 55

Austral, viii. 55

beautiful, viii. 55

brown, viii. 54

brown African, viii. 47

buff-breasted, viii. 55

Cape, viii. 48 
Partridge, Caspian, viii. 52 Ceylon, viii. 46. 47 chestnut-bellied, viii. 55 Cliukar, viii. 54

Clapperton's, viii. 46 common, viii. 51. 264

Cranch's, viii. 49

Curria, viii. 48

Damascus, viii. 51

dusky-breasted, viii. 55

dwarf, viii. 55

eye-brown, viii. 55

eyed, viii. 53

francolin, viii. 45

Gingi, viii. 57

gorget, viii. 54, 55

Greek, viii. $52.266^{\circ}$

grey, viii. 264

Guernsey, viii. 51. 265

Guiana, viii. 59. 268

Hayes, viii. 53

Javan, viii. 50

Lawrence, viii. 55

Leona, viii. 55

little, vi. 544

Malacca, viii. 33

Mexican, viii. 61

Mosambique, viii. 55

mountain, viii. 52

naked-necked, viii. 49

New Holland, viii. 65

olive, viii. 54,55

Oriental, viii. 55

pearled, viii. 46

perching, viii. 55

Pondicherry, viii. 45

red, viii. 52. 265

red-billed, viii. 55

red-necked, viii. 49

rock, viii. 65

rufous-breasted, viii. 52

rust-bellied, viii. 55

true, viii. 51

undulated, viii. 55

white-cheeked, viii. 55

wood, viii. 53

Partridges, viii. 260

American, viii. 267

Parulus ruficeps, vii. 347

Parus, vii. 120

Alpinus, vii. 123

amatorius, vii. 123

Americanus, vi. 459 ; vii. 123

ater, vii. 120. 218

atricapillus, vii. 122

atriceps, vii. 122

biarmicus, vii. 123. 222

bicolor, vii. 121

cæruleus, vii. 121
Parus Capensis, vii. 124

caudatus, vii. 121. 220

celer, vii. 123

Chinensis, vii. 123

cinerascens, vii. 123

cinereus, vii. 123

coccineus, vii. 122

cristatus, vii. 121

cyaneus, vii. 122

furcatus, vii. 122. 123

griseus, vii. 123

ignotus, vii. 123

Indicus, vii. 123

Kujœsuk, vii. 122

lugubris, vii. 122

macrocephalus, vii. 123

major, vii. 120

Malabaricus, vi, 346 ; vii. 122

Narbonensis, vii. 124

niger, vii. 123

Novæ Zelandiæ, vii. 123

palustris, vii. 121. 122. 218

pendulinus, vii. 124

peregrinus, vi. 346

Selbyensis, vii. 122

Sibiricus, vii. 122

Stömei, vii. 123

Virginianus, vii. 123

Passeres, vi. 261

third family of the, vii. 116

fourth family of the, vii. 344

Passerina, vii, 136

fusca, vii. 138

macronyx, vii. 138

maculata, vii. 138

ruficapilla, vii. 129

Pastor, vi. 392. 413. 422 ; viii. 684

candidus, vi. 423

corythaix, vi. 422

fuscus, vi. 422

griseus, vi. 424

Galla, vi. 424

ruficollis, vi. 425

setiger, vi. 396

temporalis, vi. 422

tricolor, vi. 423

Patre, le, vi. 436

Pauxi, viii. 5

mitu, viii. 119

Pavo, viii. 12

bicalcaratus, viii. 13.150

cristatus, viii. 12

Javanicus, viii. 13

muticus, viii. 12.150

spiciferus, viii. 13

Thibetanus, viii. 13

Pavonzino, viii. 462

Peacock, viii. 135

crested, viii. 12 
Peacock, iris, viii. 13

Japan, viii. 12

little, viii. 480

Luzonian, viii. 304

Malay, viii. 24

wild, viii. 305. 462

Peacocks, viii. 12

Pelecanoïdes, viii. 570

Pelican, viii. 587. 648 greater frigate, viii. 593

Pelicans, viii. 587

Pelicanus, viii. 587

Africanus, viii. 591

aquilus, viii. 593

bassanus, viii. 594

carbo, viii. 590

cristatus, viii. 591

fuscus, viii. 588

Goemardi, viii. 592

graculus, viii. 590

Manillensis, viii. 588

minor, viii. 593

nævius, viii. 591

onocrotalus, viii. 588

Palmerstonii, viii. 593

parvus, viii. 595

perspicillatus, viii. 589

Philippensis, viii. 588

piscator, viii. 594

punctatus, viii. 591

pygmæus, viii. 591

roseus, viii. 588

rufescens, viii. 589

sula, viii. 594

trachyrhynchos, viii. 589

Vieillotii, viii. 589

Pelidna, viii. 378

cinclus, viii. 379

pectoralis, viii. 379

Pendulinus chrysocephalus, vii. 167

flavigaster, vii. 167

longirostris, vii. 169

rufigaster, vii. 168

Penelope, viii. 7. 104. 120

Aburvi, viii. 10

Arracuan, viii. 8

cristata, viii. 8.120

Cumanensis, viii. 8. 123

guttata, viii. 8.

Jacquacu, viii. 8

Jacucacu, viii. 8

Jacupeba, viii. 8

Jacupemba, viii. 8

jacutinga, viii. 8

leucolophus, viii. 8

marail, viii. 8. 9. 125

obscura, viii. 9

pipile, viii. 9. 123
Penelope satyra, viii. 31 siffleur, viii. 123 superciliaris, viii. 10

Penguin, Antarctic, viii. 562 apterous, viii. 563 Cape, viii. 563

Chiloe, viii. 562

common, viii. 559

crested, viii. 561

great, viii. 559

hairy, viii. 562

little, viii. 562

Magellanic, viii. 562

New Holland, viii. 563

Papuan, viii. 561

Patagonian, viii. 560

red-footed, viii. 561

three-toed, viii. 562

woolly, viii. 562

Penguinaria cristata, viii. 561

Patachonica, viii. 560

Penguins, viii. 556. 558

Pepoaza, vi. 322

couronné, vi. 325

Dominicain, vi. 327

Percnoptera, vi. 19

Percnoptère, le, vi. 15

Percnopteri, vi. 186

Percnopterus, monk, vi. 20 of Egypt, vi. 20. 186

Perdix, viii. 45. 261

Adamsonii, viii. 46

Afra, viii. 46. 50

Arragonica, viii. 43

Asiatica, viii. 55

Australis, viii. 56

Ayamham, viii. 50

Borealis, viii. 59

Cambayensis, viii. 55

Cambienensis, viii. 33

Capensis, viii. 49

Capueira, viii. 59

Caspia, viii. 52

Chukar, viii. 54

cinereo-alba, viii. 51

clamator, viii. 48

Clappertonii, viii. 46

Cranchii, viii. 49

Damascena, viii. 51

dentata, viii. 59

ferruginea, viii. 55

fusca, viii. 54

Gardnerii, viii. 47

Gibraltarica, viii. 64

Gingica, viii. 57

Græca, viii. 52

grisea, viii. 58

gularis, viii. 50. 53 
Perdix Hardwickii, viii. 48 Hayii, viii. 53 Hudsonicus, viii. 60. 408 Le Vaillantii, viii. 49 longirostris, viii. 50 lunulata, viii. 48 Madagascariensis, viii. 46 Næcra, viii. 61 Novæ Guineæ, viii. 58 oculea, viii. 53 olivacea, viii. 54 perlatus, viii. 46 personata, viii. 53 rubiginosa, viii. 58 rubra, viii. 52 rufina, viii. 59 saxatilis, viii. 52 scutata, viii. 54 Sinensis, viii. 46 Sonnini, viii. 60 spadiceus, viii. 47 sylvatica, viii. 53 textilis, viii. 57 thoracica, viii. 50 torqueola, viii. 55 varia, viii. 65 ventralis, viii. 55 Zeilonicus, viii. 47 Pernis, vi. 59

Petrel, American, viii. 568 broad-billed, viii. 573 brown-banded, viii. 566 cinerea, viii. 565 diving, viii. 570 dusky, viii. 569 echasse, viii. 567 fulmar, viii. 565 giant, viii. 565 grey, viii. 566 hartie, viii. 565 Mank's, viii. 569 Pacific, viii. 569 pintado, viii. 565 shear water, viii. 568 stormy, viii. 566. 642 turtle, viii. 566

Petrels, viii. 564. 639 giant, viii. 641

Petrocincla, vi. 375

Petrodroma, vi. 420 ; vii. 352 Bailloni, vii. 353 sanguinea, vii. 353

Petroica, viii. 685

Pettichaps, vi. 446 lesser, vi. 472

Pezopous, vii. 505

Pezoporus, vii. 559

Pezus, viii. 67
Pezus niamba, viii. 68 serratus, viii. 67 Yapura, viii. 68 zabele, viii. 68

Phæne, vi. 21 gigantea, vi. 22

Phænicophœus, vii. 464 calorhenchus, vii. 465 Javanicus, vii. 465 superciliosus, vii. 465 viridis, vii. 464

Phænicopterus, viii. 418.543 antiquorum, viii. 420 leucogaster, vii. 464 minor, viii. 420 ruber, viii. 419

Phæopus, viii. 365 arcuatus, viii. 365

Phaetons, viii. 596. 656 ætherius, viii. 596 phænicurus, viii. 597

Phalacrocorax, viii. 589

Phalarope, gray, viii. 392 hyperborean, viii. 391 plain, viii. 384 red, viii. 391

Phalaropus, viii. 383. 391.532 fimbriatus, viii. 392 frenatus, viii. 392 fulicarius, viii. 384 rufus, viii. 384 Welleamsii, viii. 391 Wilsonii, viii. 392

Phaleris, viii. 557. 635 cerorhynca, viii. 558

Phasianus, viii. 18 Africanus, vii. 507 ; viii. 33 Amherstii, viii. 25 Argus, viii. 24 castaneus, viii. 28 Colchicus, viii. 22. 225 Crawfurdii, viii. 27 cristatus, viii. 11. 32.33 cruentatus, viii. 47. 264 Diardi, viii. 23. 235 erythropthalmus, viii. 28 fuscus, viii. 13 gallopavonis, viii. 22 gallus, viii. 19 Gardneri, viii. 47 garrulus, viii. 129 Hamiltonii, viii. 27 ignitus, viii. 30. 240 Impeyanus, viii. 14. 33 Lathami, viii. 26 leucomelanos, viii. 14. 33 melanocephalus, viii. 29 Mexicanus, viii. 33 
Phasianus motmot, viii. 10. 33. 129 muthura, viii. 27

Nepaulensis, viii. 29

nycthemerus, viii. 233

Parraka, viii. 10

Parragua, viii. 33. 129

pavoninus, viii. 13

pictus, viii. 23

Pucrasse, viii. 26

Reevesii, viii. 25

rufus, viii. 28

satyrus, viii. 31

superbus, viii. 25

torquatus, viii. 22

varius, viii. 21

veneratus, viii. 25

versicolor, viii. 23.241

Pheasant, African, vi. 38

Argus, viii. 24. 235

bar-tailed, viii. 26

black-headed, viii. 29

brown Nepaul, viii. 29

brown Penang, viii. 28

Butrol, viii. 21

Chittagong, viii. 27

coloured, viii. 14

common, viii. 225

Crawford's, viii. 27

fire-backed, viii. 240

golden, viii. 23

horned, viii. 241

hybridal, viii. 22. 232

Impeyan, viii. 14

lineated, viii. 27

motmot, viii. 10

Nepaul, viii, 241

painted, viii. 232

Parraka, viii. 10

Pukraas, viii. 26

Reeves', viii. 25

ring, viii. 22. 232

rufous-tailed, viii. 28

sanguine, viii. 47. 264

silver, viii. 23. 233

Sumatra, viii. 28

superb, viii. 21. 239

Sylhet, viii. 26

Turkey, viii. 22

variegated, viii. 231

white, viii. 232

Pheasants, viii. 18. 21. 37. 170. 225

Phene ossifrage, vi. 190

Phibalura, vi. 433

flavirostris, vi, 433

Philedon, vi. 411.546

auriculatus, vi. 416

musicus, vi. 413

Pharoïdes, vi. 413

xanthotis, vi. 416
Philemon, vi. 414. 417. 418

albifrons, vi. 417

chrysopterus, vi. 414.417

chrysotis, vi. 416

cinereus, vi. 414

cucullatus, vi. 417

erythrotis, vi. 416

icterops, vi. 415

marmoreus, vi. 415

nævius, vi. 418

nigricollis, vi. 419

sagittatus, vi. 266

viridis, vi. 415

Philydor albogularis, vii. 347

ruficollis, vii. 347

Phimus, vii. 507

Phrenotrix, vii. 184

Physeta, vi. 52

Phy totoma, vii. 319 rara, vii. 319

Piauhaus, vi. 503

Pibe, vii. 56

Pica, vii. 178

albicollis, vii. 180

chrysops, vii. 180

olivacea, vii. 180

vagabunda, vii. 180

Picchion baillon, vii. 355

Pico de pungo cola de pala, vi. 432

Picuculi, vii. 350.389

obscura aplomado, vi. 430

Picumnes, vii. 452

Picumnus abnormis, vii. 453

cirrhatus, vii. 452

exilis, vii. 453

Picus, vii. 438.509

Picus affinis, vii. 449

albirostris, vii. 442

arator, vii. 451

aurantius, vii. 443

auratus, vii. 451

aurulentus, vii. 444

Bengalensis; vii. 443

bicolor, vii. 447. 449

Borei, vii. 449

brachyurus, vii. 449

Brasiliensis, vii. 449

Cafer, vii. 451

campestris, vii. 448,449

Canadensis, vii. 446

canus, vii. 439

cardinalis, vii. 448

Carolinus, vii. 447

Ceylonus, vii. 444

Chilensis, vii. 443

chlorocephalus, vii. 445

chrysochloros, vii. 449

coronatus, vii. 450

candidus, vii. 449 
Picus Cayanensis, vii. 448 chlorolophos, vii. 449 cinnamoneus, vii. 445 concretus, vii. 442 cristatus, vii. 449 Dominicanus, vii. 443. 449 erythraucen, vii. 449 erythrocephalus, vii. 442 erythrops, vii. 449 exalbidus, vii. 445 fasciatus, vii. 449 flavescens, vii. 448 flavicans, vii. 446.450 flavicollis, vii. 449 flavifrons, vii. 450 fucescens, vii. 449 galeatus, vii. 441 Goensis, vii. 443 Goertan, vii. 444 hirsutus, vii. 450 hirundinaceus, vii. 446 icterocephalus, vii. 449,450 icteromelas, vii. 449 ischnorynchos, vii. 449 Javanensis, vii. 449 Jumana, vii. 446 Latlımii, vii. 450 leucogaster, vii. 449 leuconotus, vii. 440 leucotis, vii. 449 Lichtensteinii, vii. 449 lineatus, vii. 44] Luzonicus, vii. 445 Macei, vii. 449 Machloti, vii. 449 maculatus, vii. 449 maculifrons, vii. 450 maculipennis, vii. 449 Mahrattensis, vii. 449 major, vii. 440 Malaccensis, vii. 447 Malacunus, vii. 450 Manillensis, vii. 444 martius, vii. 439 medius, vii. 440. 446 melanochloris, vii. 448 melanoleucos, vii. 441 melanopterus, vii. 449 mentalis, vii. 444 Meropirostris, vii. 449 macrocephalus, vii. 444 miniatus, vii. 445 minor, vii. 449 Moluccensis, vii. 449 mystaceus, vii. 449 olivaceus, vii. 449 palalaca, vii. $\mathbf{4 4 5}$ passerinus, vii. 445 pectoralis, vii. 449
Picus peralaimus, vii. 449 percussus, vii. 443 philophenarum, vii. 450 pileatus, vii. 441 poicilophos, vii. 451 poicirolophus, vii. 449 principalis, vii. 441 pubescens, vii. 447 pulverulentus, vii. 442 punctatus, vii. 449 punctiligerus, vii. 449

Punicus, vii. 444 querulus, vii. 448,449 radiolatus, vii. 449 robustus, vii. 442 rotatus, vii. 449 ruber, vii. 449 rubescens, vii. 450 rubiginosus, vii. 449 rubricollis, vii. 442.449 rubriventris, vii. 446 ruficeps, vii. 449 rufus, vii. 447 Ruppelii, vii. 449 scutatus, vii. 449 Senegalensis, vii. 444 spilogaster, vii. 449 squamosus, vii. 449 striatus, vii. 448,449 superciliaris, vii. 448 tiga, vii. 450 torquatus, vii. 443 tridactylus, vii. 450 tristis, vii. 449 undatus, vii. 447 validus, vii. 442 varius, vii. 446 variegatus, vii. 449 Vieillotii, vii. 449 villosus, vii. 446 viridis, vii. 439 vittatus, vii. 449 xanthotænia, vii. 449

Pie-grièche de la Louisiane, vi. 485 Pie-grièches, vi. 262

Pie houpette, vii. 180

Piep, vii. 56

Pies, vii. 178

Pigeon, aromatic, viii. 94 azure, viii. 83 biset, viii. 76. 277 black-banded, viii. 80 black-capped, viii. 82 black-winged, viii. 93 bronze-winged, viii. 78 brown, viii. 90 Cape, viii. 92 carunculated, viii. 72. 273 chestnut-shouldered, viii. 77 
Pigeon, domestic, viii. 277 double-crested, viii. 86 double-ringed, viii. 84 Egyptian, viii. 89 elegant, viii. 80 ferrugineous-vented, viii. 79 garnet-winged, viii. 82 Geoffroy's, viii. 84 great-crowned, viii. 72. 274 great-tailed, viii. 85 grey, viii. 79 hackled, viii. 80 hook-billed, viii. 95

Hottentot, viii. 75 Hurrial, viii. 291 jamboo, viii. 81 Madagascar, viii. 80.93 magnificent, viii. 86 Martinico, viii. 74 naked-cheeked, viii. 80 New Zealand, viii. 89 Nicobar, viii. 73. 275 nutmeg, viii. 78 ordinary, viii. 76 painted, viii. 88 parrot, viii. 94 partridge, viii. 73 passenger, viii. 90 passerine, viii. 75 picazuro, viii. 89 picui, viii. 75 Pompadour, viii. 95 porphyry, viii. 85 purple-breasted, viii. 90 purple-crowned, viii. 90 purple-shouldered, viii. 95 red-crowned, viii. 80 ring, viii. 76. 275 ring-tailed, viii. 79 russet, viii. 81

St. Domingo, viii. 92

St. Thomas, viii. 94 scallop-necked, viii. 79 scarlet, viii. 89 silky, viii. 82 spotted green, viii. 89 spotted-ring, viii. 78 squameous, viii. 85 stock, viii. 76.276 Talpacoti, viii. 74 tambourin, viii. 83 Temminck's, viii. 83 tiger, viii. 89 triangular-spotted, viii. 79 turtle, viii. 289 varied, viii. 84 vinaceous, viii. 84 violet-naped, viii. 81 Waaba, viii. 93
Pigeon, Wallia, viii. 291 whiskered, viii. 83 white-bellied, viii. 74 white-crowned, viii. 79 white-eared, viii. 87 white-faced, viii. 78 white-fronted, viii. 74 white-masked, viii. 82 white nutmeg, viii. 78 white-winged, viii. 81 yellow-faced, viii. 95

Pigeons, viii. 71. 270 gallinaceous, viii. 72. 272 ordinary, viii. 275

Pillurion, vi. 488

Pinguin, Cape, viii. 639 crested, viii. 639 Patagonian, viii. 638

Pinguins, viii. 633. 636

Pinson des bois, petit, vi. 502

Pintado, viii. 163 crested, viii. 17. 168 Guinea, viii. 17 mitred, viii. 18.169

Pintados, viii. 16

Pipi des buissons, vii. 56

Pipit, bush, vii. 56 Javan, vi. 480

Pipits, vi. 477 ; vii. 55

Pipra, vi. 427,428 ; vii. 1 albifrons, vi. 409 aureola, vi. 428 aurocapilla, vi. 428 caudata, vi. 432 chloris, vi. 429 coronata, vi. 431 crisopogon, vi. 433 cristatus, vi. 433 cyanocephala, vi. 430 Desmaresti, vi. 432 elata, vi. 431 erythrocephala, vi. 428,429 filicauda, vi. 431 galeata, vi. 429,430 gularis, vi. 432 gutturalis, vi. 429 gutturosa, vi. 430 herbacea, vi. 431 leucocapilla, vi. 429 longicauda, vi. 432 ; viii. 685 manacax, vi. 429 melanocephala, vi. 433 militaris, vi. 433 ; viii. 685 musica, vi. 298 nævia, vi. 338. 409 nigra, vi. 430 Papuensis, vi. 345 pareola, vi. 428 pectoralis, vi. 430 
Pipra Peruviana, vi. 427

pileata, vi. 429

plumbea, vi. 430

polyglotta, vi. 355 ; viii. 683

punctata, vi. 432

rubrifrons, vi. 433

rupicola, vi. 427

serena, vi. 298,429

striata, vi. 431

strigilata, vi. 429

superba, vi. 428

Pitpits, vii. 171

Pitta, vi. 400

Angolensis, vi. 402

Australis, vi. 401

brachyura, vi. 401

cyanoptera, vi. 402

erythrogastra, vi. 401

gigas, vi. 401

glaucina, vi. 403

hippocrepis, vi. 400

melanocephala, vi. 401

pileata, vi. 402 ; viii. 684

strepitans, vi. 400

superciliaris, vi. 401

thoracica, viii. 684

versicolor, vi. 401

Pitylus, vii. 157. 311 atrochalybeus, vii. 158

Plantain-eater, vii. 507. 508

Pauline, vii. 507

Touraco, vii. 507. 585

varied, vii. 507

Plastron noir, le, vi. 473 ; viii. 687

Platalea, viii. 356

aiaia, viii. 357

leucorodia, viii. 357. 512

pygmæa, viii. 383

tenuirostris, viii. 357

Platycercus, vii. 488.579

Playtypus, viii. 607. 611

Borealis, viii. 612

Leisleri, viii. 612

Platyrhynchus, vi. 320

albicollis, vi. 331

aurantius, vi. 337

barbatus, vi. 332

Borbonicus, vi. 330

brachyurus, vi. 338

brevirostris, vi. 335

cancromus, vi. 337

Ceylonensis, vi. 337

chrysoceps, vi. 335

cinereus, vi. 335

collared, vi. 357

collaris, vi. 329 . 357

coronatus, vi. 338 ; viii. 682

cyanoleucus, vi. 331

Desmarestis, vii. 357
Platyrhynchus Dupontii, vi. 339

filicauda, vi. 336

flavigaster, vi. 331. 335

hirundinaceus, vi. 335

leucocephalus, vi. $\mathbf{3 3 8}$

leucophaius, vi. 336

leucotes, vi. 338

maculatus, vi. 338

melanoleucus, vi. 331

melanops, vi. $\mathbf{3 3 7}$

murinus, vi. 336

musicus, vi. 337

mystaceus, vi. 337

nævius, vi. 338

olivaceus, vi. 337

paganus, vi. 336

perspicillatus, vi. 329

platurus, vi. 336

polychopterus, vi. 331

pusillus, vi. 357

regius, vi. 337

rubecula, vi. 331

rufescens, vi. 338

ruficauda, vi. 335

ruficaudatus, vi. 338

ruficollis, vi. 332

rufiventris, vi. 331

sulphurescens, vi. 335

velatus, vi. 331

virescens, vi. 337

xanthopygus, vi. 335

Plectrophanes, vii. 128. 227 ; viii. 600

melanotus, viii. 601

Ploceus, vii. 130. 229

alecto, vii. 133

aurantius, vii. 131

aurifrons, vii. 132

Baglafecht, vii. 132

bicolor, vii. 135

collaris, vii. 135

cristatus, vii. 131. 231

flavocapillus, vii. 135

jonquillaceus, vii. 135

malimbic, vii. 131

nigerrimus, vii. 134

nigricollis, vii. 132

pensilis, vii. 231

textor, vii. 320

velatus, vii. 135

Plotus, viii. 595. 655

anhinga, viii. 656

Levaillantii, viii. 596

melanogaster, viii. 595

Surinamensis, viii. 551

Plover, viii. 455

alwargrim, viii. 308

Asiatic, viii. 311

black-headed, viii. 316

black-throated, viii. 318 
Plover, chestnut-billed, viii. 318 collared, viii. 460 crested spur-winged, viii. 316 curonian, viii. 309 dotterel, viii. 459 dusky, viii. 314 dwarf, viii. 312 fulvous, viii. 314 golden, viii. 308. 458 gregarious, viii. 311 grisled, viii. 314 half-webbed, viii. 312 hooded, viii. 317 Indian, viii. $\mathbf{3 1 0}$ Kentish, viii. 309 long-legged, viii. 307. 392. 533 Mongolian, viii. 311 New Holland ringed, viii. 318 New Zealand, viii. 317 noisy, viii. 309. 460 piping, viii. 312 red-chested, viii. 314 red-headed, viii. 317 red-necked, viii. 312 ringed, viii. 309. 460 rusty-crowned, viii. 317 sombre, viii. 315 spur-winged, viii. 316 three-banded, viii. $\mathbf{3 1 0}$ ticksee, viii. 318 white-bellied, viii. 315

Wilson's, viii. 310 wreathed, viii. 316

Plovers, viii. 305. 307. 455

Plumipeda, vi. 44

Pluvianus, viii. 316 chlorocephalus, viii, 316 melanocephalus, viii. 316

Plyctolophus, vii. 493

Pochard, black and white, viii. 616 duck, viii. 668 Mexican, viii. 615

Podarges, vii. 80. 113 Podargus auritus, vii. 114 Australis, vii. 81 cornutus, vii. 81 Cuvierii, vii. 80 great-headed, vii. 81 horned, vii. 81 humeralis, vii. 81 Javanensis, vii. 80 red, vii. 80

Podiceps, viii. 547. 628 Americanus, viii. 550 Arcticus, viii. 548 Carolinensis, viii. 549 Chilensis, viii. 550 kalipareus, viii. 550 Ludovicianus, viii. 549

Cuv. Index.
Podiceps minor, viii. 549 occipitalis, viii. 550 Philippensis, viii. 549 poliocephalus, viii. 549 Rollandi, viii. 550

Podoa, viii. 550

Podophilus, vii. 460

Pogonias, vii. 466 erythromelas, vii. 466 hæmatops, vii. 467 lævirostris, vii. 467 leuconotus, vii. 467 niger, vii. 467 personatus, vii. 467 rubicon, vii. 467 rubrifrons, vii. 467 Stephensii, vii. 467 sulcirostris, vii. 466 Vieillottii, vii. 467

Polochion, vi. 414

Polyborus, vi. 40 vulgaris, vi. 40

Polyplectron, viii. $\mathbf{1 5 0}$

Polyplectrum, viii. 13 albocellatum, viii. 13 chalcurum, viii. 13 chenquis, viii. 13

Pomatorhinus, vii. 355 montanus, vii. 355 trivirgatus, vii. 355 turdinus, vii. 355

Pompadour, le, vi. 360

Porphyrio, viii. 412 albus, viii. 414 green-mantled, viii. 413 Indicus, viii. 414 irete, viii. 409 mealy, viii. 414 melanotus, viii. 413 pulverulentus, viii. 414 smaragdinus, viii. 414 smaragnotus, viii. 413 tavoua, viii. 413

Porte lambeaux, le, vi. 425

Porzana, viii. 406

Pouillo, vii. 41

Poulette d'eau, viii. 411

Poultry, common, viii. 19

Pratincole, Australasian, viii. 417 Austrian, viii. 417 cream-coloured, viii. 418. 543 Oriental, viii. 418

Pratincoles, viii. 416. 543

Pressirostres, viii. 449

Prinia familiaris, vii. 348

Prinops, vi. $286^{\circ}$ Geoffroyii, vi. 286

Prionites, vii. 406. 423 Brasiliensis, vii. 400 
Prionites marcii, vii. 407

Pririt, le, vi. 331.340

Pritandun, vii. 393

Procellaria, viii. 564

æquinoctialis, viii. 569

Anglorum, viii. 569

Bérard, viii. 565

Brasiliana, viii. 591

cærulea, viii. 571

Capensis, viii. 640. 565

cinerea, viii. 565

desolata, viii. 566

fregatta, viii. 568

fuliginosa, viii. 569

furcata, viii. 568

gigantea, viii. 565

glacialis, viii. 565

grallaria, viii. 567

grisea, viii. 566

hastata, viii. 570

hiemalis, viii. 570

Leachii, viii. 567

leucocephala, viii. 570

lugens, viii. 566

marina, viii. 568

obscura, viii. 569

oceania, viii. 567

Pacifica, viii. 569

pelagica, viii. 566

puffinus, viii. 568

turtur, viii. 566

urrinatrix, viii. 570

velox, viii. 566

vittata, viii. 571

Wilsonii, viii. 567

Procnias, vi. 365.503

cucullata, vi. 366 ; viii. 683

cyanotropeus, vi. 365

hirundinacea, vi. 365

melanocephalus, vi. 507

ventralis, vi. 365

Promerar, vii. 383

Promepie, vii. 451

Promerops, vii. 381.399

bleu, vii. 382

cæruleus, vii. 382

Cape, vii. 381

crested, vi. 330

moqueur, vii. 405

Namaquois, vii. 405

olivatre, vii. 356

red-billed, vii. 405

siffleur, vii. 383

Promerupe, vii. 383

Psaris, vi. 290 ; viii. 679.680

cayanus, viii. 680

cristatus, vi. 291

Cuvierii, vi. 291 ; viii. 679

erythrogenys, vi. 291
Psaris Jardinii, viii. 680 niger, vi. 292 spotted, vi. 488

Psarocolinus leucopteryx, vii. 168

Psaroides, vi. 392

Psittacirostra icterocephala, vii. 162

Psittacules, vii. 501

Psittacus, vii. 481 accipitrinus, vii. 499 æruginosus, vii. 489 æstivus, vii. 497 albifrons, vii. 498 Alexandri, vii. 485.486 Amazonicus, vii. 497 ambiguus, vii. 483 Amboinensis, vii. 488 annulatus, vii. 485 Aracanga, vii. 482 Ararauna, vii. 482 aterrimus, vii. 505 atropurpureus, vii. 488 aureus, vii. 489 auricapillus, vii. 484 Australis, vii. 493 autumnalis, vii. 498 Banksii, vii. 495 barbulatus, vii. 485 barrabandi, vii. 485.502 Bengalensis, vii. 485 bitorquatus, vii. 486 Borneus, vii. 492 Brasiliensis, vii. 497 Brownii, vii. 487 buccalis, vii. 490 cærulifrons, vii. 497 canicularis, vii. 489 canus, vii. 502 capistratus, vii. 491 Carolinensis, vii. 489 ehlorolepidotus, vii. 493 chrysostomus, vii. 492 coccii, vii. 495 coccineus, vii. 493 Columbinus, vii. 497 concinnus, vii. 492 cornutus, vii. 506 cristatus, vii. 494 cyanocephalus, vii. 486 cyanogaster, vii. 486 cyanomelas, vii. 488 cyanotis, vii. 497 cyanurus, vii. 493.501 diadema, vii. 500 discolor, vii. 492 Domicella, vii. 493. 500 Dominicensis, vii. 497 Dufresnianus, vii. 497 elegans, vii. 487 eos, vii. 495 
Psittacus erithacus, vii. 584 erythacus, vii. 496 erythrocephalus, vii. 485 erythropterus, vii. 487 eupatria, vii. 485 eximius, vii. 487 festivus, vii. 498 flavirostris, vii. 496 formosus, vii. 505 fringillaceus, vii. 503 funereus, vii. 495 galeatus, vii. 495 galeritus, vii. 494 galgulus, vii. 502 garrulus, vii. 493.501 Geoffroyii, vii. 499 gigas, vii. 505 gingianus, vii. 485 gloriosus, vii. 487 goliath, vii. 505 gramineus, vii. 499 grandis, vii. 491 gregarius, vii. 504 guaruba, vii. 488 guebiensis, vii. 493 guyanensis, vii. 483.489 hæmatopus, vii. 486 Havanensis, vii. 498 humeralis, vii. 492 hyacinthinus, vii. 482 icterotis, vii. 488 Illigeri, vii. 483 incarnatus, vii. 491 Indicus, vii. 502 infuscatus, vii. 499 inornatus, vii. 484 Leachii, vii. 495 Levaillantii, vii. 499 lori, vii. 500 leucocephalus, vii. 498 leucotis, vii. 484

Lichtensteinii, vii. 493 Ludovicianus, vii. 489 lunatus, vii. 493 luteus, vii. 488 macao, vii. 482.577 macawuana, vii. 483 macrognathus, vii. 489 macrorhynchus, vii. 491 maitaca, vii. 500 Malaccensis, vii. 485 marginatus, vii. 491 melanocephalus, vii. 496 melanopterus, vii. 501 menstruus, vii. 496 micropterus, vii. 503 militaris, vii. 482 mitratus, vii. 500 Moluccanus, vii. 486
Psittacus Moluccensis, vii. 494 multicolor, vii. 488 murinus, vii. 490 muscarinus, vii. 487 nasicus, vii. 494 niger, vii. 486 Novæ Guineæ, vii. 492 Novæ Zelandiæ, vii. 506 olivaceus, vii. 491 ornatus, vii. 491 Papuensis, vii. 486 passerinus, vii. 501 Patagonicus, vii. 484 Pennanti, vii. 487 personatus, vii. 499 pertinax, vii. 489 phigy, vii. 503 Philippensis, vii. 502 Philippinarum, vii. 494 pileatus, vii. 501 Pondicerianus, vii. 490 porphyrocephalus, vii. 503 porphyrus, vii. 503 pulchellus, vii. 493 pullarius, vii. 503 pulverulentus, vii. 498 purpureus, vii. 496 pusillus, vii. 492 radhea, vii. 493 riciniatus, vii. 493 roseus, vii. 495 ruber, vii. 493 rufirostris, vii. 486 scapulatus, vii. 488.580 Senegallus, vii. 499 setarius, vii. 493. 582 severus, vii. 483 signatus, vii. 496 simplex, vii. 503 sinensis, vii. 499 solstitialis, vii. 484 sordidus, vii. 497 sosova, vii. 490 Sparmanni, vii. 503 spurius, vii. 488 squamosus, vii. 484 stavorini, vii. 493 sulfureus, vii. 494 swindericianus, vii. 502 Tabuensis, vii. 488 Taitianus, vii. 503 Temminckii, vii. 495 tenuirostris, vii. 495 torquatus, vii. 484.502 tovi, vii. 490 tricolor, vii 482 tui, vii. 50] undulatus, vii. 492 unicolor, vii. 493.500 p 2 
Psittacus vasa, vii. 487 venustus, vii. 488.578 vernalis, vii. 502 versicolor, vii. 484 vinaceus, vii. 497 virescens, vii. 490 viridissimus, vii. 493 vittatus, vii. 484 xanthops, vii. 499 xanthopterigius, vii. 504 xanthosomus, vii. 490 zonarius, vii. 493

Psophia, viii. 327 crepitans, viii. 328.470 leucoptera, viii. 329 undulata, viii. 302 viridis, viii. 328

Psoplodes, vi. 296

Ptarmigan, viii. 253

Ptarmigans, viii. 39

Pterocles, viii. 41. 256 arenarius, viii. 43.259 bicinctus, viii. 43 coronatus, viii. 44 exustus, viii. 42. 258 guttatus, viii. 42 Lichtenstenii, viii. 43 quadricinctus, viii. 44 setarius, viii. 42 tachypetes, viii. 42.258

Pteroglossus, vii. 479 culik, vii. 480 Langsdorffii, vii. 480 Humboldtii, vii. 480 Reinwardtii, vii. 480

Ptilinopus, viii. 90

Ptilonorhynchus, vi. 294. 400. 548 holosericeus, vi. 294 Macleayii, vi. 295; vii. 1 Smithii, vi. 295 viridis, vi. 295

Ptiloris paradiseus, vii. 384

Puffinaria, viii. 570

Puffin, viii. 556 Garnotii, viii. 570

Puffins, viii. 568

Puffinus, viii. 568. 642 sericeus, viii. 570

Pygargus, vi. 36

Pyranga, vi, 315 chlorocephalus, vi. 316 cinerea, vi. 316 cyanictensis, vi. 316 erythromelas, vi. 315 erythropis, vi. 316 icteromelas, vi. 316 icteropus, vi. 316

Pyrgita, vii. 134
Pyrrhocorax, vi. 395 leucopterus, vi. 396 violaceus, vi. 294

Pyrrhula, vii. 158. 312 auranticollis, vii. 158 azurea, vi. 302 cinereola, vii. 159 crispa, vii. 160 falcirostris, vii. 159 frontalis, vii. 160 longicauda, vii. 160 mysia, vii. 160 sanguinirostris, vii. 314 social, vii. 160 superciliosa, vii. 158 synoica, vii. 160

Quail, Andalusian, viii. 62

Balen, viii. 64 black-fronted, viii. 62 black-necked, viii. 62 Californian, viii. 60 Chinese, viii. 56 common, viii. 56. 267 Coromandel, viii. 57 crescent, viii. 64 crested, viii. 60 Dubkee, viii. 65 elegant, viii. 55 fighting, viii. 62 Gibraltar, viii. 64 grey-throated, viii. 58 Hottentot, viii. 64 Luzonian, viii. 63 Madagascar, viii. 57 Malouine, viii. 60 marbled, viii. 55 Maryland, viii. 59 montercy, viii. 61 mottled, viii. 64 New Guinea, viii. 58 New Holland, viii. 56 Oriental, viii. 53. 55 reddish, viii. 58 Sonnini's, viii. 60 Southern, viii. 65 spotted-necked, viii. 65 sultry, viii. 65 white-necked, viii. 58

Quails, viii. 266

American, viii. 267

Quan, viii. 8 bishop of the, viii. 267

Querula cinerea, vi. 325 lumachelli, vi. 503 Quiscalus versicolor, vii. 168 
Rachama, vi. 187

Rachamah, vi. 20

Rale, viii. 540

Rail, viii. 540

banded, viii. 403

blue-necked, viii. 402

brown, viii. 404

Cape, viii. 405

Ceylon, viii. 406

clapper, viii. 402

dark, viii. 410

dusky, viii. 406

fasciated, viii. 410

four streaked, viii. 405

gular, viii. 405

land, viii. 541

little, viii. 408

long-billed, viii. 403

New Holland, viii. 410

Otaheite, viii. 406

Pacific, viii. 406

Philippine, viii. 403

red-breasted, viii. 406

rufous, viii. 410

rufous-headed, viii. 410

Sandwicl, viii. 406

striated, viii. 403

Tabuan, viii. 406

Troglodytes, viii. 406

Virginian, viii. 402

water, viii. 402.541

Rails, viii. 401

Rallus, viii. 401. 540

aquaticus, viii. 402. 541

Ardeoïdes, viii. 333

Australis, viii. $406^{\circ}$

Baillioni, viii. 407

Barbaricus, viii. 406

Bengalensis, viii. 372

cærulescens, viii. 402

Capensis, viii. 405

Carolinus, viii. 408

Cayannensis, viii. 408

Ceylonicus, viii. 406

chericote, viii. 405

crepitans, viii. 402

crex, viii. 406

dubius, viii. 406

fasciatus, viii. 410

ferrugineus, viii. 406

fuscus, viii. 404

giganteus, viii. 333.405

gigas, viii. 333. 404

gularis, viii. 405

Jamaicensis, viii. 408

limicola, viii. 402

longirostris, viii. 403

maximus, viii. 404

melanurus, viii. 405
Rallus minutus, viii. 408

nigra, viii. 409

nigricans, viii. 405

nigrolateralis, viii. 408

Noveboracensis, viii. 408

Pacificus, viii. 406

parvus, viii. 408

Pennsylvanius, viii. 402

phænicurus, viii. 411

Philippensis, viii. 403

Porzana, viii. 407

pusillus, viii. 407

quadristriatus, viii. 405

ruficollis, viii. 408

Sandwicensis, viii. 406

striatus, viii. 403

Tabuensis, viii. 406

Taitiensis, viii. 406

torquatus, viii. 403

Troglodytes, viii. 406

variegatus, viii. 403

Virginianus, viii. 402

Ramphastos, vii. 477

Aldrovandi, vii. 478.479

ambiguus, vii. 479

Aracari, vii. 479

Azaræ, vii. 480

Baillioni, vii. 480

callorhynchos, vii. 478

carinatus, vii. 478

chlororhynchos, vii. 479

Cuvieri, vii. 479

dicolorus, vii. 479

erythrorynchos, vii. 478

erythrosema, vii. 478

inscriptus, vii. 480

maculirostris, vii. 480

maximus, vii. 478

momota, vii. 406

pectoralis, vii. 478

piscivorus, vii. 478

piperivorus, vii. 480

Stors, vii. 479

sulcatus, vii. 480

Temminckii, vii. 479

Toco, vii. 477

Tucai, vii. 478

Tucanus, vii. 478

Vaillantii, vii. 479

viridis, vii. 479

Ramphocelus, vi. 316

coccineus, vi. 317

purpureus, vi. 317

Ramphopis, vi. 316

Razor-bill, viii. 559

Raven, vii. 175

white-necked, vii. 177. 330

Ravens, vii. 326. 327

Recurvirostra, viii. 393 
Recurvirostra Americana, viii. 394 avocetta, viii. 393 Novæ Hollandiæ, viii. 394 Orientalis, viii. 394 rubricollis, viii. 394

Redbreast, vi. 439 ; vii. 9

Redpoll, yellow, vi. 460

Redstart, vi. 440 American, vi. 350

Redwing, vi. 528

Regina aurarum, vi. 164

Regulus, vi. 471 ; vii. 39

Remiz, vii. 123

Rhaad, viii. 455

Rhynchaspis, viii. 616669

Rhynchops, viii. 586. 648 albirostris, viii. 587 brevirostris, viii. 587 cinerascens, viii. 587 flavirostris, viii. 586 nigra, viii. 586

Rhynchotus, viii. 70 fasciatus, viii. 71

Riphidura, vi. 368 motacilloïdes, vi. 369

Robin, American, vi. 385 yellow, vi. 370

Rocar, vi. 376

Roc, vi. 175

Roitelet, vi. 473 ; vii. 7

Roller, Abyssinian, vii. 186 African, vii. 188 Bengal, vii. 186 blue-striped, vi. 266 Cayenne, vi. 300 garrulus, vii. 185. 337 Madagascar, vii. 188 Oriental, vii. 188 red-breasted, vi. 359 varied, vi. 295

Rollers, vii. 185. 337, 338

Rolles, vii. 187. 338

Rollier à masque noir, vi. 293

Rook, vii. 176

Rostratula Capensis, viii. 372

Rougri, le, vi. 63

Rouloul, viii. 241 de Malacca, viii. 31

Roupenne, le, vi. 393

Rousseau, le, vi. 265

Rousseline, vii. 101

Rousset, le, vi. 272

Rubiettes, vi. 439 ; vii. 7

Runner, velvet, viii. 402

Rupicola, vi. 427 ; vii. 2

Rusticola, viii. 367

Rynchæa, viii. 371.528 Hilarea, viii. 372 variegata, viii. 372
Sacre Egyptien, vi. 186

Saf-saf, viii. 455

Salangane, vii. 62. 96

Saltator, vi. 299. 317

albicollis, vi. 301

atricollis, vi. 303

aurantiarostris, vi. 300

cœrulescens, vi, 300, 301

cyanopterus, vi. 300

flavus, vi. 300

maculatus, vi. 302

melanoleucus, vi. 300

niger, vi. 302

olivaceus, vi. 299

ruber, vi. 302

rubicus, vi. 301

ruficapillus, vi. 301

validus, vi. 302

virescens, vi. 300

viridis, vi. 301

Sanderlings, viii. 378. 529

Sandpiper, viii. 319

ash-coloured, viii. 376

Bartram's, viii. 390

booted, viii. 390

Cayenne, viii. 321. 464

common, viii. 389

equestrian, viii. 382

goa, viii. 321

green, viii. 388

green-legged, viii. 388

grisled, viii. $\mathbf{3 7 5}$

Hebridal, viii. 385

Lapwing, viii. 462

little, viii. 376

long-legged, viii. 380.388

Louisiane, viii. 321

northern, viii. 377

pectoral, viii. 379

purple, viii. 376

purre, viii. 379. 530

red, viii. 380

ruff, viii. 381. 531

Schenz's, viii. 379

Selninger, viii. 376

semipalmated, viii. 383

Senegal, viii. 321

southern, viii. $\mathbf{3 7 6}$

Temminck's, viii. 375

white-winged, viii. 377

wood, viii. 388

Sandpipers, viii. 375

Sarcelles, viii. 673

Sarcoramphus, vi. 18. 169

Saria, viii. 326. 468

Sassegniat, viii. 401

Saurothera, vii. 459

Savacou de Cayenne, viii. 481

huppé de Cayenne, viii. 481 
Savacous, viii. 480

Saxicola, vi. 434 ; vii. 3 aurita, vi. 436 fruticola, vi. 436 Jardinii, vi. 439 ; viii. 685 lugens, vi. 438 mœsta, vi. 438 obscura, vi. 439 superciliaris, vi. 438 ; viii. 685 thoracina, vi. 438

Saxicolinæ, viii. 689

Scaler brown, vii. 352

Scalers, vii. 352

Scansores, vii. 435

Scolopax, viii. 358. 366. 512 ægocephala, viii. 373 alba, viii. 375 arcuata, viii. 364. 381.520

Australia, viii. 390

Australis, viii. 370

Belgica, viii. 373

Borealis, viii. 365

Brehmii, viii. 369

calidris, viii. 382

candidus, viii. 390

candida, viii. 525

Cantabrigiensis, viii. 387

Capensis, viii. 371

Cayanensis, viii. 369

cinerea, viii. 374

curonica, viii. 387

falcinellus, viii. 363.518

fedoa, viii. 374

fusca, viii. 386

gallanaria, viii. 368

gallinago, viii. 368,369

gallinula, viii. 369

gigantea, viii. 370

glottis, viii. 373 . 386

grisea, viii. 370

helias, viii. 480

incana, viii. 390

Laponica, viii. 372

leucophea, viii. 371

major, viii. 368. 527

media, viii. 369

minor, viii. 367

nigra, viii. 390

Noveboracensis, viii. 370

nutans, viii. 390

obscura, viii. 402

Paikulli, viii. 370

paludosa, viii. 369

phœopus, viii. 365. 521

pusilla, viii. 370. 379

pygmæus, viii. 380

rubra, viii. 362

rusticola, viii. 367.521

Sabini, viii. 368
Scolopax saturata, viii. 370 semipalmata, viii. 389 subarcuata, viii. 380

Terek, viii. 374

Totanus, viii. 387 vociferus, viii. 390

Scops, vi. 83. 255 of Carolina, vi. 256

Scopus, viii. 352 umbretta, viii. 353. 509

Scoter, black, viii. 665

Scoters, viii. 665

Screamer, chaja, viii. 399 horned, viii. 398. 538

Screamers, viii. 397

Scythrops, vii. 465.538 Novæ Hollandiæ, vii. 465

Secretarius reptilivorus, vi. 69

Secretary, vi. 68. 247

Seïsura, vi. 369

Seiürus tenuirostris, vi. 454

Sericulus, vi. 398 chrysocephalus, viii. 684

Serin Mozambique, vii. 143

Serins, vii. 142

Serpentarius, vi. 68

Setophaga, vi. $\mathbf{3 5 0}$ miniata, viii. 682 rubra, vi. 351 ruticilla, vi. 351 ; viii. 682

Shag crested, viii. 591 dwarf, viii. 591 spotted, viii. 591

Sheath-bill white, viii. 416

Sheath-bills, viii. 416

Shieldrake, viii. 617

Shieldrakes, viii. 669

Shoveler, viii. 616 New Holland, viii. 617

Shrike African, vi. 267 American, vi. 266 Antiguan, vi. 271 Barbary, vi. 270 ; viii. 678 barred, vi. 275 bay-backed, vi. 268 black and white, vi. 275 black-bush, vi. 275 black-headed, vi. 269 ; viii. 677 black-topped, vi. 272 blanchot, vi. 266 ; viii. 678 blue, vi. 266

Cape, vi. 265 ; viii. 677 Chinese, vi. 268

cinereous, vi. 371.483 collared, vi. 265 corvine, vi. 267 crested, vi. 268. 273 cruel, vi. 268 ferruginous-bellied, vi. 267 
Shrike fork-tailed, vi. 372 fork-tailed crested, vi. 371 frontal, vi. 295

Geoffroy's, vi. 286. 485 great cinereous, vi. 263 grey-backed, vi. 268 hooked-billed, vi. 285 Hottuiqua, vi. 267 Indian, vi. 268 Keroula, vi. 268 large bush, vi. 272 Leach's bush, vi. 274 lesser grey, vi. 263 Louisiana, vi. 270 ; viii. 678 Madagascar, vi. 266 Malabar, vi. 371 Malimbic, vi. 271 mustachio, vi. 268 natka, vi. 270 northern, vi. 269 ; viii. 677 olive, vi. 270

Pacific, vi. 268

Panayan, vi. 269

pied, vi. 272

red, vi. 263

red-backed, vi. 264

red-crested, vi, 268

red-headed bush, vi. 274

red-throated, vi. 271

rufous, vi. 294

rufous-crowned bush, vi. 274

rufous-winged bush, vi. 273

Senegal, vi. 267. 678

silent, vi. 268 ; viii. 678

spotted, vi. 273

swallow, vi. 286. 487

Tabuan, vi. 269

Vigors' bush, vi. 274

white, vi. 269

white-clieeked, vi. 268

Shrikes, vi. 262.482

Sicrin, vi. 396. 423

Siffleur, le, vi. 326

Sirli, vii. 119. 212

Siskin Arkansaw, vii. 141 common, vii. 142

Siskins, vii. 142. 268

Sison, viii. 454

Sitta, vii. 344. 385

Caffra, vii. 345

Canadensis, vii. 344

chloris, vi. 345

chrysoptera, vii. 345

Europæa, vii. 344

frontalis, vii. 345

fusca, vii. 345

melanocephala, vii. 345

minor, vii. 387

nævia, vi. 408
Sitta pusilla, vii. 345.387

Sittines, vii. $\mathbf{3 4 5}$

Skimmer, viii. 648

ashy, viii. 587

black, viii. 586

short-billed, viii. 587

yellow-billed, viii. 586

Skimmers, viii. 586

Snake-eater, vi. 68

Snipe, black-head, viii. 387

brown, viii. 370

Burka, viii. 370

Cape, viii. 371. 528

Cayenne, viii. 369

common, viii. 368

Courland, viii. 387

double, viii. 527

dusky, viii. 386.387

Finmark, viii. 386

gambet, viii. 387

great, viii. 368

green-shank, viii. 368

Jadreka, viii. 373

jack, viii. 369

little, viii. 527

red-breasted, viii. 373

red-shank, viii. 387

saturated, viii. 370

spotted, viii. 387

stagnatile, viii. 387

stone, viii. 390

telltale, viii. 390

Terek, viii. 374

yellow-shanked, viii. 390

Snipes, viii. 366.526

Soco, viii. 344

Sonateria, viii. 611

Souchets, viii. 616. 669

Sonimanga à gorge violette, et à poitrine rouge, vii. 366

long-billed, vii. 393

rayé, vii. 366

Souimangas, vii. 359. 390

Sparrow chipping, vii. 148

field, vii. 148

fire coloured, vii. 137

fox coloured, vii. 138

hedge, vi. 443. 465

house, vii. 135. 234

scarlet, vi. 315

song, vii. 148

swamp, vii. 148

tree, vii. 148

yellow-winged, vij. 148

white-throated, vii. 148

Sparrows, vii. 130. 234 proper, vii. 134

Spatula, viii. 616

Spatula blanche sans huppe, viii. 357 
Sparvius, vi. 236

bicolor, vi. 56

cærulescens, vi. 56

cinereus, vi. 52. 56

cirrocephalus, vi. 56

gilvicollis, vi. 56

guttatus, vi. 56

major, vi. 56

melanoleucus, vi. 56

monachus, vi. 52

niger, vi. 54

rufiventris, vi. 56

semitorquatus, vi. 56

subniger, vi. 56

superciliaris, vi. 56

tricolor, vi. 56

Spelectos, vii. 507

Sphecothera, vi. 411

viridis, vi. 411

Sphecotheres, vi. 297

viridis, vi. 297

Spheniscus, viii. 561. 563

Sphænura Coraya, vi. 408

striolata, vii. 347

superciliaris, vii. 346

tibicen, vi. 470

Spizaetus, vi. 43.44

Spoonbill dwarf, viii. 383. 532 roseate, viii. 357.512 white, viii. 357.512

Spoonbills, viii. 356. 510

Spreo, le, vi. 393

Squatarola, viii. 318

Stapazina, vi. 436

Stare Cape, vii. 173

cockscomb, vi. 425

collared, vi. 465 ; vii. 37

common, vii. 172

Louisiana, vii. 325

Magellanic, vii. 173. 326

wattled, vi. 413

Stares, vii. 172. 321

Starling Persian, vii. 37

Starykes, viii. 635

Staryque cristatella, viii. 557

Steatornis caripennis, vii. 81

Stercoraires, viii. 579

Stercoreus pomarinus, viii. 580

Sterna, viii. 580

aranea, viii. 584

affinis, viii. 583

Anglica, viii. 582

Arctica, viii. 582

argentia, viii. 581

argentata, viii. 582

Oahuensis, viii. 585

Bergii, viii. 584. 648

Caritiaca, viii. 581

Caspia, viii. 581

Cuv, Index.
Sterna Cayana, viii. 583

Dougalii, viii. 582

fissipes, viii. 581

fuliginosa, viii. 584

fuscator, viii. 586

galericulata, viii. 584

grisea, viii. 583

hirundo, viii. 580

inea, viii. 582

infuscata, viii. 585

leucopareia, viii. 582

leucoptera, viii. 582

magnirostris, viii. 585

media, viii. 583

melanauchen, viii. 583. 647

melanogaster, viii. 584

minuta, viii. 581

nævia, viii. 581

nigra, viii. 581

Nitzschii, viii. 585

Philippensis, viii. 586

senex, viii. 585

stolida, viii. $\mathbf{5 8 5}$

tenuirostris, viii. 583. 586

Stonechat, vi. 435

Stonechats, vi. 434

Stork, American, viii. 349. 507

black, viii. 349. 504

small black, viii. 350

violet, viii. 340 . 350

white, viii. 349. 495

Storks, viii. 348. 494

with naked-neck, viii. 350

Stournelle à collier, vii. 173

Stournes, vi. 392

Strepsilas, viii. 384, 385. 532 collaris, viii. 385

Strix, vi. 70. 74

Arcadica, vi. 81

accipitrina, vi. 72.78

Africana, vi. 72.78

alba, vi. 75.84

albifrons, vi. 84

albomarginata, vi. 86

aluco, vi. 76

Americana, vi. 71

Arctica, vi. 72

ascalaphus, vi. 71

asio, vi. 83. 256

atricapilla, vi. 84

badria, vi. 75

brachyotus, vi. 71

brachyura, vi. 72

Brama, vi. 85

bubo, vi. 76.79

bubo Magellanicus, vi. 72

castanoptera, vi. 81

Cayanensis, vi. 82

choliba, vi. 85 
Strix crucigera, vi. 86 cunicularia, vi. 79. 258 dasypus, vi. 80 decussata, vi. 85 ferruginea, vi. 81 flammea, vi. 75. 257 funerea, vi. 78. 80 grallaria, vi. 79 griseata, vi. 77 Hardwickii, vi. 77 hirsuta, vi. 85 Hudsonia, vi. 78 huhula, vi. 82 hylophila, vi. 76 Javanica, vi. 75 lactea, vi. 73 Lapponica, vi. 74 larvata, vi. 83 lempyi, vi. 84 leucotis, vi. 84 Leschenaultii, vi. 84 lineata, vi. 82 litturata, vi. 74. 78 longirostris, vi. 73 macrorhynchus, vi. 73 macroura, vi. 78 maculosa, vi. 72 Maugei, vi. 85 Mexicana, vi. 71 nævia, vi. 83 nebulosa, vi. 74 nisoria, vi. 78 nisuella, vi. 78 noctua, vi. 75.80 noctula, vi. 84 nudipes, vi. 80.83 nyctea, vi. 79. 256 occipitalis, vi. 82 Orientalis, vi. 73 otus, vi. 71 pagodarum, vi. 85 palustris, vi. 72 passerina, vi. 79, 80, 81 passerinoïdes, vi. 82 perlata, vi. 75. 81 perspicillata, vi. 83 personata, vi. 83 phalænö̈des, vi. 81 pumila, vi. 81 pusilla, vi. 81 pygmæa, vi. 79, 81 rufa, vi. 75 scops, vi. 83. 255 seloputo, vi. 85 Soloniensis, vi. 75 Sonnerati, vi. 85 strepitans, vi. 73 stridula, vi. 72.76 superciliosa, vi. 77
Strix sylvestris, vi. 75 Tenglmalmi, vi. 79.80 torquata, vi. 82 . tripennis, vi. 72 Tuidara, vi. 75 ulula, vi. 71.78 Uralensis, vi. 78 Virginiana, vi. 72

Strobiliphaga, vii. 161

Struthio, viii. 295. 422 camelus, viii. 295 casuarius, viii. 297 rhea, viii. 296. 438

Sturnella, vii. 326

Sturnus Capensis, vi. 424 ; vii. 173 carunculatus, vi. 413 ; vii. 173 cericeus, vi. 425 cinclus, vi. 410 ; vii. 173 collaris, vi. 465 ; vii. 37.173 contra, vi. 424 crispicollis, vi. 416 Dauricus, vi. 425 ; vii. 174 gallinaceus, vi. 425 Ludovicianus, vii. 173 Mauritanus, vi. 465 ; vii. 37 militaris, vii. 173. 326 olivaceus, vii. 174 predatorius, vii. 169 saularis, vi. 294 sericeus, vii. 174 unicolor, vii. 173 viridis, vii. 174 vulgaris, vii. 172 Zeylanicus, vi. 425

Sucrier cardinal, vii. 364

Sugar-eater, vii. 390.353

Suiri pardo y blanco, vi. 333 negro peco celesto, vi. 333

Suiriri, vi. 321 brun et rouge, vi. 320 cabeza y rabadilla de canela, vi. 334

choreado, vi. 334 chorreado debazo, vi. 327

chorreado sin roxo, vi. 322 chorreado todo, vi. 321 guau, vi. 319 obscuro y amarillo, vi. 334 ordinario, vi. 352 pardo aplomado, vi. 324 pitador, vi. 334 puteado, vi. 334 roxo, vi. 334

Sula, viii. 653

roxo obscuro, vi. 321

Brasiliensis, viii. 595

Sultanas, viii. 412.542

Surnia, vi. 78

Swallow, vii. 57. 60 
Swallow, aculeated, vii. 70. 103, 104 ambergris, vii. 64

ash-bellied, vii. 69

barn, vii. 62

bat, vii. 103

black, vii. 64

black-bellied, vii. 62

blue or white-bellied, vii. 65

Brazilian, vii. 64

brown-collared, vii. 67

Cape, vii. 63. 101

chalybeate, vii. 63

chimney, vii. 61. 90

cliff, vii. 62

common, vii. 90

crag, vii. 61

Daurian, vii. 64

domestic, vii. 94

esculent, vii. 96

fulvous, vii. 62

grey rock, vii. 94

green, vii. 65

grey-rumped, vii. 67

Oonalaschka, vii. 66

Otaheitan, vii. 66

Panayan, vii. 63

Peruvian, vii. 66

proper, vii. 60

purple, vii. 92

red-headed, vii. 69

river, vii. 61.91

rock, vii. 61

rufous-fronted, vii. 102

rufous-headed, vii. 63

rufous-rumped, vii. 68

Senegal, vii. 63

sharp-tailed, vii. 69.103

St. Domingo, vii. 67

Ternate, vii. 342

wheat, vii. 68

white-bellied, vii. 63

white-rumped, vii. 93

white-winged, vii. 67

window, vii. 60

wire-tailed, vii. 66

Swallows, sea, viii. 580 sharp-quilled, vii. 102

Swallowers, wind, vii. 105

Swan, viii. 597. 657

black, viii. 559. 661

black-backed, viii. 601

black-necked, viii. 599. 661

Chinese, viii. 600

common, viii. 659

mute, viii. 598. 659

spur-winged, viii. 600. 661

tame, viii. 598

whistling, viii. 598. 660

wild, viii. 598
Swift, vii. 58

Balasian, vii. 60

Chinese, vii. 59

white-bellied, vii. 59

white-collared, vii. 93

white coloured, vii. 60

Sycalis, vi. 339

Sylvia, vi. 439 ; vii. 7

Equinoctialis, vi. 451

agills, vi. 458

albicollis, vi. 436

albini, vi. 443

annulosa, vi. 453

Asiatica, vi. 451

atricapilla, vii. 30

autumnalis, vi. 458

azurea, vi. 458

bifasciata, vi. 458

Borbonica, vi. 449

cærulea, vi. 458 ; viii. 683

Cambaiensis, vi. 451

castanea, vi. 456

celata, vii. 172

certhiola, vi. 442

Cetti, vi. 443

cinerea, vi. 444

Cingalensis, vi. 448

cisticola, vi. 446.466

citrinella, vi. 460

conspicillata, vi. 446

cristata, vi. 431

cucullata, vi. 455

cyanecula, vi. 439

Dartfordiensis, vi. 446

discolor, vi. 457

domestica, vi. 473

elata, vi. 431

flavicollis, vi. 460

flaviventris, vi. 448

fluviatilis, vi. 442

formicivora, vi. 437

formosa, vi. 458

furva, vi. 473

galactotes, vi. 442 ; viii. 685

gracilis, vi. 467

grisea, vi. 281

Guzurata, vi. 451

hirundinacea, vi. 432 ; viii. 685

hortensis, vi. 446 ; vii. 34

imitatrix, vi. 437

Javanica, vi. 451

lateralis, vi. 469

leucopogon, vi. 446

locustella, vi. 443

longicauda, vi. 467

Ludoviciana, vi. 474

luscinia, vii. 12

luscinoïdes, vi. 442

Macloviana, vi. 452

Q 2 
Sylvia macroura, vi. 467

maculata, vii. 356

magnifica, vi. 470

magnolia, vi. 455

Marylandica, vi. 459

melanocephala, vi. 445

melanopogon, vi. 444

minuta, vi. 457

montana, vi. 452.457

nævia, vii. 30

nigra, vi. 437

nisoria, vii. 35

Noveboracensis, vi. 454

obscura, vi. 439

Orphea, vi. 444

pileata, vi. 437

palpebrosa, vi. 451

palustris, vi. 442.458

Pardalina, vi. 455

parus, vi. 456

peregrina, vi. 346 ; vii. 172

Philadelphia, vi. 459

philomela, vii. 21

phragmitis, vi. 442

pileata, viii. 685

pinus, vi. 464

Platensis, vi. 474

plumbea, vi. 451.464 ; viii. 686

pusilla, vi. 458,459

rara, vi. 457

rubecula, vii. 9

rubicola, vii. 5

ruficapilla, vii. 172

rufigastra, vi. 449

Ruppeli, vi. 444

salicaria, vi. 443

Sarda, vi. 446

schænobanus, vi. 443.466

sericea, vi. 441

solitaria, vii. 172

sperata, vi. 437

sphagnosa, vi. 458

Stapazina, $\beta$, vi. 436

subalpina, vi. 446

Suecica, vii. 11

superciliosa, vi. 432

sylviella, vi. 445

torquata, vi. 460

trichas, vi. 459

venusta, vi. 464 ; viii. 686

Sylvicola inornata, vi. 460

Sylvoïdes, vi. $\mathbf{2 7 6}$

Synallaxis, vii. 347. 388

albescens, vii. 347

cinerascens, vii. 347

ruficapilla, vii. 347

ruficauda, vii. 348

rutilans, vii. 348

setaria, vii. 348
Synallaxis, tessellata, vii. 348

Syndactyli, vii. 400.420

Syrrhaptes, viii. 65. 268

Syrnii, vi. 75

Syrnium, vi. 75

Tachard, le, vi. 62

Tachydromus, viii. 324

collaris, viii. 325

Senegalensis, viii. 325

Tachypetes, viii. 592

leucocephalus, viii. 593

minor, viii. 593

Tachyphonus, vi. 311

chloristerus, vi. 313

Desmarestii, vi. 311

fringiloides, vi. 312

olivaceus, vi. 312

ruber, vi. 313

rubescens, vi. 312 ; viii. 681

Suchii, vi. 313

tenuirostris, vi. 313

Vigorsii, vi. 312

Tadorna, viii. 669

Bellonii, viii. 618

familiaris, viii. 618

rutila, viii. 622

Tadornes, viii. 617

Talegalla, viii. 33

Cuvieri, viii. 33

Tamatia, vii. 472

black-banded, vii. 473

black-eared, vii. 472

lesser pied, vii. 472

maculata, vii. 472

pied, vii. 472

spotted-bellied, vii. 472

Tamatias, vii. 472

Tanager, archbishop, vi. 304

bishop, vi. $\mathbf{5 0 4}$

black and blue, vi. 303

black-faced, vi. 299

black-headed, vi. 315

black-necked, vi. 299

black-throated, vi. 310. 317. 463

blue-headed, vi. 307.491 ; viii. 687

blue-shouldered, vi. 303

blue-winged, vi. 300

Brazilian, vi. 316

Cayenne, vi. 298

crested, vi. 311

golden, vi. 297. 299

golden-crested, vi. 314 ; viii. 681

golden-forehead, vi. 314

grand, vi. 299

grey-headed, vi. 285 
Tanager green, vi. 309

green-headed, vi. 303. 316

hooded, vi. 310

Jacarine, vi. 298

Malimbic, vii. 131. 23]

Mississippi, vi. 315

negro, vi. 298

orange-billed, vi. 300

palm, vi. 305

Paradise, vi. 303. 490

Paraguayan, vi. 317

poppy, vi. 317

red, vi. 315

red-bellied, vi. 298. 314

red-breasted, vi. 317

red-headed, vi. 304.310

red-necked, vi. 311 . 314

red-throated, vi. 310

rufous-headed, vi. 304

sayacu, vi. 304

Schrauk's, vi. 309

showy, vi. 304

silent, vi. 318

spotted-emerald, vi. 305

spotted-green, vi. 305

St. Domingo, vi. 317

variable, vi. 299

yellow-throated, vi. 310

Tanagers, vi. 297. 488

cardinal, vi. 315

euphonian, vi. 297

grossbeak, vi. 299

oriole, vi. 311

ramphoceline, vi. 316

true, vi. 303

Tanagra, vi. 297

æstiva, vi. 315

albifrons, vi. 317

albirostris, vi. 317

Amboinensis, vi. 317

archiepiscopus, vi. 304

atra, vi. 299

atrata, vi. 317

atricapilla, vi. 270.315

aurata, vi. 299

auricapilla, vi. 314

aurifrons, vi. 314

axillaris, vi. 309

Bonariensis, vii. 134. 169

Brasilia, vi. 316

Brunnea, vi. 313

cærulea, vi. 317

celestis, vi. 304

canicapella, vi. 308

canora, vi. 306. 317. 489

Capensis, vi. 271. 363

capistrata, vi. 302

capitalis, vi. 317 ; vii. 134

Cayana, vi. 298, 304, 307. 490
Tanagra chlorocyanea, vi. 30 ?

chloroptera, vi. 305

chlorotica, vi. 298

cirrhomelas, vi. 312

citrinella, vi. 308

cristata, vi. 311

cristatella, vi. 315 ; vii. 127

cyanocephala, vi. 307

cyanoventris, vi. 308

decumana, vi. 300

Desmarestii, vi. 306

Diademata, vi. 302

Dominica, vi. 317

episcopus, vi. 304, 305

fasciata, vi. 307

flammea, vi. 312

flammiceps, vi. 302

flavicollis, vi. 310

flaviventris, vi. 303

formosa, vi. 305

graminea, vi. 309 ; vii. 127

Guianensis, vi. 270. 285. 317 ; viii. 679

gularis, vi. 310

gyrola, vi. 304

Jacapa, vi. 317

jacarina, vi. 298

jugularis, vi. 303

leucophæa, vi. 307

leucocephala, vi. 306

Ludoviciana, vi. 316

magna, vi. 299

martialis, vi. 311

melalictera, vi. 306

melanopis, vi. 300

melanotha, vi. $\mathbf{3 0 5}$

Mexicana, vi. 303

militaris, vi. 317

Mississippiensis, vi. 315

multicolor, vi. 306

musica, vi. 489

nigerrima, vi. 311

nigricollis, vi. 299. 310.463

nigrogularis, vi. 317

olivacea, vi. 299. 311. 315. 355

olivascens, vi. $\mathbf{3 0 5}$

ornata, vi. 304 . 307

palmarum, vi. 305

penicillata, vi. 313

Peruviana, vi. 307

pileata, vi. 310.315

psittacina, vi. 301 ; vii. 158

punctata, vi. 305

quadricolor, vi. 312

rubra, vi. 299

rubricollis, vi. 307

rudis, vi. 307.317 ; viii. 680

ruficollis, vii. 127. 311.314

rufiventris, vi. 308.314 
Tanagra Saira, vi. 313

sayaca, vi. 304, 305

Schraukii, vi. 309

silens, vii. 136. 318

Sibirica, vii. 118

Sinensis, vi. 317

speculifera, vi. 310.311

striata, vi. 306. 489

superciliaris, vi. 300

tatao, vi. 303. 490

tephrocephala, vi. 308

thoracita, vi. 308

tricolor, vi. 303.307

varia, vi. 305

variabilis, vi. 299.317

variegata, vi. 315

violacea, vi. 297. 298

virens, vi. 303. 309.317

viridis, vi. 298. 309

vittata, vi. 308

Tangara de Malimbe, vii. 131

Tantali, viii. 510

Tantalus, viii. 354

Æthiopicus, viii. 359

African, viii. 355

albicollis, viii. 361.519

albus, viii. 363

American, viii. 355

calvus, viii. 361

Cayennensis, viii. 361

Ceylon, viii. 356

coco, viii. 363

cristatus, viii. 363

hogedash, viii. 362

ibis, viii. 355. 510

lacteus, viii. 356

leucocephalus, viii. 356. 363.510

loculator, viii. 355.510

melanops, viii. 362

niger, viii. 361

pillus, viii. 355

plumicollis, viii. 355

rhodinopterus, viii. 355

ruber, viii. 362

Tanypus Australis, vi. 388

Tanysiptera, vii. 411

Tardivola, vii. 130 ; viii. 688

Tarier, vi. 435

Tariers, vii. 3

Tavon, viii. 400

Tchagra, le, vi. 271

Tchong, le, vi. 68

Teal, viii. 623

backal, viii. 624

blue-winged, viii. 671

Chinese, viii. 619

common, viii. 623

Coromandel, viii. 606

gerra, viii. 620
Teal hind, viii. 624

Madagascar, viii. 606

Manilla, viii. 624

spinous-tailed, viii. 614

Teals, viii. 673

Tectur kelaun, viii. 55

Temia, vii. 184

Tenuirostres, vii. 344 suppl. on the, vii. $\mathbf{3 8 5}$

Tern, allied, viii. 583

Arctic, viii. 582

bearded, viii. 582.647

black, viii. 581

black-naped, viii. 583. 647

Caspian, viii. 581

Cayenne, viii. 583

great, viii. 580

gull-billed, viii. 582

hoary, viii. 583

lesser, viii. 581

marsh, viii. 584

mustachio, viii. 582

noddy, viii. 585

rose-coloured, viii. 582

Sandwich, viii. 581

slender-bill, viii. 583

sooty, viii. 584

white-winged, viii. 582

Terns, viii. 580

Tersina cærulea, vi. 365

Terutero, viii. 321. 464

Teteu, viii. 464

Tetrao, viii. 33. 34. 242

albus, viii. 40

alchata, viii. 41. 257

Andalusicus, viii. 62 arenarius, viii. 43

betulinus, viii. 36

bicalcaratus, viii. 46

Bonasia, viii. 36

cachinans, viii. 40

Californius, viii. 60

Canadensis, viii. 36. 251

Canace, viii. 36

canus, viii. 36

caudacuta, viii. 42

Chinensis, viii. 56

cinereus, viii. 51. 67

Coromandelicus, viii. 57

coturnix, viii. 56. 267

Coyoleos, viii. 60

cristatus, viii. 60

Cupido, viii. 37

curvirostris, viii. 50

Falklandicus, viii. 60

Francolinus, viii. 45. 263

Franklinii, viii. 39

Guianensis, viii. 59

hybridus, viii. 35 
Tetrao Indicus, viii. 43 intermedius, viii. 35 Javanicus, viii. 50 lagopus, viii. 40 Lapponicus, viii. 40 Luzoniensis, viii. 63, 64 Madagascarensis, viii. 62 major, viii. 67

Manillensis, viii. 56

Marilandus, viii. 59 medius, viii. 35

Mexicanus, viii. 59 montanus, viii. 52 mutus, viii. 40

Namaqua, viii. 43

Nemesianus, viii. 36 nigricollis, viii. 62

Novæ Hispaniæ, viii. 33 nudicollis, viii. 49 obscurus, viii. 38 ocellatus, viii. 32 Orientalis, viii. 43. 45.53 paradoxa, viii. 66 petrosus, viii. 52 phasianellus, viii. 44 Pondicerianus viii. 45 Richardsonii, viii. 39 rubricollis, viii. 49 rufus, viii. 51 rupestris, viii. 40 Sabini, viii. 39 saliceti, viii. 40 Scoticus, viii. 41. 256 Senegalus, viii. 42 Sinensis, viii. 46. 64 Sovi, viii. 69 striatus, viii. 57 tetrix, viii. 34. 247 togatus, viii. 37 umbellus, viii. 37 urogallus, viii. 34. 243 urophasianellus, viii. 38 urophasianus, viii. 38 variegatus, viii. 67 Virginianus, viii. 59, 60 viridis, viii. 32

Tetras à plumage variable, viii. 35 à queue pleine, viii. 35

Textor alecto, vii. 232

Thalassidroma, viii. 566

Thamnophilinæ, viii. 678. 683

Thamnophilus, vi. 272 affinis, vi. 277 agilis, vi. 276 albicollis, vi. 280 albiventer, vi. 275 albonotatus, vi. 275. 278 atricapillus, vi. 280 auratus, vi. 280
Thamnophilus bicolor, vi. 275 cinnamomeus, vi. 275 caudatus, vi. 279 cærulescens, vi. 279 choloropterus, vi. 279 cinereus, vi. 274. 283 cristatellus, vi. 283 cyanocephalus, vi. 282 fasciatus, vi. 275

ferrugineus, vi. 274 griseus, vi. 278 gularis, vi. 278 guttatus, vi. 276.281 Leachii, vi. 274 leucocephalus, vi. 285 leuconotus, vi. 278 lineatus, vi. 275.281 longicaudatus, vi. 281 maculatus, vi. 276 melanogaster, vi. 277 melanoceps, vi. 278 myotherinus, vi. 279 niger, vi. 275 radiatus, vi. 276.281 rubicus, vi. 282 ruficapillus, vi. 282 ruficeps, vi. 274 ruficollis, vi. 277 rufinus, vi. 283 rutilus, vi. 282 stellaris, vi. 277 striatus, vi. 279 strigilatus, vi. 276 Swainsonii, vi. 275 torquatus, vi. 273 Vigorsii, vi. 274 virescens, vi. 282 viridis, vi. 282

Thick-knees long-legged, viii. 307 spotted, viii. 306

Thrush, vi. 510 barred-tail, vi. 408 black and scarlet, vi. 357 black-chinned, vi. 390 black-crested, vi. 380 black-crowned, vi. 434 black-headed, vi. 381 black-necked, vi. 376 blue-tailed, vi. 400 brown-eared, vi. 376 buff-winged, vi. 408 Ceylon, vi. 271 ; viii. 678 chiming, vi. 407 cyaneous, vi. 403 emerald, vi. 381 golden-crowned, vi. 454 gular, vi. 382 hermit, vi. 376.386 Indian, vi. 381 
Thrush Mindanao, vi. 294

missel, vi. 384

mocking, vi. 539

musician, vi. 406 ; vii. 47

orange-breasted, vi. 434

pagoda, vi. 423

palm, vi. 312. 381

of Paraguay, vi. 523

pigeon, vi. 394

pine, vii. 141

punctated, vi. 529

red, vi. 385

red-winged, vi. 384

reed, vi. 441

rose coloured, vi. 392

ruby-throat, vi. 440

rufous, vi. 380

solitary, vi. 375

song, vi. 522

songster, vi. 394

Sonnerat's, vi. 390

tawny, vi. 386

thick-billed, vi. 271

varied, vi. 381

Volatile, vi. 369

water, vi. 454

white-backed, vi. 408

wood, vi. 386

yellow-billed, vi. 403

yellow-fronted, vi. 390

Thrushes proper, vi. 515

rock, vi. 536

Thryothorus, vii. 48

lateralis, vi. $\mathbf{4 7 4}$

littoralis, vii. 48

Tichodroma, vii. 352.389

Tigrosoma, viii. 337

Timalia pileata, vi. 402 ; viii. 684.685 thoracica, vi. 402 ; viii. 685

Tinamou barred-tailed, viii. 71 cinereous, viii. 67

dwarf, viii. 69

great, viii. 67

Isabelle, viii. 71

little, viii. 69

Macaco, viii. 68

nocturnal, viii. 68

oariana, viii. 69

obsolete, viii. 68

rufescent, viii. 71

spotted, viii. 70

Tao, viii. 69

undulated, viii. 67

variegated, viii. 67

vermicule, viii. 68

Tinamous, viii. 269

Tinamus, viii. 66

adspersus, viii. 68

Apequia, viii. 68
Tinamus Boraquira, viii. 70

Brasiliensis, viii. 67

Canape, viii. 70

maculosus, viii. 70

magoua, viii. 67

major, viii. 70

medius, viii. 70

minor, viii. 70

nanus, viii. 69

noctivagus, viii. 68

obsoletus, viii. 68

pavoninus, viii. 70

plumbeus, viii. 68

rufescens, viii. 71

Sovi, viii. 69

strigulosus, viii. 69

Tataupa, viii. 68

undulatus, viii. 67

vermiculatus, viii. 68

ynambui, viii. 70

Tisserin alecto, vii. 133

Tisserins, vii. 130

Titmice, vii. 120

penduline, vii. 123. 223

reed, vii. 221

Titmouse, Alpine, vii. 123 amorous, vii. 123 azure, vii. 122

bearded, vii. 123. 222

black, vii. 123

black-cap, vii. 121. 122

blue, vii. 12l. 219

Cape, vii. 124

Chinese, vii. 123

cole, vii. 120.218

crested, vii. 121

fork-tailed, vii. 123

great, vii. 120. 214

great-headed, vii. 123

greyish, vii. 123

Guiana, vii. 123

Javan, vii. 122

long-tailed, vii. 121. 220

Malabar, vi. 346

marsh, vii. 218

New Zealand, vii. 123

Norway, vii. 123

Siberian, vii. 122

white-cheek, vii. 123

Titria cinereus, vi. 290

Tityra viridis, vi. 291 ; viii. 680

Tlauhqueuil, viii. 512

Toads, flying, vii. 105

Tocro, vii. 268

Todies, vii. 414

Todus, vii. 414. 429

auritus, vi. 338

brachyurus, vi. 338

cæruleus, vii. 415 
Todus cinereus, vii. 415

ferrugineus, vii. 415

flavigaster, vi. 331

fuscus, vii. 415

leucocephalus, vi. 338 ; vii. 415

macrorhynchos, vi. 337 ; vii. 415

maculatus, vi. 332

nasutus, vi. 337 ; vii. 415

obscurus, vi. $\mathbf{3 3 7}$

Paradisiacus, vi. 330

Paradiseus, vii. 415

platyrhynchus, vi. 336 ; vii. 415

plumbeus, vi. 332 ; vii. 415

regius, vi. 332 . 336 ; vii. 415

rostratus, vi. 336 ; vii. 415

rubecula, vi. 331

sylvia, vii. 415

varius, vii. 415

viridis, vii. 414. 429

Tody great-billed, vi. 337

green, vii. 414. 429

white-headed, vi. 338

Tonysiptera, viii. 690

Torticella, viii. 63

Totanus, viii. 385. 532

acuminatus, viii. 390

Bartramius, viii. 390

calidris, viii. 387

caligatus, viii. 390

chloropygus, viii. 390

Damascensis, viii. 390

fasciatus, viii. 311

fistulans, viii. 386

flavipes, viii. 390

fuscus, viii. 387

glareolus, viii. 390

grallaturius, viii. 388

griseus, viii. 386

Javanicus, viii. 390

macularius, viii. 389

maculatus, viii. 387

melanoleucus, viii. 390

nævius, viii. 387

natans, viii. 387

Noveboracensis, viii. 371

ochropus, viii. 388

petrificatus, viii. 391

semipalmatus, viii. 389

solitarius, viii. $\mathbf{3 9 0}$

speculiferus, viii. 389

stagnatilis, viii. 387

tenuirostris, viii. 390

variegatus, viii. 390

Totipalmatæ, viii. 648

Totipalme, viii. 587

Toucan, vii. 544

Aldrovandine, vii. 478

Aracari, vii. 479.547

Azara's, vii. 480

Cuv. Index.
Toucan, Baillon's, vii. 480

black and yellow, vii. 479

black and green, vii. 480

Brazilian, vii. 478

carinated, vii. 478

great, vii. 478

green, vii. 479

piperine, vii. 480

red-billed, vii. 478

red-breasted, vii. 478

spotted-billed, vii. 480

toco, vii. 477

Vaillant's, vii. 479

yellow-breasted, vii. 478

Toucans, vii. 477

Toucnamcourvi, vii. 131. 230

Touraco brun, vii. 507

géant, vii. 507

Pauline, vii. 507. 585

violet, vii. 508

Touracos, vii. 507

Touyouyou, viii. 352. 508

Tracal, vii. 119

Tragopan, viii. 30. 241

Traquet d'Angleterre, vi. 498

familier, vi. 437

imitateur, vi. 437

montagnard, vi. $43 \%$

proprement dit, vii. 5

Traquets, vii. 3

Treron, viii. 93

Trichas, vi. 459

Trichoglossi, vii. 578

Tricophorus, vi. 296 ; viii. 680 barbatus, vi. 297

Tridactyles, viii. 61

Tringa, viii. 318. 375

alba, viii. 378

albescens, viii. 377

Alpina, viii. 379

atra, viii. 387

Australis, viii. 376

Bartramia, viii. 390

brevirostris, viii. 383

canutus, viii. 375

cinctus, viii. 530. 379

cinerea, viii. 375

coot-footed, viii. 391

equestris, viii. 382

falcinellus, viii. 378

ferruginea, viii. 376

fulicaria, viii. 384

fusca, viii. 387

gambetta, viii. 382. 387

glacialis, viii. 384

glareola, viii. 388

Grenovicensis, viii. 382

grisea, viii. 375

Helvetica, viii. 319

R 
'Tringa hiatula, viii. 312

Hyperborea, viii. 384. 391

hypoleucos, viii. 389

interpres, viii. 385

Islandica, viii. 375

itimantopus, viii. 380

leucoptera, viii. 377

littorea, viii. 382

lobata, viii. 384. 392. 532

Macei, viii. 378

macroptera, viii. 321

maculosa, viii. 377

maritima, viii. 376

minuta, viii. 376.383

nævia, viii. 375

nigricans, viii. 376

Oakuensis, viii. 390

ochropus, viii. 388

platyrhynchus, viii. 380,381

pugnax, viii. 381. 531

pusilla, viii. $376,377.383$

religiosa, viii. 378

rubra, viii. 378

rufa, viii. 375

rufescens, viii. 377

ruficollis, viii. 379

Schenzii, viii. 379

semipalmata, viii. 383

squatarola, viii. 319

striata, viii. 376. 387

subarcuata, viii. 380

Temminckii, viii. 376

totanus, viii. 387

Urvilii, viii. 320

vanellus, viii. 319.462

varia, viii. 319

variabilis, viii. 379

Triorchis, vi. 39

Trochilus, vii. 367

albicollis, vii. 379

albus, vii. 371

ametlıystinus, vii. 375

ater, vii. 372

aureo viridis, vii. 371

auritus, vii. 378

aurulentus, vii. 371

bilophus, vii. 37.4

Brasiliensis, vii. 369. 371

brevicauda, vii. 372

carbunculus, vii. 377

chalybeus, vii. 373

chrysurus, vii. 369

cinereus, vii. 370

collaris, vii. 378

colubris, vii. 375

cristatus, vii. 372

elegans, vii. 369

enicurus, vii. 376

onsipennis, vii. 374
Trochilus forficatus, vii. 360

fulgens, vii. 372

furcatus, vii. 375

gigas, vii. 377

gutturalis, vii. 370

hirsutus, vii. 371

holosericeus, vii.. 371

jugularis, vii. $\mathbf{3 7 0}$

Langsdorfii, vii. 376

latipennis, vii. 374

leucogaster, vii. 378

leucurus, vii. $\mathbf{3 6 8}$

longirostris, vii. 378

maculatus, vii. 378

magnificus, vii. $\mathbf{3 7 3}$

mango, vii. 370

margaritaceus, vii. 372

Maugeanus, vii. 375

melanogaster, vii. 370

melanotus, vii. 372

mellisuga, vii. 377

mellivorus, vii. 375.378

mesoleucos, vii. $\mathbf{3 7 4}$

minimus, vii. 376

moschitus, vii. 377

multicolor, vii. 372

nævius, vii. 370

ornatus, vii. 373

ourissia, vii. 377

pectoralis, vii. 371

pella, vii. 368

petasophorus, vii. 373

pileatus, vii. 373

platurus, vii. 376

platycerius, vii. 372

polythmus, vii. 369

punctatus, vii. 371

pygmæus, vii. 372

rubineus, vii. 377

saphyrinus, vii. 379

scutatus, vii. 373

smaragdo-saphyrinus, vii. 375

squalidus, vii. 369

squamosus, vii. 379

superbus, vii. 378

superciliosus, vii. 368

Taumautias, vii. 370

thalassinus, vii. 372

violaceus, vii. $\mathbf{3 7 0}$

viridis, vii. 371

Troglodytes, vi. 473 ; vii. 42

CEdon, vi. 473 ; vii. 44

arundinaceus, vi. 474

Europæus, vi. 473

hiemalis, vi. 473 ; vii. 44

obsoleta, vi. 409

Trogon, vii. 473

albiventris, vii. 475

ardens, vii. 476 
Trogon atricollis, vii. 474 aurantius, vii. 476 black-headed, vii. 474 castaneus, vii. 474 cinereus, vii. 474 cinnamomeus, vii. 476 collaris, vii. 474 condea, vii. 475 curucui, vii. 474 domicellus, vii. 475 Domingo, vii. 475 Duvaucel's, vii. 475 fasciatus, vii. 475 indented, vii. $\mathbf{5 4 1}$ lesser red-bellied, vii. 474 mountain, vii. 475 narina, vii. 476 oreskios, vii. 475 pavoninus, vii. 476.541 red-bellied, vii. 474 Reinwart's, vii. 475 rosalba, vii. 474 rufus, vii. 474 strigilatus, vii. 474 sulfuraceus, vii. 474 Temminckii, vii. 476 temnusus, vii. 476 variegatus, vii. 474.476 violaceus, vii. 474 violet-headed, vii. 474 viridis, vii. 474 white-bellied, vii. 475 white-collared, vii. 475 yellow-bellied, vii. 474 Trogons, vii. 539

Tropic-bird, common, viii. 596 red-tailed, viii. 597

Tropidorhynchus, vi. 412.415

Troupiale, vii. 168 à calotte rousse, vii. 171 grand, vii. 166 noir et varié, vii. 17] noir, le petit, vii. 134

Troupiales, vii. 167. 319. 320

Trumpeter, undulated, viii. 302

Tschachert, vi. 288

Tuglek, viii. 631

Tuidara, vi. 75

Turdoïdes, vi. 388 ; viii. 684 leucocephala, vi. 390

Turdus, vi. 374. 510 Abyssinicus, vi. 382 æneus, vi. 395

Africanus, vi. 382 Alaspi, vi. 279. 408 albicapillus, vi. $\mathbf{3 8 2}$ albicollis, vi. 383. 387 albifrons, vi. 383.392 albiventris, vi. 387
Turdus amœnus, vi. 392

Americanus, vi. 383

analis, vi. $\mathbf{3 8 8}$

aquaticus, vi. 454

Arada, vi. 406

arcuatus, vi. 382

ardosiaceus, vi. 380.384

Arsinoë, vi. 382

arundinaceus, vi. 392. 441.514; vii. 29

Asiaticus, vi. 382

atricapillus, vi. 379.381

atriceps, vi. 389

atricullis, vi. 379

atrigularis, vi. 376

auratus, vi. 393.409

aurigaster, vi. 389

aurocapillus, vi. 392

Australis, vi. 378

azureus, vi. 389

Badius, vi. 383

bamble, vi. 406

Barbaricus, vi. 383

bicolor, vi. 393

bimaculatus, vi. 388

Borbonicus, vi. 382

brachypterus, vi. 383.468

brachypus, vi. 383

Brasiliensis, vi. 379

brevicaudatus, vi. 383

Cafer, vi. 388

Calliope, vi. 392.440

campanella, vi. 407

Camtschatkensis, vi. 440

canorus, vi. 382

cantans, vi. $406^{\circ}$

cantor, vi. 394

Capensis, vi. 389

carbonarius, vi. $\mathbf{3 7 9}$

Cayanensis, vii. 388

certhiola, vi. 442

Ceylonus, vi. 271

chalybeus, vi. 394

Chochi, vi. 383

chrysogaster, vi. 378 . 394

chrysorhœus, vi. 389

cinclus, vi. 410

cinereus, vi. 383

cinnamomeus, vi. 280. 380. 409

cirrhatus, vi. 281

Cochinsinensis, vi. 390. 419

colma, vi. 404

colma $\beta$, vi. 404

columbinus, vi. 382

Columbus, vi. 394

concolor, vi. 381.389

Coraya, vi. 408

coronatus, vi. 454

crassirostris, vi. 271. 383

R 2 
Turdus crotopegus, vi. 387

curæus, vi. 383

cyaneus, vi. 375. 383. 392. 403

cyanocephalus, vi. 384

cyanurus, vi. 400

Daoma, vi. 383

dentirostris, vi. 383

dilutus, vi. 383

disparis, vi. 389

Dominicanus, vi. 377. 425

Dominicus, vi. 385

dubius, vi. 376.383

eremita, vi. 376

erythropterus, vi. 378

explorator, vi. $\mathbf{3 7 6}$

Falklandii, vi. 382

felivox, vi. 385

flavipes, vi. 383.387

flavirostris, vi. 403

flavus, vi. 382.397

formicivorus, vi. 404

frivolus, vi. 383

fuliginosus, vi. 384

fumigatus, vi. 387

fuscatus, vi. 386

fuscipes, vi. 408

fuscus, vi. 383

Ginginianus, vi. 423

griseus, vi. 382. 402

gularis, vi. 382

gutturalis, vi. 383. 434

Guyanensis, vi. 387

hæmorrhousa, vi. 381

harmonicus, vi. 384 .

Hispaniolensis, vi. $\mathbf{3 7 9}$

Hudsonius, vi. 392

iliacus, vi. 384

importunus, vi. 378

Indicus, vi. 381

inquietus, vi. 383

Jamaïcensis, vi. 387

Javanicus, vi. 381

jugularis, vi. 392

Kamtsckensis, vi. 312

Labradorius, vi. 392

Leschenaultii, vi. 382

leucocephalus, vi. 382. 392. 425

leucogaster, vi. 394

leucogenus, vi. $\mathbf{3 8 3}$

leucomelas, vi. 383

leucophrys, vi. 383

leucopterus, vi. $\mathbf{3 8 3}$

leucotis, vi. 383.419

leucurus, vi. 375. 436

lineatus, vi. 404

lividus, vi. 385

longirostris, vi. 383

lunulatus, vi. 384. 419

Macei, vi. 383
Turdus Madagascariensis, vi. 379

Malabaricus, vi. 390. 392. 423

Manillensis, vi. 376

maxillaris, vi. 383 . 419

melanicherus, vi. 378

melanocephalus, vi. 383. 423

melanophrys, vi. 383

melanops, vi. 383.418

melinus, vi. 384. 419

melodus, vi, 386

merula, vi. 374

migratorius, vi. 385.524

Mindanaensis, vi. 294

miniatus, vi. 378

minor, vi. 383.386

monachra, vi. 382. 392.397

morio, vi. 393

motacilla, vi. 392. 454

musicola, vi. $\mathbf{3 8 3}$

mustelinus, vi. 386

musicus, vi. 384. 522

nævius, vi. 383

Naumanni, vi. 376

nigricapillus, vi. 377

nigricans, vi. 389

nigricollis, vi. 382

nitens, vi. 393

Novæ Hollandiæ, vi. 383

Noveboracensis, vi. 392

obscurus, vi. 382

ochrocephalus, vi. 392. 425

olivaceus, vi. 383.386

Oonalaschka, vi. 382

Orientalis, vi. 294. 363. 392

ornatus, vi. 377

Orpheus, vi. 385

ouravang, vi. 378

Pacificus, vi. 383

pagodarum, vi. 392. 423

palmarum, vi. 312. 381. 392. 490

pectoralis, vi. 380. 409

Peronii, vi. 383

Persicus, vi. 383

perspicillatus, vi. 377

phænicopterus, vi. 389

phænicurus, vi. 377

Philippensis, vi. 383

pilaris, vi. 384

plumbeus, vi. 380

poliocephalus, vi. 383

polyglottus, vi. 385

pratensis, vi. 379

praursus, vi. 383

punctatus, vi. 384

reclamator, vi. 379

rex, vi. 403

roseus, vi. 392

rubiginosus, vi. 280 
Turdus ruficaudus, vi. 383

ruficollis, vi. 382

rufifrons, vi. 283. 380. 409

rufiventris, vi. 383. 386. 387

rufus, vi. 385

rupestris, vi. 376

rupicola, vi. 376

Sandwichensis, vi. 383

saturninus, vi. 285

saxatilis, vi. 375

sellacis, vi. 392

Senegalensis, vi. 377. 379

shannu, vi. 383

Sibiricus, vi. 382

silens, vi. 286

Sinensis, vi. 387.425

solitarius, vi. 375.386

sordidus, vi. 287

speciosus, vi. 357.382 .392 ; vii. 122

splendidus, vi. 382

squammeus, vi. 377

striatus, vi. 387

Suerii, vi. 383

Suratensis, vi. 382

tenebrosus, vi. 383

tibicens, vi. 377

tinnicus, vi. 403

tintinnabulatus, vi. 407

torquatus, vi. 375. 534

trichas, vi. 391

tricolor, vi. 378. 383

triostechus, vi. 400

Tripolitanus, vi. 382

triurus, vi. 383

Ulietensis, vi. 383

Vaillantii, vi. 389

validus, vi. 383

variegatus, vi. 383.387

varius, vi. 381.383

violaceus, vi. 376

virescens, vi. 383

viridi olivaceus, vi. 382

viridis, vi. 381

viscivorus, vi. 384. 524

volitans, vi. 369

Wilsonii, vi. 386

Turkey, viii. 154

common, viii. 16

Honduras, viii. 163

horned, viii. 31

marail, viii. 9

Turkies, viii. 15

wild, viii. 16

Turnix, viii. 62. 268

Africanus, viii. 62

Dussumier's, viii. 65

maculatus, viii. 63

white-spotted, viii. 63
Turtle, African, viii. 83

blue-headed, viii. 73

Cambayan, viii. 84

Chinese, viii. 77

collared, viii. 77. 290

common, viii. 77

green, viii. 82

Javan, viii. 81

Luzonian, viii. 77

Malacca, viii. 85

Malabar, viii. 85

red-breasted, viii. 74

spotted-necked, viii. 77. 290

striated, viii. 82

Surat, viii. 84

Surinam, viii. 90

white, viii. 85

Tutre, vii. 407

Tuyuyu-guazo, viii. 507

Twit, vii. 387

Tyranula, viii. 681

affinis, vi. 349

barbirostris, vi. 349

musica, vi. 350

nigricans, vi. 349

obscura, vi. 349

pallida, vi. 349 ; viii. 682

Tyrannus, vi. 318 ; viii. 681

albicollis, vi. 322

ambulans, vi. 321

atricapillus, vi. 272.323

bellicosus, vi. $\mathbf{3 2 1}$

bellulus, vi. 319

calcaratus, vi. 321

carnivorus, vi. 320

Cayanensis, vi. 320

coronatus, vi. 325

crassirostris, vi. 323

crudelis, vi. 324

Dominicanus, vi. 327

griseus, vi. 324

intrepidus, vi. 323

irritabilis, vi. 320

irupero, vi. 326

leucotis, vi. 324

longipennis, vi. 319

magnanimus, vi. 320

matutinus, vi. 324

melancholicus, vi. 319

pentaveo, vi. 320

pepoaza, vi. 322

pipiri, vi. 323

pyrrhophaius, vi. 321

rixosus, vi. 321

rufescens, vi. 321

rufiventris, vi. 323

rufus, vi. 321

Savanna, vi. 319

solitarius, vi. 32 
Tyrannus sulphuratus, viii. 68] verticalis, vi. 324 violentus, vi. 320 vociferus, vi. 323 vorax, vi. 324

Tyrant, black and white winged, vi. 322 noisy, vi. $\mathbf{3 2 3}$ red-brown, vi. 321 spiny-footed, vi. 321 St. Domingo, vi. 324 thick-billed, vi. 323

Tyrants, vi. 318. 491 bald, vi. 357

Tytira, viii. 679

\section{Umbre, viii. 509} tufted, viii. 353.509

Umbres, viii. 352

Upupa, vii. 379.380 aurantia, vii. 382 Capensis, vi. 424 ; vii. 381 epops, vii. 380.398 erythrorhynchos, vii. 382. 405 fusca, vii. 382 Indica, vii. 382 Madagascariensis, vi. 424 magna, vii. 382 Mexicana, vii. 382 minor, vii. 381

Papuensis, vii. 382 Paradisea, vi. 330 ; vii. 382 promerops, vii. 365. 381. 405 superba, vii. 382

Uria, viii. 553

Balthica, viii. 554

Brunnichii, viii. 554

Francesii, viii. 554

grylloïdes, viii. 554

lachrymans, viii. 554

Lomvia, viii. 554

Mandtii, viii. 555

marmorata, viii. 555

scapularis, viii. 554

Troile, viii. 554

Uru, viii. 59

Urubitinga, vi. 44. 236

Urucurea, vi. 79

Urutau, vii. 78. 112

Urutaurana, vi. 45

Vanga, vi. 285

destroying, vi. 286

destructor, vi. 286

striata, vi. 274

Vaginalis, viii. 416
Vaginalis alba, viii. 416 chionis, viii. 416

Vanellus, viii. 318,319 Egyptius, viii. 320 albicapillus, viii. 321 Cayanensis, viii. 464 cinctus, viii. 320 cristatus, viii. 319.462 flavipes, viii. $\mathbf{3 2 0}$ gallinaceus, viii. 321 . 322 lobata, viii. 322 Senegalus, viii. 465 tricolor, viii. 322

Vanneau, viii. 462

Vautour de Norwège, vi. 186 grand, vi. 15 petit, vi. 186

Vermivora, vi. 465

Vest doré, vi. 395

Vauve à épaulettes, la, vii. 150 chrysoptère, la, vii. 151

Vidua, vii. 149 longicauda, vii. 149

Vinago, viii. 93. 273

Vinette, vi. 478

Vireo flavifrons, vi. 355 musicus, vi. 356 virescens, vi. 356 rufescens, vi. 436

Viuva, vi. 327

Vouroudrions, vii. 461 Vultur, vi. 15

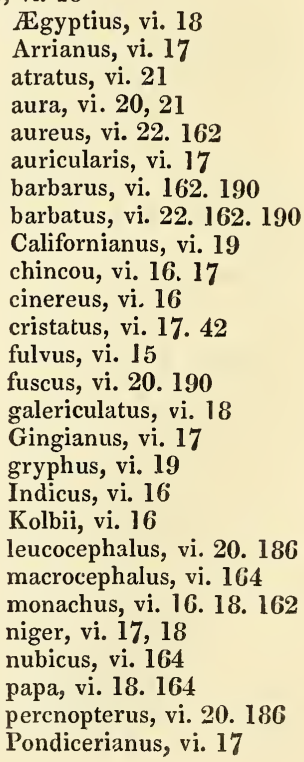


Vultur sacra, vi. 18. $166^{\circ}$ serpentarius, vi. 69 trencalos, vi. 15 uruba, vi. 21 vulturinus, vi. 21

Vulture, vi. 15

Angola, vi. 17. 186. 190

Arabian, vi. 17

ashy, vi. 16

bearded, vi. 22.190

black, vi. 16. 21.162

black-crowned, vi. 162

brown, vi. 17

Californian, vi. 21

carrion, vi. 20

Cheriway, vi. 21

chocolate, vi. 17

cinereous, vi. 16. 162

crested, vi. 42

crested-black, vi. 16

Gingi, vi. 20

Egyptian, vi. 18. 187

fulvous, vi. 15.160

golden, vi. 22

great of the Andes, vi. 19

Indian, vi. 15, 16

Kolben's, vi. 16

little, vi. 187

of Lambs, vi. 190

of Malta, vi. 190

of New Holland, vi. 21

of Norway, vi. 187

painted, vi. 18.166

plaintive, vi. 21

Pondicherry, vi. 17

sociable, vi. 17. 163

tawny, vi. 21. 23

white-tailed, vi. 166

Vultures, vi. 15.157

king of the, vi. 18. 164

Waders, viii. 293

Wagtail, Cape, vi. 477

green, vi. 475

mournful, vi. 475

pied, vi. 475

scapular, vi. 448

white, vi. 475

yellow, vi. 476

yellow-headed, vi. 476

Wagtails, vi. 474 ; vii. 50

proper, vi. 474

white, vii. 51

yellow, vii. 54

Wallikikili, viii. 224

Walyvogels, viii. 444

Wapaw, vi. 485
Warbler, African, vi. 470

Alpine, vi. 465 ; vii. 35.37 .173

aquatic, vi. 443

autumnal, vi. 458

Awatcha, vi. 464

babbling, vi. 445 ; vii. 34

Batavian, vi. 453

bay-breasted, vi. 456

black and white, vi. 436

black-headed, vi. 333 . 445

black-jawed, vi. 447

black-necked, vi. 451

black-poll, vi. 456

black-throated, vi. 456

black-throated blue, vi. 457

Blackburnian, vi. 460

blue, vi. 440

blue-eyed yellow, vi. 460

blue green, vi. 457

blue-headed, vii. 172

blue-striped, vii. 172

blue-tailed, vi. 447

blue-throated, vi. 439 ; vii. 11

blue yellow-backed, vi. 459

blue winged yellow, vii. 172

bog, vi. 442

banana, vi. 463

Bourbon, vi. 449

brown, vi. 473 ; vii. 44

brown-throated, vi. 462

buff-faced, vi. 447

cærulean, vi. 458

Cape May, vi. 455

Cetti's, vi. 443

chestnut-bellied, vi. 447

chestnut-sided, vi. 456

China, vi. 449

chret, vi. 452

Cingalese, vi. 448

citron, vi. $\mathbf{4 5 2}$

citron-bellied, vi. 449

Connecticut, vi. 458

crested, vi. 333

Dartford, vi. 446

Daurian, vi. 447

dusky, vi. 450

dwarf, vi. 470. 471

equinoctial, vi. 451

exile, vi. 469

flaxen, vi. 469

grasshopper, vi. 443

gray, vi. 444

green and white, vi. 462

green Indian, vi. 448

golden-crest like, vi. 471

golden-winged, vii. 172

grisly, vi. 461

hang-nest, vi. 463

hawk-like, vi. 445 ; vii. 35 
Warbler, hemlock, vi. 456 Jamaica, vi. 462 Javan, vi. 451 Kentucky, vi. 458 Lambert's, vi. 468 long-billed, vi. 463 long-legged, vi. 452 long-tailed, vi. 467 Luzonian, vi. 436 Madagascar, vi. 449 Magellanic, vi. 461 Maurice, vi. 473 mountaineer, vi. 453 mourning, vi. 459 murine, vi. 447 Nashville, vii. 172 olive, vi. 448 orange-bellied, vi. 472 orange-crowned, vii. 172 orange-headed, vi. 462 orange-rumped, vi. 469 Orpheus, vi. 444 palm, vi. 454 passerine, vi. 445 ; vii. 34 Patagonian, vi. 461 pensile, vi. 472 Philippine, vi. 436 pine creeping, vi. 464 pine swamp, vi. 458 pink, vi. 448 plumbeous, vi. 45] Prairie, vi. 457 protonothary, vii. 172 red-bellied, vi. 305 red-tail, vi. 440 reed, vi. 466 of the reeds, vi. 443 rufous and black, vi. 462 rufous-tailed, vi. 461 rusty-headed, vi. 461 rusty-side, vi. $\mathbf{4 5 2}$ Sarda, vi. 446 sedge, vi. 442 sibyl, vi. 437 silky, vi. 441 simple, vi. 463 sooty, vi. 436 spectacled, vi. 446 spotted, vi. 443 ; vii. 30 spotted yellow, vi. 457 St. Domingo, vi. 462 stone chat, vii. 5 subalpine, vi. 446 superb, vi. 468 taylor, vi. 450 Tenessee, vii. 172 undated, vi. 449 wheat-ear, vii. 4 white-breasted, vi. 445
Warbler, white-chinned, vi. 450 white-crowned, vi. 448 white-eyed, vi. 453 winter, vii. 37 worm-eater, vi. 462 worm-eating, vii. 172 yellow-bellied, vi. 461 yellow-poll, vi. 472 yellow-rump, vi. 454 yellow-throated, vi. 460 yellow wren, vi. 472

Warblers, vi. 434. 440.465 ; vii. 3

Water-hens, viii. 411

Wattle-bird, vii. 336

Weavers, vii. 229

Wheatear, vi. 435 black-hooded, vi. 437 Rousset, vi. 435

Whenchat, vi. 435

Whip-poor-Will, vii. 74. 109

Whisky John, vi. 485 white, vi. 485

Whitetail, vi. 435

White-throat, vi. 444 lesser, vi. $\mathbf{4 4 5}$

Widow Chuck Will's, vii. 74

Wigeon, American, viii. 622 Cape, viii. 624

Wimbrel, viii. 365

Woodcock, viii. 367.521 little, viii. 367 Savanna, viii. 369

Woodcracker, vii. 387

Woodpecker, vii. 509 Azara's, vii. 444 banded, vii. 451 Bengal, vii. 443 black-bearded, vii. 512 blue, vii. 387 buff-crested, vii. 441 Canada, vii. 446 cardinal, vii. 448 Carolina, vii. 447 Cayenne, vii. 448 Ceylonese, vii. 444 Chili, vii. 143 concrete, vii. 442 Crawford's, vii. 513 crimson-rumped, vii. 444 digging, vii. 451 dusty, vii. 442 encenade, vii. 447 ferruginous, vii. 445 field, vii. 448 gaudy, vii. 444 Goa, vii. 443 gold-back, vii. 444 gold-winged, vii. 451 golden-crested, vii. 448 
Woodpecker, great black, vii. 439 greater spotted, vii. 440 green, vii. 439

hairy, vii. 446

helmet, vii. 441

Lewis's, vii. 443

lineated, vii. 441

little, vii. 447

Mace's, vii. 449

Malacca, vii. 447

Manilla, vii. 444

middle spotted, vii. 440

minute, vii. 451

northern three-toed, vii. 450

passerine, vii. 445

pierced, vii. 443

pileated, vii. 441

rayed, vii. 448

red, vii. 444,445

red-bellied, vii. 446

red-cheeked, vii. 447

red-beaded, vii. 442

red-necked, vii. 442

robust, vii. 442

rufous, vii. 447

sklit, vii. 448

Sphix's white-billed, vii. 442

St. Domingo, vii. 443

strong-billed, vii. 442

supercilious, vii. 448

Surinam, vii. 445

swallow, vii. 446

white-billed, vii. 441

yellow, vii. 445

yellow-bellied, vii. 446

yellow-crested, vii. 448

yellow-headed, vii. $\mathbf{4 4 5}$

yellow-shafted, vii. 451

yellowish, vii. 446

Woodpeckers, vii. 438

Worabee, vii. 132. 232

Worail, viii. 304

Wren, Buenos Ayres, vi. 474 ; vii. 47 Byron's golden-crested, vii. 42 common, vi. 473 ; vii. 42 gold-crested, vi. 472 ; vii. 39 great Carolina, vi. 474 ; vii. 48

house, vi. 473 ; vii. 44

marsh, vii. 49

reed, vi. 442 ; vii. 30
Wren, winter, vi. 473 ; vii. 44 yellow-crested, vii. 41

Wrens, vi. 471

Wryneck, vii. 452 anomalous, vii. 453

crested, vii. 452

least, vii. 452

little, vii. 453

Wrynecks, vii. 451. 513

Xanthornus, vii. 168,169

Xenops, vii. 345.388

Anabatoïdes, vii. 346. 388

genibarbis, vii. 346

Hoffmanseggii, vii. 346

rutilus, vii. 346

Xiuhtototl, vi. $306^{\circ}$

Yacous, viii. 7

Yacu carraguata, viii. 129

Yacucha, viii. 10

Yahana bleu de ciel, viii. 413

Yapou, vii. 318

Yapu, vii. 166

Yellow-throat, Maryland, vi. 459

Yetapa, vi. 320

Yiperu, vi. 319

Ynambu bleuâtre, viii. 68 canapé, viii. 69 rayé, viii. 67

Ynambus, viii. 66

Ypecaha, viii. 409 brun, viii. 409 noirâtre, viii. 409

Yphantes, vii. 168

Yunx, vii. 451. 513 minutissima, vii. 451, 452 torquilla, vii. 452.513

Yzguautzli, vi. 42

\section{Zapornia, viii. 406}

minuta, viii. 408

pusilla, viii. 408

Zaramagullen noir, viii. 591

Zopilote papa, vi. 164

Zosterops, vi. 453

dorsalis, vi. 453 



\title{
R E P T I L I A.
}

\author{
VOL. IX.
}

* Letter $s$ in this index refers to the Synopsis, of which the folios are at the foot of the pages.

Авома, vol. ix. 254

Abou Burs, 148

Abranchus, 410 ; s. 109

Alleghaniensis, s. 109

horridus, $s .109$

Acanthopis, 277 ; $s .82$

Brownii, 277 ; s. 82

cerastinus, $277 ; s .82$

Acanthosaura, $s .56$

Acontias, 244 ; s. 76

cæcus, $245 ; s .76$

lineatus, $s .77$

meleagris, s. 76

reticulata, s. 76

Acrochordus, 264. $336 ; s .99$

dubius, $s .99$

fasciatus, 281 s. 99

Javensis, 264

Javanicus $s .99$

Ada, 111. 203 ; s. 28

bicarinata, s. 29

common, s. 28

crocodilus, $s .28$

double-crested, s. 29

Adder, Bordelaise, s. 262

collared, $s .261$

four-striped, 262

great, 257

green and yellow, 262

viperine, 261

Adders, 256. 331

Æsping, 272

Agama, 122; s. 54

aculeata, 124

agilis, s. 58

Aralensis, $s .58$

arenaria, $s .58$

armata, s. 56

atra, $124 ; s .57$
Agama azurea, vol. ix ; s. 42

barbata, $122 ; s .60$

brevicaudata, $s .42$

calotes, $s .56$

catenata, $127 ; s .40$

collaris, $s .31$

colonorum, 123. 216

cornuta, 124. $217 ; s .45$

cristatella, $127 ; s .55$

cristata, 127; s. 55

cyclura, $126 ; s .42 .43$

deserta, s. 58

dorsalis, $s .56$

Douglassii, $s .44$

flavigularis, $s .55$

gigantea, 128

grandoculis, $s .60$

gutturosa, 127 ; s. 56

Indica, s. 55

isodactyla, $s .58$

Jacksoniensis, $s_{\mathrm{e}} 60$

minor, $s .55$

Molinaii, s. 47

nigricollis, s. 41.43

nigrocollaris, 126

occipitalis, s. 56

ocellata, s. 59

ophiomachus, 127

orbicularis, $s .57,58$

picta, $127 ; s .40$

platura, s. 52

plica, $s .41$

Pondiceriana, s. 57

ruderata, $s .58$

semitæniata, $s .41$

sinuata, $s .58$

spinosa, 216;s. 57

straminea, $s_{0} 58$

subspinosa, s. 57 
Agama taraguira, vol. ix ; s. 41 tigrina, $s .55$ tuberculata, $136 ; s .41 .56$ umbra, 124 ; s. 41 undulata, $126 ; s .43$ versicolor, $s .55$ vultuosa, $127 ; s .56$

Agama, Aral, s. 58

Cape, $s .57$ common, $122 ;$ s. 57 muricated, 123 mutable, $s .58$ obsoletely-crested, $s .56$ occipital, s. 56 ocellated, 122 Ruppel's, s. 58 sand, s. 58 slender, $s_{0} 58$ tubercular, $s .56$ yellow-striped, $s .57$

Agamæ, 215 orbiculariæ 124

Agame arlequiné, 127 à pierreries, 124 à queue prenante, 129 poìetué, 151

Ahætula $259 ; s .93$

Linnei, s. 93

liocercus, s. 93 periophthalmica, $s .93$ Richardi, s. 93

Aispysure, 280

Alecto, $s .79$

Alygyra, 117 ; s. 35

Cuvieri, s. 35 common, s. 35

Alligator, 105; s. 21 lucius, $s .21$ palpebrosus, $s .21$ sclerops, $s .21$ eye-browed, s. 21 pike-muzzled, s. 21 spectacled, s. 21

Amblicephalus, s. 94 carinatus, $s .94$ coccineus, s. 94 levis, $s .94$ mikani, s. 94 Nattereri, s. 94

Amblyrhynchus, $s .37$ ater, $s .37$ cristatus, $133 ; s .37$

Ameiva, 113. 205 ; s. 29 argus, 114 ; s. 29 cæruleocephala, $114 ; s .30$ collaris, s. 31

cyanomelas, $s .30$ lateristriga, $114 ; s .30$ lemniscata, $114 ; s .30$
Ameiva litterata, vol. ix. 114 ; s. 29 tessellata, $s .30$

Teyus, $114 ; s .31$

trilineata, $s, 30$

viridis, $s .31$

vulgaris, $s .29$

Ameiva, black marked, 205

blue, $s .30$

blue and black, $s .30$

blue-headed, $s .30$

checkered, s. $\mathbf{3 0}$

collared, $s .31$

green, $s .31$

graphique, 114

lettered, $s .29$

striped, s. 30

three-streaked, s. 30

Amphibia, s. 99

Amphipneusta, s. 107

Amphisbœna, 246. 324 ; s. 66

alba, 247. 325 ; s. 66

blana, s. 66

cæca, 247 ; s. 67

cinerea, s. 66

flavescens, $247 ; s .66$

fuliginosa, $247.326 ; s .66$

oxyura, s. 66

pathyura, s. 66

punctata, 247 ; s. 66

vermicularis, 247 ; s. 66

Amphisbœena, blind, $s .67$ white, 325 ; s. 66

Amphiuma, 411. 474; s. 109 means, 411. 474 ; s. 109 tridactylum, 411. $475 ; s .109$

Anacondo, 253

Ancistodon, s. 79

Anguis, 241, 242, 243. 306 ; s. 74

alba, s. 91

ater, $251 ;$ s. 75

bipes, 161 ; s. 74

calamaria, $s .91$

clivica, $244 ; s .74$

corallinus, $251.327 ; s .75$

crocotatus, s. 77

erix, $244 ; s .74$

fragilis, 244. 306 ; s. 74

granulatus, 264

laticauda, s. 98

lineata, s. 74

lumbricalis, 248

maculatus, 251

melanosticus, s. 75

Meleagris, 245; s. 76

platurus, 280

quadrupes, 159. 240

reticulatus, 248 ; $s .76$

rufa, $s .75$

scytale, 251 ; s. 75 
Anguis striatus, vol. ix ; s. 75 tessellatus, $251 ; s .75$ ventralis, $243 ; s .65$ vermicularis, 247 zyphura, $264 ;$ s. 98

Anilius, 250 ; s. 75

corallinus, $s .75$

latta, $s .75$

maculatus, $s .75$

melanotis, $s .75$

melaustictus, $s .75$

rufus, s. 75

scytale, $s .75$

Anolis, 137. 205. 227 ; s. 46

bimaculata, $s .46$

Cuvierii, s. 46

Edwardsii, 228; $s .46$

equestris, $s .46$

gracilis, $s .46$

lineatus, s. 46

podargicus, s. 46

punctatus, $s .46$

velifer, $s .46$

violaceus, $s$. 46

viridis, $s .46$

Anolis of Carolina, 139

Cuvier's, $s$. 46

de terre, 158

dotted, $s .46$

Edwards, s. 46

equestrian, s. 46

gigantesque, 157 ; s. 68

gouty, s. 46

great-crested, 138

green, s. 46

lined, s. 46

marbré, 158 ; $s .68$

parcé, $s .68$

pavé, 157

roquet, 138

slender, s. 46

small-crested, 138

striped, 139

two-spotted, s. 46

violet, $s .46$

with white points, 140

Anolius, $137 ; s .46$

equestris, 138

gracilis, 140

Leachianus, 145

velifer, 138

viridis, 140

Aquaquetzpallia, 132

Ascalabotes, 140 stenodactylus, $s .49$

Aspic of Cleopatra, 275

$$
\text { of Egypt, } 275
$$

Aspidura brachyorrhis, s. 92

Aspis, $270 ; s .83$
Aspis Cleopatræ, vol. ix ; s. 81 ocellata, s. 81

Asterodactylus, $s .105$

Atractos, s. 91 trilineatus, $s .91$

Atropos, s. 79 puniceus, $s .80$

Auletris, s. 101

Axolotl, 411 ; s. 108 common, s. 108

Axolotls, 411

Basilisk, 226

banded, s. 45

mitred, s. 45

Basilisks, 134

Basiliscus, 134 ; $s .45$ Americanus, $s .45$ vittatus, $s .45$

Bathrops, 267

Batracians, 5

Batrachia, 5

Berus, 271 ; s. 81 ammodytes, $s .82$ vulgaris, $s .82$

Bimane wormlike, s. 66

Bipède cannelé, 163 .

Bipedes, 160 lepidopode, 160

Bipeds, 240

Bipes, $160 ; s .73 .74$ anguinus, $s .74$

Fraseri, $s .73$

lepidopus, s. 74

lineata, $s .73$

Bipes Brasilian, $s .73$

Cape, s. 74

Fraser's, $s .73$

New Holland, s. 73

Blindworm American, s. 74

common, s. 74

Otto's, s. 74

Boa, 251. 327; s. 96

aculeata, $s .82$

albicans, 257

amethystina, 257

anacondo, $253 ;$ s. 96

Antarctica, $s .82$

aquatica, 253 ; s. 96

canina, $254 ; s .96$

carinata, $255 ; s .97$

castanea, 257

cenchris, $253 ;$ s. 96

cenchrya, $254 ; s .96$

conica, s. 97

constrictor, $253 ; s .96$

contortrix, s. 85

elegans, $254 ; s .97$ 
Boa empereur, vol. ix. 253 ; s. 96 fasciata, 279

hiprale, 254 ; s. 96

horatta, 278 ; $s .82$

hortulana, $254 ; s .96$

krait, s. 82

latotecta, s. 95

lateristriga, $s .96$

lineata, 279

Merremii, 254

murina, $253 ; s .96$

mutus, $s .79$

ocellata, 255 ; s. 97

orbiculata, $257 ; s .97$

ordinata, 257

ornata, $s .97$

palpebrosa, 277 ; s. 82

porcaria, $s .85$

regia, s. 97

reticulata, 257 ; s. 97

rhombeata, 257 ; s. 97

scytale, $253 ;$ s. 96

Tartarica, s. $\mathbf{9 8}$

Boas, 251

riperina, $255 ;$ s. 97

Boicininga, 345

Boiquira, 345

Bojobi, 253

Bombina obstetricans, s. 103

Bombinator, 402; s. 104

Daudini, s. 104

igneus, s. 104

Bong bleu, 279

Bongare à anneaux, 279

Boodroopam, 269

Bora, 257; s. 97

Bothrops, s. 79

furia, $s .79$

leucostigma, s. 79

leucurus, $s .80$

Megæra, s. 79

suruacea, s. 79

tæniatus, $s .79$

Brachycephalus, 403 ; s. 104

Brachylopus, 129 ; s. 37

fasciatus, $s .37$

Brachyorrhos, s. 91

albus, $s .91$

badius, $s .91$

brachyurus, $s .91$

decussatus, $s .91$

dimidiatus, s. 91

flammigerus, $s .91$

Kuhlii, s. 91

schach, $s .91$

torquatus, $s .91$

trilineatus, $s .91$

Brachypus, $162 ; s .66$

abdominalis, $s .66$
Brachypu, common, vol. ix ; s. 66

Breviceps, 403 ; s. 104

albifrons, s. 104

gibbosus, s. 104

globosus, s. 104

granosus, s. 104

Surinamensis, $s .104$

marmoratus, s. 104

Brodro pam, s. 80

Bronchocela, s. 55

Bufo, 398. 451 ; s. 102

acutirostris, 402 ; s. 103

agua, 402 ; s. 103

albifrons, s. 104. 403

Bengalensis, $402 ; s .103$

bombina, 402

calamita, 461 ; s. 103

chlorogaster, $s .103$

cinereus, $s$. 102

cinctus, $402 ; s .103$

clamosus, $s .103$

cognatus, $s_{\bullet} 103$

cruciatus, $s .103$

cyanophlyctis, $s .100$

ephippium, s. 104

flaviventris, s. 103

fuscus, s. 103. 399

gibbosus, 403 ; s. 104

globulosus, 403 ; s. 104

granulosus, 402; s. 103

ictericus, 401 ; $s .103$

levis, $s .104$

Lazarus, 401 ; s. 103

maculiventris, 401 ; s. 103

margaritiferus, $s .104$

marinus, $s .103$

melanostrictus, $s .103$

musicus, 402. 463 ; s. 103

nasicus, 402

nasutus, 402

obstetricans, 400. 462;s. 103

Palmarum, 401

prætextatus, $s .103$

proboscideus, $402 ; s .103$

pustulosus, $s .103$

Roeselii, s. 103

roseus, $s .103$

rudibunda, $s .100$

salsus, s. $] 03$

scaber, 401 ; s. 103

Schreberianus, $s .103$

semilineatus, 402; s. 103

spinosus, 462

sitibundus, $s .103$

stellatus, $401 ; s .103$

variabilis, s. 103

ventricosus, 402 ; s. 104

viridis, $s .103$

vulgaris, $s .103$ 
Bumbos, vol. ix. 193

Bungari, 279

Bungarum pamma, 279

Bungarus, 259. 279. 385 ; s. 95

annularis, s. 95

cæruleus, s. 95

filiformis, $259 ; s .93$

filum, s. 94

semifasciatus, s. 95

Cæcilia, 281.386; s. 109, 110 albiventris, 283 ; s. 110 annulata, 283,284 ; s. 110 bivittata, 284 ; s. 110 glutinosa, 284 ; s. 110 hypocyana, $s .110$ interrupta, 283 ; $s .110$ lumbricoïdes, 284 ; s. 110 nasuta, $s .110$ rostrata, $284 ; s .110$ tentaculata, s. 110

Cæcilia, annulated, 283 bearded, $s .110$ glutinous, 284 ; s. 110 interrupted-ringed, s. 110 Javanese, s. 110 ringed, s. 110 sharp-nosed, s. 110 tentaculated, 283 two-banded, s. 110 wormlike, s. 110

Caiman, 105

Calamaria, s. 91

Calamita, 396 ; s. 101

bilineatus, $s .101$

boans, $s .101$

Carolinensis, s. 102

cinerea, s. 101.102

maximus, s. 101

melanorabdotus, $s .102$

Surinamensis, s. 102

Caliscertula, 211

Calotes, 126 ; $s .55$

gutturosa, $s .56$

lepidogaster, 127 ; s. 56

ophiomachus, s. 55

Tiedemanni, s. 55

tympanistra, s. 56

Calotes, armed, s. 56

blue, $s .55$

common, s. 55

Indian, $s .55$

Kuhl's, s. 56

long-legged, $s .56$

smaller, $s .55$

Cameleo trapes, 153

Cameleonians, 152
Caretta nasicornis, vol. ix ; s. 21

Cascavela, $\mathbf{3 4 5}$

Cataphracta, $s .2$

Caudisona, 267 ; s. 78

Causus, s. 83

Cayman, s. 21

pike-muzzled, 106. 197

spectacled, 105. 198

with osseous-eyelids, 107. 200

Caymans, 105. 196

Cecella, 159

Cenchris, 255 ; s. 79.97

mockeson, $s .79$

ocellatus, s. 97

regia, s. 97

Centropyx, 115

intermediate, $s .31$

spurred, s. 31

striated, s. 31

Cerapes carinata, s. 92

Cerastes, 271 ; s. 81

plicatus, s. 96

Ceratophris, 395

Ceratophys, s. 101

boiei, s. 101. 104

clypeata, 395 ; s. 101

cornuta, s. 101

Daudini, 395

dorsata, 395 ; 101

granosa, 395 ; s. 101

granulata, s. 104

montana, s. 101

scutata, s. 101

Spixii, 395; s. 101

varius, 395 ; s. 101

Cerberus, 257

Chalcide, $162 ; s, 66$ monodactyle, 162

Chalcides flavescens, $s .66$ monodactylus, $162 ; s .66$ tridactylus, $s .66$ zygnis, $s .72$

Chalcides, $161.240 ; s .65$ annulated, s. 66 common, $s .65$

Chalcis, 162 ; s. 66

Chamæleopsis, s. 45 Hernandesii, $s$. 45

Chamæsaura, 160 ; s. 73 cophias, 162 ; s. 66 propus, 163 ; s. 66

Chamelion, 232

dwarf, 154

of Molucca, with forked nose, 155

of Senegal, 154

Chameleons, 152

Chameleo, 152

bifidus, s. 54 
Chameleo bifurcus, vol. ix. 155. 237 ; s. 45

bilobus, 155 ; $s .53$

Brookesii, s. 53

carinatus, 153

cucullatus, $s .54$

dilepis, 155. 237 ; s. 53

fimbriatus, $s .53$

gymnocephalus, $s .53$

lateralis, $s .53$

margaritaceus, 154 ; s. 53

monilifer, $s .53$

Owenii, s. 54

pardalis, $154 ; s .53$

Parsonii, 155; s. 54

planiceps, 154 ; s. 53

pinnulus, 154

pumillus, $s .53$

Seichellensis, 154

Senegalensis, $s .53$

subcroceus, 153

superciliaris, $s .53$

tigris, 153 ; $s .53$

verrucosus, 154 ; s. 53

vulgaris, s. 53

Chameleon, $s .52$

banded, s. 53

Brookesii, s. 53

Cape, s. 53

common, 153 ; s. 53

eared, s. 53

hooded, s. 54

panther, s. 53

Parson's, s. 54

Senegal, s. 53

streaked-sided, s. 53

three-horned, s. 54

two-horned, s. 54

Champses, 102

Chaunus marmoratus, s. 104

Chelodina, 12 ; s. 15

longicollis, s. 15

Chelonia, 4. 14 ; s. 20

caretta, s. 20

coriacea, $s .20$

imbricata, s. 21

lachrymata, 15 ; s. 20

maculosa, 15 ; s. 20

Mydas, s. 20

olivacea, s. 20

radiata, $16 ; s .20$

virgata, 16 ; s. 20

Chelonians, 4. 81

Chelonura, 13

Chelydes, 17

Chelydra, 13 ; s. 14

lacertina, s. 14

serpentina, $s .14$

Chelys, 17 ; s. 14
Chelys Adansonii, vol. ix ; s. 15

castaneus, s. 14

Cayennensis, s. 17

depressa, $s .16$

Dumeriliana, s. 17

expansa, s. 17

fimbriata, $s .17$

lata, s. 17

longicollis, s. 15

Macquarii, s. 15

Matamata, s. 17

planiceps, s. 16

radiolata, s. 16

rufipes, $s .16$

subrufa, s. 15

viridis, $s .16$

Chelys, Adanson's, s. I5

Cape, s. 15

Cayenne, s. 17

chestnut, $s .14$

Demerara, s. 17

depressed, s. 16

Dumeril's, s. 17

expanded, s. 17

flat-headed, $s .16$

green, s. 16

long-necked, s. 15

Macquarie's, s. 15

radiated, s. 16

red-footed, s. 16

Chersina, s. 5

$$
\text { angulata, s. } 5
$$

Chersine, 9

Chersydrus, $28 \mathrm{I}$; s. 99

granulatus, s. 99

Chirotes, 162 ; s. 66

Chlorosoma viridissima, s. 90

Chlamydosaurus, 217 ; s. 60

Chrysodonta larvæformis, 411. 474 ; $s$ 。 109

Chysopelea, s. 94

Paradisi, s. 94

rhodopleura, s. 94

Cicigna, 159. 240; s. 63

Burnettii, 240

Madagascariensis, s. 64

sepiformis, s. 63

Cicigna, common, s. 63

Madagascar, s. 64

smooth, s. 63

Cista sternalis, 404

Cistuda, 13. 77. 80 ; s. 7

Amboinensis, s. 7

Carolinæ, $s, 7$

Europea, s. 7

odorata, s. 13

trifasciata, s. 7

Clælia, s. 89

aurora, s. 90 
Clælia dorsata, ix. s. 90 melanocephala, $s .90$ occipitalis, s. 90

Clamydosaurus, 247 ; s. 60 Kingii, $s .60$

Clothonia, s. 98

Cobra, 270 di capello, 274.376

Coclopeltis lacertina, s. 94 tigrina, s. 94

Colobus, 162 ; s. 66 Daudini, s. 66

Coluber, 256. 330 ; $s, 85$ acuminatus, s. 93 æneus, s. 96 æstivus, $s .86$ Esculapii, s. 87. 89

Africanus, s. 90

agilis, $s .89$

ahætula, $259 ; s .93$

albus, s. 91

alvearius, s. 92

ambiguus, s. 79

ammobates, s. 88. 90

ammodytes, 271 ; s. 82

anastomosatus, $s .86$

angulatus, $s .96$

annulatus, s. 90

aspis, s. 82

atratus, 264

atriventer, $s .88,89$

atrofuscus, $s .90$

atropos, $s .81$

atrovirens, 262 ; $s .87$

atrox, s. 79. 95

audax, s. 92

aulicus, s. 92

aurora, $s .90$

Austriacus, 262. 335

azureus, s. 87

baliedeira, $s .91$

barbarus, s. 87

berus, 270. 272.354 ; s. 82

bicarinatus, $s .89$

bicinctus, s. 90

bilineatus, s. 87. 91

binatus, $s .89$

bitis, $s .81$

boæformis, 257; 97

Boddartii, s. 90

Borealis, s. 85

bracyurus, $s .91$

Bramminus, s. 86

Brasiliensis, s. 81

buccatus, s. 95

bucephalus, 259 ; s. 95

cacodæmon, s. 80

cæruleus, s. 94

calamaria, s. 91

Cuv. Index.
Coluber cancellatus, ix. s. 88. 92

candidus, $s .95$

caninana, s. 87

canus, s. 87, 88

capistratus, $s .87$

capite niger, s. 90

capite triangularis, $s .80$

Caracaras, 259 ; s. 93

carinatus, s. 89

carinicaudatus, $s .95$

castaneus, $s .83$

catenulatus, 260

catenularis, s. 92

Catesbii, $s .95$

caudiolus, $s .90$

cenchoa, $s .95$

cerastes, 271 ; s. 81

Cerberus, 257 ; s. 95

chersea, 272 ; s. 82

cinerascens, $s .88$

Clælia, s. 89

clamacophorus, $s .88$

cobella, s. 90

coccineus, s. 94

compressus, $s .95$

conspicillatus, $s .88$

constrictur, 335 ; s. 85

corais, s. 87

cornutus, s. 81

coronatus, s. 87

crassicaudatus, $s .90$

crucifer, s. $88 ; 93$

cyaneus, s. 87

decorus, 259 ; s. 94

dictyodes, s. 90

dimidiatus, s. 91

doliatus, s. 90

domicella, $s .83$

domicellarum, s. 83

dora, s. 86

dubius, s. 81

Egypticus, s. 81

elaphis, $s .87$

elegans, s. 94

Epidaurius, s. 90

erythrogrammus, s. 95

erythrogaster, $s .86$

Esculapii, 263

fasciolatus, s. 92

fasciatus, $s .86$

flavescens, 264; s. 87

flavilineatus, s. 88

flavius, 276

formosus, $s .89$

fulgidus, $260 ; s .93$

fuliginosus, s. 87

fuscūs, $s .89$

Galathea, s. 92

Gallicus, s. 93 
Coluber geminiatus, ix. 5,88 Geoffroyii, $s .87$

getulus, s. 87

Girondicus, 262; s. 93

glaucus, s. 97

grammineus, $s .80$

hæmachates, 273

haje, 274 ; $s .83$

halys, s. 80

Hebe, s. 92

Hebriacus, $s .81$

Helena, s. 88

heterodon, $s_{0} 85$

hippocrepis, s. 87

hitambocia, s. 90

horridus, 263

humanus, s. 87

hybridus, $s .85$

hydrus, $s$. 86

ibibcea, $s$. 94

ignobilis, $s .90$

inornatus, $s .88$

intumescens, $s .81$

jaculatrix, 263

janthinus, 263; s. 90

Jara, s. 96

Javanicus, 257. 330 ; s. 97

korros, s. 88

lacertina, $s .94$

lacteus, 276 ; $s .83$

lævicollis, $s .89$

lævis, s. 91

lanceolatus, s. 79

laticaudatus, 275 ; s. 84

Latonius, 276 ; $s .84$

latratus, $s .83$

lebetinus, $s .80$

lemniscatus, 275 ; $s .83$

leucocephalus, s. 92

leucomelas, s. 87

Lichtensteinii, $s .87$

lineatus, 263 ; s. 90

lineolatus, s. 94

Linnæi, s. 91.96

liocercus, s. 93

lubricus, 276 ; s. 84

lumbricoïdes, $s .91$

lutrix, s. 89

macrorhinus, s. 92

maculosa, s. 91

malignus, $s .92$

malpolon, $s .86$

margaritaceus, s. 88

Maximiliana, s. 92

Megæra, s. 79

Meifrenii, s. 87

melanocephalus, s. 90

melanopis, s. 88

melanurus, s. 88
Coluber Meleagris, ix. s. 90

Meridionalis, s. 91

Merremii, s. 90

mikani, s. 94

miliaris, s. 90

minoclironis, s. 87

monilis, s. 95

molurus, $s .96$

multipunctata, $s .91$

murorum, s. 85

Naia, 274; s. 83

nasicornis, $s .81$

nasutus, 260 ; $s .93$

natrix, 261. 333 ; $s, 85$

Nattereri, s. 94

nebulatus, $s .95$

Nicandri, s. 90

Novæ Hispanicæ, s. 87

nympha, s. 89

Bahaiensis, s. 87

obscurus, s. 88

octolineatus, s. 91

Olfersii, s. 87

olivaceus, $s .87$

Oppelii, s. 85.88

ordinatus, $s .86$

Orientalis, $s .90$

ornatus, s. 94

oxycephalus, s. 88

pæcilogyrus, $s .89$

pæcilostoma, s. 86

palustris, $s .86$

Parias, s. 88

parietalis, $s .86$

personatus, $s .87$

Peruvianus, s. 87. 95

pethola, s. 90. 92

pictus, s. 94

pileatus, s. 87

plicatus, s. 96

plumbeus, s. 87

Plutonicus, s. 87

porcatus, $s .86$

porphyraceus, $s .83$

prester, 273 ; $s .82$

proximus, $s .86$

pseudo-echidna, s. 85

pullatus, s. 87

punctatus, s. 87

purpurascens, s. 93

pyrrhonotus, $s .89$

quadricarinatus, $s .80$

radiatus, s. 88

raninus, $s .90$

reginæ, 263 ; s. 90

reticulata, s. 91

rhabdocephalus, s. 88

rhombeatus, $s, 91.94$

rufescens, s. 90 
Coluber rufulus, ix. s. 90

Russellii, s. 81. 92

Saturninus, s. 88

saurocephalus, $s .88$

scalaris, $s .87$

scandens, s.94

schokari, s. 94

scurrula, s. 87

Sebæ, s. 97

serpentinus, $s .90$

severus, $s .88$

sexcarinatus, $s .89$

sibilans, 264; s. 86.93

Siculus, 261

simus, $s .85$

stolatus, s. 86

subcinctus, $s .92$

subfuscus, $s .89$

subirides, $s .86$

subminiatus, s. 86

Sumatranus, s. 80

Surinamensis, s. 84

tæniolatus, $s .91$

trilineatus, 264

tessellatus, $s .91$

Tisiphone, 268; s. 79

trabalis, $s .87,88$

trianguliferus, s. 86

triangulum, s. 87

tricolor, $s .88$

trigonocephalus, $s .80$

trinoculus, $s .81$

triseriatus, $s .81$

tristis, s. 24

trivirgatus, s. 88

typhlus, $s .90$

Tyria, s. 94

variabilis, s. 86.87

varius, $s .96$

venustissima, $s .89$

versicolor, $s .88$

vip. nigrum, 273 ; s. 83

vipera, $s .81$

viperinus, 261; s. 85,86

virgulata, $s .91$

viridicæruleus, s. 87

viridiflavus, s. 87

viridissimus, 263 ; s. 90

vittatus, $s .86^{\circ}$

vulneratus, s. 88

Colubro uccellatore, 335

Constrictor, s. 97

Cophias, 162. 267; s. 72.79

atrox, $269 ; s .79$

bilineatus, 269 ; $s .80$

bothrops, s. 79

crotalinus, $s .79$

flavescens, s. $66^{\circ}$

furia, s. 79
Cophias hypnale, ix. s. 80

jacaraca, 269 ; s. 79

lanceolatus, s. 79

leucostigma, s. 79

leucurus, s. 80

Megæra, s. 79

Neuweidii, $s .79$

punicea, $s .80$

Russellii, s. 80

Sumatranus, s. 80

tæniatus, s. 79

tessellatus, $s .79$

triangulum, $s .79$

trigonocephalus, $s: 80$

viridis, s. 80

Wagleri, $s .80$

Coralle, 254

Corallus obtusirostris, $s .97$

Cordylea, 120

Cordylus, 118, 213

dorsalis, 119

griseus, 119; s. 63

lævigatus, s. 63

microlepidotus, 119

niger, 119

stellio, 214

Coronella, 260; s. 89

Austriaca, s. 93

cervina, $s .86$

kotamboya, s. 90

petolaria, s. 90

pethola, s. 92

tessellata, $s .86$

venustissima, $s .89$

Coui, 65

Craspedocephalus, 268 ; s. 79

Crest-bearer, 131

Criket, Savannah, $s, 100$

Crocodile armed, $s .23$

common, $102 ; s .22$

double-crested, 103.104; s. 22

double-shielded, 105

flat-headed, $s .23$

helmeted, 105

Indian, s. 22

intermediate, $s .23$

lozenged, 105

of St. Domingo, 131

of the Nile, 102

rhombic, s. 22

Ruppell's, s. 22

Siam, s. 22

slender-muzzled, 104

two-shielded, s. 22

West Indian, $s .22$

with armed-nape, 10 :

Crocodiles, 98. 102. 181

Egyptian, 190

Crocodilians, 98 
Crocodilurus, ix. 111

Amazonicus, $112 ; s .29$

ocellatus, $s .29$

Crocodilus, $98 ;$ s. 21,22

acutus, $104 ; s .22$

biporcatus, 103 ; s. 22

biscutatus, 105 ; s. 22

cataphractus, $105 ; s .23$

complanatus, 103

Cuvieri, s. 21

fissipes, 106

galeatus, 105 ; s. 22

Gangeticus, s. 23

intermedius, $s .23$

lacunosus, 103

longirostris, 200

lucius, 106. 197 ; s. 21

marginatus, 103

octophractus, $s .22$

palpebrosus, $107.200 ; s .21$

flanirostris, $s .23$

punctulatus, 106

rhombifer, 105 ; s. 22

sclerops, 105. $198 ; s .2 \mathrm{l}$

suchus, 103

tenuirostris, 101. $201 ; s .23$

trigonatus, $107 ; s .21$

vulgaris, $s, 22$

Crocodilea, 120

Crotalophorus, 267 ; $s .78$

miliaris, $s .78$

Crotalus, 266. $336 ; s .78$

atricaudatus, $s .78$

cascavella, s. 78

Catesbii, $s .78$

confluentus, $s .78$

durissus, 267.346 ; s. 78

Dryinus, $s .78$

horridus, 267.345 ; s. 78

Loeflingii, $s .78$

miliaris, 267. 348 ; s. 78

mutus, $268 ; s .79$

rhombifer, $s .78$

strepitans, $s .78$

tergeminus, $s .78$

triseriatus, $s .78$

Cryptobranchus, 410

Ctenosaura, $s .37$

acanthura, s. 38

armata, $s .38$

Bellii, $s .38$

cycluroïdes, $s .37$

lanceolata, $s .38$

similis, $s .38$

Curuacea, $s .79$

Curucu, 463

Cyclodus, s. 67

flavigularis, $s .67$

Cyclura, s. 37. 39
Cyclura carinata, ix. $134 ; s .39$

nubila, $s .39$

Shawii, s. 38

teres, $s .39$

Cylindrophis, $s .75$

melanotis, $s .75$

resplendens, $s .75$

Cynosaura, $s .62$ punctata, s. 62

Cyrtodactyle, beautiful, $s .51$ Brasilian, $s .52$ eyed, $s .51$ flat-tailed, s. 52 marbled, $s_{0} 5$ ] Nilius's, $s .52$ piping, $s .52$

Cyrtodactylus, s. 51 ocellatus, $s .51$ pipiens, $s .52$ platura, $s .52$ pulchellus, $s .51$ Spixii, s. 52

Cyrtophanes, $s .55$ cristatus, $s .55$

Cystignathus, $s .100$ mystaceus, $s .100$ pachypus, s. 100 pygmæus, $s .100$

Daboia, $s .81$

Dactylethra, 396 ; s. 104

Dacypeltis scaber, s. 85

Delma, s. 73

Dendrophis, 259 ; s. 94 ahætula, s. 93

chaireacos, s. 94

formosa, s. 94

liocercus, s. 93

maniar, s. 94

picta, s. 94

polychroa, s. 94

scandens, $s .94$

tristis, $s .94$

Devin, 253; s. 96

Dipsas, 259; s. 95

bucephalus, $s .95$

carinata, $s .94$

Catesbii, $s .95$

cenchoa, s. 95

compressus, s. 95

cynodon, $s .95$

dendrophila, s. 95

Drapiezii, $s .95$

Indica, 259 ; $s .95$

irregularis, $s .95$

multomaculata, s. 95

nebulatus, s. 95

Savignii, s. 95

Schokari, s. 94 
Dipsas Scholtii, ix. $s .88$

Disteyre, 280 trigonatus, $s .95$

Distera doliatus, $s .98$

Disteria valakadin, $s .99$

Doryphorus, $120 ; s .42$ long-tailed, s. 42 short-tailed, s. 42

Dracæna, 203

Draco, s. 59 abbreviata, $s .59$ fimbriatus, $s .59$ fuscus, 223 ; $s .59$ hæmatopogon, $s .59$ lineatus, 222 ; $s .59$ quinquefasciatus, $s .59$ Timorensis, $s .59$ viridis, s. 59

Dragon, banded, $s .59$ black-pouched, $s .59$ brown, 223 flying, 222 great, 112 ; $s .28$ lined, $s .59$ short-pouched, $s .59$ Timor, s. 59 winged, 222

Dragonne, 111

Dragons, 98. 111. 217

Dryinus, 260 ; s. 93 æneus, 260 ; s. 93 auratus, $s .93$ nasutus, $s .93$ oxyrhinchus, s. 93 rostratus, s. 93

Dryophis, 260 ; $s .93$ fulgidus, s. 93 nasutus, $s .93$ panthoraria, s. 93 pavoninus, s. 93 prasina, $s .93$ Russellii, s. 93 xanthozonica, s. 93

Dryophyllax Nattereri, s. 94

Duberia, 260 arctiventris, $s .89$

Echidna, 270 ; s. 81 arietans, $s .81$ cærulescens, s. 97

Echis, 255. 278 ; $s .82$ arenicola, s. 82 krait, $s .82$ carinata, $s .82$

Ephimotes, 136

Eft, common, s. 106 web-footed, $s .106$

El adda, 156

Elaphoïdes fusca, $s .84$
Elaps, ix. 275. 384 ; s. 83

anguiformis, 276 ; $s .83$

bilineatus, s. 94

bivirgata, s. 84

Boie, $s .84$

Duberria, s. 89

chalybea, s. 84

coccineus, s. 84

collaris, $s .84$

corallinus, s. 84

flavius, $s .84$

furcatus, s. 84

fuscus, $s .83$

ibaboca, s. 84

lacteus, $s .83$

Latonius, $s .84$

lemniscatus, 275. 384 ; s. 83

lutonia, $s .89$

melanocephalus, $s .90$

micrurus, $s .84$

octolineatus, $s .91$

plicatilis, s. 96

Surinamensis, 276 ; s. 83

triscalis, s. 90

Empereur, s. 96

Emyda, s. 19

punctata, s. 19

Emydidæ, 79

Emydosauri, s. 21

Emydosauria, 100

Emys, 11. 71 ; s. 6.8

Adansonii, $12 ; s .15$

Amazonica, s. 17

Amboinensis, s. 7

annulifera, s. 12

barbatula, $s .17$

Batagur, s. 9

Bellii, s. 12

bipunctata, s. 10

bitentaculata, s. 17

canaliculata, $12 ; s .16$

Carolinæ, $s .7$

carunculata, 12 ; s. 16

Caspia, s. 9

castanea, $s .14$

crassicollis, $s .8$

Cayennensis, s. 17

centrata, 12

cinerea, 12

concentrica, 12 ; s. 11

concinna, 12

contracta, 12

constricta, s. 17

couro, s. 7

crassicollis, s. 8

decussata, 76 ; $s .11$

depressa, 12

Dhor, $s .8$

discolor, s. 16 
Emys dorsata, ix. 12; s. 10 erythrocephala, $s .17$ Europea, 71 ; s. 7 expansa, $12 ; s .17$ fusca, 12

Geoffroyana, s. 16 geographica, 75 ; s. 12 gibba, s. 17

guttata, s. 10

Hamiltonii, $s .9$

Kinosternoides, s. 12

icterocephala, $s .17$

insculpta, 12

leprosa, 12

Lesueurii, s. 12

lineata, $s .9$

longicollis, 12.76

lutaria, 12. 76

lutescens, 12

Macquarii, $12 ; s .15$

marmorea, s. 10

Maximiliana, s. 17

Muhlenbergii, s. 10

nasuta, 12

occilifera, s. 9

occipitalis, 75

odorata, 12 ; s. 13

olivacea, s. 15

ornata, $76 ; s .12$

Pennsylvanica, s. 13

picta, 73 ; s. 10

planiceps, 12

platicephala, 12

pseudogeographica, 12

pulchella, 12

punctata, 12

punctularia, s. 10

radiolata, s. 16

reticulata, 12 ; s. 11

rubriventris, 12

rugosa, s. 12

scabra, 12; s. 10

scripta, 12; s. 11

scorpoïdes, s. 13

Senegalensis, 12

serpentina, $s .14$

serrata, 12. $76 ; s .11$

speciosa, s. 10

Spengleri, $s .8$

spinosa, s. 8

stenops, s. 16

subrufa, 12

tecta, s. 9

thuryi, s. 8

tracaxa, s. 1'

trifasciata, s. 7

tritentacula, 12

triporcata, s. 13

viridis, s. 16

\section{Emys vittata, ix. s. 11 vulgaris, $s .9$}

Enaliosauri, s. 23

Euchydus piscator, $s .86$

Engyrus, s. 97

Engystoma, 396. 403 ; s. 104 dorsatum, 403 ; s. 104 granosum, 403 ; s. 104 marmoratum, 403 ; s. 104 ovale, 403 ; s. 104 ovalis,

Surinamensis, 403 ventricosum, 403 ; s. 104

Enhydres, 264

Ephimotes, s. 41

Epicrates, s. 96 cenchria, s. $\mathbf{9 6}$

Epicrium, s. 110 Hasseltii, s. 110

Erpetodryas, s. 89 exoletus, $s .89$ lævicollis, s. 89

Erpeton tentacule, 256

Erpetons, 256

Erythrolampus, $s .89$ formosus, s. 89

Erix, 255 ; s. 98

Eryx anguiformis, s. 98 Braminus, 248 ; s. $76^{\circ}$ jaculus, $s .98$ Indica, $s .98$ melanostictus, s. 75 Meleagris, s. 76 rufus, $s .75$ Turcica, s. 98

Etrobenae, 166

Eublepharis, s. 49 Hardwickii, s. 49

Eubydris rhynchops, s. 95

Eumerodes, 165. 220

Eunectes, s. 96 lateristrigota, s. 96 murina, s. 96

Exypuestes, $s .29$

Famo-cantrata, 149

Father of the leprosy, $\$ 48$

Frog, Argus, s. 100 bell, 446 blue and yellow, 397 blue tree, $s .102$ bull, 394.445 common, 393. 443 ; s. 100 green, 392. 442 ; s. 100 grunting, s. 100 land, s. 103; paradoxical, 393 shad, 445 ; s. 100 
Frog spring, ix. s. 100 tinging, 397 tree, 396. 449 zebra, 397

Frogs, 390. 422

Galleywasp, 239

banded, s. 71

Bell's, s. 70

black-spotted, $s .69$

common, $s .69$

coppery, $s .70$

double streaked, $s .70$

doubled, s. 69

eyed, $s .68$

fine-scaled, $s .71$

five-lined, s. 69

four-lined, $s .71$

Lacepede's, $s .69$

large, s. 68

lateral, $s .70$

many-scaled, s. 68

red-headed, s. 70

ribbon, $s$. 68

seven-striped, $s .69$

thin-scaled, $s .71$

two coloured, $s .70$

Gariocephalus, s. 55

Gavial, common, s. 23

great, 200

little, 101. 201

of the Ganges, 101

Gavialis, $s .23$

Gangeticus, s. 23

Gavials, 101. 200

Gecko, 144 ; $s .48$

aculeatus, $146 ; s .51$

Ægyptiacus, 144

ascolobates, $s .50$

fascicularis, 143. 146

guttata, $s .48$

inunguis, $142 ; s .47$

Leachianus, $s, 49$

levis, 147. 229

lobatus, 148

Mabuia, 146;s. 51

Madagascariensis, $s .49$

marginatus. 147

rapicauda, 148

Reevesii, $s .48$

squatydus, 148

spinicauda, 230

trihedrus, $s .51$

verrucosus, $s .50$

verruculatus, 140

vittatus, $s .49$

Gecko, American, s. 48

annulaire, s. 48
Gecko annulated, ix. $\delta .48$

à queue épineuse, 147

à tubercules trièdres, 147

banded, $144 ; s .49$

cepedian, 143

Chinese, $s .48$

common, s. 48

fasciculated, $s .48$

fringed, 149

Hardwicke's, $s .49$

Horsfield's, s. 49

house, 148. $23 \mathrm{l}$

Leach's, $s .49$

Mabuia, s. 51

Madagascar, s. 49

Peruvian, 149

porphyré, 150

sarroube, $s .50$

smooth, 147.229

spitting-banded, 150

of Surinam, 148

tuberculous, 119

wall, 143. 229

warty, s. 50

Geckos, 228. 140

Geckotians, 140

Geckotte, 119. 143

Geitje, 229

Gemmatophora, s. 59

barbata, s. 60

muricata, $s .60$

Gemmatophore bearded, s. 60 muricated, s. 60

Geosaurus, 140

Gerrhonote, blue, $s .64$

Burnet's, s. 64

Depp's, $s .64$

imbricate, $s .64$

retired, s. 64

rough-necked, s. 64

smooth-headed, $s .65$

Gerrhonotus Burnettii, $s .64$

cæruleus, $s .64$

Deppii, s. 64

leiocephalus, $s .65$

imbricatus, s. 64

rudicollis, s. 64

tæniatus, $s .64$

Gerrhosaurus, $s .63$

flavigularis, $s .63$

Goitreux, 114

Gonocephala, 128

Gonyodactylus, $s .51$ marmoratus, s. 51

Gonyosoma viride, $s_{\circ} 88$

Gongylophis, s. 97

Guana, allied, s. 38

armed, $s .38$

banded, s. 37 
Guana, Bell's, ix. s. 38

black, $s . \mathbf{3 7}$

clouded, $s .39$

common, s. 36

cycluroid, $s .3 \ell$

keeled, $s .39$

lancet-toothed, s. 38

rough-headed, $s .37$

round, $s .39$

smooth-necked, s. 37

spiny-tailed, s. 38

Gymnodactylus, $151 ; s .51$

geckoïdes, 151 ; $s .52$

Gymnotholamus, $s .71$ quadrilineatus, $s .71$

Gyrinus, $s .108$

Hæmacates, $s .83$

Hardun, 120

Haje, 274. 376

Hellbender, .110 large, $s .109$

Hemidactyle, margined, $s .51$

Hemidactylus, 146 ; $s .50$ granosus, $s .51$ marginatus, $s .51$

Hemiphractus Spixii, s. 101

Herpeton, $s .99$ tentaculatus, s. 99

Herpotodryas, s. $\mathbf{8 9}$

Heterodactyle Spix's, s. 66

Heterodactylus, $s .66$ imbricatus, 162 ; $s .66$

Heterodermata, 286

Heterodon, 258 ; s. 85 nasua, $s .85$ platyrhynchos, $s .85$ simus, s. 85

noirâtre, 258

tacheté, 258

Hognose, 258

Holoderma, s. 28 horridum, s. 28

Homalopsis, 260 ; s. 95 asperus, $s .96$ carinicaudatus, $s .95$ erythrogrammicus, $s .95$ Jara, $s .96$

moluroides, s. 96 obtusatus, 257 ; s. 95 plumbea, $s .96$ rhynchops, s. 95

Homalosoma arctiventris, $s .89$ punctatum, $s .89$

Homodermata, 286

Horatta, 278

Hurria, 258; s. 89 carinata, $s .92$
Hurria bilineata, ix. s. 89 nympha, $s .89$ pseudotriga, $s .95$

Hyalinus, $s$. 65

Hydochthon, s. 108

Hydras 279

Hydraspis, 12. 76

Adansonii. s. 15

Cayennensis, $s .17$

depressa, $s .16$

Dumerilliana, $s .17$

expansa, s. 17

lata, $s .17$

Macquarii, s. 15

pachyura, s. 17

planiceps, s. 16

radiolata, s. 16

rufipes, s. 16

subrufa, $s .15$

viridis, $s .16$

Hydrophis, 279, 280. $385 ; s .98$

chloris, 386

melanurus, 386

nigrocinctus, 386

obscurus, 386

Hydrus, 279. 280. 385 ; s. 98

atricapillus, $s .98$

bicolor, 280

Brugmansii, s. 98

cærulescens, $s .98$

carinatus, $s .99$

chloris, s. 98

cinereus, s. 95

colubrinus, 276 ; s. 84

curtus, $280 ; s .99$

cyanocinctus, $s .98$

doliatus, s. 98

fasciatus, $s .99$

gracilis, $s .98$

lævis, $s .98$

lanceolatus, s. 98

leiolepis, $s .98$

major, $280 ; s .98$

melanurus, $s .99$

nigrocinctus, $s .98$

obscurus, $s .98$

palustris, $s .86$

piscator, $s .86$

platura, $s .98$

rhynchops, $s .95$

schistosus, $s .98$

Shawii, $s .98$

spiralis, 280.386 ; $s .98$

striatus, $s .98$

Hyla, $396 ; s .101$

abbreviata, 398 ; s. 102

affinis, 397 ; $s .102$

albomarginata, 397 ; s. 102

arborea, s. 101 
Hyla aurantiaca, ix. s. 102 bicolor, $397 ; s .101$ bifasciata, s. 102 bilineata, 397 ; s. 101 Blochii, s. 101 boans, s. 101, 102 breviata, s. 102 Buffonia, 397 ; s. 102 bufonoïdes, $s .102$ centripoda, s. 102 cinerascens, 397; s. 102 cyanea, 398 ; s. 102 delitescens, $s .102$ erythropoda, s. 102 femoralis, 397 ; s. 102 frontalis, 397 ; s. 102 Gaimardii, s. 102 geographica, 397 ; s. 102 hypochondrialis, s. 102 Indica, $s .101$ intermixta, s. 102 lactea, s. 102 lateralis, 397.449 ; s. 102

Leschenaultii, s. 101 leucophyllata, s. 102 marmorata, 397; s. 101 oculata, 397 ocularis, $s .102$ palmata, 397 ; s. 101 papillaris, 397 ; s. 102 pardalis, 397 ; s. 102

Peronii, $s .101$ pulchra, s. 101 Quoyii, s. 102 ranæformis, $s .101$ rubeola, $s .102$ ruber, $s .102$ scelaton, s. 102 Scychellerii, s. 101 Seurii, s. 102 squirella, 398 ; s. 102 Surinamensis, s. 102 tibiatrix, 450 ; s. 102 tibiceus, 397 tinctoria, 450 ; s. 101 trivittata, 398 ; s. 102 variegata, s. 102 venulosa, 397 ; s. 101 vermicularia, s. 101 verrucosa, 397 ; s. 102 violacepoda, s. 102 viridifusca, $s .101$ viridis, s. 102

Hylæ, 448

Hypochton, 412

Ichthyophis, $s .110$

Ichthyosaurus, $164 ; s .23$

Cuv. Index.
Ichthyosaurus communis, ix. $s .23$ grandipes, s. 24 intermedius, $s .24$ latifrons, $s .24$ platydon, $s .24$ tenuirostris, $s .24$

Iguana, 131. 223 acanthura, s. 38 armata, $s .38$ atra, $s .37$ Belli, s. 38 cærulea, 225 ; s. 36 carinata, $s .39$ cornuta, 133 ; s. 36 cristata, s. $\mathbf{3 7}$ cyclura, 133 ; s. 39 cycluroïdes, $s .37$ delicatissima, 133 ; s. 37 emarginata, s. 36 fasciata, 225 ; s. 37 gutturosa, $s .56$ lanceolata, s. 38 lophyroïdes, $s .36$ nubila, $s .39$ nudicollis, $133 ; s .37$ similis, $s .38$ squamosa, s. 36 teres, $s .39$ tuberculata, $131 ; s .36$ viridis, $s .36$

Iguana, banded, 129 common American, 131. 223 horned, 133, 225

mud, s. 108 slate coloured, 432. 225 with armed-tail, 133 with armed-neck, 133

Iguane goitreux, 139

Iguanians, 117. 140. 213

Iguanodon, 140

Ilysia, $250 ; s .75$

rufa, $s .75$

Istiurus, 130

Jacaratinga moschifer, 107

Jacaré, 199

Jurura, 68

Kalo-shouter-sun, 386

Katla tutta, s. 92

Kentropyx, $s .31$ calcaratus, $s .31$ intermedius, $s .31$ striatus, s. 31

Keril patee, 386

Kinixys, 11. 69 ; s. 6 Belliana, s. 6 
Kinixys castanea, ix. $6 ? ; s, 6$ erosa, $s .6$

homeana, $s .6$

Kinosternon, 13. 77. 79; s. 13

Amboinense, 79

brevicaudatum, 79

longicaudatum, 79 ; s. 13

nigricans, 79

odoratum, s. 13

Pennsylvanicum, 79 ; s. 13

scorpoïdes, $s .13$

Shawianus, 79 ; s. 13

triporcatum, s. 13

Kinosternon musky, s. 13

Pennsylvanian, s. 13

three-keeled, s. 13

Koscordylos, 120

three-ridged, s. 13

\section{L'A tropos, 270}

La Bossue, s. 101

La Brazilienne, 270

La Daboré, 270

L'Elégant, 254

La Rouge, s. 102

La Vipère élégante, 270

Lacerta, $s .31$

acanthura, $s .38$

Africana, $153 ; s .53$

agilis, 116. 208. 211

alegyra, 117 ; s. 35

Amboinensis, 131

Ameiva, 114. 205; s. 29

anguina, 160 ; s. 73

apoda, 242 ; s. 65

arenicola, 116

Argus, $116 ; s .26$

arguta, $s .34$

aurita, s. 58

azurea, $s .42$

Basiliscus, 134 ; s. 45

bicarinata, 112. 204 ; s. 29

bilineata, 116 ; s. 32

bimaculata, 138 ; s. 46

bipes, 161 ; $s .74$

bivittata, 110

Boscii, s. 34

bosquien, 116

bullaris, 138, 139. 227

calotes, 127 ; s. 55

Capensis, 109; s. 27

caudiverbera, $149 ; s .50$

caudavolvula, s. 59

chalcides, 159

chameleon, 154

cordylus, 119 ; s. 63

crocodilus, 102

cruenta, s, 34
Lacerta Cuvieri, ix. s. 35

cyprius 157

dracæna, $111 ; s .26$

Dugesii, s. 33

Dumerillii, s. 33

Edwardsiana, s. 33

exanthematica, 111 ; s. 26

facetanus, 143. 229

fasciata, s. 43.69

fusca, $s .32$

Gangetica, 101

gecko, 148. 231 ; s. 48

geitje, 229

grammica, $s .35$

guttata, 129 ; s. 59

guttulata, s. 34

gutturosa, s. 30

Hispanicus, $s .35$

homalocephala, $145 ; s .49$

hyacinthina, $s .43$

iguana, 13I. 223

Jamaicensis, $s .32$

Javanica, s. 60

Knoxii, $s .34$

Lalandii, s. 32

lemniscata, 114; s. 30

lepida, $s .32$

Leschenaultii, s. 35

litterata, 205

lineata, $s .71$

lobata, s. 58

lophura, s. 60

lumbricoïdes, 163 ; s. 66

marmorata, 135 ; s. 47

monitor, s. 27

monodactyla, 160 ; s. 73

montanus, s. 35

muralis, s. 32

muricata, 123 ; s. 60

mystacea, s. 58

nebulosa, 217 ; s. 39

Nilotica, 109 ; s. 27

occidua, 157. 239 ; s. 69

ocellata, 116. 208 ; s. 32

Olivieri, s. 33

orbicularis, 124. 216

pardalis, s. 34

pipiens, $s .52$

platura, 151 ; s. 52

plica, $s .41$

pumila, 154

quadrilineata, s. 71

quinquelineata, $115 ; s .69$

rapicauda, 147

rhombica, s. 32

rubropunctata, s. 34

salamandra, 406; s. 105

sanguinolenta, $s, 58$

Savignii, s. 33 
Lacerta Schreibersiana, ix. s. 32 scincoïdes, $158 ; s .67$ scincus, 110.156 scutata, 128 ; s. 54 scutellata, $s .33$ sepium, 116 seps, $162: s .63$ sericea, 116 serpens, 159 ; s. 72 sputator, $230 ; s .52$ stellio, 120. $214 ; s .57$ stirpium, 210 strenuosa, 139 striata, $s .31$ strumosa, s. 46 superciliosa, 134 tæniolata, $s .68$ Teguixin, 113 ; s. 29 terrestris, 116 tetradactyla, $162 ; s .65$ Teyou, $s .31$ tiliguerta, 211 tuberculata, 224 Turcica, s. 50 Uralensis, $12.9 ; s .59$ varia, $110 ; s .25$ variabilis, $s .34$ velox, $116 ; s .34$ viridis, 116. 209; $s .32$ viridula, 211 vittata, 144 vivipara, $s .32$

Lacertians, 107. 201

Lachesis, $s .79$ mutus, $s .79$ rhombeata, 269 ; s. 79

Lagarto, 105

Langaha, 278

Le geleote, $s .56$

Lecolepis, s. 62 guttatolineatus, s. 62 guttatus, $s .62$ maculatus, s. 62

Leiocephalus, s. 42 carinatus, $s .42$

Leiocephalus, keeled, s. 42

Leiolepis, 125 guttatus, 125

Leioseloma obscura, s. 98

Lepidosoma, $s .71$

Leposoma, 126

Leposternons, 247

Leposternon, s. 67 microcephalus, 247 ; s. 67 oxyrhynchus, $s .67$

Leposternon dotted, s. 67 sharp-nosed, s. 67

Leptophis, s. 93 mancas, s. 94
Leyoselasme, ix. 280

Liophis, s. 89

Fosteri, s. 90

miliaris, s. 90

pœcilogyrus, $s .89$

reginæ, $s .90$

Lizard, Bosc's, $s .34$

Cape, $s .32$

clouded, $s .39$

common toad, $s .45$

Douglas' toad, s. 44

Duges', s. 33

Dumeril's, $s .33$

Edwards', s. 33

eyed, $s .32$

frilled, $s .60$

gentil, 116

of Gibraltar, 210

grammic, $s .35$

great green, 116. 208

green, s. 32

horned toad, s. 45

of Jamaica, 201

Indian, $s .35$

Knox's, s. 34

mountain, $s .35$

nimble, 211

Olivier's, $s .33$

orbicular, $s .45$

orbicular-toad, s. 45

of the Pandang, 144

panther, $s .34$

red-spotted, $s .34$

Savigny's, s. 33

scaly, 211

Schreibers', s. 32

scutated, s. 54

serpent, 160

shielded, $s .33$

Spanish, $s .35$

spotted, s. 34

spotted green, 209

striped, 115

Ural toad, s. 59

variable, $s .34$

wall, $s .32$

Lizardet, 112. 204

Lizards, 5. 98. 115. 206

Lophura, $130 ; s .60$

Amboinensis, s. 60

concinna, s. 61

Cuvieri, s. 60

Lesueuri, s. 60

Lophura, beautiful, $s .61$

Lophyrus, 127

agamoïdes, 217 ; $s .41$

albomaxillaris, $127 ; s .40$

auronitens, 127

gigas, $s .55$

U 2 
Lophyrus Kuhlii, ix. s. 55

Margaritaceus, 127 ; $s .40$

ochrocollaris, 127 ; s. 40

panthera, 127 ; s. 40

rhombifer, 127 ; s. 40

xiphurus, $134 ; s .40$

Lophyrus with forked-casque, 128

Loricata, 100. 164

Luchensis, 269

Lycodon, $s .91$

aulicus, $s .92$

capucinus, $s .92$

fuliginosus, s. 92

Galathea, s. 92

Hebe, $s .92$

leucocephalus, $s .92$

macrorhinus, $s .92$

malignus, $s, 92$

pethola, s. 92

Russellii, s. 92

subcinctus, s. 92

unicolor, s. 92

Lygosoma, 159; s. 71

aurata, $s .72$

punctata, $s .71$

scincoïdes, s. 72

serpens, $s .72$

Lygosoma, dotted, s. 71

golden, $s .72$

short-footed, s. 71

Lyriocephala, 128; $s .54$

Margaritaceus, 128

Maboina, 156. 158

Mabouia, 239

des Bananiers, 147

des Murailles, 146

Mabuia, 150

Macrosoma elegans, s. 93

Malpolon, 260

Marble, common, s. 47

Mastigura, s. 61

Matamata, 17

Brazilian, s. 17

Megæra, s. 79

Megalophrys montana, s. 101

Megalosaurus, 140

Menobranchus, 412 ; s. 108

Lacepedii, s. 108

lateralis, 412 ; $s .108$

Menobranchus Lacépède's, $s .108$ Say's, s. 108

Menopoma, 410. 475

Alleghaniensis, s. 109

Metaxa, s. 87

Microps unicolor, s. 104

Micruri, 276

Micrurus Spixi, s. 84
Mokeson, ix. 258

Molge, s. 106

gigantea, $s, 109$

glutinosa, s. 106

tridactylus, $s .105$

Monitor, s. 25

albogularis, 109 ; s. 28

Bengalensis, 111 ; s. 26

bivittatus, $s .25$

chlorostigma, s. 26

crocodilurus, 112. 203

exanthematica, $s .26$

flavescens, $s .25$

heraldicus, s. 27

marmoratus, 111

nebulosus, $s .27$

nigricans, 111

Niloticus, s. 27

ocellatus, $s .25$

ornatus, 109 ; $s .27$

pulcher, 202; s. 27

scincus, $s .27$

Timorensis, $s .26$

varius, $s .25$

viridis, $s .26$

Monitor, brown spotted, s. 26

clouded, s. 27

of Congo, 202

double-banded, 110

of Egypt, 110

elegant, $s .26$

eyed, s. 25

green, $s .26$

green spotted, s. 26

heraldic, $s .27$

laced, $s .25$

land, 202

of the Nile, 109. 202; s. 27

ornamented, $s .27$

Timor, $s .26$

two-banded, $s .25$

varied, s. 27

yellowish, s. 25

Monitors, 108. 201

Monodactyle, Anguine, s. 73

Monodactylus, s. 73

Monoxillo, horrid, s. 28

Mosasaurus, 140

Muræna, s. 108

Mutabilia, s. 99

Naia, 273; s. 82

hæmachates, s. 83

haje, s. 83

latratus, $s .83$

nivea, $s .83$

rhombeata, s. 83

spectatrix, s. 83 
Naia tricuspidans, ix. $s .83$ tripudians, 274

Natrix ahætula, $s .93$ alamensis, $s .90$ aspera, $s .96$ aulica, $s .92$ bicarinatus, $s .89$ bivittatus, $s .86$ cherscoïdes, $s .86$ chiametla, $s .90$ cinnamomea, $s .89$ exoleta, $s .89$ Forsteri, $s .90$ lappiata, s. 92 occipitalis, $s .90$ ocellata, $s .86$ punctatissima, $s .94$ sculptura, $s .86$ scurrula, s. 87 semilinea, s. 90

Necturus, s. 108

Oligdonon, 260

Oligodon, $s .88$ atriventus, $s .88$ bitorquatus, $s .88$ punctatus, s. 89

Ophidia, 5

Ophidians, 5. 241

Ophidii, $s .78$

Ophiodes Gronovii, s. 73

Ophiorhyza mungos, 274

Ophiosauri, s. 62

Ophiosaurus, 243

Ophiosaurus, $s .65$

punctatus, 243 ; s. 65 striatulus, 243 ; $s .65$ ventralis, $243 ; s .65$

Ophis, 273 ; s. 83.88 Merremii, s. 83 subcinctus, $s .92$

Ophoïdes, s. 73

Ophrias, 277; s. 82 acanthopis, $s .82$

Ophryessa, 134; $s .39$

Ophyessa, $s .39$ azurea, $s .42$ Braziliensis, s. 40 brevicaudatus, s. 42 Margaritaceus, s. 40 picta, $s, 40$ plica, s. 41 rhombifer, s. 40 superciliosa, $s .40$

Ophyessa Brazilian, s. 40 lozenge, s. $\mathbf{4 0}$ painted, $s .40$ pearly, s. 40 sword-tailed, s. 40
Ophyessa umber, ix. $s .41$

Oplocephali, 277

Cuvieri, $s .84$

Oplurus, 136 ; $s .41$ torquatus, $137 ; s .41$

Otilopha, s. 103 ocellata, s. 104 typhonia, $s .103$

Otilophes, 403

Ouaran, 109. 202 el hard, 110

Oular caron, 264 limpé, 281

Ouran grey, s. 27 white-throated, $s .28$

Oxybelis, $s .93$ æneus, $s .93$

Oxyrhopus annulatus, $s .90$ pethola, s. 91

Oxyrhynchus, 402 ; $s .103$

Padani cootoo, s. 97

Paludicola albifrons, s. 104

Pareas, s. 94

Passericki, s. 93

Passerita, 260 ; s. 93

Pedda-poda, 257 ; s. 97

Pelamides, 279. 280

Pelamis, s. 98

bicolor, s. 98

carinatus, $s .99$

curtus, s. 99

fasciatus, $s .99$

gracilis, $s, 98$

Lindsayi, $s .99$

melanurus, $s .99$

ornatus, $s .98$

Rupelii, s. 99

Valakadin, s. 99

Pelias, 272; $s .82$

berus, 272 ; $s .82$

chersea, $s .82$

niger, s. 79. 81

Pelobates fuscus, $s .103$

Pareas carinata, s. 94

Periops hippocrepis, $s .87$

Phanerobranchus, $s .108$

Phelsuma, 143 ; s. 47

Philodryas Olfersii, $s .87$

Phrynocephale, eared, s. 58 eyed, $s .59$

Pallas's, s. 59

Phrynocephalus, 129 ; $s .58$ auritus, $s .58$

caudevolvula, $s .59$

ocellatus, $s .59$

Uralensis, $s .59$

Phrynosoma, s, 44 
Phrynosoma Buffonium, ix. s. 45 cornuta, $s .45$ Douglassii, $s .44$ orbiculare, s. 45

Phyllodactylus, s. 50 marmoratus, $s .49$ pulchellus, $s .50$

Phyllomedusa, $s .101$ Phyllhydrus, s. 108 pisciformis, $s .108$

Phyllurus, 151 ; s. 52 Cuvieri, s. 52 Nilii, $s .52$

Physalamis Cuvieri, s. 104 Physignathus, 130 Cocin Cinus, 130 concinnus, s. 61 iguanoides, $s .61$

Pipa, 403, 463; s. 105 Bufonia, 396 ; s. 104 curururu, 404 ; s. 105 lævis, 396 ; s. 104

Pipa, common, s. 105 of Surinam, 463

Plica, s. 40

Platuri, 276

Platurus, $s .84$

fasciatus, $s .84$ laticaudatus, $s .84$ semifasciatus, $s .84$

Platydactyle cepedian, $s .47$ clawless, $s .47$ eyed, $s .47$ ornamented, s. 48

Platydactylus, 142;s. 47 Egyptiacus, s. 48 Americanus, s. 48 cepedianus, $s .47$ fascicularis, s. 48 ocellatus, $s .47$ ornatus, s. 48

Pleurodeles, $s .106$

Plesiosaurus, 164 ; s. 24 auxois, $s .24$ carinatus, $s .24$ dolichodeirus, s. 24 pentagonus, $s .24$ recentior, s. 24 trigonus, $s .24$

Polychrus, 135. 227; s. 47 acutirostris, 136 ; s. 47 fasciatus, $s .47$ of Guiana, 135 marmoratus, s. 47 strigiventer, $s .47$ virescens, $s .47$

Porte anneau, 254

Pneustes, 129

Pneustoides, 129
Proteus, ix. 412. 475; s. 107 anguinus, $412.476 ; s .108$ tetradactylus, $s .108$ tritonius, 408

Proteus, common, s. 108 of the lakes, $s .108$

Protonopsis, 410 horrida, s. 109

Psammodromus, $s .35$ Hispanicus, $s .35$

Psammosaurus, $110 ;$ s. 27 albogularis, s. 28 scincus, $s .27$

Psammophis, 260 ;

Psamnophis, s. 93 bicolor, s. 94 Girondicus, $s .93$ lacertina, s. 94 pulverulenta, $s .94$ rhombeata, s. 94 schokari, s. 94

Pseudo Ameiva, 115 striata, $s .31$

Pseudo-Boa, 255. 279. 385 carinata, 278 coronata, s. 92 krait, $278 ; s .82$

Pseudobranchus, 414 ; s. 109 striatus, $s .109$

Pseudoelaps, 260

Pseudopus, $242 ; s .65$ Olfersii, $s .73$ Pallasii, 242 ; s. 65 serpentinus, $s .65$

Pseudopus, Durville's, $s .65$

Pteropleura, s. 49 Horsfieldii, 145 ; s. 49

Ptychozoon, 145 ; s. 49

Ptygopleura, s. 62

Ptyodactylus, 148 ; s. 50 guttatus, $s .50$

Pygodactylus, 161 ; s. 74 Gronovii, s. 73

Pygopus, $160 ; s .73$ cariococca, $161 ; s .73$ lepidopus, $s .73$ striatus, $161 ; s .73$

Python, $330 ; s .97$ amethystinus, $330 ; s .97$ bivittatus, s. 97 bora, 330 Javanicus, $s_{.} 97$ Peronii, s. 97 poda, s. 97 punctatus, s. 97 rhynchops, s. 95 Schneideri, $s .97$ tigris, $s .97$

Python amethyste, 257 
Python tigre, ix. 257

Pythons, 256

Pyxis, 11.69 ; s. 6 aranoïdes, $s .6$

Quetzpalco, 121. 136

Rainette bleu, 398

à tapiner, $s .101$

Rana 390. 422; s. 99

arborea, 396. 449 ; s. 101.102

Austrasi, s. 102

bilineata, $s .102$

boans, s. 101

bombina, 399. 461 ; s. 103. 104

bufo, 398. 460 ; s. 102

bufo calamita, 399

Bufonia, s. 104

cærulea, s. 102

Catesbiana, s. 100

clamitans, 394. 445 ; s. 100

coriacea, 395 ; s. 100

cornuta, 395

cultripes, s. 100

cutricularis, s. 100

cyanophlyctis, s. 100

Daudini, s. 100

dorsalis, s. 100

dubia, s. 103

esculenta, 392. $442 ; s .100$

fætidissima, $s .103$

fasciata, $s .104$

flaviventris, s. 100

fontinalis, $s .100$

gibbosa, $s .101$

gigas, 395 ; s. 100

grunniens, 446 ; s. 100

gryllus, s. $\mathbf{J} 00$

halecina, 394. 447 ; s. 100

hypscaphelia, $s .100$

labyrinthica, 395 ; $s .100$

lactea, s. 102

maculata, 395 ; $s .100$

margaritifera, 403; s. 104

marina, 401 ; s. 103

maxima, 397 ; s. 100.101

megastoma, 395 ; $s .101$

melanota, s. 100

mephitica, s. 103

meriana, s. 101

miliaris, 395 ; s. 100

mugiens, $s .100$

musica, s. 103

mystacea, 395 ; s. 100

nigrita, s. 100

obstetricans, $s .103$

ocellata, 394. 447 ; s. 100
Rana ovalis, ix. s. 104

pachypus, 395 ; s. 100

palmipes, 394 ; s. 100

palustris, $s .100$

paradoxa, 393. 447 ; s. 100

pardalis, s. 100

pentadactyla, $s .100$

pipa, 404 ; $s .105$

pipiens, 394.445 ; s. 100

plicata, s. 100

protentosa, s. 103

pumilla, s. 100

punctata, 395. 444 ; s. 100

pygmæa, 395 ; s. 100

ridibunda, $462 ; s .100$

rubella, $395 ; s .100$

scapularis, $s .100$

scutata, 395 ; s. 101

sibilatrix, 395 ; s. 100

sitibunda, $s .103$

sonans, s. 100

squamigera, s. 101

Surinamensis, s. 104

sylvatica, s. 100

systoma, $s .104$

temporaria, 393.443 ; s. 100

tigrina, 394 ; $s .100$

tinctoria, 397 ; s. 101

typhonia, $395 ; s .100 .103$

variabilis, $400 ; s .103$

variegata, s. 104

venulosa, s. 101

vespertina, $s .100$

Virginica, 394, 395 ; s. 100

viridis, s. 103

zebra, $s .101$

Rattlesnake, 266

banded, 345

miliary, 348

striped, 348

Reptilia, 1

Rhinella, s. 103

acutirostris, s. 103

granulosus, s. 103

nasicus, $s .103$

proboscideus, s. 103

semilineata, s. 103

Rhinophis, $s .77$

Rhinopirus, 256

Rhinostoma nasua, s. 85

proboscideum, s. 85.94

Rondas tataloopam, 248

Safeguard, great American, 113. 204 variegated, $s .29$

Safeguards, 112. 204

Saiphos, $s \cdot 72$ equalis, s. 72 
Saiphos, lacertine, ix. s. 72

Sal à trois doigts, 406

Salamander, 464

Alpine, s. 105

ashy, s. 106

banded, $s .106$

Beechey's, s. 107

black, s. 105.107

brown, s. 107

brownish, s. 107

common, 406. 465 ; s. 105

crested, 408

cylindrical, s. 107

dorsal, s. 107

great, 410

Green's, s. 107

glutinous, s. 106

long-tailed, s. 107

marbled, 408 ; s. 105

painted, s. 107

palmipedes, 409

punctated, 409 ; s. 107

red, s. 107

red-backed, s. 106

Spanish, s. 106

spotted, s. 107

tigrine, s. 107

two lined, s. 107

variolated, s. 107

violet, $s .106$

white-faced, $s .107$

with spotted-sides, 408

yellow, s. 107

Salamanders, 404

aquatic, 407.473

land, 405

Salamandra, 404, 405 ; s. 105

Alleghaniensis, s. 108.109

Alpestris, 408 ; s. 106

atra, 406 ; s. 105

Beechii, s. 107

bilineata, 407 ; s. 107

cinerea, s. 106

cristata, 408

cylindrica, s. 107

dorsalis, $s .107$

erythronota, 407 ; s. 106

fasciata, $407 ; s .106$

fenestrata, $s .106$

flavissima, s. 107

frontalis, s. 107

fusca, s. 105. 107

gigantea, 410 ; $s .109$

glutinosa, s. 106

Greenii, s. 107

horrida, s. 109

Japonica, 407 ; s. 106

longicaudata, s. 107

maculata, s. 107
Salamandra maculosa, ix. s. 105

major, s. 106

marmorata, 408. 474 ; s. 105

nigra, s. 107

palmata, 409 ; s. 106

palustris, s. 105

perspicillata, 406

picta, s. 107

platydactyla, s. 107

punctata, 409 ; s. 106.107

rubra, 407; s. 107

rubriventris, $s .107$

savi, 406

sinciput alba, s. 107

subfusca, s. 107

subviolacea, 407 ; s. 106

symmetrica, $409 ; s .107$

tigrina, $407 ; s .107$

variegata, $s .107$

variolata, 407 ; s. 107

venenosa, 407 ; s. 106

vulgaris, $s .105$

Salamandrina, s. 105

perspicillata, s. 105

tridactyla, s. 105

Salem maculosa, 406

Sarruba, 149

Sauri, s. 24

Sauria, 5.97

Saurians, 5

Saurocephalus, $s .24$

lanciformis, $s .24$

leanus, $s .24$

Saurodon, s. 24

Saurophis, 162 ; s. 65

Lacepedii, $s .65$

New Holland, s. 65

Scargus Valeno, s. 106

Scinc, shop, s. 67

à fleurs noirs, 158

Scincoïdians, 155

Scincus, 155 ; s. 67

bicolor, 157

bistriatus, 158

brachypus, $s .71$

crotophomelas, 158

cyanurus, s. 71

cyprinus, 157

decemlineatus, s. 68

erythrocephalus, 157 ; s. 70

fossar, s. 69

lateralis, s. 70

lineatus, s. 68

Mabouya, s. 69

multicarinatus, $s .68$

multiseriatus, 157

nigrofasciatus, s. 68

nigroluteus, $158 ; s, 68$

ocellatus, 158 
Scincus octolineatus, ix. s. 68 officinalis, 156, $237 ; s .67$ pachyurus, $s .67$ rufescens, 157 ; s. 68 sepiformis, s. 63 tiliguyus, s. 69 tiliqua, 239 trivittatus, 157 ; s. 68 tuberculatus, $s .67$ unicolor, s. 70 undecim striatus, s. 68 variegatus, $158 ;$ s. 68 vittatus, $s .69$

Scelophorus, s. 42 ; s. 43 æneus, $s .44$ grammicus, s. 43 pleurostictus, s. 43 scalaris, $s .44$ spinosus, $s .43$

Scheltopusik, 242 Pallas's, s. 65

Scytale, 255. 278; s. 82. s. 92 bizonata, 278 ; s. 82 brachyorrhis, s. 92 catenata, s. 79 coronata, 255 ; s. 92 krait, 278 ; s. 82 niger, s. 81

Semiphora, $s .57$

Senembi, 132

Sepedon, 273 ; s. 83 hœmachates, s. 83 Merremii, s. 83 porphyraceus, $s .83$ rhombeata, $s .83$

Seps, 158 ; s. 72 equalis, $s: 72$ fragilis, s. 73 Peronii, 159 ; s. 72 ruber, 211 scincoïdes, 159 sexlineata, $161 ; s .74$ striata, $s .72$ tridactylus, 240 varians, 210 zyginis, s. 72

Seps, common, s. 72 four-toed, s. 72 Gronovien, 161 monodactyle, 161 Peron's, s. 72 striated, s. 72

Serpent, Asculapian, 263 of Congo, 475 spectacled, 274 venomous, $s .78$ yellow, 268. 349

Serpents, 5. 241. 307. s. 78. naked, 281

Cuv. Index.
Serpents, queen of, ix. 345 true, 245

Sheathclaw, beautiful, s. 50 Egyptian, s. 50 fimbriated, s. 50 porphyry, s. 50 smooth, $s .50$

Shoutur sun, 386

Sibons, 260

Siguana, s. 74 Ottonis, $s .74$

Siphonops, s. 110

Siredon, $s, 108$ axolotl, s. 108

Siren, 413. 478; s. 108 anguina, 412. 476; s. 108. intermedia, $414 ; s .108$. lacertina, 414. 478; s. 108 pisciformis, 411 ; s. 108 striata, 414;s. 109

Siren, common, s. 108 four-footed, s. 109 intermediate, 414 ; s. 108 lacertine, 414 striated, s. 109 striped, 414

Sitana, s. 57 Pondicherry, s. 57

Skink, common, 237

Skinks, 155. 237

Slow-worm, common, 306

Snake, black, 335 black coral, $s .75$ black eared coral, $s .75$ blind pintado, s. 76 Cape pintado, s. 76 common, 333 common Congo, s. 109 common coral, $s .75$ common glass, $s .65$ common rattle, s. 78 dottled coral, $s .75$ French, 334

Indian coral, s. 75 Javan, 330 lozenge spotted rattle, $s .78$ miliar rattle, s. 78 netted pintado, s. 76 rattle, 336 ; s. 78 ribbon, $s .86$ scytale coral, $s .75$ spectacled, 376 spotted coral, $s .75$ three-toed Congo, s. 109 wampum, s. 86

Snakes, $242 ; 243$ sea, s. 98 water, $s .98$

Sourancou, 268 
Spœnops capistrata, ix. s. 71 Sphæriodactylus, 150 ; s. 52 sputator, s. 52

Sphargis, 16 ; $s .20$

Spheriodactyle, banded, s. 52

Squamata, s. 24

Stellio, 118. 140. 143. 213; s. 57 argyropus, $s .51$

azureus, 120

brevicaudatus, 120

fimbriatus, 149

Hasselquistii, 141

Mauritanicus, 147

niger, s. 62

perfoliatus, 147

phyllurus, 151

platyurus, 147 ; s. 51

spinipes, 121.215; s. 61

vulgaris, $214 ; s .57$

Stellio, common, s. 57

gecko, 144

of the Levant, 120. 214

Stelliones, common, 119 bâtards, 12]

Steneosaurus, 102

Stenodactylus, 151 ; s. 49.51 guttatus, $15 \mathrm{l}$; $s .49$ pipiens, s. 52

Stenostoma, $\mathbf{2 4 7}$; s. 76 albifrons, s. 77

Stercus lacerti, 120

Sternotherina, $\mathbf{7 9}$

Sternotherus, 78. 80 ; s. 14

Boscii, 80

castaneus, s. 14

Leachianus, 79, 80

odoratus, 80 ; s. 13

trifasciatus, $80 ; s .7$

Stombus, 395 ; s. 104

Tachydermum horridum, $s .28$

Tachydrome, Chinese, $s .36$

Tachydromus, 117 ; s. 36 quadrilineata, s. 36 sexlineata, s. 36

Tadpole, 415, 416

Talectée, 200

Tapayaxin, 217

Tapayes, 216

Tar tutta, s. 92

Taraguira, 114

Tarente, 143

Tarentola, $143 ; s .48$

Tarentole, 119

Tayapaxin, 124

Tecoixin, s. 43

Tedo, 463
Teguixin, ix. s. 29

monitor, s. 29

Teius, 111 ; $s .28$

ameiva, 114

bicarinata, s. 29

cæruleocephalus, s. 30

calcaratus, s. 31

collaris, $s, 31$

crocodilus, $s .28$

cyaneus, 114

cyanomclas, $114 ; s .30$

intermedius, $s .31$

lateristriga, $s . \mathbf{3 0}$

lemniscatus, s. $\mathbf{3 0}$

litteratus, s. 29

monitor, s. 29

ocellifer, 114 ; s. 29

striatus, $s .31$

tessellatus, $s .30$

tritæniatus, $114 ; s .30$

viridis, $s .31$

vulgaris, $s .29$

Temapara, 113

Terrapene, 13. 69. 77. 80

bicolor, $s .7$

Carolinæ, 80 ; s. 7

clausa, 77

Europæa, 80

guttata, s. 7

nebulosa, $s .7$

triporcata, s. 13

Terrapin, American box, s. 7 banded, s. 11

batagur, s. 9

Bell's, s. 12

Caspian, s. 9

common, s. 9

concentric, s. 11

dhor, s. 8

dotted, s. 10

eastern box, $s .7$

Europæan box, s. 7

eye bearing, $s .9$

furrowed, $s .11$

Hamilton's, $s .9$

Lake Erie, s. 12

lettered, s. 11

lineated, $s .9$

marbled, s. 10

Muhlenberg's, s. 10

ornamented, s. 12

painted, s. 10

reticulated, s. 11

ring-bearing, s. 12

rough, $s .10$

rugose, $s .12$

serrated, s. 11

Thury's, s. 8

specious, s. 10 
Terrapin, Spengler's, ix. s. 8 spinous, $s .8$ spotted, s. 10 tented, $s .9$ thick-necked, $s .8$ three banded box, $s .7$ white spotted, s. 12

Terrentola, 143

Testudinata, $s .2$

Testudo, 8. 9 ; s. 2 actinodes, $68 ; s .4$ Amboinensis, $s .7$ angulata, 10 ; s. 5 aranoïdes, $s .6$ areolata, $10.67 ; s .5$ armata, $s .4$ atra, s. 20

Belliana, s. 6

Bellii, s. 5

biguttata, 10

bipunctata, $s .4$

Boscii, s. 13

brevicaudata, $s .7$

Brongniartii, 9; s. 18

Cafra, 10 ; s. 5

cagado, 10 ; s. 3 calcarata, s. 4

Testudo, Californica, s. 3 campanulata, s. 4 carbonaria, 10 ; s. $\mathbf{3}$ caretta, $16.92 ; s .20$ carinatus, s. 18

Carolina, 10 ; s. 3. 5.7 Caspia, s. 9

centrata, s. 11

ceppediana, s. 20

clausa, 13 ; s. 7

coriacea, 17.93 ; s. 20

coui, s. 4

denticulata, 10 ; s. 3.6 .

depressa, 10 ; $s .3 .16$

Dussumierii, s. 3

elegans, $s .4$

elephantopus, s. 3

erosa, s. 6

Europæa, 11

ferox, 19. 96 .

fimbriata, 17.95

flava, 71 ; s. 7

galeata, $s .15$

geometrica, 10. 64. s. 5

gigantea, s. 3

glutinosa, s. 13

Graji, s. 4

granosa, 19

Græca, 9; s. 4. 5

Hercules, 10 ; $s .3$

Hermanni, s. 5
Testudo, Homeana, ix. s. 6

imbricata, 15.89 ; s. 21

Indica, 10.63 ; $s .3 .15$

irregulata, $s .7$

Japonica, s. 20

juvencella, $s .5$

Leveriana, $s .7$

longicollis, s. 15

lutaria, 71 ; s. 7.9

luteola, s. 5

marginata, 10 ; s. 4

martinella, s. 16

matamata, s. 17

Meleagris, $s .7$

miniata, s. 5

multiscutata, s. 20

Mydas, 15. 57. 81 ; s. 20

nasicornis, s. 20

nigra, $10 ; s .3$

nigricans, s. 15

odorata, s. 13.

orbicularis, 11.71 ; s. 7

palustris, $s .11$

pardalis, 68 ; $s .4$

Pennsylvanica, 13 ; s. 13

picta, 12

planiceps, $s .16$

platycephala, s. 16

Polyphemus, s. 3

pulchella, s. 7

punctata, s. 7. 10

pusilla, $s .5$

radiata, 10,$65 ; s .4$

Rascht, s. 19

reticulata. $s .11$

retusa, s. $\mathbf{3}$

Retzii, s. 13

rugosa, s. 12

scabra, s. 15

Schweigeri, s. 3

scorpoïdes, $s .13$

sculpta, $10 ; s .3$

Senegalensis, $s .15$

serpentina, 13 ; s. 14

serrata, s. 8. 11. 14

signata, $10 ; s .5$

Spengleri, $s .8$

stellata, $10 ; s .4$

subnigra, 13 ; s. 14.15

subrufa, s. 13. 15

sulcata, s. 4

tabulata, 10. 65 ; $s .3$

teutoria, $\mathbf{6 8}$; $s .5$.

tricarinata, $13 ; s .8 .13$

triunguis, $18.95 ; s .18$

truncata, $s .3$

viridis, 15

zolhafa, s. 5

$\times 2$ 
Tête plate, ix. 149 rouge, 115

Tetradactylus, $162 ; s .65$

Chalcidices, s. 65

Decresiensis, 159

Tetraonyx, s. 19

Cuvieri, s. 19

Teuhtlacot zauhqui, 345

Teguguazu, 113

Thammodactylus punctissimus, $s .94$

Thecadactylus, $147 ; s .49,50$

fimbriatus, $s .50$

lævis, $s .50$

lobatus, $s .50$

poli, $146 ; s .51$

porphyreus, $s .50$

Theleosaurus, 102

Tiliguerta, 116. 211

Tiliqua, $157 ; s .67$

ænea, s. 70

Bellii, s. 70

bicolor, s. 70

bistriata, s. 69. 70

Capensis, s. 68

carinatus, $s .68$

crotaphomelas, $s .68$

Cyprinas, $s .68$

erythrocephala, $s .70$

fasciatus, $s .71$

homolocephala, s. 69

lateralis, $s$. 70

microlepis, $s$. 71

multiscutata, s. 68

nigrolutea, $s .68$

nigropunctata, $s .69$

occidua, s. 69

ocellata, s. 68

quinque lineata, $s .69$

Sloanii, s. 70

tæniolata, s. 68

tenuis, $s .70$

trivittata, s. 68

Whitii, s. 67

Tiliqua, black and yellow, $s .68$

Cape, $s .68$

Indian, $s .68$

New Holland, s. 67, 68

three streaked, $s .68$

Tisiphone, 263; $s .79$

cupœa, s. 79

Shausii, $s .79$

Catesbii, $s .79$

Toad, brick coloured, s. 103

brown, 399. 461

common, 399. $460 ; s .102$

marine, 401

obstetric, 400

pearled, 403

reed, 399
Toad, variable, ix. 400 yellow bellied, 402

Toads, 398. 451

Tomacolin, 210

Torquatrix, 250;s. 75

Tortoise, 8

alligator, 80

angular, s. 5

areolated, 67 ; s. 5

Bell's, $s .6$

bell-shaped, $s, 4$

box, 13.77

box, of Amboina, 13

cinereous, 75 .

common, 9

European fresh-water, 71

fierce, 19. 96

fimbriated, 17

geometrical, 10.64;s. 5

Gopher, s. 3

Greek, 57 ; s. 5

Hercules, $s .3$

Hicatée, 67

Home's, s. 6

Indian, $10.63 ; s .3$

Lake Erie, 75

leopard, s. 4

long necked, 76

marked, s. 5

mud, $76 ; s .9$

painted, 12. 73

radiated, $10.65 ; s .4$

radiated box, $s .6$

Schweiger, s. 3

serrated alligator, s. 14

snake, 13

soft, 18. 95

soft of the Nile, 18

speckled, 11. 71

starred, s. 4

sulcated, s. 4

tabular, $65 ; s .3$

terrestrial, 57

worn, $s .6$

Tortoises, 4 ; s. 2

fresh-water, 11. 71

land, 9

sea, 14

Tortrix, $250 ; s .74$

corallinus, 327

latta, 251 ; s. 75

punctata, $251 ; s .75$

rufa, $251 ; s .75$

Russellii, 248 ; s. 76

Trachydosaurus, $s .67$

rugosa, s. 67

Tragops nasutus, $s .93$

Trapelus, $125 ; s .57$

ÆEgyptius, 125 ; s. 58 
Trapelus mutabilis, ix. s. 58

hispidus, s. 57

Savignii, s. 58

subhispidus, $s .57$

'Trapelus, of Egypt, 125

Tridactylus Decresiensis, 159 ; s. 72

Trigonocephalus, $267.349 ; s .79 .80$

Blomhoffi, s. 80

Boeii, s. 80

cacodæmon, s. 80

halys, $s .80$

hypnale, $s .80$

lanceolatus, 268. 349

Megæra, 349

orophyas, $s .80$

rhodostoma, s. 80

Trigonocephalus, lozenged, 269 yellow, 268

Trimeresure petite tête, 277

Trimeresuri, 277

Trimesurus, s. 84

leptocephalus, $s .84$

viridis, s. 80

Trionyx, 18. 95 ; s. 18

Egyptiacus, 18.95 ; s. 18

carinatus, 18

Coromandelicus, s. 19

Cuvieri, s. 19

Euphraticus, 19 ; s. 19

ferox, s. 18

Gangeticus, s. 18

Georgicus, s. 18

granosus, 19

Indicus, $s .18$

Javanicus, 19 ; s. 19

muticus, s. 18

punctatus, s. 19

spiniferus, s. 18

stellatus, 19

subplanus, $s .19$

Trionyx, armless, $s .18$

Egyptian, s. 18

Euphratic, s. 19

fierce, s. 18

flat, s. 19

Hurum, s. 18

Indian, s. 18

Javan, s. 19

punctuated, s. 19

Triton, 407

Alleghaniensis, s. 109

Gesneri, 408 ; s. 105

lateralis, $412 ; s .108$

Tropidolepis, 125 ; s. 42

aculeatus, s. 43

æneus, $s .44$

fasciatus, s. 44

grammicus, s. 43

pleurostictus, s. 43
Tropidolepis scalaris, ix. $s .44$ spinosus, s. 43 torquatus, s. 43 undulatus, $s .43$

Tropidolepis, æneous, s. 44 banded, s. 44 Bell's, s. 44 collared, s. 43 green, $s .43$ northern, s. 43 side streaked, s. 43 spinous, s. 43 thorny, s. 43

Tropidolæmus, s. 79 Wagleri, s. 80

Tropidonotus, $s .85$ æstirus, $s .86$ chrysargus, $s .85$ cyanocephalus, $s .86$ dimidiatus, $s .86$ dorsa, s. 86 hypostictus, s. 86 lacrymans, $s .86$ lugubris, s. 86 melanozostus, $s .85$ mortuarius, s. 86 natrix, $s .85$

Nattereri, s. 86 ordinatus, s. 86 pœcilostoma, s. 86 parietalis, s. 86 piscator, $s .86$ porcatus, $s .86$ proximus, $s .86$ rhodomelas, s. 86 spilogaster, $s .86$ subminiatus, s. 85 tessellatus, $s .86$ trigrinus, $s .86$ trianguliferus, s. 86 vibikari, s. 86 vittatus, $s .86$

Tropidurus, s. 41

Cuvieri, s. 41

cyclurus, s. 42 semitæniatus, $s .41$ torquatus, $136 ; s .41$ tuberculata, $s .41$

Tropidurus, collared, $s .41$ cycluroid, s. 42 half-banded, s. 41 tubercular, s. 41

Tropidosaurus, $126 ; s .35$ montanus, $s .35$

Tupinambis, 108. 111. 201, 202

albogularis, $s .28$

bivittatus, s. 25

caudivertus, s. 26 cepedianus, s. 26 
Tupinambis exilis, ix. $s .25$

griseus, $s .27$

lacertinus, $s .29$

maculatus, s. 29

marmoratus, s. 25

monitor, 113 ; s. 29

nigropunctatus, 113 ; s. 29

ornatus, 202 ; s. 27

stellatus, s. 27

viridomaculatus, s. 26

Tupinambis à taches vertes, 113

bigarré, 110

cepedien, 111

élégant, 111

étoilé, 111

variegated, 202

Turtle, coriaceus, 17.93 ; s. 20

green, $15.81 ; s .20$

imbricated, 15.89 ; s. 21

loggerhead, 16. 92 ; s. 20

Turtles, 14. 81

Typhlops, 247 ; s. 76

albifrons, 248 ; s. 77

Braminus, 248 ; s. 76

cinereus, 248 ; s. 76

crocotatus, 248 ; s. 77

fasciatus, s. 77

leucogaster, s. 77

leucorrhous, 248 ; s. 77

lineatus, s. 77

oxyrhynchus, 249 ; s. 77

Philippinus, 249 ; s. 77

reticulatus, s. 76

rostralis, $s .77$

septemstriatus, 248 ; s. 77

undecimstriatus, 248 ; $s .77$

vermicularis, s. 77

Typhlops, ash, $s .76$

banded, s. 77

Bralımin, s. 76

lined, s. 77

netted, $s .76$

Philippine, s. 77

seven streaked, $s .77$

sharp-nosed, s. 77

white-bellied, $s .77$

white-fronted, $s .77$

worm-like, $s .77$

yellow, s. 77

Tyria, 260

Tyrse, 18

Ular sawa, 257

Uræus, $s$. 83

Uran elegans, s. 26. 27

punctatus, $s .26$

Russellii, s. 25
Uranocentron, ix. s. 42 azureus, s. 42

Uromastix, 121 ; $s .61$ acanthinurus, 121 ; s. 61

dispar, s. 61

griseus, 121 ; s. 62

Hardwickii, s. 62

maculatus, $s .62$

niger, $s .62$

ocellatus, s. 61

ornatus, 121 ; $s .61$

Reevesii, s. 62

reticulatus, 121 ; s. 62

spinipes, s. 61

Uromastix, Bell's, s. 62

black, $s .62$

common, s. 61

of Egypt, 121

ornamented, s. 61

spotted, $s .62$

two coloured, $s .61$

Uronectes, 165

Uropeltis, 251 ; s. $\mathbf{5 0 . 7 6}$

Ceylonicus, 251

Philippinus, 25]

Varanus, 109

Viper, black, 273

brown, 268

commun, 270. 354

horned, 271

listle, 272

of Charas, 271

plumed, 272

red, 365

short-tailed, 270

Swedish, 365

with horned-muzzle, 271

Vipers, 269

Vipera, 269, 270; s. 81

Ægyptica, s. 81

arietans, $s .81$

aspis, s. 80

atrox, s. 79

Atropos, s. 81

berus, 272 ; $s .82$

brachyura, 270 ; s. 81

Brasiliana, s. 79

buccata, s. 95

cærulescens, s. 97

cerastes, $s .81$

chloris, s. 80

Clotho, 270

Daboia, s. 81

elegans, $s .81$

flava, s. 81.83

hœmachates, s. 83

haje, s. 83 
Vipera halys, ix. s. 80

Illyrica, 271. 368 ; $s .82$

inflata, $s .81$

Lachesis, 270

lanceolata, s. 79

lebetina, $s .80$

lophophris, 272 ; s. 81

moderans, s. 97

nasicornis, $s .81$

nasua, s. 85

ocellata, $270 ; s .81$

Pallasii, $s .80$

pretextata, $s .80$

Pysche, s. 84

Russellii, s. 81

semifasciata, s. 96

severa, $s .81$

stolata, s. 86

trigonocephala, $s .80$

Tigrina, s. 79

Weigelii, s. 79

Vipère fer de lance, 268

Psyche, 276

Walker's, double, 246

Wasp, galley, 157

Woodslave, 230

Xenodon, $260 ; s .88$ æneus, s. 88

inornatus, s. 88

Merremii, s. 88

ocellatus, s. 88
Xenodon saurocephalus, ix. s. 88

Xenopeltis, 258 ; s. 92 concolor, 258 ; s. 92

leucocephalus, s. 92

unicolor, s. 92

Xenopus, s. 104

Boiei, s. 104 ovalis, s. 104

Xiphes dorsale, s. 97 ornatum, s. 97

Xiphosoma, 255 ; s. 96 aramboja, 254 ; s. 96 canina, s. 96 dorsale, 254 ; s. 97 hortulana, s. 96

Xiphura, $s .39$

Yacaré, 199

Zacholus Austriacus, s. 93 Girondicus, s. 93

Zamerinus Æsculapii, s. 87 viridiflavus, $s .87$

Zolhafa, 58

Zonurus, 119 ; s. 63 cataphractus, s. 63 microlepidotus, s. 63

Zonurus, armed, s. 63 common, s. 63 small-scaled, s. 63

Zygnis, 159 striata, 160 ; s. 72 



\section{P I S C E S.}

VOL. $\mathrm{X}$.

Abacatuia, (Marcgr.) x. 198

Abramis, (Cuv.) $\mathbf{3 8 0}$

Abusseau, 227

Acanthicus hystrix, 414

Acanthinions, (Lacep.) 191

Acanthinion, bleu, 191 orbiculaire, 176 rhomboïde, 191

Acanthopode argenté, 177

A canthopterygians, what called, 18

Acanthopterygii, 8. 100

fifth family of, 168

six th family of, 171

seventh family of, 180

supplement to, 270

Acanthure argenté, 215 zébre, 215

Acanthurus, (Lacép. \& Bl.) 214

chirurgus, 359

ctenodon, 215

glauco-pareius, 215

guttatus, 215

harpuras, 216

Meleagris, 214

nasus, 216

scalprum, 215

scopas, 215

striatus, 215

suillus, 215

velifer, 215

Acara aia, 115

pitamba, 115

Acaramucu, 575

Acarauna longirostris, 259

Acarne, the (Rondel.) 165

Acerina, (Cuv.) 115

Achire barbu, 499

fascé, 499

marbré, 499

orné, 499

Cuv. Index.
Achirus, (Lacep.) x. 499

Acinacée bâtarde, 186

Acipenser, $(L$. $) \mathbf{5 8 8}$

brevirostris, 591

helops, $\mathbf{5 9 0}$

huso, 590. 627

maculosus, 591

oxyrhyncus, 591

pygmæus, 590

rubicundus, 591

Ruthenus, 590

steuatus, 590

sturio, 589

Ageneiosus, (Lacép.) 407

Agonus, $(B l ., S c h)$. acipenserinus, 140 decagonus, 141 dodecaedrus, 140 lævigatus, 140 rostratus, $\mathbf{1 4 0}$ stegophthalmus, 140

Agriopes, 146

Agriopus torvus, 284

Aiereba, (Marcgr.) 615

Aiguillat, Blainville, 604

Aken parah, 191

Alabes (Cuv.) 530

Alalonga. of the Italians, 183. 343

Albicore, (Sloane) 184

Albula, (Plumieri ?) 40 gonorynchus, 444 nobilis, 421

Plumieri, 444

Alepocephalus, (Risso) 390 rostratus, 390

Alicorti, the, 183

Alosa, (Cuv.) 438

Alphestes gembra, 115

Alticus, (Commers.) 231

Aluteres, (Cuv.) 575 
Aluteres cryptacanthus, x. $\mathbf{5 7 5}$

Ambassis, (Commers.) 108

Americanus, 117

Australis, 117

oxygeneios, 117

Amia, (Gronov.) 107

(L.) 447

(Salv.) 190

of the Ancients \& of Rond, 185

of Browne, 444

immaculata, 444. 447

Ammocœtes, (Dum.) 622

Ammodytes, (L.) 557

cicerelius, 538

lancea, 538

tobianus, 538

Amore guaçu, 241 pixuma, 240, 241

Amphacanthus, (Bloch.) 214

magniahac, 214

marmoratus, 214

nebulosus, 214

Amphiprion bifasciatus, 160

ephippium, 160

leucurus, 160

scansor, 218

(Bl., Schn.) 160

matejuelo, 123

percula, 160

polymnus, 160

Amphisile, (Klein) 268

Anabas, (Cuv.) 217

the genus, 360

testudineus, 218

Anableps, $(B l$. $) 385$ tetrophthalmus, 386

Anacanthus, (Ehrenb.) 615

Anampses, (Cuv.) 256

Anarrhichas, (Len.) 234

lupus, 235

minor, 235

Anchovies, the, 441

Ancylodon, 152, 299

Angel-fish, 640

Angler, the 246

Anguilla, 539

(Thurnberg and Shaw), 523

Anguille long-bec, 524

marbré, 525

pimperneaux, 524

plat-bec, $\mathbf{5 2 4}$

verniaux, 524

Anguilliformes, 522

Anodus, (Spix) 424

Anostomus, (Cuv.) 425

Antennarius, (Commers.) 247

Anthias, (Bl. in part.) 111

of Block, the 112

bilineatus, 159
Anthias boops, x. 120

caballerote, 115

catalufa, 120

cherna, 113

diagramma, 158

grunniens, 157

Japonicus, 159

jocu, 115

Johnii, 115

macropthal 'us, 120

maculatus, 157

Orientalis, 113

rabirrubia, 110

sacer, 111. 275

saponacea, 116

striatus, 113

testudineus, 218

Vosmeri, 159

Apalike, the, 443

Aphyes, of the Ancients, 227

Apistes, 145

alatus, 146

minoüs, 146

tænianotus, 146

Apistus fusco-virens, 283 Israelitorum, 283

Apisurus, (Lacép.) 215

Apogon, (Lacép.) 106 rex mullorum, 107 rouge, 107

Apterichthes, the (Dum.) $\mathbf{5 2 9}$

Apteronotus, (Lacép.) 534

Argentina, (L.) 423

bonak, 444

Carolina, 423. 443

glossodonta, 424.444

machnata, 423, 443

sphyræna, 423, 432

Argyctius quadrimaculatus, 210

Argyreiosus, (Cuv.) 198

Arnoglossum, (Rondelet) 496

Aspidophori, (Lacép.) 140

Aspidophoroïde, (Lacép.) 141

Aspredo, (Lin.) 411

Aspro, (Cuv.) 105 vulgaris, (Cuv.) 105

Astroblepus grixalvii, 468

Astrodermus, (Bonelli.) 206 guttatus, 206

Atherina, (Lin.) 226 Australis, 442

Boyer, 227

Brasiliensis, 228

the dwarf, 290

endrachtensis, 228

hepsetus, 227

Jacksoniana, 228

lacunosa, 228

mochon, 227 
Atherina mænidia, x. 228

Neso gallica, 228

notata, 228

pinguis, 228

presbyter, 227

sihama, 123

Aulopus, (Cuv.) 432

Aulostomus, (Lacép.) 267

Aurata, of the Latins, 313

Ausonia Cuvieri, 203

Auxis, (Cuv.) 184 the genus, 344

Axinurus, (Cuv.) 216 thynnoides, 216

Badi motta, 431

Bagrus, 403

Baliste armé, 573

à brosses, 574
bourse, 573
bridé, 573
cendré, 573
écharpe, 573
étoilé, 573
grandes tâches, 572
Lamouroux, 573
noir, 572
pralin, $\mathbf{5 7 3}$
sillonné, 573
verdâtre, 573

Balistes, (L.) 571

the, 584

aculeatus, 573

Américain, 573

arcuatus, 573

biaculeatus, 575

buniva, 572

bursa, 573

capriscus, 572

Chinensis, 574

conspicillum, 573

forcipatus, 572

fuscus, 572

geographicus, 574

guttatus, 574

hispidus, 574

Japonicus, 574

jellaka, 572

Kleinii, 575

lævis, 575

lineatus, 573

longirostris, 574

maculatus, 572

medinilla, 573

monoceros, 574, 575

niger, 572,573

papillosus, 57.1
Balistes pelleon, x. 574 penicilligerus, $\mathbf{5 7 4}$ punctatus, 572 rectangulus, 573 ringens, 572,573 scopas, 574

stellaris, 572, 573

sufflamen, $\mathbf{5 7 2}$

tomentosus, $\mathbf{5 7 4}$

verrucosus, 573

vetula, 572

villosus, 574

viridescens, 573

viridis, 573

Barbier, 517

Barbus, (Cuv.) 378

caninus, 378

eques, $\mathbf{3 7 8}$

plebeius, 378

Basse, the 102. 273

the common, 102

Betrachoïde gangene, 249 varié, 249 verneul, 249

Batrachoïdes, (Lac.) 249 blennioides, 489 Gmelini, 489

Batrachus, (Bl. Schn.) 249 conspicillum, 250

Diemensis, 250

dubius, 250

grunniens, 249

porosissimus, 250

quadri spinis, 250

Surinamensis, 250

tau, 249, 250

Bandroge Ferguson, 247

Beaumaris, the, 601

Belone, (Cuv.) 393 argalus, 393

cancila, 393

Caribæa, 393

caudimacula, 393

crocodila, 393

truncata, 393

Beryx decadactylus, 124

lineatus, 124

Besugo, of the Spaniards, 165

Black fish, 409

Black grunts, 306

Blennie pointillé, 232

Blennius, (L.) 228

(Proper) Cuv. 223

the, 367

acuminatus, 232

argenteus, 232

Audifredi, 232

C'apensis, 232

cavernosus, 230

Y 2 
Blennius chubs, x. 489 cornutus, 230 edentulus, 231 fasciatus, 230 fenestratus, 232 gadoïdes, 489 galerita, 230 gattorugine, 229. 231 gunnellus, 233

labrosus, 234

labrus, 487

lumpenus, 232

lupus, 487 quinque maculis, 261 mustelaris, 232 ocellaris, 229 palmicornis, 230 pavo, 230 pholis, 230 phycis, 488 pilicornis, 230 punctatus, 232 raninus, 489 rubriceps, 230 saliens, 367 simus, 231 spadiceus, 232 superciliosus, 232 tentacularis, 229 torvus, 146 truncatus, 231 villosus, 145 viviparus, 234. 488

Blepharis, (Cuv.) 198

Blepsias, the, (C'uv.) 145. 283

Bodian Aia, 115

hiatule, 110

cyclostome, 114

grosse tête, 113

lutjan, 166

melanoleuque, 114

six-raies, 105

tetracanthe, 127

Bodians, of Bloch, 112

Bodianus apua, 112 argenteus, 170 argyroleucus, 153 bœnak, 112

bodianus, 257 guativere, 112 guttatus, 112 maculatus, 114 melanurus, 113 pentacanthus, 123 sebæ, 127 stellifer, 153 triourus, 306 triurus, 158

Bogmarus, (Bl. Schn.) 210
Bokee sorra, x. 598

Bola chaptis, 153 coiton, 153 cuja, 153 pama, 299

Bonaci arara, 112

Boniton, the, 184

Bonita of the tropics, the, 183

Bontah, the, (Rus.) 224

Boops, (Cuv.) 167. 320 salpa, 167 vulgaris, 167

Borassus flabelliformis, 36 I

Bostriche tacheté, 222

Bostrichoïde œillé, 222

Bostryche Chinois, 238

Bothriocephalus solidus, 289

Botlah parah, 191

Bouclier, 519 porte écuelle, 517

Bouco-rougo, 317

Brama, (Bl. Schn.) 178 marina cauda forcipata, 327 the genus, 326

Branchiostegi, old order of, 19

Bream, little, 380

Brochet de Bantam, 393

Brosmius, (Cuv.) 487

Brotula, (Cuv.) 488

Buffalo-fish, the, 175. 324

Bullhead, the river, 280

Buro, (Commer.) 214 brunneus, 214

Butirin banane, 446,447

Butirinus of Commerson, 424. 444

Butrinus banane, 444

Cabassoude, (Rondel.) 227

Cabassous, of Provence, 227

Cabrilla, 112

Cæcula pterygea, 529

Cæsio, (Lacép,) 169 asuror, 170 poulain, 200 smaris, 170

Cæsiomore, 191

Cæsiomores, (Lacép.) 191

Cæsiomore Baillon, 191

Cailleu, (Duham.) 436 Tassard, of the Antilles, 439

Calamoia pota, 161

Callarias galarias, 484

Callichthys, (Lin.) 410

Calliodon, (Cuv.) 265

Calliomore Indien, 142

Callionymus, (Lin.) 241 Baicalensis, 243 
Callionymus cithara, x. 243

diacanthus, 243

dracunculus, 242

Indicus, 142. 243

jaculus, 243

lacerta, 242

lyra, 242

ocellatus, 243

Orientalis, 243

pusillus, 242

sagitta, 243

Callorhynchus, (Gron.) 593

Callorinchus Australis, 97

Camaripu guaçu, 443

Campilodon, (Oth. Fab.) 192

Camuri of Marcgrave, 104

Canthari, the, 319

Cantharus, (Cuv.) 167 brama, 167 vulgaris, 167

Canthère douteux, 170

Capelan, the, (Duhamel) 420. 484

Capenna, (Marcer.) 156

Capros, (Lacép.) 199

Caranges, (L.) 195

Caraangues franches, $\mathbf{3 5 5}$

Carangues, of French sailors, 196 lunaires, 355

Carangus, of the Antilles, 196 the bastard, 196 petaurista, 196 sexfasciatus, 196

Caranna, 112

Caranx, (Cuv.) 194

Daubenton, 195

Dumerilii, 354

lune, 196

punctatus, 195

trachurus, 355

Caranxomorus, (Lacép.) 205

Carapus, (Cuv.) 533

Carcharias, (Cuv.) 599. 635

Cardine, the, 517

Carp, 144

the common, 451

fecundity of the, 454

food of the, 453

golden, 377

long life of the, $\mathbf{4 5 2}$

the preserving alive of, 455 spawning of the golden, $\mathbf{4 5 7}$

Carpione, of Lombardy, 418

Carps, queen of the, 376

Carrelet of the French, 492

Cataphractus, (Lacép.) 410 Americanus, 408

Catastomus, (Lesueur) 380 cyprinus, 380,381

Ceixupira, (Marcgr.) 189
Centranodon, (Lacép.) x. 142

Centrarchus, (Cuv.) 119

Centrarcluus æneus, 119

Centriscus, (Lin.) 268

Niloticus, 398

proper, 268

pungitius, 290

scolopax, 268. 372. 412

scutatus, 269

velitaris, 269

Centrogaster, (Hout.) 214

argentatus, 214

equula, 170. 200

fuscescens, 214

rhombeus, 177

Centrolophe nègre, 205

Centrolophus, (Lacép.) 205

Centronotus, (Lacép.) 188 (Schn.) 232 spinosus, 189

Centropode rhomboïdal, 177

Centropome ambasse, 108 macrodon, 107

Plumier, 102

de Roche, 120

rouge, 124

six-raies, 105

Centropomeœillé, (Lacép.) 152

Centropomus, (Lacép.) 104 rubens, 107

undecimalis, 104

Centropristris, (Cuv.) 117 nigricans, 117

Cephalacanthes, (Lacép.) 138

Cephalopholis, 112 Argus, 112

Cephaloptera, (Dum.) 617. 653

Cephalopteram assena, 617

Cephalus, (Sh.) 569

Cepola, (Lin.) 212 cæcula, 239

gladius, 209

iris, 209

Japonica, 212

rubescens, 212

tænia, 209. 212

trachyptera, 209

Cestracion, (Cuv.) 603

Chætodon, (Lin.) 171

the genus, 322

acuminatus, 174

alepidotus, 203

annularis, 176

arcuatus, 176. 198

argenteus, 177

Argus, 175

armatus, 106

arthriticus, 176

aruanus, 161 
Chætodon aureus, x. 176 auriga, 173

baronessa, 172

Bengalensis, 161

biaculeatus, 160

bicolor, 176

bifascialis, 172

bifasciatus, 164

bimaculatus, 173

canescens, 174

capistratus, 173

Chinensis, 218

chirurgus, 215

ciliaris, 175

cornutus, 174

couronné, 175

curassao, 161

cyprianaceus, 178

decussatus, 172

ensis, 178

faber, 174. 198

fasciatus, 173.176

flavus, 173

Fremblii, 323

frontalis, 173

geometricus, 176

glaucus, 191

guttulatus, 176

guttatus, 214

imperator, 176

Kleinii, 172

lanceolatus, 155

lineatus, 215

longirostris, 174. 323

longimanus, 175

macrolepidotus, 174

maculatus, 161

marginatus, 161

Mauritii, 161

mesoleucos, 176

mesomelas, 176

meta, 215

Meyeri, 172

miliaris, 172

monodactylus, 158

nasogallicus, 173

Nicobariensis, 176

nigricans, 215

nigrofuscus, 215

orbicularis, 176

orbis, 174

ocellatus, 173

octofasciatus, 172

ornatus, 323

paru, 176

pavo, 160

pentacanthe, 176

plebeius, 173

Plumieri, 174
Chætodon principalis, x. 173 properly so called, 17 punctatus, 175 quinquecinctus, 176 rhombeus, 177 rhomboïdes, 191 rostratus, 173. 180. 323 sargoïde, 161 saxatilis, 161 sebæ, 172 sebanus, 173 setifer, 173 sohal, 215 sordidus, 161 strigangulus, 172 striatus, 172 Suratensis, 161. 262 teïra, 176 tetracanthus, 175 tricolor, $\mathbf{1 7 5}$ triostegus, 215 unicornis, 216 unimaculatus, 173 vagabundus, 172 vespertilio, 176 vittatus, 172 zèbre, 215

Chalceus, (Cuv.) 426 angulatus, 427 fasciatus, 426 macrolepidotus, 427 opalinus, 427

Chane (X $\alpha \nu \eta)$ of the Greeks, 110

Characin nefasch, 430

Characinus Amazonicus, 429 of Artedi, 424 dentex, 429

Charax, of Risso, 163

Chatoessus, (Cuv.) 438

Chauliodus, (Schn.) 392 Sloanï, 392

Cheiline scare, 253 trilobé, 253

Cheilnus, (Lacép.) 253 auratus, 253

Cheilodactylus, (Lacép.) 158 of Carmichael, 158 carponemus, 158 fascé, 158 zonatus, 158

Cheilodipterus, (Lacép.) 107 Arabicus, 107 octovittatus, 107 quinquelineatus, 107

Cheilodiptère chrysoptère, 102 cyanoptère, $\mathbf{1 5 4}$ heptacanthe, 194 rayé, 107

Chela of Buchanan, 383 
Chelmons, x. 323

Chelmon, (Cuv.) 173

Chewoola-parah, 198

Chimæra, (L.) 592

the Antaretic, 593

the Arctic, 593

callorhynchus, 593

monstrosa, 593

proper, (Cuv.) 593

Chirivita jaune, 176 noire, 176

Chirocentrus, (Cuv.) 444

Chironectes, the, 369

biocellatus, 248

Commersonii, 247, 248

furcipilus, 248

hispidus, 248

lævigatus, 247

histrio, 370

marmoratus, 248

mummifér, 248

ocellatus, 248

pictus, 247

punctatus, 248

scaber, 248

tuberosus, 248

tumidus, 247

unipinnis, 248

variegatus, 248

Chironemus, (Cuv.) 119

Georgianus, 119

Chirus, (Stell.) 244

Chitchillée, (Russell) 163

Chnees, of the Moluccas, 445

Chondropterygians, branchiæ of, 47 cerebellum of, 72

Chondropterygii, 6. 586 supplement on the, 624 with fixed gills, 629

Chromis, (Cuv.) 261 Nilotica, 262. 371 vulgaris, 261. 371

Chrysophris, (Cuv.) ]63. 312 of the Ancients, 163 aurata, 163. 314 chrysargyra, 163 microdon, 163

Chrysostromus, (Lacép.) 202

Chrysotose lune, 200

Chrysotosus, (Lac.) 200

Chrysurus, 115

Cichla macroptera, 158

Cicilia, (Lacép.) 529

Cirrhibarba, (Cuv.) 232

Cirrłinùs, (Cuv.) 379

Cirrhite pantherin, 118 tacheté, 118

Cirrhites, (Commers.) 118 aprinus, 118
Cirrhites vittatus, x. 118

Citharinus, (Cuv.) 430 Geoffrœi, 430

Citharus, (Rondel.) 495 asper, 495 pinguis, 495

Citula Banksii, 196

Citulæ, (Cuv.) 197

Clarias, (Gronov.) 409

Clepticus, (Cuv.) 259 genizara, 259

Clinus, (Cuv.) 231

Clupanodon Champol, 438 Chinois, 437 ilisha, 438 Jussieu, 437

Clupea, 434 (L. Cuv.) 435 the genus, 474 æstivalis, 438 atherinoïdes, 442 alosa, 438. 479 Brasiliensis, 444 clupola, 437 coval, 437 cyprinoïdes, 443 dentex, 445 dorab, 445 encrasicholus, 441 fasciata, 200 finta, 438 gigantea, 443 harengus, 435. 474 haumela, 208 humeralis, 437 kélée, 438 latulus, 436 matowka, 438 melanura, 437 melastoma, 437 menhaden, 438 mystax, 442 mystus, 442 nasus, 439 palasah, 438 phasa, 442 pilchardus, 437 Sardina, 437. 480 setirostris, 442 sprattus, 436 synura, 441 telara, 442

Thrissa, 439 tuberculense, 442 vernalis, 438 villosa, 420

Clupée macrocéphale, 444 raie d'argent, 442

Coal-fish, the, 485 
Cobitis, (L.) x. 384

anableps, 386

barbatula, 384

fossilis, 384

geta, 385

heteroclita, 387

maialis, 388

tænia, 385

Cock-up, of the English of Calcutta, 103

Cod-fish, 484

Cod, fishery of the, 508

flesh of the, 507

habitat of the, 506

Isle of Man, red, 509

the well-known, 505

Coffre (ostracion) quatorze piquans, 577

Cogrus, (Rafin.) 526

Coius catus, 115

chatareus, 180

coboius, 218

datnia, 121

nandus, 157

Colas of Guadaloupe, 115

Colisa, of Bengal, 362

Colin, the French, 485

Comephorus, (Lacép.) 243

Conger, (Cuv.) 524

the, 544

à chapelet, 525

fishing for the, 545

niger, 546

Cora motta, 222

Coracinus, of the Ancients, 261 the white or Egyptian, 262

Corbeau, of the Mediterranean, 226

Cordonnier, of Martinique, 198

Coregonoïdes Amboinensis, 426

Coregonus, (Cuv.) 421

albus, 423

fera, 422

hyemalis, 422

palæa, 422

thymalloïdes, 421

quadrilateralis, 423

signifer, 421

Coricus, (Cuv.) 258

rubescens, 258

Coris, (Lacép.) 255

aigrette, 255

angulé, 255

Corvina, (Cuv.) 152

the, 292

acuminata, 155

the black, 153

formosa, 156

furcæa, 153

katchelée, 153

miles, 153

nageb, 157
Corvina obliqua, x. 153 oscula, 153

Corvo di fortiera, 297

Corvulus, (Ges.) 107

Corvus Niloticus, 226

Corydorus edentulus, 408

Coryphæna aurata, 359

cærulea, 261. 265

chrysurus, 359

from what derived, 357

equisetus, 359

fasciolata, 205

(Lin.) 204

hippurus, 205. 357

lineata, 261

lineolata, 261

nigrescens, 117

novacula, 260

pelagica, 262

pentadactyla, 261

Plumieri, 263

pompilus, 205

psittacus, 261

torva, 146

velifera, 206

Coryphæne Plumier, 263

Cotte madegasse, 142

Cottus, (Lin.) 133. 138

Acadianus, 141

anostomus, 140

Australis, 146

bubalis, 139

cataphractus, 140

diceraus, 139

gobio, 139. 280

grunniens, 249

hemilepidotus, 142

hispidus, 141

insidiator, 142

Japonicus, 140

macrocephalus, 238

monopterygius, 141

octodecim spinosus, 139

pistilliger, 140

polyacanthocephalus, 139

quadricornis, 139

scaber, 142

scorpio, 281

scorpius, 139

spatula, 142

trachurus, 142

tripterygius, 141

Virginianus, 139

Courpata, of the Mediterranean, 226

Crenideus, 320

Crenilabrus, (Cuv.) 256

Roissal, 257

tinca, 257

Curimate, (Gilbert) 424 
Curimatus, (Cuv.) x. 424

Curvata pinima, 195

Cybia, the, 344

Cybium, (Cuv.) 185 cavalla, 185

Commersonii, 185. 344

guttatum, 185. 345

lineolatum 185

maculatum, 185

regale, 185

Cychla, (Bl. Schn.) 262

ænea, 119

Argus, 262

argyrea, 170. 262

bimaculata, 262

brama, 262

Brasiliensis, 262

cuning, 170. 262

cynodon, 262

erythrura, 262

guttata, 262

Japonica, 262

macropthalma, 262

maculata, 262

ocellaris, 262

pelagica, 205. 262

punctata, 262

saxatilis, 262

sparoïdes, 119

Surinamensis, 262

variabilis, 117

Cyclogaster of Gronovius, 521

gelatinosus, 521

liparis, 521

Cyclopterus, (L.) 501

bimaculatus, 501

Cornubicus, 518

gelatinosus, 502

gibbosus, 502

liparis, 502

littoreus, 501

lumpus, 502, 518

minutus, 502. 521

Montagui, 502

nudus, 501

pavonius, 502

souris, 502

spinosus, 502.521

ventricosus, 502

Cyclostomata, 7 (Dum.) 617

Cynœdus, (Gronov.) 158

Cynodon gibbus, 429 vulpinus, 429

Cyprin sucet, 381

Cyprinodon, (Lacép.) 388

flavulus, 388

ovinus, 388

umbra, 388

Cuv. Index.
Cyprinodon, variegatus, $\mathbf{x} .388$

Cyprinoïdes, 374

Cyprinus alburnus, 382

(Cuv.) 375

amarus, 378

Anne Caroline, 376

aspius, 382

ariza, 382

auratus, 377,456

bacaila, 383

ballerus, 380

barbus, 378

basbora, 382

bendelesis, 379

binny, 378

bipunctatus, 382

blicca, 389

boga, 382

brama, 389

Buggenhagii, 380

bulatmai, 378

calbasu, $\mathbf{3 7 9}$

capoeta, 379

carassius, 376

carpio, 376. 451

catla, 377

cirrhosus, 379

clupeoïdes, 383

cocsa, 379

cotis, 380

cultratus, 383

curmuca, 379

daniconius, 379

distica, 383

dentex, 428

devarid, 377

dobula, 381

erythrophthalmus, 382

fimbriatus, 380

gibelio, 377

gobio, 379

gonius, 379

gonorynchus, $\mathbf{3 8 4}$

grislagine, 382

gros yeux, 377

idus, 381

jeses, 382

kunama, 379

latus, 380

lepidotus, 378

leuciscus, 382

macrophthalmus, 377

mola, 382

morar, 383

mordoré, 376

morula, 379

mrigala, 379

mursa, 378

nandina, 379 
Cyprinus, nasus, $x .382$

Niloticus, 380

pala, 382

phoxinus, 382

quatre lobes, 377

rex cyprinorum, 376

Rohita, 379

rouge-brun, 376

rutilus, 381

sophore, 382

teres, 381

tinca, 379

tinca auratus, 379

tolo, 382

vimba, 380

vert-violet, 376

Dactyloptera, (Lac.) 137

Orientalis, 138

Dalophis, (Rafin.) 528

Dalatias nocturnus, 604

Dangiri mangelang, 183

Dascyllus, (Cuv.) 161

Datnia Buchanani, 121 cancellata, 121

Daurade, whence derived, 313

Deddi-jallah, 403

Delatias sparophagus, 605

Demi-bec de Baggewaal, 395

Dentale, of the Italians, 166

Dentex, (Cuv.) 165

the genus, 316

hexodon, 166

macrocephalus, 166

macropthalmus, 166

vulgaris, 166.317

Derbio, the (Rond.) 190

Diabolus marinus, 617

Diabase, striped, 156

Diacope, (Cuv.) 114

calveti, 114

macolor, 114

notata, 114

octolineata, 114

quadriguttata, 114

rivulata, 114

Sebæ, 114

Diagramma (Cuv.) 157

lineatum, 157

pertusum, 158

pictum, 158

pœecilopterum, 158

Diana semilunata, 206

Diodon, (L.) 566

antennatus, 566

atinga, 566. 579

histrix, 566. 581

holocanthus, 581

jaculiferus, 566

mola, 570

multimaculatus, 566
Diodon nictemerus, $\mathrm{x} .566$

novem-maculatus, 566

orbiculatus, $\mathbf{5 6 6}$

pilosus, 567

punctatus, 566

rivulatus, 566

sex-maculatus, 566

spinosissimus, 566

tigrinus, 566

triodricus, 566

Diploprion, (Kuhl \& Van Hasselt) 106 bifasciatum, 106

Dipterodon, 178

Capensis, 178

héxacanthe, 107

ruber, 107

Discoboli, 499

Ditchæe, (Schn.) 437

Dondoo-paum, 530

Dog-fish, 631

Dondrum yellakah, 573

Donzelles, (French) 535

Doras, (Lacép.) 407

granulosus, 408

niger, 408

oxyrhyncus, 408

Dorosoma, (Rafin.) 438

Dorsch, of the Baltic, 484

Dorse, the 510

Dorsuaire, (Lacép.) 177

Drum, the 303

Dules, (Cuv.) 120

auriga, 120

rupestris, 120

tæniurus, 120

Echelus, (Rafin.) 523

Enchelyopus colias, 127

Echeneis, (L.) 503

lineata, 504

osteochir, 504

naucratus, 504

remora, 504

Echiquier, l', (Lacép.) 255

Eel, the common, 539

the conger, 524

eggs of the, 543

the electric, 532. 549

food of the, 542

habitat of the, 541

organization of the, 540

the snake, 526

tenkée, 616

Eels, (properly so called,) 523

Ekalah parah, 196

Elacate, (Cuv.) 189

Americana, 189

motta, 189 
Eleotris, (Gronov.) x. 241

dominatrix, 241

lanceolata, 238

noir, 241

Mauritii, 1.93

Elops, (L.) 443

(Commers.) 259

saurus, 423

Emoï, 130

Emissole commune, 602 tachetée de blanc, 602

Enchelyopus Americanus, 489 barbatus, 488

Engraulis, (Cuv.) 441 edentulus, 442 lemniscatus, 442 meletta, 441

Enoplosus, (Lacép.) 106 armatus, 106

Ephippii of India, 175

Ephippus, (Cuv.) 174

Argus, 324

gigas, 174

terla, 175

Epibulus, (Cuv.) 258

Epidesmus maculatus, 210

Epinephelus, 112

ciliatus, 144

marginalis, 113

merra, 112

ruber, 113

Epinoche, of the French, 287

Eques, $(B l$.) 155

acuminatus, 155

Americanus, 155

balteatus, 155

punctatus, 155

Equula, (Cuv.) 200

caballa, 170

cara, 200

coma, 201

daura, 201

ensifera, 200

fasciata, 200

insidiatrix, 201

minuta, 201

ruconius, 201

splendens, 201

totta, 201

Eregoodoo-tenkée, 617

Erythrinus, (Gronov.) 446 tæniatus, 446

Esclave à six lignes, 121

Esoces, 388

Esox, (L.) (Cuv.) 389 alepidotus, 390 argenteus, 444 belone, 393. 449 boa, 391
Esox Brasiliensis, x. 395

chirocentre, 445

estor, 390

flavulus, 388

gymnocephalus, 446

hepsetus, 395

Lewini, 465

lucius, (pike.) 274. 389. 460

Malabaricus, 446

osseus, 449

ovinus, 388

pisciculus, 388

reticularis, 390

saurus, 394

spliyræna, 130

stomias, 392

synodus, 431. 443. 447

truttaceus, 390

viridis, 449

vulpes, 444

zonatus, 388

Etelis, (Cuv. \& Val.) 106

carbunculus, 106

Etmopterus aculeatus, 604

Exocetus, (L.) 395. 465

appendiculatus, 397

comatus, 397

exiliens, 396. 466

fasciatus, 396

furcatus, 397

mesogaster, 279. 396

Nuttalii, 397

volitans, 396. 465

Fahaca, the, 582

of the Arabs, 567

Fanfre of the sailors, 189

Faux merlan, 484

Falx Venetorum, 209

Fegaro, of the Genoese, 296

Fierasfer, 537

gymnotus, 534

Filar, why called, 544

Fi-fi, of the Isle of France, 286

Fish, the, 1. 661

the abdominal, 8

angel, 608

the apodal, 8

with armed cheeks, 133

articulation of the bones of, 5]

balloon, 564

bones of, 58,59

the brain of, 28.71

Branchial system in osseous, 65 cartilaginous, 6

cartilage of the bones of, 52 of St. Christopher, 356

circulation of blood in, 87.89

classification of, 10

the coal, 512

Z 2 
Fish, the common, $x .7$

the cranium of, 54

deglutition of aliment of, 85

the ear of, 77

egg-roes of, 98

external organs of the senses of, 74

exterior of, 47

eyes of, $\mathbf{3 6}$

the eye of, 75

flat, 514

food supplied by, 33

fresh-water, $\mathbf{4 5}$

in general, supplement on, 25

globe, 564

growth of, 99

habitation of, 35

habits of, 34

head of, 3

hermaphrodites among, 96

Malacopterygian, 8

intestinal canal of, 85

manducation and teeth of, 82

the modern definition of, 27

moor, 569

muscles of, 67

muscle of the branchial apparatus of, 70

muscle of the jaws of, 68

muscles of the operculum of, 69

the myology of, 66

nerves of, 73

the nostrils of, 78

organs of generation of, 94

organs of nutrition of, 82

the organs of taste of, 79

the organs of touch in, 80

the ovaria of, 95

pelagian, 38

periodical blindness of, 37

of St. Peter, the, 199

the pharyngeal bones of, 60

properly so called, 6

pupil of the eye of, 76

respiration of, 90, 91

the scales of, 81

secretions of, 92

the senses in, 29

sexual emotions of, 31

skeleton of osseous, 53. 64

snout of, $\mathbf{5 7}$

spleen of, 87

spawn of, 97

spiny globe, 566

stock, 514

the structure of, 49

sternum of, 63

sun, 569

the subbrachian, 8

taking of a flying, 41
Fish, taste in, x. 4

teeth of, 84

temperature of, 30

texture of the bones of, 50

trunk, 575

use of swim-bladder of, 93

vertebræ of, 3.61

the vertical fins of, 62

Fishes, the nature of, 25 organization of, 25

flat, 490

Fistularia, (Lin.) 266

Chinensis, 268

immaculata, 267

proper, (Lacép.) 267

paradoxa, 561

serrata, 267

tabacaria, 267

Fistularidæ, 266

Flag-man, 324

Flasco psaro, 567

Flounder, the rose-coloured, $\mathbf{4 9 2}$

Fundulus, (Lacép.) 387

Brasiliensis, 388

cænicolus, 387

fasciatus, 388

Gachua, of India, 364

Gadoïdes, 482

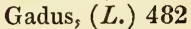

Eglefinus, 484

albidus, 489

Bacchus, 486

barbatus, 484. 511

blennoïdes, 484

brosme, 487

callarias, 484

carbonarius, 485

Cimbricus, 487

furcatus, 489

gracilis, 484

longipes, 489

luscus, 484

macrocephalus, 474

maculosus, 487

Magellanicus, 486

maraldi, 486

merlangus, 485

merluccius, 485

minutus, 484

molua, 486

morrhua, 483

mustela, 487

pollachius, 485

quinque-cirrhatus, 487

raninus, 489

Saida, 484

salarias, 230

lota, 486,514

tau, 249 
Gadus tomcodus, x. 484 tricirrhatus, 487

trifurcatus, 489 virens, 485

Galanga, 246

Gal d'Alexandrie, 197

Galaxias, (Cuv.) 390

Galeus, (Cuv.) 601

Gallus, (Cuv.) 198

Gardon Le, 381

Gar-fish, 393

Gasterocephalus, ( $R l$.) 425

Gasterostei, the, 287

Gasterosteus, (Cuv.) 148 aculeatus, 149. 288. argyropomus, 149 biculeatus, 149 brachycentrus, 149

Canadensis, 189 cataphractus, 149 ductor, 189 gymnurus, 149 Japonicus, 148 leiurus, 288 niger, 149 Noveboracensis, 149 Occidentalis, 191 ovatus, 191 quadracus, 149 spinarella, 138 spinochia, 149 trachurus, 149, 288 tetracanthus, 149

Gastrobranche dombey, 622 Gastrobranchus, $(B l)$. cœcus, 622.660

Gastropelecus sternicla, 425

Gempylus the, 186. 346 coluber, 346

Prometheus, 346 serpens, 186. 346 with what connected, 207

Germon of the Biscayans, the, 183 of Gascony, 343

Gerres, the genus, 321 aprion, 170 argyreus, 170

- filamentosus, 170 lineatus, 170 Plumieri, 322 poieti, 170 rhombeus, 322

Gerrus, (Cuv.) 170 rhombeus, 170

Gherad el bahr, 283

Gilt-head, (of Pennant) 314

Girelle Duperry, 256 Gaymard, 255 Labiche, 255
Glut-eel, of the English, x. 524

Glyphisodon, (Lacêp.) 161 azureus, 161 lachrymatus, 161 melanurus, 161 sparoïdes, 161 uniocellatus, 161

Gnaibi Coara, 156

Gnathobolus, (Schn.) 439

Gobiésoce testar, 501

Gobiesox, (Lacép.) 500

Gobio, (Cuv.) 379

Gobioïde rubicunda, 239 Smyrnéen, 502

Gobioïdes, 228. 367 (Lacép.) 238

Gobiomore dormeur, 241 Gronovien, 193

Gobiomoroïde pison, 240

Gobiomore taiboa, 241

Gobius, (Lin.) 235 proper, (Lacép. \& Schn.) 236 the genus, 368 alepidotus, 238 auratus, 241 bato, 238

Boddarti, 238 bosc, 230

Broussonet, 238

boulereau, 368

capito, 237

changua, 238

cruentatus, 237

elongatus,

fluviatilis, 237

Gronovii, 193. 240

guiris, 238

jozzo, 237

Kælreuteri, 240

lagocephalus, 238

lanceolatus, 238

lota, 238

macrocephalus, 238

minutus, 237. 502

niger, 237. 368

ocellaris, 238

pisonis, 240, 241

oblongatus, 238

Plumieri, 238

Russellii, 238

Schlosseri, 240

striatus, 240

strigatus, 24 l

viridi-pallidus, 238

Gode mollet, 484

Ghomphosus, (Lacép.) 259

cæruleus, 259

Lacépède, 259

viridis, 259 
Gold-fish, x. 377. 456

Gonorynchus, (Gron.) 383

Gourami, the, 363

(Lacép.) 220

Grammistes, (Cuv.) 104

acuminatus, 155

Orientalis, 104

trivittatus, 156

Grig-eel, of the English, 524

Grumblers, (what called,) 134

Grystes, (Cuv.) 117 Macquariensis, 117

Guaperva, the, 198, 248 (Marcgr.) 176

Guarapuca, (Marcgr.) 185

Guaratereba, (Séb.) 196

Guavina, (Pam.) 241

Gudgeon, the, 458

Guebucu, (Marcgr.) 188

Gurgur, of Upper Egypt, 406

Gurnard, the grey, 279 the red, 278 the sapphirine, 279

Gurrah-parah, 198

Gymnarchus, (Cuv.) 534 Niloticus, 535

Gymnètre cépédien, 210

Gymnetrus, (Bloch.) 209 cepedianus, 211

Grillii, 211

Hawkenii, 211

Mediterraneus, 210

Russellii, 2] I

Gymnocephalus ruber, 112

Gymnodontes, 7

Gymnogaster, (Gronov.) 208 Arcticus, 210

Gymnomuréne cerclée, 528

Gymnothorax, $(B l)$.

Gymnotus, (L.) 530

the genus, 547

acus, 534.537

albifrons, 534

æquilabiatus, 533

albus, 533

anatomy of, 553

brachiurus, 533

electric power of, 550

electricus, 532. 548

fasciatus, 533

fierasfer, 534

macrourus, 533

mode of taking, 554. 557

notopterus, 441.534

proper, (Lacép.) 532

rostratus, 534

shocks given by, 552

Gomphosus variegatus, 259

Gynodontes, 564
Haddock, the, x. 509

English, 484

Halé, the, 409

Hake, the, 513

Hæmulon, (Cuv.) 156

the genus, 305

caudimacula, 156

capenna, 156

chrysopterum, 156

elegans, 156

heterodon, 156

Harder, the, 193

Harpurus, (Forst.) 214

Hausen, 627

Hadou, of the French, 484

Heliasus, 161

Helostoma, 362

(Kuhl.) 219

Temminckii, 219

Helotes, (Cuv.) 121 sexlineatus, 121

Hemilepidotus, (Cuv.) 141

Hemiramphus, (Cuv.) 394

Brasiliensis, 395

brevirostris, 394

Commersonii, 395

hepsetus, 395

longirostris, 394

marginatus, 394

Hemitripterus, (Cuv.) 141

Heniochus, (Cuv.) 174 macrolepidotus, 323

Hepatus of Rondelet, 312

Heptatremus, 659

Herder, (Shepherd) 193

Herrings, the, 474 migration of, 475,476 proper, the, 435

Heterobranchus, (Geoff.) 408 bidorsalis, 409

Hippoglossus, (Cuv.) 494

Hippocampus, (Cuv.) 560 brevirostris, 560 guttulatus, 560

Hippos of Linnæus, 195

Histrophori, the, 351

Histrophorus Americanus, 352 Indicus, 352

Holocanthe, jaune \& noir, 172

Holocanthus, (Lacép.) 175 arcuatus, 325

ciliaris, 325

imperator, 325

Lamarckii, 325

Holocentre, argentinus, 110

bleu, 113

calcarifer, 103

Chani, 110

cilié, 159 
Holocentre diacanthe, x. 160 gymnose, 113 heptadactyle, 103 jaune, 113 léopard, 114 négrillon, 160 noire, 205 océanique, 113 panthérin, 112 rabagi, 164 résmare, 113 siagonotus, 110 Sonnerat, 160 Thunburg, 124

Holocentres, of Bloch, 112 Holocentrum, (Artedi) 123 diadema, 124 hastatum, 123 leo, 123 longipinne, 123

Orientale, 123 rubrum, 123 sammara, 124 sogho, 123

Holocentrus albofasciatus, 144 amicalis, 176 Argus, 110 auratus, 112 Bengalensis, 114 cæruleo punctatus, 112 fasciatus, 110 Lamarck, 176 marinus, 110 lanceolatus, 113 Maroccanus, 110

' ongus, 113 punctatus, 112 quinquelineatus, 114 quadrilineatus, 121 salmonoïdes, 113 servus, 120

Surinamensis, 158

tigrinus, 113 unicolor, 114 virescens, 110

Hologymnoses, (Lacép.) 255

Huro, (Cuv. \& Val.) 105 nigricans, 105

Hydrargyre swampine, 388

Hydrocyon, (Cuv.) 428 brevidens, 429 falcirostris, 428 faucille, 428 hepsetus, 428 lucius, 429 scomberoïdes, 429

Hyodon, (Lesueur) 445 clodalus, 446

Hypopthalmus edentatus, 404
Hypopthalmus nuchalis, x. 404

Hypostoma, (Lacép.) 414 etentaculatum, 414

Ikan-Karbauw, 324

Ikan-layer, (fan-fish,) 352

Ikan-sumpit, 328

Isabelita, $\mathbf{1 7 5}$

Isakurrah Tenkee, $\mathbf{6 1 4}$

Ischnosoma bicirrhosum, 448

Istiophorus, (Lacép.) 188

Isurus oxyrhynchus, 601

Jacaraqua, 161

Jacob Evertsen, 112

Jaguaraca of Marcg. 123

Jangartoo, (Russell) 437

Jara dandrée parah, 196

Jerré potoo, 498, 499

Jinagow, (Russ.) 443

Joel, (Rondel.) 227

Johnius, (Bl.) 153 anei, 153

of Bloch, 302

cataleus, 153

guttatus, 112

humeralis, 153

karutta, 153

maculatus, 153

pama, 153

regalis, 152

ruber, 152

saxatilis, 154

Senegalensis, 153

xanthurus, 154

Julis, (Cuv.) 254

cæruleo-cephalus, 256

crotaphus, 255

Gioffredi, 254

Turcica, 254. 256

vulgaris, 254

Jura sucker, 500. 518

Karasche, (Geoffr.) 406

Katalai, 302

Katchelé, 302

Katelé, 302

Kingfisch, of the Anglo-Americans, 154

Kiphose double bosse, 178

Kitt, of the English, 496

Klip bagre, 408

Kohl-fisch, 485

Kolinska, of the Russians, 290

Koma sorra, 607

Komé, (Russell) 439

Konam, (Russell) 185 
Kuddera, (Russ.) x. 394

Kuguroo-parah, 196

Kunnesee, the, 224

Kurra Wodagahwah, (Russ.) 195

Kurtus, (Bloch) 357

(Cuv.) 203

argenteus, 180

cornutus, 204

Indicus, 204

Kurwa, (Russell) 166

Kyphose, 177

Labeo, (Cuv.) 380

Labrax, (Pall.) 244

(Cuv.) 102

decagrammus, 244

hexagrammus, 244

of the Greeks, 103

Japonicus, 103

monopterygius, 244

lagocephalus, 244

lineatus, 103

lupus, (Cuv.) 102. 273

mucronatus, 103

octogrammus, 244

superciliosus, 244

Waigiensis, 103

Labre argenté, 256

anguleux, 124

ceinturé, 255

chapelet, le, 164

Commersonien, 157

deux croissants, 253

diagramme, 253

Diane, 253

ennéacanthe, 253

fuligineux, 253

hébraique, 256

hérissé, 253

hololépidote, 152

iris, 119

large queue, 253

large raie, 146. 263

lisse, 114

long museau, 170

macrocéphale, 166

macroptère, 119

macrogastère, 161

malaptéronote, 253

marbré, 118

neustrien, 252

parterre, 255

filamenteux, 262

Plımiérien, 156

quinze épines, 262

sagittaire, 180

salmoide, 117

six-bandes, 161
Labre sparoïde, x. 119

tenioure, 255

tetracanthe, 127

trilobé, 255

Labrus, (Lin.) 251

the genus, $\mathbf{3 7 0}$

Adriaticus, 110

albovittatus, 255

Americanus, 253

auritus, 119

ballan, 252

bergilta, 251

bifasciatus, 256

bivittatus, 255

Brasiliensis, 255, 256

burgal, 258

carneus, 252

chanus, 110

chloropterus, 255

chogset, 258

chromis, 155. 303

chrysops, 258

cruentatus, 120

cyanocephalus, 255

exoletus, 257

fasciatus, 253

fuscus, 257

gibbus, 257

grunniens, 155

guttatus, 255

hassec, 253

hepatus, 110

hiatula, 263

inermis, 253

jaculator, 180

Japonicus, 158

julis, 254

Lapina, 257

leopardus, 113

lunaris, 256

lineatus, 252

lunula, 253

macrolepidotus, 255

maculatus, 251, 252

malopterus, 255

Margaritiferus, 255

melagaster, 253

melops, 257

merula, 252

mola, 255

niger, 306

Niloticus, 262

obliquus, 153

ocellatus, 257

octolineatus, 114

ornatus, 255

palloni, 257

perca, 257

punctatus, 262 
Labrus quinquemaculatus, x. 257 rubens, 257

scarus, 253

Sinkii, 258

spalatensis, 257

squeteague, 152. 300

tetrodon, 256

tinca, 252. 257

trichopterus, 220

trimaculatus, 252

turdus, 252

unimaculatus, 257

variegatus, 252

venosus, 257

versicolor, 164

vetula, 252

violaceus, 258

virescens, 258

viridis, 252. 256

Labyrinthiform Pharyngeals, 217

Lachnolaimus, (Cuv.) 254

caninus, 254'

suillus, 254

Læviraia, (Salv.) 613

Lamayellaka, 572

Lamies, (Fr.) 600

Lampreys, the, 618. 654

Lamprillon, 623

Lampries, (Retzius) 200 guttatus, 200

Iamproyon, 623

Lates, (Cuv.) 103 of the Ancients, 103 Niloticus, 103. 273 nobilis, 103

Latté, (Russ.) 175

Latus, of the Ancients, 103

Lavaret, false name of, 421

Lebias, (Cuv.) 387

ellipsoïdea, 387

fasciata, 387

rhomboïdalis, 387

Leiche, the, (Brouss.) 605

Laborde, 606

Leiostome queue jaune, 154

Lepadogaster, 517

(Gouan.) 499. 500. 517

balbis, 500.518

Decandolle, 500

dentex, 501

Candolii, 518

proper, 500

rostratus, 500. 517

Wildenou, 500

Lepidoleprus, (Risso) 489

cœlorhyncus, 490

trachyrhyncus, 490

Lepidope argenté, 207 of Péron, 207

Cuv. Index.
Lepidopus, (Gouan.) x. 207 argyreus, 207. 346

Lepiscanthus, of Lacépède, 147. 287

Lepisostée spatule, 449

Lepisosteus, (Lacép.) 448

Lepodus saragus, 178, 179

Leptocephalus, (Pennant) 535 Morissii, 535

Leptocéphale Spallanzani, 529

Lepturus, (Artedi) 208

Lethrinus, the, 166. 318

Leyognathe argenté, 200

Leuciscus, (Klein.) 381

Liche, the, (Brouss.) 605 sineuse, 190

Lichia, (Cuv.) 190 sinuosa, 190

Licone de mer, 519

Ling, the, 486. 514

Linguatula, (Rondel.) 498

Liparis, 521

(Artedi) 502

Loache, pond, 384

Lobotes, (Cuv.) 158

Surinamensis, 306

Loche d'étang, 384

Lonchuri, the, 154

Lonchurus ancylodon, 152. 301 tarbatus, 154. 301

Lophe cheronecte, 248

Lophie fauyas, 249

Lophius barbatus, 247

budecassa, 246

bufo, 249

Cornubicus, 247

dubius, 250

gibbus, 247

histrio, 247, 248

histrio ocel. 248

histrio pictus, 247

monopterygius, 247

parvipinnis, 246

pictus, 248

piscatorius, 246. 369

raninus, 248

ruber, 249

setigerus, 246

stellatus, 249

vespertilio, 248

viviparus, 246

Lophobranchii, the, 7. 558

Lophotes, (Giorna) 212 cepedianus, 213

Lopius, proper, (Lin.) 245

Loricaria cataphracta, 414

(L.) 413

cirrhosa, 414

plecostomus, 414

proper, 414

A a 
Loricaria rostrata, x: 414 setigera, 414

Lota, 514

$$
\begin{aligned}
& \text { (Cuv.) } 486 \\
& \text { elongata, } 486 \\
& \text { molva, } 514
\end{aligned}
$$

Lucio-perca, (Cuv.) 109

Americana, 109 sandra, 109. 274

Lumpsucker, 519

Lumpus, 501

Lupus, of the Romans, 103

Lutjan of Cayenne, 152 diacanthe, 153 gymnocéphale, 108 hexagone, 124 microstome, 157 triangle, 152 perchot, 160 trilobé, 117

Lutjans (of Bloch, the, 112 Lutjanus acutirostris, 115

bidens, 257 erythropterus, 115 hasta, 157 Lamarckii, 258 lunulatus, 112 luteus, 157 lutjanus, 115 Mediterraneus, 257 Norvegicus, 257 notatus, 258 rostratus, 257 rupestris, 257 Surinamensis, 157 trifasciatus, 160 verres, 257 virescens, 257 viridens, 258

Luvarus imperialis, 203 Lymne (Lacép.) 615

Macabi, (Parra) 444

Mackerel, the, 181 the bastard, 355 cerebellum of, 72 the common, 38.181 derivation of, $\mathbf{3 3 3}$ the genus, $\mathbf{3 3 0}$ the little, 182 swim-bladder of, 93

Macquaria, from Australia, 307 Macrognate armé, 191 Macrognathus, 354 pancalus, 191 (Lacép.) 191

Macropode vert doré, 219 Macropodus, 362 (Lacép.) 219
Macropteronote brun, x. 409

Macropteronotes, (Lacép.) 409 magur, 409

Macroramphose (Lacép.) 268

Macrourus, (Bloch.) 489 rupestris, 490

Maculato-striatus, 566

Mœna, (Cuv.) 168 the genus, 321 jusculum, 169 radiata, 169 vulgaris, 168

Mœnidia, of Brown, 228. 395

Maigre, the, 292 at Aunis, 151 brulé, 152 of the Cape, 152

Mais-parah, 197

Makaira, (Lacép.) 188 blackish, 188

Mal, of the Swedes, 401

Malacanthus, (Cuv.) 263

Malacopterygian family, the, 21

Malacopterygians, what called, 48

Malacopterygii, 7 abdominales, 279. 374. 451 apodes, 522. 539 subrachiati, 482

Malapterurus, (Lacép.) 411

Mallotus, (Cuv.) 420

Malthe, (Cuv.) 248 angusta, 249 nasuta, 248 notata, 249 stellata, 249 truncata, 249

Manatia, (Lac.) 617

Mangelang, (Russell,) 185

Mango-fish, 129

Manti-bukaropaumu, 529

Marqueraux batardo, 195

Marulke, 144

Mastacembelus, 354 (Gronov.) 192

Matejuelo blanco, 263

Mégalope filamenteuse, 443

Megalops, (Lacép.) 442 cepedianus, 439 notatus, 439 oglina, 439

Meleagris, (Sh.) 577

Mené (Lacép.) 201

Mené, Anne Caroline, 201

Merlan, 486

Merlangus, 485 the, 5] I carbonarius, 512 pollachius, 513 vulgaris, 511 
Merle, the, x. 205

Merluccius, 485. 513 barbatus, 489

Merous, the, 111

Menides, 168

Mesoprion, 115 (Cuv.) 114 buccanella, 115 cynodon, 115 lunulatus, 115 monostygma, 115 quinque lineatus, 115 rangus, 115 rapilli, 115 sillao, 115 unimaculatus, 115 uninotatus, 115. 253

Meunier, le, 381

Microptére Dolomieu, 159

Micropterus, (Lacép.) 159

Microstoma, (Cuv.) 391

Micrura, $(B l)$.

Miller's-thumb, the, 280

Mobular, 617

Mocharra, in South America, 170

Molinesia, (Lesueur.) 388 latipinna, 388

Molle, 488

Monacanthus, (Cuv.) 573

Monk-fish, 640

Monocentris, (Bl.Schn.) 147 of Bloch, 287 Japonica, 148

Monoceros, (Bl. Sch.) 216

Monochir, (Cuv.) 498 théophile, 499

Monodactyle falciforme, 177

Monopterus, (Com. \& Lacép.) 529 Javanensis, 529

Mookalee parah, 191

Mookarrah tenkée, 616

Moon-fish, the, 200

Moringa, of the Antilles, 527

Mormyrus (L.) 397

anguilloïdes, 398

bané, 399

de Belbey's, 399

cannume, 398

caschive, 398

cyprinoïdes, 399

de Denderah, 398

dorsalis, 399

d' Hasselquist, 398

labiatus, 399

oxyrinque, 398

de Salheyhe, 399

Morrhua, 483

Motella, (Cuv.) 487

AEglefinus, 509
Mourines, the, x. 615

Mucu, the, 208

Mud-fish, 388

Mugil, (Lin.) 222

the genus, 365

albula, 224. 367

appendiculatus, 225. 443

auratus, 224

cæruleo-maculatus, 225

capito, 224

cascasia, 225

cephalus, 223. 365

chanos, 225

chelo, 225

Christian, 225

cinereus, 225

cirrhostomus, 225

corsula, 224

of Forsk, 224

crenilabis, 225

Ferrandi, 225

labeo, 225

lineatus, 224

niger, 226

parsia, 225

peradak, 225

Plumieri, 224

saliens, $\mathbf{3 6 7}$

salmoneus, 225. 443

saltator, 224

Mugiloïdes, the, 222

Mugilomore, Ann Caroline, 255. 443

Mullet, the mountain, 367

Mullets, king of the, 107

Mullers, (Lin.) 131

Mullus, (properly so called,) 132

barbatus, 132. 277

bifasciatus, 132

imberbis, 107

multibande, 132

Russellii, 132

surmuletus, 132. 278

trifasciatus, 132

vittatus, 132

Muræna, (L.) 522

(Lacép.) 523

(Thunb.) 526

the genus, 539

anguilla, 524

annulata, 526

Balearica, 525

cæca, 529

Cassini, 525

catenatus, 528

Christini, 528

colubrina, 522. 526

conger, 524. 554

the common, 527. 546

favaginea, 527

A a 2 
Muræna fasciata, x. 526 grisea, 547

Helena, 527. 546

longicollis, 524

maculosa, 526

Meleagris, 527

moringa, 527

myrus, 524, 525

mystax, 525

nebulosa, 528

nigra, 525

ondulé, 528

ophis, 526

patherina, 527. 547

picta, 527

pintade, 527

pratbernon, 527

proper, 546

punctata, 527

saga, 528

savanna, 525

serpens, 526

sordida, 528

strongylodon, 525

talabou, 525

unicolor, 527

zebra, 528

Murænoïdes, (Lacép.) 232

Murænophis, (Lacép.) 526, 527

saga, 528

colubrina, 526

Murenophis étoilé, 528

$$
\text { gris, } 527
$$

Mustela fusca, 487 maculata, 487

Mustelus, (Cuv.) 601

Muttah, (Russell,) 222

Myletes, (Cuv.) 427

brachypomus, 427

duriventris, 427

Hasselquistii, 428

macropomus, 427

paco, 427

rhomboïdalis, 427

Myliobatis, (Dum.) 615

bovina, 616

marginata, 616

Myripristris, (Cuv.) 124

Jacobus, 124

Japonicus, 124

parvidens, 124

Mysticetus, of Aldrovandus, 490

Mystus, (Arted. \& Lin.) 402

Myxine, (L.) 620

glutinosa, 622

Myxodes, (Cuv.) 231

Nallatemerce, 6. 11

Naseus, the genus, 359
Naseus brevirostris, $\mathrm{x} .216$

Carolinarum, 216

(Commer.) 216

fronticornis, 216

incornis, 216

tandock, 216

tuber, 216

tumifrons, 216

Nason loupe, 216

Natoo, (Russ.) 442

Naucrates, (Rafin.) 189

the genus, 352

ductor, 352

$\mathrm{Nez}$, le (French), 600

Niphons, (Cuv. \& Val.) 106

Niphon spinosus, 106

Niqui, of Pison, 250

Nettasoma melanura, 528

Nomeus, (Cuv.) 193

Maurit:, 193

with what confounded, 193

Norée nalaka, 495

Nonnat, what called, 227

Notacanthus, 354

(Bl.) 192

nasus, 192

Notaptère kapirat, 441

Notemigonus, (Rafin.) 438

Notidanus, (Cuv.) 602

Notistium, (Herman), 188

Notoptère capirat, 534 fontanes, $\mathbf{5 3 7}$

Notopterus, (Lacép.) 440

Novacula coryphæna, 261

Oblada, (Cuv.) 168, 320 communis, 168

Odax, (Cuv.) 265

Odontognathe aiguillonné, 440

Odontognathus, (Lacép.) 439

Oligopodus, (Lacép.) 206

Officier, l', 484

Olistus, (Cuv.) 197

Olivaceus, (Brunn.) 257

Ombrina Fournieri, 155

Omalius de Hallois, 623

Ompok siluroïde, 402

Opah, of Pennant, the, 200

Ophicephali, the, 364

Ophicephalus auranticus, 222

(Bl.) 221

barca, 222

chena, 222

lata, 222

gachua, 222

marginatus, 222. 364

marulius, 222

punctatus, 222

sola, 222 
Ophicephalus sowara, x. 222 striatus, 222

Ophidie, chinoise, 211

Ophidium, (L.) 535 aculeatum, 191

barbatum, 536, 537

blacodes, 537

brevibarbe, 536

dentatum, 537

glesne, 211

imberbe, 537

ocellatum, 537

Vassali, 536

virile, 537

Ophiognathus, (Harwood,) 531 ampullaceus, 531

Ophis hyala, 526 long-museau, 526

Ophisurus, (Lacép.) 525

atternan, 526

guttatus, 526

ophis, 526

Opistognathus, (Cuv.) 233

Sonnerati, 233

Orcynus, (Cuv.) 183

Oreosoma, the, 290

(Cuv.) 150

Orphie, (Belone, 393

Orthagoriscus, (Schn.) 569

oblongus, 569.570

hispidus, 569

spinosus, 570

varius, 569

Osmere à bandes, 431 galonné, L', 431

Osmeri, (Lacép.) 428

Osmerus, (Arted.) 419 the sub-genus, 474

Osphromene Gal, 220

Osphromenus, (Commerson.) 219. 363 notatus, 220

olfax, 220

vittatus, 220

Osteoglossum, (Vandellii) 448

Osteorinque fleurieu, I07

Ostracion, (L.) 575

arcus, 577

auritus, 577

bicaudalis, 576

bicuspis, 576

concatenatus, 576

cornutus, 577

cubicus, 577

diaphanus, 577

gibbosus, 577

lentiginosus, 577

nasus, $\mathbf{5 7 7}$

punctatus, 577

quadricornis, 576

stellifer, 576
Ostracion trigonus, $\mathrm{x} . \mathbf{5 7 6}$

triqueter, 576, 577

tuberculatus, 577

turritus, 577

Otolithi, the, 152

Otolithus, 299

(Cuv.) 152

guatucupa, 152

regalis, 152

rhomboïdalis, 152

ruber, 152. 300

striatus, 152

versicolor, 152

Otter pike, of the English, 126

Ovum, Commersonii, 569

Ovoïde fusée, 569

Oxycephas scabrus, 490

Pacu, (Spix.) 424

Pagellus, 315

(Cuv.) 164

ery thrinus, 316

Pagrus, 164

acarne, 165

bogaraveo, 165

calamus, 164

centrodontus, 165

erythrinus, 165

lithognathus, 164

mormyrus, 165

penna, 164

totus argenteus, 322

vulgaris, 164. 315

Palasorrah, the, 600

Pamples, what called, 202

Panieri, 218

Paningu, (Renard,) 215

Pantouflier, (Res.) 607

Panura, (Marcgr.) 230

Paralepis, (Cuv.) 131

Parring, of the Moluccas, 445

Parrot-fishes, 264

Pastinaca, (Adans.) 613

grabatus, 615 runsua, $6 \mathrm{I} 5$

Pêche lait, the, 193 naire, of Pondicherry, 103 pierre, 152. 299

Pectorales pediculati, 245

Pedakomé, (Russell,) 439

Pedda mottah, 189 poorawah, 442

Pegasus, (L.) 561 draco, 562

laternarius, 562

natans, $\mathbf{5 6 2}$

volans, 562

Pégouze, (Rondel.) 498 
Pégouze, of Risso, x. 499

Peis-rei of Languedoc, 294

Pei S. Pierre, 518 poure, 517

Peisrey of Languedoc, 151

Pelates, (Cuv.) 121 quinque lineatus, 121

Pelor filamentosum, 285 horrida, 285

Pelors, 146 obscurum, 147

Pempheris, (Cuv.) 179 the genus, 328 mangula, 180 Molucca, 180 touea, 179

Pennatula filosa, 187

Pentapoda, 166

Pentapus vittatus, 318

Peprilus, (Cuv.) 202 crenulatus, 203 Kurtus allied to, 203

Perca, (Cuv.) 102 (Linnaus), 101 acerina, 119 acuta, 102

Antarctica, 194 asper, (Lin.) 105 aurata, 159

bilineata, 105

bimaculata, 262

cabrilla, 110

ciliata, 102

cernua, (Lin.) 116

chrysoptera, 156

cirrhosa, 143

cottoïdes, 146

diagramma, 158

elongata, 103

flavescens, 102

fluvialis, 102

fluviatilis (Perch), 271. 574

formosa, 156

gigas, (Gm.) 112

gracilis, 102

granulata, 102

grunniens, 157

guttata, 112

juba, 157

labrax, (Lin.) 102. 273

lineata, 107

lucio-perca, 109. 274

maculata, 112

marginata, 102

marina, 110. 144

Mitchilli, 103

nigra, 205

Nilotica, 103. 273. 294

Norvegica, 144

ocellata, 152
Perca pertusa, x. 158

picta, 158

Plumieri, 102

prognathus, 117

pusilla, 199

saltatrix, 194

saxatilis, 103

scandens, 218

Schraitzer, 116

scriba, 110

sorrato granulata, 102

trifurca, 117

undulata, 153

unimaculata, 163

varia, 117

variabilis, 144

Volgensis, 100

zingel, 105

Perch, the, 101

black, 117

cerebellum of, 72

the common, 102

cranium of the, 55

the pond, 119

the, (properly called so,) 102

sea, (commonly called,) 110

triple-tailed black, 306

Percha pulchella, 124

Percis, (Bl. Sch.) 126

cancellata, 127

colias, 127

cylindrica, 127

maculata, 127

ocellata, 127

semifasciata, 127

Percoiddes, the, 101

the abdominal, 129

genera linked to the, 19

the jugular, 125

supplement on, 270

thoracic, 101

Percophis, (Cuv.) 127

Brasilianus, 127

Fabre, 127

Periopthalmus, (Schn.) 239

papilio, 243

ruber, 240

Peristedion, (Lacép.) 137

Perro colorado, 257

Persèque pentacanthe, 105 triacanthe, 105 vanloo, (Risso) 296

Pesce balestra, 571

Pes-Espada, of Monte Video, 347

Petromyzon, (L.) 618. 657

argenteus, 620

branchialis, 620.623

cirrhatus, 622

fluvialis, 620

marinus, 620 
Petromyzon planeri, x. 620.623 rouge, 623 sucet, 620

Phaëton æthereus, 42

Phalangista, (Pall.) 140

Phalangistes acipenserinus, 140 fusiformis, 140 loricatus, 140

Pharyngeals, labyrinthiform, 360

Pholis, (Artedi) 230

Phycis, (Art. \& Schn.) 488 of the ancients, 236 blenoïdes, 489 fusca, 489 Mediterraneus, 488 ranina, 489 tinca, 488, 489

Piabucus, (Cuv.) 425

Picarel commun, le, 169 raillard, 170

Pike, the, 460 of America, 104 of Chalons, 461 the common, 389 frog, 464, 465 length of the, 463 perch, the, 109. 274

Pikes, king of the, 465

Pilot, the, 352 the common, 194

Pilot-fish, 189

Piméloptère bosquien, 178

Pimelepterus, (Lacép.) 177

Pimélode aboureal, 403 scheilan, 406

Pimelodus, (Lacép.) 177 aor, 403

bilineatus, 403

biscutatus, 406

carasius, 403

carcio, 403

Cranchii, 468

conirostris, 406

Cyclopum, 468

gagata, 406

genidens, 405

Herzbergii, 405

gulio, 403

maculatus, 404

membranaceus, 406

namdia, 405

nangra, 403

octo-cirrhus, 405

pirinamp, 405

properly so called, 404

sebæ, 405

silondia, 407

synodontes, 400

Piméloptère du Cap, 178
Pimelopterus marciac, x. 178

Pinguipes, (Cuv.) 127

Brasilianus, 127

Pinnæ spuriæ, 181

Piquitinga, (Marcg.) 395. 442

Pira aca, 574

Plagiostomi (Desm.) 594

Plaice, the, 492

Platax, the, 176.326

Platessa, (Cuv.) 492. 514 achirus, 499 aquosus, 496 aramaca, 496

Argus, 497

bilineatus, 499

Borealis, 493

Boscii, 496

cardina, 496

Commersonien, 498

flesus, 514

hippoglosus, 494

hirtus, 496

jerreus, 498

lævis, 496

lascaris, 498

latus, $\mathbf{4 9 3}$

limanda, 494

limanoïdes, 495

lineatus, 499

macrolepidotus, 495. 496

maculosus, 496

mancus, 597

maximus, 495

mollis, 499

nudus, 496

ocellatus, 498

Orientalis, 498

Pan, 498

passer, 493

plagiusa, 498

platessa, 514

pola, 493

punctatus, 496

rhombus, 326. 495

Rondeletii, 491

solea, 497

trichodactylus, 499

triocellatus, 496

unimaculatus, 496

zebra, 491

Platycephalus, (Bl.) 142

dormitator, 142. 241

endractensis, 142

sexatilis, 142

sihamus, 123

spatula, 243

undecimalis, 142

Platyptera melanocephala, 244 trigonocephala, 244 
Platypterus, (Kuhl. \& Van Hasselt.) x. 244

Platystacus anguillaris, 410

(Bl.) 411

cotylephorus, 413

lævis, 413

verrucosus, 413

Plectognathi, the, 7.563

what called, 19

Plectropoma, (Cuv.) 114 puella, 114

melanoleucum, 114

Plectorynque, (Lacép.) 157

Plectropome ponctué, 114

Plesiops, (Cuv.) 263

Pleuronectes, (L.) 491

adalah, 495

arel, 499

diaphanus, 497

erumei, 495

flesus, 493

lunatus, 497

mangilii, 498

microchirus, 498

nalaka, 496

ornatus, 517

planus, 494

plagiusa, 499

plagusiæ, 499

platessa, 492

podas, 497

potoüs, 499

rhomboïdes, 497

rhombus, 516

stellatus, 494

Plotosus, (Lacép.) 409 cæsius, 410

Pœcilia, (Schn.) 387 cænicola, 387, 388

calaritana, 387

multilineata, 387

fasciata, 388

Schneideri, 387

Surinamensis, 387

unimacula, 387

vivipara, 387

Pogonate doré, 305

Pogonathe courbine, 155 doré, 154

Pogonatus auratus, 412 courbin, 412

Pogoniæ, the, 302

Pogonias, (Lacép.) 154 fascé, 154

Poisson loup, 462

St. Pierre, 356

Poissons, histoire naturelle des, 11. 12

Pole of Belon, the, 498

Pollock, the, 485. 513
Polooso-parah, x. 196

Polyacanthus, 362

(Kuhl.) 218

Polydactyle plumier, 130

Polynemus, (Lin.) 129

Americanus, 130

decadactylus, 130

enneadactylus, 130

Niloticus, 378

Paradiseus, 129

plebeius, 130

quinquarius, 129

sextarius, 130

tetradactylus, 130

uronemus, 130

Polyodon, (Lac.) 591 feuille, 591

Polyprion, (Cuv.) 116 cernium, 117

Polypterus, (Geoffr.) 449 bichir, 450

Senegalus, 450

Pomacanthus, (Cuv.) 176

Pomacentre croissant, 173 paon, 160

Pomacentrus, (Quoy \& Gaym.) 160 (Lacép.) 160 emarginatus, 160 punctatus, 160

Pomatome Skib, 194

Pomatomus, (Riss.) 107 telescope, 108

Pomolobus, (Rafin.) 438

Pomotis vulgaris, 119 (Cuv.) 119

Poorwa, (Russ.) 442

Porcus bayad, 403

Pounder, the, 443

Poure, 572

Premnas, (Cuv.) 160 unicolor, 160

Prêtre, le, 227

Priacanthus, (Cuv.) 119

Prieglers of Holstein, 332

Priodon, (Cuv.) 216 annularis, 216

Prionotes, 136

Prionure microlépidote, 215

Prionurus, (Lacép.) 215

Pristigaster, (Cuv.) 440

Cayanus, 440 tardoore, 440

Pristopoma, (Cuv.) 156 caripa, 157

coro, 157

guoraca, $15 \%$

hasta, 157

nageb, 157

paikelli, 157 
Pristis, (Lath.) x. 608 antiquorum, 608. 641 cirrhatus, 608 cuspidatus, 608 microdon, 608 pectinatus, 608 semi-sagittatus, 608

Psettus Commersonii, 177 rhombeus, 177 sebæ, 177

Pteraclis, (Gronov.) 206 velifer, 206

Pterois, (Cuv.) 144

Pyra pixanga, 112

Quiebra-acha, 191

Raasch, the, 411

Raie, fabronienne, la, $\mathbf{6 1 7}$

Raia, (Cuv.) 612 aquila, 616. 651. 653 aspera, 613 asterias, 613

Banksiana, 653 batis, 612 . 650

cephaloptera, $\mathbf{6 1 7}$

clavata, 612. 614. 649

flagellum, 616

fullonica, 613

giorna, 617

Gesneri, 614

halavi, 610

Jamaicensis, 615

Jussieui, 616

lymna, 614

maclura, 615

manatia, 653

miraletus 613

Nieuhowii, 616

narinari, 616

oculata aspera, 612

orbicularis, 615

oxyrhincus major, 612,613

pastinaca, 614.651

quadriloba, 616

rostellata, 613

rubus, 612

rudula, 613

sabina, 614

sephen, 614

timlei, 611

torpedo, 611

undulata, 613

Raie Banksienne, 617

bordée, 613

chardon, 613

chinoise, 610,611

croisée, la, (i15

Cuvier, 613

Cuv. Index.
Raie giorna, x. 617

lentillat, $61: 3$ ondée, 613 tuberculée, $\mathbf{6 1 4}$

Raii, of the Nile, 428

Ramado of Nice, the, 224

Raniceps, 489

Rason l' écluse, 261

Ray, rough, 612. 649 the sting, 614

Rays, 643

Razor-fish, 261

Regalec lancéolé, 211

Regalecus glesne, 211 maculatus, 210 remipes, 211

Requin (French), 599 à nageoires noires, 600

Rhina, (Sch.) 610 ancylostomus, 610

Rhinchobdella, (Bl. Schn.) 191 aral, 191

Rhinobatus, (Schn.) 609 Djiddensis, 609 electricus, 610 lævis, 609 rhinobatus, 610 thouin, 610

Rhinoptera, the, $\mathbf{6 1 6}$

Rhombus, 515 (Cuv.) 495 barbatus, 516 candidissimus, 497 polus, 498 maximus, 515

Rhynchobdella, 354 halepensis, 192 Orientalis, 191

Riband-fish, 206

Rinelepis aspera, 414

Roblots of Picardy, 332

Roschal, the, 429

Rouge-gueule, 305

Roussette, of Gunner, 598

Roussea at Marseilles, the $\mathbf{1 6 5}$

Rypticus, (Cuv.) 116 arenatus, 116

Sabran of Commerson, 445

Sabre of Commerson, 445

Saccopharynx, (Mitchell) 531 flagellum, 531

Sagre, the, (Brouss.) 604

Saida, the, 484

Sailors, the, 35]

Saïris hians, 394 (Rafin) 394

Salanx, (Cuv.) 392 B b 
Salarias, (Cuv.) x. 231 quadripinnis, 231

Salema, (Marcgr.) 163

Salmlet, of the English, 418

Salmo, (L.) 415. 468

Rgyptius, 430

alpinus, 418

albula, 422

anostomus, 425

argentinus, 425

Badi, 431

bimaculatus, 425,426

falcatus, 428

clupeoïdes, 422

conirostris, 431

curima, 424

cyprinoïdes, 424. 430

dentex, 428. 429

edentulus, 424

eperlanus, 420. 474

foetens, 431

fario, 418

filamentosus, 433

fasciatus, 424

Frederici, 424

gibbosus, 425

Grœnlandicus, 420

hamatus, 416

hucho, 417

internedius, 431

Lemanus, 417

marœnula, 422

marœna, 422

marmoratus, 418

melanurus, 425

Mexicanus, 431

microps, 431

minutus, 431

myops, 431

Niloticus, 428. 430

odoe, 428

ophiodon, 431

oxyrhincus, 421

peled, 423

punctatus, 418

rhomboïdes, 426

Schiefermulleri, 416

salar, 416. 468

salvelinus, 418

saurus, 431.447

sikus, 423

silus, 423

tæniurus, 424

thymallus, 421

thymallus latus, 422

truncatus, 431

trutta, 417

tumbil, 431

umbla, 419
Salmo unimaculatus, x. 424

Wartmanni, 422

Salmon, entrance into rivers of, 460 growth of, 473

maxillary bones of, 56

shoals of, 470

spawning of, 472

rapid swimming of, 471

Salmon-trout, 417

Salmone varié, 431

Salmonidæ, 415

Saltator, (Commers.) 231

Saluth of the Swiss, 401

Salviani, 162

Sandkruyper of Renard, 142

- Sansonnets, of the French, 182 of Normandy, 332

Sarda, (Cuy.) 184 of Rondelet, 185

Sar de Toulon, 310

Sardes à plumes, 164

Sardine de la Martinique, 437

Sargue de Rondelet, Le, 162

Sargus, (Cuv.) 162 annularis, 162

raucus, 162

Rondeletii, 309, 310 vulgaris, 162

Sarikulla, (Russ.) 153

Sauclet, of Languedoc, 227

Saumoneau, of the Rhine, 418

Saurel, the, 355

Saurels, 195

Saurus, (Cuv.) 430 maximus, 443

Savala, (Russell) 208

Savalli, the, 443

Savonnier, 116

Saw-fish, 641

Scampirro, of Sardinia, 336

Scarcina argyrea, 207 imperialis, 210 quadrimaculata, 210

Scare bridé, 265 denticulé, 265 ennéacanthe, 265

Scarus, (Lin.) 263 cæruleus, 265

Capitaneus, 265

Catesby, 265

chadri, 265

chloris, 265

chrysopterus, 265

coccineus, 265

Cretensis, 264

creticus, 264. 371

gallus, 220. 256

with blue jaws, great, 265

guacamaia, 265 
Scarus loro, x. 265

psittacus, 265

pullus, 266

Schlosseri, 180

spinidens, 265

tæniopterus, 265

vetula, 265

viridis, 265

Scatharus, 320

Sclerodermata, 7

Scheid of the Germans, 401

Schelfisch, (Anderson) 484

Schilbe, 402

Sciæna proper, (Cuv.) 151

the, 292

aquila, 293. 296

argyrea, 170

barbata, 302

cirrhosa, 154. 294, 295

coro, 157

cylindrica, 127

diacantha, 102

formosa, 113

fusca, 155

guterina, $\mathbf{1 5 7}$

ghanam, 159

gigas, 155

grandoculis, 164

harak, 166

hamruhr, 120

hololepidota, 298

imberbis, 152

jaculatrix, 180

Japonica, 148

jerbua, 120

kasmira, 114

lineata, 103

macrolepidota, 241

maculata, 241

mahsena, 166

Malabarica, 123

murdjan, 124

nageb, 157

nebulosa, 154

nigra, 152, 153. 294. 295

pama, 299

Plumieri, 102

sammara, 124

shotaf, 157

spinifera, 123

umbra, (Cuv.) 151. 295

undecimalis, 104

Sciéne rayée, 105

Sciænoïdes, the, 150. 291 genera linked to the, 19

Sclerodermes, $\mathbf{5 7 0}$

Scolopsides, the, (Cuv.) 159. 300

kurita, 159

lycogenis, 159
Scolopsides kate, x. 159

Schomber, the genus, $\mathbf{3 3 0}$

(Cuv.) 181

aculeatus, 191

alalonga, 183

amia, 190

atun, 186

bisus, 184

brachypterus, 183

calcar, 190

canagurta, 182

carangus, 196

chloris, 193

chrysos, 196

cobias, 182

colias, 335

Commersonii, 185

cordyla, 195

coretta, 183

crumenopthalmus, 195

dentatus, 186

dentex, 196

ductor, 189

edentulus, 200

equula, 170. 200

falcatus, 191

fasciatus, 193

Forsteri, 190

gladius, 188

glaucus, 190

grex, 182

guttatus, 185

hippos, (Lin.) 195, 196

ignobilis, 196

Kleinii, 196

lactarius, 193

lysan, 191

maculatus, 185

Malabaricus, 196

minutus, 201

niger, 189

palamitus, 185

pelagicus, 200. 205

pelamys, 183

Plumieri, 195

pneumatophorus, 182. 331

ponticus, 185

regalis, 185

rhombeus, 177

Rotleri, 195

ruber, 195

saliens, 191

sansun, 196

sarda, 184

scombrus, 181

speciosus, 196

thunina, 183

thynnus, 182. 335

trachurus, 195 
Scomber vernalis, x. 182 zonatus, 193

Scomber-esox equirostris, 394 Scomber-esox (Lacép.) 394

Scomber-esox scutellatus, 394

Schomberoïde Commersonien, 190

Schomberoïdes, the, 181. 328

Schomberomore plumier, 185

Scombre Laroche, 184

Scombrésoce campérien, 394

Scomibri, the, 181

Scopelus, (Cuv.) 432 of Pennant, 424

Scorpæna, (Lin.) 133. 143

(Cuv.) 143

Americana, 141

antennata, 145

brachion, 147

bufo, 143

Capensis, 144

carinata, 146

cirrhosa, 143

diabolus, 143

dactyloptera, 144

didactyla, 147. 285

flava, 141

horrida, 147

Kœnigii, 145

Malabarica, 144

monodactyle, 146

papillosa, 144

plumier, 144

porcus, 143. 282

scropha, 143. 281

venosa, 144

volitans, 145. 282

Scorpène aiguillonnée, la, 160

de Waigiou, 117

Scyllium, (Cuv.) 597.631

Scymnus, (Cuv.) 605

Scyris, (Cuv.) 197

Sea-devil, 246

Sea-eagle, the, 616

Sea-gudgeons, 235

Sea-horse, the, 560 of the Cape, (Dutch,) 284

Sea-locust, 283

Sea-pike, 393

Sea-snipe, 268

Sebastes, (Cuv.) 144 imperialis, 144

Norvegicus, 144

Seer-fish, what called, 345

Seepaard, the, 284

Selache, (Cuv.) 603

Selachii, 7

Selacii, 594

Selène argentée, 198 quadrangulaire, 198
Sept-œil, x. 620

Seriola, the, 354

(Cuv.) 192

bipinnulata, 193

cosmopolita, 193

Dumerilii, 354

Sériole, (Dum-Ris.) 193

de Rafinesque, 193

Serpe balbo, 432

crocodile, 432

Humboldt, 432

microstome, 391. 432

of Risso, 425

Serpens marinus compressus lividus, 186

Serpes, (Lacép.) 425 of Risso, 432

Serra-salmes, 426

Serra-salmes aureus, 426

Serra-salmes mento, 426

Serra-salmes piraya, 426

Serra-salmo chalceus, 426

Serra-salme citharine, 430

Serran bourignon, 113

Serrani, the, (properly so called,) 110

Serranus, (Cuv.) 109

altevelis, 113

bontoo, 112

cabrilla, 110

flavo-cæruleus, 113

fascicularis, 110

flavus, 110

geographicus, 113

oculatus, 111

phaëton, 113

radialis, 110

sarnicus, 276

suillus, 112

vitta, 110

Seserinus, (Cuv.) 203

Rondeletii, 203

Sey, the, 485

Shark, hammer-headed, 607. 640

basking, 638

white or common, 599. 631

fox, 635

Sharmuth, 409

Sheephead, of the Anglo Americans, 162

Sheep's-head, of the United States, 311

Siganus, (Forsk.) 214

rivulatus, 214

stellatus, 214

Sillago, (Cuv.) 122

domina, (Cuv.) 122

maculata, 123

Silurus, (L.) 399.466 anguillaris, 409

asotus, 401

ascita, 407 
Silurus aspredo, x. 413

$$
\text { attu, } 401
$$

auritus, 402

batrachus, 409

bimaculatus, 401

bagre, 404

bayad, 403

callichthys, 410

cæcutiens, 402

candiru, 402

carinatus, 408

cataphractus, 408

chinois, 401

catus, 405

clarias, 404.406

cornutus, 268. $41 \mathrm{l}$

costatus, 407

docmac, 403

the electric, 411

electricus, 411

erythropterus, 403

fasciatus, 403

fossilis, 401

glanis, 401

hemioliopterus, 404

hexadactylus, 413

imberbis, 142. 412

inermis, 407

marinus, 404

lima, 403

mystus, 402

quadrimaculatus, 405

militaris, 407

schal, 406

proper, (Lacép.) 401

Skate, the, 612.649

Smaris, the genus, 321

(Cuv.) 169

alcedo, 169

cagarella, 169

lineatus, 170

vulgaris, 169

Smelt, the, $\mathbf{4 7 4}$

Soap-maker, 116

Soffieta of Rome, 373

Sole, the common, 517

Solea, 517

cornue, 498

(Cuv.) 497

oculata, 498

Solenostoma, (Seb.\& Lacép.) 561

Somdrum-kara-mottee, 204

Soring, (Russ.) 123

Sorrakowah, 600

Spada maxima, 209

Spare Atlantique, 112

branchion, 255

breton, 170

chrysomelane, 113
Spare demilune, x. 115

hemisphère, 255

holocyanose, 265

lepisure, 114

rougeor, 265

sanguinolent, 112

sauteur, 194

Sparoides, the, 162. 303

Sparus, (Cuv.) 162

Abildgardii, 265

acutirostris, 163

argenteus, 164. 179

argyrops, 164

aurata, 163

boops, 167

berda, 164, 165

bogaraveo, 165

brama, 167

bufonites, 163

calamara, 164

castaneola, 178, 179

cantharus, 167

centrodontus, 165. 314

chærorhynchus, 166

chlorurus, 253

chromis, 261

chrysurus, 115

compressus, 328

cretus, 255

crenidens, 163

cuning, 170

cynodon, 166

dentex, 166. 317

erythrinus, 115.165. 316

fasciatus, 253

formosus, 252

haffara, 163

hasta, 163,164

insidiator of Pallas, 30. 259

macropthalmus, 166.317

mæna, 168

Malabaricus, 115

melanurus, 168

mormyrus, 165.397

ovis, 162. 311

mylio, 164

oblongus, 263

pagrus, 164. 165

pantazzo, 162

perroquet, 163

puntazzo, 163

radiatus, 169. 253

Raii, 178. 179

rayé d'or, 166

salpa, 167. 316

sarba, 163

smaris, 169

spinifer, 164

Surinamensis, 262 
Sparus synagris, $\mathrm{x} .115$ tetracanthus, 115 tricuspidatus, 169 virginicus, 157 vittatus, 157. 166

Spatularia, $(S h)$.

Sphagebranchus, $(\mathrm{Bl}$.) 528 imberbis, 529 rostratus, 529

Sphéroïde tuberculé, 568.569

Sphyræna, $(B l)$. (Bl. Sch.) 130 barracuda, 131 picuda, 131

Spyrène ouvert, 104 spet, 130

Sphyrna, (Rafin,) 606

Spinax, (Cuv.) 604

Spirobranchus Capensis, 221 (Cuv.) 221. 363

Squale bouclé, 606 glauque, 600 liche, 606

Squalus, (L.) 596 acanthias, 604

Africanus, 598

Americanus, 605

barbatus, 598

barbillon, 598

brevipinnis, 606

canicula, 597, 598. 630

carcharias, 599. 605. 631

carcharia minor, 600

catulus, 597, 598. 631

centrina, 605

ciliaris, 600

cinereus, 602

Cornubicus, 599, 600

écailleux, 606

of Edwards, $\mathbf{5 9 8}$

elephas, 603

fasciatus, 598

galeus, 601

galonné, 598

glaucus, 600

griseus, 602

lobatus, 598

longicaudus, 598

maximus, 603. 638

Monensis, 601

mustelus, 602

nez, 601

nicéen, 605

Philippi, 604

pointillé, 598

prionurus, 598

pristis, 608

punctatus, 598

spatula, 591
Squalus spinax, x. 604 spinosus, 606 squamosus, 606 squatina, 607.640 stellaris, 597, 598

tiburo, 607

tigre, 598

tigrinus, 598

ustus, 599,600

uyatus, 604

vacca, 602

vulpes, 599. 635

zygæna, 607

Squamipennes, 171

Squatina angelus, 607. 640

aculeata, 608

Dumerilii, 607, 608

Star of the night, of the Arabs, 430

Sternarchus, (Schn.) 534

Sternoptyx, (Herman.) 433

diaphana, 433

Olfersii, 434

Stickleback of the English, 287 with armed tail, 288 with naked tail, 288 small sea, 290

Stock-fisch, 486

Stolephore Commersonien, 442

Stomias, (Cuv.) 391

barbatus, 391

Schneideri, 392

Stone-bass, 170 of Jamaica, the, 171

Stromateus, $(\operatorname{Lin})$.

aculeatus, 202

albus, 202

argenteus, 202

candidus, 202

cryptosus, 203

fiatola, 202

griseus, 202

longipennis, 203

niger, 202

paru, 202, 203

stellatus, 202

Sturgeon, great, 627 outward form of the, 624

Sturgeons, the, 588. 624

Sturiones, 7. 588. 624

Stylephorus, (Shaw.) 211 chordatus, 211

Sucker, 384

Suckers, the, 617

Sudis, (Cuv.) 447

Adansonii, 448

gigas, 448

Niloticus, 448

pirarucu, 448

Surgeons, vulgar name of, 215 
Suttivara, (Rus.) x. 610

Sword-fish, habits of the, 350

Sword-fish, the common, 187

Synanceia, (Bl.Schn.) 147

bicapillata, 147

brachio, 286

cervus, 139

verrucosa, 147

Synbranchus, (Bl.) 530

immaculatus, 530

marmoratus, 531

Syngnathus, (L.) proper, 559

acus, 559

barbarus, 559

æquoreus, 560

fasciatus, 560

foliatus, 560

longirostris, 560

ophidion, 560

papacinus, 560

pelagicus, 559

Rondeletii, 559

rubescens, $\mathbf{5 5 9}$

segaliensis, 140

typhle, $\mathbf{5 5 9}$

viridis, 559

Synode chinois, 443

fascé, 443

renard, 444

Synodontis, (Cuv.) 406

Synodus erythrinus, 446

maturaque, 446

palustris, 446

synodus, 447

tareira, 446

vulpes, 446

Tacaud, the, 484. $51 \mathrm{I}$

Tænia altera, 209

falcata, 209

prima, 209

Tæniarote, large raie, 146. 263

Tænianotes, the, 144

Tænioïde Hermannien, 239

Tænioïdes, 206

(Lacép.) 239

Tafel-visch, of the Dutch, 324

Talan-parah, 196

Tasard, (Lacép.) 184

Tauche de mer, 441

Taurichtes, the, 175

Taurichthys varius, 175 viridis, 175.325

Tautoga, (Mitch.) 253

Tchawil-parah, 197

Tella katchelee, 153

Temerée, (Rus ) 611
Temnodon, (Cuv.) x. 194

saltator, 194

Tench, the common, 459

the sea, 488

Tenkée kunsu, 615 Shindraki, 614

Tetragonopterus, (Artedi) 426 argenteus, 426

Tetragonurus Cuvierii, 226 (Risso.) 225

Tetrapturus, (Rafinesque) 187

Tetraodon, (L.) 567

argentatus, $\mathbf{5 6 8}$

Commersonii, 568. 582

cutcutia, 568

electricus, 569. 584

fahaca, 568

fluviatilis, 568

geometricus, 568

Gronovii, 569

hispidus, 568. 582

Honkennii, 568

immaculatus, 568

lævissimus, 568

lævigatus, 568

lagocephalus, 568

lineatus, 567. 569.582

lunaris, $\mathbf{5 6 8}$

mola, 569

moucheté, 568

patica, 568

physa, 567, 568

Plumieri, 568

psittacus, $\mathbf{5 6 8}$

ocellatus, 568. 583

reticularis, 568

rostratus, $\mathbf{5 6 8}$

Spengleri, 568

testudineus, 568

turgidus, 568

Tetrapturus belone, 187

Therapon, (Cuv.) $\mathbf{1 2 0}$

puta, 121

theraps, 121

Theutis, javus, 2]4 hepatus, 215

Theutyes, 213

Thornback, 649

Thryssa, (Cuv.) 442

Thunder of the Arabs, the, 411

Thymallus, (Cuv.) 420

Thynnus, the genus, $\mathbf{3 3 5}$ pelamys, 343

Thyrsites atun, 345 the genus, 345 with what connected, 207

Timucu of Marcgrave, 393

Tinca, (Cuv.) 379 the, 459 
Tinca vulgaris, x. 459

Tolparah, (Russell) 191

Tomcod, the, 484

Tonilles, $(F r)$.

Torpedo, (Dum.) 610 the galvanic, 611, 642 marmorata, 611

narke, 611

the numbing, 611 unimaculata, 611

Torpille vulgaire à cinques taches, 611

Toxotes, the genus, 328

(Cuv.) 180 jaculator, 180

Trachichtys, (Shaw.) 125

Australis, 125

Trachinotus, (Lacép.) 191

Trachinus, (Lin.) 125. 138

araneus, 126

cirrhosus, 145

draco, 125. 276

lineatus, 125

radiatus, 126

trichodon, 122

vipera, 126

Trachurus aquilus, 193 imperalis, 196

Trachypterus, (Gouan.) 209 cristatus, 210

Tree-climber, 218

Triacanthus, (Cuv.) 575

Trichodon, Stelleri, (Cuv.) 121, 122

Trichogaster fasciatus, 218 trichopterus, 220

Trichonotus, (Schn.) 243 setigerus, 243

Trichopode, mentonnier, 220 trichoptère, 220

Trichopodus, (Lacép.) 220

bejeus, 218

chuna, 218

colisa, 218

cotra, 218

lalius, 218

sola, 218

Pallasii, 220

satyrus, 220

Trichopus, 363

Trichiurus, the electric, 348

Trichiurus armatus, 349

caudatus, 207

ensiformis, 207

gladius, 207

haumela, 208. 348

lepturus, 208. 347

medius, 349

muticus, 349

savala, 209. 348

Trigla, (Lin.) 133, 134
Trigla Adriatica, x. 134

aspera, 136

Carolina, 136

cataphracta, 137

cuculus, 134, 135

evolans, 136

gurnardus, 135. 279

hirundo, 134. 136. 279

lineata, 134. 136

lucerna, 136. 279

lyra, 135. 279

obscura, 136

palmipes, 136

papilio, 136

phalæna, 136

pini, 134. 278

(properly so called,) (Cuv.) 134

punctata, 136

rubicunda, 147

sphinx, 136

strigata, 136

tribulus, 136

volitans, 279

Triodon, 570

bursarius, 570

maci: tè̀re, 570

Tromietta of Genoa, 373

Trout maxiliary bones of, 56

Trumpet-fish, 373

Trygon (Adans.) 613. 651

Tub-fish, the, 279

Tubleu, of the Isle of France, 263

Tunny, cerebellum of, 72

the common, 182

the genus, 335

fishery of the, $\mathbf{3 3 8}$

striped-bellied, 183

Turbot, $\mathbf{5 1 5}$

Turdus perbelle pictus, 252

Umbra, (Cramer.) 388

Ubarana of Marcgrave, 444

Ubirre of Laet, 208

Umbrina, (Cuv.) 154. 292

of the Romans, 151

Russellii, 154

Unibranchoperturi, (Lacép.) 530

Unibranchoperture lisse, 529

Unibranchoperturus cachia, 530

Upeneus, (Cuv.) 132

Uranoscopus, (Lin.) 127

affinis, 128

cirrhosus, 128

filibarbis, 128

guttatus, 128

inermis, 128

lævis, 128

lebeck, 128 
PISCES。

Uranoscopus marmoratus, x. 128 monopterygius, 128 scaber, 128 Y græcum, 128 (Marcgr.) 156

Vackum, (Valent.) 170 mare, 170

Vadigo, 190

Vana motta, 431

Vandellius Lusitanicus, 207

Vassili-massi, 345

Vielle tachetée, 252

Vingeram, (Russell) 185

Vivanet gris, 115

Vlag-man, 324

Vogmar, of the Icelanders, 210

Vomer, (Cuv.) 197. 356 properly so called, 198

Vraie Limandelle, 493

Wachnia, the, 184

Wahla kuddera, 393

Wallagoo, (Russell) 401

Wallah, (Russ.) 445

Water-dog, the, 429

Weak-fish, the, 300

Weaver, common, 126 great, 125

Weavers, the, 125

Wels, of the Germans, 401

Whiff, of the English, 496

Whiting, the, 486. 511

Whiting, of the Anglo Americans, 154 of Bengal, 153

Wootin parah, 196

Wordawahah, (Russ.) 170

Xiphias, (Lin.) 186
Xiphias, the genus, x. 349 gladius, 187

Xiphias imperator, 187 makaira, 188 platisterus, 188 (proper) (Cuv.) $\mathbf{1 8 7}$ velifer, 188

Xiphostoma Cuvieri, 429

Xisteres, 177

Xyrichthys, (Cuv.) 260 novacula, 260

Zeus, (Linn.) (Cuv.) 199 argentarius, 201 ciliaris, 198 faber, 199 gallus, 198 guttatus, 200 imperialis, 200 insidiator, 201 luna, 200 maculatus, 201 niger, 198 proper, 356 pungio, 199 regius, 200 rostratus, 198 setapinnis, 199

Zeus sutor, 198

Zeus vomer, 198

Zeyl-vish, (sail-fish,) 352

Ziphotheca tetradens, 207

Zoarcus, (Cuv.) 233 labrosus, 234 viviparus, 234

Zygæna, (Cuv.) 606 malleus, 607. 640 nobilis, 607 tudes, 607 



\section{FOSSIL REMAINS.}

VOL. XI.

Adapis, the, Fossils, 112

Ætobates, 430

Alligator, 180, 181. 185

Alligatores, 202

Alluvial, what strata called, 24

Ammodytes, 424 tobianus, 424

Ammonicus striatus, 482

Ammonites, the, 442, \&c. 479 sphæricus, 482

Amphibia, Classis, 202

Anarrhycas lupus, 426

Anenchelum, (Blainv.) 393 Glarisianum, 393

Anguis, 282

Animal of the Ohio, 63

Animal of Paraguay, 132

Anoplotheria, the, 109. 250 proper, the, 110.

Anoplotherium, (Cuvier) 105 commune, 110 gracile, 110 leporinum, 111 medium, 110

Antracotheria of the lignites, 250

Antracotherium, the, 112

Aperea, 131

Astraïtes, 502

Astreææ, the fossil, 502

Astræa dendroïdea, 503

Astropodium elegans, 513

Auricula ringens, 463

Baculites, the, 441

Balæna, 228 fossil, 144

Balinius terebellatus, 463

Balistes, Fossirs, 404 dubius, 404

Batracian reptiles, fossil, 332

Bats, humerus of, 160

Bauman, cavern of, 13

Bears, fossil, 118

Behemoths of the Arabs, 50

Belemnites, the, 437. 444. 483 macronatus, 449

Bellerophon, 483

Birds, femora of, 157

fossil, 151

head of, 158

humerus of, 160

tibia, 159

Blennius, 423 cuneiformis, 423 ocellaris, 423

Blochius 423 longirostris, 423

Breccie, the, 447

Bronita, 495

Cachalots, the, 143

Callionymus, 423 Vestenæ, 423

Cameleons, articulation of, 156

Cameleon, head of the, 292

Campagnol, 129 hypudæus, 130

Cancer Mænas, (Lin.) 389

Carcinite, 49]

Cardia rustica, 459

Carnivora, the fossil, 117

Caryophylli, 514

Cayman, the, 177, 178. 180. 185 with bony eyelids, 190 
Cayman, the pike-muzzled, Fossils, 187

the spectacled, 188

Caymans, distinction of the, 222

Centriscus, 405 aculeatus, 405

longirostris, 405

velitaris, 405

Ceratophytes, 498

Cerithium, 489

$$
\text { gigas, } 490
$$

Cetacea, fossil, 143 teeth of, 307

Chæropotamus, the, 111

Chætodon, 417

arcuatus, 418

Argus, 418

asper, 418

aureus, 420

canescens, 419

canus, 420

chirurgus, 419

ignotus, 419

lineatus, 420

macrolepidotus, 419

mesoleucus, 419

nigricans, 419

orbis, 420

papilio, 420

pinnatiformis, 417

pinnatus, 417

rhomboïdes, 4.01

rhombus, 419

rostratus, 420

saxatilis, 419

striatus, 418

subarcuatus, 418

subaureus, 420

substriatus, 418

subvespertilio, 417

triostegus, 420

velifer, 421

vespertilio, 417,418

Champses, (Herodotus) 193

Chamses of the Ancient Egyptians, 186

Chelonia Mydas, 260

Chelonian reptiles, 260

Chelydes, coracoïd bone of the, 267

Clupea, 393. 397. 399. 410

Bernardi, 425

brevissimus, 425

cyprinoïdes, 410

Davilei, 399

dentesse, $\mathbf{4 2 5}$

dubia, 399

elongata, 399

evolans, 410

Knorrii, 399

Lametherii, 397
Clupea, megaptera, Fossils, 394

murænoïdes, 410

Scueuchzeri, 394

sprattiformis, 399

Clypeastra, 495

Cornua ammonis, 479

Coryphæna apoda, 421

hippurus, 426

Cottus, 423

bicornis, 423

Crinoïdea, 514

Crocodile, 180

Crocodile of Adanson, the black, 177, 178. 198

the American, 177

articulation of, 156

of Caen, 236

of Ceylon, 177

the common, 178. 181

the double-crested, 197

of the Ganges, 177. 200

of the Ganges, common, 175

of the Ganges, long-backed, 178

of Lacépède, 178

the lozenge, 197

of the Nile, common, 191

properly so called, 186

the sacred, 194, 195, 196

of Siam, 183

slender-muzzled, 190

Crocodiles, fossil, 174 osteology of living, 204, \&c.

Crocodili, 203

longirostres, 204

Crocodilus, (genus) 202

acutus, 203

acutus, (Cuv.) 198

Africanus, 177

Africanus of Laurenti, 178

biporcatus, 183. 197. 203

biscutatus, (Cuv.) 198. 203

cataphractus, (Cuv.) 199. 204

cazrinatus, 183

galeatus, (Cuv.) 198. 203

Gangeticus, 204

Lacerta, 178

lucius, 187. 202

longirostris, 183

longirostris, (Sch.) 200

Niloticus, (Daud.)

oopholis, 183

palmatus, 183

palpebrosus, 183. 190. 192. 202

palpebrosus, head of the, 222

pentonix, 183

priscus, 233, 234. 241. 316

porosus, 183. 197

priscus, 241

rhombifer, 197. 203 
Crocodilus sclerops, FossIls, 183. 188. 192. 212

tenuirostris, (Cuv.) 201. 204 terrestris, 177

terrestris, of Laurenti, 178

trigonatus, 183. 191. 202

vulgaris, (Cuv.) 191

Crustacea, 490 vulgaris, 203

Crustacite, 491

Cuckoo, the petrified, 152

Cyclopterus lumpus, 404

Cylindrita, 507

Cyprinus, 426

bipunctatus, 426

capito, 426

jeses, 426

squamosseus, 426

Dapedium, 392

politum, 235

Delphinus frontatus, $] 42$

Depedium politum, 390

Dichobunes, the, 110

Diluvial, what strata called, 24

Diodon, 405 orbiculatus, 405 reticulatus, 405

Dolphins, 250 fossil, 141

Dormouse, 129

Dracæna, of Lacépède, 290

Draco volans, 326

Dracona, of M. Lacépède, 327

Edentata, fossil, 131

Echinus, 495

Echinitis, 495

Echinometra, 495

Echinodermata, 495

Elasmotherium, the, 89 named by Fischer, 34

Elephant found in America, the fossil, 55

the fossil, 10. 41

carnivorous, 63

found by the Tungoose fisherman in $1799,8.52$

Elephas primigenius, 59

Elops macropterus, 397

Emydes, the, 257

Emys of Aristotle, 269

centrata, 276

expansa, 257. 273. 275,276

serpentina, 258. 273

serrata, 265. 273, 274.

Encrinus, 504
Encrinus liliiformis, fossins, 508 ramosus, 5] 1

Encrinites testudinarius, 512

Eschare, 496

Esox, 397.400. 407

acutirostris, 400

bellone, 400.407

Eislebensis, 397

falcatus, 408

incognitus, 398

longirostris, 407

lucius, 408,426

macropterus, 408

syphræna, 407

vulpes, 408

Exocœtus, 410, 411

exiliens, 411

Father of oxen, what called, 64

Felis spelæa, 127 antiqua, 127

Felinæ, fossil, 126

Fibularia, 495

Fish, flying, 410 fossil, 382

Fistularia, 407

Bolcensis, 407

Chinensis, 407

dubia, 407

petunha, 407

Flustrum, 496

Fossil balænæ, 144

batracian reptiles, 332

bears, 118

birds, 151

carnivora, the, 117

cetacea, 143

crocodiles, 174

dolphins, 141

edentata, 131

fish, 9. 382

felinæ, 126

hyæna, 124

insects, 20

lamantins, 140

mammalia, 1

man, pretended, 333

marine mammalia, 139

narwhals, 143

phocæ, 139

remains of vertebrated animals, 1. 432

reptiles, 19. 166

rodentia, 129

ruminantia, 113

saurians, 299

tortoises, 252

vegetables, 20 
Fossils, marine, fossius, 6

Fouette-queue, 178. 181

Frogs, articulation of, 156

Gadus, 423. 426

merluccius, 423

Gavial, the 178. 186

the great, 200

the little, 201

noticed by Elian, 175

Gavials, distinction of the, 222

Geckos, 291

Geosaurus, of Cuvier, 316

Gobius, 423 barbatus, 423

Smyrnensis, 409. 423

Veronensis, 423

Gryphææ, 488

Gryphites, 488

Herring, 393. 397. 399. 410

Hieracites, 151

Hipponyces, the, 440

Hippopotamus, the fossil, 10. 77 the little fossil, 82 the middle fossil, 83

Holocentrus, 414 calcarifer, 414, 415

lanceolatus, 415

macrocephalus, 415

maculatus, 415

sogho, 415

Horses, the fossil, 91

Hyæna, the fossil, 124

Hyperoodon, of M. Lacépède, 305

Hyperoodontes, the, 143

Hypudæus, the, 130

Ichthyodontes, 427

Ichthyolites, of Glaris, 385

of Iceland, $\mathbf{3 9 0}$

localities of, 388

Ichthyosaurus, the, 29. 286. 343, 344.

abundant in England, 347

communis, 351, 352. 357. 364

intermedius, 352.358

platyodon, 352. 358. 365

ribs of, 361

shoulder and sternum of, 361

tenuirostris 352. 358. 364

vertebræ of, 359

Ichth yospondyles, 426

Iguanas, 29]

Iguanodon, 31

Insects, the fossil, 493

Invertebrated animals, fossil remains of, 432

Jurassic, what strata called, 27
Koslordylos, (modern Greek,) FossiLs, 186

Krokodile noir du Niger, 191

Kurtus velifer, 421

Labrus, 416

bifasciatus, 417

ciliaris, 416,417

Julis ? 398

melapterus, 417

merula, 416

punctatus, 416

rectifrons, 417

turdus, 416

Lacerta of Linnæus, 281

agilis, 290

alligator, 178

ameiva, 284. 290. 294. 309

bicarinata, 284.290

caudiverbera, 178

crocodilus, 178

crocodilus, (Lin.) 192

dracæna, 294

Gangetica, 178. 200

gigantea, 318

monitor, 302

Nilotica, 284

teguixin, 284. 289. 294. 309. 317

Lagomys, 35, 129

Lamantins, 140. 250

Land-tortoises, 252

articulation of, 156

Lapis pentagonus, 507

Lilium lapideum, 508. 509

Lirostrites, (Lamarck) $\mathbf{4 4 0}$

Litliophytes, 497

Lizard, 186

Lizards, articulation of, $\mathbf{1 5 6}$ caudal vertebræ of, 296 osteology of existing, 281 pelvis and humerus of, 298

Loir, 129 the sternum, 297

Lophiodon, the, 94

Lophiodonta, 250

Lophius, 407 ganelli, 407 piscatorius, 407

Loricaria, 409

Lutjanus, 414 ephippium, 414 lutjan, 414

Madrepora, 515

muricata, 516

ramosa, 516 
Madrepore, Fossils, 496

Mammalia, fossil marine, 139

Mammotl, derivation of the word, 50 of the Russians, 41

Man, no fossil remains of, 21

Man, pretended fossil, 333

Manati, 433

Marine tortoise, articulation of, 156

Masasaurus, of Maestricht, 365

Mastodon, of the Cordilleras, 77 cranium of the, 71

femur of the, 72

the fossil, 10

with narrow teeth, the, 73

of Humboldt, 77

the genus, 62

the great, 62

the great, where found, 67

the little, 77

tapirosdian, 77

osteology of the great, 69

Matamata, the, 260

Megalonyx, 35. 131. 135

Megalosaurus, 318

Megalonix, remains of the, 15

Megatherium, 35.

$$
\text { (Cuv.) } 131
$$

proper, 131

Mehemoth, of the Arabs, 50

Milleporæ, 517

Monitor, of the Nile, 284

Monitors, the, 300

Monopterus, 422 gigas, 422

Mosasaurus, of Mr. Conybeare, 31. 316

Moschi, the, 208

Mouse, the domestic, 131

Mugil, 411

cephalus, 426

brevis, 411

Mullus barbatus, 426

Muræna, 424

cæca, 424

conger, 424

Murænophis, 424

Mus arvalis, 130

Muscardin, the, 131

Musk, the, 21]

Narinari, of Marcgrave, 431

Narkobatus giganteus, $\mathbf{4 0 3}$

Narwhals, fossil, 143

Naturforscher, 330

Nautilus, 481

Deshayesii, 482

imperialis, 482

pompilius, 482

umbilicatus, 482

zigzag, 481
Ombrias, Fossils, 495

Oolites, the, 445

Ophidium, 424 barbatum, 424

Orthoceratites, 483

Ostracion turritus, 404

Ostracites, 486

Ouaran, of the Arabs, 284 nose of the, 289

Ovarium, 495

Oysters, the, 486

Pachydermata, Cuvierian fossil, 99

Pæcilia, 401 of Bloch, 402 dubia, 401

Palæobalistum, 405 orbiculatum, 405

Palæoniscum, 396 Freieslebense, 396

Palæorhynchum, 393 Glarisianum, 393

Palæotheria, 250

Palæotherium, (Cuv.) 105

Aurelianense, 109

the broad-footed, 108

crassum, 107, 109

curtum, 108

the great, 107

Isselanum, 109

magnum, 107

jaw of, 79

latum, 108

medium, 107. 108

minus, 105. 108

the middle-sized, 107

the short, 108

the small, 108

the thick-footed, 107

velaunum, 10 .

Palæothrissum, 396 inæquilobum, 396

macrocephalum, 396

magnum, 396, 397

parvum, 397

Pegasus lesiniformis, 406

Pentacrinos, 507

Pentacrinus, 514

basaltiformis, $\mathbf{5 1 4}$

briareus, 514

caput Medusæ, 514

subangularis, 514

Perca, 413

Americana, 413

formosa, 413

minuta, 426

Perch, 413

Perdicites, 151 
Perna maxillata, Fossics, 457

Pesce capone, 152

Petrosilex molaris, (Millstones) 451

Phocæ, 250

$$
\text { fossil, } 139
$$

Physter, 228

Pike, 397. 400

the common, 408

Planospirites, $\mathbf{4 5 1}$

Planulites, 480

Plesiosaurus, the, 29. 343. 346. 365. bones in the, 369

carinatus, 375

of Mr. Conybeare, 371

dolichodeirus, 375

pentagonus, 376

recentior, 375

signification of, 366

trigonus, 376

Pleuronectes, 422

maximus, 398

quadratulus, 422

platessa, 395. 422

Pleurotoma dentata, 459

Polychrus, 309

Polynemus quinquinarius, 411

Polyparia, 496

Polyparies, the, 436

Polyparium, defined, 497

Polypi, 496

Porpus, supposed fossil of a, 225

Proteosaurus, 345

Pterodactyli, 230

Pterodactylus, the, 153. 326

brevirostris, 330

longirostris, 326

Rabbit, two species of the, 129

Rafcht, of the Euphrates, 269

Raia aquila, 430, 431

Reptiles, Chelonian, 260 fossil, 166

Reptile, wing-toed, 326

Rhinoceros Cuvierii, 88 entire fossil, 85

the fossil, 10. 83

of Siberia, fossil, 87

incisivus, 89

leptorhinus, 88

minutus, 89

tichorinus, 87

Rhombus minor, 397

Rodentia, fossil, 129

Rorquals, subgenus of the, 144

Ruminantia, fossil, 113

Saimiri, the, 208
Salagraman, Fossics, 480

Salamanders, articulation of, 156

Salmo cyprinoïdes, 410 muræna, 408. 410

Samiri, thc, 211

Sauri, (Ordo) 202

Saurian order, 281

Saurians, fossil, 299

Sauvegardes, (Cuv.) 284

Sciæna, 413

jaculatrix, 413

Plumieri, 414

Scomber, 411

attalunga, 411

chloris, 413

cordyla, 412.414

glaucus, 412

ignobilis, 412

Kleinii, 412

orcynus, 413

pelagicus, 412

pelamis, 411

pneumatophorus, 412

speciosus, 412

thynnus, 411

trachurus, 412

Scorpæna, 415

scrofu, 415

Scutella, 495

Sea-tortoises, 265

Sephen ray, 403

Seps tetradactylus, 156 tridactylus, $\mathbf{1 5 6}$

Serpulæ, the, 436

Silurus, 333. 409

ascita, 410

bagre, 409

cataphractus, 409

cattus, 410

glanis, 334, 335, 336

Sparus, 415

volcanus, 417

dentex, 415, 416

macrophthalmus, 415

sargus, 415

vulgaris, 415

Squalus, the, 144. 402

catulus, 403

auriculatus, 430

carcharias, 402

columbinus, 430

Cornubicus, 429

ferox, 429

glaucus, 403

grineus, 430

lamia, 430

inominatus, 402

pristodontus, 430

pristobatys dubius ? 430 
Squalus tricuspidens, FossiLs, 430 vacca, 439

Stellaria columellæ, 507

Stellerides, 496

Stellion of Linnæus, 186

Stellio uromastyx, 291

Strata, the primitive, 2 the transition, 3

Stromateus, 397. 400 gibbosus, 397 hexagonus, 397.401 major, 397

Suchis, 177

Suchus, 177. 184 of M. Geoffroi, 193

Sulcata, littoralis rostrata of Lind, 151

Sygnathus, 406 breviculus, 406 typhle, 406

Synbranchus, 424 immaculatus, 424

Tapirö̈dian Mastodon, 77

Tapirs, the gigantic, 91

gigantic, why used in the plural, 93

Tardigrada, 131

Temsach, of the modern Egyptians, 186

Terebratules, 485

Terebratula vitrea, 460

Terebratulites, the, 436

Testudo centrata, 257

clausa, 257

dorsata, 257

Europæa, 257. 258

fimbriata, 260. 262

Græca, 257

Indica, 280

Mydas, 276

picta, 257

scabra, 257

scripta, 257

triunguis, 269

virgulata, 257

Tetraodon, 404

hispidus, 405

Konckennii, 404

ocellatus, 404

Thirsé, of the Nile, 269

Thornback, 393

Tien-schu, of the Chinese, 50

Toads, articulation of, 156

Tonitru, 495

Tortoise, the great Indian, 252
Tortoise, head of the, FossiLs, 252 humerus of the, 267

Tortoises, the fossil, 252

land, 252

marine, 259

sea, 259. 265

soft, 258

Trigla, 411

cataphracta, 426

culusus, of Linnæus, 152 cyra, 411

Trionyx, the, 258. 269

Triton cristatus, 339

Trongotherium, (Fischer) $\mathbf{1 3 0}$ of Russia, 35

Trygonobatus crassicaudatus, 403 vulgaris? 403

Turtle, temporal of the sea, 354

Tubipore, $496^{\circ}$

Ursus arctoïdeus, 35. 120. 124

arctos, 120

augustidens, 36

cultridens, 36. 124

Etruscus, 124

priscus, 35.124

spelæus, 35. 120. 124

spelæus of the Germanic caves, 15, 16

Volvolæ, 507

Xiphodons, the, 110

Yacaré, of M. d' Azara, 189

Zeus, 394. 421

gallus, 420

platessa, 395, 421

reglesianus, 394

rhombeus, 422

spinosus, 395

triurus, 421

vomer, 421

Ziphius, the, 150

cavirostris, $] 43$

longirostris, 144

named by Cuvier, 33

planirostris, 143 



\title{
MOLLUSCA. ZOOPHY'TES.
}

\author{
VOL. XII.
}

Abyle, xii. 492

Acalephæ, the, 437. 481 hydrostaticæ, 489 simplices, 482. 561

Acalephæ, supplement on the, 561 umbrella of the, 562

Acamarchis, 503

Acanthocephala, (Rud.) 469

Acarda, (Brug.) 92

Acasta Montagui, 137

Acastæ, the (Leach), 137

Acephala, the, 5. 89. 369. 413 nuda, 125 salivary apparatus of, 197

Acetabulum, (Lam.) 507

Achatina, (Lam.) 32

Acmea, (Hartm.) 62

Acrostoma, (Le Sauvage) 479

Actæon, (Montf.) 63

Actinia, (L.) 494

effota, 495

equina, 495

plumosa, 495

proper, 495

senilis, 495

Actiniæ, the, 572 food of the, $\mathbf{5 7 4}$

Actinocamax, of Miller, 15

Actinocrinites, the $\mathbf{4 4 4}$

Adeona, (Lamour.) 513 grisea, 513

Equorea, 482

Agaricina, 512

Agastraria, of Blainville, 594

Agathistega, 17

Aggregata, 128

Aglaophenia, (Lamour) 502

Akera, (Müller) 45

Akera, proper, xii. 47

Alcinoë, (Rang.) 487

vermiculata, 488

Alcyonium, (Linn.) 516

asbestinum, 530

digitatum, 516

epipetrum, $\mathbf{5 1 5}$

exos., 516

mamillosum, 496

Alecto of Leach, $\mathbf{4 4 3}$

Amatia, (Lamour) 502

Ambulacraria, 541

Ammonie, (Montf.) 13

Ammonites, (Brug.) 15 (Lam.) 15

Amorpha of Blainville, 594

Amphiroë, (Lamour.) 505

Amphistoma (Rud.) 471

Ampulla carinata, 61

Ampullaria, (Lam.) 61

Ampullinæ, (Blainv.) 62

Anadiomene, (Lamour.) 506 flabellata, 506

Anambulacraria, the, 541

Ananchites, (Lam.) the, 449

Anatifa, the, 426

$$
\text { (Brug.) } 135
$$

Anatifæ, locomotion of the, 188

Anatina, (Lam.) 119

Anchasella, (Cuv.) 466

Ancillaria, (Lam.) 74 cinnamomea, 75

Anguinaria, (Lam.) 501

Animal-plants, 436. 523

Animalia radiata, 435

Animals, apathetic, 153 intelligent, 153 sensible, 153

\section{D d 2}


Animals, vertebrated, xii. 153

Anodontes, 400 (Brug.) 105

Anomia, (Brug.) 96 of Linnæus, 421 craniolaris, 133 scobinata, 132 tridentata, 20 turbinata, 133

Antennularia, (Lam.) 502

Antineæ, tentacula of the, 572

Antipathes, (Lin.) 508 spiralis, 508

Anula, (Brug.) 70

Aplysia, the genus, 351

Apiocrinites, the, 444

Aplysia, (Lin.) 43

Brasiliana, 44

depilatus, 44

fasciata, 44 punctata, 44

Apolles, (Montf.) 80

Aquilla, (Montf. 79

Arca, (Lin.) 102 cucullata, 102 pellucida, 103 tortuosa, 102

Argonauta, (Lin.) 9 Arctica, 20

Argo, 10 vitrea, 50

Argus, (Poli.) 94

Arions, the, 27

Asamæ, the (Ranzani), 137

Ascaris, 461 lumbricoïdes, 461 renalis, 462 vermicularis, 462 visceralis, 462

Ascidia, (Lin.) 128 pedunculata, 128

Aspergillium, (Lam.) 125 Javanensis, 125

Aspidogaster, (Boer.) 474 conchicola, 474

Astartæ, the, (Sowerb.) 116

Asteriæ, the, 525. 538

Asterias, (L.) 440 aurantiaca, 442 caput Medusæ, 443 discoïdea, 441 glacialis, 442 membranacea, 441 multiradiata, 443 nigra, 442 ophiura, 443 paposa, 442 rubens, 441 . tessellata, 441
Astoma, xii. 485

Astræa, 511

Atlanta, (Lesueur) 51

Keraudrenii, 51 Peronii, 51

Auricula, (Lam.) 35 myosotis, 36

Avicul̉a, 101

$$
\begin{aligned}
& \text { (Brug.) } 100 \\
& \text { the genus, } 382 \\
& \text { margaritacea, } 387
\end{aligned}
$$

Aximea, (Poli) 103

Baculites, (Lam.) 15

Balani, the, 429 locomotion of the, 188 shell of the, 430

Balanus, (Brug.) (Proper.) 136 (Lepas) tintinnabulum, 282 squamosus, 282 of the tortoise, 431

Batolithes, (Montf.) 92

Belemnites, 14

Bellerophon, (Montf.) 10

Berenice, (Peron) 485

Bernacles, the, 426

Beroë, (Müll.) 486 globular, 486 hexagone, 487 ovatus, 487

Biphora, (Brug.) 126

Biphores, the, 413

Bonellia, (Rolando) 455 viridis, 455

Botrylli, the, 417

Botryllus, (Gartn.) 129 conglomeratus, 129 stellatus, 129

Bothryocephalus, (Rud.) 477 corollatus, 478 nodosus, 557 solidus, $\mathbf{5 5 7}$ young of the, 556

Brachiella, (Cuv.) 467 Thynni, 467

Brachiocephala, locomotion of the, 189

Brachionus, (Müll.) 520

Brachiopoda, 5. 131 supplement on the, 419

Bressoïdes, $(K l)$.

Brissus, $(K l)$.

Brontis, (Montf.) 79

Buccina, (Gm.) 77

Buccinoïda, 68

Buccinum, (Brug.) 74 (Lin.) 73 arcularia, 74 caudatum, 77 
Buccinum, concholepas, xii. 76

dolium, 280

echinophorum, 77

glabratum, 74

harpa, 75

lapillus, 367

maculatum, 77

monodon, 76

olearium, 75

perdix, 75

Persicum, 76

strigosum, 77

tritonis, 61

undulatum, 74

vibex, 77

Bulimus avenaceus, 31

glans, 33

labrosus, 31

proper, $\mathbf{3 0}$

terrestris, 30

zebra, 31

Bulla, (Lin.) 34. 46

ampulla, 47

aperta, 46

carnosa, 47

ficus, 81

fontinalis, 35

hydatis, 47

hypnorum, 35

lignaria, 46

naucum, 47

ovum, 71

physis, 47

verrucosa, 71

virginea, 32

volva, 71

zebra, 32

Bullæa, (Lam.) 45 aperta, 46

Bursatella, (Blainv.) 45 Leachii, 45

Busiris, Risso, 40 griseus, 40

Byssomia, (Cuv.) 121

Cœnurus, (Rud.) 479

Calamary, the, 283. 301 prey of the, 302

Calcar, (Montf.) 54

Calceolaria, 92

Callianira, 487 didiploptera, 487

Callianyra, (Lamour.) 502

Calmarets, the, 10

Calpe, 492

Calpurnæ, (Montf.) 71

Calyptræa, (Lam.) 66

Camerines, (Brug.) 16

Campanularia, (Lam.) 501
Cancellaria, ( $\operatorname{Lam}$.$) xii. 73$

Cantharis, (Montf.) $\mathbf{5 5}$

Capillarïa, (Zeder) 460

Capsa, (Brug.) 117

Capuloida, 65

Capulus, (Montf.) 65

Cardia, the, 402

Cardiacea, 111

Cardita, (Brug.) 107

Cardium, (Lin.) 111 cardissa, 112 edule, 111. 402 rusticum, 402

Carinaria, (Lam.) 50 cymbium, 50 depressa, 50 fragilis, 50

Carybdea, (Peron) 486

Carychium myosotis, 36

Caryophyllæus, (Bl.) 471

Caryophyllia, (Lam.) 511

Cassidaria, (Lam.) 77

Cassidulus, (Lam.) 448

Caribæorum, 449

Cassiopææ, the, 485

Cassis, (Brug.) 76

Castalia, (Lam.) 107 ambigua, 107

Catillus, (Brong.) 99

Cavitaria, (Rudolphi) 458

Cavolina, (Abild.) 20 (Brug.) 40 natans, 20

Cellepora hyalina, 504

Cellularia, (L.) 503 salicornia, 503

Cephæa, (Peron) 485

Cephalopoda, the, 4, 5 branchiæ of the, 6 supplement on the, 283

Cephalophora, salivary apparatus of, 197

Cerastes, (Poli) 111

Ceratites of Haan, 15

Ceratophyta, 507

Ceratophytes, the, 504

Cercaria, (Müll.) 521

Cerebratula, (Renieri) 468

Cerithium, (Brug.) 77 atrum, 78

Cestoïdes, 480

Cestum, (Lesueur) 488 Veneris, 488

Chama, (Brug.) 110 (Lin.) 108 the genus, 401 antiquata, 107 coralliophaga, 108 cor., 111 gigas, 109 
Chama Lazarus, xii. 110 oblonga, 107

Chamacea, 108

Chicoracea, (Montf.) 79

Chimæra, (Poli) 101

Chinese-parasol, the, 352

Chiton, 368

$$
\text { (Lin.) } 88
$$

raripilosus, 282

squamosus, 282

Chondracanthe lisse, 466

Chrondracanthus, (Laroch) 467 zei, 467

Chondrosepia, (Leukard) 11

Chondrus, (Cuv.) 31

Cidaris, (Klein.) 446

Cimber, (Mont.) 66

Cineras, (Leach) 136 vittata, 136

Cirropoda, 5. 134 supplement on the, 426

Clausilia, (Drap.) 32

Clavagella, (Lam.) 125 echinata, 125

Clavatulæ, the, 80

Clavella, (Oken.) 467

Cleodora, (Péron) 20

Clio, the genus, 315

(Lin.) 18

amati, 48

Borealis, 18. 230. 316

helicina, 20. 316

Clione, the genus, 315

$$
\text { (Pall.) } 18
$$

Clithon, (Mont.) 65

Clypeaster, (Lam.) 449

Clythia, (Lamour.) 501

Cockle, the common, 402

Colombella, (Lam.) 73

Comatula, (Lam.) 443

Concholepas, (Lam.) 76

Conchology, observations on, 249, \&c.

Coniæ, the, (Blain.) 137

Conia radiata, 137

Conocarpus, (Lin.) 370

Conovulus, (Lam.) $\mathbf{3 6}$

Conulus, $(K l$. $) 448$

Conus, (L.) 69 cedonulli, 70 litteratus, 70

Coquillages, of Adanson, 147

Coral, black, 508

of commerce, 509 residence of the, 583

Coralium, (Lam.) 509

Corallina, (L.) 504 conglutinata, 506 marginata, 506 obtusata, 506
Corallina officinalis, xii. 505 proper, $\mathbf{5 0 5}$ rigens, 505 rubens, $\mathbf{5 0 5}$ tuna, 506

Corallines, the, 581

Coralliophaga, (Blain.) 107

Corallium Gothlandicum, 500

Corbis, (Cuv.) 113

Corbula, (Brug.) 117 gallica, 117

Corbule, 119

Corine, (Gartner) 497

Coriocella, (Blain.) 68

Coriocelle noire, 68

Cornua-ammonis, 15

Cornularia, (Lam.) 501

Coronulæ, (Lam.) 137

Corsican moss, $\mathbf{5 0 6}$

Crania, (Brug.) 133 antiqua, 133 personata, 133

Crassatella, (Lam.) 108

Crassinæ, the, (Lam.) 116

Crenatula, (Lam.) 99

Crepidula, (Lam.) 66

Creseis, 20

Creusiæ, the, (Leach) 137

Creusia spinulosa, 137

Cristatella, 498 mucedo, 498

Criopus, (Poli) 133

Crisia, 503

Cryptostoma, (Blain.) 68 Leachii, 68 Carolinum, 68

Cuboïdes, 493

Cucullæa, (Chemn.) 102

Cucullanus, 460 ascaroïdes, $\mathbf{4 6 4}$ lacustris, 460

Cupulites, 491

Cuvieria eurisochroma, 485

Cuvieries, 20

Cyanea, (Cuv.) 483 cærulea, $\mathbf{5 6 8}$

Cyathocrinites, the, 444

Cyclades, the, 403

Cyclas, (Brug.) 112 fluviatilis, 148

Cyclidium, 521

Cyclobranchia, the, 367

Cyclobranchiata, the, 25. 87

Cyclolithes, 511

Cyclostoma, the, 53. 354

(Lam.) 58

achatinum, 60

elegans, 281. 354

Cyclocotyles, (Otto) 473 
Cyclocotyles bellones, xii. 473

Cymba, (Sowerb.) 72

Cymbium, (Montf.) 72 what called, 308

Cymbulia of Péron, the, 19

Cymopolia, (Lam.) 505

Cypræa, (L.) 70

Cypricardia, (Lam.) 107

Cyprina, (Lam.) 113

Cyrena, (Lam.) 112

Cyrtodaria, (Daud.) 120

Cysticercus, (Rud.) 479

Cystidicola, (Fisch. Monogr.) 461

Cythereæ, the, (Lam.) 116

Cytherea lutea, 282

Dactylopora, 516

Dagysa, (Gm.) 126

Dail commun, 123

Delphinula, (Lam.) 56

Demarestia, (Lesueur) 51

Dentalium entalis, 281

Derostoma, 475

Dianchora, (Sower.) 96

Diazona, (Sav.) 130

Dibothryorhyncus, (Blain.) 477

Diceras, (Lam.) 110 arietina, 110

Didema, (Ranz.) 138

Diphyes, (Cuv.) 492

Diphyllidia, (Cuv.) 42 Brugmansii, 42

Dipsada, (Leach) 106

Discinæ, the, (Lam.) 133

Discosoma, (Rup.) 495 nummiforme, 495

Distichopora, 513

Distoma, (Retz \& Zéder) 471

Dolabella, (Lam.) 44 Rumphii, 45

Doliolum, (Otto) 487 Mediterraneum, 487

Dolium, (Lam.) 75 maculatum, 280

Donax, (Lin.) 112 rugosa, 112

Doridion Meckelii, 47

Doridium, (Meck.) 47

Doris, the genus, 349

(Cuv.) 37

affinis, 40

Argo, 37

atromarginata, 37

lacera, 37

obvelata, 37

papillosa, 39

peregrina, 40

quadrilineata, 38
Doris radiata, xii. 39

verrucosa, 37

Dynamenes, (Lamour.) 502

Eburna, (Lam.) 74

Echinanthus, (Klein.) 449

Echini, locomotive apparatus of the, $\mathbf{5 4 2}$ proper, (Lam.) 446

Echinococcus, (Rud.) 480

Echinocyamus, (Leske) 450

Echinodermata, the, 437, 438 supplement on the, $\mathbf{5 3 7}$ apedicellata, 453

Echinorhyncus, ( $\mathrm{Gm}$.) 469 gigas, 470 hæruca, 471

Echinone, (Phelsum \& Leske) 447

Echinostoma, (Rud.) 472

Echinus, (Lin.) 445. 539 altus, 449 atratus, 447

auritus, 448

bicordatus, 449

cyclostomus, 447

decadactylus, 448

depressus, 447

esculentus, 446

hexaporus, 448

lucunter, 447

locomotion of the, 543

mamillatus, 446

miliaris, 446

nucleus, 450

orbiculatus, 450

orbiculus, 448

ovatus, 449

oviformis, 450

purpureus, (Müll.) $\mathbf{4 5 0}$

quadrifasciatus, 448

quadriradiatus, 449

rosaceus, 449

sinuatus, 447

spatangus, 450

teres, 450

tetraporus, 448

vulgaris, 448

Echion, (Poli) 97

Echiurus, 456

Egeria, (Roiss.) 113

Electra, (Lamouroux) $\mathbf{5 0 3}$

Eledon (Aristotle) 9

Emarginula, (Lam.) 87

Enallostega, 17

Enchelis, (Müll.) 521

Encrinites, the, 444

Encrinus of Ellis, 539

(Guett) 443

asterias, 444 
Entozoa, (Rudolphi) xii. 456 nomatoïdea, 458 body of the, 548 digestive apparatus of the, 551 locomotion of, 554 mouth of the, 550 residence of, 553 nervous system of, 552 nutriment of, 555 skin and muscles of the, 549 supplement on the, 547

Entrochi, 445

Eolidia, (Cuv.) 39

Eschara, (Lam.) 513

Escharæ, the, 130

Etheria, (Lam.) 100

Eucœlium, (Sav.) 130

Eucratia, 503

Eudora, (Peron) 485 moneta, 485

Eugeniacrinites, the, 445

Eunicea, (Lamour.) 508

Euryale, (Lam.) 443 palmiferum, 443

Eurybia, 20

Evomphatus, (Sowerby) 56 pentangulatus, 56

Explanaria, 511

\section{Fasciola, (L.) 471} hepatica, 472

Fasciolariæ, the, 81

Favonia, (Per.) 485

Favosites, the, 500

Festucaria, (Schr.) 471

Fibularia, (Lam.) 450

Filaria, (L.) 459 Medinensis, 459.558

Fimbria, (Megerl.) 113

Firola, (Péron) 51 gibbosa, 51 mutica, 51

Firolæ, the, 352

Firoloïdæ, the, 51

Fissurella, (Lam.) 86

Fistulana, (Brug.) 124

Flabellaria, (Lam.) 506

Flabellina, (Cuv.) 40

Floriceps, (Cuv.) 478

Flustra, $\left(L_{\text {. }}\right) 504$

foliacea, 504 verticillata, 503

Foramenifera, (D'Orbigny) 17

Foveolia, (Peron) 483

Fulgur, (Montf.) 81

Fungia, (Lam.) 510

Furcularia, (Lam.) 519

Furia infernalis, 558
Fusus, (Brug.) xii. 80

Galathæa, (Brug.) 113

Galaxaura, (Lamour.) 506

Galeæ, $(K l)$.

Galerites, (Lam.) 448

Gasteropoda, the, 5. 21 pectinibranchiata, 68 supplement on the, 322

Gastrochæna, (Spengler) 124

Gastroplax, (Blain.) 48. 352

Gastropteron Meckelii, 47

Gervillia, (Defr.) 99

Geryonia-proper, (Peron) 485

Glaucus, (Forster) 39 the genus, 350

Globites, of Haan, 15

Glossus, (Poli) 110

Glycymeris, (Lam.) 120

Gonialites, of Haan, 15

Gonium, 521

Gorgonia, (L.) 508 antipathes, 508 crassa, 508 pinnata, 508 reseda, 508

Gorgonocephala, (Leach) 443

Gryphæa, (Lam.) 94 tricarinata, 94

Guinea-worm, the, 459. 558

Gymnorynchus reptans, 480

Hæruca, $(G m)$. muris, 471

Haliotides, discus of the, 258

Halymede, (Lamour) 506

Halyotis, (Lin.) 85 proper, (Lam.) 85

Hamites, (Sowerb.) 16

Harpa, (Lam.) 75

Hectocotyle argonautæ, $\mathbf{4 7 4}$ octopodis, 474

Hectocotyles, (Cuv.) 474

Helices, the, 322. 329 detriment from, 345 eggs of the, 337 food of the, 335 locomotion of the, 331 reproduction of, 341 reptation of the, 334 respiration of the, 332 where found, 333 young of the, 338

Helicina, 53 (Lam.) 6] striata, 62

Helico-Limax, (Férus.) 30 
Helicostega, (D`Orbigny) xii. 17 ammonoïda, 17 nautiloïda, 17

Helix, 29

(Lin.) 34

aculeata, 29

ægopthalmus, 29

albella, 29

ampullacea, 61

arbustorum, 29

aspersa, 29

brevipes, 30

(Bulimus) zebra, 280

castanea, 29

cicatrosa, 29

cincta, 29

citrina, 29

cornea, 34

cretacea, 29

decollata, 30

epistylium, 29

extensa, 29

fragilis, 35

fruticum, 29

fulva, 29

fuscescens, 29

glauca, 29

globulus, 29

grisea, 29

hæmastoma, 29

hortensis, 29

Itala, 29

janthina, 64

lapicida, 29

lactea, 29

ligata, 29

limosa, 35

lucerna, 29

lucorum, 29

Lusitanica, 29

nemoralis, colour of, 162

nemorensis, 29

neritella, 62

nivea, 29

oblonga, 31

ovalis, 31

palustris, 35

the peculiar organ of the, 336

pellucida, 30

picta, 29

pomatia, 29. 339

pulla, 29

putris, 32

rapa, 29

ringens, 30

rosacea, 29

rufa, 30

scarabæus, 35

Cuv. Index.
Helix sinuata, xii. 29

spirorbis, 34

stagnalis, 35

striata, 62

terrestris, 29

turturum, 29

venusta, 29

vertigo, 31

vittata, 29

vivipara, 59

vortex, 34

Hellicostega turbinoïda, 17

Hemicardium, 112

Heteropoda, the, 25. 352

(Lam.) 49

Hexastoma, 473

Hiatella, (Daud.) 121

Himantopus, 521

Hinnita, (Defr.) 95

Hippocrenes, (Montf.) 82

Hippopus, 110 (Quoy \& Gaim.) 491

Hipponyx, (Defr.) 65

Hippurites, 92

Holostoma, (Nitzsch) 472

Holothuria, (Lin.) 450

frondosa, 452

papillosa, 453

phantapus, 451

physalis, 490

Priapus, 454

regalis, 452

spirans, 489

squamata, 452

Thalia, 127

tremula, 452

zonaria, 128

Holothuriæ, the, 544

Hortoles, of Montfort, 14

Hyalæa, the, 316

Hyalea, (Lam.) 20

cornea, 20

the body of the, 317

digestive apparatus of the, 320

foot of the, 321

mantle of the, 318

trunk and eyes of the, 319

Hydatid, the globular, 479

Hydatides, 479

Hydnophora, (Fisc.) 512

Hydra, Linn. 497

the, 575

fusca, 497

sociata, 496

viridis, 497

Hydræ, locomotion of the, 576 reproduction of the, 577

Hydræ, residence of the, $\mathbf{5 7 8}$

E e 
Hyria, (Lam.) xii. 107

Hyria rugosa, 107

Inclusa, 118

Ianira, (Oken.) 487

Inferobranchia, the, 35]

Inferobranchiata, the, 24. 41

Infundibulum, (Montf.) $\mathbf{5 5}$

Infusoria, 518

body of the, 592

homogenea, 520 supplement on the, 591

Infusoriæ, the, 438

Inoceramus, (Sowerb.) 99

Intestina, (Rudolphi) 456 cavitaria, $\mathbf{4 5 8}$ parenchymata, 458

Iridina, 106 exotica, 106

Isis, (L.) 509 aster, 444 dichotoma, 510 hippuris, 509 nobilis, 509 ocracea, 509 proper, (Lam.) 509

Isocardia, (Lam.) 110

Jania, (Lamour.) 505

Janira, (Oken.) 487

Janthina, (Lam.) 63

Janthinæ, the, 355 food of the, 357 habitation of the, 356

Judaic stones, 446

Kerona, 521

Kolpoda, 521

Laganum, (Klein.) 450

Laniogerus, (Blain.) 39 Elfortii, 39

Lanistæ, the, (Montf.) 61

Lathira, (Brug.) 80

Lavignons, the, 118

Lenticular stones, $\mathbf{1 6}$

Lepas, (Lin.) 134

anatifera, 135

aurita, 429

balanoïdes, 432

balænaris, 137

balanus, 137. 432
Lepas diadema, xii. 138. 282. 433

mitella, 136

leporina, 429

pollicipes, 135

porosus, 137

spongites, 433

Stræmii, 137

testudinaria, 431

tintinnabulum, 432

Lernæa, (L.) 465

adunca, 467

branchialis, 465

cornuta, 467

multicornis, 466

ocularis, 466

radiata, 467

triglæ, 467

unciata, 467

Leucophra, 520

Liagora, (Lamour.) 506

Licorne, (Montf.) 76

Ligula, (Bloch) 481 abdominalis, 481 cingulum, 481

Liguus, (Mon.) 32

Lima, (Brug.) 95

Limaces, eggs of the, 325

food of the, 324

mischief done by the, 327

locomotion and tentacula of the, 323

Limacina, (Cuv.) 19

Limax, the genus, 322

agrestis, 27.328

antiquorum, 27

griseus, 326

maximus, 27

noctiluca, 328

proper, 26

rufus, 27

sylvaticus, 27

tergipes, 40

Limnæa, the genus, 347 (Poli) 106

Limnææ, habits of the, 348

Limnæus, (Lam.) 34

Linguatula, (Rud.) 464

Lingula, (Brug.) 131 anatina, 131

Liorhyncus, (Rud.) 464

Lithodermis, (Cuv.) 454 cuneus, 454

Lithodomus, (Cuv.) 105

Lithophyta, 509

Littorina, (F'éruss.) 60

Lituus, of Breyn, 13

Lobaria, (Blain.) 47 quadriloba, 46

Loligo, the, 283. 301 
Loligo, (Lam.) xii. 10 ink of the, 289 media, 11 sagittata, 10 vulgaris, 10

Loligopsis, (Lam.) 10

Loricula, 503

Loripes, (Poli) 114

Lotorium, (L.) 79

Lucernaria, (Müll.) 496 auricula, 496 quadricornis, 496

Lucina, (Brug.) 114 saxorumi, 115

Lumbricus echiurus, 456 edulis, 455

Lunulites, the, 516

Lutraria, (Lam.) 119

Lymnorea, (Peron) 485

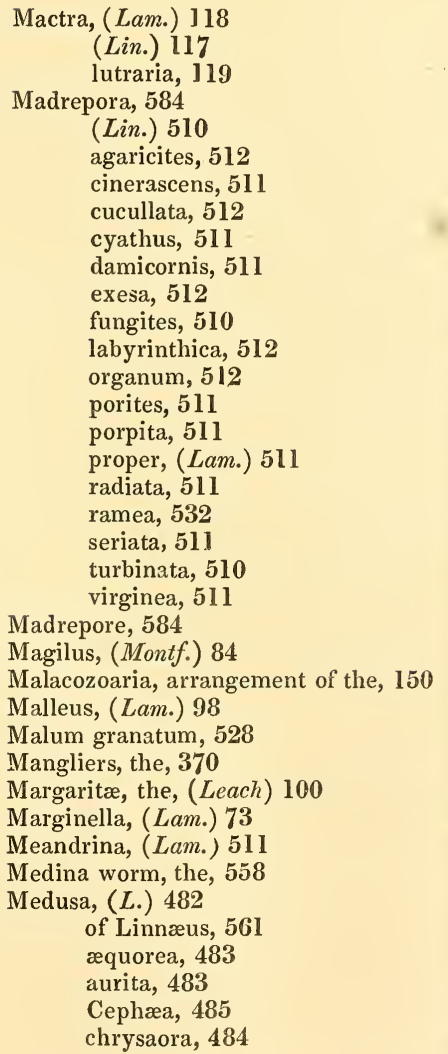

Medusa, frondosa, xii. 485 infundibulum, 487 marsupialis, $486^{\circ}$ minima, 485 mollicina, 483 pileus, 486 proboscidalis, 485 umbella, 489 velella, 489

Medusæ, food of the, 565 organization of the, 564 phosphorescence of the, 567 the tissue of the, 563

Medusariæ of M. Delaniarck, 561

Melampes, (Montf.) 36

Melania, (Lam.) 62 amarula, 62 trunctata, 62

Mélanie thiare, 62

Melanopsis, (Féruss.) 62 buccinoïdea, 62

Meleager, (Montf.) 56

Melite, (Lam.) 509

Millepora, (L.) 512 alcicornis, 513 cellulosa, 513 foliacea, 513 informis, 513 proper, (Lam.) 513 tubulosa, 504 violacea, 513

Minyas, (Cuv.) 453

Mitra, (Lam.) 73

Modiola caudigera, 105

Modiolus, (Lam.) 105

Mollusca animalia, 1 apparatus of locomotion in, 181 the aquatic, 231 aquatic pulmonaceous, 346 arteries of the, 212 brachiata, 151 circulatory apparatus of the, 213 circulating system of the, 1. 210 classification of the, 4.243 coloration of shells of, 163 definition of the, 141 diseases of the, 227 edges of the mantle of, 158 eggs of the, 219,220 food of the, 201. 203 foot of the, 182. 185 form of the, 154 generation of the, 216 gills of the, 206, 207 heart of the, 211 injury done by, 240 intestinal canal of the, 198 lamellibranch acephalous, 157 the lithophagous, 406

E e 2 
Mollusca, liver of the, xii. 200

locomotion of the bivalve, 186

mantle of the, 156

the marine, 232

motion of the bivalve, 193

movements of the acephalous, 192

muscles of the, 173

the naked, $\mathbf{3}$

nervous system of the, 221. 222

operculum of the, 175.177

organ of vision in the, 180

ovary of the, 217

peculiarity in the acephalous, 187

relation with the mineral king-

dom of, 241

reptantia, 151

respiration of the, 204, 205. 208, 209

sailing of certain, 190

sense of smelling in, 179

sense of touch in, 178

shells of the, 159

shell of the, $160-167.225-229$

shell of the acephalous, 172

skin of the, 155

stomach of the, 199

strata resulting from, 243

subsilentia, 151

supplement on the, 139

teeth of the, 171. 195

the terebrant, 406

the terrestrial, 233

tongue of the, 196

utility of the, 237, 238, 239

veins of the, 214

where found, 234, 235

Molpadia, (Cuv.) 453

holothurioïdes, 453

Monas, (Müll.) 522

Monoceros, (Lam.) 76

Monodon, (Lam.) 60

Monophora, 52

Monostoma, (Zeder.) 471

Monticularia, (Lam.) 512

Mopsea, (Lamour.) 510

Morio, (Montf.) 77

Murex, (Brug.) 78

(of Linnaus) 77, 78

Babilonius, 80

brandaris, 366

bufo, 80

cochlidium, 80

cretaceus, 79

gyrinus, 80

haustellum, 79

Islandicus, 80

lotorium, 79

Macellanicus, 79
Murex perversus, xii. 81 proper, (Montf.) 79 ramosus, 79

rapa, 81

ricinus, 76

scolymus, 81

stramineus, 80

tribulus, 79

tripterus, 79

tritonis, 79

trunculus, 366

tubifer, 79

tulipa, 81

vespertilio, 80

Muricea, (Lamour.) 508

spicifera, 508

Mussel, the common, 223

the pearl, 387

Mussels, the, 393

the fishing of, 398

the fresh-water, 400

malady from eating, 395

where found, 395

Mya, (Lam.) 119

(Lin.) 118

australis, 118

glycimeris, 120

Hispanica, 118

margaritifera, 106

pictorum, 107

siliqua, 120

truncata, 119

Mynias, cyanea, 454

Mytilacea, 104

Mytilö̈da, (Brong.) 105

Mytilus, (Lin.) 104

(L.) 393

anatinus, 106

barbatus, 105

cygneus, 106. 400

edulis, 104, 223

hirundo, 101

lithophagus, 105

margaritaceus, 387

margaritiferus, 100. 382

pholadis, 121

Nassa, (Lam.) 74

Natica, (Lam.) 64

Nautilus, (Lin.) 12 of the ancients 303

of Aristotle, 305

the papyraceous, 309

(Pliny), 9

Pompilius, 13. 311, 312, 313

properly so called, 13

spirula, 12. 280. 313

Navicella, (Lam.) 66 
Navicula, xii. 493

Nematoïdea, (Rudolphi) 458

Nemertes, (Cuv.) 468

Nemertesia, (Lamour.) 502

Nerita, (Lin.) 64

corona, 65

fluviatilis, 65

peloronta, 280

perversa, 64

pulligera, 65

turrita, 65

Neritina, (Lam.) 64

Nermertes Borlasii, 468

Nesea, (Lamour.) 505

Notarchus, (Cuv.) 45 gelatinosus, 45

Nucleolites, 447

Nucula, (Lam.) 103

Nudibranchia, the, 349

Nudibranchiata, the, 24. 36

Nullipora, 513

Nummulites, (Lam.) 16

Octhosiæ, the, (Ranzani) 137

Octopi, duration and size of the, 294 fishing for the, 293

food of the, 291

long arms of the, 290

size of the, 295 utility and injury of the, 296

Octopus, (Lam.) 8

the argonaut, 299

the genus, 289

habitation of the, 292

the navigating, 298

Oculina, (Lam.) 511

Ocyröe, (Rang.) 488 maculata, 488

Ocythöe antiquorum, 297

Oliva, (Brug.) 71 (Rafinesque) 297

littorata, 280 subuluta, 71

Olygiræ, (Say.) 62

Ombellularia, (Cuv.) 515

Ombrella, (Lam.) 352

Onchidia, the, 346 typhæ, 346

Onchidium, (Buch.) 33

lævigatum, 33 typhæ, 33

Onchidora, (Blain.) 37 (Leachii) 37

Onychotheuthis, (Lichtenst.) 11

Onykia, (Lesueur) 11

Ophiocephalus, (Quoy \& Gaim.) 468

Ophiostomus, 460 cystidicola, 461
Ophiures, (Lam.) xii. 442

Orbicula, (Cuv.) 133

Orbicula, (Lam.) 424

Orbitulites, (Lam.) 15 the, 516

Orythia, (Peron) 485

Oscabriones, the, 368 locomotion of the, 188 motion of the, 194 nervous system of the, 224

Ostracea, the, 91

Ostracita, (Le Peyr.) 92

Ostrea, (Lin.) 93

(L.) 369

cristata, 93

edulis, 93.380

folium, 93

lima, 95

malleus, 98

maxima, 94

parasitica, 93. 370

solea, 94

Otion, 429

spondyloïdea, 95

Cuvierii, 136

(Leach) 136

Ovulæ, (Montf.) 71

Ovulites, (Lin.) 516

Oxyuris, (Rud.) 460 curvula, 460

Oyster, the, 369 the common, 93

the leaf, 93

the little Mediterranean, 93

the parasite, 93 the pearl, 387

Oysters, art of greening, 377 eggs of the, 371 the green, 376 heart of the, 215 mode of parking, 373 nutritious quality of, 379 utility of, 372

Pachytes, (Defr.) 96

Padollæ, the, (Montf.) 86

Pagurus Bernhardus, 9

Paludina, (Lam.) 59

Paludinæ, the, 354

Pandora, (Brug.) 120

Panope de Faujas, 120

Panopea, (Mesnard) 120

Pantacrinus Europæus, 444

Paphia, (Roiss) 108

Paramecium, 52l

Parenchymata, 469

Parmacella, (Cuv.) 28

Olivieri, 28 
Parmacella palliolum, xii. 28 Parmophorus, (Lam.) 87 Patella, (Lin.) 88 anomala, 133 contorta, 67 cornucopiæ, 65 cymbalaria, 281 equestris, 67 fornicata, 66 Hungarica, 65 neritoïdea, 66 sipho, 67

Patellæ, the, 367

Pavonia, 512

Pavonaria, (Cuv.) 515

Pecten, (Brug.) 94

Pectines, the, 381

Pectinibranchia, the, 353

Pectinibranchiata, the, 25. 52

Pectunculus, ( $\mathrm{Lam}$.) 103

Pedicellaria, 498

Pedicellata, the, 439

Pedum, (Brug.) 95

Pelagia, 483 panopyra, 483

Pelaguses, (Montf.) 15

Peloris, (Poli) 93

Peloronta, (Oken.) 64

Penicillus, (Lam.) 505

Pennatula, 585

(L.) 513

antennina, 515

cynomorium, 515

encrinus, 515

filosa, 466

grisea, 514

juncea, 514

mirabilis, 514. 515

mode of growth in the, 586

phospliorea, 514

proper, (Cuv.) 514

reniformis, 515

rubra, 514

Pennella, (Oken.) 466 filosa, 466

Pentacrinites, the, 444

Pentalasmis, (Leach) 135

Pentastoma, (Rud.) 464

Perdix, 75 tænioïdes, 464

Perna, (Brug.) 98

Peronæa, (Poli) 112.114

Petricola, (Lam.) 117

Phasianella, (Lam.) 61

Phœnicuri, 475

Pholades, the, 406 phosphorescence of the, 409

Pholadidoïdes Anglicanus, 282

Pholas, (Lin.) 122
Pholas candida, xii. 410 dactylus, 123.282 hians, 124

Phorcynia, of Lamarck, 483

Phyline quadripartita, 46

Phyllidia, (Cuv.) 41 trilineata, 41

Phylliroe, 52

Physa, (Drap.) 35 acuta, 35

Fhysalia, (Lam.) 490

Physaliæ, the, 569 sting of the, 570

Physaloptera, 463

Physsophora, (Forsk.) 490 filiformis, 492 hydrostatica, 491 proper, (Peron.) 491

Pileolus, (Sowerby) 66 plicatus, 66

Pileopis, (Lam.) 65

Pinna, (Lin.) 101 the genus, 391 nobilis, 101

Pinnæ marinæ, 528 where found, 392

Pintadinæ, the, (Lam.) 100

Pintadina margaritacea, 387

Pirena, (Lam.) 63 terebralis, 63

Placobranchus, (Van Hasselt.) 40, 41

Placuna, (Brug.) 97

Plagiostoma, (Sowerb.) 96

Planaria, (Müll.) 474 aurantiaca, 475

Brocchii, 475

cornuta, 475 lactea, 475

Planites of Haan, 15

Planorbes, habits of the, 347

Planorbis, (Brug.) 34

Platycrinites, the, $\mathbf{4 4 4}$

Pleurobranchæa, (Meckel.) 43

Pleurobranchidium, (Blain.) 43

Pleurobranchus, (Cuv.) 42 Peronii, 43

Pleurotoma, 57 (Lam.) 80

(Murex, Lin.) Babylonia, 280

Plexaurus, (Lamour.) 508

Plicatula, (Lam.) 98

Plocameros, (Leuckard) 37 ocellatus, $\mathbf{3 7}$

Plumatella, (Bosc.) 500

Plumularia, (Lam.) 502

Pneumodermon, (Cuv.) 19 Peronii, 19

Pneumon, of Aristotle, 526

Pocillopora, (Lam.) 511 
Podopsis, (Lam.) xii. 96

Pollicipes, (Leach) 135. 429

Polpo moscardine, 307 moscarolo, 307

Polycera, (Cuv.) 37

Polyclinum, 130

Polylepe vulgaris, 282

Polyparia, 499 nantia, 513

Polyphemus, 33

Polyphysa, (Lam.) 507 aspergillum, 507

Polypi, the, 130. 438. 493.499 carnosi, 493 cellulosi, 502 the coralliferous, 581 corticati, 507 gelatinosi, 496 supplement on the, $\mathbf{5 7 2}$ tubiferi, 499

Polypus, of the Ancients, 8. 289 (Aristotle) 8.

Polystoma, (Zeder) 473 integerrimum, 473 tænioïdes, 464

Pompilus, (Pliny) 9

Pompylos of Pliny, 304

Porites, the, 511

Porpita, (Lam.) 488

Potamida, (Brong.) 78

Pompylos, Lamarckii, 78

Poteriocrinites, 444

Poulpe musqué, 9

Priapulus, (Lam.) 454

Primnoa, (Lamour.) 508

Prionoderma, (Rud.) 464

Prostoma, 475

Proteus, $(L)$. diffluens, 522

Proto, (Defr.) 57

Pterocera, (Lam.) 82

Pteropoda, the, 4.18 supplement on the, 315

Pterobrachea, (Forsk.) 50 coronata, 5]

Psammobia, (Lam.) 122

Psammothea, (Lam.) 122 violacea, 122

Psilopus, (Poli) 110

Psyche, 20

Pudenda marina, 545

Pudendum regale, 452

Pulmonaria, the, 24. 26 the aquatic, 33 the terrestrial, 26

Pulmonariæ, the, 322

Pulvinites, (Defr.) 100

Pupa, (Lam.) 31. 281

Purgamenta maris, 527, 528. 545
Purpura, (Brug.) xii. 75

(Lam.) 358

monoceros, 169

Purpuræ, colours obtained from, 363. 365

the dye obtained from, 362

habits of the, $\mathbf{3 5 9}$

many species of the, 360

mode of taking the, 362

Pyrgo, (Defrance) 21

Pyrgomæ, the, (Savig.) 137

Pyrgoma cancellata, 137

Pyrosoma, (Péron) 129

the genus, 418

Atlanticum, 130

Pyrula, (Lam.) 81

Racemides, (Cuv.) 491

Radiolites, (Lam.) 92

Ranella, (Lam.) 80

Renilla, (Lam.) 515

Retepora, (Lam.) 513

Rhizophyza, (Peron) 491

Rhizostoma proper, (Cuv.) 484 suckers of, 566

Rhizostome bleu, 484

Rhodocrinites, the, 444

Rhyncobothrium, (Blain.) 478

Ricinula, (Lam.) 76

Rissoa Freminvilii, 62

Rostellaria, (Lam.) 82

Rotella, (Lam.) 54

Rotifera, 518

Rotifère des toits, 519

Rupellariæ, the, 408

Salicornaria, 503

Salpa, ( $G m)$. democratica, 127

maxima, 127

ostofera, 128

scutigera, 127

Tilesii, 127

tricuspis, 127

Salpæ, the, 127, 413

circulation of the, 414

eggs of the, 416

nutriment of the, 415

Sanguinolaria, (Lam.) 122

Sarcinula, (Lam.) 512

Scalaria, the, 353 (Lam.) 57

Sclephites, (Sowerb.) 15

Scarabæus, (Montf.) 35

Scaturiginum, (Drap.) 35

Scelorostoma, (Blain.) 463

Scirpearia, (Cuv.) 515

Scissurellæ, (D'Orbigny) 57 
Scolex, (Müll.) xii. 480 gigas, 480

Scutella, (Lam.) 448

Scutibranchiata, thie, 25. 85

Scyllæa, (Lin.) 38 pelagica, 39

Scyllææ, the, $\mathbf{3 5 0}$

Sea-acorns, 429

Sea-anemones, 494.572

Sea-hare, the, 351

Sea-hedge-hogs, 540

Sea-nettle, 561

Sea-nettles, the, 437. 481 the fixed, 493.572

Sea-stars, the, 440.525

Sea-urchins, 445

Sepia, (Lam.) 11

(Lin. 8

the genus, 283

hexapus, 303

loligo, 10

media, 11

octopodia, 8

octopus, 297

officinalis, 12

organ of hearing in, 143

rugosa, 8

sepiola, 11

tuberculata, 12

tunicata, 303

unguiculata, 303

Sepiæ, eggs of the, 286, 287

fecundation of the, 285

fishing for the, 293

food of the, 284

Sepiola, (Cuv.) 11

vulgaris, 11

Sepiotheutes, (Blain.) 11

Septaria, (Fer.) 66

Serialaria, (Lam.) 502

Serialopora, 511

Serpula anguina, 281

Sertularia, (Lin.) 501

abietina, 502

anguina, 501

antennina, 502

chelata, 503

dichotoma, 501

eburnea, 503

hœlicina, 502

lendigera, 502

loricata, 503

Myriophyllum, 502

neritina, 503

operculata, 502

proper, 502

verticillata, 501

Ship-worm, 144

Siderolithes, the, 16
Sigaretus, (Adans.) xii. 67

Sigillina, (Sav.) 130

Siliquaria, (Brug.) 84 anguina, 281

Simplegades, (Montf.) $\mathbf{1 5}$

Siphonaria, (Sowerb.) 67

radiata, 281.

tristensis, 67

Siponculus, $(\mathrm{Gm}$.) 454

edulis, 455

levis, $\mathbf{4 5 5}$

verrucosus, 455

Sistra, (Montf.) 76

Slug, the phosphorescent, 328

Slugs, the common, 322

Snail, the common, 29

Snails, the, 322. 329 medicinal use of, 340 sense of touch in, $\mathbf{3 3 0}$

Solarium, (Lam.) 56

Sole of thie Indian Ocean, the, 94

Solemya, 120

Solen, the, 403

(Lin.) (Cuv.) 121

anatinus, 119

legumen, I22

minutus, 121

sanguinolentus, 122

strigilatus, 122

Solens, history of the, 404,405

Sormet, of Adanson, 46

Sparus raii, 483

Spatangus, $(K l)$.

brissus, 524

depressus, 448

Sphærulites, (Lameth) 92

Sphyrions, (Cuv.) 466

Spirifer, (Sowerb.) 132

Spiroptera, 463 strumosa, 463

Spirula, (Lam.) 12 Australis, 280. 313

Peronii, 313

Spondylus, (Lin.) 97

Sponge, the common, 517

Sponges, the, 587

Spongia, 587

$$
\text { (L.) } 517
$$

officinalis, 517

Star-fish, the, 440.538

Stephanomia, (Peron) 492 amphitritis, 492

Sternaspis, (Otto) 456

Strigea, (Abildg.) 471

Stromatia, (Lam.) 86

Strombus, (Lin.) 81

amplus, 82

fusus, 82

lambis, 82 
Strombus pes pelicani, xii. 82 proper, (Lam.) 82

Strongylus, (Müll) 462 armatus, 462 equinus, 462 gigas, 462

Struthiolariæ, the, 80

Stycostega, 17

Stylinæ, 512

Subsilien, what named, 403

Succinea, (Drap.) 31 amphibia, 32

Synoïcum, (Lam.) 130

Tabularia acetabulum, 507

Tænia apri, 479

bovina, 479

caprina, 479

cellulosa, 479

cerebralis, 479

cordata, 479

elliptica, $\mathbf{5 5 7}$

ferarum, 479

finna, 479

globosa, 479

lanceolé, 464

lata, 476

ovilla, 479

pisiformis, 479

utricularis, 479

vulgaris, 476

Tectaria, (Montf.) 54

Tectibranchia, the, 351

Tectibranchiata, the, 24

Telescopium, (Montf.) 55

Tellina, (Lin.) 113

cornea, 112

fluminea, 113

gari, 122

inæquivalvis, 121

lactea, 114 togata, 120

Tellinides, (Lam.) 114

Tenia vulgaris, 476 nodulosa, 477 solium, 477

Teniæ, 476

Tenioïdes, 476

Tentacularia, (Bosc.) 478

Terebellum, (Lam.) 71 subulatum, 71

Terebra, (Brug.) 77

Terebratula, (Brug.) 131

Terebratulæ, the, 316. 421 gills of the, 422 muscular apparatus of the, 423

Teredina, (Lam.) 124 personata, 124

Cuv. Index.
Teredines, the, xii. 410

habits of the, 412

Teredo, (Lin.) 123

clava, 124

injurious nature of the, 144

navalis, 123. 282

nigra, 282 palmulatus, 124

Tergipes, (Cuv.) 40

Testacea, 90 definition of, 141

Testacella, (Lam.) 28 haliotoïdea, 28

Te tribranchiata, 42

Tetralasmis, (Cuv.) $\mathbf{1 3 6}$ hirsutus, 136

Tetrarhyricus, (Rud.) 478 lingualis, 478

Thæa, (Lamour) 502

Thalassema, (Cuv.) 456 Neptuni, 456

Thalassema scutatum, 456

Thalassianthus Asterias, 495 (Ruppel,) 495

Thalia, (Brown) 126 salpa, 413

Thecidea, (Defr.) 133 Mediterranea, 133

Thethya, (Lam.) $\mathbf{5 1 7}$ (Lin.) 38 fimbria, 38.475

Theyton, of the ancients, 128

Tibiana, (Lamour) 501 fasciculata, 501

Timorienna, (Quoy \& Gaim.) 52

Tornatella, (Lam.) 63

Tremadotes, (Rud.) 471

Trianophora, (Rud.) 477

Trichocephalus, 459 acetabularis, 474 dispar, 459

Trichocera, (Lam.) 519 innata, 519

Trichoda paxillum, 519

Trichodæ, $\mathbf{5 2 0}$

Trichostomus, (Rus.) 460

Tricuspidaria, (Rud.) 477

Tridacna, (Brug.) (Lam.) 109

Trigonia, (Brug.) 103 scabra, 104

Trigonie nacrée, 103

Tristoma, (Cuv.) 473 coccineum, 473

Triton, (Lin.) 134

Tritonia, (Cuv.) 38 Hombergii, 38 elegans, 38 glauca, 38 rubra, 38 F f 
Tritonium, ( $L a m$.$) xii. 79$

Trochoïda, 54

Trophona, (Montf.) 79

Trochus, (Lin.) 54

agglutinans, 55

cinerarius, 55

concavus, 55

dolabratus, 63

foveolatus, 55

inermis, 54

Indicus, 55

iris, 55

labeo, 61

maculatus, 55

perspectivus, 56

telescopium, $\mathbf{5 5}$

tessellatus, 60

vestiarius, 54

virgatus, 55

Tubicinella, (Lam.) 137

Tubicinellæ, the, 137

Tubicolaria, (Lam.) 519

Tubipora, $(L$. $)$

catenulata, 500

musica, 500

Tubularia, (L.) 500

campanulata, 500

cornucopia, 501

coryna, 498

indivisa, 501. 530

marina, 500

Tubulibranchiata, 83

Tubulipora, (Lam.) 504

Turbinella, (Lam.) 81

Turbinolia, (Lam.) 510

Turbinolopses, (Lamour.) 511 ocracea, 511

Turbo, (Lin.) 32. 56

(Montf.) 56

the genus, 353

calcar, 54

clathrus, 58

delphinus, 57

elegans, 58

imbricatus, 57

lincina, 58

littoreus, 60

marmoratus, 353

nodulosus, 57

petholatus, 56

pica, 56

scalaris, 58

thermalis, 230

uva, 31

Turcocerca, (Lam.) 521

Turrilites, (Montf.) 16

Turritella, (Lam.) 57

Typhis, (Mont.) 79
Umbrella, (Lam.) xii. 48

Unguis odoratus, 56

Ungulinæ, the, 115

Ungulina transversa, 115

Unio, (Brug.) 106

the, 401

the genus, 386

littoralis, 106

margaritifera, 389

Urchins, 540

Urceolaria, (Lam.) 520

Urtica marina, 561

Vaginicola, (Lam.) 519

Vaginulus, (Férus.) 27

Valvata, (Müll.) 58 cristata, 59 planorbis, 59

Velata, (Montf.) 64

Velella, 489

Venericardia, (Lam.) 108

Venus, the genus, 403

(Lin.) 115

chione, 282

deflorata, 117

Dione, 116

dionea, 169

dysera, 116

exoleta, 116

fimbriata, 113

gigantea, 116

imbricata, 108

Islandica, 113

lapicida, 117

litterata, 116

meretrix, 116

monstrosa, 117

pectinata, 117

Pennsylvanica, 115

ponderosa, 108

puerpera, 116

scripta, 116

scotica, 116

tigerina, 117

Veretillum, (Cuv.) 515

Vermetus, (Adans.) 83

(Serpula) Adansonii, 281

Vermiliæ, the, 84

Vertumnus Otto, 475 thethidicola, 475

Vibrio, (Müll.) 521 aceti, 521 glutinis, 521

Vigneau, le, 60

Virgularia, (Lam.) 514

Vitrina, (Drap.) 30

Voluta, (Lin.) 34. 71 
Voluta Æthiopica, xii. 72 auris Judæ, 36 auris Midæ, 36 cancellata, 73 glabella, 73 mercatoria, 73 minuta, 36 musica, 72 musicæ, 280 proper, 72

Volvæ, (Montf.) 71

Volvaria, (Lam.) 72 bulloïdes, 72 monilis, 72

Volvox, 522

Vorticella, 498
Vorticella, of Müller, xii. 579 racemosa, 580 tetrapetala, 520

Vulsella, (Lam.) 98

Worm, the ship, 410

Worms, the intestinal, 437 the molluscous, 152 testaceous, 152

Zoanthus, (Cuv.) 495

Zoomorphoses of Guettard, 146 Zoophytes, the, $\mathbf{4 3 5}$ supplement on the, 523 



\section{AR'TICULA'TA.}

VOL. XIII.

Abranchia, the, xiii. 28. 112 asetigera, 76 distinction of, 7 setigerous, 28 without setæ, 31

Acanthonyx, (Latr.) 165

Acarides, the, 502. 529 proper, 503

Acarus, (Fab.) 505 (Lin.) 502 the genus, 529 autumnalis, 508 domesticus, 529 exulcerans, 532 farinæ, 531

hairs of the, 530 longicornis, 505. 518 marginatus, 502 passerinus, 533 proper, (Lat.) 529 reduvius, 507

ricinus, 507 telarius, 504 testudinarius, 502

Achæus, (Leach) 171 Cranchii, 171

Alciopa Renaudii, 22

Aclysia, (Aud.) 509 dytisci, 509

Acoëtes, (Aud. \& $E d w)$. Pleei, 27

Actinea, the, 11

Admetus, (Herbst.) 149

IEga, 218

Eglea, (Leach) 184

Æthra, (Leach) 173 depressa, 173

Aglaura, (Sav.) 20

A glaura fulgida, 20
Agonata, of Fabricius, xiii. 227 Agnostus, (Brogn.) 380 heteromorphous genus of, 379

Agrab, (a scorpion) 491

Albiona, (Sav.) 34 verrucosa, 34

Albunea, (Fab.) 178

Alciopa, (Aud.\& $E d w)$.

Alima, (Leach) 201 hyalina, 201

Alpheus, (Fab.) 192 elegans, 192 Malabaricus, 192 sivado, 194 thyrenus, 192

Amphictenæ, (Sav.) 14

Amphinome, the, 93 (Brug.) 18 chevelue, 18

Amphipoda, 204 order of, 315

Ampithoë, (Leach) 209

Amphitrite, (Cuv.) 14 auricoma, 14 auricoma Belgica, 14 auricoma Capensis, 15 cristata, 13 dorée, 15 infundibulum, 12 nereïs, to what belongs, 43 ostrearia, 15 pectinaria, 88 penicillus, 10 plumosa, 15 proboscidea, 10 reniformis, 12 ventricosa, 13 vesiculosa, 12 volutacornis, 12 
Amphitritea chetopoda heterocrisina, xiii. 9

Amphitrites, skin of the, 86

Amymone, (Müller) 332

Ancœus, head of the, 236

Anceus, (Riss.) 212 forficularis, 212

Animal-flower, the, 11

Annelida, the, 3. 6. the setigerous, 41 supplement to the, 37

Annelides, when first named, 48

Anomala, 177

Anthosoma, (Leach) 374 Smithii, 374

Anthura, (Leach) 222 antennæ of, 219

Antonomea, (Risso) 193 Olivii, 193

Aphrodita, (Lin.) 25 aculeata, 26. 76

cirrhosa, 26

clava, 27

hystrix, 26

lepidota, 26

plana, 27

punctata, 26 squamata, 6. 26

Aphroditæ, of Pallas, 18 blood of the, 6

Aphrodite aculeata, 67 carunculata, 18

Apoda, (Blain.) 76

Apseudes, (Leach) 211

Apus, (Scop.) 355

annulated body of, 238

canciformis, 359

Montagui, 359

vert, 359

Aquatic-fleas, what called, 270

Arachneida, egg of the, 2

Arachnida, the, 4. 131

abdomen of the, 439

body of the spinning, 440

feet of the, 442

organs of manducation of, 441

supplement to the, $\mathbf{4 3 8}$

supplement on the trachean, 510

web of the, 447

Arachnides, the, 383

the pulmonary, 385

the trachean, 385. 495

Araignée à bandes blanches, 432

à chevrons, 432

domestique à longues pattes, 412

maçonne, 399

mineuse, 399

Araignées-crabes, 398
Aranea, (Lin.) xiii. 387

aquatica, 410

avicularia, 397

avicularia, bite of, 554

cancer, 448

cementaria, 464

cicatrosa, 416

citrea, 424

conica, 417

cucurbitina, 417

diadema, 416

esuriens, 415

Florentina, 408

formicaria, 432

globosa, 424

impressa, 416

irregularis, 424

labyrinthica, $\mathbf{4 6 8}$

levipes, 422

mactans, 411

picea, 401

saccata, 428

sanguinolenta, 431

Sauvagesii, 400, 464

scalaris, 416

scenica, 432. 485

senoculata, 417

smaragdula, 419

tarantula, 428

tredecim guttata, 411

viridissıma, 419

Araneïdes, 387

bite of the, 394

body of the, 452

diving bell of the, 469

the wandering, 449

the young, 451

Araneus amentatus, 428

margaritarius, 422

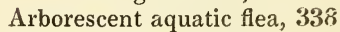

Arcania, (Leach) 162

Arcturus, (Latr.) 223 tuberculatus, 223

Arcuata, 151

Arenicola, 91

(Lam.) 17

clavata, 18

gills of the, 92

œsophagus of the, 93

piscatorum, 17

what called, 41

Argas, (Lat.) 507

perricus, 507

Argule foliacée, 370

Argulus, (Müller) 366

charon, 369, 370

delphinus, 370

foliaceus, 365

Argyroneta, (Lat.) 410 
Argyroneta, the genus, xiii. 468 aquatica, 468

Aricia, (Sav.) 24

$$
\text { Cuvierii, } 24
$$

Aristenia, (Sav.) 18

Armadillo, 225

Artemia, (Leach) 350 salina, 350

Articulated animals, the, 1

Asaphus, (Brogn.) 381 of Brogniart, 381

Aselle d'eau douce, 223

Asellota, (Lat.) 223

Asellus, (Geof.) 223

Aspidiphora, (Latr.) 355

Astaci, colour of the, 298 eggs of the, 297

fishing for, 303

food of the, 302

the fresh water, 301

ganglia in the, 244

liver of, 256

reproduction of parts in the, 299

Astacini, (Latr.) 183. 185 mandibles of the, 234

Astacus, 177

(Fab.) $\mathbf{3} 88$

the genus, 296

marinus, 176

eyes of, 246

organization of, 230

Atelecyclus, 152

Athanas, (Leach) 194

Athax, (Fab.) 507 nitescens, 194

Atoma, (Latr.) 509

Atte fourmi, 432

Attus, (Walck.) 431

Atya, (Leach) 190 scabra, 190

Atylus, (Leach) 209 carinatus, 209

Atypus, (Latr.) 401

Sulzeri, 401 rufipes, 402

Aulastoma, (Mog. Tan.) 33 nigrescens, 33

Axius, (Leach) 187 stirhynchus, 187

Bdella, (Latr.) 505 (Sav.) 33

Nilotica, 33

Binocle à queue en filet, 359 à queue en plumet, 373

Binoculus, (Geoff.) 325. 355

Bipettata, 202

Birgus, (Leach) 180
Bopyrus, (Latr.) xiii. 216

crangorum, 216

cymothoë, 319

Branchellion, 34 torpedinis, 35

Branchiarius quadrangulatus, 27

Branchiobdella, (Blain.) 34 (Odier) 33 astaci, 33

Branchiobdellion, (Rud.) 34

Branchiopoda, the, 324 what called, 241

Branchipe des marais, 352. 354

Branchipes, annulated body of, 238

Branchipus, (Lat.) 350 stagnalis, 354

Buthus, (Leach) 436 occitanus, 437

Bychorco, of the Calmucs, 514

Cænobita, (Latr.) 180

Calanus, (Leach) 333

Calappa, (Fab.) 173 fornicata, 173 granulata, 173

Caligides, (Lat.) 365

Caligus, (Müll.) 371 curtus, 372

elongatus, 372

imbricatus, 374

Mulleri, 372

piscinus, 372

productus, 374

proper, 372

risculus, 372

Callianassa, (Ireach) 187 subterranea, 187

Calymene, (Brogn.) 380

Campecopea, (Leach) 221

Camposcia 167

Cancelli, what called, 180. 304

Cancer, the, 147. 285

(Fab.) 151

of Linnæus, 137

angulatus, 156

araneus, 168

arctus, 183

articulosus, 210

asper, 164

astacus, 189

Bernhardus, 181

bimaculatus, 148

cervicornis, 167

corallinus, 152

corrugatus, 150

cristatus, 167

cursor, 158

cymodoce, 155 
Cancer dentatus, xiii. 151 dodecos, 171 dormia, 175 dromia, 175. 296. fluviatilis, 155 gammarus, 189 grossimanus, 209 spinosus, 210 germanus, 327 granulatus, 173 grassipes, 211 hexapus, 185 homarus, 304 lanatus, 174 latipes, 147. 150 latro, 180 macrouri, 287 maculatus, 152 mænas, 150. 273. 280 maia, 172

mantis, 200 marinus, 304 monoculoïdes, 206 Norvegicus, 188 organization of, $\mathbf{2 3 0}$ pagurus, 151, 152. 273 palmatus, 209 pedatus, 214 platychiles, 185 puber, 150. 273 pulex, 209 rhomboïdes, 156 rubricatus, 210 ruricola, 160. 294 salinus, 350 setiferus, 190 spinifrons, 154 squinado, 166 superciliosus, 168 symnista, 178 uca, 159 what called, 275

Canolira, (Leach) 218

Caprella, (Lamarck) 214

Carcinoïda, (Latr.) 325

Carcinion, what called, 180. 304

Carcinos potamios, 278

Carcins of Dr. Leach, 276

Cardisoma, (Latr.) 159

Carides, 189

Caris, (Lat.) 508

Carpilius, (Leach) 152

Cecrops, de Latreille, 375 (Leach) 375

Cephalothorax, (Lat.) 372

Cerapus, (Say) 210 tubularis, 210

Ceriques, the, 288 Cheliceres, (Lat.) $\mathbf{3 6 0}$
Chelifer, (Geoff.) xiii. 497 feet of the, 520 the genus, 518 cancroïdes, 518

Cheliferi, body of the, $\mathbf{5 1 9}$

Chetopoda, the, 41 arrangement of the, 49 body of the, 53 colour of the, 58 difference in the rings of, 57 digestive apparatus of, 63 distinction of the, $\mathbf{5 0}$ food of the, 74 gill of the, 55 internal organization of the, 60 intestinal caual of the, 59 rings of the, 54 liver of the, 66 mastication of the, 65 nervous system of the, 71 observations on the, 47 organization of the, 52 setæ of the, $\mathbf{5 6}$ where placed, 51

Chetopods tubicolous, 75

Cheyletus, (Lat.) 504

Chilodorus, (Leach) 347

Chirocephale diaphane, 351, 352. 354.

Chirocephalus, (Jurine) $\mathbf{3 5 0}$

Chloeia, (Sav.) 18

Chætopterus, (Cuv.) $2 \%$

Pergamentaceus, 28

Chorinus, (Leach) 166

Chrysodontes, (Aken.) 14

Cilicæa, (Leach) 221

Latreillii, 22]

Cirè apoa, 278, 288

Cirolana, (Leach) 219

Cirrhatulus (Lam.) 25 Lamarckii, 25

Cirrhinère filigère, 25

Cistenæ (Leach) 14

Citigrades, the, 425

Cladocera, (Cuv.) 337 (Lat.) 326

Clepsina, (Sav.) 35 paludosa, 35

Clitellio, (Sav.) 29

Cloportes, 318

Cloportides, (Latr.) 318

Clorodius of Dr. Leach, the, 151

Clotho, (Walck.) 404 Durandii, 405

Clubiona, (Lat.) 409

Clymena, (Sav.) 31 amphistoma, 31 Ebiensis, 31 lumbricalis, 31

Condylura, (Lat.) 328 
Condylure de Dorbigny, xiii. 328

Conilira, (Leach) 218

Contractiles, (Calymena) 381

Coronis, (Latr.) 201

Corophium, (Latr.) 211

the subgenus, $\mathbf{3 1 7}$

longicorne, 317

Corystes, (Latr.) 161

Crab, the, 147

of our coasts, common, 150

the Molucca, 361

what called, 275

Crab-spiders, bite of the, 394

Crabes boursières, 292 de paletuviers, 294

Crabs, courage of, 271

the earth, 283

the land, 158.271. 288

the painted, 289

pouched, 292

properly so called, the, 151

the violet, 288.291

the white, $288,289,290$

Crangon, 313

(Fab.) 191

vulgaris, 191

Craw-fish, 177

Crustacea, the, 3. 131

division of the, 137

egg of the, 2

eyes of the, 232

food of the, 272

generation of, 262

gills of the, 259

hearing of the, 248

heart of the, 257

local habitations of, 268

modes of motion of, 269

moulting of the, 252

muscles of the, 242

nervous system of the, 134

nutrition of the, 253

products of generation of, 265

reproduction of lost members of, 266

respiration of the, 258

sense of smell in, 249

sense of taste in, $\mathbf{2 5 0}$

skin of the, 251

stomach of the, 254

supplement on the, 226

Cryptopoda, 172

Cryptopus (Latr.) 195

Defrancii, 195

Ctenus, (Walck.) 426

Cyamus, (Latr.) 214 proper, (Lat.) 215

Cyclops, (Müll.) 329 castor, 333

Cuv. Index.
Cyclops exiliens, xiii. 328 quadricornis, 333 staphylinus, $\mathbf{3 3 2}$

Cymodocea, (Leach) 221

Cymothoa, (Fab.) 217 œstrum, 217 paradoxa, 217

Cymothoada, (Lat.) 216

Cynoræsthes, (Hermon) 506

Cynorhæstes pictus, 507

Cypris, (Müll.) 334 carapace of, 237 strigata, 335

Cytherea, (Müll.) 334

Cytherina, (Lam.) 334

Dactylocera, 206

Daphnia, (Müll.) 340 cristallina, 338 gigas, 349

longispina, 347

magna, 345

pulex, $345,346,347$

setifera, 338

sima, 340. 347

Daphnides, (Straus) 326. 337

Daphnis, carapace of, 237

Decapoda, 140 in general, 139

Decapods, brachyurous, 146 circulation of the, 141 the macrourous, 176

Decempedes, (Latr.) 211

Dentalium, 16 aprinum, 17 arcuatum, 17 dentalis, 17 elephantinum, 16 entalis, 17 fasciatum Martini, 17 habits of the, 91 rectum, 17 sexangulum, 17 striatulum, 17

Dexamine, (Leach) 210

Dichelestium, (Hermann) (Son.) 375 sturionis, 376

Dinemoura, (Lat.) 373

Diplotes hyalina, 27

Doclæa, (Leach) 168 Rissonii, 169

Dolomedes, (Latr.) 426

Dorippe, (Fab.) 174 lanata, 174

Dorsibrancliia, 17.91 distinction of, 7

Drassus, (Walck.) 406 lucifugus, 408 relucens, 408 
Drassus segestriformis, xiii. 407 viridissimus, 407

Dromia (Fab.) 175 Indica, 296

Dromiæ, the, 296

Dynamene, (Leach) 221

Dynomene, (Latr.) 175 hispida, 175

Dysdera, (Latr.) 402 erythrina, 411

Ebalia, (Leach) 162

Egeon, (Risso) 191

Egeria, (Leach) 168 Indica, 169

Elmins of Hippocrates, 39

Emerita, (Gronov.) 178

Enterion, (Sav.) 29

Entomolithus paradoxus, 378

Entomostraca, the, (Müll.) 320 antennæ of the, 322 characters of, 137

Entomostracites, what called, 380

Entomozoa apoda, 7 chetopoda, 7 what called, 95

Entomozoaria, what called, 95

Epeïra, (Walck.) 415

apolisca, 476

cicatrosa, 416. 475

conica, 417

curcubitina, 415. 417

diadema, 390. 416

diadema, cocoon of the, 444

diadema, web of the, 448

fasciata, 417

fusca, 417

scalaris, 416

sericea, 388,416

Epeïræ, the, 474

Epeïre de l' Opuntia, 418

Epibdella, (Blain.) 35

Epicarides, (Lat.) 216

Episinus, (Walck.) 411

Eresus, (Walck.) 431

Ericthus, (Latr.) 201 vitreus, 201

Eriodon, (Latr.) 402 occatorius, 402

Eriphia, (Latr.) 154

Erpobdellæ, (Blain.) 33

Eryon, (Desm.) 188 Cuvierii, 188

Erythræus, (Lam.) 503

Esox belone, 372

Eteone, (Sav.) 19. 22

Etreilles, (Cuvier) 276

Eulalia, (Sav.) 21

Eulai, what named, 38
Eulimene, xiii. 354 blanchâtre, 355

Eumolpe, (Oken) 26 maxima, 27

Eunice, (Cuv.) 19 the genus, 111 gigantea, 19 harassii, 19

Eunomia, (Risso) 21

Eupheus, (Risso) 211 ligioïdes, 211

Euphrosyne, (Sav.) 13 laureata, 18 mirtosa, 18

Eurydice, 219

Eurynoma, (Leach) 164

Exochnata, (Fab.) 176

Extensiles, (Asaphus, Ogygia, Paradoxides,) $38 \mathrm{I}$

Eylais, (Lat.) 508

Filiforma, (Latr.) 214

Filistata, (Latr.) 403

Fish-lice, 371

Flea, arborescent aquatic, 338

Fly, St. John's, 473

Galathadeæ, (Leach) 183

Galathea, (Fab.) 184 gregaria, 184 rugosa, 184

Galeodes, the genus, 510 (Oliv.) 496

Arabs, 516 araneoïdes, 511. 517 bite of the, 515 body of the, 511 dorsalis, 517 feet of the, 513 jaws of the, 512 melanus, 516 phalangium, 516 setifera, 517

Gargonali, (a scorpion,) 491

Gamasus, (Latr.) 504

Gammarinæ, (Latr.) 207

Gammarus, the genus, 315 (Fab.) 205

eyes of, 247 padatus, 214 proper, (Latr.) 209 salinus, 350

Gebia, (Leach) 186 stellata, 186

Gecarcini, the, 288

Gecarcinus, (Latr.) 160 pictus, 289 
Gelasimus, (Lat.) xiii. 157 the genus, 281

Gelasima maracoani, 282

Gelasimus pugillator, 282 vocans, 282

Geo-bdella, (Blain.) 33 trochetii, 33

Glosso-bdella, (Blain.) 35

Glossopera, (John's) 35

Glycera, (Sav.) 23 Meckelii, 23

Gnathia, (Leach) 212

Gnathophyllum, (Latr.) 192

Gomeza, new genus of, 296 bicornis, 296

Gonodactylus, (Latr.) 200

Gonoleptes, (Kirby) 501

Gonoplax, (Leach) 156

Gordius, 35 aquaticus, 36

Grancio, of the Italians, 278 Granzo, of the Italians, 279 Grapsi, the, 294

Grapsus, (Lam.) 160 cinereus, 295 pictus, 289. 294. 458 varius, 160. 295

Grimotea, (Leach.) 184

Hæmocharis, (Sav.) 34

Hæmopsis, (Sav.) 32 sanguisorba, 32

Halitheæ, (Sav.) 26

Helluo, (Oken.) 33

Hermits, what called, 305

Hesione, 24 festiva, 24 pantherina, 24 splendida, 24

Hepatus, (Lat.) 153 fasciatus, 154

Heteropa, 211

Hippa, (Fab.) 178 adactyla, 179

Hippeus, what called, 283

Hippides, (Latr.) 178

Hyppolyte, (Leach) 193

Hipponöe, (Aud. \& Edw.) 19 Gaudichaudii, 19

Hirudo, (Lin.) 31 branchiata, 27. 35

complanata, 35 grossá, 35

hippoglossi, 35

hyalina, 35

medicinalis, 32

medicinal utility of, 120
Hirudo muricata, xiii. 34 octoculata, 33 piscium, 34 sanguisuga, 32 sexoculata, 35 trioculata, 35 vittata, 34 vulgaris, 33

Holetra, 526 (Hermann) 500

Homola, (Leach) 174 spinifrons, 174

Homard, 304

Hyas, (Leach) 168

Hydrachna, (Lat.) 508 (Mill.) 507

Hydrachnellæ, 507

Hymenocera, (Latr.) 192

Hymenosoma, (Leach) 170 orbicularia, 170

Hyperia, (Latr.) 206

Hypobdella, (Blain.) 32

Hypogæon, (Sav.) 30 hirtum, 30

Ibacus, (Leach) 183 Peronii, 183

Icthyobdella, (Lamarck) $\mathbf{3 4}$

Icthyophilus, (Latr.) 217

Idotea, (Fab.) 222 aquatica, 223

Idoteïdes, (Leach) 222

Ilia, (Leach) 162 nucleus, 162

Inachus, $(F a b)$. cornutus, 166 Dorsettensis, 171 sagittarius, 171

Inequiteles, 410

Infusoria, what named, 46

Insects, the, 4. 131

Intestinal worms, what named, 39

Iöne, 207

Iphis, (Leach) 162

Isopod, the order, 318

Isopoda, 215

Ixa, (Leach) 162

Ixodes, (Latr.) 506 ricinus, 507 reflexus, $\mathbf{5 0 7}$ reticulatus, 507

Jæra, (Leach) 224 albifrons, 224

Janira, (Leach) 223 maculosa, 223

Jassa, (Leach) 211

G $\mathrm{g} 2$ 
Jassa pulchella, xiii. 211

Jatrobdella, (Blain.) 32

Kabutogani, of the Japanese, 364

Kalimus, (Latr.) 168

Karis, what named, 313

Kleistagnatha, ( $F a b)$.

Labinia emarginata, 169

Læmodipoda, 213

the order, $\mathbf{3 1 8}$

Lambrus, (Leach) 164

Land-crabs, the, 271

Larunda, (Leach) 215

Latona, 338

Leech, the, 31

food of the, 129

nervous system of the, 128

sexual organs of the, 127

the stomach of the, 125

the horse, 32

the black, 33

the body of a, 121

venous system of the, 126

Leeches, apparatus of digestion of, 124 apparatus of locomotion of, 123 the envelope of the, 122 residence of, 130

Leodice, (M. de Blain.) 19 attennuata, 19

Gallica, 19

Hispanica, 19

Lepidia, (Sav.) 21

Lepidurus, (Leach) 356

Leptomera, (Lat.) 214

Leptopodia, (Leach) 171

Leptopus, (Lam.) 169

Leptus, (Lat.) 508

the subgenus, 529

autumnalis, 508

Lernæiformes, (Lat.) 375

Leucosia, (Fab.) 161 (Leach) 162 cylindrus, 162

Leucothoë, (Leach) 210

Libinia, (Leach) 168, 169 canaliculata, 169

Ligia, (Fab.) 224

Limnadia, (Adolph. Brgn.) 348 Hermani, 349

Limnatis, (M. Moquin-Tandon) 33

Limnochares, (Lat.) 508

Limnoria, (Leach) 219 terebrans, 219

Limulæ, segmentary division of, 231 blanc, 365

des Moluques, 365
Limulus, serricaude, 359

(Fab.) xiii. 361

(Müll.) 355

cyclops, 363, 364

heterodactylus, 364

palustris, 359

Polyphemus, 365

Sowerbii, 364

tridentatus, 365

Lingyphia, (Latr.) 413 triangularis, 473

Lissa, (Leach) 165

Lithodes, (Latr.) 172

Lobster, the, 177. 304

Lobsters, gigantic, 310

Locusta, why named, 182

Lombrinère brillant, 23

Lophyropa, the, 324, 325. 337

Louvette, 506

Lucanus cervus, 236. 354

Lumbrici, 37

circulatory apparatus of, 115

dorsal vessel of the, 69

general envelope of the, 114

little utility of the, 118

mouth of the, 64

nervous system of the, $\mathbf{1 1 6}$

organization of the, 113

Lumbricus, the genus, 112

proper, (Cuv.) 28

armiger, 24

cirrhatus, 25

fragilis, 23

lineatus, 30

marinus, 17. 41.91

minutus, 29

to what restricted, 39

sabellaris, 31

squamatus, 23

terrestris, 29. 41

tubicola, 30

tubifex, 30

Lumbrinera, (Blain.) 23

Lupa, of Dr. Leach, 149

Lycastis, (Sav.) 23

Lycoris, (Sav.) 20

Egyptia, 21

nuntia, 21

Lycosa, (Latr.) 427

allodroma, 480

amentata, 481

fabrilis, 428

littoralis, 481, 482

perita, 480

ruricola, 482

of the South of France, 483

tarantula, 388. 427, 428. 480

Lycosæ, the, 480

eggs of the, 393 
Lycose Narbonnaise, xiii. 428 ouvrière, 428

Lyncæus, carapace of, $\mathbf{2 3 7}$

Lynceus, (Müll.) 347

Lysidice, (Sav.) 20

Galatina, 20

Olympia, 20

Valentina, 20

Lysmata, (Risso.) 193 seticauda, 193

Macrocheles, (Latr.) 502

Macropa, (Latr.) 185

Macropodia tenuirostris, 171 (Leach) 171

Macrophthalmus, (Leach) 156

Macrourous decapods, 296

Mæra, (Leach) 209

Maia, the, 295

(Leach) 166

longipes, 170

retuja, 167

squinado, 163. 273

taurus, 166

Malacobdellæ, (Blain.) 35

Malacostraca, characters of, 137 the natural division of, 138 with sessile eyes, the, 202 what called, 228

Marmagnato, (spider) 472

Marmignatto, (spider) 472

Marphisæ, (Sav.) 19

Matuta, (Fab.) 147

Megalope maculata, 312 sculpta, 312

Megalopus, (Leach) 185

Melanogaster, (Cuv.) 408

Melita, (Leach) 209

Meria glabra, 165

Micippus, (Leach) 166

Micrommata, (Lat.) 419 argelas, 390 smaragdina, 477

Micrommate argelas, 419 à tarses spongieuses, 420 smaragdine, 419

Micropthira, (Lat.) 508

Miclyris, (Latr.) 158

Misis, (Latr.) 195

Missulena, (Walck.) 402

Mite, the common, 529 the domestic, 529 in flour, the, 531 of the itch, 531

Mites, 502. 529

Mithrax, (Leach) 165 spinicinctus, 165

Monoculus, 215
Monoculus of Linnæus, xiii. 137. 320. 325

apus, 359

foliaceus, 370

gyrini, 371

pediculus, 339

piscinus, 372

Polyphemus, 325. 365

pulex, 347

quadricornis, 333

Salmoneus, 374

taurus, 327

Monolepis, (M. Say) 185

Muleion, (Latr.) 195

Lesueurii, 195

Mygale, 395

the genus, 556

ariana, 466

avicularia, 397. 459,460

avicularia, abode of, 462

cæmentaria, 399. 463

calpeiana, 398

cancerides, 458

cardense, 400

carminans, 399. 463

dwelling of the, 465

eggs of the, 462

fasciata, 398

fodiens, 400

the mason, 462

nidulans, 400

nipples of the, 443

poison of the, 461

recluse, 462

Sauvagesii, 400

Valentina, 398 proper, (Walck.) 396

Myriana, (Sav.) 27

Myrmecia, (Lat.) 429

fulva, 429

Mysis Fabricii, 195

Næsa, (Leach) 221

bidentata, 221

Naides, the, 119

Naïs, (Lin.) 30

cæca, 30

digitata, 30

elinguis, 30

littoralis, 30

proboscidea, 30

Rathke, 22

serpentina, 30

tubifex, 31

vermicularis, 30

Nauplius, (Müll.) 332

Naupredia, (Latr.) 241

Naxia, (Leach) 165 
Nayades, (Walck.) xiii. 409

Nebalia, 195

$$
\text { (Leach) } 327
$$

Herbstii, 328

Nebalie ventrue, (Risso.) 328

Nelocira, (Leach) 219

Nephelis, (Sav.) 33 atomaria, 33

Nephrops, (Leach) 188

Nephthys, (Cuv.) 23

Hombergii, 23

Nereides, abdominal appendages of the, 101

body of the, 97

circulation of the, 107

dorsal muscular bands of, 62

external parts of the, 96

eyes of the, 102

food of the, 110

habitation of the, 109

rings of the, 98

locomotive apparatus of the, 103

mouth of the, 104

proboscis of the, 99

teeth of the, 100

Nereïphylla, (Blain.) 21

Nereiphylle de Pareto, 21

Nereïs of Linnæus, 94

alba, 23

aphrodisoïdes, 27

armillaris, 22

Beaucoudraisii, 21

bifrons, 24

cæca, 27

conchilega, 82

crassa, 19

cuprea, 19

ebranchiata, 23

fimbriata, 20

flava, 22

gigas, 68. 105. 106

incisa, 21

lamellifera Atlantica, 21

longa, 22. 27

lumbricoïdes, 91

noctiluca, 72

Norwegica, 19

pelagica, 20. 108

pinnata, 19. 105

pinnigera, 21

prismatica, 24

proper, (Cuv.) 20

pulsatoria, 21

sanguinea, 19

stellifera, 21

tubicola, 19

verrucosa, 21

versicolor, 20

viridis, 21
Nerocila Livoneca, xiii. 217

Nhamdu, 557

Nhamdu-guaçu, (great spider, 457

Nicothoë, $(A u d . \& E d w$.$) 328. 376$ astaci, 377

Nika, (Risso.) 191 edulis, 192

Nogaus, (Leach) 373

Notaspis, (Herm.) 504

Notopoda, 173

Nursia, (Leach) 162

Nymphon, (Fab.) 499 the genus, 524

Obisium, (Illig.) 497. 519

Ocypete, (Lat.) 509

Ocypode, $(F a b)$.

the genus, 283

alba, 283

ceratophthalma, 284

rhombea, 158

cursor, 284

hippeus, 284

Enone lucida, 20

Ogygia, (Brogn.) 381

Oletère atype, 401

Oletra, (Walck.) 401

Olencira, (Leach) 218

Oniscides, (Lat.) 224. 318

Oniscoda, (Latr.) 223

Oniscus, 216. 318

of Linnæus, 137

arenarius, 211

armadillo, 225

asellus, $\mathbf{2 2 5}$

cæruleatus, 212

ceti, 215

the two cords in, 245

entomon, 222

eyes of, 247

gammarellus, 208

locusta, 208

lutosus, $\mathbf{3 7 3}$

murarius, 225

Oceanicus, 224

proper, (Lin.) 225

sylvestris, 225

thoraïcus, 207

Ophelina, (Sav.) 24

Orbiculata, 160

Orbiteles, 412

Orchestia, (Leach) 208

Oribata, (Lat.) 504

Orithyia, $(F a b$. $) 148$ mamillaris, 148

Ostracoda, (Cuv.) 333

(Latr.) 326

Ostrapoda, (Straus.) 326. 333 
Ovalia, (Lat.) xiii. 214

Oxyopes, (Lat.) 425

Ozolus gasterostei, 37]

Pactolus, (Leach) 172

Boscii, 172

feet of, 239

Pæcilopoda, 359

Paguri, eggs of the, 309 ganglia of the, 142 residence of the, 307 tail of the, 238

Pagurini, (Latr.) 179

Pagurus, the genus, 304 (Fab.) 180 clypeatus, 181 latro, 310

Palæmon, 313

(Fab.) 193

diversimana, 193

penæus, 273

serratus, 193

squilla, 319

sulcatus, 190

Palinuri, the, 310 eyes of the, 246

Palinurus, (Fab.) 183 quadricornis, 183 size of, 275

Palmyra, (Sav.) 25 aurifera, 25

Pálpimane bossu, 431

Palpimanus, (Dufour) 430

Panalus, (Leach) 193

Pandalus annulicornis, 193

Pandarus, (Leach) 373 bicolor, 373 Boscii, 373

Pan-fish, 363

Paradoxides, (Brogn.) 381

Parthenope, ( $F a b$. $) 164$ fornicata, 173

Pasiphæa, 194

Pectinariæ, (Lam.) 14

Pedipalpi, the, 432

Penæus, the genus, 314

(Fab.) 190 monodon, 190

Pericera, (Latr.) 166

Pernys, what called, 317

Perroquet d'eau, 347

Persephona, (Leach) 162

Phalangia, legs of the, 528

Phalangita, (Lat.) 500

Phalangium, 497.501

the genus, 526

araneoïdes, 516
Phalangium cancroïdes, xiii. 498 cornutum, 501.527. 529 opilio, 501

Pherusa, (M. de Blainville) 15 (Leach) 210 fucicola, 210

Philodrome tigré, 422

Philodromus, (Walck.) 421 oblongus, 423 rhombiferus, 422

Philoscia, (Latr.) 225

Pholcus, (Walck.) 411 phalangioïdes, 412 phalangista, 412

Pholque, à queue, 412

Phoxichili, oviparous feet of, 523

Phoxichilus, the genus, 522 (Latr.) 499 Phalangioïdes, 524

Phronima, 315 (Latr.) 205

Phrosina semilunata, 206

Phrosine, (Risso) 206 macrophthalma, 206

Phrynus, 433

Phylira, (Leach) 162

Phylline, (Oken.) 35

Phyllodoce, (Sav.) 21 maxillosa, 27

Phyllopa, 325. 347 what called, 241

Phyllosoma, (Leach) 202

Pilumnus, (Leach) 155

Pince, (Geoffroy) 518 rouge, La, 505

Pinna, the, 285

Pinnipedes, 147

Pinnophylax, 284

Pinnoter, 284

Pinnotheres, (Latr.) 158

Pinnotheres, the, 284 of Pliny, 287

Pirimela, (Leach) 152 denticulata, 152

Pisa, (Leach) 165 aurita, 166 chiragra, 166 heros, 166 xyphias, 166

Piscicolæ, (Blain.) 34 cephalota, 34 tessellata, 34

Plagusia, (Latr.) 160 depressa, 160

Platyonichus, 150

Pleione, (Sav.) 18 alcyonia, 18 vagans, 73

Podocerus, (Leach) 211 
Podocerus variegatus, xiii. 2 Il

Podophthalmus, (Lam.) 149

Poecilopoda, the, 321

Polybius, (Leach) 147 Henslowii, 148

Polydonte, (Reni.) 27

Polydoræ, the, 22

Polydorus, (Oken.) 34

Polygonata, (Fab.) 215

Polyphemus, (Müll.) 338 oculus, 339 of the ponds, 339

Polynöe (Sav.) 26

impatiens, 27

lævis, 26 muricata, 27

Pontobdella, (Leach \& Blain.) 34 areolata, 34

spinulosa, 34 verrucata, 34

Pontonia, (Latr.) 192

Pontophilus, (Leach) 191

Porcellana, (Lam.) 184

hirta, 185

hirsuta, 312 polita, 312

Porcellio, (Lat.) 225

Portuni, the, 147. 276

Portunus, (Fab.) 149

bimaculatus, 277

depurator, 277

Dufourii, 150

guttatus, 277

hastatus, 278

longipes, 277

pelagicus, 276

plicatus, 277

puber, 277

Tranquebarius, 150

Pou du gastéroste, 371

Praniza, (Leach) 212

Prawn, 177

Prawns, the, 313

Probosci-plectanas, 10

Processa, (Leach) 191

Prophylax, 181

Proto, (Leach) 214

Protula Rudolphii, 12

Pseudobdella, 33

Pseudo-scorpiones, 495

Psocus pulsatorius, 521

Pterygocera, (Lat.) 211

Pterygopoda, (Latr.) 373

Pulex aquaticus arborescens, 347

Pulmonariæ, the, 386

Pycnogona, the, 521

Pycnogonides, the, 498. 521

Pycnogonum, (Brun.) 499

balænarum, 522
Quadrilatera, xiii. 154

Ranina, (Lam.) 175

Raninæ, the, 296

Rectigrades, the, 403

Red-blooded worms, the, 3

Reniformes, (Agnostus) 381

Remipes, (Latr.) 179 testudinarius, 179

Rhyncoprion, (Herm.) 507

Riciniæ, (Latr.) 505

Ricinus, 506

Rocinela, (Leach) 218

Rouget, what called, 509

Sabella, (Cuv.) 11

(Linn. \& Gmel.) 11

of Linnæus, 80

alveolata, 15

Belgica, 14

bispiralis, 12

Capensis, 15

chrysodon, 15

grandis, 12

Indica, 12. 15

protula, 12

unispira, 12

villosa, 12

Sabellæ astartæ, 12 spirographicæ, 12

Saltigrades, 429

Salticus, the genus, 485

(Latr.) 431

formicarius, 432

grossipes, 487

Saltique de Sloane, 431 chevronné, 432

Sanguisuga, (Sav.) 32

Sarcoptes, (Latr.) 505

Sauce-pan-fish, 363

Schizopoda, 194

Scirus, the genus, 518

(Herm.) 505

vulgaris, 505

Scoletoma, (Blain.) 23

Scolex of Aristotle, 38

Scolezia of Athenæus, 38

Scolopendræ, marine, 41

Scolopodes, (Blain.) 23

Scorpio, 434

the genus, 487

australis, 491

cancroïdes, 498. 519

cimicoïdes, 498. 519

crassicauda, 491

Europæus, 437

occitanus, $436,437.489 .492$, 493 
Scorpio, (proper) xiii. 437 Tunitanus, 437

Scorpion, the African, 437 the aquatic 488 araignée, 518. 521 the book, 498 d'Europe, (Faux) 518 the European, 436, 437 des livres, 498 of Maupertius, 436 the reddish, $436,437.492,493$ of Souvignargues, 436

Scorpiones, pseudo, 495

Scorpions, the gestation of, 494 of Languedoc, 490 the winged, 488

Scyllarus, (Fab.) 182 (Leach) 183

Sea-spiders, 163

Sea-spider, what named, 295

Sea mantes, wliat called, 315

Segestria, (Latr.) 408

Senelops, (Dufour) 421 omalosoma, 421

Serolis, (Leach) 217

Serpula, (Lin.) 9 characters of the genus, 78 contortuplicata, 10 gigantea, 10. 12 spirillum, 11 spirorbis, 11 vermicularis, 10

Serpulæ cymospiræ, 11 the genus, 76 manners and habits of the, 79 spiramellæ, 11

Seta, what called, 42

Seytodes, (Latr.) 410 blonde, 411 thoracica, 411

Shrimp, 177

Shrimps, the, 313

Sida, 338

Sigalion, (Aud. \& Edw.) 27

Siphonostoma, 365

Siphostoma diplochaïtes, 16. 87. 90 uncinata, 16

Siphostomæ, œsophagus of the, 89

Siro, (Latr.) 501

Smaridia, 505

Smerdis, (Leach) 201

Soldiers, what called, 305

Solifuga, 497

Solpuga, (Fab.) 496 the genus, 510 ararhnoïdes, 517 fatalis, 518

Sparassus, (Walck.) 419

Sphæroma, (Latr.) xiii. 220 Cuv. Index.
Sphæroma dentata, 220

Sphæromides, 219

Sphasus, (Walck.) 425

Spider, bite from a, 453 the bush, 459 à chevrons blancs, the, 429 the female mason, 399 the hunting, 460 the mason, 395. 463 proper, web of the, 467 threads of the, $\mathbf{4 4 5}$

Spiders, the, $\mathbf{3 8 7}$ combats of, 555 the crab, 398. 478.557

the erratic, 425 fecundation of the female, 450 the laterigrade, 418 long-legged, 526 proper, the, 409.466 the sedentary, 403 the wolf, 425

Spio, (Fab.) 22

filicornis, 22 seticornis, 22

Spirographis Spallanzanii, 12 Spirorbis, (Lam.) 11 the genus, 80

Squilla, (Fab.) 199 eusebia, 201 lobata, 214 proper, (Latr.) 200 scyllarus, 200 what named, 313

Squillæ, gills of, 260 liver of, 256

Stenocionops, (Leach) 167

Stenopus, (Latr.) 190

Stenorhynchus, (Lam.) 171 (Leach) 222 lineare, 222

Stomapoda, 196 in general, 139 the order of, 315

Stomapods, what called, 240

Storena, (Walck.) 424

Stylaria, (Lamarck) 30

Swimmers, the, 147

Syllis, (Sav.) 22 monilaris, 22

Synodus, (Latr.) 219

Syphostoma, (Otto) 16

Tachypleus, (Leach) 364

Talitri, the, 316

Talitrus, (Latr.) 208

Tarantula, the, 427 bite of the, 433

$\mathrm{H} \mathrm{h}$ 
Tarantula, (Fabr.) xiii. 433

Terebella, (Cuv.) 12

bicornis, 10

carunculata, 18

cirrhata, 13

complanata, 18

conchilega, 13. 82

constrictor, 13

description of, 81

flava, 18

gigantea, 13

intestinal canal of, 84

Medusæ, 13

nebulosa, 13

reniformis, 12

rostrata, 18

rubra, 20

stellata, 11

tentaculata, 25

tube of the, 85

variabilis, 13

venusta, 13

Terebellæ, of Gm. 18

Idaliæ, 13

organization of the, 83

phyzellæ, 13

the ovary of the, 70

Tessarops, (Rafinesque) 430

Tetragnatha, 497 (Latr.) 414

Thalassina, (Latr.) $] 86$ scorpionides, 186

Thelphusa, (Latr.) 155

the genus, 278

fluviatilis, 279

Thelyphonus, (Latr.) 433

Thenus, (Leach) 183

Indicus, 183

Theraphosæ, of M. Walcknaer, 396

Theridion, (Walck.) 411

benignum, 471

tredecim guttatum, 472

wounds from a, 554

Thia, (Leach) 153

polita, 153

Thomisus, the genus, 478

(Walck.) 423

citratus, 480

citreus, 424.479

cristatus, 424.478

globosus, 424

tigrinus, 478

Thomisi, threads produced by, 391

Thomise tigrée, 422

Tics, the, 505

Tourlourous, 288 the, 289

Trachean Arachnides, 495

Trapezia, (Latr.) 155
Trigona, xiii. 163

Trilobites, the, 378

Trochetia, (Dutrochet) 33

Trogulus, (Latr.) 502

Trombidia hexapoda, 509

Trombidium holosericeum, 503 (Fab.) 503

tinctorium, 503

Trophonia, (Aud. \& Edw.) 30 barbata, 30

Tubicolæ, 76

the, 9

distinction of, 7

Tubifex, (Lamarck) 31

Tubipora arenosa, 15

Tubiteles, the, $\mathbf{4 0 4}$

Tubularia Fabricia, 12 magnifica, 12 penicillus, 12

Tylos, (Latr.) 224

Typhis, (Risso) 212 ovoïdes, 212

Uca, (Latr.) 159 (Leach) 157

Uloborus, (Latr.) 413

Walckenarius, 414

Unipeltata, 199

Unkia, of the Japanese, 364

Unogata, (Fab.) 386

Uroctea, (Dufour) 404 quirique-maculata, 405

Uropoda, (Latr.) 504

Uroptera, (Latr.) 205

Vermes, division of the class of, 45 the name of, 37

Vitalis aquaticus, what called, 42

Weavers, the, 404

Wolf-spiders, eggs of the, 393

Wood-lice, 318

( $V u l g)$.

Worm, the common earth, 29

Worms, division of the class of, 44 the name of, 37

the red-blooded, 40

what called, 38

Xantho, (Fab.) 152

Xirika, 288

Xyphosura, 360

Zoea, (Bosc) 327

Zoë pelagica, 327

Zuzara, (Leach) 220 


\section{N S E C A.}

Abax Corsicus, xiv. 217

metallicus, xiv. 215

Abeille-perce-bois, xv. 570 tapissière, xv. 573

Abræus, (Leach) xiv. 389

Acanthia, $(F a b$.$) xv. 220.236$ of what composed, xv. 219

(Lat.) xv. 220

clavicornis, xv. 432

Acanthocerus, (Macl.) xiv. 466 æneus, xiv. 466

Acanthocinus, of Meg. \& Dej. xv. 108

Acanthomera, xiv. 552 (Wied.) xv. 699

Acanthopoda, xiv. 409

Acanthoptera, (Lat.) xv. 103

Acanthopus, (Klüg.) xv. 387 (Meg.) xv. 13

Acanthoscelis, description of, xiv. 201

Acari, attach themselves to dogs, xiv. 164

diseases produced by, xiv. 161

Acarides, or ticks, xiv. 164

Acarus, resembles a pediculus, xiv. 162 gnopterorum, xv. 564

Acerous, insects called, xiv. 41

Acheta, of Fabricius, xv. 192. 196

Achias, $(\mathrm{Fab}$.) xv. 710

Acilius, xiv. 247

Acinopus, description of, xiv. 208 maculipennis, xiv. 208 megacephalus, xiv. 208

Acmaeodera, xiv. 357

Acœnitus, (Lat.) xv. 362

Acræa, (Fab.) xv. 586

Acrida, of Mr. Kirby, xv. 202

Acridium, of Geoffroy, xv. 202 of Geoffroy, habits of, xv. 204

Acrocera, (Meig.) xv. 692

Acrocinus, (Illig.) xv. 108
Acrydium, (Geoff.) xv. ${ }^{8} 176$ armatum, xv. 177 cœcum of, xv. 180

Actora, (Meig.) xv. 717

Aculeata, xv. 370

Acupalpus, description of, xiv. 210

Adela, (Lat.) xv. 627

Degeerella, xv. 627

Reaumurella, xv. 628

Adelium, $(F a b.) \times v .13$ eupreum, xv. 22

Adelocera, (Lat.) xiv. 317

Adelostoma, (Dup.) xiv. 547

Adephages, description of the, xiv. 172

Adesmus, xv. 110

Adolestoma sulcatum, xiv. 548

Adorium, $(F a b.) \times v .141$

IEdes, (Hoff.) xv. 683

IEgialia, xiv. 459 (Latr.) xiv. 460

IEgithes, of Fab., xv, 155

Egocera, xv. 599

Æsalus, (Fab.) xiv. 498 scarabœoïdes, xiv. 498

Eschrotes, xiv. 454

Æshna, xv. 296. 312

Etalion, (Latr.) xv. 225

Agacephala, (Manh.) xiv. 469

Agaon, (Dalm.) xv. 366

Agarista, (Leach) xv. 595

Agarus, (Leach) on what founded, xiv. 247

Agathidium, (Illig.) xv. 155

Agathis, (Lat.) xv. 362

Aglæ cærulea, xv. 576

Aglaope, $\left(F a b_{0}\right) \times v .600$

Aglia, xv. 605

Aglossa, (Lat.) xv. 623

Agonum, description of, xiv. 223 rotundatum, xiv. 223

Agra, description of, xiv. 194

$$
\text { H h } 2
$$


Agrilus, xiv. 357

Agrion, xv. 296

Agriones, xv. 312

Agyrtes, (Froch.) xiv. 397 castaneus, xiv. 397 subniger, xiv. 397

Akis, (Fab.) xiv. 545, 546. 548 abbreviata, xiv. 546 angustata, xiv. 546 collaris, xiv. 546 glabra, xiv. 546 orbiculata, xiv. 546 punctata, xiv. 546

Aleochara, description of, xiv. 298, 299

bipunctata, xiv. 299

fumata, xiv. 299

lanuginosa, xiv. 299

nana, xiv. 299

nitida, xiv. 299

Aleyrodes, wings of, xv. 230

Alomya, (Panz.) xv. 362

Alpæus, description of, xiv. 237

Altica, (Geoff.) xv. 141 marginella, xv. 142

Alticæ, xv. 153

Altisæ, xv. 36 proper, xv. 142

Altitarsus, xv. 142

Alucita, (Fab.) xv. 627 (Lat.) xv. 625

Alurnus, of Fab, xv. 136

Alydas pellucidus, xv. 241

Alydus, ( $F a b$.$) xv. 218$

Alysia, (Latr.) xv. 363

Alyson, (Jur.) xv. 378

Amara, observations upon, xiv. 213

Amarygmus, (Dalm.) xv. 13

Amathusia, (Fab.) xv. 590

Amazons, the neuters of red ants, xiv. 120

Amblirhinus, (Schoen.) xv. 57

Amblychus, description of, xiv. 226

Amblyteres, xiv. 476

Amerhinus, xv. 61

Ametobolia, what so called, xiv. 7

Ammobates, (Latr.) xv. 385

Ammophilus, of Mr. Kirby, xv. 375

Amphicoma, (Latr.) xiv. 487 abdominalis, xiv. 488

Ampulex, (Jur.) xv. 376

Amycterus, (Scha.) xv. 56

Amydetes, (Hoffm.) xiv. 333 apicalis, xiv. 333

Anacolus sanguineus lugubris, xv. 100

Anaspis, (Geoff.) xv. 29

Anax imperator, xv. 304

Anchomenus, description of, xiv. 223

Anchonus, (Schoen.) xv. 63
Ancylochira, xiv. 357

Ancylorhyncus, (Lat.) xv. 690

Ancycloscelis, (Latr.) xv. 387

Andrena, (Lat.) xv. 882

Andrenes, (Fab.) xv. 381

Andrenetæ, xv, 44

(Lat.) xv. 381

Andrenoïdes, xv. 383

Anelastes Drurii, xiv. 325

(Kirby,) xiv. 325

Anelytra, remark of the ancients on, xv. 389

Anisomera, (Meig.) xv. 686

Anisonyx, xiv. 489

Anisoplia histrio, xiv. 474

Anisoscelis, xv. 218

Anisotoma, (Illig.) xv. 4. 155

Anobia, xv. 89

Anobium, (Fab.) xiv. 84. 351 where placed, xv. 379

larvæ of, xv. 90

minutum, xiv. 352

paniceum, xiv. 352. 382

pertinax, xiv. 352

reticulatum, xv. 92

striatum, xiv. 351, 352

tessellatum, xiv. 351

Anocolus lugubris, xv. 116 quadripunctatus, xv, 116

Anomala, (Meg. Dej.) xiv. 484

Anopheles, xv. 683

Anoplognathus, (Leach) xiv. 477

Anoplura, resemble the Parasita, xiv. 148

Anotia, (Kirby) xv. 224

Anthaxia, xiv. 358

Antherophagus, (Knoch) xiv. 402 antennæ of, xiv. 403

Anthia, where found, xiv. 93. 96 description of the, xiv. 184, 185 Burchellii, xiv. 270

Anthicus, (Fab.) xiv. 385 ; xv. 30. 123. 166

Helwigii, xiv. 386 minutus, xiv. 386

Anthidium, (Fab.) xv. 384

Anthipna, (Escholtz.) xiv. 487

Anthobii, xiv. 486

Anthomizides, xv. 710

Anthomyia, (Meig.) xv. 711

Anthophagus, description of, xiv. 297

Anthophila, (Lat.) xv. 381, 382

Anthrax, (Scop.) xv. 694. 753 marginicollis, $\mathrm{xv} .780$

Anthreni, change in food of, xiv. 66

Anthrenus, (Fab.) (Geoff.) xiv. 406 elongatus, xiv. 406

hirtus, xiv. 408

ruficornis, xiv. 406 
Anthrenus versicolor, xiv. 406 Viennensis, xiv. 406

Anthribus, $x v$. 66

(Fab.) xv. 20. 53

(Geoff.) xv. 53. 155

Anthrophora, (Fab.) xv. 384 (Lat.) xv. 387. 571

Antichides, xv. 29

Anteon, (Jur.) xv. 367

Antlia, (Kirby) xv. 613

Antliata, (Fab.) xv. 681

Ant-lion, instinct of, xiv. 104 larvæ of the, xv. 324 manner of feeding of larvæ of the, xiv. 66

Ant-lions, eyes of, xiv. 45 metamorphosis of, xiv. 81. 83

Ants, acid of, xiv. 73 acid produced by, xiv. 72 anecdotes of exotic, $\mathrm{xv} .487$ anecdote of fire, xv. 487 architecture of, $\mathrm{xv} .506$ ash-coloured, nests of, xv. 451 attack upon some ash-coloured, $\mathrm{xv} .491$

attack of the wood, xv. 470

auxiliaries of the Amazon, xv. 499

battle between, xv. 468

carpenter, xv. 444

campaign of rufescent, xv. 491

comparison of, xv. 194

conjugal affection of working, xv. 458

domestic labours of, xv. 507

domestic life of, xv. 502

domestic economy of, xv. 459

turf, emigrations of, xv. 453

enemies of the, $x v .488$

error respecting the Amazon, $\mathrm{xv} .501$

the Ethiopian, xv. 446

experiment upon, xv. 510

experiment upon yellow, xv. 457 . 465

family affection of, xv. 464

fate of the male, xv. 455

flight of, xv. 454

fondness for drink of, xiv. 70

food of, xiv. 68

food of the Amazon, xv. 498

the fuliginous, xv. 457

gymnastic exercise of wood, xv. 471

habits of the, xv. 434

habits of the female, xv. 456

habits of the mining, xv. 500

habits of the sugar-cane, xv. 485

habits of the yellow, xv. 473
Ants, instinct of, xiv. 67. 104

instinct of the brown, $x v \cdot 464$

larvæ of, xv. 448

proper, larvæ of, xv. 450

lethargic state of, $\mathrm{xv} .476$

marvellous account of the Amazon, $\mathrm{xv} .490$

metamorphosis of, xiv. 118

migration of, $\mathrm{xv} .462$

mixt society of, $x v .502$

neuter, xv. 451

neuters among, xiv. 19. 78. 114

neuter, live how, xiv. 119

are omnivorous, xiv. 23

our limited knowledge of foreign, xv. 483

political economy of, xv. 511

preservation of the societies, $\mathrm{xv}$. 509

prey of, xv. 466

prophetic of rain, xiv. 33

ravages of, $\mathrm{xv} .484$

recruiting system of, $\mathrm{xv}, 464$

appearance of reason in, xiv. 111

of Linnæus, the red, xv. 443

riches of the, $\mathrm{xv} .474$

societies of, xiv. 120 ; xv. 477

some called negroes, $\mathrm{xv} .504$

size of, xiv. 78. 96

societies of sanguine, xv. 480

society of, xiv. 115

temperature developed by, xiv. 75

the visiting, $\mathrm{xv} .486$

warlike expedition of, xv. 495

wars of the, xv. 467

wars of the Amazon, xv. 483

wars of the ash-coloured, xv. 482

(working, without metamorphosis, xiv. 21 yellow, xv. 443

Apalus, (Fab.) xv. 33, 34

Apanisticus, xiv. 357

Apate, (Fab.) xv. 84

Apatomza, (Wied.) xv. 693

Aphæna, of M. Guerin, xv. 260

Aphaneura rufescens, xv. 575

(Westwood, ) xv. 575

Aphanisticus, xiv. 313

Aphides, abdomen of, xv. 231

fluid of the, xv. 472

food of, xv. 232

habits of, xv. 267

viviparous and oviparous, xiv. 33

without wings, $\mathrm{xy}, 231$

Aphidii, xv. 227

family of the, xv. 264

Aphidiphagi, xv. 158 
Aphidiphagi supplemeut on the, xv. 160

Aphidivorous worms, xv. 760

Aphis, xv. 161

(Lin.) xv. 227

populi, xv. 270

proper, xv. 227

quercus, xv. 270

salicis, xv. 271. 273

ulmi, xv. 270

wings of, xv. 230

Aphodius, (Fab.) xiv. 460

(Illig.) xiv. 458

arenarius, xiv. 460

Aphodus, xiv. 450

Aphritis, xv. 704

Apiariæ, xv. 385

(Lat.) xv. 382

insect on, xv. 42

subgenera of the, xv. 566

Apion, xv. 54

Apiropodes, of M. Savigny, xiv. 25

Apis, of Linnæus, xv. 381

the genus, xv. 530

bicornis, xv. 572

muraria, xv. 571

proper, (Lat.) xv. 388

rostrata, $\mathrm{xv} . \mathbf{3 7 6}$

tricornis, xv. 573

venea, xv. 573

violacea, xv. 570

Apius, (Jur.) xv. 377

Apoda, xv. 54

Apogonia, (Kirby) xiv. 477 genellata, xiv. 477

Apollo Linnæi, xiv. 86

A pomecyna, xv. 110

Aporus, (Spin.) xv. 375

Apotomus, description of, xiv. 206 rufus, xiv. 207 testaceus, xiv. 207

Apsis, (Germ.) xv. 60

Aptera, stigmata of, xiv. 4

Apterogyna, (Lat.) xv. 372

Ehrenbergii, xv. 515

Apterous insects, eyes of, xiv. 8 insects, tarsi of, xiv. 65 insects, thorax of, xiv. 10

Aptinus, description of the, xiv. 185 Balista, xiv. 186 Pyrenæus, xiv. 186

Apus, thoracic feet of the genus, xiv. 9

Aquatic-flea, xiv. 251 scorpions, xv. 249

Arachnida, of M. de Lamarck, xiv. 25 have no antennæ, xiv. 39 jaws of, xiv. 9 thorax of, xiv. 10 vision of, xiv. 8
Arachnides antennistes, of M. de Lamarck, xiv. 25

Aradi, habits of, xv. 243

Aradus, of Fab., xv. 218 antennæ of, Xv. 220

Araneïda, differ from insects proper, xiv. 38

Araneïdæ, size of, xiv. 96

Arcopagus, (Leach) xv. 167

Arctia, (Schr.) xv. 611

Arenicoli, xiv. 459. 466

Areodes, (Leach) xiv. 482

Argos, xv. 593

Argus, (M. Lamarck) xv. 591 bleu d', xv. 592

Argynnis, xv. 583 (Fab.) xv. 587

Argyrites (Latr.) xv. 710

Aristolochus, xv. 413

Aristus, xiv. 566 description of, xiv. 206. 274 bucephalus, xiv. 275 fulcatus, xiv. 275

Armideus, of M. Ziegler, xiv. 463

Arpactus, (Jur.) xv. 378

Arpeneures, what called, xv. 579

Arrhenodes, of Stevens, xv. 55

Articerus, (Dalm.) xv. 168

Ascalaphus, xv. 327

(Fab.) xv. 298

Barbarus, xv. 328

Ascia, (Meg.) xv. 704

Asida, (Lat.) xiv. 555 grisea, xiv. 555

Asilus, xv. 758 the genus, xv. 752

(Lin.) xv. 690 proper, xv. 691 forcipatus, xv. 753

Asilici, (Lat.) xv. 690 the division, $x v .752$

Asindulum, xv. 687

Asiraca, xv. 224

Aspidiphorus, (Zieg. Dej.) xiv. 403

Aspistes, xv. 689

Astata, (Lat.) xv. 377

Astemma, xv. 219

Astomella, (Dufour) xv. 692

Astrapæus, description of, xiv. 290

Ateuchus, xiv. 451 of the Egyptians, xiv. 452 whence derived, xiv. 504 Bacchus, xiv. 454 cupreus, xiv. 453

flagellatus, xiv. 453

Hollandiæ, xiv. 454

Kœnigii, xiv. 453

laticollis, xiv. 453

Leei, xiv. 453 
Ateuchns miliaris, xiv. 453

Ateuchus, pilularius, story of an, xiv. 1]1. 453

profanus, xiv. 453

sacer, xiv. 92.452 .505

sanctus, xiv. 453

Schæfferi, xiv. 454

semi-punctatus, xiv. 453

sinuatus, xiv. 453

sex-punctatus, xiv. 454

tmolus, xiv. 458

triangularis, xiv. 454

variolosus, xiv. 453

violaceus, xiv. 454

volvens, xiv. 454

Athalia, (Leach) xv. 357

Athericera, xv. 701. 706

Athericeræ, the, xv. 758

Atherix, (Meig.) xv. 695

Athryrœus, (Macl.) xiv. 464

Atlas, of China, xv. 605

Atopa, (Fab.) xiv. 327 cervina, xiv. 327

Atractocerus, (Palis.) xiv. 352 necydaloïdes, xiv. 352

Atta, (Fab.) xv. 372 cephalotes, xv. 486

Attacus, xv. 602

Attagenus, (Lat.) xiv. 405 serra, xiv. 406

Attelabus, xiv. 374; xv. 66, 67 (de G.) xv. 99

(Lin.) Xv. 99

of Lin. and of Fab., xv. 54

apiarius, xiv. 346

formicarius, xiv. 345

mollis, xiv. 345

Pennsylvanicus, xiv. 189

Atychia, (Hoff.) xv. 600

Auchenia, (Thunb.) xv. 124

Aulacus, (Jur.) xv. 360

Auletes, (Schœnh.) xv. 54

Axina, (Kirb.) xiv. 344 analis, xiv. 344

Axinophorus, characters of, xiv. 271 Brasiliensis, xiv. 271

Badister, description of, xiv. 226

Barbicornis, (Godart) xv. 593 basalis, xv. 677

Baccha, (Meig.) xv. 703 (Fab.) xv. 715

Bagous, xv. 60

Balæna urticata, xv. 622

Balaninus, xv. 60

Banchus, (Fab.) xv. 361. 364

Baridius, xv. 61

Bed-bug, habits of, xv. 237 without wings, xv. 230
Beemares, of Geoff. xv. 54

Bee, intellect of the, xiv. 100 intellect and industry of the, xiv. 101

quotation relating to the, xiv. 112

sting of the, xv. 389. 531

the upholsterer, xv.571. 573

Bees, the, xv. 381. 530

abdomen of, xv. 534

appearance of reason in, xiv. 109

care of nursing, xv. 542

the carpenter, xv. 570

cells of, xv. $\mathbf{5 5 2}$

crowd plants and flowers, when, xiv. 61

culture of, $\mathrm{xv} .565$

diseases of, xv. 561

dislike oil of turpentine, xiv. 63

the drones, or male, xv. 531

dwellings of, xiv. 119

eggs of, xv. 541

how to prevent the emigration of, xv. 558

enemies of, xv. 563

English, anterior tarsi of, xiv. 18

M. Huber's experiment upon, xv. 535

eyes of, xiv. 46

fecundation of, $\mathrm{xv} .539$

feeding of, xv. 560

the female, xv. 531

fondness for drink of, xiv. 70

food of, xiv. 68

formation of the cells of, xv. 553

formation of swarms of, xv. 546

the hives of, $\mathrm{xv} .550 .556$

glass hives for, $\mathrm{xv} .557$

how to clear a hive of, $\mathrm{xv} .559$

honey of, xv. 554

the humble, xv. 567

humming of, xiv. $76^{\circ}$

instinct of, xiv. 67. 104-108

the king, xv. 531

labours of, xv. 551

larva of, xv. 543

length of life of, xv. 562

the mason, xv. 571

metamorphosis of, xiv. 118

method of forming swarms of, xv. 549

method of making fruitful, $x v$. $\mathbf{5 4 5}$

method of taking a swarm of, xv. 547

neuters among, xiv. 19. 78. 114

organs of the, xv. 533

perspiration of, xiv. 74 
Bees, prophetic of rain, xiv. 33

the queen, xv. 531

quotation from Virgil respecting, xiv. 34 ; xv. 532

remark of Aristotle respecting, xiv. 80

respiration of, $\mathrm{xv} .537$

sensation of, xiv. 103

sense of touch in, xv. 538

sex of working, xv. 544

best situation for, $\mathrm{xv} . \mathbf{5 5 5}$

sting of, xv. 389, 531

taste of, xv. $\mathbf{5 3 7}$

temperature developed $\mathrm{ky}$, xiv. 75

use of antennæ of, xiv. 44

utility of, xiv. 120

wings of, xiv. 16

the working, xv. 530

Beetle, black, xv. 187

common burying, organs of smell of, xiv. 63

senses in the eye of a, xiv. 48

Beetles burying, why called, xiv. 393 frequent the snake-root and staphelia, xiv. 60

Belostoma, xv. 222

marginata, xv. 248

Belus, (Schœn.) xv. 54

Belyta, (Jur.) xv. 368

Bembecides, (Lat.) xv. 376

Bembidion, description of, xiv. 240. 280

Beris, (Lat.) xv. 699. 757

Berosus, xiv. 431

Bethylus, (Lat.) xv. 367

Berytus, (Fab.) of what composed, xv. 241

Bibio, (Geoff.) xv. 689 (Fab.) xv. 695

Biblis, (Fab.) xv. 589

Biches, what called in French, xiv. 500

Bitoma, (Herbs.) xv. 86

Bipartiti, description of, xiv. 197

Bithynus, (Leach) xv. 167

Bittacus, (Lat.) xv. 298

Blaps, xiv. 552. 567

(Fab.) xiv. 553. 559

clathrata, xiv. 556

gages, xiv. 553

glabra, xiv. 559

lævigata, xiv. 553

mortisaga, xiv. 553

punctata, xiv. 556

sulcata, xiv. 553. 568

tibialis, xiv. $\mathbf{5 5 4}$

tibidens, xiv. 558

voice of, xiv. 76

Blatta, (Lin.) xv. 171. 192

cœcum of, xv. 180
Blatta acervorum, xv. 175

Americana, xv. 172

Germanica, xv. 172

gigantea, xv. 185, 186

Lapponica, xv. 172

Orientalis, xv. 172. 187

rugicollis, xv. 187

Blattæ, xv. 184

Blapsides, whence named, xiv. 551

Blaptinus, (Deg.) xiv. 558

Blastanus, (Hoff.) xv. 30

Blepharis of M. Sevoille, xv. 190 elegans, xv. 190

Blethisa, description of, xiv. 238

Blue meat-fly, ovoviparous, xiv. 77

Bocydium, (Lat.) xv. 225

Bolboceras Australasiæ, xiv. 465 Bilbergii, xiv. 508 (Kirby) xiv. 465. 508

Boletophagus xv. 9

(Fab.) xv. 5 cornutus, xv. 9

Bolitophila, (Hoff.) xv. 687

Bombardiers of M. Latreille, xiv. 266

Bombidium, description of, xiv. 240

Bombus, xv. 567

lapidarius, xv. 568

(Lat.) xv. 388

Bombyces, the, xv. 679 antennæ of, xiv. 78

Bombycites, xv. 605

Bombylii, the, $x v .753$

Bombylius, (Lin.) xv. 692 proper, (Aleig.) xv. 693

Bombyx, xv. 602

abietis, xv. 609

anachoreta, xv. 609

antiqua, xv. 610

antiqua, represented by Kirby and Spence, xiv. 43

Atlas, where found, xiv. 96

betulifolia, xv. 606

Bucephala, xv. 609

caja, xv. 611

caja, represented by Kirby and

Spence, xiv. 42

Cecropia, xv. 605

chrysorrhæa, xv. 611

compressa, xv. 613

coryli, xv. 609

cyllopoda, xv. 615

dispar, xv. 609

illicifolia, xv. 606

Jacobeæ, xv. 612

luna, xv. 605

mori, xv. 607. 679

mylitta, xv. 605

Neustria, xv. 608

populifolia, xv. 606 
Bombyx pavonia major, xv. 606 potatoria, xv. 606 processionnea, xv. 608 proper, xv. 607 pudibunda, xv. 609 pythio-campa, xv. 608 quercifolia, xv. 606 species of wild, xv. 634 tau, xv. 605

venulia, xv. 599 versicolora, xv. 609 verticalis, xv. 623

Borborus, (Meig.) xv. 712

Boreus, (Latr.) xv. 298

Boros, (Herbs.) xiv. 561

corticalis, xiv, 561

thoracicus, xiv. 561

Bostrichi, Larvæ of, xv.90

Bostrichus denticollis, xv. 91 proper, (Geoff.) x v. 84 thoracicus, $\mathrm{xv} .91$ (Fab.) xv. 82, 83

Botys, (Lat.) xv. 622

Bourdon, xv. 567

Bousiers, what called, xiv. 507

Brachelytra, description of, xiv. 288

Brachinus, description of the, xiv. 185 bimaculatus, xiv. 267 bombarda, xiv. 187 causticus, xiv. 187 complanatus, xiv. 186 crepitans, xiv. 187. 266, 267 exhalans, xiv. 187 displosor, xiv. 186. $267,268.270$ explodens, xiv. 187 glabratus, xiv. 187 scolepeta, xiv. 187

Brachini, Helluo approximated to the, xiv. 191

Brachycera, where found, xiv. 93

Brachycerus, xv. 68 (Fab.) xv. 56

Brachyopa, (Hoff.) xv. 705

Brachypus, xv. 60

Brachyrhinus, xv. 69

Brachystoma, (Meig.) xv. 691

Bracon, (Jur.) xv. 363 (Fab.) xv. 361

Braula, (Netz.) xv. 719

Brassicariæ, what called, xv. 411

Brassolis, (Fab.) xv. 590

Brentus, xv. 68 (Fab.) xv. 55 Temminckii, xv. 68

Brevirostræ, xv. 5 7

Brontes, (Fab.) xv. 95

Broscus, description of, xiv. 218

Bruchus, (Fab.) xv. 20

(Geoff.) xiv. 348
Bruchus of Lin. xv. 53

larvæ of, xiv. $381 ;$ xv. 64

acaciæ, xv. 65

granarius, food of, xiv. 68

palmarum, xv. 65

pisi, xv. 66

properly so called, xv. 53

Bucentes, xv. 705

$$
\text { (Lat.) xv. } 706
$$

Bucephalus Antenor, where found,xiv.92 Bug, families of the, xv. 233

bed, where placed, xv. 236

land, xv. 232

Bugs, proper, xv. 220

difference between Nymph and

Larvæ of, xiv. 21

growth of young, xv. 239

Bulbifer, (Deg.) xv. 63

Bupreste bleu, xiv. 196 écunoné, xiv. 358

Buprestides, xiv. 310 hepatic conduits of, xiv. 308

Buprestis, xiv. 356 ; xv. 39

(Lin.) xiv. 310

(proper) xiv. 311

dangerous to oxen, xiv. 183

peculiar formation of, xiv. 255 signification of, xiv. 265

Buprestis acuminata, xiv. 357 adspersa, xiv. $\mathbf{3 5 7}$

æreicollis, xiv. 357

affinis, xiv. 312

alni, xiv. 357

amœna, xiv. 358

appendiculata, xiv. 358

Berolinensis ænea, xiv. 357

bicornis, xiv. 357

bifasciata, xiv. 358

canaliculata, xiv. $\mathbf{3 5 8}$

carbonaria, xiv. 357

cariosa, xiv. 357

castanea, xiv. $\mathbf{3 5 7}$

chrysis, xiv. 312.357

chrysostigma, xiv, 312

chrysostigma affinis, xiv. 358

conspersa, xiv. $\mathbf{3 5 7}$

cuprea, xiv. 357

cupressis, xiv. 357

cyanicornis, xiv. 358

cylindrica, xiv. 357

elata, xiv. 357

emarginata, xiv. 313

equestris, xiv. 358

exasperata, xiv. $\mathbf{3 5 7}$

eximia, xiv. 357

fasciculata, xiv. 311

fascicularis, xiv. 357

flavomarginata, xiv. 358

flavomaculata, xiv. 357

Cuv. Index. 
Buprestis Florentina, xiv. 357

gibbosa, xiv. 357

gigas, xiv. 257. 312

grandis, xiv. 357

hirsuta, xiv. 357

hirta, xiv. 357

hœmorrhoïdalis, xiv. 357

impressa, xiv. 358

interrupta, xiv. 357

leucostica, xiv. 357

leucogaster, xiv. 357

lineola, xiv. 313

lugubris, xiv. 357

macularia, xiv. 357

manca, xiv. 358

mariana, xiv. 357

mumata, xiv. 313

nitida, xiv. 358

novem-maculata, xiv. 357

ocellata, xiv. 312

octo-guttata, xiv. 357

ornata, xiv. 357

penicillata, xiv. 357,358

pilosa, xiv. 357

principialis, xiv. 358

punctata, xiv. 357

quadrimaculata, xiv. 358

rubi, xiv. 357

rustica, xiv. 357

rutilans, xiv. 357

scutellaris, xiv. 358

sternicornis, xiv. 311. 357

stigma, xiv. 358

strigosa, xiv. 357

tæniata, xiv. 357

tarda, xiv. 358

tarterica, xiv. 357

tenebricosa, xiv. 357

tenebrionis, xiv. 357

variabilis, xiv. 357

variolaris, xiv. 357

viridis, xiv. 312

vittata, xiv. 312

Whitehillii, xiv. 358

Butterflies, the, xv. 582. 671

properly so called, the, xv. 583

alpine, xiv. 90

diurnal, colour of, xiv. 999

manner of feeding of, xiv. 66

metamorphosis of, xiv. 83

prophetic of rain, xiv. 33

remark of Aristotle respecting, xiv. 80

seek plants and flowers, when, xiv. 61

wings of, xiv. 16

Butterfly, eggs of the, xiv. 20

flight of the, xv. 672

lenses in the eye of a, xiv. 48
Butterfly, mouth of a, xiv. 22

Byrrhi, xiv. 423

Byrrhis, (Geoff.) xiv. 351

of Linnæus, xiv. 406. 407 ; xv. 22

proper, (Lin.) xiv. 407

crinaceus, xiv. 408

pilula, xiv. 408.423

pygmæus, xiv. 405

sericeus, xiv. 405

setiger, xiv. 408

striato-punctatus, xiv. 408

verbasci, xiv. 406

Byturi, xiv. 420

Byturus, (Lat.) xiv. 402

Cadelle, xv. 92, 93

Calandra, $(F a b$.$) xv. 59. 62, 63$

eggs of, xv. 73

granaria, xv. 72

granaria, food of, xiv. 68

palmarum, xv. 80

Calandræ, xv. 72 remedy against, xv. 78

Calathus, description of, xiv. 221

Calleida, description of, xiv. 195

Callicera, (Meig.) xv. 704

Callicerus, (of M. Gravenhorst) xiv. 300

Callichroma, (Lat.) xv. 103

Callidium, (Fab.) xv. 102. 105 where found, xv. 117

Callimorpha, (Lat.) xv. 611

Callirhipis, (Lat.) xiv. 325

Childreni, xiv. 365

Dejeunii, xiv. 326

ruficornis, xiv. 365

Callisthenes, description of, xiv. 235

Callistus, description of, xiv. 223

Callomyia, (Meig.) xv. 696

Calobata, (Fab.) xv. 715

Calobates, (Lat.) xv. 715

Calopus, (Fab.) xv. 19

Calosoma, $(F a b.) \times v .13$

(Fab.) description of, xiv. 206. 235

calidum, xiv. 235

indagator, xiv. 235

inquisitor, xiv. 235.267

longicornis, xiv. 206

porculatum, xiv. 235

reticulatum, xiv. 235

scrutator, xiv. 235

Calosomi, conical appendages of the, xiv. 174

Calopes, antennæ of, xv. 18

Calymbetes Lowei, xiv. 284

Calyptocephalus, xiv. 370

Campsia, (Lep. \& Ser.) xv. 12

Camptocerus, (Dej.) xv. 82 
Camptodontus, description of, xiv. 204

Cayennensis, xiv. 204

Camptognetha testacea, xv. 516

Camptorhynchus, xv. 61

Campylus, (Fisc.) xiv. 322

Campyolmyza, (Wied.) xv. 688

Canopseus, (Meig.) xv. 703

Canopus punctatus, xv. 233

Cantharis, (Geoff.) xv. 27. 33

(Degeer.) xv. 41

(Lin.) xiv. 330. 337. 340. 353

ænea, xiv. 340

bipustulata, xiv. 340

dermestoïdes, xiv. 353

fusca, xiv. 337

livida, xiv. 338

officinalis, xv. 43,44

Orientalis, where found, xiv. 93 qualities of, xv. 47

Cantharides, xv. 45

what called by the Ancients, xv. 50

elytra of, xiv. 17

mode of collecting, xv. 49

mode of preserving, xv. 49

Cantharidia, xv. 31

Cantharus, with what synonymous, xiv. 510

Capnodes, xiv. 357

Capricorns, xy. 116

Capricornus, coleoptera of, xiv. 68

Capsus, xv. 219

Carabi, conical appendages of the, xiv. 174

dwelling of, xiv. 183

majores, xiv. 276

Carabici, description of the, xiv. 182 grandipalpous, xiv. 191

hunters and carnivora, xiv. 175 subgenera of, xiv. 190

succeed to Drypta, xiv. 192

Carabus, xv. 68 where placed by Dejean, xiv. 188 derivation of the word, xiv. 265 description of, xiv. 182. 195. 233 ( $F a b$.$) description of, xiv. 206$. 236

(Lin.) description of, xiv.220. 234 æneus, xiv. 209

affinis, xiv. 234

agricola, xiv. 226

agrorum, xiv. 224

albipes, xiv. 223

ammon, xiv. 224

angulatus, xiv. 228

angustatus, xiv. 235

angusticollis, xiv. 223

articulatus, xiv. 242

arvensis, xiv. 234
Carabus auratus, xiv. 234 auro-nitens, xiv. 234 azureus, xiv. 225

biguttatus, xiv. 242

bipunctatus, xiv. 242

bipustulatus, xiv. 226

borealis, xiv. 239

brevicollis, xiv. 237

buprestis, xiv. 277

Calydonius, xiv. 206

cancellatus, xiv. 234

carnifex, xiv. 224

cassideus, xiv. 226

catenatus, xiv. 234

cephalotes, xiv. 218

cinctus, xiv. 224,225

cisteloïdes, xiv. 22]

cælatus, xiv. 234

cæruleus, xiv. 234

concolor, xiv. 234

consitus, xiv. 234

convexus, xiv. 234

coriaceus, xiv. 233

crepitans, xiv. 266

Creutzeri, xiv. 233

Crœsus, xiv. 225

crux-major, xiv. 228

cursor, xiv. 242

cyaneus, xiv. 233

cyanocephalus, xiv. 196

decorus, xiv. 242

depressus, xiv. 215. 226. 233

dorcus, xiv. 501

Doris, xiv. 242

emarginatus, xiv. 226

excavatus, xiv. 229

Fabricii, xiv. 233

festivus, xiv. 224

flavicornis, xiv. 223

frigidus, xiv. 221

fuscus, xiv. 221

gemmatus, xiv. 234

gibbus, xiv. 211

gigas, xiv. 232

glabratus, xiv. 234

granulatus, xiv. 234

guttula, xiv. 242

helopiodes, xiv. 224

Helwigii, xiv. 237

hirtipes, xiv. 209

hispanus, xiv. 233

hæmorrhoïdalis, xiv. 196

Hoffmansegii, xiv. 226

holosericeus, xiv. 224

hortensis, xiv. 234

impressus, xiv. 225

irregularis, xiv. 234

leucophthalmus, xiv. 214. 220

lunatus, xiv. 224

I i 2 
Carabus madidus, xiv. 216 marginatus, xiv. $2 z \bar{z} 3$ Megerlei, xiv. 232 melanocephalus, xiv. 221 Meridianus, xiv. 210 merulianus, xiv. 242 micans, xiv. 224 micros, xiv. 242 minutus, xiv. 242 modestus, xiv. 242 monilis, xiv. 234 monticola, xiv. 214 morbillosus, xiv. 234 multipunctatus, xiv. 239 nigricornis, xiv. 224 nitens, xiv. 234 nodulosus, xiv. 234 notulatus, xiv. 228 oblongo-punctatus, xiv. 216 oblongus, xiv. 223 occulatus, xiv. 224 osseticus, xiv. 233 parum-punctatus, xiv. 223 Paykulii, xiv. 217 peltatus, xiv. 226 picicornis, xiv. 237 picimanus, xiv. 214 pilicornis, xiv. 229 planus, xiv. 186. 220 politus, xiv. 225 posticus, xiv. 224 prasinus, xiv. $22: 3$ psammodes, xiv. 237 puchellus, xiv. 242 purpurascens, xiv. 234 pygmæus, xiv. 242 Pyræneus, xiv. 234 qua dricolor, xiv. 224 quatuor-punctatus, xiv. 223 quatuor-sulcatus, xiv. 224 quatuor-guttatus, xiv. 242 rufescens, xiv. 237 rufipes, xiv. 229 sabulosus, xiv. 237 saponaceus, xiv. 224 scabrosus, xiv. 232, 233 Scheidlerii, xiv. 234 scorbiculatus, xiv. 223 selphoïdes, xiv. 226 semipunctatus, xiv. 240 sex-punctatus, xiv. 223 signatus, xiv. 209 spinibarbis, xiv. 237.278 spinilabris, xiv. 237 spoliatus, xiv. 224 stigma, xiv. 224 striola, xiv. 215 sycophanta, xiv. 235. 278 sylvestris, xiv. 234 tenebrioïdes, xiv. 214
Carabus tenuicollis, xiv. 224 terminatus, $x i v .237$ terricola, xiv. 220 tricolor, xiv. 242 uliginosus, xiv. 239 ustulatus, xiv. 242 vaporariorum, xiv. 210 vespertinus, xiv. 210 violaceus, xiv. 234 vivalis, xiv. 221

Cararia, (Lep. \& Ser.) xv. 12

Carcophagus, xv. 126 Banksiæ, xv. 126

Carenum, description of, xiv. 199

Caris, description of the, xiv. 180

Carnivora, description of the, xiv. 172

Carpenter-ants, xv. 444

Carpenter-bees, the, xv. 570

Carpomyzæ, xv. 715

Caruns of M. Nitzsch, xv. 706

Casnonia, description of the, xiv. 189

Cassida, xv. 143

$$
\text { (Lin.) xv. } 136
$$

bicolor, xv. 772

Cassidæ, habits of the, xv. 131

Cassidariæ, the, xv. 135

Cassignetus geotrupoïdes, xiv. 499

Castnia, (Fab.) xv. 595, 596

Catadromus, xiv. 214

Catascopus, description of, xiv. 218 (of M. Kirby) xiv. 188

Catops, (Fab.) xiv. 398 (Payk.) xiv. 398 blapoïdes, xiv. 398

Catascopus elegans, xiv. 219 Hardwickii, xiv. 218

Caterpillar of Bombyx Neustria, xv. 655

the common, $\mathrm{xv} .650$

of large cabbage butterfly, xiv. 5 mouth of a, xiv. 22 nest of the, $x v .651$

Caterpillars, attitudes of, xv. 647 change of skin of, xv. 580 chrysalis from, xiv. 20 colours of, $\mathrm{xv} .638$ experiment upon, xv. 662 eyes of, xiv. 46 false, xv. 391. 397 the fish-formed, xv. 617 food of, xiv. 67 ; xv. 580.642 general history of, xv. 635 the geometer, xv. 665 the hairy, xv. 640 horn of, xv. 639 intestines of, xv. 581 labours of certain, xiv. 113 leaves of apple trees feed, xv. 658 of lepidoptera, xiv. $\mathbf{6 6}$ metamorphosis of, xv. 667 
Caterpillars, mode of life in, xv. 641 moulting of, xv. 666 muscles of, xiv. 54 no blood vessels in, xiv. 71 pine forests nourish, xv. 652 prejudice against, xv. 648 the processionary, xv. 657 procession of, xv. 653 remark of Aristotle respecting, xiv. 80

the rolling, $\mathrm{xv} .661$

the shorn, xv. 640

skin of, $\mathrm{xv} .638$

societies of, xv. 580

spinning of, xv. 654 unsocial nature of, xv. 659 winter retreats of, xv. 649

Catheretes, (Herbst.) xiv. 401

Cebrio, (Oliv.) xiv. 323

(proper,) xiv. 324

bicolor, xiv. 352

brevicornis, xiv. 324

femoratus, xiv. 325

fuscus, xiv. 325

gigas, xiv. 324,325

longicornis, xiv. 324

ruficollis, xiv. 325

Cebriones, xiv. 365 peculiarity of the, xiv. 309 where found, xiv. 92

Cebrionites, xiv. 323

Cecidomye, (Lat.) xv. 429

Cecidomyia, (Meig.) xv. 685 the, xv. 748

Cecydomyiæ, food of, xiv. 68 larvæ of, xv. 749 nymphs of, xv. 749

Celerio, sphinx, xv. 597

Celonites, (Lat.) xv. 379

Celyphus, (Dalm.) xv. 716 obtectus, (Dalm.) xv. 716. 773

Cemonus, (Jur.) xv. 378

Cenopalpus, (Gyll.) xv. 18

Cenops, (Lin.) xv. 705

Centrinus, xv. 61

Centris, (Fab.) xv. 387

Centrotus, (Fab.) xv. 225 furcatus, xv. 261 globularis, xv. 261

Cephaleia, (Jur.) xv. 357

Cephalia, (Meig.) xv. 715

Cephalocera, (Lat.) xv. 698

Cephalotes, description of, xiv. 218

Cephus, (Lat.) xv. 358

Cerambycinæ, size of, xiv. 96

Cerambycini, xv. 100

Cerambyx, xv. 101. 116

$$
\text { (Deg.) xv. } 99
$$$$
\text { (Fab.) xv. 102, 103.112 }
$$

Cerambyx, (Oliv.) xv. 19. 102 ambrosiacus, xv. 103 carcharias, xv. 110 cerdo, xv. 104 coriaceus, xv. 100 Fichteli, xv. 117 fuliginator, xv. 109 heros, xv. 104. 117 linearis, $\mathrm{xv} .110$ longimanus, xv. 108 moschatus, $x v, 103$ proper, xv. 104 regular, xv. 10] sanguineus, xv. 105 textor, xv. 109

Cerapterus, xv. 83

Ceramus, (Lat.) xv. 380

Ceraphron, (Jur.) xv. 368

Ceraspis, (Lepel.) xiv. 481 pruinosa, xiv. 482

Ceratophya, (Wied.) xv. 704

Ceratophyus, of M. Fischer, xiv. 463 dispar, xiv. 463

Ceratopogon, (Meig.) xv. 685

Ceraturgus, (Wied.) xv. 691

Cerceris, (Lat.) xv. 379

Cerocoma, (Geoff.) xv. 31 description of, xv. 49 cantharides distinct from, $\mathrm{xv}$. 46

Cercopis, xv. 226

fulvo scutellata, xv. 262 spumaria, xv. 261

Cercus, (Lat.) xiv. 401

Cercydion, xiv. 432

Cereatina, (Lat.) xv. 383

Ceria, (Fab.) xv. 703

Ceriæ, the, xv. 763

Cermatia, description of, xiv. 133 livida, $\mathrm{x}$ iv. 134

Ceropales, (Lat.) xv. 375

Cerophytum, xiv. 318

Ceroplateus, (Bosc.) xv. 688

Certallum, (Meg.) xv。 105

Cerura, (Schr.) xv. 612

Cerylon, (Gyll.) xv. 85 (Lat.) xv. 85

Cethosia, xv. 583 (Fab.) xv. 587

Cetonia, of Fabricius, xiv. 473. 494, 495 aurata, xiv. 496.516 Chinensis, xiv. 495 cornuta, xiv. 495 cruenta, xiv. 495 elongata, xiv. 493 fastuosa, xiv. 496 gagates, xiv. 494 geotrupinus, xiv. 494 imperialis, xiv. 495 
Cetonia Kirbii, xiv. 516

plana, xiv. 495

regia, xiv. 495

vencosa, xiv. 495

Cetoniæ, where found, xiv. 530

Ceutocerus, xiv. 390

Ceutorhynchus, xv. 62

Chalcidæ, xv. 364

Chalcides, larvæ of, xv. 581

Chalcidites, the, xv. 433 larvæ of the, xv. 433

Chalcimon, (Dalm.) xiv. 499 Humboldtii, xiv. 499

Chalcis, (Fab.) xv. 364 proper, xv. 365

Chalepus, xv. 136 (Macleay,) xiv. 472

Chasme, (Lep. \& Ser.) xiv. 488

Chasmodia, (Macl.) xiv. 474

Chasmopterus, (Dej.) xiv. 488

Chauliodes, (Lat.) xv. 299 maculipennis, $\mathrm{xv} .332$

Chelobasis, new subgenus of, xv. 143

Chelonarium, xiv. 364 (Fab.) xiv. 317

Chelonia, (Godart) xv. 611

Chelonus, (Jur.) xv. 363

Chelostoma, (Lat.) xv. 384

Chermes, (Lin.) xv. 227

(Geoff.) xv. 228

(Lin.) habits of, xv. 264

Chernnum, (Latr.) xv. 166

Chigoe, xiv. 156

Chilognatha, description of, xiv. 126. 137 motion of the, xiv. 127

Chilopoda, description of, 151. 140

Chionea, (Dalman) xv. 687

Chirocera, (Latr.) xv. 365

Chiromyza, (Wied.) xv. 698

Chiron, xiv. 502

(Mac L.) xiv. 460

digitatus, xiv. 461

Chironomi, the, xv. 743 larvæ of, xv. 745

Chironomus, (Meig.) xv. 684 curious appearance of, xv. 318 plumosis, xv. 744

Chiroscelis, (Lam.) xiv. 560 bifenestra, xiv. 561

Chlamys, (Knoch.) xv. 137

Chlænius, description of, xiv. 224 azureus, xiv. 225 velutinus, xiv. 224

Chlorima, xv. 57

Chlorion, (Lat.) xv. 375

Chlorops, (Meig.) xv. 714

Chœridium, xiv. 454

Choleva, xiv. 397
Choleva, (Latr.) xiv. 398

Choragus, (Kirb.) xv. 138

Chrysides, (Lat.) xv. 369 the, xv. 434

Chrysina auripes, xiv. 517 of Kirby, xiv. 516

Mexicana, xiv. 517 Peruviana, xiv. 516

Chrysis, xv. 526

of Linnæus, xv. 369

of Spinola, xv. 370

organs of touch of, xiv. 64

Chrysobotus, xiv. 358

Chrysochlora, (Lat.) xv. 700

Chrysomela, xv. 139. 148

(Fab.) xv. 13

(Lin.) xv. 27. 123

Calmariensis, xv. 141

cerealis, $\mathrm{xv} .140$

elongata, xiv. 343

larvæ of, xv. 152

nitidula, xv. 142

oleracea, xv. 142

populi, xv. 140

populi, fluid emitted by, xiv. 73

proper, xv. 140

sanguinolenta, $\mathrm{xv} .140$

tanaceti, xv. 141

Chrysomelinæ, xv. 136

Chrysomelina, size of, xiv. 96

Chrysophora, (Dej.) xiv. 473

Chrysopilus, (Fab.) xv. 695

Chrysops, (Meig.) xv. 697

Chrysotus, xv. 696

Chrysotoxa, the, xv. 763

Chrysotoxum, (Meig.) xv. 703

Chyliga, (Fall.) xv. 713

Chylognatha, lower lip of the, xiv. 132

Cicada, (Lin. Fab.) xv. 226

(Oliv.) xv. 223

Kirby's remarks on, xv. 254

mannifera, xv. 252

of what composed, xv, 252

orni, xv. 252

septendecim, xv. 255

spumaria, xv. 261

Cicada tibicen, xv. 255

Cicadæ, larvæ of, xv. 257

Cicadaria chrysis, xv, 682

Cicadariæ, xv. 223 psylus, xv. 682

Cicadella, xv. 224 proper, (Oliv.) xv. 226

Cicadellæ proper, xv. 263

Ciccus, (Lat.) xv. 226

Cicindela aquatica, xiv. 240

bipustulata, xiv. 177

campestris, xiv. 179

Carolina, xiv. 177 
Cicindela coarctata, xiv. 180 compared with Ditomos Bucephalus, xiv. 198 description of the, xiv. 176. 178 dromica, xiv. 180 flavipes, xiv. 241 for cantharides, $\mathrm{xv} .48$ Germanica, xiv. 179 Hybrida, xiv. 179 manticora, xiv. 263 megalocephala, xiv. 177 octonotata, xiv. 264 quadrimaculata, xiv. 242 quadrinotata, xiv. 178 riparia, xiv. 239 sinuata, xiv. 179 sylvatica, xiv. 179. 262 tristis, xiv. 177 what called, xiv. 366 connected with what, xiv. 191 radial articulation of the, xiv. 183

what indicated by, xiv. 261

Cicindeletæ, xiv. 191 description of the, xiv. $\mathbf{1 7 6}$

Cigala mantes, xiv. 92 noise made by, xiv. 76 wings of, xv. 230

Cigalæ, abdomen of female, xv. 231 food of, xiv. 67. 232 epimoros of, xiv. 51 metamorphosis of, xiv. 81

Cigales chanteuses, xv. 252

Cimbex, xv. 400 (Oliv.) xv. 355 amerinæ, larvæ of, xv. 401

Cimbices, flight of, xv. 401

Cimex, of Linnæus, xv. 217. 232

Cimex betulæ, xv. 234 baccarum, xv. 235 families of, xv. 233 griseus, xv. 235 lectularius, xv. 237 ornatus, xv. 236 personatus, xv. 243 tipularius, xv. 242 upper wings of, xv. 229 vagabundus, xv. 244

Cimices scutellati, xv. 232

Cinetus, (Jur.) xv. 368

Cinips, xv. 526

Cionus, (Clair.) xv. 61

Circellium, (Latr.) xiv. 454

Cis, (Lat.) xv. 84 approximate to what, xv. 5 boleti, xv. 91 of what composed, xv. 91

Cissites, xv. 31

Cistela, of Geoffroy, xiv. 407 ; xv. 22
Cistela, (Fab.) xv. 15, 16 cervina, xiv. 327

Cistelides, xv. 15

Cistogaster, (Lat.) xv. 708

Citellaria, (Meig.) xv. 700

Cixius, xv. 224

Cladius, (Klüg.) xv. 356

Cladophorus, xiv. 371

Clambus, xv. 168

Clavicornes, xiv. 398 family of the, xiv. 384

Claviger proper, xv. 168

Clavipalpi, xv. 154

Clavipes, (Fab) xv. 365

Cleonus, xv. 59

Cleonymus, (Latr.) xv. 366

Cleopatra Papilio, (where found,) xiv. 88

Cleptes, (F'ab.) xv. 366

of Latreille, xv. 370

Cleri, of Olivier, xiv. 399

Clerus, xiv. 374

(Fab.) xiv. 345

(Geoff.) xiv. 342

alvearius, xiv. 375

apiarius, xiv. 374 .

formicarius, xiv. 345

Kirbyi, xiv. 376

mollis, xiv. 345

mutillarius, xiv. 345

proper, (Geoff.) xiv. 345

unifasciatus, xiv. 343

Clitus, (Fab.) xv. 105

Clivina, description of, xiv. 204 Arctica, xiv. 205

Clypeaster, (Andersche) xv. 159

Clythra, (Fab.) xv. 137 quadripunctata, xv. 137

Clytus Haiji, xv. 118

Cnemacanthus gibbosus, xiv. 276

Cnemidotus, description of, xiv. $25^{\prime}$

Cnodalon, (Lat.) xv. 12 (Fab.) xv. 13

a new species of, $x v .21$ nodosum, xv. 22

Cobax, of M. Germar, xv. 223

Cocci, abdomen of, xv. 231 female without wings, xv. 23 granum, (Pliny) xv. 291 injury done by, xv. 276

Coccinella, xv. 160 bipunctata, xv. 159. 162 for cantharides, $\mathrm{xv} .48$ larvæ of, xv. 152 pustulata, xv. 159 septempunctata, 159. 162

Coccinellæ, food of the, xv. 161 larvæ of, xv. 162

Cœlioxys, (Lat.) xv. 385 
Cœnomyia, (Lat.) xv. 699

Cœnosia, (Meig.) xv. 711

Coccus, xv. 229. 273

(Geoff.) xv. 274

of Lin., xv. 228

cacti, xv. 277

caraccias, $\mathrm{xv} .37$

farinarius, $\mathrm{xv} .285$

ficus caricæ, xv. 284

tinctorius Polonicus, xv. 282

ulmi, xv. 282, 283

Cochineal, (Geoff.) xv. 274

cultivation of the, $\mathrm{xv} .273$

insects, xv. 273

insects, maternal stratagem of, xiv. 77

insects, size of, xiv. 78

Cochineals, habits of the, xv. 275

Cochleoctonus, xiv. 336

Cochonnes, what called, xv. 597

Cock-chafer, xiv. 519

Cock-chafers, larvæ of, xiv. 521

method for destruction of, xiv. 525

ravages of, xiv. 521

Cock-roaches, xv. 184

Cocytia, (M. Bois Duval) xv. 595

Codrus, (Jur.) xv. 368

Colaphus, (Meg.) xv. 140

Colapses, xv. 149

Colaspis, $(F a b.) \times v .139$

Coleoptera, aquatic, winglet of, xiv. 17

of Capricornus, food of, xiv. 68 carnassial, buccal apparatus of, xiv. 6

carnivorous, where found, xiv. 96

corslet of, xiv. 12

description of, xiv. 26.170

derivation of, xiv. 254

eyes of, xiv. 45

fluid emitted by fiesh-eating, xiv. 72

food of, xiv. 67

herbivorous, size of, xiv. 78

lamellicorneous, different from other hexapodes, xiv. 4

masticating insects, xiv. 40

mesothorax of, xiv. 51

metamorphosis of, xiv. 82

microptera, xiv. 290. 292

nicerium of, xiv. 15

nymphæ of, xiv. 21

stigmata on first segment of, xiv. 3

tarsi of, xiv. 261

thoracic segments of, xiv. 13

thorax of, xiv. 11
Coleoptera, what so called, xiv. 17

Colletes, (Lat.) xv. 382

Colliuris emarginata, xiv. 182 description of the, xiv. 181 longicollis, xiv. 182

Collyris, description of the, xiv. 181

Colobicus, (Latr.) xiv. 399 tarsi of, xiv. 400

Colobothea, xv. 110

Colophæna, description of, xiv. 193

Colophon, of Mr. Westwood, xiv. 533, 534

Colpodes, description of, xiv. 219 Brunneus, xiv. 220

Colydium, (Hellw.) xv. 84. 86

Colymbetes, description of, xiv. 247

Comasinus, (Deg.) xv. 60

Coniatus, (Schoen.) xv. 59

Conognathus, xiv. 358

Conops, the, xv. 766. 766 (Lin.) xv. 701. 706 rufipes, xv. 766

Conopsariæ, xv. 706, 766

Cophenes, (Lat.) xv. 706

Cophosus cylindricus, xiv. 215

Copris, xiv. 450.511 of Fabricius, xiv. 455,456 (Oliv.) xiv. 458

Antenor, xiv. 457

Bucephalus, xiv. 457

gigas, xiv. 457

hamadryas, xiv. 457

hispanus, xiv. 457

jachus, xiv. 457

lunaris, xiv. 450

Midas, xiv. 457

Molossus, xiv. 457

nemestrinus, xiv. 457

proper, xiv. 506

proper, (Geoff.) xiv. 457

Sabæus, xiv. 457

Coprobius, xiv. 454

Copromyza, (Fall.) xv. 712

Coprophagi, xiv. 447. 449

Coprophilus, description of, xiv. 296

Coptocephalus Brasiliensis, xv. 113

Coptodera, description of, xiv. 197

Cordistes, description of, xiv. 193 cinctus, xiv. 272

Cordyla, (Meig.) xv. 689

Cordylura, xv. 713

Corei, xv. 240

Corethra, xv. 742

(Meig.) xv. 684

culiciformis, $x v .742$

Corethræ, nymphs of, xv. $\mathbf{7 4 3}$

Coreus bicolor, xv. 241

histrix, xv. 240

(Fab.) xv. 218 
Corixa, (Geoff.) xv. 222

Corixæ, habits of the, xv. 251

Coronis, (Lat.) xv. 596

Corslet rouge, xiv. 196

Corsyra, between Brachini \& Catascopi, xiv. 188

Corticus, (Dej.) xiv. 560

Corydalis, (Lat.) xv. 299

Corynetes, ( $F a b$.$) xiv. 346, 347$ sanguinicollis, xiv. 347

Cossiphenes, xv. 5

Cossonus, (Clairv.) xv. 63

Cossus, (Fab.) xv. 603, 604 œsculi, xv. 605

ligniperda, xv. 579. 604

no blood vessels in, xiv. 71

palpi of, xv. 601

pyrinus, xv. 605

scalaris, xv. 605

Cossyphus proper, (Fab.) xv. 6

$$
\text { (Gyll.) xv. } 159
$$

(Oliv.) xv. 5

depressus, xv. 9

Coxelus, xv. 5

Crabro, (Fab.) xv. 378

(Geoff.) xv. 355

improper name of, xv. 400

Crabronitæ, xv. 377

Crambi, (Fab.) xv. 620

Crambus, $(F a b$.$) xv. 625$ carnellus, xv. 625 carneus, xv. 626 erigatus, xv. 624

Craspedosome, xiv. 131

Cremastocheilus, (Knoch.) xiv. 493

Creophilæ, xv. 707

Crepuscularia, xv. 594

Cricket, bill of the, xiv. 15 house, xv, 199

Crickets, appendages of the trachea of abdominal segment, xiv. 16 field, xv. 198

field, habits of, xv. 201

how destroyed, xv. 200

sound produced by, xiv. 76

stigma of the metathorax of, xiv. 3

why called, xv. 197

Criocerides, xv. 123

Crioceris, xv. 125. 141

(Fab.) xv. 122

(Geoff.) xv. 123

how produced, $x v .430$

larvæ of, xv. 132.754

Crioceres, xv. 126

larvæ of, xv. 128

Criquets proprement dits, xv. 202

of Geoffroy, habits of, xv. 204

Cuv. Index.
Crocisa nitidula, xv. 575

(Jur.) xv. 386

Crustacea, of M. de Lamarck, xiv. 25

amphipod, segments of abdomen of, xiv. 9

decapode, heart of, xiv. 2

decapode, segments of abdomen of, xiv. 9

description of, xiv. 38

form a part of insects, xiv. 24

insects differ from, xiv. 1

isipod, segments of abdomen of, xiv. 9

jaws of, xiv. 9

made a particular class by $\mathbf{M}$. Cuvier, xiv. 39

thorax of, xiv. 10

Crydia, appendages of the trachea of abdominal segment, xiv. 16

Crypticus, (Iat.) xiv. 559

Cryptocephali, xv. 146

Cryptocephalus, xv. 137

proper, xv. 138

quadripunctata? xv. 146

rugicollis, xv. 146

Cryptocerus, (Latr.) xv. 372

Cryptodus, xiv. 467

Cryptophagus, (Herbst.) xiv. 402 silaceus, xiv. 403

Cryptorhynchus, xv. 62. 72

Cryptosoma, (Dej.) xiv. 319

Cryptus, (Fab.) xv. 361

(Jur.) xv. 356

Lavignii, xiv. 135

(of Dr. Leach) xiv. 135

hortensis, xiv. 135

Crytops, (Sch.) xv. 56

Ctenipes, description of, xiv. 22l

Ctenicera, (Lat.) xiv. 320

Ctenistes, (Reich.) xv. 167

Ctenodactyla, description of, xiv. 194 Chevrolatii, xiv. 194 of Cayenne, xiv. 194

Ctenodes, (Oliv.) xv. 103

Ctenophora, (Meig.) xv. 685

Ctenostoma, description of the, xiv. $\mathbf{3 0}$

Cucujus, $(F a b$.)description of, xiv. 95.199 bifipes, xiv. 199

Cuculinæ, xv. 385

Culex of Linnæus, xv. 683. 725

annulatus, xv. 732

ciliatus, xv. 684

reptans, xv. 752

Culices, the, xv. 726 annoyance from, xv. 729 corslet of the, $\mathrm{xv} .728$

Culicides, (Lat.) xv. 683

Culicoïdes, (Lat.) xv. 685 $\mathrm{K}$ k 
Cupes, (Fab.) xiv. 354 capitata, xiv. 354

Curculio, xv. 68 of Fabricius, xv. 56.58. 69 (Lin.) xv. 20. 55 properly so called, xv. 57 larvæ of, xv. 93 acuminatus, xv. 57 argentatus, xv. 69 chrysis, xv. 69 cyanipes, xv. 70 fastuosus, xv. 69 ligustici, xv. 70 regalis, xv. 69 spectabilis, xv. 70 taniarisci, xv. 69 viridis, xv. 69

Curculiones leaping, xv. 69

Cursoria, xv. 171

Cychrus, description of, xiv. 231 attenuatus, xiv. 231 elevatus, xiv. 231 Italicus, xiv. 231 reflexus, xiv. 228 related to Dryptæ, xiv. 191 rotatus, xiv. 231

Cyclica, xv. 134 supplement on the, xv. 143

Cyclocepha, (Latr.) xiv. 472

Cyclomus, xv. 56 (Schoe.) xv. 56

Cyclosomi, anatomical observation, xiv. 208

Cylas, (Lat.) xv. 55

Cylidrus, (Lat.) xiv. 343

Cyllenia, (Lat.) xv. 694

Cymatodera, xiv. 375

Cymex, (Lat.) xv. 220

Cymindis anomæus, description of, xiv. 195 fusula, xiv. 188

Cynips, xv. 419 of Lin. xv. 364 eggs of, xv. 423 fagi, gall of, xv. 429 galls produced by, xv. 425 ficus caricæ, xv. 424 food of, xiv. 68 glecome, gall of, xv. 429 psenes, xv. 424 quercûs folii, gall of the, xv. 427

gall produced by, xv. 427

Cynthia, description of, xiv. 227

Cyphicerus, (Schoe.) xv. 57

Cyphomyia, (Wied.) xv. 699

Cyphon, (Fab.) xiv. 328 palustris, xiv. 329

Cyrtus, (Lut.) xv. 692
Dacne, proper, (Lat.) xiv. 402. 420

Dacus, $(F a b.) \times v .716$

Dalcar, (Deg.) xiv. 561

Damoiselles, what called, xv. 304. 312

Danaï festivi, xv. 583

Danaïs, (Fab.) xv. 586

Dapsa, (Zieg.) xv. 157

Daptus, description of, xiv. 208 pictus, xiv. 208 vittatus, xiv. 208

Darcacerus, (Dej.) xv. 102

Darnis, (Fab.) xv. 225 camelus, xv. 260

Dascillus, (Lat.) xiv. 327

Dasycerus, (Brong.) xv. 87

Dasygastræ, (Cuv.) xv. 384

Dasygnathus, where placed, xiv. 469

Dasypoda, (Lat.) xv. 382

Desypogon, (Meig.) xv. 690

Dasytes, xv. 35

(Fab.) xiv. 340

cæruleus xiv. 340

Dasyus, (Serv.) xiv. 482

Death's head moth, xv. 677

Death-watch, xiv. 351

Decatoma, (Lat.) xv. 699

Delpliax, (Fab.) xv. 224

Deltoïdes, (Lat.) xv. 620

Demetrias, description of, xiv. 195

Dendari, (proper) xiv. 557

Dendarus, xiv. 556

Dendroïdes, (Lat.) xv. 28

Dendrophagus, (Gyll.) xv. 95

Dendrophilus, (Leach) xiv. 389

Denticrura, description of, xiv. 294

Deracanthus, (Schoe.) xv. 56

Derbe, (Fab.) xv. 224

Dermaptera of Kirby, xv. 179. 229

Dermeste à oreilles, xiv. 411

Dermestes, xiv. 379. 403. 420

(Fab.) xiv. 401. 405; xv. 87, 88

(Geoff.) xiv. 391. 402.426

(Lin.) xiv. 340. 401, 402. 404 ; $\mathrm{xv} .84$

adstrictor, xiv. 318

affinis, xiv. 404

atomarius, $\mathrm{xv} .168$

emarginatus, xiv. 405

hirtus, xiv. 340

laniarius, xiv. 404

lardarius, xiv. 404

means of removing, xiv. 422

Megatoma, xiv. 405

murinus, xiv. 404

pellio, xiv. 405

rufitarsis, xiv. 407

scarabæoïdes, xiv. 432

serra, xiv. 406 
Dermestes subfasciatus, xiv. 406 tessellatus, xiv. 404 trifasciatus, xiv. 404 undatus, xiv. 404, 405 violaceus, xiv. 347.377 vulpinus, xiv. 404 where found, xiv. 421

Desmocerus, (of Dej.) xv. 11]

Dermoptera, description of, xiv. 26

Dexia, (Meig.) xv. 709

Dialyta, xv. 713

Diaperales, xv. 2. 7

Diaperis, $\mathrm{xv} .2$

Boleti, xv. 8

nigro-punctatus, xv. 8 proper, (Geoff.) xv. 3 whence named, $\mathrm{xv} . \boldsymbol{8}$

Diaphorus, xv. 696

Diapria, (Latr.) xv. 368

Diasomus digitatus, xiv. 461

Diateina holymenoïdes, xv. 774

Dibolia, (Lat.) xv. 142

Dicerea, xiv. 357

Dicerous, insects called, xiv. 41

Dices, (Dej.) xv. 32

Dicheles, (Lep. \& Serv.) xiv. 488

Dicrania, (Lep. \& Serv.) xiv. 485

Dicranoura, (God.) xv. 612

Dictya, (Fab.) xv. 716

Diesia, xiv. 542

Diggers, description of, xiv. 197 ; xv. 374

Diglobicerus, xiv. 342

Dinaelateroïdes, xiv. 322

Dilophus, (Meig.) xv. 689

Dinetus, (Jur.) xv. 376

Dineutes, xiv. 253

Dinidor, sub-genus of, xv. 218

Dioctria, (Meig.) xv. 691

Dinodes rufipes, xiv. 225

Dionæa muscipula, xv. 771

Dionix, (Dej.) xv. 166

Diopsii, (Lin.) xv. 715

Diopsis brevicornis, xv. 774 fasciata, xv. 773

Diosomus, (Dalm.) xiv. 460

Diphucephala, (Dej.) xiv. 482

Diplolepariæ, xv. 363

Diplolepis, (Fab.) xv. 366 (Geoff.) Xv. 364

Diplopides, of Geoffroy, xv. 419 Diploptera, xv. 379

Dipsa, (Fall.) xv. 714

Diptera, the, xv. 681 description of, xiv. 28 duration of life of, xv. 723 eyes of, xiv. 45 larvæ of, xv. 724 regions of the back of, xiv. 51 muscles of, xiv. 54
Diptera, metathorax of, xiv. 11 respiration of, xiv. 74 stigmata of the thorax of, xiv. 12 sucker of, xiv. 40 supplement on the, $x v .721$ wings of, xiv. 16

Dirceæ, tarsi of, xv. 11

Dircæa of Fab. xv. 16, 17.18 barbata, xv. 24 ruficollis, xv. 23

Discælius, (Lat.) xv. 381

Dissectors, what called, xiv. 404

Ditichocera, (Kir.) xv. 107

Ditoma chloroticus, xiv. 208 vittiger, xiv. 208

Ditomus bucephalus, larva of, xiv. 198 calydanius, xiv. 206 description of, xiv. 206. 274

Diurna, xv. 582

Dixa, (Meig.) xv. 686

Dog-flies, what named, xv. 775

Dolichocera, xv. 714 Childreni, xv. 65

Dolichoceres, xv. 712

Dolichopi, the, xv. 755

Dolichopides, xv. 695

Dolichopus, (Lat.) xv. 695 ungulatus, $\mathrm{xv} .755$

Dolichurus, (Latr.) xv. 376

Dolichus, description of, xiv. 221 proper, xv. 696

Donacia, xv. 125 (Fab.) xv. 123 crassipes, xv. 133

Donaciæ, xv. 121. 133

Dorcacerus, xv. 98

Dorcadion of Dalman, xv. 109

Dorcatoma, (Herbst.) xiv. 350 Dresdensis, xiv. 351

Doritis, $(F a b$.$) xv. 584$

Dorthesia, xv. 37

Dorylas, (Fab.) xv. 372

Doryphora, (Illig.) xv. 139 princeps, xv. 149

Dung-beetles, what called, xiv. 507

Dragon-flies, respiration of, xiv. 74 wings of, xiv. 16

Dragon-fly, experiment upon eyes of, xiv. 46

lenses in the eye of a, xiv. 48

Drapetes, (Meg. Dej.) xiv. 317 castaceus, xiv. 317

Drapetis, (Meig.) xv. 692

Drepana, xv. 612

Drilus, (Oliv.) xiv. 335 ater, xiv. 337 ruficollis, xiv. 337 subflavus, xiv. 336

Dromias, description of, xiv. 195 k $\mathbf{k} 2$ 
Dromica tuberculata, xiv. 265

Drummer, what called, xv. 186

Dryinus, (Lat.) xv. 367

Drymela, (Meig.) xv. 711

Dryomyza, xv. 714

Dryops, (Fab.) xv. 19, 20 of Olivier, xiv. 410

(Payk.) xv. 14

(Schoenh.) xv. 19

affinity with gyrini of, xiv. 409

picipes, xiv. 411

(proper) (Oliv.) xiv. 411

Dryopthorus, xv. 63

Drypta emarginata, xiv. 192 description of the, xiv. 192

Dryptæ, related with Cychrus, xiv. 19]

Dynastes, lenses in the eye of a, xiv. 48

Dyschirius, description of, xiv. 204

Dytilus, (Fisch.) xv. 19

Dyctioptera, (Lat.) xiv. 330

Dynastes, type of, xiv. 470

Dytisci, xiv. 433

Dytiscus, xiv. 424

(Lin.) xiv. 429

abbreviatus, xiv. 248

aciculatus, xiv. 247

adspersus, xiv. 248

agilis, xiv. 248

ater, xiv. 248

bimaculatus, xiv. 248

bipunctatus, xiv. 248

bipustulatus, xiv. 248

castalis, xiv. 247

chalconatus, xiv. 248

cinereus, xiv. 248

circumflexus, xiv. 247

confluens, xiv. 249

crassicornis, xiv. 250

depressus, xiv. 249

description of, xiv. 243,244

dorsalis, xiv. 249

duodecim-pustulatus, xiv. 249

erythrocephalus, xiv. 249

fenestratus, xiv, 248

fuliginosus, xiv. 248

fulvus, xiv. 250

fuscus, xiv. 248

geminus, xiv. 249

general description of, xiv. 281

granularis, xiv. 249

griseus, xiv. 247

griseus, where found, xiv. 90

guttatus, xiv. 248

halensis, xiv. 249

hyalinus, xiv. 248

hyberni, xiv. 247

impressus, xiv. 250

inæqualis, xiv. 249

interruptus, xiv. 248
Dytiscus lævigatus, xiv. 247

lateralis, xiv. 247

latissimus, xiv. 246

Leander, xiv. 248

lineatus, xiv. 249

lituzatus, xiv. 249

maculatus, xiv. 248

marginalis, xiv. 245.246

marmoreus, xiv. 248

minutus, xiv. 248

nigrita, xiv. 249

obliquus, xiv. 250

(proper) xiv. 246

palustris, xiv. 249

picipes, xiv. 249

pictus, xiv. 249

planus, xiv. 249

punctatus, xiv. 247

reticulatus, xiv. 249

Ræselli, xiv. 247

ruficollis, xiv. 247

serricornis, xiv. 247

sex-pustulatus, xiv。 249

stagnalis, xiv. 248

stagnalis quatuor-vittatus, xiv. 247

sticticus, xiv. 247

sulcatus, xiv. 247

transversalis, xiv. 247,248

tripunctatus, xiv. 247

varius, xiv. 248

vittatus, xiv. 247

zonatus, xiv. 248

Earwig, the common, xv. 171

the common, description of, $x v$. 182

Earwigs, description of, xv. 181

pylorus of, xv. 180

Eccoptogaster, (Herbst.) xv. 82

Echinomya, (Dum.) xv. 707

Eciton, (Lat.) xv. 372

hamata, xv. 516

Ecomerus, (Zeig.) xv. 705

Edessa, (Fabr.) xv. 217 amethystina, xv. 218 papillosa, xv. 218

Egeria, xv. 598

Engis, $(\mathrm{Fab}$.) xiv. 402

Elampus of Spinola, xv. 370

Elaphorus, (Fab.) xiv. 426 crenatus, xiv. 426

Elaphri, description of, xiv. 280

Elaphrus, (Fab. Oliv.) description of, xiv. 240

aquaticus, xiv. 240

biguttatus, xiv. 240

description of, xiv. 238 
Elaphrus cupreus, xiv. 239 impressus, xiv. 242 paludosus, xiv. 239. 242 riparius, xiv. 239 rupestris, xiv. 242

Elater, (Fab.) xiv. 319

(Lin.) xiv. 314.318

æneus, xiv. 321

auratus, xiv. 363

aureolus, xiv. 363

Borealis, xiv. 322

buprestoïdes, xiv. 313.359

castaneus, xiv. 321

clavicornis, xiv. 318

cinctus, xiv. 322

cruciatus, xiv. 321

cupreus, xiv. 320

denticornis, xiv. 319

dermestoïdes, xiv. 318

equestris, xiv. 317

fascicularis, xiv. 320

ferrugineus, xiv. 321

flabellicornis, xiv. 320

fuscus, xiv. 317

germanus, xiv. 321

hæmatodes, xiv. 320

lævigatus, xiv. 317

linearis, xiv. 322

lusciosus, xiv. 363

noctilucus, xiv. 320.362

occulatus, xiv. 363

ovalis, xiv. 317

pectinicornis, xiv. 320

phosphorus, xiv. 363

(proper) xiv. 320

ruficollis, xiv. 321

striatus, xiv. 315

undulatus, xiv. 315

what called, xiv. 359

Elaterides, xiv. 314

Elateroïdes, xiv. 359

(Schoeff.) xiv. 353

Eledona, (Latr.) xv. 5. 9

Elenophorus, (Meg.\& Dej.) xiv. 547

Elephastomus, (Macl.) xiv. 465

Eleutherata, description of the, xiv. 170

Elmis, (Lat.) xiv. 411

Elodes, (Lat.) xiv. 328

Elophori, xiv. 444

Elophorus, (Fabricius) xiv. 425, 426 ; $\mathrm{xv} .85$

aquaticus, xiv. 445

brevis, xiv. 426

elongatus, xiv. 426

marinus, xiv. 426

minimus, xiv. 426

pygmæus, xiv. 426

Elpenor sphinx, xv. 597
Embia, (Lat.) xv. 347

Emesa filum, xv. 244

Empides, the, xv. 753

Empis, (Lin.) xv. 691

Empusa, (Illig.) xv. 172

Enceladus, description of, xiv. 198 gigas, xiv. 199

Encynetus, xiv. 328 hæmorrhoïdalis, xiv. 328

Encyrtus, (Lat.) xv. 367

Endomychus, (Web.) xv. 157

Endromis, xv. 609

Engidites, xiv. 402

Engis, of Fabricius, xiv. 400

Enicodes, xv. 117

Enoplium, (Lat.) xiv. 347

Enterides, hepatic conduits of, xiv. 308

Entimus, xv. 57

Entodon, (Dalm.) xv. 367

Entomostracea, thorax of, xiv. 10

Epeolus, (Lat.) xv. 385

Ephemera, (Lin.) xv. 296 eggs of, xiv. 79 eyes of, xiv. 45 instinct of, xiv. 67 metamorphosis of, xiv. 22. 81 why named, xv. 313 females of the, xv. 320 food of, xv. 316 gills of, xiv. 75 great numbers of, xv. 315 habits of the, xv. 314 larvæ of the, xv. $32 \mathrm{l}$ motions of the, xv. 318

Ephydra, (Fall.) xv. 711

Epiphasis cornutus, xiv. 364

Epippium, (Meig.) xv. 700

Epicharis, (Klüg.) xv. 387

Epistenia ceruleate, xv. 432 of $\mathrm{Mr}$. Westwood, xv. 432

Epitragi, xiv. 558

Epitragus, (Lat.) xv. 12

Epomis, description of, xiv. 225 circumscriptus, xiv. 225 gorgi, xiv. 276

Erebus, (Latr.) xv. 613

Erioptera, (Meig.) xv. 686

Eriphia, (Meig.) xv. 711

Eristales, the, xv. 760

Eristalis, (Fab.) xv. 705 (Lat.) xv。704 (Meig.) xv. 702 intricarius, xv. 42.44

Erodius, (Fab.) xiv. 544 bilineatus, xiv. 544 gibbus, xiv. 544 levigatus, xiv. 544 proper, (Latr.) xiv. 544

Erotylus, (Fab.) xiv. 402 ; xv. 155 
Erycina, (Lat.) xv. 592

Xanthippe, xv. 677

Ethiopian ant, $\mathrm{xv} .446$

Eubria, (Zieg.) xiv. 328

Eucera proper, xv. 386

Euceræ, of America, xv. 386

Eucharis, xv. 364

(Lat.) xv. 365

Euchræus, of Latreille, xv. 369

Euchlora, (Macl.) xiv. 484

Eucnemis, $(A r h$.$) xiv. 316$ capucinus, xiv. 316

Eudamychus, (Fab.) xv. $\mathbf{1 5 7}$

Euglossæ, (Lat.) xv. 388

Eulopa, of Fallen, xv. 226

Eulophus, (Geoff.) xv. 367

Eumenes, (Lat.) xv. 380 proper, xv. 381

Eumenia, (Fab.) xv. 590 Childrenæ, xv. 677

Eumolpus, xv. 147 (Fab.) xv. 138 vitis, xv. 138. 147

Eumorphus, xv. 157

Euparia, xiv. 459 castanea, xiv. 459

Eupelix, (Germ.) xv. 226

Eupelmus, (Dalm.) xv. 366

Euploca, (Fab.) xv. 586

Euplocamus, (Lat.) xv. 625

Euplectus, (Leach) xv. 167

Eupoda, xv. 121 supplement on the, xv. 125

Euprosopi, resembling the Cindelæ, xiv. 178

Euprosopus, description of the, xiv. 177 quadrinotatus, xiv. 178

Eurhinus, (Kirby) xv. 54 (Schoen.) xv. 61

Eurybia, (Illig.) xv. 591

Eurycephalus niger, xv. 119 new subgenus of, xv. 119

Eurychile, description of the, xiv. 181

Eurychora, (Thunb.) xiv. 547

Eurychorus, xiv. 565

Eurynotus of Mr. Kirby, xiv. 557 muricatus, xiv. 557

Euryope, (Dalm.) xv. 138

Euryptera, of M. Guerin, xv. 260

Eurypus, (Kirb.) xiv. 344 rubens, xiv. 344

Eurysternus, xiv. 454 of M. Dalman, xiv. 459

Eurytoma, (Illig.) xv. 366

Eusthenia spectabilis, xv. 348

Euthycera, (Lat.) xv. 714

Evæstheti, xiv. 305

Evæsthetus, description of, xiv. 294
Evætsthetus scaber, xiv. 294

Evania, (Fab.) xv. 359

Evaniales, xv. 359. 406

Exophthalmus, (Lat.) xiv. 322

Eyprepia, (Ochs.) xv. 611

Feronia, description of, xiv. 212 (Leach) xv. 719

Figites, (Lat.) xv. 364

Figulus, xiv. 501

Fiscipennes, xv. 628

Forbicina, description of, xiv. 145

Forficula Brasiliensis, xv. 184 pylorus of, xv. 180

Forficulæ, eyes of, xiv. 171

Formica, xv. 434 of Linnæus, xv. 371 emarginata, xv. 464 fuliginosa, $\mathrm{xv} .444 .446$

Formica-leo, xv. 324 proper, xv. 371 quotation from Virgil, xiv. 34 rufa, xv. 435. 464. 480 rufa, acid of, xv. 489

Formicæ rufæ, habitation of, xv. 436

Formicariæ, larvæ of the, xv. 450

Flata, (Fab.) xv。 224

Flea, common, remarks upon the, xiv. 156

penetrating, xiv. 156

metamorphosis of the, xiv. 83 without metamorphosis, xiv. 21

Fleas, description of the, xiv. 154 garden, xv. 153

general remarks upon the, xiv. 165

little carriage drawn by, xiv. 167 parasitical insects, xiv. 166

wonderful structure of, xiv. 168, 169

Flesh-fly, deceived by smell, xiv. 103 deposits its eggs in flowers, xiv. 43

Flies, the, xv. 773

nymphæ of, xiv. 22

tarsi of, xiv. 65

Fly-bug, xv. 243

Fly, the domestic or common, xv. 767

the, (hop) xv. 161

Fœnus, xv. 359 proper, (Fab.) xv. 360 habits of, xv. 406

Forficula, of Oliv. xv. 179 (Lin.) xv. 171 description of $\mathrm{xv} .18 \mathrm{I}$ auricularia, xv. 171. 181 minor, xv. 171

Forked-tails, what named, xv. 418 
Fossores, xv. 373

Fulgora lanternaria, xv. 258 nigromaculata, xv. 260 pallida, xv. 260 punctata, xv. 259

Fulgoræ, description of the, xv. 258 proper of Fab. xv. 223

Fulgorella, xv. 223

Fungicolæ, xv. 156

Fungivora, xv. 687

Gad-fly, the, xv. 763

Galba, (Lat.) xiv. 316

Galerita, description of, xiv. 193

(Fab.) description of, xiv. 199

Africana, xiv. 193

depressa, (Fab,) xiv. 199

fasciolata, xiv. 191

flejus, (Fab.) xiv. 199

hirta, xiv. 191

occidentalis, xiv. 193

olens, xiv. 190

Schupelii, (Dej.) xiv. 199

Galeruca, xv. 141

Calmariensis, xv. 150

nympheæ, xv. 150, 151

tanaceti, xv. 150

Galerucitæ, xv. 140

the leaping, $\mathrm{xv} .141$

Galerucæ, xv. 149

Galgulus, (Latr.) xv. 221

Galleria, (Fab.) xv. 624 alvearia, xv. 624 cereana, xv. 563.624

Gallicolæ, (Lat.) xv. 363. 419

Gallinsecta. xv. 228

Gall insects, xv. 274

Gardener, the, xiv. 234

Gastropacha, (Ochsenheimer) xv. 606

Geniates, xiv. 477 barbatus, xiv. 478

Genuchus, xiv. 496

Geocorisæ, xv. 217

food of, xv. 232

the sheath of the sucker of, $x v$. 230

Georissi, xiv. 423

Georissus, xiv. 410

(Lat.) xiv. 412

pygmæus, xiv. 412

Géomètres, what called, xv. 579

Geometricians, what called, xv. 637

Geomyza, of Fallen, xv. 713

Geophilus, of Dr. Leach, xiv. 135

longicornis, xiv. 135

maritimus, xiv. 135

Geotrupes, xiv. 507

(Fab.) xiv. 466. 470. 475
Geotrupides, (Macl.) xiv. 460

Geotrupes arata, xiv. 466

bicolor, xiv. 469

boas, xiv. 469

depressus, xiv. 471

didymus, xiv. 471

dispar, xiv. 463

excavatus, xiv. 476

Momus, xiv, 463

Egeon, xiv. 469

orphnus, xiv. 469

oryctes, xiv. 470

phalangista, xiv. 463

proper, (Lat.) xiv. 463

rhinoceros, xiv. 469

silenus, xiv. 469

stentor, xiv. 469

stercorarius, xiv. 259. 463

valgus, xiv. 471

vernalis, xiv. 464

Geotrutes, xiv. 462

Geron, xv. 693

Gerris, (Lat.) xv. 221

description of, xv. 245

lacustris, xv. 246

laticauda, xiv. 248

where found, xv. 232

Gibbium, (Scop.) xiv. 349

Glaphyrus, (Latr.) xiv. 487

Glaucopis, (Fab.) xv. 600

Globaria, xiv. 427

Leachii, xiv. 428

Globicornis, (Latr.) xiv. 407

Gloma, (Meig.) xv。692

Glomeris, description of, xiv. 129 marginata, 130

Glossata, (Fab.) xv. 577

Glow-worm, phosphoric secretion of, xiv. 73

Gnathesia glabra, xiv. 545

Gnathium, (Kirb.) xv. 34

Gnathocera, xiv. 496

Gnats the, xv. 726

food of, $\mathrm{xv} .727$

gills of, xiv. 75

instinct of, xiv. 67

larvæ of, xv. 735

moulting of larvæ of, $\mathrm{xv} .736$

nymph of, xv. 737

prolific nature of, xv. 734

quotation from Spenser respect-

ing, xv. 730

where found, $\mathrm{xv} .731$

Gnoma, (Dej.) xv. 104

of Fab. xv. 109

ruficollis, $\mathrm{xv} .104$

sanguinea, $\mathrm{xv} .104$

suturalis, xv. 120

Gnovista, (Meigen.) xv. 687 
Goliath, (Liam.) xiv. 494

what called, xiv. 529

of Lamarck, where found, xiv. 93

Goliathidæ, xiv. 492

Gomphocerus, xv. 177

Sibiricus, xv. 177

Gonatopus, (Klüg.) xv. 36\%

Gonia, (Meig.) xv. 708

Gonius, (Jur.) xv. 376

Goniodès, peculiar to the Gallinacea, xiv. 153

Goniopleura, xv. 149 auricoma, xv. 149

Gonopus, Lat.) xiv. 554

Gonypus, (Lat.) xv. 691

Gorytes, (Lat.) xv. 378

Graine d'écarlate, xv. 291

Gramma, the, xv. 588

Grandipalpi, description of, xiv. 230

Graphipterus, description of the, xiv. 185

Graphiptera, where found, xiv. 92, 93 not found in Spain or Italy, xiv. 184

Grass-hoppers, muscles of, xiv. 54 what, xv. 202 where found, xv. 203

Grinding-insects, mouth of, xiv. 8

Gryllo-talpæ, (Lut.) xv. 174 description of, xv. 192

Gryllo-talpa, digestive organ of, xv. 180 resemblance of, $\mathrm{xv} .196$ stomachs of, xv. 179

Gryllus, xv. 192

of Fabricius, xv. 202

of Fabricius, habits of, xv. 204

(Geoff. \& Oliv.) xv. 174

of Lin. xv. 173

acheta, $(F a b$.$) xv. 192$

acrida, xv. 176

Egyptius, xv。 177

bulla, xv. 176

bulla, (Lin.) xv. 177

campestris, xv. 187. 196, 197

campestris, description of, $\mathrm{x} v$. 198

cærulescens, xv. 177

carinatus, xv. 176

cristatus, $x v .214$

domesticus, xv. 197

dux, xv. 214

gallinaceus, xv. 176

general name of, xv. 202

lineola, xv. 177

locusta, xv. 176

locusta, of Linnæus, xv. 202

migratorius, 177. 214

monster, xv. 175
Gryllus monticollis, xv. 215 pedester giornæ, xv. 177 proper, xv. 176

striaculus, xv. 177

tataricus, xv. 177

tetigonia, xv. 175

umbraculatus, xv. 174

Gymnetis, of Macleay, xiv. 495

Gymnomyza, (Fall.) xv. 717

Gymnomyzides, xv. 716

Gymnopleurus, (Illig.) xiv. 453

Gymnosomia, (Meig.) xv. 708

Gypona, of M. Germal, xv. 226

Gyrini, general description of, xiv. 285 eyes of, xiv. 45 size and habits of, xiv. 251

Gyrinus, description of, xiv. 243. 250 bicolor, xiv. 253

minutus, xiv. 253

natator, xiv. 252

politus, xiv. 253

Gyropi, female, xiv. 153

Gyropus, food of the, xiv. 152

Hæmatopota, (Meig.) xv. 697

Halictus, (Lat.) xv. 382

Haliplus, description of, xiv. 250

Hallomeni, tarsi of, xv. 23

Hallomenus, (Payk.) xv. 17

Haltica, (Geoff.) xv. 141

Hamaticerus, xv. 104

Hamaxobium, xiv. 328

Hammionus, (Mühf.) xiv. 322

Hammonie, (Cuv.) xiv. 324

Haplides, xiv. 484

Harpali, conical appendages of the, xiv. 174

Harpalus of M. Bonelli, xiv. 208 analis, xiv. 209

anthracinus, xiv. 214

aterrimus, xiv. 214

binotatus, xiv. 209

caliginosus, xiv. 209

circumfuscus, xiv. 212

description of, xiv. 209

flavilabris, xiv. 209

collaris, xiv. 242

heros, xiv. 209

livens, xiv, 223

luridipennis, xiv. 212

megacephalus, xiv. 208

nigrita, xiv. 214

prasinus, xiv. 267

ruficornis, xiv. 209

saponarius, xiv. 210

tardus, xiv. 209

viduus, xiv. 223

Harpers, what called, xv. 255 
Harpyia, (Ochs.) xv. 612

Hedobia, xiv. 349

Hedychrum, xv. 370

Hegeter, (Lat.) xiv. 545

Heleomyza, (Fall.) xv. 714

Helcon, (Nees d'Es.) xv. 363

Helicani, xv. 583

Heliconia Cyrene, xv. 674

Heliconius, (Latr.) xv. 586

Helicorni, xv. 583

Heliophili, xiv. $\mathbf{5 5 7}$

Heliophilus, of Dejean, xiv. 557

Helix nemoralis, xiv. $\mathbf{3 3 6}$

Helluo, of Bonelli, xiv. 271 of Brazil, xiv. 191 costatus, xiv. 191 description of the, xiv. 191 pleuracanthus, xiv. 272 sulcipennis, xiv. 272 where found, xiv. 94

Helodes, (Fab.) xv. 140

Helœus, (Lat.) xv. 6 Brownii, xv, 6 where found, xv. 9

Helophilus, (Meig.) xv. 702

Helophoridea, (Leach) xiv. 425

Helopii, xv. 11

Helops, xv. 10.2] (Fab.) xv. 12, 13, 14. 19 aterrimus, xv. 22 glaber, xiv. 559

Helorus, (Latr.) xv. 368

Helwigia, xv. 361

Hemelytra, what so called, xiv. 17

Hemerobia, eyes of, xiv. $\mathbf{4 5}$

flight of, xv. 331

larvæ of, xv. 270

Hemerobiæ, eggs of, xiv. 79 metamorphosis of, xiv. 81. 83

Hemerobini, xv. 298

Hemerobius, (Lin.) xv. 299. 300

longicornis, $\mathrm{xv} .331$

maculatus, xv. 299

larvæ of the, xv. 324

pulsatorius, xiv. 381

fulvicephalus, xv. 299

why ramed, $x v .328$

Hemerodromia, (Hoff) xv. 692

Hemipeplus, xv. 27

Hemiptera, the, xv. 216

corslet of, xiv. 12

description, of. xiv. 27

eyes of, xiv. 45

hearing of, xiv. 56

metamorphosis of, xiv. 81,82

neuters among, xiv. 78

organ of suction of, xiv. 40

second family of, xv. 221

supplement on the, xv. 229

Cuv. Index.
Hemiptera, why named, xv. 229

Hemirhipus, (Lat.) xiv. 320

Henaps, (Illig.) xv. 692

Hepiales asellus, xv. 610 bufo, xv. 610 testudo, xv. 610

Hepialites, xv. 601. 603

Hepialus, (Fab.) xv. 603 humuli, xv. 603

Hepiolus, xv. 603

Hercules, (ant so called, xv. 446

Heriades, (Spin.) xv. 384

Hermes, sub-genus of, xv. 332

Hermetia, (Lat.) xv. 699

Hermina, (Lat.) xv. 620

Hesperia, (Fab.) xv. 593 malvæ, xv. 593 rurales, (Fab.) xv. 582

Hesperies, (Lin.) xv. 591

Hesperi sphinges, xv. $\mathbf{5 9 5}$

Heteroceri, xiv. 423

Heterogyna, xv. 370. 434

Heterocerus, xiv. 409 lævigatus, xiv. 410 marginatus, xiv. 410

Heteromera, xiv. $\mathbf{5 3 8}$

Heteroptera, xv. 217

Heteroscelis, xv. 217 (Lat.) xiv. 554

Heterotoma, xv. 219

Heterotarsus, (Lat.) xiv. 563

Hexaphyllum, xiv. 536 Brasiliensis, xiv. 536

Hexapode insects, stigmata of, xiv. 3

Hexapodes, majority of insects, xiv. 4

Hexapod insects, segments of abdomen of, xiv. 9 insects, thorax of, xiv. 10.11

Hexatoma, (Lat.) xv. 686 (Meig.) xv. 698

Hexodon, (Oliv.) xiv. 472 what have relations with, xiv. 515

Hexogonia, (Kirby) where to be placed, xiv. 197

Hilara, xv. 691

Hippobosca, (Lin.) xv. 719

equina, xv. 719

ovina, xv. 719

Hippobosci, the, xv. 775 bite of the, xv. 777

Hippoboscus, generation of the, xv. 776 nearly full-grown at their birth, xiv. 33 ovoviparous, xiv. 77

Hirmoneura, xv. 694

Hispa, xv. 143

(Lin.) xv. 135

atra, xv. 136 
Hispa mutica, xiv. 560

mystacina, xiv. 327

proper, xv. 136

spinipes, xv. 136

Hister, xiv. 386. 388. 413 ; xv. 66

(Fab.) xiv. 391

cadaverinus, xiv. 414

flavicornis, xiv. 389

glabratus, xiv. 391

globosus, xiv. 390

hispidus, xiv. 390

minutus, xiv. 390

oblongus, xiv. 389

picipes, xiv. 389

proboscideus, xiv. 389

proper, xiv. 388

punctatus, xiv. 389

sinuatus, xiv. 387

striatus, xiv. 390

sulcatus, xiv. 390

unicolor, xiv. 389

Histeroïdes, xiv. 386

Hithyia, (Fab.) xv. 626

Hœmonia, (Meg.) xv. 123

Holhymenia, (Lepel \& Serv.) xv. 218

Hololepta, xiv. 388

Holoptilus, (Lep. \& Serv.) xv. 220

Homalura, (Meig.) x v. 717

Homoptera, bite of, xv. 222

Homotenes, what so called, xiv. 7

Hoplia, (Illig.) xiv. 485 formosa, xiv. 485

Hoplides, xiv. 528

Hopliæ, by whom named, xiv. 528

Hoplitus, description of, xiv. 250

Horia, xv. 38

(Fab.) xv. 31

cantharis, xv. 38

Horiales, xv. 30

Hornet, the, xv. 517

Humble-bees, xv. 567 dwellings of, xiv. 119

insect on, xv. 42

metamorphosis of, xiv. 118 society of, xiv. 114

Hyblæa, (Fab.) xv. 620

Hyboma, xiv. 454

Hybos, (Meig.) xv. 691

Hybosorus, (Macl.) xiv. 466

Hybotini, xv. 691

Hycleus, (Lat.) xv. 32

Hydaticus, xiv. 247 ; xv. 62 marmoratus, xiv. 284

Hydera, xiv. 410

Hydrachna, description of, xiv. 248 gibba, xiv. 249

Hermanni, xiv. 248

ovalis, xiv. 249

scripta, xiv. 249
Hydrachna uliginosa, xiv. 248

Hydræna, (Illig.) xiv. 426

(Kugel) xiv. 426

longipalpis, xiv. 426

margipallens, xiv. 426

riparia, xiv. 426

Hydrobius, xiv. 431

griseus, xiv. 431

luridus, xiv. 43]

melanocephalus, xiv. 431

scarabæoïdes, xiv. 431

truncatellus, xiv. 431

Hydrocampe, (Lat.) xv. 623

Hydrocanthari, xiv. 433

description of, xiv. 243

Hydrochus, (Germ.) xiv. 426

Hydrocorisæ, the, xv. 221. 248

Hydrometra, the, x v. 244

of Fabr. xv. 221

habits of, xv. 245

where found, xv. 232

Hydromyzides, xv. 711

Hydrophili, xiv. 433

extraordinary change in food of, xiv. 66

manner of laying its eggs,xiv. 435 larvæ of, xiv. 443

Hydrophilus, (D. G.) xiv. 426

of Geoffroy, xiv. 424

ater, xiv. 430

brown, prey of dytiscus, xiv. 247

caraboïdes, xiv. 434

olivaceus, xiv. 430

piceus, xiv. 429.434

properly so called, xiv. 429

proper, of Dr. Leach, xiv. 430 rufipes, xiv. 430

Hydroporus, description of, xiv. 249

Hydroptila, of Mr. Dalman, xv. 302

Hydroüs, of Dr. Leach, xiv. 429

Hygrobia, description of, xiv. 248

Hylecoetus, (Lat.) xiv. 353 dermestoïdes, xiv. 353

Hylesinus, (Fab.) xv. 82

Holobius, xv. 59

Hylœus, (Fab.) xv. 381 proper, (Lat. \& Fab.) xv. 382

Hylotoma, (Lat.) xv. 356 enodis, larvæ of, xv. 403 rosæ, xv. 402

Hylotomæ, larvæ of, xv. 402

Hylurgus, (Lat.) xv, 82

Hymenoptera, xv. 353

aculeata, xv. 379

description of, xiv. 27

eyes of, xiv. 45

food of, xiv. 67 ; xv. 395

masticating insects, xiv. 40

metamorphosis of, xiv. 82 
Hymenoptera, metathorax of, xiv. 11 neuters among, xiv. 77 nymphæ of, xiv. 21 peculiar characters of, xv. 391 regions of the back of, xiv. 51 shield of, xiv 17

stigmata of the thorax of, xiv. 12

sting of females of, xiv. 18

supplementary segments of, xiv. 3 supplement on the, xv. 389 tegula of, xv. 578 wings of the, xiv. 15

Hypantus, xv. 58

Hypera, (Germ.) xv. 59

Hyphydrus, description of, xiv. 249 lyratus, xiv. 249

Hypocera, xv. 717

Hypophlæus, xv. 8 (Fab.) xiv. 561 ; xv. 4 boros, xiv. 561

Hypulus, (Payk.) xv. 18

Ibalia, (Latr.) xv. 361

Ichneumon, xv. 407

de Laponie, $x v .404$

(Lin.) xv. 360

aphidum, xv, 418

organs of touch of, xiv. 64

fly, xv. 429

habits of the female, xv. 410

luteolus, xv. 418

proper, xv. 362

rufus, larvæ of, xv. 417

utility of the, xv. 408

Ichneumonidæ, larvæ of, xv. 407

Ichneumonides, the, xv. 360. 406 larvæ of, xv. 581

Ichneumons, eggs of, $\mathrm{xv} .415$ galls of, $\mathrm{xv} .421$

Idea, (Fab.) xv. 586 (Meig.) xv. 710

Imatidium, $x \mathbf{v}$. 136

Incendula, what called, xiv. 366

Insects, brief description of, xiv. 38

Ips, of Degeer, xv. 82. 89

(Fab.) xiv. 400

(Oliv.) xiv. 402 ; xv. 4.84

with what classed, xv. 8

with what confounded, xiv, 420

Irenæus, (Leach) description of, xiv. 296

Isocerus, (Deg.) xiv. 557

Issus, $(\boldsymbol{F a b}$.) xv. 224

Iuli, description of, xiv. 129

feet of, xiv. 139

long growth of the, xiv. 126

metamorphosis of, xiv. 82
Iuli, parts of generation of, xiv. 18 proper, description of, xiv. 130 stigmata of the, xiv. 125

Iulus araneoïdes, xiv. 134 copulation of the, xiv. 128 complanatus, xiv. 131 depressus, xiv. 131 fasciatus, xiv. 130

Indus, xiv. 130 maximus, xiv. 130.140

ovalis, xiv. 130

sabulosus, xiv. 130

slight notice of the genus, xiv. 137

stigma, xiv. 131

terrestris, xiv. 130

food of the, xiv. 140

tridentatus, xiv. 131

with pencilled tail, xiv. 131

Jalodis, xiv. 357

Jarpeada, (cochineals) xv. 280

Jassus, (Fab.) xv. 226

Joppa, (Fab.) xv. 362

Kakerlacs, what called, xv. 486

Kermes, (Geoff.) xv. 274

habits of the, xv. 286

harvest of, xv. 290

three different states of, xv. 289 ulmi, xv. 293

the young, $\mathrm{xv} .288$

Labidus, (Jur.) xv. 372

Labourers, what called, xv. 333. 340

Lacophilus, (Leach..) xiv. 248

Lady-birds, xv. 160

Lady -cow, xv. 161

Lædie, (Cuv.) xiv. 320

Læna, (Meg.) xv. 14

Lœmosthenus, (Bon.) description of, xiv. 220,221

Lagria, xv. 35

(Fab.) xv. 27

hirta, xv. 35

metallonotus, xv. 35

Lagriariæ, xv. 26

Lamellicornes, xiv. 446

Lamia, xv. 97. 106

(Fab.) xv. 108

ædilis, xv. 108

curculionoïdes, xv. 109

funesta, xv. 109

lugubris, xv. 109

nebulosa, xv. 109

tristis, $\mathrm{xv} .109$

\section{12}


Lamia, where placed, xv. 118

Lamiæ, of Fab, xv. 109 where found, xiv. 88

Lamiariæ, xv. 107

Lamprias, description of, xiv. 196

Lamprima, (Latr.) xiv. 498 Humboldti, xiv. 499 (Schœen.) xiv. 499

Lampyri, hepatic vessels of, xiv. 308

Lampiris, (Lin.) xv. 27

Lamprosoma, (Kirb.) xv. 138

Lampryas ruficeps, xv. 272

Lampyris, (Lin.) xiv. 329

Italica, xiv. 332

Italica, experiments upon, xiv. 368

noctiluca, xiv. 334

plumicornis, xv. 333

proper, xiv. 334

sanguinea, xiv. 330

splendidula, xiv. 333, 334

what called, xiv. 366

Languria, (Lat.) xv. 155

Laphria, (Meig.) xv. 690

Larra, (Jur.) xv. 376

Larrates, (Cuv.) xv. 376

Lasiocampa, xv. 606;

Lasioptera, (Meig.) xv. 686

Lasius, (Wied.) xv. 693

Latridius, (Deg.) xv. 123 (Herbs.) x v. 87

Lathrobia, xiv. 304

Lathrobium, description of, xiv. 292 elongatum, xiv. 293

Lauscania, (Lat.) xv. 717

Leapers, the, xv. 135. 173

Lebia, description of, xiv. 196 dorsalis, xiv. 272

Ledra, xv. 224. 226

Legionaries, (neuters of red ants,) xiv. 120

Leias, (Meig.) xv. 688

Leiodes, (Latr.) xv. 4

Leioïdes, xiv. 445

Leistus, description of, xiv. 236 cæruleus, xiv. 237 rufescens, xiv. 237

Lema, of Fab., xv. 123

Lepidoptera, xv. 577

antennæ of the nocturnal, $x v$. 721

anterior stigmata of, xiv. 12

description of, xiv. 27

the diurnal, xv. 582

diurnal, eyes of, xiv. 45

food of, xiv. 67

food of caterpillars of, xiv. 66

holes near the eyes of, xiv. 5

instinct of, xiv. 67
Lepidoptera, larvæ of, xv. 578 metamorphosis of, xiv. 83 mouth of, xiv. 40 nymphæ of, xiv. 21 pterygodes of, xiv. 28 regions of the back of, xiv. 51 size of, xiv. 96 supplement on the, xv. 630 thorax of, xiv. 11 where found, xiv. 93.95

Lepisia, (Lepel \& Serv.) xiv. 484

Lepisma, description of the, xiv. 143. 145

Forbicini, xiv. 145

polypoda, xiv. 145

saccharina, xiv. 145

sugar, xiv. 145

thezeana, xiv. 145

vittata, xiv. 145

Lepisme, banded, xiv. 145

Forbicine cylindrique, xiv. 145

Lepismenæ, description of the, xiv. 143

Lepitrix, (Lep. \& Serv.) xiv. 489

Leptides, the, xv. 754 wings of, xv. 694

Leptis, (Fab.) xv. 695 scolopacea, xv. 754 vermileo, xv. 754

Leptocera, (Dej.) xv. 107

Leptocerus, $\mathrm{xv} .57$

Leptochire, (Germ.) description of, xiv. 2.96

Leptochirus scoriaceus, xiv. 296

Leptocorisa, (Lut.) xv. 219

Leptopodites, xv. 715

Leptopus, (Latr.) xv. 220

Leptosomus, xv. 57

Leptotrachelus, description of the, xiv. 189

Leptura, xv. 106

(Dej.) xv. 112

(Fub.) xv. 20

(Kirb.) xv. 112

(Lin.) xv. 111.123

armata, xv. 112

arcuata, xv. 105

calcarata, xv. 112

tomentosa, xv. 112

Lepturæ, where found, xv. 120

Lepturetæ, xv. 110

Lesteva, description of, xiv. 297

Lethri, xiv. 507

Lethrus, (Scop.) xiv. 462 æneus, xiv. 499 cephalotes, xiv. 462.507

Leucospis, $(F a b$.$) xv. 365$

Leucothyreus, (Macl.) xiv. 477

Lexocera, (Lat.) xv. 713

Lexoceres, xv. 712 
Libellula, (Lin.) xv. 295 depressa, xv. 311

Libellulæ, episternum of, xiv. 5̃ food of the, xv. 304 growth of, xv. 305 metamorphosis of, xiv. 81 nymphs of, xv. 306 parts of generation of, xiv. 18 proper, xv. 296 respiration of, xiv. 74 stigma of the metathorax of, xiv. 3

stigmata of, xiv. 4 ; xv. 309

Libellulinæ, instinct of, xiv. 67

Libythea, (Fab.) xv. 589

Lice, bird, xiv. 164

description of the, xiv. 148

general observations upon, xiv. 157

proper, description of the, xiv. 149

Licinæ, conical appendages of the, xiv. 174.

Licini, where found, xiv. 88

Licinus, description of, xiv. 226

Lierman, what called, xv. 255

Ligniperdæ, what called, xv. 348

Limacodes, (Lat.) xv. 610

Limnebius, of Dr. Leach, xiv. 431

Limnobia, xv. 686

Limnichus, xiv. 404

(Zieg. Dej.) xiv. 405

Limnius, (Illig.) xiv. 411

Limulæ, heart of, xiv. 2

Lion of the Aphides, what called, xv. 324. 329

Lions des pucerons, xv. 329

Lion-worm, xv. 755

Liothea, female, xiv. 153 tarsi of the, xiv. 152

Liparis, xv. 609

Liparus, xv. 59. 69

Lipotena, (Nitz.) xv. 719

Lipsa, (Lat.) xv. 710

Liris, (Fab.) xv. 376

Lissa, (Meig.) xv. 713

Lissodes, (Lat.) xiv. 317

Lissomus, (Dalm.) xiv. 317 punctulatus, xiv. $\mathbf{3 1 7}$

Lithobius, description of, xiv. 134 the forked, xiv. 134 variegatus lævilabrum, xiv. 134

Lithophilus, (Frôhl.) xv. 158

Lithosia, (Fab.) xv. 612

Lithurgus, xv. 384

Livia, (Latr.) xv. 266

Lixus, xv. 69

(Fab.) xv. 59.63

odontalgicus, $\mathrm{xv} .72$
Lixus paraplecticus, xv. 70 rhynchœus, xv. 59

Lobæderus, xiv. 364 moniliformis, xiv. 365

Locust, application of the name of, $\mathrm{xv}$. 202

migratory, xv, 214

Locusta, of Geoffroy, what, xv. 175. 202 cæcums of, xv. 180 dux, xiv. 258

ephippiger, xv. 175 migratoria, xv. 207 tatarica, xv. 207. 214

verrucivora, $\mathrm{xv} .175$ viridissima, xv. 175

Locustæ, stomachs of, xv. 179

Locusts, destruction of, $x v .213$ difference between nymph and larva of, xiv. 21

fecundity of, xv. 212

flight of, xv. 209

fondness for drink, xiv. 70

habits of the true, xv. 204 metamorphosis of, xiv. 81 migratory, xv. 176

proper, what, xv. 202

record of ravages of, xv. 206

sound produced by, xiv. 76 the true, xv. 202

Lomechusa, description of, xiv. 299 dentata, xiv. 299 paradoxa, xiv. 299

Lonchæa, (Fall.) xv. 717

Lonchophorus, (Germ.) xiv. 456

Lonchoptera, (Meig.) xv. 714

Longicornes, xv. 97

Longilabres, xv. 217

Longipalpi, xiv. 293

Longirostres, $\mathrm{xv}$. 69

Longitarsus, (Lat.) xv. 142

Long-legs, what called, xv. 738

Lophonocerus, (Lat.) xv. 102

Lophosia, (Meig.) xv. 708

Lophyrus, (Latr.) xv. 357

Loricera, description of, xiv. 228 ænea, xiv. 229

Louse of the Buffalo, xiv. 150 crab, xiv. 150 the human body, xiv. 149 the human head, xiv. 149 of the pubis, xiv. 150 of the stag, xiv. 150

Lucane chèvre, xiv. 501 chevreuil, xiv. 501

Lucani, metamorphosis of the, xiv. 532 what called by Pliny, xiv. 530 description of the, xiv. 532

Lucanidæ, xiv. 496

Lucanus, xiv. 497 
Lucanus caraboïdes, xiv. 501 cervus, xiv. 500.531 female of, xiv. 533 nebulosus, xiv. 499 parallelipipedus, xiv. 501.533 (proper) (Lin.) xiv. 500

Lucernuta, what called, xiv. 366

Lucifuga, what named, xv. 184

Lucifugous insects, wanting the choroid, xiv. 8

Lucio, what named, xiv. 366

Lucciola, xiv. 335 what called, xiv. 366

Luperus, (Geoff.) xv. 141

Lycoperdina, (Fab.) xv. 157

Lyctus, $(F a b.) \times v .85,86$

Lytta, (Fab.) xv. 32, 33 (Klüg.) xv. 33

Lycus, $(F a b$.$) xiv. 330 ;$ xv. 36 aurora, xiv. 331 bicolor, xiv. 331 fasciatus, xiv. 331 hepatic vessels of, xiv. 308 latissimus, xiv. 330 minutus, xiv. 86 . 33 I proboscideus, xiv. $\mathbf{3 3 0}$ rostratus xiv. $\mathbf{3 3 0}$ reticulatus, xiv. 331 serraticornis, xiv. 331

Lyda, (Fab.) xv. 357

Lygæi, the, xv. 242 some without wings, $\mathrm{xv} .230$

Lygæus, (Fab.) xv. 219 apterus, xv. 242 grandis, xv. 242 what referred to, $\mathrm{xv}, 218$

Lymexylon, (Fab.) xiv. 352, 353 abbreviatum, xiv. 353 dermestoïdes, xiv. 353 flabellicorne, xiv. 354 flavipes, xiv. $\mathbf{3 5 3}$ larvæ of, xiv. 382 morio, xiv. 353 navalis, xiv. 353 proboscideum, xiv. 353, 354 proper, (Fab.) xiv. 353

Lyrops, (Illig.) xv. 376

Lystra, xv. 223 pulchra, xv. 260

Lystronichus, (Lat.) xv. 15

Macellarius, xiv. 405

Machilis, description of, xiv. 144

Machla, (Herbs.) xiv. $\mathbf{5 5 5}$

Macraspis, (Macl.) xiv. 473

Macrocephalus, of Olivier, xv. 65, 66

Macrocera, (Meig.) xv. 687

Macrochira, (Meig.) xv. 711
Macrodactyla, xiv. 410

Macrodactylus, (Lat.) xiv. 483

Macrogaster abbreviatus, xiv. 353

Macroglossum, xv. 597

Macronota of Weidmann, xiv. 496

Macronychus, (Müll.) xiv. 412 quadrituberculatus, xiv 412.

Macrorynus, (Schoe.) xv. 57

Mæchidius, xiv. 467

Macropeza, (Meig.) xv. 686

Macropus, (Thunb.) xv. 108

Mækistocera, (Wiedmann) xv. 686

Maggots, what vulgarly called, xv. 770

Malachi, description of the, xiv. 373

Malachius, ( $F a b$.) xiv. 340

Malacoderme, xiv. 322

Malacodermi, xiv. 309

Mallophagi, what are called, xiv. 152

Mallota, (Meig.) xv. 702

Malthinus, (Lat.) xiv. 339

Manticora, description of the, xiv. 176 236

maxillosa, xiv. 176 pallida, xiv. 176

Mantes, xv. 192

(Fab.) xv. 300

causta, xv. 190

religiosa, xv. 172

siccifolia, xv. 173

whence named, xv. 187

viatoria, xv. 189

Mantispa, (Illig.) xv. 300

Mars, xv. 589

Masarides, (Lat.) xv. 379

Masaris, $(F a b$.$) xv. 379$

Masomelas, xiv. 322

Mason-ants, proceedings of the, $\mathrm{xv}_{\text {. }}$ 443

Mason-bees, xiv. 346

Masons, what and why called, xv. 438

Mastigocerus, (Klüg.) xv. 357

Mastigus, xiv. 413

(Hoff.) xiv. 385

palpalis, xiv. 385

May-bug, larva of, xiv. 447

May-bugs, elytra of, xiv. 17

May-worms, xv. 39

Meal-worm, the, xiv. 562

Meat-fly, blue, ovoviparous, xiv. 77

frequents the snake-root and taphelia, xiv. 60

Mechanitis, $(F a b$.$) xv. 586$

Medeterus, (Fisc.) xv. 696

Megacephala, description of the, xiv. 177

Carolina, xiv. 263

Euphratica, xiv. 177

Mexicana, xiv. 263

size of the, xiv. 181 
Megacephali, xv. 696

Megacephalus, xv. 175

Megachile, (Lat.) x v. 384. 571 apiaria, xiv. $\mathbf{3 7 5}$ muraria, xiv. 374

Megaderus, (Dej.) xv. 102

Megagnathus, (Mej.) xv. 88

Megalodentes, (Latr.) xv. 357

Megalophthalmus, xiv. 371

Megalopus, (Fab.) xv. 122

Megarhinus, the genus, xv. 684

Megascelis, (Dej) xv. 124

Megatoma, (Herbs.) xiv. 404 (Lat.) xiv. 405 rufitarsis, xiv. 407 serra, xiv. 406

Megilla, (Fab.) xv. 383 muraria, xv. 526

Melanarius, xiv. 214

Melandrya, (Fab.) xv. 17 arrangement of, xv. 22

Melandryæ, xv. 24

Melanitis, (Fab.) xv. 589

Melanophila, xiv. $\mathbf{3 5 8}$

Melanophora, (Meig.) xv. 709

Melasis, xiv. 313. 359

buprestö̈des, xiv. 313. 359

elateroïdes, xiv. 313

flabellicornis, xiv. $\mathbf{3 5 9}$

picoa, xiv. 318

sphondyloïdes, xiv. 318

tuberculata, xiv. 316

Melasoma, xiv. 539. 558 supplement of, xiv. 564

Melipona, xv. 388

Melecta, (Lat.) xv. 386

Melissodes, xv. 386

Melitæa, (Fab.) xv. 587

Melites, of Kirby, xv. 381

Melitophili, xiv. 490

Mellinus, xv. 378 oxybelus, xv. 378

Melliturga, (Latr.) xv. 387

Meloë, xv. 38, 39

of Lin. xiv. 353 ; xv. 31, 32, 33 Marci, xiv. 353

cantharides distinct from, xv. 46

used for the cantharides, xv. 48 peculiar formation of, xiv. 255 properly so called, (Lin.) xv. 33 proscarabæus, xiv. 153 ; xv. 43 scarabæus, xv. 41

Melolontha, xiv. 464. 471 of Fab. xiv. 472. 474, 475. 489

(Geoff.) xv. 137

(Illig.) xiv. 488

(Oliv.) xiv. 488

abdominalis, xiv. 341
Melolontha æquinoctialis, xiv. 481

Alpina, xiv. 488

atra, xiv. 481

atriplicis, xiv. 476

Ankæteri, xiv. 481

aurata, xiv. 484

barbata, xiv. 472

bicolor errans, xiv. 484

bivulnerata, xiv. 482

brunnea, xiv. 482

castanea, xiv. 472

chrysochlora, xiv. 515

farinosa, xiv. 485

ferruginea, xiv. 472

fervida, xiv. 481

Frischii, xiv. 484

germinata, xiv. 472

hippocastani, xiv. 480

hololeuca, xiv. 481

holosericea, xiv. 484

Julii, xiv. 484

chrysomelina, xiv. 464

colaspidoïdes, xiv. 483

cornuta, xiv. 476

crysochlora, xiv. 473

cyanocephala, xiv. 484

lanata, xiv. 478

marginata, xiv. 484

melanocephala, xiv. 472

nigrifrons, xiv. 478

obscura, xiv. 478

Occidentalis, xiv. 481

pallens, xiv. 472

pilosa, xiv. 481

pini, xiv. 481

(Fab.) properly so called, xiv. 479

ruricola, 482

ruficornis, xiv. 481

signata, xiv. 472

serrata, xiv. 481

solstitialis, xiv. 481

subspinosa, xiv. 483

sulcicollis, xiv. 477

variabilis, xiv. 482

variegata, xiv. 482

villosa, xiv. 480

viridis, xiv. 484

vitis, xiv. 484.518

vulgaris, xiv. 259. 519

Melolonthæ, xiv. 517

food of, xiv. 68

Melophagus, xiv. 150

(Nitz.) xv. 719

Melophila, (Nitz.) xv. 719

Melyrides, hepatic vessels of, xiv. 308 where found, xiv. 373

Melyris, (Fab.) xiv. 339. 341 viridis, xiv. 341 
Membracis of Fabricius, xv. 224 proper, $(\boldsymbol{F a b}.) \times \mathrm{xv} .225$ wings of, $x v, 230$

Menelaus papilio, where found, xiv. 95 Mergamerus, xv. 126 Kingii, xv. 126

Meria, (Illig.) xv. 374

Merodon, (Meig.) xv. 704

Meryx, (Lat.) xv. 87

Mesembrina, (Meig.) xv. 709

Mesosa, of Meg. xv. 109

Methoca, of Lat. xv. 373

Metopius, (Panz.) xv. 362

Microcephala, fifth section of, xiv. 298

Microcephalus, description of, xıv. 227

Microdon, (Meig.) xv. 704 englossoïdes, xv. 779

Microgaster, (Lat.) xv. 363

Micropeplus, description of, xiv. 297 porcatus, xiv. 297

Micropeza, (Meig,) xv. 715

Microptera, description of, xiv. 288

Midas, where found, xiv. 92

Milesia, (Lat.) xv. 704, 705

Millipedes, approximate to many crus-. tacea, xiv. 7 description of, xiv. 124 mouth of, xiv. 10

Miltogramma, (Meig.) xv. 708

Mimela of Mr. Kirby, xiv. 484

Miopa nigripennis, xv. 779

Miris, (Fab.) xv. 219

Misocampe, (Lat.) xv. 366

Miscophus, (Jur.) xv. 377

Misolampus, (Lat.) xiv. 552

Mites, diseases produced by, xiv. 161 found on insects, xv. 44

Mitosata, description of, xiv. 124

Moine, what called, xiv. 509

Mole-crickets, xv. 192 whence ramed, xv. 193

Molobrus, xv. 688

Molops Corinthius, xiv. 214 elatus, xiv. 216

striolatus, xiv. 215

terricola, xiv. 216

Mollusca, remarks upon, xiv. 37

Molorchus of Fab. xv. 106 peculiar formation of, xiv. 255

Moluris, (Lat.) xiv. 551

Monachamnus, of Dej. xv. 109

Monedula, (Lat.) xv. 376

Monk, what called, xiv. 509

Monocheles, (Illig.) xiv. 486

Monomera, xv. 168

Monophleba of Dr. Leach, xv. 228

Monotoma, xv. 84

(Herbst.) xv. 85

Mordella, xv. 28. 36
Mordella (Lin.) xv. 29

Mordellonæ, xv. 28. 36

Morio, description of, xiv. 205

Brasiliensis, xiv. 205

monilicornis, xiv. 205

Orientalis, xiv. 205

Mormolyce, description of, xiv. 220 phyllodes, xiv. 120

Morphi, xv. 583

Morpho, $(F a b$.$) xv. 590$

Morpion, xiv. 150

Mosillus, (Lat.) xv. 717

Mosquito, manners of the, xv. 751

Mosquitos, xv. 733

Moths, food of, xiv. 69 proper, habits of, xv. 679

Mouches à scie, xv. 397 à corslet armé, xv. 758 papilionacées, xv. 348 scorpions, $x v .323$

Mulio, $(F a b$.$) xv. 703, 704$ (Meig.) xv. 694

Murmidius, (Leach) xiv. 408

Musca, xv. 707

(Deg.) xv. 710

(Fab.) xv. 705

of Linnæus, xv. 694. 701

711. 713. 757

(Panz.) xv. 712

Cæsar, xv. 709

cardui, xv. 716

carnaria, xv. 709. 766

cellaria, xv. 711

cerasi, xv. 716

cynipsea, xv. 715

cynophila, xv. 712

domestica, xv. 709

filiformis, xv. 715

fungorum, xv. 711

grossa, xv. 707

pluvialis, xv. 711

proper, xv. 773

proper, (Lin.) xv. 709

pyrastri, xv. 761

stercoraria, xv. 713

tripilis, what called, $\mathrm{xv} .407 .410$

vanitoria, xv. 709

vibrans, xv. 716

Muscæ vibrantes, what named, xv. 407

Muscides, the, xv. 707. 767

the larvæ of the, $\mathrm{xv} .768$

Mutilla, (Lat.) xv. 372

of Linnæus, xv. 372

(female) without metamorphosis, xiv. 21

Klugii, xv. 516

(proper), xv. 372

Myas, description of, xiv. 217 
Myas chalybæus, xiv. 217

Mycetobia, (Meig.) xv. 688

Mycetochares, (Lat.) xv. 16

Mycetophagus, (Fab.) xiv. 397 ; xv. 17. 86

(Gyll.) xv. 87

castaneus, xiv. 397

proper, (Fab.) xv. 87

spinipes, xiv. 397

Mycetophila, (Gyll.) xv. 16 (Meig.) xv. 688

Mycterus, xv. 20

(Clairv.) xv. 20

Mydas, proper, (Fab.) xv. 698 stylet of, xv. 698

Mydasii, (Lat.) xv。 698

Mygalæ, where found, xiv. 92

Mygale, respiratory organs of, xiv. 6

Mylabris, xv. 41. 50

(Geoff.) xv. 53

(Oliv.) xv. 32

(Schoeff.) xv. 20

cantharides, distinct from, xv. 46

used for cantharides, xv. 48

chicorii, xv. 47. 51

the Chinese employ, xv. 47

pustulata, xv. 50

where found, xiv. 94

Mylabrum of Fab. xv. 32

Mylœchus, (Latr.) xiv. 398

Myniops of Schœenherr, xv. 58

Myodites, xv. 37

(Lat.) xv. 28

Myodocha, (Latr.) xv. 219

Myopa, (Fab.) xv. 706

Myorhinus, (Schoen.) xv. 60

Myosodus, xiv. 216

Myriapoda, description of, xiv. 124 jaws of, xiv. 9

Myriapodes, approximate to many crustacea, xiv. 7

mouth of, xiv. 10

Myriapods, abdomen of, xiv. 18

a passage from insects to crustacea, xiv. 136

description of, xiv. 26

dwelling of, xiv. 137

sketch of metamorphosis in, xiv. 22

Myrina, (Fab.) xv. 592

Myrmecoda, (Lat.) xv. 373

Myrmecodes Australis, xv. 516

Mermecophila, xv. 175

Myrmeleon, xv. 298

erythrocephalum, xv. 327

larvæ of the, xv. 324

Myrmeleonides, xv. 298

Myrmex, what called, xv. 435

Myrmica, (Latr.) xv. 371

Cuv. Index.
Myrmica emigrations of, xv. 453 instinct of, xv. 443

Myrmicæ, what called, xv. 435

Myrmice hamata, xv. 503

Myrmosa of Latr. xv. 373

Mystacida, xv. 302

Myzine, (Latr.) xv. 374

Nabis, (Lat.) xv. 220

Naucores, xv. 248

Naucori, where found, xv. 232

Naucoris, (Geoff.) xv. 221

Nebria, description of, xiv. 237 arenaria, xiv. 237 Borealis, xiv. 239

Necrobia, (Lat.) xiv. 346 classification of, xiv. 376 violacea, xiv. 347

Necrodes, xiv. 418 (Wilk.) xiv. 394

Necrophilus, (Lat.) xiv. 397

Necrophori, xiv. 415 larvæ of, xiv. 418

Necrophorus, (Fab.) xiv. 391 Germanicus, xiv. 393 grandis, xiv. 393 humator, xiv. 393 mortuorum, xiv. 393 vespillo, xiv. 393.416

Necydalis, (Fab.) xv. 19. 20 (Geoff.) xiv. 339 (Lin.) xiv. 352 ; xv. 37.106 brevicornis, xiv. 352 proper, xv. 106

Negra, (cochineals, xv. 280

Neides, (Lat.) xv. 219 (Lat.) of what composed, xv. 241 tipularia, xv. 242

Nematocera, (Meig.) xv. 686

Nematodes, xiv. 319

Nematopus rufoscutellatus, xv. 241

Nemestrina, (Lat.) xv. 694

Nemestrinæ, the, xv. 754

Nemocera, the, xv. 682. 721

Nemoceræ, xv. 725

Nemognathus, (Lat.) xv. 34 jaws of, xv. 46

Nemoptera Africana, xv. 324 (Latr.) xv. 297 extensa, xv. $\mathbf{3 2 4}$ where found, xiv. 92

Nemopteræ, the, xv. 324

Nemosoma, (Desmar.) xv. 84

Nemotelus, (Geoff.) xv. 700

Nemoura, (Lat.) xv. 301

Nepa, of Linnæus, xv. 221. 248 cinerea, xv. 249 proper, (Lat.) xv. 222

M m 
Nepæ proper, labrum of, xv. 249 where found, $x v .232$

Nephortoma, (Meig.) x v. 685

Nepides, xv. 221

Neptis, (Fab.) xv. 589

Nerii sphinx, xv. 597

Nermomyia; (Nitzch.) xv. 719

Neuroptera, xv. 294 description of, xiv. 27 eyes of, xiv. 45 masticating insects, xiv. 40 metamorphosis of, xiv. 81, 82 neuters among, xiv. 78 nymphæ of, xiv. 21 supplement on the, xv. 303

Nigidius, xiv. 501

Nilio, (Lat.) xv. 6

Nilo, where found, xv. 9

Nirmi, (of Hermann) xiv. 152

Nirmidea, (Leach) xiv. 150

Nirmus, a name adopted from Hermann, xiv. 164 description of the, xiv. 150

Nitela, (Lat.) xv. 377

Nitidula, xiv. 399 ; xv. 66

(Gyll.) xiv. 391

(Herbst.) xiv. 401

(Oliv.) xiv. 400, 401

ænea, xiv. 401

grisea, xiv. 420

orbiculata, xiv. 404

(proper,) (Fab.) xiv. 401

viridescens rufipes, xiv. 401

why named, xiv. 419

what resemble, xiv. 419

Nitidularia, xiv. 398

Noctiluca, what called, xiv. 366

Noctua, xv. 614

(Fab.) xv. 613

absinthii, xv. 616

Artemisiæ, xv. 616

chrysitis, xv. 616

gamma, xv. 615

pacta, xv. 615

Patroclus, xv. 594

sponsa, xv. 615

verbasci, xv. 616

Nocturna, xv. 601

Nomada, (Fab.) xv. 385

Nomia, (Lat.) xv. 382

Nosodendron, (Lat.) xiv. 407

Notacantha, the, xv. 698.757

Noterus, description of, xiv. 249

Nothus, (Zieg.) xv. 19

Notiophilus, description of, xiv. 240

Noctualites, xv. 613

Notoclea, marsh, xv. 140

Notodonta, (Ochs.) xv. 610 palpina, xv. 610
Notonecta, (Lin.) xv. 222

glauca, xv. 251

minima, xv. 251

habits of the, xv. 251

Notonectes, where found, xv. 232

Notonectides, xv. 22.2

Notopeda, elater, xiv. 314 what called, xiv. 359

Notoxi, xv. 38

Notoxus, (Fab.) xiv. 345

of Geoff. xv. 30

how allied to odacantha tripustulata, xiv. 189

Illigeri, xiv. 342

ninutus, xiv. 386

Notophila, (Fab.) xv. 711

Nyctelia, (Lat.) xiv. 544

Nycteribia, (Lat.) xv. 720

Nycteribix, the, xv. 778

Nycteus, (Lat.) xiv. 328

Nymphales, xv. 583

Nymphalis, (Lat.) xv. 589

Annetta, xv. 676

chrysites, xv. 675

pitheas, xv. 675

Thamyris, xv. 675

Nymphipara, xv. 718

Nysson, (Lat.) xv. 377

Nyssoniani, xv. 377

Nymphes, (Leach) xv. 299

Obrium, (Meg. Dej.) xv. 105

Occitanus, where found, xiv. 96

Ochthebius, (Leach) xiv. 426

Ochina, xiv. 350 hederæ, xiv. 350

Ochodæus, (Meg.) xiv. 464

Octogonotes, xv. 141

Ocydromia, (Hoff.) xv. 691

Ochtera, (Lat.) xv. 711

Ocyptera, (Meig.) xv. 708

the, xv. 771

bicolor, xv. 772

cassidæ, xv. 772

Odocantha, description of, xiv. 193 cynocephala, xiv. 189 dorsalis, xiv. 189 melanura, xiv. 189. 270 tripustulata, xiv. 189

Odonata, xv. 294

Odonptera palpina, xv. 614

Odontæus, (Ziegl.) xiv. 465

Odontomachus, (Lat.) xv. 371

Odontomyia, (Meig.) xv. 700

Odynerus, (Lat.) xv. 380

habits of, xv. 524

Ecophora, (Lat.) xv. 627

(Edalia, (Meig.) xv. 691

Edemera, $(F a b.) \times v .106$ 
CEdemera, (Oliv.) xv. 19. 20 classification of, xv. 24 distinguished from cantharides, $\mathrm{xv}, 45$

CLdemeræ, salivary vessels of, xv. 11

Emerites, xv. 18

Edionychis, (Lat.) xv. 142

CEdipoda, xv. 177

CEgus, xiv. 501

CEnas, (Oliv.) xv. 32

(Estrides, xv. 705

CEstrus, (Lin.) xv. 701. 705

the, $x v .763$

bovis, xv. 765, 766

food of, xiv. 69

hæmorrhoïdalis, xv. 764

hominis, xv. 766

ovis, xv. 764

Oiceptoma, xiv. 395

Oides, (Weber) xv. 141

Oiketicus Kirbyi, xv. 679

Olites, $(F a b$.$) xv. 714$

Olyntha, (Mr. Gray) xv. 347

Brasiliensis, xv. $\mathbf{8 4 7}$

Omalia, xiv. 306

Omalium, description of, xiv. 296,297

macropterum, xiv. 298

ovatum, xiv. 297

porcatum, xiv. 297

rugosum, xiv. 297

staphylinoïdes, xiv. 297

Omalisus, (Geoff.) xiv. 331 suturalis, xiv. 331

Omalopia, (Dej.) xiv. 482

Omaloptera, (Leach) xv. 717

Omalus, (Jur.) xv. 367

Omaseus, xiv. 214

elongatus, xiv. 215

melanarius, xiv. 215

Ometis, (Lat.) xiv. 474

Omias, xv. 58

Ommatius, (Illig.) xv. 691

Omophron, description of, xiv. 238 limbatus, xiv. 279, 280

Omophronta, head of the, xiv. 174

Omus Californicus, xiv. 263

Oniscus pustulatus, xiv. 130 zonatus, xiv. 130

Oniticellus, xiv. 455

Onitis, xiv. 455

(Fab.) xiv. 456

what comprehended under the name, xiv. 506

where found, xiv. 92

Onthophagus, (Lat.) xiv. $\mathbf{4 5 5}$

Onthophilus, xiv. 389, 390

Oödes, description of, xiv. 224

Opatrini, xiv. 557

Opatrinus, xiv. 556
Opatrum, xiv. 569 ; xv. 9

(Fab.) xiv. 559

granulatum, xiv. 341

sabulosum, xiv. 560.570

Ophionæa, description of the, xiv. 189

Ophion, $(F a b$.$) xv. 361$

Ophions; of Fabricius, xv. 418

Ophiusa scapulosa, xv. 614

Ophoni, anatomical peculiarity of, xiv. 207

Ophonus, description of, xiv. 210

Opilo, (Lat.) xiv. 345

Opili, xiv. 374

Oplognathus, (Kirby) xiv. 473

Orchesia, (Lat.) xv. 16

Orchestes, (Illig.) xv. 61

Orgyia, (Ochs.) xv. 609, 610 gnostigma, xv, 610

Orneodes, xv. 629

Ornithomyia, (Lat.) xv. 719

(Leach, ) xv. 719

Ornithomyiæ; the, xv. 778

Orobitis, xv. 62

Orsodacna, xv. 125

(Lat.) xv. 122

chlorotica, xv. 126

Orsodacnæ, xv. 126

Ortalis, (Fall.) xv. 716 paludium, xv. 716

Orthiorynchus, xv. 58

Orthocætes, (Germ.) xv. 63

Orthocerus, xiv. 570 (Lat.) xiv. 560

Orthogonius, description of, xiv. 197 Hopei, xiv. 273

Orthoptera, description of, xiv. 26 ; xv. 169

of Olivier, xv. 229

corslet of, xiv. 12

eyes of, xiv. 45

hearing of, xiv. 56

leaping division of, $\mathrm{xv} .192$

masticating insects, xiv. 40

mesothorax of, xiv. 51

metamorphosis of, xiv. 81, 82

muscles of, xiv. 54

palpi of, xiv. 9

size of, xîv. 96

stomachs of the, xv. 179

supplement on the, xv. 178

Orthorapha cassidioïdes, xv. 263

Ortocheile, (Lat.) xv. 696

Oryctes, (Illig.) xiv. 468 type of, xiv. 509

Oryssus, (Lat.) xv. 358

Oscines, food of, xiv. 68

Oscinis, (Lat.) xv. 714

Osmia, (Lat.) xv. 571

(Panz.) xv. 384

M m 2 
Osmia cærulescens, xv. 573 fulviventris, xv. 575

Osmylus, (Latr.) xv. 299

Osorius, description of, xiv. 295

Osphya, (Illig.) xv. 19

Otiocerus, antennæ of, xiv. 41 or Kirby, xv. 223

Ourapteryx, (Leach) xv. 619

Oxæna, description of, xiv. 205

Braziliensis, xiv. 274

brunnea, xiv. 205

dentripes, xiv. 205

goniotropis, xiv. 274

Gyllhenalii, xiv. 205

Rogeri, xiv. 205

Oxea, (Klüg.) xv. 386

Oxiuri, (Latr.) xv, 367 the, xv. 434

Oxura, (Kirb.) xiv. 552 setosa, xiv. 552

Oxybelus, (Lat.) xv. 377

Oxycera, (Meig.) xv. 700

Oxycheila, description of the, xiv. 177 binotata, xiv. 264 distigma, xiv. 264 tristis, xiv. 177

Oxygnathus, description of, xiv. 203 elongatus, xiv. 203

Oxypori, xiv. 304

Oxyporus abdominalis, xiv. 299 analis, xiv. 299

bipustulatus, xiv. 299

chrysomelinus, xiv. 299

description of, xiv. 290

lunulatus, xiv. 299

marginatus, xiv. 299

marginellus, xiv. 299

maxillosus, xiv. 290

merdarius, xiv. 299

pygmæus, xiv. 299

rufipes, xiv. 299

subterraneus, xiv. 299

suturalis, xiv. 299

Oxypterum, (Leach) xv. 719

Oxystomus, description of, xiv. 203 cylindricus, xiv. 204

Oxyteli, xiv. 305

Oxytelus, xiv. 295

Oxytelus, (Oliv.) description of, xiv. 296

bicornis, xiv. 296

penicillatus, xiv. 296

Pachychnemus, xiv. 489

Pachyderes, ruficollis, xiv. 364

Pachygaster, (Meig.) xv. 701

Pachylis, (Lep. \& Ser.) xv. 218
Pachymere, (Lep. \& Ser.) xv. 219

Pachymosa Esculapius, xiv. 506

Pachypus, (Dej.) xiv. 475

Pachyrhynchus, xv. 58

Pachysoma, xiv. 452

Hippocrates, xiv. 506

Pachystomus, (Lat.) xv, 698

Pachyta, (Meg.) xv. 112

Prcilonotus, xiv. 357

Pæciloptera, (Lat.) xv. 224 antica, xv. 260

Pæcilus, description of, xiv. 213

Pælobius, description of, xiv. 248

Palarus, (Lat.) xv. 376

Palm-weevil, xv. 80

Palpadores, where placed, xiv. 385

Palpicornes, xiv. 424. 433

Pamborus, description of, xiv. 230 alternans, xiv. 230

Pamphagus, (Thunb.) xv. 176

Pamphilus, (Latr.) xv. 357

Pamphedron, (Lat.) xv. 378

Panache jaunâtre, xiv. 336

Panagée à quatre taches, xiv. 228

Panagæus, description of, xiv. 228

Pangonia, (Lat.) xv. 697

Panaps, (Lamarck) xv. 692

Panorpa, (Lin.) xv. 297 proper, (Lat.) xv. 298 rufa, xv. 324

Panorpæ, of the, xv. 323

Panorpatæ, (Latr.) xv. 297

Pallurgus, (Panz.) xv. 383

Papilio, of Linnæus, xv. 582

Alexanor, xv. 584

Alexis, xv. 592

Antiopa, xv. 587

Apollo, xv. 585

Atalanta, xv. 588

bombyliformis, xv. 598

brassicæ, xv. 585

C. album, xv. 588

cardui, xv. 588

cardui, where found, xiv. 90

cardumines, xv. 585

Childrenæ, xv. 673

Chrysippus, where found, xiv 92

Cleopatra, xv. 585

Cleopatra, where found, xiv. 88

cleotas, xv. 673

cratægata, xv. 632

daplidice, xv. 585

farinalis, xv. 623

fuciformis, xv. $\mathbf{5 9 8}$

graminis, xv. 616

hyale, xv. 585

hysipyle, xv. 585

Iö, Xv. 588 
Papilio Iö, represented by Kirby and Spence, xiv. 42

Jason, xv. 589, 590

Lavinia, xv. 594

leilus, xv. 594

lemnata, xv. 623

Machaon, xv. 584

napi, xv. 585

noctua, xv. 596. 608, 609. 611, 612.615. 617

nymphalis, xv. 586

nympheata, xv. 623

Orontes, xv. 594

paludata, xv. 623

pelicani, xv. 586

Phidippus, xv. 590

pinguinalis, xv. 623

podalirius, xv. 584

polychloros, xv. 588

potamogata, xv. 623

Priamus, where found, xiv. 96

rapæ, xv. 585

rhammi, xv. 585

riphæus, xv. 594

rumina, xv. 585

sinapis, xv. 585

stellatarum, xv. 598

stratiolata, xv. 623

urticæ, xv. 588. 631. 670

urticæ, tongue of, xv. 633

urticæ, description of figure of, xiv. 47

Papiliones, Danaï candidi, xv. 585

Danaï festivi, xv. 586

equites, xv。 $\mathbf{5 8 3}$

milites, xv. 590

plebei, xv. 591

plebei urbicolæ, xv. 593

Papillons bourdons, (Degeer) xv. 595 estropiés, xv. 593

Paragus, (Lat.) xv. 703

Parandra, (Lat.) xv. 99. 113

Parasita, description of the, xiv. 26. 148

Parmena, of Meg, xv. 109

Parnasii, xv. $\mathbf{5 8 3}$

Parnassius, (Lat.) xv. 584

Parnus, of Fabricius, xiv. 410, 411 acuminatus, xiv. 411 obscurus, xiv. 412

Parnopes, (Latr.) xv. 369

Paropsis, xv. 140

Pasimachus, description of, xiv. 200 Mexicanus, xiv. 274

Pasites, xv. 385

Passali, description of, xiv. 537

Passalus, (Fab.) xiv. 502 of Olivier, xiv. 497 interruptus, xiv. 537
Passalus, where found, xiv. 94

Passandra, xv. 93

(Dalm.) xv. 88

fasciata, xv. 94

Patellimani, description of, xiv. 221

Patrobus, description of, xiv. 229

Paussus, (Lin.) xv. 83

Pavonia, xv. 590 morpho, xv. 590

Pavarii, xv. 583

Paxillus, xiv. 502

Paxylloma, (Brebisson) xv. 360

Pedicia, (Lat.) xv. 685

Pediculea, (Leach) xiv. 149

Pediculi corporis, xiv. 163 diseases produced by, xiv. 161 metamorphosis of, xiv. 82 proper, xiv. 164

Pediculus, (Lin.) xv. 717 description of the, xiv. 148,149 ardew, xiv. 151 corvi coracis, xiv. 151 humanus corporis, xiv. 149 humanus capitis, xiv. 149 melittæ, xiv. $153 ; x v .42,43$, 44

pavonis, xiv. 151 (properly so called) xiv. 152 pubis, xiv. 150 resembles an acarus, xiv. 162 stermæ hirundinis, xiv. 151 suis, xiv. 150

tinnunculi, xiv. 151 vespertilionis, $\mathrm{xv} .779$

Pedilus, (Fisch.) xv. 19

Pedini, (proper) xiv. 558

Pedinus, (Lat.) xiv. 556

Pelecinus, (Latr.) xv. 359

Pelecium, description of, xiv. 227 cyanipes, xiv. 227

Pelecocera, (Hoff.) xv. 705

Pelidnota, (Macl.) xiv. 473

Pelocophorus, xiv. 342

Pelocotoma, (Fisch.) xv. 29

Pelogonus, (Lat.) xv. 220

Pelopæus, (Fab.) xv. 378 (Lat.) xv. 376

Pelophila, description of, xiv. 238

Pelor, (Bon.) description of, xiv. 211

Peltastes, (Illig.) xv. 362

Peltes, (Fab.) xiv. 400

Peltis, (Fab.) xiv. $346^{\circ}$

(Geoff.) xiv. 390. 394. 398

Pemeliæ, where found, xiv. 96

Pentamera, tarsi of the, xiv. 172

Pentamerous coleoptera, xiv. 308

Pentaphyllus, xv. 3

Pentatoma, xv. 217

baccarum, xv, 235 
Pentatoma betulæ, xv. 234 grisea, $\mathrm{xv} .772$ ornata, xv. 236

Penthetria, (Meig.) xv. 689

Penthimia, (Germ.) xv. 226

Pepsis, (Fab.) xv. 375 apicalis, xv. 516

Percus ebenus, xiv. 217

Perga of Dr. Leach, xv. 355. 402 scutellata, xv. 402

Pericalus, description of, xiv. 219 cicindeloïdes, xiv. 219

Perilampus, xv. 366

Perla of Geoffroy, xv. 301 with what confounded, xv. 347

Perlides, xv. 300

Petalocheiris Pariss, xv, 220

Petauristes, (Lat.) xv. 123

Petits porte-queue, les, xv. 592

Petrobius, description of, xiv. 144 maritimus, xiv. 145

Phædon, (Meg.) xv. 140

Phagiocephalus tubularis, xv. 774

Phalacri, (Payk.) xv. 66

Phalæna, the, xv. 601.602. 678

alpinalis, xv. $6 \overline{2} 3$

alucites, xv. 628

antiqua, xv. 679

à six ailes, xv. 619

attacæ, xv. 605

betularia, xv. 602

bombyx caja, xiv. 49

bombyx crategata, xiv. 49

bombyx geom. grossulariata: xiv. 49

bombyx lubricepeda, xiv. 48

brumata, xv. 619

Cynthia, xv. 605

falcataria, xv. 613

forficalis, xv. 623

grossulariata, xv. 619

hexadactyla, xv. 632

hirtaria, xv. 602

lacertinaria, xv. 613

margaritalis, $\mathrm{xv} .623$

margaritaria, xv. $6 \mathrm{~J} 9$

mineus, xv. 605

prodromia, xv. 602

proper, xv. 619

purpuraria, xv. 623

pyralides, xv. 620. 622

sambucaria, xv. 619

sanguinalis, xv. 623

scalaris, $\mathrm{xv} .605$

tinea semiargentella, xv. 632

syringaria, xv. 619

Phalenæ, xv. 348

tortrices, xv. 616

the large shouldered, $\mathrm{xv} .616$
Phalænites, xv. 618

Phalæri, xiv. 445

Phalarcrus, (Payk.) xv. 155

Phalène de l'éclair, xv. 230

Phalènes tipules, xv. 628

Phaleria, xv. 7

(Lat.) xv. 2

bicolor, xv. 3

peltoïdes, xv. 3

Phanæus, (Macl.) xiv. 456

Phania, xv. 709

Phasgonophora sulcata, xv. 433

Phasia, (Meig.) xv. 708

Phasma of Fab. xv. 173 arrangement of, xv. 191

gigas, $\mathrm{xv} .191$

rossia, xv. 173

where found, xiv. 96

Phatypterix, (Lacép.) xv. 612

Phengodes, (Hoffm.) xiv. 333

Philanthus, (Fab.) xv. 379 (Jur.) xv. 379

Phileremus, (Latr.) xv. 385

Phileurus, (Lat.) xiv. 470

Philopteri, biliary vessels of the, xiv. 153

Philopterus, proper to birds, xiv. 152

Phlæa, (Lep. et Ser.) xv. 218

Phleboptera, what called, xv. 391

Phoberus, xiv. 467

Phœnicocerus, Dejeanii, xv. 103 (Lat.) xv. 103

Pholidotus, (Macl.) xiv. 499

lepidosus, xiv. 499

Phora, (Lat.) xv. 717 proboscis of, $\mathrm{xv} .707$

Phrenapates, Bennettii, xv. 91

Phrenapetes, xv. 91

Phryganea quadrifasciata, xv. 302

Phryganea, (Lin.) xv. 301

filosa, $\mathrm{xv} .302$

hirta, xv. 302

longicornis, xv. 302

nigra, xv. 302

proper, xv. 302

Phrygania, gills of, xiv. 75

larvæ of, xv. 350.611

with what confounded, xv. 347

eyes of, xiv. 45

Phryganiæ, what called, xv. 348

Phthiria, (Meig.) xv. 693

Phthridium, (Herm.) xv. 720

Phthiromyia, (Lat.) xv. 719

Phtirus, (Dr. Leach) xiv. 150

Phycis, (Fab.) xv. 625

Coleti, xv. 625

Phylam, (Meg.) xiv. 559

Phyllia, form of the, xv. 191

Phyllium, of Illiger, xv. 173 
Phyllium, arrangement of, xv. 191 bioculatum, xv. 191

Phyllocharis, (Dalm.) xv. 139

Phyllobius, (Schœn.) xv. 57

Phyllocerus, xiv. 322

Phyllophagi, xiv. 474. 484

Physodera Desiani, xiv. 273

Physodactylus, (Fisch.) xiv. 324 Henningii, xiv. 324

Phytonomus, (Schœn.) xv. 59

Phytoscapus, (Schon.) xv. 57

Pieris, $(S c h r$.$) xv. 585$

Nemesis, xv. 674

Swainsonii, xv. 674

Piestus, (Grav.) description of, xiv. 296 sulcatus, xiv. 296

Piezata, (Fab.) xv. 353

Pilulariæ, wliat called, xiv. 451 why called, xiv. 504

Pimelia of Fab. xiv. 412. 541. 551, 552 (Herbs.) xiv. 552 (Lin.) xiv. 542 anomala, xiv. 543 bipunctata, where found, xiv. 89 , 542. 565

ciliata, xiv. 565

coronata, xiv. 543

cryptochyle, xiv. 543

dentipes, xiv. 552.555

gibba, xiv. 551

gibbosa, xiv. 553

glabra, xiv. 566

hispida, xiv. 543

longipes, xiv. 543

maculata, xiv. 543

minuta, xiv. 543

morbilosa, xiv. 543

obscura, xiv. 555

of what formed, xiv. 565

pygmæa, xiv. 412

Payraudii, xiv. 543

scrabriuscula, xiv. 566

silphoïdes, xiv. $\mathbf{5 4 5}$

striata, xiv. 551

striatula, xiv. 566

trachyderma, xiv. 543

unicolor, xiv. 551

Pimeliariæ, xiv. 564

Pimpla, (Fab.) xv. 361

Pine tenthredo, xv. 399

Pinicola, (Breb.) xv. 357

Pinophilus latipes, xiv. 292

Pipiza, (Meig.) xv. 705

Pison, (Spin.) xv. 377

Planiceps, xv. 375

Planipennes, xv. 297

Plant-lice, habits of, xv. 267

Platinatus striatus, xiv. 557 crenatus, xiv. 557
Platinatus excavatus, xiv. 557

Platycerus, xiv. 501

(Geoff.) xv. 88

signification of, xiv. 530

Platychile, resembling manticora, xiv. $176^{\circ}$

Platygaster, xv. 369

Platygenia, (Macl.)xiv. 493

Platynotus dilatatus, xiv. 556 lævigatus, xiv. 555 reticulatus, xiv. 555 serratus, xiv. 555 undatus, xiv. 555

Platynus, description of, xiv. 223 blandus, xiv. 223 complanatus, xiv. 223

Platyope, xiv. 542

Platypeza, (Meig.) xv. 696

Platypus, (Herbs-) xv. 83

Platyscelis, (Latr.) xiv. 558

Platysma nigra, xiv. 214

Platysoma, xv. 95 (Leach) xiv. 389

Platystoma, (Meig.) xv. 716

Platyura, (Meig.) xv. 688

Plectes osseticus, xiv. 233

Plectris, (Lepel. \& Serv.) xiv. 483

Plicipennes, xv. 301

Ploas, (Meig.) xv. 694 ægeriformis, xv. 779

Plochionus, descripticn of, xiv. 196 quadrimaculatus, xiv. 273

Ploeiræ, the, xv. 244

Ploiaria, (Scop.) xv. 220

Ploiotribus, (Lat.) xv. 82

Plusia concha, xv, 614

Podidum, (Lat.) xv. 376

Podisma, xv. 177

Podium nigripes, xv. 516

Podontia, (Dalm.) xv. 139

Podura, description of the, xiv. 145 alvalis, $\mathrm{xv}, 146$ ambulans, xiv. 146 annulata, xiv. 146 arborea, xiv, 146 aquatica, xiv. 146 aquatica grisea, xiv. 146 atra, xiv. 147 cincta, xiv. 146

Podurellæ, description of the, xiv. 145

Poduria fimetaria, xiv. 146

Podura lignorum, xiv. 146 metamorphosis of, xiv. 82 minuta, xiv. 147 plumbea, xiv. 146 polypoda, xiv. 147 proper, description of the, xiv. 146 pusilla, xiv. 146 
Podura signata, xiv. 147 vaga, xiv. 146 villosa, xiv. 146 viridis, xiv. 147

Pœderi, xiv. 305

Pœderus, description of, xiv. 292. 293 proboscideus, xiv. 294

Pogonocherus, xv. 108

Pogonocerus, (Fisch.) xv. 28

Pogonophorus, description of, xiv. 236

Pogonus, description of, xiv. 211 halophilus, xiv. 212 pallidipennis, xiv. 212

Polistes apicalis, xv. 575

Polistichus, description of the, xiv. 190

Polisticus discoïdeus, xiv. 191

Polocrium, (Spin.) xv. 374

Polyergi, larvæ of the, xv. 450

Polyergus, (Lat.) xv. 371 account of, xv. 490

Polymera, (Wiedmann) xv. 686

Polyommatus, xv. 592

Polyommates, xv. 610

Polydesmus, description of, xiv. 13]

Polytomus femoratus, xiv. 327 marginatus, xiv. 327 mystacinus, xiv. 327 (Dalm.) xiv. 326

Polyxenus, description of, xiv. 131 lagurus, xiv. 131

Pompilus, (Fab.) xv. 375

Pompilius bifasciatus, xv. 516

Pompylus, (Fab.) xv. 378

Ponera, (Lat.) xv. 371 size of, $x v .471$

Pontia, (Fab.) xv. 585

Popilia, (Leach) xiv. 483 bipunctata, xiv. 483

Porcellus sphinx, xv. 597

Porphyrops, (Meig.) xv. 696

Potamophili, xiv. 409

Potamophilus, (Germ.) xiv. 410

Pou de bois, $\mathrm{xv} .345$

Pneumora of Thunb. xv. 176

Prasocuris, (Lat.) xv. 140

Priocera, (Kirby) xiv. 344 variegata, xiv. 344

Prioni, xv. 114

Prionii, xv. 98

Prionus, (Geoff.) xv. 99 arvicornis, xv. 115 depsarius, xiv. 86 coriarius, xv. 115 nitidus, xv. 100 sabricornis, xv. 100

Procerata saldonana, xv. 617

Procerus, description of, xiv. 232

Caucasicus, xiv. 233

tauricus, xiv. 233
Procirrus Lefekuri, xiv. 294

Procris, (Fab.) xv. 600

Procrustes, description of, xiv. 233

Proctotrupes, (Latr.) xv. 368

Proteus, (carabus æneus) xiv. 209

Progalles-insectes, xv. 274

Prognatha, description of, xiv. 296

Pronæus, (Lat.) xv. 375

Proscarabæi, xv. 38

Proscarabæus, fecundity of the, xv. 40

Proscopia, (Klïg.) xv. 176

Prosopis, (Fab.) xv. 383 (Jur.) xv. 381

Prostomis, (Lat.) xv. 88

Proteinus, description of, xiv. 297

Psalidium, xv. 58

Psalidognathus, xv. 115 Friendii, xv. 116

Psammetichus, (Lat.) xiv. 549

Psammodes, (Kirby) xiv. 551 longicornis, xiv. 551

Psammodius, (Gyll.) xiv. 458 arenarius, xiv. 460 sulcicollis, xiv. 459

Psammœcus Boudier, xv. 123

Psammotherma, (Lat.) xv. 372

Psarus, (Lat.) xv. 703

Pselaphii, xv. 166

Pselaphus, (Herbs.) xv. 166 (Illig.) xiv. 385 proper, (Herbs.) xv. 167

Psen, (Lat.) xv. 378

Pseudo-bombyces, xv. 609, 610

Pseudo-tineæ, xv. 621

Psila, (Meig.) xv. 713

Psilodera Capensis, xv. 779

Psilomyia, (Lat.) xv. 713

Psilopus, xv. 696

Psilota, (Fab.) xv. 705

Psilus, (Jur.) xv. 368

Psoa, (Fab.) xv. 84

Psocus, (Latr.) xv. 300

Psorophora, xv. 684

Psyche, (Schr.) xv. 611

Psychoda, (Lat.) xv. 685

Psychomylæ, xv. 302

Psylla, (Geoff.) xv. 227 alni, xv. 266

buxi, xv. 267

ficus, xv. 266

Psyllæ, of Geoffroy, habits of, xv. 264

Psylliodes, (Lat.) xv. 142

Pterochile, (Klüg.) xv. 380

Pterodontia flavipes, xv. 779

Pteromalus, (Latr.) xv. 366

Pterophorites, (Lat.) xv. 628

Pterophorus, xv. 628 hexadactylus, xv. 628, 629 pentadactylus, xv. 628 
Pterostichus, xiv. 214. 216

Pterotarsus, xiv. 363

histrio, xiv. 363

Pterygophorus, (Klüg.) xv. 357

Ptilinus, xiv. 335

(Fab.) xiv. 350

(Geoff.) xiv. 350

larvæ of, xiv. 379

pectinatus, xiv. 350

pectinicornis, xiv. 350

pollens, xiv. 350

serratus, xiv. 350

Ptilium, xv. 168

Ptilodactyla, (Illig.) xiv. 327

Ptilodactylus, (Wied.) xv. 699

Ptini, general description of, xiv. 378

Ptiniores, of what composed, xiv. 377

Ptiniori, hepatic vessels of, xiv. 308

Ptinus, (Fab.) xiv. 385

(Fab.) (Oliv.) xiv. 349

(Lin.) xiv. $347,348.350 .351$

denticornis, xiv. 350

far. xiv. 349

Germanus, xiv. 349

imperialis, xiv. 349

latro, xiv. 349

pertinax, xiv. 351

proper, (Lin.) xiv. 348

scotias, xiv. 350

serricornis, xiv. 350

striatus, xiv. 349

sulcatus, xiv. 350

testaceo-villosus, xiv. 327

Ptyocerus, (Hoff.) xiv. 326

Ptychoptera, (Meig.) xv. 685

Ptylodactyla elaterina, xiv. 327

Pucerons, food of, xiv. 68

liabits of, xv. 267

neuters among, xiv. 78

ovoviparous, xiv. 77

Pulex, description of the, xiv. 154 general remarks upon the, xiv. 165

irritans, xiv. 156

penetrans, xiv. 156

Pupipara, xv. 717

the family of the, xv. 774

Pupivora, the, xv. 359. 406

Purpuricenus, (Dej.) xv. 103

Pygæra, xv. 609

Pyrales, metamorphosis of, xiv. 83

Pyralis, (Fab.) xv. 617

Heracleana, xv. 617

pomana, xv. 617

prasinaria, xv. 618

rutana, xv. 617

tapezana, xv. 625

umbellana, xv. 617

vitis, xv. 617

Cuy. Index.
Pyrochroa, xv. 28. 36

(Degeer) xiv. 327

(Geoff.) xv. 27

nitida, xiv. 327

Pyrochroïdes, xv. 27

Pytho, (Lat.) xv. 15

Quadrimani, description of, xiv. 207

Ramphhomyia, xv. 691

Ramphus, (Clairv.) xv. 61

Ranatra, (Fab.) xv. 222

Ranatræ, habits of the, xv. 250

labrum of, xv. 249

where found, xv. 232

Raphidia, (Lin.) xv. 300

Raphium, (Meig.) xv. 696

Red ant, of Linnæus, xv. 443

Reduvii, food of, xv. 232

Reduvius, $(F a b$.$) xv. 220$ dorsalis, xv. 243 personatus, xv. 243 spinidorsis, xv. 244 tuberculatus, xv. 244

Rembus, description of, xiv. 225 impressus, xiv. 225 politus, xiv. 225

Renagrida, (Cochineals) xv. 280

Rhagio, of Fab., xv. 754

Rhagium, (Dalh.) xv. 112 (Fab.) xv. 111

Rhagocrepis Riedelii, xiv. 271

Rhamnusium, (Meg.) xv. 112

Rhæbus, of Fischer, xv. 19. 53

Rhicipera marginata, xiv. 327

Rhina, (Lat.) xv. 63

Rhingia, (Scop.) xv. 705 rostrata, $\mathrm{xv} .763$

Rhingiæ, the, xv. 763

Rhinomacer, xv. 66, 67 (Fab.) xv. 20

Rhinomacera, of Oliv., xv. 53

Rhinosimi, xv. 25

Rhinosimus, (Lat.) xv. 20. 53

Rhinotia, (Kirb.) xv. 54

Rhinotragus, (Dalm.) xv. 106

Rhipicera, (Lat.) xiv. 326

Rhipidia, (Meig.) xv. 686

Rhipiptera, the, xv. 681 antennæ of, xiv. 78 description of, xiv. 27 metathorax of, xiv. 11 otherwise called stresiptera, xiv. 25 supplement on the, xv. 683

Rhisotrogus, (Lat.) xiv. 481

Rhynchite, xv. 54

$\mathrm{N}$ n 
Rhynchænus, xv. 60. 69 (Fab.) xv. 59

Rhynchostoma, xv. 20

Rhyncophorus, xv. 19

Rhyngota, of Fabricius, xv. 230

Rhysodes, (Lat.) xiv. $\mathbf{3 5 4}$ exaratus, xiv. 355

Rhytirrhinus, xv. 58

Rhyzophagus, (Herbs.) xv. 85

Richard, what called, xiv. 310 origin of the name, xiv. 356

Ricini, of Degeer, xiv. 152 or bird-lice, xiv. 164 very numerous, xiv. 165

Ricinus, description of the, xiv. 150 canis, xiv. 151 emberizæ, xiv. 151 fringillæ, xiv. 151 gallinæ, xiv. 151 genus approximating to, xv. 43 pavonis, xiv. I65 philoptère, xiv. 153 mergi, xiv. 151

Ripidius, (Thunb.) xv. 28

Ripiphori, xv. 36 larvæ of, xv. 44

Ripiphorus, xv. 37

$$
\begin{aligned}
& \text { (Payk.) xv. } 29 \\
& \text { paradoxus, xv. } 37 \\
& \text { (Oliv.) xv. } 28
\end{aligned}
$$

Robert the devil, what called, xv. 588

Ropalomera, (Wied.) xv. 711

Ryngota, (Fab.) xv. 216

Runners, the, xv. 171

Ruricola, (Fab.) xv. 591

Rutela, (Lat.) xiv. 473 cerata, xiv. 474 cetoniö̈des, xiv. 474

Rutilæ, how confounded, xiv. 517

Rhynchani, of Fab., xv. 61

Rhyncophora, short billed, xv. 56

Ryssonotus, xiv. 499

Sabethes, (Robineau Desvoidy) xv. 683 Sagaris, (Panz.) xv. 364

Sagra, xv. 122. 125 tristis, $\mathrm{xv} .125$

Sagrides, xv. 122

Salda, (Fab.) xv. 219

Saldes, of Fabricius, xv. 220

Saltatoria, xv. 173

Salius, (Germ.) xv. 61

Sandalus, (Knock) xiv. 326 niger, xiv. 326 petrophya, xiv. 326

Sanguine ants, attack upon, xv. 470 Saperda, xv. 108. 119 (Fab.) xv. 105
Saperda carcharias, xv. 120 cylindricollis, xv. 119

Sapyga proper, (Latr.) xv. 374

Sapygatæ, (Lat.) xv. 374

Sapromyza, (Fall.) xv. 714

Sarapus, xiv. 391 (Fisch.) xiv. 391

Sarcophaga, xv. 709

Sargus, (Fab.) xv. 700. 701

Saropoda, xv. 387

Sarrotrium, (Germ.) xiv. 560 (Illig.) xiv. 560 celtis, xiv. 560

Saturnia, (Schrank) xv. 605

Satyri, xv. 583

Satyrus, xv. 590 (Lat.) xv. 591

Sauterelles, xv. 202

Saw-flies, eyes of, xiv. 46

Scæva, (Fab.) xv. 703

Scarabæi, of the earth, xiv. 511 of the flowers, xiv. 511. 529 of the trees, xiv. 511 horns of, xiv. 533 silphiö̈des, xiv. 509 unctuous, xv. 39

Scarabæidæ, xiv. 448 size of, xiv. 96

Scarabæus, xiv. 451 of Fabricius, xiv. 462.466 (Lin.) xiv. $449.456,457,458$. 465. 468

Actæon, xiv. 470 appearance of reason in the, xiv. 111 bellicosus, xiv, 457 auratus, xiv. 496 Belzebut, xiv. 457 boas, xiv. 509 candidæ, xiv. 476 carnifex, xiv. 457 cyclops, xiv. 465 cylindricus, xiv. 498 dichotomus, xiv. 470 emarginatus, xiv. 457 eremita, xiv. 492 Esculapius, xiv. 452 fasciatus, xiv. 492 femoralis, xiv. 506 festivus, xiv. 457 fimetarius, xiv. 458 formed from larvæ, how, xiv. 56 fulgïdus, xiv. 453 fullo, xiv. 479 globosus, xiv. 460 hemipterus, xiv. 492 Hercules, xiv. 470

Hippocrates, xiv. 452 Jasius, xiv. 457 
Scarabæus lancifer, xiv. 457

Lazarus, xiv. 465

longimanus, xiv. 470

longipes, xiv. 454

Iunaris, xiv. 475

macropus, xiv. 473

melolontha, xiv. 479

melolontha, alimentary tube of, xiv. 480

mimas, xiv. 457

mobilicornis, xiv. 465

Momus, xiv. 463

nasicornis, xiv. 468.509

negasoma, xiv. 470

nobilis, xiv. 491

proboscideus, xiv. 465

nuchicornis, xiv. 456

properly so called, xiv. 470

punctatus, xiv. 470

quadridens, xiv. 465

sacer, xiv. 452

Silenus, xiv. 509

stercorarius, xiv. 463

sticticus, xiv. 496

tarandus, xiv. 509

taurus, xiv. 455

testaceus, xiv. 465

to what applied, xiv. 512

typhœus, xiv. 463

vernalis, xiv. 464

what named by Pliny, xiv. 510

Scaphidites, xiv. 397

Scaphidium proper, xiv. 398

Scaphinotus, description of, xiv. 231

Scapterus, (Dej.) description of, xiv. 201

Scaritides, description of, xiv. 197

Scarites, of Fabricius, xiv. 200

description of, xiv. 202

(Ross) description of, xiv. 206 arenarius, xiv. 204

Bonelli's division of, xiv. 273

Bucephalus, xiv. 275

for cantharides, $\mathrm{xv} .18$

cyaneus, xiv. 199

depressus, (Fab.) xiv. 201

elongatus, xiv. 203

gagates, xiv. 216

Georgiæ, xiv. 205

gigas, xiv. 202

Hottentota, xiv. 216

lævigatus, xiv. 199. 203

marginatus, (Fab.) xiv. 201

piceus, xiv. 216

pyracmon, xiv. 202

ruficornis, xiv. 202

rufus, xiv. 207

sabulosus, xiv. 203

terricola, xiv. 202
Scarites, where found, xiv. 92

Scaurus, xiv. 566

$$
\begin{aligned}
& \text { (Fab.) xiv. } 549 \\
& \text { (Sterm.) xv. } 14 \\
& \text { (Fab.), description of, xiv. } 206 \\
& \text { sulcatus, xiv. } 566
\end{aligned}
$$

Scotobius, (Germ.) xiv. 549

Scatomyzides, xv. 712. 714

Scatophaga, (Lat.) xv. 713

Scathopse, (Geoff.) xv. 689

Scelion, xv. 368

Scenopinus, (Lat.) xv. 696

Schizochilus Brasiliensis, xiv. 304

Schizorhina, xiv. 496

Schneider, what called, xiv. 462

Schyzocera, (Lat.) xv. 356 Peleterii, xv. 403

Sciophila, xv. 688

Scleroderma, of Klüg, xv. 373

Scolia proper, (Fab.) xv. 374 fulva, xv. 516

Scolietæ, (Latr.) xv. 374

Scolopendra, description of, xiv. 134 cingulata, xiv. 135 coleoptrata, xiv. 134 dorsalis clypeata, xiv. 131 electrica, xiv. 135

forficata, xiv. 134

forked, xiv. 134

gigantea, xiv. 135

lagura, xiv. 131

longicornis, xiv. 134

maleficent, xiv. 133

morsitans, xiv. 126. 133. 135

occidentalis, xiv. 135

phosphorea, xiv. 135

witl twenty-eight feet, xiv. 134

Scolopendræ, bite of the, xiv. 142 description of the, xiv. 141 metamorphosis of, xiv. 82 proper, stigmata of, xiv. 132 rings of the body of the, xiv. 124

Scolytus, xv. 89

(Geoff.) xv. 82

description of, xiv. 238

destructor, $\mathrm{xv}, 89$

flexuosus, xiv. 213

proper, (Geoff.) xv. 82

Scopulipodes, xv. $\mathbf{3 8 6}$

Scorpion, European, xiv. 92 flies, xv. 323

Scorpions of Cayenne, size of, xiv. 96

Scotimus, (Kirb.) xiv. 555 crenicollis, xiv. 555

Scutellaria, stigmata of, xiv. 4

Scutellera, xv. 232

(M. de la Marck) xv. 36 r

(Lam.) xv. 217

$\mathrm{N} \times 2$ 
Scutellera basalis, xv. 233

Hottentota, xv. 233

nigro-lineata, xv. 233

Scutelleræ, description of, xv. 232

Scutigera, description of, xiv. 133

Scydmœnus, (Lat.) xiv. 385

clavatus, xiv. 386

Helwigii, xiv. 386

hirticollis, xiv. 386

where found, xiv. 385

Godarti, xiv. 386

minutus, xiv. 386

Scyrtes, (Lat.) xiv. 328

Seatophaga, $(F a b$.) xv. 715, 716

Securifera, xv. 354

Seleropterus, (Schoen.) xv. 62

Semblis, xv. 299

the genus, $x v .331$

Separda of Fab. xv. 109

Sepedon, $(F a b.) \times v .715$

Sepidium, xiv. 566

(Fab.) xiv. 550

acuminatum, xiv. 550

cristatum, xiv. 550.567

reticulatum, xiv. 550

rugosum, xiv. 550

tricuspidatum, xiv. 550. 567

variegatum, xiv. 550

vittatum, xiv. 550

Sepsis, (Fall.) xv. 715

Serica, (Macl.) xiv. 482

Seraptia, (Lat.) xv. 23. 30

Sericaria, (Lat.) xv. 609

Sericomyia, (Meig.) xv. 702

Serricornes, xiv. 308

Sericostoma, xv. 301

Serropalpides, xv. 16

Serropalpus, (Illig.) xv. 30

(Payk.) xv. 18

fusculus, $x v .23$

striatus, xv. 23, 24

Sesia, xv. 598

$$
\text { (Fab.) xv. } 595
$$

Sesiades, xv. 598

Shegalyra, to what it belongs, xv. 418 fasciipennis, $\mathrm{xv} .418$

Siagona, description of, xiv. 199 depressa, xiv. 199

description of, xiv. 296

atrata, xiv. 199

fuscipes, xiv. 199

rufipes, xiv. 199

sejus, xiv. 199

Siagonum quadricorne, xiv. 296

Sialis, (Lat.) xv. 299

Sicus, (Lat.) xv. 692

Sigalphus, (Latr.) xv. 363

Silis, (Meg. Dej.) xiv. 338

rubricollis, xiv. 338
Silis spinicollis, xiv. 338

Silk-worm, change undergone by,xiv. 80 manner of feeding of the, xiv. 66

Silk-worms, acid from, xiv. 73 the cocoons of, xv. 668

Silpha, (Lin.) xiv. 390, 391. 394. 398. $400,401.426$

atrata, xiv. 396

dispar, xiv. 395

excrementitious, apparatus of the, xiv. 172.308

Indica, xiv. 394

lachrymosa, xiv. 394

lævigata, xiv. 396

Laponica, xiv. 396

littoralis, xiv. 394

opaca, xiv. 396

obscura, xiv. 396

(proper, ) xiv. 394

Pedemontana, xiv. 396

quadripunctata, xiv. 396

reticulata, xiv. 396

rugosa, xiv. 396

sabulosa, xiv. 560.570

sinuata, xiv. 395

subterranea, xiv. 397

Surinamensis, xiv. 394

thoracica, xiv. 395

tristis, xiv. 396

vespillo, xiv. 393

with what confounded, xiv. 414

Silphæ, larvæ of, xiv. 415

Silphales, xiv. 390.398

Silvanus, (Lat.) xv. 88

Silvius, (Meig.) xv. 697

Simblephilus, (Jur.) xv. 379

Simplicimana, probably include catas. copus, xiv. 188

Simplicimani, description of, xiv. 211

Simulia, xv. 733

Simulium, (Lat.) xv. 689. 752

Sinodendron, $(F a b$.$) xiv. 460.498 .501$ cornutum, xiv. 502 digitatum, xiv. 461

Siphonaptera, description of the, xiv. 154 Sirex, xv. 404

of Linnæus, xv. 358

fusicornis, xv. 405

gigas, xv. 404, 405

juvencus, $x v .405$

spectrum, xv. 405

Sispes, $(F a b$.$) xv. 365$

Sisyphus, xiv. 453

Sitaris, xv. 51

elytra of, xv. 46

(Lat.) xv. 34

Smerinthus, (Lat.) xv. 598

Smynthurus, description of the, xiv. 146

Soldiers, what called, xv. 333 
Solenorhinus, (Schœen.) xv. 60

Sparasion, xv. 368

Sparedrus, (Meg.) xv. 19

Spærium, (Charpeut) xv. 175

Specomyia, (Lat.) xv. 703

Spercheus, (Fab.) xiv. 427

Speridium, xiv. 401

Spectrum, xv. 191 of Stoll, xv. 173 where found, xiv. 96

Sphæridiota, xiv. 431

Sphæridium, (Fab. Gyll.) xiv. 401 of Fabricius, xiv. 432. 445

Sphærites, (Dufst.) xiv. 391

Sphærocera, (Lat.) xv. 712

Sphæroderus, xiv. 231

Sphecodes, (Lat.) xv. 382

Sphegina, (Meig.) xv. 704

Sphegydes, (Lat.) xv. 374

Spheniscus, (Kirb.) xv. 13 erotyloïdes, $\mathrm{xv} .13$

Spherites, denticulation of, xiv. 390

Sphex, (Lat.) xv. 374, 375 (of Linnaus) xv. 359. 365. 373. 377 organs of touch of, xiv. 64

Sphinx, (Lin.) xv. 595

Atropos, xv. 597. 677

achemænides, xv. 680

celerio, where found, xiv. 90

euphorbiæ, xv. 596

fenestrina, xv. 599

filipendulæ, xv. 600

nerion, where found, xiv. 90

ocellata, xv. 598

proper xv. 596

populi, xv. 598

statices, (Lin.) xv. 600

Sphinxes, seek plants and flowers, when, xiv. 61

Sphinx tilæ, xv. 598

Sphodrus, description of, xiv. 220 complanatus, xiv. 221 janthinus, xiv. 221 terricola, xiv. 220

Sphondylus, of Cordus, xv. 193

Sphryracephala, (Say.) xv. 774

Spider, instinct of, xiv. 104

Spiders, heart of, xiv. 2

Spinneret, what named, xv. 636

Spondylis, (Fab.) xv. 99 where placed, xv. 113

Squillæ, heart of, xiv. 2

Staphylini, general description of, xiv. 301

head of the, xiv. 302

Staphylinos, of Aristotle, xv. 193

Staphylinus, (Lin.) xv。 166 description of, xiv. 288
Staphylinus æneus, xiv. 292 alternans, xiv. 292 amœnus, xiv. 292 atricapillus, xiv. 299 aureus, xiv. 292 bi-pustulatus, xiv. 299 biguttatus, xiv. 294 boleti, xiv. 298 brunnipes, xiv. 292 canaliculatus, xiv. 298 clavicornis, xiv. 294 collaris, xiv. 298 cupreus, xiv. 292 cyaneus, xiv. 292 dilatatus, xiv. 291 elegans, xiv. 292 elongatus, xiv. 292,293 emarginatus, xiv. 299 erythrocephalus, xiv. 292 erythropterus, xiv. 292 fulgidus, xiv. 292

fulmineus, xiv. 292

fuscipes, xiv. 299

hirtus, xiv. 291

hæmorrhoïdalis, xiv. 292

impresses, xiv. 298

Juno, xiv. 294

linearis, xiv. 293

lunulatus, xiv. 299

maxillosus, xiv. 291

melanocephalus, xiv. 292

minutus, xiv. 298

murinus, xiv. 291

ochraceus, xiv. 292

oculatus, xiv. 292

olens, xiv. 291

pilosus, xiv. 292

politus, xiv. 292

(proper, description of, xiv. 291

pyropterus, xiv. 292

pubescens, xiv. 292

riparius, xiv. 294

rufus, xiv. 290

similis, xiv. 292

socialis, xiv. 298

stercorarius, xiv. 292

striatus, xiv. 299

strumosus, xiv. 299

ulmi, xiv. 290

Statyra, xv. 27

Stelis, (Panz.) xv. 385

Stenelytra, xv. 10

supplement on the, xv. 21

Stenepteryx, (Leach) xv. 719

Steni, xiv. 305

Stenocorus, (Dalm.) xv. 103

(Lin.) xv. 112

(Oliv.) xv. 111

Cyaneus, xv. 111 
Stenoderus, (Dej.) xv. 112

Stenolophus,(Deg.)descriptionof,xiv.210 vaporariorum, xiv. 210

Stenopterus, (Illig.) xv. 106

Stenosis, (Herb.) xiv. 548

Stenosthetus, of M. Megerle, xiv. 300

Stenostoma, (Lat.) xv. 20

Stenotrachelus, (Payk.) xv. 14

Stenus, description of, xiv. 294

Stephanus, (Jur.) xv. 361 Brasiliensis, xv. 418

Sternocera, xiv. 357

Sternoxi, xiv. 309 hepatic conduits of, xiv. 308 how divided, xiv. 310

Steropes, (Stev.) xv. 30

Steropus Hottentota, xiv. 216

Stigmatium, xiv. 375 cicindeloïdes, xiv. 376

Stigmodera, xiv. 357

Stigmus, (Jur.) xv. 378

Stilbium princeps, $x v .434$

Stilbum, of Spinola, xv. 369

Stilichus, xiv. 294

Stilponotus euripiformis, xv. 22 new subgenus of, $x v .22$

Stizus, (Lat.) xv. 376

Stomis, description of, xiv. 218 pumicatus, xiv. 218

Stomoxys, xv. 705 (Geoff.) (Fab.) xv. 706 the, xv. 767 bite of the, xv. 722

Stratyomys, xv. 758 (Geoff.) xv. 700 the, xv. 757 a kind of aigrette in, xiv. 74

Stratyomydes, of Latreille, xv. 757 (Lat.) xv. 699

Strebla, (Dalm.) xv. 719

Stresiptera, of Mr. Kirby, xv. 681 * a vicious denomination, xiv. 25

Strongylium, (Kirb.) xv. 14

Strongylus, (Herbst.) xiv. 401

Stygia, (Draparnaud) xv. 601 Australis, xv. 601. 604

Stygides, (Lat.) xv. 694 antennæ of, xv. 694

Stylops Childreni, xv. 684 *

Styphlus, (Schoen.) xv. 60

Subdipterus, xv. 37

Subulicornes, xv. 304 (Lat.) xv. 295 neuroptera, xv. 296

Suckers, xv. 391

Sucking-insects, organs of manducation of, xiv. 9

Suctoria, general remarks upon the, xiv. 165
Suctoria, description of the, xiv. 26. 154

Suctorial insects, food of, xiv. 40

Surveyors, what called, xv. 637

Swimmers, description of, xiv. 243

Sybines, $\mathrm{xv}, 60$

Sybistroma, xv. 696

Sygnatha, xiv. 133

Sylphs, frequent the snake root and staphelia, xiv. 60

Sylvanis, xv. 589

Sylvanus cænobites, xv. 589

Synagris, of Fabricius, xv. 575 (Lat.) xv. 380 cornuta, xv. 575

Synapha, (Meig.) xv. 688

Synchita (Helv.) xv. 85

Syndesus, (Macl.) xiv. 501 cornutus, xiv. 502

Synistata, of Fab., xv. 294

Synistates, of Fabricius, xv. 390

Synodendron, (Fab.) xv. 84

Synotomis, (Illig.) xv. 600

Synuchus, description of, xiv. 221

Syphona, (Meig.) xv. 706

Syrphiæ, larvæ of, xv. 270

Syrphidæ, the, xv. 701.759

Syrphides, proboscis of, $\mathrm{xv} .705$

Syrphus, xv. 702.760

(Fab.) xv. 704

food of the larvæ of, $x v .762$ proper, (Lat.) xv. 703

Syrtes, (Fab.) xv. 219

Syrtis fasciatus, xv. 242

Systropha, (Illig.) xv. 383

Systropus, (Wied.) xv. 706

Syzygops, xv. 58

Tabani, the, xv. 755

bite of, $x v .722$

Tabanides, $\mathrm{xv} .696$ the family of, xv. 755

Tabanus, (Lin.) xv. 696 autumnalis, xv. 757

bovinus, $\mathrm{xv} .756$ proper, xv. 697

Tachina, $\left(\boldsymbol{F} a b_{0}\right)$ xv. 707, 708, 709

Tachinus, description of, xiv. 299

Tachypori, with what associated, xiv. 307

Tachyporus, description of, xiv. 299 chrysomelinus, xiv. 307

Tachypus, description of, xiv. 233 eyes of, xiv. 241

Tagenia, (Lat.) xiv. 548

Tagona, of Fis., xiv. 546

Tailors, what named, xv. 738

Tamnophilus, xv. 60 
Tanypus, xv. 746

(Meig.) xv. 684

Tanyrhynchus, (Schøen.) xv. 60

Tanystoma, xv. 690

Tanystomata, wings of, xv. 695

Taphria, description of, xiv. 221

Tarpa, (Fab.) xv. 357

Tarus, description of, xiv. 195

Taupin, xiv. 324 double-croix, xiv. 320

Taxicornes, the, xv. 1

Tefflus, of Megerle, xiv. 232 description of, xiv. 232

Teleas, (Lat.) xv. 368

Telephori, change in food of, xiv. 66 hepatic vessels of, xiv. .308 where found, xiv. 371

Telephorus, (Schœff.) xiv. 337

biguttatus, xiv. 339

fuscus, xiv. 372

minimus, xiv. 339

with what confounded, xiv. 366

Temnoscheila splendens, xv. 93

Temporanceros, xv. 733

Tenecrium chamædrys, xv. 243

Tenbrio, xiv. 559. 568; xv. 68

(Fab.) xv. 99

(Lin.) xiv. 542 ; xv. 87

cadaverinus, $\mathrm{xv} .3$

for cantharides, $x v .48$

chrysomelinus, xv. 3

culinaris, $\mathrm{xv} .3$

digittatus, xiv. 561

dubius, xiv. 324

fossor, xiv, 204

grandis, xiv. 562

how distinguished from Blaps, xiv. 567

impressus, xv. 3

lævigatus, xv. 140

Mauritanicus, xv. 92

molitor, xiv. 562.569

mortisága, xiv. 553

muricatus, xiv. 543. 566

nitidulus, $\mathrm{xv} .3$

numerous changes of, xiv. 256

proper, (Lin.) xiv. 562

retusus, $\mathrm{xv} .3$

Tenebrionites, xiv. 558. 568

Tengra, (Lat.) xv. 374

Tenthredinæ, xv. 357. 397

Tentliredineta, xv. 354

Tenthredo, xv. 430

of Linnæus, xv. 354. 397

egg's of, xv. 423

ovalis, xv. 399

pine, $x v .399$

proper, xv. 356. 400

rosæ, xv. 398
Tentyria, xiv. 566

(Lat.) xiv. 545

Tenypeza, (Meig.) xv. 713

Tephrites violacea, xv. 779

Tephritis, (Fab.) xv. 711.715

(Lat.) xv. 716

dictya, (Fab.) xv. 716

Terebrant hymenoptera, xv. 394

Terebrantia, xv. 354

'Termes, xiv. 92

(Lin.) xv, 300

arborum, xv. 337,338

atrox, xv. 337, 338

bellicosus, xv, 333. 337. 339, 340

destructor, xv. 337. 345

fatalis, xv. 338. 345

lucifugus, xv. 342

mordax, xv. 337,338

morio, $x v .345$

pulsatorius, xiv. 381

Termites, society of, xiv. 118 arborum, xv. 339

are omnivorous, xiv. 23

bellicosi, xv. 341

enemies of the, $\mathrm{xv} \cdot 335$

habits of, xv. 332

infancy of, xiv. 117

military, xv. 344

neuters among, xiv. 19. 78

society of, xiv. 115

soldiers among the, xiv. 114

viatores, $\mathrm{xv} .340$

Termitinæ, xv. 299, 300

Tesseratoma of M. Peltier, xv. 218 ossa cruenta, xv. 239

Tetanocera, (Dumeril) xv. 714 (Lat.) xv. 715

Tetanops, (Meig.) xv. 716

Tetragonoderus, description of, xiv. 212

Tetramera, xv. 52

Tetraonyx, (Lat.) xv. 33

Tetraopes, xv. 108

Tetraptera of Geoffroy, xv. 389

with farinaceous wings, xv. 230

Tetratoma, xv. 8

(Herbs.) xv. 4

fungorum, xv. 9

Tetryx, (Lat.) xv. 177

Tetyra, xv. 232 (Fab.) xv. 217

Tettigometra, xv. 224

Tettigonia, xv. 263

(Fab.) xv. 223

rosæ, xv. 263

(Germ.) xv. 226

wings of, xv. 230

Tettigoniæ, food of, xv. 232

gallinsectæ, food of, xiv, 68 
Tettix, Kirby's, remarks on, xv. 254

Teucer papilio, where found, xiv. 95

Thanatophilus, (Leach) xiv. 395

Thais, xv. 583

$$
\text { (Fab.) xv. } 585
$$

Thanasimi, xiv. 374

Thanasimus, (Lat.) xiv. 345

Therates, description of the, xiv. 181

Theratus marginatus, xiv. 180

Thereva, (Fab.) xv. 708

$$
\text { (Lat.) xv. } 695
$$

Thoracanta, (Lat.) xv. 365

Throscus, (Lat.) xiv. 318

Thrips, (Lin.) xv. 227 habits of, xv. 267

Thylacites, xv. 58

Thymalus, (Latr.) xiv. 400 limbatus, xiv. 400

Thynnus, (Fab.) xv. 374

Tyhreophora, (Lat.) xv. 712

Thyris, (Hoff.) xv. 599

Thysania, (Dalm.) xv. 613

Thysanoura, description of the, xiv. 143

Thysanura, description of, xiv. 26

Ticks, attach themselves to dogs, xiv. 164

Tilli, where found, xiv. 376

Tillus, (Oliv.) xiv. 343. 347 damicornis, xiv. 347

dermestoïdes, xiv. 347

Wiberi, xiv. 347

elongatus, xiv. 343

serraticornis, xiv. 347

Timarcha, (Meg.) xv. 140

Timia, (Meis.) xv. 717

Timiæ, (Wied.) xv. 716

Tinea, xv. 625

(Lin.) xv. 227

colonella, xv. 624

the corn, xv. 627

evonymella, xv. 626

flavifrontella, xv. 626

Geoffroyella, xv. 627

granella, xv. 626

granella, food of, xiv. 68

habits of, xv. 679

harisella, xv. 627

hordei, food of, xiv. 68

larvæ of, xv, 93

majorella, xv. 627

padella, xv. 627

pellionella, xv. 626

rufimitrella, xv. 627

sarcitella, xv. 626

tribunella, xv. 624

Tineæ, (Lin.) xv. 620

metamorphosis of, xiv. 83

Tineites, (Lat.) xv. 620

Tingis, (Fab.) xv. 219
Tingis clavicornis, xv. 243 peculiarity of, xv. 242

Tiphia, (Fab.) xv. 374

Tipula, (Lat.) xv, 684 of Linnæus, xv. 725 erythrocephala, xv. 752 oleracea, xv. 750 proper, (Lat.) xv. 685.750 variegata, xv. 746 variegata, nymph of, xv. 747

Tipulæ caliciformes, xv. 738 food of, xiv. 69 metamorphosis of the, xiv. 83

Tipularia, resemble the suctoria, xiv. 154

Tipulariæ, the, xv. 738 (Lat.) xv. 684 of Latreille, where found, $\mathrm{xv}$. 731

eggs of, xv. 740

larvæ of the, xv. 739

prolific nature of, $\mathrm{xv} .741$

Tipules couturières, $\mathrm{xv} .738$

Titanura, (Meig.) xv. 713

Tmesisternus, (Lat.) xv. 107

Tomicus, (Lat.) xv. 82

Tortrices, xv. 617

Tortrix dentata, xv. 617

Tourniquet, xiv. 251

Toxotus, (Dej.) xv. 112

Toxicum, (Lat.) xiv. 561 richesianum, xiv. 561

Toxophora, (Meig.) xv. 693 fulva, xv. 779

Trachelides, xv. 26. 35

Trachelus, (Jur.) xv. 358

Trachodes, (Schoen.) xv. 60

Trachusa, (Jur.) xv. 384

Trachyderes, (Dalm.) xv. 102

Trachynotus, (Lat.) xiv. 550

Trachys, xiv. 357 in what compreliended, xiv. 313

Trachyscelis, (Latr.) xv. 4

Tragocerus, (Dej.) xv. 107

Tragopa, (Lat.) xv. 225

Trechus, description of, xiv. 242 rubens, xiv. 242

Tryphyllus, of Megerle \& Dejean, xiv. 403

Trémaeres, what so named, xiv. 4

Trephritis, (Lat.) xv. 716

Tresia, Lacordairei, (Dej.) xiv. 264

Trichiopoda, (Lat.) xv. 708

Trichius, xiv. 483 of Fabricius, xiv. 489.491 barbatus, xiv. 493 changes and habits of, xiv. 529 eremita, xiv. 492

fasciatus, xiv. 492.529 
Trichius hemipterus, xiv. 492 nobilis, xiv. 491 succinctus, xiv. 529

Trichocera, (Meig.) xv. 686

Trichocoryne, xiv. 306

Trictenotoma, xiv. 534 Childreni, xiv. 534

Tricodes cyaneus, xiv. 343

Trichodes, biliary vessels of the, xiv. 153

food of the, xiv. 152

(Fab.) xiv. 345

alviarius, xiv. 346 apiarius, xiv. 346.374

Tricognatha, description of the, xiv. 192 margipennis, xiv, 192

Tricondyla, description of the, xiv. 182 size of the, xiv. 181

Trydactyli, xv. 196

Tridactylus, (Oliv.) xv. 174

Trigonosoma perilampiformis, xv. 774

Trilobites, between myriapoda and crustacea, xiv. 137

Trimera, xv. 156

Trineura, (Meig.) xv. 717

Triongulin des andrenettes, xiv. 153

Triplax, (Fab.) xv. 155

Trips, palpi of, xv. 216

Tritomia, xiv. 401

(Fab.) xv. 155

(Geoff.) xv. 87

bipustulatum, xv, 155

Trixa, (Meig.) xv. 708

Trixagus, (Kugel) xiv. 318

Trogides, (Macleay) xiv. 466

Trogoderma, (Latr.) xiv. 406

Trogosita, (Fab.) xiv. 561; xv. 88.155 (Oliv.) xv. 88

calcar, xiv. 562

squamosa, xv. 93

blue, xv. 92

carraboïdes, xv. 92

Trogus, xiv. 247

Trojan-knights, of Linnæus, xv. 584

Tropidia, (Meig.) xv. 705

Trox, xiv. 508

of Fab. and Olivier, xiv. 467

dubius, xiv. 412

horridus, xiv. 467

spinicornis, xiv. 466

Truncatipennes, description of the, xiv. 183

Truxales, stigma of the metathorax of, xiv. 3

Truxalis, (Fab.) xv. 176

Trypananeus, xv. 91

Tryphyllus, (Dej.) xv. 87

Trypoxylon, (Fab.) xv. 378 (Lat.) xv. 377

Cuv. Index.
Trypeta, (Meig.) xv. 716

Tubicenus, (Dej.) xv. 54

Turdus gryllivora, xv. 214

Turf-ants, emigration of, xv. 45.3 instinct of the, $\mathrm{xv} .464$

Tychus (Leach.) xv. 167

Tylode, xv. 62

Uleoïota, (Lat.) xv. 95 flavipes, xv. 96

Ulidia, (Meig.) xv. 717

Ulocerus (Schoen.) xv. 55

Uloma, (Dej.) xv. 2

Ulomo, (Meg. \& Dej.) xv. 3

Ulonata, of $\mathrm{Fab}$, xv. 169. 178

Ulosomus, (Schon.) xv. 62

Upis, (Fab.) xiv. 562 ceramboïdes, xiv. $\mathbf{5 6 2}$

Urania, (Fab.) xv. 593. 594

Urocerata, xv. 358.403

Urocerus, (Jur.) xv. 358

Urophora Hardwickii, xv. 261

Usia, (Lat.) xv. 693

Vaguevague, what called, xv. 346

Vanessa, (Fab.) xv. 587 morio, xv. 587

Vappo, (Lat.) xv. 701

Velia, (Lat.) xv. 221

Veliæ, the, xv. 248

Vermillon, xv. 291

Vers polypes, xv. 744

Vertebralia, insects next in rank to, xiv. 33

Verrucivora, experiment upon, xv. 203

Vespa, (Lin.) xv. 365. 380

chartaria, xv. 528

crabro, xv. 517

habits of, $x y .516$

minuta, xv. 365

muraria, xv. 524

nidulans, xv. 527

proper, (Lat.) xv. 381

vulgaris, $x v .517$

Vespariæ, xv. 380. 516

Vesperus, (Dej.) xv. 111

Vipion, xv. 363

Volucella, xv. 759

(Geoff.) xv. 702

Vrillettes, xiv. 379

Walking-leaves, what named, xv. 191

Wasp, appearance of reasor in a, xiv. 109

the common, xv. 517

O 0 
Wasp, curious experiment upon tlie eyes of, xiv. 46 nest of the common, xv. 519

Wasps, the, xv. 380

combats amongst the, xv. $\mathbf{5 2 3}$ crowd plants and flowers, when, xiv. 61

habits of the, xv. 516

how destroyed, xv. 200

instinct of the paste-board, $x v$. 528

nests of the pastemboard, xv. 529

metamorphosis of, xiv. 118

method of destroying, xv. 523

nests of, xiv. 119

neuters among, xiv. 19. 78

pedicle of, xiv. 52

size of, xiv. 96

society of, xiv. 114

sting of, xv. $\mathbf{3 8 9}$

temporary society of, xiv. 120

wings of, xiv. 16

Water-scorpions, xv. 248

Wax-moth, xv. 563

White-ants, xv. 332

White-worm, what so called, xiv. 447. 480

Wimbles, xiv. 379

Winged insects, thorax of, xiv. 11

Wood-ants, xv. 464

Wood-lice, xv. 346

metamorphosis of, xiv. 82

moulting of the, xiv. 142

roll themselves in a ball, xiv. 129

what called, xv. 345

Worms, description of, xiv. 38

of flies, (improperly so called,) xiv. 84

remark of Aristotle respecting, xiv. 80

Xantholinus, xiv. 292

Xenops, lenses in the eye of, xiv. 48

Xestomyza, (Wied.) xv. 693

Xiphydria, (Lat.) xv. 358

Xorides, (Latr.) xv. 361

Xya, (Illig.) xv. 174
Xya variegata, xv. 174

Xyela, (Dalm.) xv. 357

Xyla variegata, xv. 196

Xylita, (Payk.) xv. 17

Xyphicera, (Lat.) xv. 176

Xylocopa, (Fab.) xv. 384 (Lat.) xv. 383

Xylophagi, xv. 81. 83

Xylophagus proper, xv. 699

Xylophili, xiv. 467

Xylophilus, of Bonelli, xv. 53

Xyluta, xv. 705

Xylotrogi, xiv. 309.352

Xysta, (Meig.) xv. 709

Xytelinus, (Lat.) xiv. 350

Yellow ant, xv. 443

Ypsolophus, (Fab.) xv. 625

Yponomeuta, (Lat.) xv. 626

Zabrus, description of, xiv. 211 gibbus, xiv. 211

Zancudos culices, xv. 733

Zeli, the, xv. 244

Zelima, (Fab.) xv. 584

Zelus, (Fab.) xv. 220 rufescens, xv. 244

Zethus, (Fab.) xv. 381

Zeuzera, (Lat.) xv. 604

Zirophorus coriaceus, xiv. 306

Zodion, (Lat.) xv. 706

Zonitis, xv. 51

(Fab.) xv. 33, 34

larvæ of, xv. 44

Zoophytes, description of, xiv. 37

Zophosis, xiv. 566

(Lat.) xiv. 544

(Germ.) xiv. 544

Zuphium, description of, xiv. 190

Zygæna, xv. 599

(Fab.) xv. 595

Zygœnides, xv. 599

Zygia, (Fab.) xiv. 341

oblonga, xiv. 341

Zygops, xv. 62

Zyrophorus, description of, xiv. 296

Zygothrica dispar, xv. 774 . 


\title{
ALPHABETICAL TABLE
}

\author{
OF

\section{AUTHORS QUOTED IN THIS WORK.}

In explaining the abbreviations employed to indicate the numerous writers necessarily referred to in this work, it has been thought useful to give the reader a general idea of their profession, the period of their birth and decease, and of the character of their writings.

Abild. - Abildganard (Peter Christian), a Danish naturalist ; Professor at Copenhagen, died in 1808.

One of the continuers of the Zoologia Danica of Muiller, and author of various Memoirs published among those of the Society of Natural History, and of The Royal Society of Sciences of Copenhagen, as well as of the Society of Naturalists of Berlin.

ACAD. DES Sc.

I thus quote the "Mémoires de l'Académie des Sciences," of Paris, of which one quarto volume was annually published from 1700 to 1790 .

I have also occasionally quoted the "Mémoires des Savans Etrangers à l'Académie," eleven volumes, from 1750 to 1786.

I have also frequently quoted the "Memoirs of the Academy of Berlin" from 1819 , and the new ones of the Academia Naturæ Curiosorum of Bonn, from Vol. IX. 1818, at which period they assumed their new form.

For those of the Academy of Petersburg, see Peterob. or Petrop.

Acosta or rather Mendez DA Costa (Emmanuel), a Portuguese naturalist, resident in London.

"Historia Naturalis Testaceorum Britannix," l vol. 4to. London, 1778.

Adanson (Michael), born at Aix in 1727, and died in Paris 1806, Member of the Académie des Sciences, and one of the first naturalists who attempted the classification of Shells according to their animals.

"Histoire Naturelle des Coquillages du Sénégal," 1775, 1 vol. 4to.

Agassis, a German naturalist.

Editor of the "Fishes of Spix," and author of Memoirs in the Isis.

\section{Ahr.-Ahrens.}

"Augusti Ahrensii, Fauna Insectorum Europæ, fascic. I-XII."

Alb. or Albin.-Albin (Eleazar), an English painter.

"A Natural History of Birds," 3 vols. 4to. London, 1731 - 38, containing 306 indifferent coloured plates.

"A Natural History of Spiders," 1 vol. 4 to. with plates. London, 1736 .

Albinus(Bernard-Sigefroy) Professor at Leyden, and one of the great anatomists of the eighteenth century, born at Frankfort in 1697, died in 1770 .

We have only had occasion to quote him for the description of the Pennatulæ inserted in the "Annotationes Academica," 8 Nos. in 4to. Leyden, 1754-1768.

002 
Aldrov. or Aldr.-AldrovanDI (Ulysse), a nobleman of Bologna, Professor of the University of Bologna, born 1525, died blind, 1605.

His " Natural History," in fourteen vols. folio, from 1599 to 1640 , eleven of which are on the subject of animals, was mostly published by his successors. The third volume of the Ornithology and the first of the Insects were the only ones published during his life. It is an undigested and wearisome compilation.

Amor.-Amoreux (N.), a physician of Montpellier.

"Notice des Insectes de la France, réputés Venimeux," 1 vol. folio, with plates. Paris, 1786.

"Description Méthodique d'une espèce de Scorpioll commune à Souvignargues, en Languedoc." Journal de Plysique, XXXV.

Anders.-Anderson (John), a merchant and burgomaster of Hamburg, born in 1674 , died in 1743 .

"Histoire Naturelle de l'Islande du Groënland," \&c. 2 vols. 8vo. Paris, 1750.

This work, although antiquated and superficial, is still the principal source of our information relative to the Cetacea.

Andrex (John Gerard Reinhard), druggist at Hanover, born in 1724 , died in 1793 .

"Letters written from Switzerland to Hanover, 1763," in the German Langnage. They were at first printed separately in the Hanover Magazine for $1764-65$, and republished in 1 vol. 4to. Zurich, 1776 .

Ans. Mus. or Du Mus.-_"Annales du Muséum d'Histoire Naturelle de Paris," by the professors of that establishment, 20 vols. 4to, from 1802 to 1813 .

This work is continued under the title of :

"Mémoires du Muséum d'Histoire Naturelle," \&c. Paris, 1815, et seq. Eighteen volumes have been published.

Argenv.-Argenville (Antoine Joseph Des-Alliers d'), maitre des Comptes of Paris, born 1680 , died 1765 .

"L'Histoire Naturelle Eclaircie dans une de ses principales parties, la CoNchyliologie," 4to. first edition. Paris, 1742; the second angmented by the addition of the Zoomorphose, ibid., 1757; the third augmented by M. Favaune, 2 vols, ibid., 1780.

Arted.-Artedi (Peter), a Swedish naturalist, and a friend of Linnæus, born in 1705, drowned at Amsterdam in 1735 .

His work on Fishes was published by Linnæus. "P. Artedi Ichthyologia sive Opera Omnia de Piscibus," I vol. 8vo. Leyden, 1738.

The edition of Walbaum, "Artedius Renovatus," 5 vols. 8vo. Gripswald, 1788_89 is greatly augmented, but by an injudicious compiler.

Ascan.-Ascanius (Peter), Professor at Copenhagen.

Author of five numbers in folio, the first containing "Coloured Illustrations of the Natural History of the North," from 1767 to 1779.

Audeb.-Audebert (Jean-Baptiste), a painter at Paris, born in Rochefort, 1759, died 1800.

"Histoire Naturelle des Singes et des Makis," folio, Paris, 1800, with sixtytwo plates drawn from the stuffed specimens in the Museum.

"Oiseaux Dorés ou à Reflets Métalliques," 2 vols. folio, Paris, 1802.

Aud.-Audoutn (Jean-Victor), Doctor of Medicine, sub-librarian to the Institute of France, assistant naturalist to Messrs. de Lamarck and Latreille at the Jardin du Roi, and member of various societies, born in Paris, 27th of April 1797.

"Anatomie d'une Larve Apode" (Conops), found in a Bombus lapidarius, by Messrs Lachat and $\mathrm{Au}-$ douin, 1818.

"Mémoire sur les rapports des Trilobites avec les Animaux Articulés," published with plates in the Annales Générales des Sciences Physiques, VIII.p. 233.

"Mémoires sur l'Achlysie, Nouveau Genre d'Arachnide," published with plates in the Mem. Soc. d'Histoire Naturelle, II. 497. 
"Note on a new species of Achlysie," Id. II. 497

"Lettres sur la Génération des Insectes addressée à l'Académie des Sciences," published in the Annales des Sciences Naturelles, II. p. 281.

"Recherches Anatomiques sur la Famille du Drele et sur le Mâle de cette Espéce," published witl plates in the Annales des Sciences Naturelles, II. p. 443 .

"Recherches Anatomiques pour servir à l'Histoire Naturelle des Cantharides," published with plates in the Annales des Sciences Naturelles, IX. p. 31.

"Prodrome d'une Histoire Naturelle, Chimique, \&c., des Cantharides," a medical thesis for the degree of M.D., 4to. Paris.

"Mémoire sur la Nicothoé," a new genus of the Crustacea which lives on the blood of the Lobster. Messrs Audouin and Milne Edwards, published in the Annales des Sciences Naturelles, IX. p. 345.

"Mémoire sur l'Anatomie et la Physiologie des Crustacés," published in the same work.

"Explication Sommaire, \&c." of the plates in the great work on Egypt, the publication of which had been interrupted by the indisposition of M. Savigny. To M. Audouin also, in conjunction with M. Geoffroy SaintHilaire, we are indebted for the description of the Mammalia.

"Observations pour servir à l'Histoire de la Formation des Perles," inserted in the Memoires du Muséum d'Histoire Naturelle, 1829.

"Mémoires sur plusieurs Mollusques, entre autres sur la Glycimère, sur une Clavagelle vivante, genre Siliquaire, et sur le genre Magile," presented to the Académie des Sciences in 1829, and republished from that work in the review of the Annales des Sciences Naturelles.

\section{With Milne Edwards.}

"Résumé d'Entomologie ou d'Histoire Naturelle des Animaux Articulés," 2 vols. 18mo. Paris, 1829.

"Histoire Naturelle des Animaux du littoral de la France," still in MS.

\section{Azz.-De Azzara (Don Felix)} a Spanish officer, born 1746, has given us two excellent works on the natural history of Paraguay.

"Essai sur 1'Histoire Naturelle des Quadrupèdes du Paraguay," trans- lated from the manuscript by $\mathrm{M}$. Moreau de Saint-Méry, 2 vols. 8vo. Paris, 1801.

"Voyages dans l'Amérique Méridionale de 1781, jusqu'en 1801," translated by Mr. Walckenaer, 4 vols. 8vo. Paris 1809. The last two volumes, translated by Sonnini, contain the natural history of the birds of Paraguay.

BaJon, formerly staff-surgeon at Cayenne.

"Mémoires pour servir à l'Histoire de Cayenne," \&c., 2 vols. 8vo. Paris. 1777. They contain some details relative to the animals of that country.

Barr.-Barrere (Pierre), Professor at Perpignan, died 1755.

"Essai sur l'Histoire Naturelle de la France Equinoxiale," 1 vol. 12mo. Paris, 1741 .

"Ornithologiæ Specimen Novum." 1 vol. 4to. Perpignan, 1745.

Barton (Benjam. Smith) an American naturalist and Professor at Philadelphia, died 1816.

"A Memoir on the power of fascination attributed to the Rattlesnake," 1 vol. 8vo. Philadelphia, 1796.

"Facts, Observations, and Conjectures on the generation of the Opossum," pamphlet in 8vo. Philadelphia, 1801.

"Some Notice of the Sirena lacertina, and of another suecies of the same genus," pamphlet, 8vo. Philadelphia, 1808.

"Memoir on a Reptile called the Hellbender," pamphlet, 8vo. 1812. It is the Salamandra gigantea.

\section{Bartram (William).}

"Voyage dans les partics sud de l'Ámérique Septentrionale," translated from the English by M. Benoits, Paris, 2 vols. 8vo.

\section{Baud.-Baudet de la Face (Marie-Jean).}

"Essai sur 1'Entomologie du Départment du Puy-de-Dôme," a Monograph of the Lamellicornes, 1 vol. 8vo. Clermont, 1809.

Bast.-Baster (Job), a Physician of Harlaem, fellow of the Royal Society of London, born 1711 , died 1775 .

"Opuscula Subseciva," 1 vol. 4to. 
divided into two volumes with plates, Harlaem, 1764 and 1765.

Basterot (B. de), a Lawyer.

"Mémoire Géologique sur les Environs de Bourdeaux," 8vo. Paris, 1825.

Beauv.-Beauvors (Palisot de). See Palisot.

Bechst, or BECH.-Bechstein (J. M.), a naturalist of Saxony, born 1757 .

"The Common Natural History of Germany," 4 vols. 8vo. Leipsig, 1801 -1809. in the German language. It only treats of the Quadrupeds and Birds.

\section{BeLL (Thomas).}

Author of various Memoirs on Reptiles in the Linnæan Transactions, Zoological Journal, \&c.

Bel.-Belon (Pierre), a Physician at Mans, and a Professor of the College of France, born 1517, died 1564 .

"Observations faites dans ses Voyages en Orient," 1 vol. 4to. 1553.

"Histoire des Poissons," 1 vol. 8vo. Transv., 1551.

"Histoire Naturelle des étranges Poissons Marins, et Description du Dauphin, \&c." 1 vol. 4to, 1551.

"Histoire Naturelle des Oiseaux," 1 vol. folio, 1551 .

Bennet (E. T.), an English naturalist.

Author of several Memoirs in the Zoological Journal.

Bennet (J. Whitchurch, an English naturalist.

"Natural History of the Fishes of Ceylon," of which but two numbers, in 4to. are yet published. The plates are beautiful.

Bergrus (Peter-Jonas), a Swedish naturalist, Professor at Stockholm, died 1790.

Quoted as author of certain Memoirs among those of Stockholm.

Beseke (John Melchior Theophilus), Professor at Mittau in Courland, born 1746. Author of

"Materials for the History of the
Birds of Courland" (in German), 8vo. 1792, Mittau and Leipzic.

Bendant (F. S.), a French naturalist, \&c., member of the Académie des Sciences, quoted for his

Memoirs on Shells, published in the Annales du Muséum.

Besler or Mus. Besler (Michael Robert), a physician at Nuremberg, born 1607, died 1661 .

"Rariora Musei Besleriani," folio, 1716.

Blainv.-Blainville (Henri Ducrotay de), adjunct Professor to the Faculté des Sciences, and member of the Académie des Sciences.

I quote several of his Memoirs on all the branches of Zoology, published in the Annales du Museum, Bulletin des Sciences, Journal Physique, and his articles Mollusques and Vers, in the Dictionnaire des Sciences Naturelles. The first is printed separately under the title of MALACOLOGIE. Paris and Strasb., 8vo, 1828, with 1 vol. of plates.

"Mémoire sur les Bélemnites," 4to. Paris, 1827.

"Essai d'une Monographie de la Famille des Hirudinées," 8vo. Paris, 1827.

BL.-Bцосн (Mark-Eleazer), a Jewish physician in Berlin, born at Anspach 1723, died 1799. His

"Ichthyology, or General and Particular History of Fishes," in twelve numbers, folio, with 432 plates, Berlin, $1785-1796$, is far from being general. It only contains such species as he could procure, and almost all the foreign ones are badly coloured. His

"Systema, Ichthyologiæ"-see ScHNEIDER-also includes the species of other authors, but arranged in a fantastic manner.

"A Treatise on the Generation of Intestinal Worms" (in German), 4to. Berlin, 1782.

Blum. or Blumenb.-BlumenBACH (John Frederick), Professor of Medicine and Natural History at Gottingen.

"Manual of Natural History," 8th edition (in German), 1 vol. 8vo. 
Gottingen, 1807. There is also a French translation of the same by M. Artaud, 1 vol. 8vo. Metz, 1803 .

"Plates of Natural History" (Abbildingen), 10 numbers, 8vo. each consisting of 18 plates. Gottingen, 1796 1810 .

Boccone (Paul), a Bernardine monk of Sicily, born in 1633, died 1704 .

"Recherches et Observations Naturelles," \&c., 1 vol. 12mo. Paris, 1671 .

Bodd.-Boddaert (Peter), Physician, \&c., of Flessingen, in Zealand.

"Elenchus Animalium, vol. I. sistens Quadrupedia," 8vo. Rotterdam, 1785. The sequel has not appeared.

Four letters on as many animals of the Cabinet of Schlosser, and on the Lacerta amboinensis.

Bohatsch (John Baptist), Professor at Prague, died 1772 .

"De quibusdam Animalibus," \&c. 1 vol. 4to. Dresden. 1761.

This work contains some good observations on certain Mollusca and Zoophyta.

BoIE, a young naturalist of Kiel, who died in Java. His voyage was undertaken for scientific purposes.

He had prepared extensive materials for publication on the Reptilia.

Bojanus (Louis Henry), a German naturalist, Professor at Vilna, died 1828.

"Monograph of the Fresh-water Tortoises of Europe," folio, Vilna, 1819, an excellent work. He was also the author of several Memoirs in the Isis.

Boisd.-Boisduval (J.A.), physician and curator of the cabinet of count Dejean.

"Essai sur une Monographie des Zygenides," l. vol. 8vo. with plates. Paris 1829.

"Europæorum Lepidopterorum Index Methodicus," added to the Essay, \&c.

He has lately, jointly with Major de Conte of the United States army, published the first three numbers of another work, entitled
"Histoire Générale et Iconographie des Lépidoptères or des Chenilles de l'Amérique Septentrionale," 8vo. Paris.

The same gentleman in conjunction with count Dejean, has also published the first numbers of another, called the

"Iconographie et Histoire Naturelle des Coléoptères d'Europe," 8vo. Paris, 1827.

He has also described some new species of Lepidoptera in the Annales de la Société Linnéenne de Paris.

Bomme (Leonard), a physician in Zealand.

Author of certain Memoirs published among those of the Society of Sciences of Flessingen, or Flushing.

Bon, or Bonan.-Bonanni, or rather Buonanni (Filippo), a jesuit professor at the college of Rome, born 1638, died 1725. He was an assiduous observer, but we have only quoted his work entitled

"Recreatio Mentis et Oculi in Observa tione Animalium Testaceorum," I vol. 4to. Rome, 1684.

Bonap. or Ch. Bonap.-Bonaparte (Charles Lucien), Prince of Musignano, son of the prince of Canino.

Author of an excellent Supplement to Wilson's American Ornithology, and of several memoirs in the Annals of the Lyceum of New York.

Bonnat.-Bonnaterre (the Abbé), Professor of natural history at Tulle.

He superintended the engraving of the plates of the Vertcbrata for the Encyclopedie Methodique, and gave the text for those of the Reptiles and Fishes,

His figures generally are copied from authors, and not always judiciously selected.

Bonel.-Bonelli (Francesco), director of the Cabinet of Natural History, and professor of Zoology at Turin.

"Catalogue of the Birds of Piedmont," pamphlet, 4to, 1811.

"Entomological Observations," in two parts, published in the Memoirs of the 
Academy of Sciences of Turin. They treat of the genus Carabus of Linnæus, or of the Carabici.

He also published other Memoirs, of which we may particularly notice the "Descrizione di sei nuovi Insetti Lepidopteri della Sardegna," in the thirtieth volume of the same collection.

Bonnet (Charles), a celebrated philosopher and naturalist of Geneva, born in 1720 , died 1793 . We only quote his

"Traité d'Insectologie," 2 vols. 8vo. Paris, 1745, and in the first volume of his works in 4to. Nenfehâtel, 1769.

Bont.-Bontius (Jacques, ) physician general at Batavia in the commencement of the seventeenth century.

"Historiæ Naturalis et Medicæ Indiæ Orientalis, libri VI." printed as a sequel to the work of Pisou, "De Indix utriusque re Naturali et Medica."

Borlasse (William), an English clergyman, curate in the county of Cornwall, born in 1696, died 1772 .

" Natural History of Cornwall," 1 vol. folio. Oxford, 1758.

Born (Ignatius de), a Transylvanian naturalist and celebrated mineralogist, born 1742 , died 1791 .

"Testacea Musei Cæsarei Vindobonensis," I vol. folio. Vienna, 1780.

Bory-SAINT-Vincent, a naturalist of Bordeaux, who accompanied Captain Baudin to the Isle of France, and late president of the Commission of Natural History in the Morea.

"Voyage aux quatres principales isles d'Afrique." This work, which we have quoted, contains various interesting Zoological observations.

"Essai d'une Classification des Animaux Microscopiques," 8vo. Paris, 1826.

$\mathrm{He}$ alsofurnished the explanations of the latter part of the plates of the article Vers, in the Encyclopédie Méthodique.

"Essai Monographique sur les Oscillaires," 8vo. Paris, 1827.

Various articles in the Dictionnaire Classique d'Histoire Naturelle, of which he is the principal editor.
Bosc (Louis), member of the Académie des Sciences.

Author of numerous memoirs in the Actes de la Société de l'Histoire $\mathrm{Na}$ turelle, the Bulletin des Sciences, \&c. and of the Histoires Naturelles des Vers, des Coquilles et des Crustacés, which form a sequel to Déterville's small edition of Buffon.

Bosman (Wm.), a Dutch merchant, who lived in the seventeenth century.

"A Voyage to Guinea," 1 vol. 8vo., Utrecht, 1705, containing original notes on various animals.

Boud.-Boudier (Henri Philippe), druggist,

Has published in the Annales de la Société Linnéenne de Paris, the description of a new species of Lema for the Faune Française.

Bourguet (Louis), professor at Neufchatel, born 1678, died 1742 .

"Traité des Petrifications," I vol. 4to. Paris, 1742.

Bowdich, an English naturalist.

Author of a Journey to Ashantee, and of a Voyage to Madeira, which contain various observations relative to natural history.

Bowdich (Mrs.) now Mrs. LeE.

Is publishing a History of the Fresh-water Fishes of Great Britain, with splendid plates. London 1828, 1829.

Brander (Gustavus), an English naturalist, died 1787 .

"Fossilia Hantoniensia Collecta et in Museo Britannico deposita," 4to. London, 1766. list.

Brantz, a young Dutch natura-

"Memoir of the Euriotis," the same Rat as our Otomys.

Brebis.- Brebisson, member of the Société Linnéenne of Calvados.

"Catalogue Méthodique des Crustaces Terrestres, Fluviatiles et Marins, recueillis dans le département du Calvados," 8vo. 
Brenm (Christian-Louis), a German clergyman.

"Materials for a History of Birds" (in German), 3 vols. 8vo. Neustadt, 1820. 1822.

Bremser, curator of the imperial cabinet of Vienna.

"On the Worms that inhabit living Mau" (in Germany), 4to. Vienna, 1819. It has been translated into French by Dr. Grundler, with additions by M. de Blainville, 8vo. Paris, 1824.

Breyn.-Breynuus (J. Philip), a naturalist and physician of Dantzick, born 1680, died 1764 .

"Dissertatio de Polythalamiis, nova Testaceorum classe," 4to. Dantzick, 1732.

"Historia Naturalis Cocci ralicum Tinetorii," 1 vol. 4 to. Gedaui, 1731.

Briss.-Brisson (Mathurin Jacques), professor of natural philosophy, member of the Académie des Sciences, and in his youth curator of the cabinet of natural history of Réaumur; born 1723, died 1806.

"Le Règne Animal divisé en IX classes," 1 vol. 4to. Paris, 1756 . It only contains the Quadrupeds and Cetacea.

"Ornithologie," 6 vols. 4to. Paris 1770. A useful work on account of the minute exactness of the descriptions. The plates were drawn by the same hand that furnished the figures of the Planches Enluminées of Buffon, and are frequently taken from the same specimens.

\section{Brit. Zool.}

Under this title we quote the large anonymous folio with fine plates, called "British Zoology," printed in London in 1766. It is by Pennant, and has been reproduced by him under the same title in 4 vols. 8 vo. See Pennant.

Brocchi $(G$ ), a military engineer, died 1828 at Syria, in the service of the Pacha of Egypt.

"Conchiologia Fossile Subapennina," 2 vols. 4 to. Milan, 1814.

Brongn.-Brongniart (Alexander), member of the Académie des Sciences, and professor of the

Cuv. Table of Authors.
Faculté des Sciences de Paris, and of the Jardin du Roi, born 1770 .

"Essai d'une Classification Naturelle des Reptiles," 4to. Paris, 1805.

I also quote his works on the Fossil Shells_- Coquilles Fossiles"_-both in the Annales du Museum, and our joint publication on the geology of the environs of Paris, I also refer to his

"Histoire des Crustaces Fossiles," 4to. published by him and M. Desmaret. Paris, 1812.

Brouss.-Broussonnet (PierreMarie-Auguste), perpetual secretary to the Société d'Agriculture, and member of the Académie des Sciences; born 1761, died 1807. I quote his

"Mémoire sur les Chiens de Mer," in the Mémoires de l'Académie des Sciences, 1780. Also his

"Ichthyologia," 4to. of which but one part was published. London and Paris, 1782.

Brown Jan.-Brown (Patrick), an Irish physician, resident in $\mathrm{Ja}$ maica.

"The Civil and Natural History of Jamaica," 1 vol. folio. London, 1756.

Brown or Br.-Brown (Peter), an English painter.

"New Illustrations of Zoology," 1 vol. 4to. London, 1776, with fifty coloured plates of animals of various classesall of them indifferently executed.

Bruce (James), the celebrated Scotch traveller, born 1730, died 1794.

"Travels in Abyssinia, and to the Sources of the Nile." I quote the French translation, 5 vols. 4 to. Paris, 1790.

Brug.-Brugieres (Jean-Guillaume), a physician at Montpellier, and a traveller, born 1750 , and died at Ancona on his return from Persia, 1799. I quote his

"Dictionnaire des Vers," published in the Encyclopédie Méthodique. But one volume, 4 to. has appeared. Paris, 1792. I also quote his

"Figures de Vers," for the same work, of which there are four.

P p 
Brun.-Brunnick (Martin T.), a Danish naturalist, Professor at Copenhagen.

"Ichthyologia Massiliensis," \&rc., l vol. 8vo. Copenhagen and Leipsic, 1768.

"Entomologia sistens Insectorum Tabulas Systcmaticas," 8vo. Copenhagen, 1764. Also various Memoirs published among those of the Society of Sciences, and of the Society of Natural History, of Copenhagen.

Buchan.-Buchanan (Dr. Francis Hamilton), a Scotch physician at Bengal, died in 1829 .

Author of certain Memoirs in the Transactions of the Linnæan Society, and of a Journey from Madras to the Mysore \&c. which contains several valuable observations. We are particularly indebted to him for

"A Natural History of the Fishes of the Ganges," 1 vol. 4to. with a great numbcr of excellent plates. Edinburgh, 1822 .

Buckland (William), professor of Geology at Oxford, author of the

"Reliquiæ Diluvianæ," 4to. London, 1825 , and of numerous Memoirs on fossils.

Buff.-Bufron (Georges-LouisLeclerc, Comte de), Intendant of the Jardin du Roi, and Treasurer of the Académie des Sciences, born 1707, died 1788.

"Histoire Naturclle, generale et particulière, avec la Description du Cabinet du Roi." I always quote the Paris edition of 1749-1789, in 36 vols. 4 to. of which three are general, twelve relate to Quadrupeds, seven are supplements to his general observations and to the Quadrupeds, nine treat of Birds, and five of Minerals.

Buf. Enl. or Enlum. See Planches Enluminees.

\section{Bullet. Des Sc.}

"Bulletin des Sciences par la Société Philomatique," a journal which has appeared monthly since 1791 , which contains a multitude of abridged and valuable obscrvations relative to $\mathrm{Na}$ tural History.
Burchell, an English traveller. "Travels in the Interior of Southern Africa."

Carena (Giacinto), professor at Turin.

"Monograph of the Genus Hirudo," vol. XXV. of the Memoirs of the Academy of Turin, 4to. 1820.

Carmich. - Carmichael, an English officer.

I quote his Memoir on the Fishes of Tristan d'Acunha. Lin. Trans. XII.

Carus (Charles-Gustavus), Professor at Dresden.

Author of several works on comparative Anatomy. I quote his Memoir on the circulation in the Larvæ of the Neuroptera, printed in German, 4to. Leipsic, 1827.

Cat.Catesb.-Cateshy (Mark), a traveller in North America, born 1680 , died 1749 .

"The Natural History of Carolina, Florida, and the Bahama Islands," 2 vols. folio, with an Appendix and two hundred and twenty coloured plates, London, 1731. 1743 .

Cauche (François), of Rouen, a soldier or sailor at Madagascar, died 1638.

"Une Relation de Madagascar," \&c. 1 vol. 8vo. 1631 .

Cavolini (Filippo), a physician and naturalist at Naples.

"Memorie per servire alla Storia de' Polipi Marini," 4to. Naples, 1785.

"Sulla Generazione dei Pescie dei Granchi," 1 vol. 4to. Naples, 1787.

Ceetri (Francesco).

"Storia Naturale di Sardegna," 4 vols. $12 \mathrm{mo}$. Sassari, 1774-1777.

Chabert, director of the Ecole Veterinaire at Alfort.

"Traité des Maladics Vermineuses dans les Animaux," pamphlet, 8vo. Paris, 1782. 
Chab.-Chabrier (J.) a corresponding member of the Societé d'Histoire Naturelle.

He published a series of Memoirs on the flight of Insects, in the Annales du Museum d'Histoire Naturelle. A certain number of impressions were taken separately, which form his "Essai sur le Vol des Insectes," I vol.4to. Paris, 1823.

Chamisso (Adelbert de), a distinguished literary gentleman and naturalist of Berlin, who sailed round the world with Captain Kotzebue.

I quote his Memoir on the Salpæ (in Latin), 1 vol. 4to. Berlin, 1830.

Charp. - Charpentier (Toussaint de).

"Horæ Entomologicæ," 1 vol. 4to. with plates. Breslau, 1825 .

Chemn.-Chemnitz (John Jerome), of Magdeburg, chaplain to the garrison of Copenhagen, born 1730 .

He continued the great work on Conchyliology of Martini, and is the author of various Memoirs published among those of the Society of Naturalists of Berlin, of Copenhagen, and of the Naturforscher.

Choris (Louis), a Russian painter, who accompanied Captain Kotzebue in his voyage round the world. He was assassinated near VeraCruz, when about to commence his travels in Mexico.

"Voyage Pittoresque autour du Monde," folio. Paris, 1822.

"Vucs et Paysages des Régions Equinoxiales," folio. Paris, 1826.

Clairv.-Clairville, an English naturalist, residing in Switzerland.

"Entomologie Helvetique," 2 vols. 8vo. in French and German, with excellent plates. The first volume was published in 1798 , and the second in 1806 ; both were printed at Zurich.

Clarck, an English Veterinary Surgeon.
"A Monograph of the CEstri," in the third volume of the Linnæan Transactions. He has published a second edition of it.

CLerc (Charles), a Swedish painter and a pupil of Linnæus.

"Aranei Suecici Descriptionibus et Figuris Illustrati," 1 vol. 4to. in Swedish and Latin. Holmix. 1757.

"Icones Insectorum Rariorum," 1 vol. 4to. Holmix, 1759-1764. This work is useful as an indication to the Lepidoptera described by Linnæus, from the Cabinet of Quecn Frederica Ulrica.

Cloquet (Jules), a physician and surgeon of Paris.

"Anatomie des Vers Intestinaux," 4to. Paris, 1824.

Clus.-Clusius, or l'Ecluse (Charles), born at Arras 1526, died 1609. He was physician to the Emperor, and subsequently a professor at Leyden.

"Exoticorum Libri X," I vol. folio. Anvers, 1605.

Collet Meygnet (G. F. H.), physician.

"Mémoire sur un Ver trouvé dans le rein d'un Chien" (the Strongylus gigus), inserted in the Journal de Physique, vol. LV.

Fab. Col.-Columa (Fabius), a physician at Rome, an illegitimate descendant of the illustrious house of Colonna, born 1567, died about 1660 . He was an exact and erudite observer.

"De Purpura," 4to. 1616.

"Aquatilium et Terrestrium aliquot. Animalium, aliarumque Naturalium Rerum Observationcs," printed at the end of his Ecphrasis, ib., 4to. 1616.

Com. or Commers.-Commerison (Philibert), born at Dombes in 1727, and died at the Isle of France, 1773. A most indefatigable traveller, and learned naturalist.

I quote his manuscripts and drawings deposited in the Library of the $\mathrm{Mu}$ seum.

P 2 
Cook (Captain), the celebrated navigator, born 1728, and killed at the Sandwich Islands in 1779.

His three great voyages, which have been translated into all languages, are well known to every one,

Coqueb.-Coquebert (Antoine Jean), a naturalist established at Rheims.

"Illustratio Inconographica Insectorum quæ in Museis Parisinis observavit $\mathbf{J}$. Chr. Fabricius," 3 parts, 4to. Paris, 1799-1804.

He also published various notes in the Bulletin des Sciences.

Coucr (Jonathan), an English naturalist.

I quote his paper "On the Fishes of Cornwall." Linn. Trans. XIV.

Cram.-Cramer (Peter), a merchant of Amsterdam.

"Papillons Exotiques des trois parties du Monde, l'Asie, l'Afrique, et l'Amérique," in Dutch and French, 4 vols. 4to. containing four hindred coloured plates. Amsterdam, 1779-1782.

For the Supplement, see Stoll.

Creutz.-Creutzer(Christian).

"Entomologische Versuche," or Entomological Essays, 8vo. with coloured plates, Vienna, 1799 .

\section{Crevelt, a German naturalist.}

Author of a Memoir on a Gecko, published among those of the Society of Naturalists of Berlin. 1809.

Curt.-Curtis (John), an Eng. lish naturalist and painter.

He has commenced a work illustrating the genera of Insects and plants Peculiar to Great Britain. Their characters are figured with the greatest accuracy.

This work, which is published in numbers, already forms 3 vols. 8 vo.

The same author has also published in the Zoological Journal some interesting observations on the Elater noctilucus.

Cuv.-Cuvier (George-LeopoldCretien-Frederic-Dagobert), born at Montbeliard, 1769; perpetual Secretary to the Académie des Sciences, \&c. \&c. \&c.
Of my own works, exclusive of my Memoirs contained in the Amnales du Muséum, I quote the following:

Ménag. du Mus., or "Ménagerie du Muséum d' Yistoire Naturelle," by Messrs. Lacepède, Cuvier, and Gcoffroy, with plates coloured by Marechal, and engraved by Miger, 2 vols. 8vo. Paris, 1804. There is another edition in folio.

Tab. Elem., or "Tableau Elémentaire de l'Histoire Naturelle des Animaux," 1 vol. 8vo. Paris, 1798.

Leç. d'Anat. Comp., or "Leçons d'Anatomie Comparée, recueillies et publiées par MM. Dumeril and Duvernoy," 5 vols. 8vo. Paris, $1800,1805$.

Rech. sur les Oss. Foss., or "Recherches sur les Ossements Fossiles des Quadrupèdes," 4 vols. 4to. Paris, 1812. A second edition was published in 5 vols. 4to. 1821-1823.

Mém. sur les Moll., or " Mémoires pour servir à l'Histoire des Mollusques," 1 vol. 4to. Paris, 1816.

Cuv. et Val.-Cuvier and VaLENCIENNES.

"L'Histoire Naturelle des Poissons," a work which I am now publishing in conjunction with M. Valenciennes. There are now completed 5 vols. 4 to. and 8vo. Paris and Strasburg.

Fred. Cuv.-Cuvier (Fred.), Inspector General of the University of Paris, member of the Académie des Sciences, \&c. \&c., born at Montbéliard, 1773 .

I quotehis Mémoires in the Annales du Muséum, and principally those which relate to the teeth of the Mammalia, published in 1 vol. 8 vo. Paris, 1825. I also particularly cite his

"Histoire Naturelle des Mammifères" prblished in conjunction with M. Geoffroy Saint-Hilaire in folio and 4to. with illustrations drawn from nature.

Cyrill.-Cyrillus or Cirillo (Dominico), a physician at Naples publicly executed in $\mathbf{1 7 9 6}$.

"Entomologiæ Neapolitanæ Specimen," 1 vol. folio. with coloured plates. $\mathrm{Na}$ ples, 1787.

DAHL (George).

"Coleoptera and Lepidoptera," I vol. 8vo. Vienna, 1823. 


\section{Daldorf, a Danish officer.}

Author of Memoirs on certain Fishes, published in the Linnean Transactions, and in the Journal of Gottingen.

Dalm.-Dalman (John Wm.), lately deceased at Stockholm, where he was director of the Museum.

"Analecta Entomologica," I vol. 4to. with plates. Holmix, 1823.

"Prodromus Monographiæ Castnix," 1 vol. 4to. with one plate, Holmix, 1825 .

"Om Nagra Svenska Arter of Coccus," Memoir, 4to. with plates. Stockholm, 1826.

"A Monograph of the Chalcidites, or of the Insects of his family of the Pteromalni," l vol. 8vo. Stockholm, 1820.

"A Synopsis of the Lepidoptera of Sweden," published in the Memoirs of the Academy of Stockholm, 1816.

"Ephemerides Entomologicæ," 1 vol. 8vo. Holmix, ] 824.

"A Memoir on certain Ichneumonides," 1 vol. 8vo. Stockholm, 1826 .

A second, in the Swedish language, on the Insects enclosed in Copal, I vol. 8vo. Stockholm, 1826.

Dal.-Dalyell (J. Graham), a Scotch naturalist.

"Observations on Various Interesting Phenomena of the Planaria," 8vo. Edinburgh, 1814

Dampier (William), the celebrated English mariner, born 1652.

"Voyage round the World," 2 vols. 8vo. London, 1697 and 1699. It has been translated into French, and has passed through several editions. It contains some interesting traits of the history of animals.

Daniels (Samuel), an English painter.

"African Scenery," I vol. folio, a magnificent work, which contains several beautiful figures of extremely rare animals.

Daub. - Daubenton (L.-JeanMarie), born at Montbard, 1716, died at Paris, 1800 . He was a Professor of the Museum and of the Collége de France, and member of the Institute.

I quote the descriptions of the animals with which he has eniriched the Natural History of Buffon.
Daud-Daudin (François-Marie), died at Paris in 1804.

"Traité Elémentaire et Complet d'Ornithologie," of which but 2 vols. 4 to. Paris, 1800, have yet appeared; they only contain the Birds of Prey, and a part of the Passerinæ. It is an indifferent compilation.

"Histoire Naturelle des Reptiles," 8 vols. 8vo. Paris, 1802 and 1803, a sequel to the Buffon of Sonnini.

"Histoire Naturelle des Rainettes, des Grenouilles et des Crapands," l vol. 8vo. with numerous and coloured plates. Paris, 1803.

Dej.-Dejean (Comte), peer of France, lieutenant-general, \&c.

"Catalogue de la Collection des Coléoptères de M. le Comte Dejean," l vol. 8vo. 1821.

"Species General des Coléoptères," 3 vols. 8vo. 1825-1829. The fourth volume has lately been published.

" Histoire Naturelle et Iconographie des Coléoptères d'Europe," by MM. Latreille and Count Dejean, 3 nos. 8vo. 1822

See Boisduval.

Juss.-De Jussieu (Antoine de), Professor of Botany to the Jardin du Roi; born at Lyons 1686, died 1758.

I quote some of his Memoirs on Zoology, published among those of the Academie des Sciences.

Dekay (James E.), an American physician and naturalist.

Author of several Memoirs in the Annals of the Lyceum of New York.

Delap. et Brul.-Delaporte de Brulle.

"Notice sur un Nouveau Grnre de la Famille des Charansons," published in the fourth volume of the Mémoires de la Société d'Histoire Naturelle de Paris.

Delle Chiaje (Stefano), Professor at Naples.

"Memoirs on the History of the Invertebrate Animals of the Kingdom of Naples," 2 vols. 4to. Naples, 1823 , 1825.

Deluc (John Andrew), a naturalist of Geneva, \&c. 
I have only quoted this celebrated geologist in relation to his Memoir "Sur les pierres judaiques," published in the "Mémoirs des Savants etrangers."

\section{Deshayes (G. P.) a naturalist of} Paris.

"Anatomie et Monographie du genre Dentale," in the "Description des Coquilles Fossiles des Environs de Paris," 4to. Paris, 1824, 1825.

Desm. - Desmarets (Anselme Gaetan); corresponding member of the Académie des Sciences, and Professor of Zoology to the Ecole Veterinaire of Alfort.

"Histoire Naturelle des Tangaras, des Manakins, et des Todiers," I vol. folio. Paris, 1805.

"Traité de Mammalogie," serving as an explanation to the plates of the Mammalia of the Encyclopedie Methodique, 1 vol. 4to. Paris, 1820.

He is also the author of various articles in the "Dictionnaire d'Histoire Naturelle ;" of which we will particularly designate that on the Malacostraca.

"Considérations generales sur la Classe des Crustaces," 1 vol. 8vo. with plates. Paris, 1803.

"Histoire Naturelle des Crustaces Fossiles,"published by him and M. Brongniart.

Desmounins (Charles), vice-president of the Société Linnéenne de Bourdeaux.

"Essai sur les Spherulites." Bourdeaux, 1826.

Diq. or Diquem.-Dicquemare (the Abbé Jacques François), a naturalist of Havre, born 1733, died 1789 .

An indefatigable observer, and author of various memoirs on the Zoophyta and Mollusca in the Philosophical Transactions, Journal de Physique, \&c. \&c.

Donati (Vitale), a physician at Padua, and traveller to the king of Sardinia, born 1733, and shipwrecked on his return from Egypt in 1763 .

"Natural History of the Adriatic Sea," published in Italian, 1 vol. 4to. Venice, 1750. The French translation,

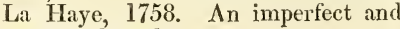
superficial work.
Donov. - Donovan (Edward), an English painter.

"The Natural History of British Fishes," 5 vols. 8vo. London, 1820 .

"The Natural History of British Insects," 8vo.

"An Epitome of the Natural History of the Insects of China," 1 vol. 4to. London, 1778.

"An Epitome of the Natural History of the Insects of India," 4to. London, 1800. I have seen but twelve nos.

"General Illustration of Entomology," Part I. "An Epitome of the Insects of $A$ sia," 1 vol. 4to. London, 1805.

Dorthes (Jacques Antoine), a physician at Montpellier, born 1759 , died 1794.

"Mémoire sur les Araignées Maçonnes," published in the second volume of the Transactions Linnéennes.

Drap. or Draparn.-DraparNaun (Jacques Philippe Raimond), Professor at Montpellier, born 1772 , died 1804.

"Tableau des Mollusques Terrestres et Fluviatiles de la France," pamphlet, 8vo. Montpellier and Paris, 1801.

"Histoire Naturelle des Mollusques Terrestres et Fluviatiles de la France," 4to. with fine engravings. Paris, 1805.

Drap.-Drapiez, Professor of Chemistry at Brussels.

Mémoirs on a new genus of tetramerus Coleoptera, and a description of new species of Mammalia, Birds, and Insects, published in the Annales Generales des Sciences Physiques.

Drur.-Drury, an English goldsmith, lately deceased.

"Illustrations of Natural History," 3 vols. 4to. with finely coloured plates representing the rarer insects of his cabinet. London, 1770-1782.

Duf.-Dufour (Leon), a physician at Saint-Sever, Landes.

"Mémoire Anatomique sur une nouvelle espece d'Insecte du genre Brachine," in the 18th volume of the Annales du. Muséum d'Histoire Naturelle.

"Various.memoirs, "Sur l'Anatomie des Coleoptères, des Cigales, des Ciaadelles, des Labidoures," on a new species of Ornithomyix, and on the genus Ocyptera, published in the Annales des Sciences Naturelles. Two Me- 
moirs inserted in the Journal de Physique, one on the Anatomy of the Scorpions, and the other on that of the Scolia. The Annales Générales des Sciences Physiques contain several others, in which he gives a description of various Arachnides, and of several ncw species of Coleoptera, together with the anatomy of the Ranatra linearis, and of the Nepa cinerea.

Dufts. - Duftschmid (Gaspard), Professor at Lintz.

"Fauna Austrix," 8vo, in German.

I lave only seen the first two volumes, one of which appeared in 1805, and the other 1812. Lintz and Leipsic.

\section{Dugez (Antoine) Professor at} Montpellier.

"Recherches sur la Circulation, la Respiration et la Reproduction des Annelides à branches," 1828.

"Espèces Indigènes du genre Lacerta," Aunales des Sc. Nat., XVI. 1828.

Duham.-Duhamel du MonCEAU, naturalist, agriculturist, \&c., member of the Académie des Sciences, born at Paris 1700 , died 1782.

"Traité general des Pêches," folio, Paris, 1769. I quote this work on account of the number of good plates of fishes which it presents.

Dum. or Dumer.-Dumeril (Constant), Professor to the Faculté de Medicine, and to the Jardin du Roi, member of the Académie des Sciences, born at Amiens, 1774.

Editor of the first two volumes of my "Leçons d'Anatomie Comparée."

"Zoologie Analytique," l vol. 8vo. Paris, 1806.

"Traité Elémentaire d'Histoire Naturelle," 2 vols. 3 vo. second edition. Paris, 1807. Fourth edition, Paris, 1830.

Various Memoirs on Comparative Anatomy, among which is one on the "Poisons Cyclostomes," \&c.

"Considérations Gémérales sur la Classe des Insectes," 1 vol. 8vo, with plates.

Also the articles in the Dictionnaire des Sciences Naturelles relative to Insects.

Duponch.-Duponchel (A.J.), continuer of Godart's Natural History of the Lepidoptera of France.

"Monographie du Genre Erotyle," 4to. with plates, printed in the twelfth volume of the Memoires du Muscum d'Histoire Naturelle.

He has continued, from the sixth volume inclusively, the work of the late M. Godart, cntitled." Histoire Naturclle des Lepidoptères de France." Tho scventh is nearly completed. He has described a new genus of Coleopterous Insects, which he calls Adelostoma, and has published obscrvations on the metamorphosis of the Nyphale Petit Sylvain.

Duport (Andrew Peter), fellow of the Royal Society of London.

Author of a Memoir on the Glaucus, in the fifty-third volume of the Philosophical Transactions.

Dutertre (Jean-Baptiste), a Dominican friar, missionary to the Antilles, born 1610 .

"Histoire Générale des Antilles habitées par les Français," 4 vols. 4to. Paris, $1666,1671$.

The second volume, or that relative to Natural History, contains some good observations. There is an edition in 1 vol. 1654 .

Dutrochet (N.), physician at Chateau-Renaud.

An accurate and ingcnious obscrver, author of certain memoirs in the Annales du Museum, \&c.

Duv.-Duvau (Augusta), member of the Société d'Histoire Naturelle.

"Nouvelles Recherches sur l'Histoire Naturelle des Pucerons," a memoir read before the Academie des Sciences on the 26th of April 1825, and published in the Memoires du Museum d'Histoire Naturelle.

Edwards (George), an English painter, member and librarian of the Royal Society.

"Natural History of Rare Birds," 4 vols. 4to.

"Gleanings of Natural History," 3 vols. 4 to. 
These two works form but one single collection of three hundred and sixtytwo plates.

Next to the Planches Enluminées, it is the richest in respect to Birds that we possess. It also contains animals of other classes. The figures are beauful, the text indifferent.

Edw.-Edwards (Milne), in conjunction with M. Victor Audouin, has published

"Recherches Anatomiques et Physiologiques sur la Circulation dans les Crustaces." Annales des Sciences Naturelles, II.

"Recherches Anatomiques et Physiologiques sur le Système Nerveux des Crustaces." Aun. des Sc. Nat., XIV.

"De la Respiration Aërienne des Crustaces et des modifications que l'appareil branchiale présente dans les Crabes Terrestres." Ann. des Sc. Nat., XV.

"Mémoire sur le Nicothoe," a singular animal that sucks the blood of the Lobster. Ibid. IX.

"Resumé des Recherches sur les Animaux sans vertebre faites aux Isles Chausay."

"Description des Annelides des Côtes de la France."

Edwards (Milne) alone.

"Description de quelques Crustaces nouveaux." Ann. des. Sc. Nat., XIII.

"Recherches Zoologiques pour servir à l'Histoire Naturelle des Lezards." Ann. des Sc. Nat., XVI.

"Monographie des Crustaces Amphipodes."

Egede (John), a Dane, Missionary to Greenland, born 1686, died 1758 .

"Description of Greenland," 1 vol. 8vo. Copenhagen and Geneva, 1763.

\section{Eisenh.-Eisenhardt (Charles} William), author of

"A Memoir on the Medusæ," in those of the Academia Naturæ Curiosorum of Bonn; and with additions by Chamisso, of a Memoir on certain animals of the class of Worms, Ibid., X. part II.

\section{Ellis (John), a London mer-} chant.

" Essay towards a Natural History of the Corallines found on the Coast ot Great Britain and Ireland," 4to. London, 1755. Translated into French, and published at the Hague, 1756.

"The Natural History of many curious and uncommon Zoophytes," 1 vol. 4to. London, 1786. This work was published by him and Solander.

Engram.-Engramelle(MarieDominique-Joseph), an Augustine friar at Paris, born in 1727, died in $\mathbf{1 7 8 0 .}$

" Papillons d'Europe peints par Ernest et décrits par le reverend pere Engramelle," 6 vols. small folio, consisting of three liundred and forty-two coloured plates. The work finishes with the Noctua inclusively.

Ernest was an artisan of Strasburg who had a great and self-acquired talent for painting lepidopterous Insects.

Erxi..-Erxleben (John Christian Polycarpe), Professor of Natural History at Gottingen, born 1744, died 1777 .

"Systema Regni Animalis, Classis I., Ảnimalia, 1 vol. 8vo." Leipsic, 1777.

Esp.-Esper (E. T. C.), Professor at Erlang.

"Europæische Schmetterlinge," or Lepidoptera of Europe, 4 vols. 4to. the first and the fourth divided into two, with coloured plates.

This work is not completed, but some additional numbers on the true Phalenites or the Geometræ, have been published.

"Die Pflanzenthiere," \&c., his work on Zoophytes, 4 vols. 4to. Nuremb., 1791, et seq.

Euphrasen (B. A.), a Swedish naturalist.

Author of a voyage to St. Bartholomew, and quoted for a Memoir inserted among those of the Academy of Stockholm.

\section{Eversh.-Eversham.}

Author of the Zoological Appendix to the "Travels in Bucharia," of the Baron de Mayendorf, with notes by M. Lichtenstein. It has been translated into French by Mr. Amédée Jaubert, 8vo. Paris, 1826. 
Fab.-Fabricius (J. C.), a pupil of Linnæus, Professor of Natural History and Rural Economy at Kiel, born at Tundern, in the Duchy of Sleswick in 1742, died 1807. He published a great many works on Entomology, of which I have particularly quoted the following.

"Entomologia Systematica emendata et aucta," 4 vols. 8vo. the first and third in two parts. Hafniæ, 1792-1794. This work contains several of his anterior ones, revised and re-modelled, such as the "Systema Entomologiæ," 1 vol. 8vo.; "Species Insectorum," 2 vols. 8vo.; "Mantissa Insectorum," 2 vols. 8vo.

"Supplementum Entomologiæ Systematicæ," 1 vol. 8vo. Hafiniæ, 1798.

"Systema Eleutheratorum," 2 vols. 8vo. Kiliæ, 1801.

"Systema Rhyngotorum," 1 vol. 8vo. Brunsvigæ, 1801.

"Systema Piezatorum," 1 vol. 8vo. Brunsvigæ, 1804

"Systema Antliatorum," 1 vol. 8vo. Brunsvigæ, 1805.

He was about to publish his, "Systema Glossatorum," when the hand of death was laid upon him. An extract from that work is given by Illiger in his Magazin für Insectenkunde.

Fab. or Fabr.-Fabricius (Otho), Clergyman in Greenland, and subsequently in Norway and Denmark.

"Fauna Groënlandica," \&c. I vol. 8vo. Copenhagen and Leipsic, 1790, a work of great value, on account of the exactness of the descriptions, but in which names are frequently improperly applied.

$\mathrm{He}$ also published certain memoirs among those of the Society of Natural History of Copenhagen.

FAlck (J. P.), a Swede, Professor of Botany at Petersburg, born 1727 ; travelled in the service of the Russian government from 1768 to 1773 , and committed suicide at Cassan in $\mathbf{1 7 7 4 .}$

His travels were published in German, 3 vols, 4to. Petersburg, 1785, 1786.

The last two relate entirely to Natural History.

Fall.-Fallen (Charles FreCuv. Table of Authors. derick), Professor of Natural History at Lund.

"Diptera Suecix," 4to. first volume. Lundæ, 1814-1817.

Farin.-Farines, a naturalist residing in the department of the Pyrenees Orientales.

Author of Observations on the larva of the Ripiphorus bimaculatus, in the Annales des Sciences Naturelles, 1826.

\section{Favanne.}

Author of a "Dictionnaire de Conchyliologie," and of a greatly enlarged edition of the Conchyliology of d'Argenville.

Fauj.-Faujas de Saint-Fond (B.), Professor of Geology at the Muséum d'Histoire Naturelle.

"Histoire Naturelle de la Montaigne de St. Pierre de Maestricht," 1 vol. 4to. Paris, 1799.

Fermin (Philip), physician at Surinam.

"Histoire Naturelle de la Hollande équinoxiale," l vol. 8vo. Amsterdam, 1765

"Description de Surinam," 2 vols. 8vo. Amsterdam, 1769.

Two indifferent works, filled with errors of nomenclature.

Fern. or Hern.-Hernandez (Francisco), physician-in-chief at Mexico, under Philip II.

"Nova Plantarum, Animalium et Mmeralium Mexicanorum Historia," folio, Rome, 165l. A singular combination of fragments of the author, figures drawn by other's, and annotations of editors. It should be read cautiously.

Feruss.--Ferussac (J. Daudebart de), a French naturalist.

Author of a new and enlarged edition of an "Essai d'une Méthode Conchyliologique," originally written by M. de Ferussac, Sen., pamphlet, 8vo. Paris, 1807.

"Histoire des Mollusques, Terrestres, et Fluviatiles," folio, with fine plates. It is not yet completed.

He is also the principal editor of that important periodical called the "Bulletin Universel des Sciences," \&c. 
Feulll.-Feuillee (Louis), the companion and plagiarist of $\mathrm{Plu}$ mier, born 1660, died 1732 .

"Journal d'Observations faites sur les Côtes Orientales de l'Amérique ;" 2 vols. 4to. Paris, 1714.

Journal, \&c., in New Spain and the islands of America, 1 vol. 4to. Paris, 1725 .

Fitch. and Moll.-Fitchel (Leopold de), a naturalist of Vienna, who in conjunction with J. P. C. De Mole, member of the Academy of Munich, published the

"Testacea Microscopica, aliaque mínuta ex generibus Argonauta et Nautilus," cum tab. XXIV. Vienna, 1803.

Fisch.-Fischer de Waldheim (Gotthelf), a German naturalist, Director of the Imperial Museum at Moscow. Of his numerous works, we quote the following :

"Fragments of Natural History," in German, 1 vol. 4to. Frankfort, 1801.

"Anatomy of the Makis," in German. Frankfort, 1804.

"Description of certain Insects," published in the Memoirs of the Naturalists of Moscow, l vol. 4to. Moscow. 1806.

"Entomographia Imperii Russici," 2 vols. 4to. with splendid engravings. Moscow, 1820 - 1822

"Observations on a carnivorous Fly called Medeterus," 4to. with plates. Moscow, 1819.

"Memoir or the Argas of Persia," 4to. with a plate. Moscow, 1823.

"Letter on the Physodactylus, a new genus of Coleopterous Insects," 8vo. Moscow, 1824.

Fitzing.-Fitzinger, a physician and naturalist at Vienna.

"A New Classification of Reptiles according in their national affinities," 4to. in German. Vienna, 1826.

Fleming (John), a Scotch pastor.

"Philosophy of Zoology," 2 vols. 8 vo. Edinburgh, 1822.

Fleuriau de Bellevue, a naturalist at Rochelle.

Author of Memoirs on the Testacea and other Mollusca, published in the Bulletin des Sciences, Journal de Physique, \&c.

Forsk.-Forskahe (Peter), a Swedish naturalist, born 1734, a pupil of Linnæus, and the companion of Niebuhr in his travels to the East, died during the journey in $\mathbf{1 7 6 3 .}$

"Descriptiones Animalium, \&c., quæ in Itinere Orientali observavit," 4to. Copenhagen, 1775.

"Icones Rerum Naturalium quas in Itinere Orientali depingi curavit," 4to. Copenhagen, 1776.

Posthumous work, and extremely precious on account of the new species described in them, although the nomenclature is incorrect.

Fortis (J. B. or Alberto), an Italian naturalist, born at Venice 1740 , died a Bookseller at Bologna 1803. I quote his

"Mémoires pour servir à l'Histoire Naturelle et principalement à l'Orictographie de l'Italie," 2 vols. 8vo. París, 1802

Forst.-Forster (John Reinhold), born at Dirchaw in Polish Prussia 1729, naturalist in the English service for the second voyage of Cook, and subsequently Professor at Halle. He died in 1798.

"Zoologiæ Indicæ Rarioris Spicilegium," 4to. London, 1790.

"Enchiridion Historiæ Naturali inserviens," 8vo. Halle, 1788.

I also quote him for the articles inserted by Bloch in his posthumous System of Fishes.

Fourcroy (Antoine Francois de), the celebrated Professor of Chemistry, Counsellor of State, and member of the Académie des Sciences; born 1755 , died 1809 . The only work we have had occasion to quote is his

" Entomologia Parisiensis," 2 vols. 8vo. Paris, 1785, a small work of his youth, and a mere abridgment of that of Geoffroy.

Fred. Cuv. See Cuv. 
Freminv.-Freminville (Baron de), an officer of the French navy; an able naturalist.

Author of various articles in the Dictionnaire Classique d'Histoire Naturelle.

Fries (B. F).

"Monographia Tanyporum Sueciæ," Lundæ, 1823.

FR.-Frisch (J. L.), Rector of the Gymnasium of Berlin, born 1666, died 1743 .

"A representation of certain German and foreign Birds" (in German) 2 vols. folio, Berlin, 1739-1763, containing two hundred and fifty-five extremely exact, but not fine plates.

"Beschreibung von Insecten in Teuschland," or a Description of the Insects in Germany, 1 vol. 4to. Berlin, 1730 .

Froel.-Froelich (J. A.), a German naturalist and physician of Elwangen.

Author of two Mcmoirs on the Intestinal Worms in the Naturforscher.

GerT.-Gertner (Joseph), a celebrated botanist of Wirtemberg, born 1732 , died 1791 .

Author of the Carpologia, and also in his youth of Zoological observations inserted in the Philosophical Transactions, and in the Miscellanea Zoologica of Pallas.

Gaillardot, a physician at Luneville, and an able naturalist.

Author of Memoirs on Fossils, published in the Annales des Sciences Naturelles, \&c.

Garden (Alexander), a Scotchman, physician at Charleston, South Carolina, born 1730, died 1771.

He transmitted various observations to Linnæus.

Gaza (Theodore de), a Greek who sought an asylum in Italy in the sixteenth century. He translated into Latin the work of Aristotle upon Animals.

Geb.-Gebler (F.), a Russian naturalist and physician.

"Observationes Entomologicæ," a Memoir in 4to.
Deg.-Geer (Charles, Baron de), Marshal of the court of the Queen of Sweden, and member o the Academy of Stockholm, born 1720 , died 1778 .

"Mémoires pour servir à l'Histoire des Inscetes," 7 vols. 4to. with plates. Stockholm, 1752-1778. An excellent work, that forms a sequel to that of Reaumur. The first two volumes are rare. Retzius has given an abridgment of this work in Latin. entitled,

"Genera et Specics Insectorum," I vol. 4to. Lipsiæ, 1783.

There is also a German translation of it, enlarged by Goez.

Geoff.-Geoffrox, a celebrated physician of Paris.

"Histoire abrégée des Inscctes," 2 vols. 8vo. with plates. Paris, 1764.

This very elementary work has been republished, and augmented by Species added to it, by Fourcroy in his abridgment of the same. See FourCROY.

"Traité sommaire des Coquilles tant Fluviatiles que Terrestres, qui se trouvent aux environs de Paris," 1 vol. $12 \mathrm{mo}$. Paris, 1767.

A small work, but remarkable for the attempt to class shells according to their animal.

Geoff.-Geofrroy Saint-HiLaIre (Etienne), Professor of the Museum d'Histoire Naturelle, and member of the Académie des Sciences, born at Etampes 1773.

I quote his numerous Memoirs published in the Magasin Encyclopedique, the Annales du Museum, and $n$ the great work on Egypt.

Various Memoirs on the organization of the Crustacea and Insects, published in different periodicals, such as the Journal Complementaire des Sciences Medicales, Mémoires du Muséum d'Histoire Naturelle, \&c., and his

"Philosophie Anatomique," 2 vols. Paris, 1818 and 1822.

Isid. Geoff.-Geoffroy SaintHilaire (Isidore), son of the preceding, assistant naturalist of the Museum of Natural History.

Author of various memoirs among those of the Muséum d'Histoire Naturelle, Q q 2 
and the Annales des Sciences Naturelles; also of the description of the Fishes of Egypt in the great work on that country.

Geor.-Georgi (J. T.), a German naturalist, who travelled in the service of the Russian government in 1772, 1773, and 1774 .

His travels are printed in German, 2 vols. 4to. Petersburg, 1775.

Gramar (Ernest Frederick), a German naturalist.

"Dissertatio sistens Bombycum Species," \&c., 4to. Halle.

He continues the "Magazin für Insectenkunde," of Illiger.

Gm.-Germar (E. Francis), Professor of Mineralogy at Halle.

"Magazin der Entomologie," 4 vols. 8vo. Halle, 1813-1821.

"Insectorum Species Novæ," first vol. 8vo. with plates. Halle, 1824. See AHrens.

Gesn.-Gesner (Conrad), a physician at Zurich, born 1516, died 1565 .

I quote his "History of Animals," 3 vols. folio, to which has been added a Treatise on Serpents, and one on the Scorpion. This work, which is arranged alphabetically, is an excellent compilation of all the knowledge of the ancients, and is enriched with useful observations, and numerous wood cuts, most of them good. ist.

Gilliams, an American natural-

Author of certain Memoirs on Reptiles and Fishes, published in the Journal of the Academy of Natural Sciences of Philadelphia.

Gioen I (Giuseppe), a Sicilian naturalist of the house of Angio.

"Description of a new family and of a new genus of the Testacea," \&c., in Italian, pamphlet, 8vo. Naples, 1783.

It is the stomach of the Bulla lignaria, which he has converted into an animal.

Giorna (M. P.), a Piedmontese naturalist, professor at Turin, born 1741 , died 1809 .

I quote some of his Memoirs, published among lthose of the Academy of Turin。
Gmelin (Samuel Theophilus), born at Tubingen 1743, a German naturalist and traveller in the service of Russia, from 1768 to 1774 , at which period he died in Persia.

His travels were published in German, 4 vols. 4to. Petersburg, $1770-1784$. They abound in valuable articles on Natural History.

Gm.-Gmelin (John Frederic), Professor of Chemistry at Gottingen, born at Tubingen in 1748, died in 18-.

The author of the thirteenth and last edition of the "Systema Naturæ" of Linnæus. His work, notwithstanding the ignorance of things, want of judgment, and crudity that it exhibits, is still necessary, as being the only tolerably complete account of what had been done down to 1790 .

Godart (J. B.), Chief of the Lyceum of Bonn under the Imperial Regime, died 1825.

Editor of the article "Papillon" of the Encyclopédie Méthodique.

" Histoire Naturelle des Lepidoptères ou Papillons de France," 5 (first) vols. $8 \mathrm{vo}$., commenced in 1822 .

Goetz. or rather Goez.-Goeze (I. A. E.), pastor of Quedlimbourg, one of the principal writers on the Intestinal Worms, born 1731, died 1793.

"Natural History of Intestinal Worms" (in German), 1 vol. 4to. Brackenberg, 1782 .

Goldfuss (G. A.), Professor at Bonn.

"A Manual of Zoology," 2 vols. 8vo. Nuremberg, 1820.

Author of various memoirs published among those of the Academia Naturæ Curiosorum.

Goran (Antoine), Professor at Montpellier.

"Historia Piscium," I vol. 4to. Strasburg, 1770.

Of the numerous works published by this learned naturalist, the above is the only one we have had occasion to quote. Strictly speaking, it is a mere description of genera, but drawn up in 
detail and in technical terms, in the manner of Liunæus. It is preceded by a sort of Ichthyological Philosophy.

Grav.-Gravenhorst (J. L. C.), member of the Physical Society of Gottingen, \&c.

"Coleoptera Microptera Brunsvicensia," \&c., 1 vol. 8vo. Brunsvigæ, 1802.

"Monographia Coleopterorum Micropterorum," 1 vol. 8vo. Gottingæ, 1806.

"Nosography of the genus Ichneumon," 1 vol. (the first) 8vo. with plates, 1814.

"Monographia Ichneumonum Pedemontanæ Regionis," forming part of the twenty-fourth volume of the Memoirs of the Academy of Sciences of Turin.

"A Monograph of apterous Ichneumons," I vol. 8vo. with plates.

The description of a new genus, Helwigia, of the same tribe, an extract of which has been published in the Bulletin Universel of Baron de Feritssac.

"Conspectus Generum et Familiarum Ichneumonidum, auctoribus J. L. C. Gravenhorst et C. G. Neg. ab Esenbeck," 4to.

Gray (J. E.), an English naturalist attached to the British $\mathrm{Mu}$ seum.

Author of Memoirs on Reptiles, in the Annals of Philosophy, 1825, and the Philosophical Magazine, 1827.

Grew (Nehemiah), celebrated for his discoveries in his "Vegetable Physiology," Secretary of the Royal Society of London, died 1711. I sometimes quote his

"Museum Regalis Societatis," folio. London, 1681.

\section{Gronov.-Gronovius (John Frederick). \\ Author of various Memoirs on Fishes, published among those of various learned bodies, the Philosophical Transactions in particular.}

Gronov.-Gronovius (L. Theodore), a municipal officer of Leyden, nephew of the preceding, born 1730 , died 1.777 .

"Museum Ichthyologicum," 1 vol. folio. Leyden, 1754.

"Zoophylacium Gronovianum," I vol. folio. Leyden, 1765-1787.
Grundeer (G. A.), painter and engraver at Halle.

Quoted for a Memoir in the Naturforscher.

Gualt.-Gualtieri (N.), physician at Florence, previously a Professor at Pisa.

"Index Testarum Conchyliorum quæ adservantur in Museo R. Gualteri," folio. Florence, 1742.

The figures are numerous and exact.

Guer.-Guerin (F. E.), member of the Société d'Histoire Naturelle.

A Memoir on a dipterous Insect of the genus Boletophila, published in the tenth volume of the Annales des Sciences Naturelles.

A second on the Eurypode, a new genus of the Crustacea, in the sixteenth volume of the Memoirs du Museum d'Histoire Naturelle.

A third on a new genus, Themisto, of the same class, in the fourth volume of the Mem. d'Hist. Nat.

"Iconographie du Règne Animal," 4to. 1829. Ten numbers have already been published.

He edited many of the articles relative to Insects in the Encyclopédie Méthodique, and gave the explanations of the plates, relative to those animals, of the same work.

Guldenst.-Guldenstedt (J. A.), of Riga, born 1745, and died at St. Petersburg in 1781; traveller in the service of Russia from 1768 to 1775 .

His travels were published in German, 2 vols. 4to. Petersburg, 17871791.

We also quote several of his Memoirs, published among those of the Academy of Petersburg.

Guild.-Lansdown Guilding。

"Natural History of the Lamia amputator." Linnæan Transactions, vol. XIII.

Gunner (J. E.), bishop of Drontheim in Norway, born 1781, died 1773.

We quote certain Memoirs published among those of the Society of Drontheim, and of the Society of Sciences of Copenhagen. 
Gyllenh.-Gyllenhal (L.), a Swedish naturalist. We quote the fourth part of the first volume of his

"Insecta Suecica," I vol. 8vo. Lipsiæ, 1827.

HaAn (William de), Curator of the Royal Museum of the Netherlands, at Leyden.

"Mornographiæ Ammoniteorum et Goniatiteorum Specimen," 8vo. Leyden, 1825 .

Hagenb. - Hagenbach (J. J.), one of the Curators of the Royal Museum of Leyden, died 1826.

"Mormolyce Novum Genus," 1 vol. 8vo. with a plate. Nurembergæ, 1825.

Hamm.-Hammel (A. D.)

"Entomological Essays," No. 1-6. 8vo. Petersburg, 1821-1827.

"Observations on the Blatta germanica," 8vo. Petersburg, 1821 .

Hammer (L. F.), Professor of Natural History at Strasburg, sonin-law of the late Hermann.

We quote his memoir on the American Ostrich, published in the Annales du Museum.

Hardw.-Hardwicke (T.), an English general who resided in India.

I quote several of his papers from the Linnæan Transactions.

Harlan (Richard), an American naturalist and physician, Professor of Comparative Anatomy at Philadelphia.

"Fauna Borealis Americana," 1 vol. 8vo. Philadelphia, 1825, a work which contains the history of the quadrupeds of that country.

He has also published various interesting Memoirs among those of the Lyceum of New York and of the Academy of Natural Sciences of Philadelphia.

Harris (G. P.), an English naturalist.

I quote his description of two new species of Didelphis, inserted in the Linnæan Transactions, vol. IX.
Harr. - Harris (Moses), an English painter.

"An Exposition of English Insects," in French and English, 1 vol. 4to, with coloured plates. London, 1781.

Hart.-Hartmann, painter and engraver of subjects of Natural His . tory at St. Gall.

Author of a System of the terrestrial and fluviatile Testacea of Switzerland.

Hasselq.-Hasselquist (Fred.) a Swedish naturalist, one of the first pupils of Linnæus, born 1722, died 1752 .

His Travels in the East were published by Linnæus in the Swedish language, with Latin descriptions of the animals and plants. Stockholm, 17-.

There is a French translation without the descriptions, 1 vol. $12 \mathrm{mo}$. Paris, 1769

Hasselt (J. C. Van), a young Hollander, physician, and naturalist, the friend and companion of Kulh, whom he survived but a few months.

Hegetsch. - Hegetschweiler (J. J.), a naturalist of Switzerland.

"Dissertatio Inauguralis Zootomica de Insectorum Genitalibus," 1 vol. 4to. Turici, 1820.

\section{Helw.-Helwigg (J. C. L.)}

"Fauna Etrusca, \&c. Petrii Rossii, iterum edita et annotatis perpetuis aucta," 1 vol. 8vo. Helmstadii, 1755. See Illiger.

Herbst (J. F. W.), a preacher at Berlin, born 1743 .

"Natursystem aller Bekanten in und Auslændischen Insekten," \&c. von Carl. Gustaf Jablousky forgesetz, von J. F. W. Herbst, 10 vols. 8vo. with an atlas of coloured plates to each volume. Berlin, 1785, et seq. It is a treatise on the Coleoptera.

"Versuch einer Naturgeschichte der Kraben und Krebse," 3 vols. 4to. with sixty-two coloured plates. Berlin, 1790-1803. A treatise on the Crustacea, and a useful compilation containing several new figures.

"Natursystem der ungeflugelten Insecten" (the genera Solpuga, Tarentula, 
and Phalangium), 1 vol. 4 to. with coloured plates. Berlin, 1797.

"Natursystem der ungeflugelten Insekten" (genus Scorpio), l vol. 8vo. Berlin, 1708 .

"Archiv der Insecten Geschichte, Herausgegeben," von J. Casp. Fuesly, I vol. 4to. with coloured plates. Zurich and Wintertkar, 1791. This work has been translated into French.

He has also published a Monograph of the Linnæan genus Papilio, representing all the species, but as most of the figures are copies, I have not quoted it.

Herm.-Hermann (John), Professor at Strasbourg, a laborious and erudite naturalist, born 1738 , died 1800.

"Tabula Affinitatum Animalium," 1 vol. 4to. Strasbourg, 1783.

"Observationes Zoologicæ Posthumæ," 1 vol. 4to. Strasbourg and Paris, 1804.

Herm.-Hermann (J. F.), son of the preceding, born in 1768, died before his father, 1793.

He has left an "Apterological Memoir," 1 vol. fol. Strasbourg, 1804

Hoev.-VAn Der Hoeven (John) Professor at Leyden.

Author of a "Manual of Zoology," in Dutch, 2 vols. 8vo. Delft, 1827; of a "Thesis de Sceleto Piscium," 8vo. Leyden, 1822; and of a Memoir on the Ornithorhynchus, \&c.

Hoffman.-Hofmansegg (C.), a learned naturalist of Saxony, and a zealous protector of the sciences.

Author of various Memoirs on the animals of the Brazils and Portugal.

Holten, a Danish naturalist.

Quoted as author of a Memoir published in the fifth volume of the Soc. of $\mathrm{Na}$ tural History of Copenhagen.

Home (Sir Everard), the cele.. brated surgeon, curator of the Hunterian Museum, at London, and fellow of the Royal Society.

"Lectures on Comparative Anatomy," 6 vols. 4to. London, 1814-1828.

I also quote several of his Memoirs published in the Philosophical Transactions.
Hopp.-Hoppe (D. H.), druggist at Ratisbon.

"Enumeratio Insectorum Elytratorum Indigenorum," 1 vol. 4to. with coloured plates. Erlangæ, 1795. It is a useful work in studying the Donaciæ.

Horns.-Hornstedt, a Swede who travelled in Java.

Quoted for a Memoir on the Acrochordus, published among those of Stockholm, 1787.

Horsf.-Horsfiecd (Dr. Thomas), an English naturalist.

"Zoological Researches in Java and the neighbouring Islands," 4to. with excellent plates. London, 1825.

He also published the first number of a "Descriptive Catalogue of the Lepidoptera in the Museum of the East India Company," 4to. London, 1828.

\section{Houtr.-Houttuyn (Martin).}

Author of certain Memoirs among those of the Academy of Haarlem; of a Dutch translation taken from the Systema of Linnæus, \&c. He is also the continuer of Noseman's History of the Birds of the Netherlands.

Hub.-Huber (Francis), corresponding member of the Academy of Sciences of Geneva. Deprived of sight, but still a most perspicacious observer.

"Nouvelles Observations sur les Abeilles," 2 vols. 8vo. with plates. Paris and Geneva, 1814. The second volume is from the pen of his son.

Hub.-Huber (Peter), son of the preceding.

"Recherches sur les mœurs des Fourmis Indigénes," 1 vol. 8vo. with plates. Paris and Geneva, 1810.

"Observations sur les Bourdons," published in the sixth volume of the Transactions de la Société Linnéenne.

Hubn.-Hubner (J.), painter at Augsburg.

His Iconographic work on the Lepidoptera of Europe is the most complete and perfect of the kind hitherto published. The text is in the German language. He is about to give us the 
Exotic Lepidoptera, of which several plates have already appeared; the whole number, in royal 8vo. will amount to near a thousand.

Humb.-Humboldt (Alexander de), member of the Académie des Sciences, of the Academy of Berlin, \&c. \&c., born 1769 .

Of the works of this illustrious savant I chiefly quote the

"Observations de Zoologie et d'Anatomie Comparée," of which there are already published fourteen numbers, 4to. Paris, $1811-1827$.

Hunter (John), the celebrated Scotch surgeon who settled in London, born 1728, died 1793.

I quote his Treatise on the teeth, and various Memoirs inserted in the Philosophical Transactions.

Huzard, Jun., who in conjunction with M. Pelletier published

"Recherches sur le genre Hirudo." Paris, 1825.

Illig.-IILliger (J. C. G.), Professor at Berlin; he died young.

"Prodromus Systematis Mammalium et Avium," 1 vol. 8vo. Berlin, 1811. A work remarkable for the precision which the author endeavours to give to the genera of these two classes, and for the elegance of its nomenclature.

"Verzeichniss der Kofer Preussens," or a Catalogue of Prussian Insects, a work commenced by Theophilus Kugelann, and terminated by I. Illiger, 1 vol. 8vo. Hall., 1798.

"Magasin far" Insectenkunde," 7 vols. 8vo. Brunswick, 1801-1807.

"Systematisches Verzeichniss von den Schmetterlingen der Wiener Gegend," 2 vols. 8vo. Brunswick, 1801. It is a new edition of the "Systematic Catalogue of the Lepidoptera of the Environs of Vienna."

Hehas also continued the "Fauna Etrusca" of Rossi, commenced by Helwigg, vol. ii. 8vo. Helmstadii, 1807.

\section{ITTIOL.VERON.-ItTIOLITOLOGIA VERONESE.}

A great work on the Petrified Fishes of Mount Bolca, where, notwithstanding its magnificence, they are neither faithfully delineated nor well characterized.
JACQ.-JACQUIN (N. J. de), a celebrated botanist and professor at Vienna, born at Leyden, 1727, died in 18 -

"Miscellanea Austriaca," 2 vols. 4to. Vienna, 1778, 1781. It contains some observations relative to animals.

$J_{A C Q}-J_{A C Q U i N}$ (J. F. de), son of the preceding.

The author of " Materials for a History of Birds" (in German), 1 vol. 4to. which contains some figures of rare birds. Vienna, 1784.

Johns.-Johnson (J. Rawlins), an English naturalist.

"A Treatise on the Medicinal Leach," 8vo. London, 1816, and the second part, Ibid. 1825 .

"Observations on the genus Planaria," Philosophical Transactions, 1822, and continued in 1825 .

Jour. d'Hist. Nat.

By this title we designate a periodical work, of which only two volumes 8vo. were published, and which were united under the name of "Choix de Mémoires sur divers objets d'Histoire $\mathrm{Na}$ turelle, par MM. Lamarck, Brugières Olivier, Haüy et Pelletier." Paris, 1792.

\section{Journ. de Phys.}

Under this appellation I quote the " $\mathrm{Ob}$ servations sur la Physique, l'Histoire Naturelle et les Arts," of which 2 vols. appeared annually, from 1773 to 1823 : at first under the direction of the Abbé Rozier, then under that of Lamatherie, physician and adjunct professor in the Collége de France, and finally under the superintendence of M. de Blainville.

Jurine (Louis), Professor of Anatomy and Surgery at Geneva.

"Nouvelle méthode de classer les Hyménoptères at les Diptères," with plates, Hymenopteres, vol. 1. 4to. Geneva, 1807; a very superior work, and indispensable for the study of this order.

"Observations sur le Zenos vesparum," a memoir in 4to. with one plate, 1816 .

"Observations sur les ailes des Hyménoptères," a memoir, with plates, published in the twenty-fourth volume of the Memoirs of the Academy of Sciences of Turin. 
"Histoire des Monocles," 1 vol. 4to. with plates. Geneva, 1820 .

His second son, whose demise is to be regretted, has published in the seventh volume of the Annales du Muséum, \&c., an excellent Memoir on the Argulus foliaceus. See Argulus in this work.

KAMPF.—K AEMPER (Engilbert), a German physician, who travelled in Persia, India, and Japan, born at Lemgo, in the county of Lippe in 1651 , died 1713 .

"Amœnitatum Exoticarum," faseic. V. 4to. Lemgo, 1712.

"A Description of Japan" in German, translated into French under the title of " Histoire Naturelle, Civile et Ecclésiastique du Japan," 2 vols. folio. La Haye, 1729.

KAUP, a German naturalist.

Author of the notes on Reptiles in the Isis of Oken.

Kirb.-Kirby (Wm.), an English clergyman, member of the Linnæan Society, rector of Barham, in the county of Suffolk, \&c.

"Monographia Apum Angliæ," 2 vols. 8vo. with plates. Ipswich, 1802 .

He has published, in the ninth volume of the Transactions of the Linnæan Society, a Monograph of the Bees of England, and in the eleventh, that of the Strepsiptera.

He has lately, in conjunction with $\mathrm{M}$. Spence, published a new edition of the "Introduction to Entomology," 4 vols. 8 vo. with plates. London, 1828.

He has also written several Memoirs on various Insects, for the Linnæan Transactions, and the Zoological Journal, most of which we have quoted.

Kleem.-Kleeman (C. F. C.), a painter at Nuremberg, born 1735 , died 1789.

"Beytræge zur Natur Oder Insectengeschichte," 1 vol. 4to. Nüremberg, 1761. A supplement to the work of Røesel his father-in-law, forming the fifth volume.

KL.-KLeIN (J. T.), Secretary of the Senate of Dantzick, a laborious author who has written on every branch of natural history, but without taste or genius ; born in 1685 , died 1759 .

Cuv. Table of Authors.
"Summa Dubiorum circa classes Quadrupedum et Amphibiorum Linnæi," 1743.

"Quadrupedum Dispositio et brevis Historia Naturalis," 1751.

"Historiæ Avium Prodromus," 1750.

"Stemmata Avium," 1759.

"Tentamen Herpetologiæ," 1755.

"Historiæ Naturalis Piscium promovendæ missus," V. 1740-1749.

"Mantissa Ichthyologica," 1746.

"Methodus Ostracologica," 1753.

"Descriptiones Tubulorum Marinorum," 1737.

"Naturalis Dispositio Echinodermatum," 1734.

KLUG.-KLUG (Francis), physician at Berlin.

"MonographiaSiricum Germaniæ, atque Gencrum illis Adnumeratorum, eum tabulis æneis coloratis VIII." 1 vol. 4to. Berlin, 1803.

Various Memoirs on different genera or species of Hymenoptera published among those of the Society of Naturalists of Berlin.

"A Critical Review of the Genera of Fabricius derived from that of Apis, Lin." in the Entomological Magazine of Illiger, 1807.

"Entomologische Monographien," I vol. 8vo. with plates. Berlin, 1824.

"Proscopia, Novum Genus Insectorum Orthopterorum," folio, with 2 plates.

"Entomologiæ Brasilianæ Specimen."

And several Monographs in the German language.

KNoch (A. G.)

"Neue Beytraegc zur Insectenkunde," 1 vol. 8vo. with plates. Leipsic, 1801.

KNORR and WALCh, or KNORR, \&c., or Walch Petrif. of Knorr.

KnoRR (George Wolgang), an engraver at Nuremberg, born 1705 , died 1761 , and

WALCh (J. E. E.), Professor at Jena, jointly published a work called the

"Collection of the Monuments of the revolutions experienced by the terrestrial globe, containing petrifactions, \&c. 4 vols. Nuremberg, 1775-1778.

I quote them in relation to certain Testacea and Lithophyta.

I also quote, under the title of KNORR Vergn., or KNorr Delic., a work of the same engraver, editions of which are to be found in various languages, styled in German, Vergnïgungen, \&e. Delicix, in Latin, and in French, Amuscments des yeux et de l'esprit, or Collection de Coquillages,

R r 
\&c. 6 vols. 4 to. Nuremberg, 1760 , 1773.

\section{Køhl.-Køhlreuter (J. G.)}

We quote several of his Memoirs, inserted in the Nov. Comment., Acad. Petrop.

\section{Krusenstern, a Russian admi- ral,}

Whose voyage round the world contains several observations relative to Natural History by Tilesius.

KuнL (Henry), a young naturalist of Hanau, born in 1797, who died at Batavia, where he was engaged in collecting for the Museum of the Netherlands, in company with a young Hollander named Van Hasselt. Their collections were immense, and included all the classes. Kuhl has left us in German

" Materials for Zoology and Comparative Anatomy, Monographs of the Parrots, Petrels, Bats of Germany," \&c.

Lac. or Lacep.-Lacepede (B. G. E. de la Ville Courte de), Professor of the Muséum d'Hist. Nat., member of the Académie des Sciences, \&c. \&c. born at Agen.

I have frequently quoted his three principal works, which form a sequel to the great "Histoire Naturelle" of Buffon.

" Histoire Naturelle, Générale et Particulière des Quadrupèdes Ovipares et des Serpents," 2 vols. 4to. Paris, $1788-1789$.

"Histoire Naturelle, \&c. des Poissons," 5 vols. 4to. Paris, 1798-1803.

"Histoire Naturelle, \&c. des Cetacés," 1 vol. 4to. Paris, 1804.

Also certain Memoirs in the Annales du Muséum.

LAET (Jean de), a geographer of Antwerp in the seventeenth century.

"Novus Orbis, seu Descriptionis Indiæ Occidentalis," lib. XVIII. 1 vol. folio. Leyden, 1633.

Laich. - Laicharting (J. N. de), Professor at Inspruck, born 1757.

"Verzeichniss der Tyroler Insecten," with plates, 2 vols. 8 vo. Zurich, 1781 -1784 .
Lam.-Lamarck (Jean-Baptiste De Monnet, Chevalier de), Professor of the Muséum d'Hist. Nat., and member of the Academie des Sciences, born at Basentin, in Picardie in 1743 ; died at Paris in December 1829 .

Of the numerous works of this celebrated naturalist I have chiefly quoted the

"Système des Animaux sans vertèbres," 1 vol. 8vo. Paris, 1801 .

"Extrait du Cours de Zoologie sur les Animaux sans vertèbres," 8vo. Paris, 1812.

" Histoire Naturelle des Animaux sans vertèbres," 7 vols. 8vo. Paris, 18151822 .

"Mémoires sur les Coquilles," published in the Annales du Muséum.

The author having become blind during the publication of this work, was aided, in the Bivalves, by M. Valenciennes, and in the following classes, by Mademoiselle Lamarck, his eldest daughter.

Lamartiniere, a French naturalist, one of the unfortunate companions of La Peyrouse.

Quoted for a Memoir on some parasitical animals, published in the Journal de Physique for 1787, and at the end of the Voyage de la Peyrouse.

Lambert, an English naturalist.

Author of Memoir on the Bos Frontalis, in the seventh volume of the Linnæan Transactions.

Lamour. - Lamouroux (J. V. F.), a naturalist of Agen, Professor at Caer..

Quoted for certain Memoirs in the Annales du Muséum, and for a "Histoire des Polypiers," of which I saw a part in MS., at the time of my first edition. It was published in 1 vol. 8 vo. 1817

"Exposition Méthodique de l'ordre des Polypiers," with the plates of Ellis and Solander, and some new ones, 1 vol. 4to. Paris, 1821.

"Dictionraire des Zoophytes," forming part of the Encyclopédie Méthodique, 4to. Paris, 1824.

LANGsd. - LANGSdorf, a German naturalist who accompanied Admiral Krusenstern, and established himself at Brazil. 
Author of certain Memoirs, and quoted as having given names to the various objects he discovered.

Lapeyr.-Lapeyrouse(Philippe Picot, Baron de), Professor of Natural History at Toulouse.

"Description de plusieurs espèces d'Orthoceratites et d'Ostracites," I vol. folio, Nuremb. 1781.

I also quote certain articles written by him for the Dictionnaire des Oiseaux of the Encyclopédie Méthodique.

Laroche (De), a young physician of Paris, prematurely snatched from the sciences by death.

Author of Memoirs in the Annales du Muséum, and one in particular, Sur les Poissons d'Ivica, in the thirteenth volume of that collection.

Lasp.-Lasperres (J。 H.), a municipal officer of Berlin.

"Sesiæ Europeæ, Iconibus et Descriptionibus illustratæ," 1 vol. 4to. Berlin, 1801.

"Critical observations on the Systematic Catalogue of the Lepidoptera of the Environs of Vienna," inserted in the Magazin für Insecktengunde of Illiger, \&c.

Lath.-Latham (John), Fellow of the Royal Society, born 1740 .

This author has enriched the science of Ornithology, in particular, with new and beautiful species, but his works, which are not written with critical accuracy, should be read with caution.

"A General Synopsis of Birds," 3 vols. 4 to. and two Supplements. London, 1782 , et seq.

"Index Omithologicus," 2 vols. 4to. London, 1790.

Lat. - Latreille (Pierre-André), Professor of the Muséum d' Histoire Naturelle, member of the Académie des Sciences, \&c. born at Brives, in 1762 .

"Histoire Naturelle des Salamandres," 1 vol. 8vo, with plates. Paris, 1800.

"Histoire Naturelle des Reptiles," forming a sequel to Deterville's Buffon, 4 vols. 12 mo. with plates.

"Précis des Caracteres Génériques des Insectes," I vol. 8vo. Brives, 1796.

"Genera Crustaceorum et Insectorum," 4 vols. 8vo. with plates. Paris, 1806 -1807 .
"Histoire Naturelle des Crustacés et des Insectes," forming a sequel to Sonnini's edition of Buffon, 14 vols 8vo. with plates. "Paris, $1802-1805$.

"Histoire Naturelle des Fourmis," 1 vol. 8vo. with plates. Paris, ]802.

His Memoirs inserted in the Annales du Muséum, \&c.

The entomological portion (partly written by him) of the Nouveau Dictionnaire d'Histoire Naturelle, and of the Encyclopédie Méthodique, and the whole of the same part in the Observations de Zoologie et d'Anatomie Comparée, or the second part of the Travels of Messrs. de Humboldt and Aimé Bompland.

"Mémoires de la Soc. d'Histoire Naturelle de Paris," 4to.

"Esquisse d'une Distribution Générale du Règne Animal," l vol. 8vo. Paris, 1824.

"Familles Naturelles du Règne Animal," 1 vol. 8vo. Paris, 1825.

Various general Memoirs on Insects, published among those of Mus. d' Hist. Nat.

The description of the Insects collected by Caillaud in his travels in Nubia, which forms part of this Narrative.

The Entomological portion of the second edition of the Nouveau Dictionnaire d'Histoire Naturelle, and various articles of the Dictionnaire Classique d'Histoire Naturelle, as well as those relative to the same subject, of the Encyclopédie Méthodique.

The description (Ann. der Sc. Gener.), of a new genus of Spider.

Laur. or Laurent.-LaurenTiNi (J. N.), a physician of Vienna.

"Specimen medicum exhibens Synopsin Reptilium emendatum," I vol. 8vo. Vienna, 1768.

This thesis is said to have been written by Winterl, since celebrated as a paradoxical chemist.

Leach (W. E.), an English physician and naturalist, one of the $\mathrm{Cu}$ rators of the British Museum.

A Monograph of the genus Meloe, with plates, inserted in the Transactions of the Linnæan Society.

"MalacostracaPodophthalma Britanniæ," 4to. with fine coloured plates. London, 1815, 1816. Eight numbers have been published.

" A General Arrangement of the Classes Crustacea, Myriapoda and Arachnides, constituting part of the eleventh voII 12 
lume of the Transactions of the Linnæan Society. An extract of this work is given in the Bulletin de lir Société Philomatique.

"On the Classification of the Natural Tribes of Insects, Notonectidea," published in the twelfth volume of the above mentioned Transactions.

"Description of some new genera and species of Animals discovered in Africa," by T. C. Bowdich, a half sheet in 4 to.

"Zoological Miscellany," 3 vols. 8vo. London, 1817.

-On the Genera and Species of Proboscideous Insects," I vol. 8vo. with plates. Edin. 1817.

"Appendix, No. 10, to a general notice of the animals taken by Mr. John Cranch, during the expedition to explore the source of the river Zaire," 4 to.

Various articles in the Dictionnaire des Sciences Naturelles, relative to the Crustacea, and Memoirs in the Linnæan Transactions.

Le CL.-Le Clerc, naturalist at Laval, author of

" Observations sur la corne du Psile de Bosc," presented to the Academie des Sciences, in 1815, and of other interesting observations.

Lec., or Le C.-Le Conte, an American naturalist, and officer in the service of the United States.

Author of various memoirs on Quadrupeds, Reptiles, \&c., published in the Journal of the Academy of Natural Sciences of Philadelphia.

Lefebv.-Lefebvre (Alexander), a French naturalist,

Has published in the Annales de la Société Linnéenne, a description of several new Insects taken by him in Sicily, and that of three Lepidoptera.

Legaut (François), a Protestant of Burgundy, who sought refuge in Holland.

"Voyages et Aventures de Fr. Leguat et de ses Compagnons," 2 vols. $12 \mathrm{mo}$. London, 1720., They contain good figures of various animals.

\section{LEISLER.}

Author of a Supplement to Bechstein's Birds of Germany. Hanau, 1812, 1813.

\section{LePel.-Lepelletier de Saint} Fargeau (Amédée), a naturalist of Paris. Author of

"Monographie des Chrysis des Environs de Paris," in the Ann. du Mus. d'Hist. Nat., No. 58.

"Mémoire sur les Araignées" in the Bulletin de la Société Philomathique, April 1813, No. 67.

"Monographia Tenthredinetarum Synonymia Extricata," 1 vol. 8vo. Paris, 1823.

Jointly with M. de Serville, of the article on Insects in the tenth volume of the Encyclopédie Méthodique.

$\mathrm{He}$ has communicated to the Académie des Sciences, Observations on the coition of different species of Volucella, a genus of Dipterous Insects.

Leske (N. G.), Professor at Leipsick, and subsequently at Marburg, born 1752, died 1786 .

"Museum Leskeanum, Regnum Animale," I vol. 8vo. with coloured plates. Lips. 1789.

I also quote him for his enlarged edition of "Klein's Treatise on the Echini," 1 vol. 4 to. Lips. 1778.

Less.-Lesson (R. P.), naturalist, jointly with M. Garnot, of Duperrey's Expedition in the Coquille.

These two naturalists have edited the Zoological part of the narrative of the above expedition; that part is not yet completed. M. Lesson is also the author of the

"Manuel de Mammalogie," 1 vol. 12 mo. Paris. 1827.

"Manuel d'Ornithologie," 2 vols. $12 \mathrm{mo}$. Paris, 1820.

"Manuel de l'Histoire des Mollusques et de leurs Coquilles," 2 vols. $12 \mathrm{mo}$. Paris, 1829. His

"Histoire des Oiseaux Mouches," with excellent plates, now being published.

Lesuer (C. A.), a French naturalist, from Havre.

One of the draughtsmen who accompanied Baudin, and one of Péron's most efficient and zealous co-operators in Zoological researches. $\mathrm{He}$ has published some Zoological Observations in the Bulletin des Sciences, and the prospectus of a great work on the Medusæ, accompanied by specimens of sevcral of the plates. $\mathrm{He}$ has also furnished various papers for 
the Journal of the Academy of Natural Sciences of Philadelphia, the Mémoires du Mus. d'Hist. Nat., \&c.

Leukard (F. S.), author of

"Zoological Fragments," Helmstadt, 1819.

The Mollusca of the Voyage of Ruppel.

Lew.-Lewins (J. W.), author of the

" Natural History of the Lepidopterous Insects of New South Wales," with coloured plates, 1 vol. 4to. London, 1805.

"Natural History of the Birds of New Holland."

Licht-Lichtenstein (A. A. H.), Professor of the Oriental Languages at Hamburg, born in 1753 .

A dissertation on the genus Mantis of Linnæus, in the sixth volume of the Linnæan Transactions.

Lichtenstein (H.), Professor at Berlin.

"Voyage to the Cape of Good Hope," 2 vols. 8 vo. Berlin, 1811.

Various Memoirs on the Antilopes, the genus Dipus, the Animals of Marcgrave, \&c. published among those of the Academy of Berlin.

Lindroth, a Swedish naturalist.

Author of a paper in the nineteenth volume of the New Stockholm Memoirs.

LINk (J.H.), a physician at Leipzic, born in 1674, died in 1734 .

"De Stellis Marinis, liber singularis," published by Christ. Gabr. Fischer, 1 vol. folio. Leipzic, 1733.

L. or Lin.-LinNæeus or LinNe (Charles de), Professor of Natural History at Upsal, and author of the great reform in the nomenclature of natural History. He was born in 1707 , and died in 1778. I quote his

"Systema Naturæ," particularly the tenth edition of 1757 ; the twelfth of 1766 ; and above all, the thirteenth edition, published by Gmelin, 7 vols. 8vo. Leipzic, 1788.

"Amønitates Academicæ," a collec- tion of theses, in 10 vols. $8 \mathrm{vo} .749$ et seq. -1790 .

"Museum Adolphi Frederici Regis," with thirty-three plates, 1 vol. folio. Stockholm, 1754.

The author himself, in his other works, quotes a second volume of this latter one; it is a small octavo.

"Musæum Ludovicæ Ulricæ Reginæ," 1 vol. 8vo. Stockholm, 1764 .

"Fauna Suecica," 1 vol. 8vo. first edition, 1746; second, 1761 ; the third by Retzius, Leipzic, 1800, only containing the Vertebrata.

Lin. Trans., or Trans. Lin. Soc., or Lin. Soc.

"Transactions of the Linnæan Society of London," 13 vols. 4to. London, 1791, et seq.

Lister (Martin), an English naturalist, and physician to Queen Anne, died 1711.

"Historia sive Synopsis Methodica Conchyliorum," with 1059 engravings, 1 vol. folio. London, 1689-1693.

There is another edition, with the synonymes of Linræus, published by William Huddesford. London, 1770.

" Historia Animalium Angliæ, de Araneis, de Cochleis, tum Terrestribus tum Fluviatilibus, de Cochleis "Marinis." London, 1678.

The part relating to the Spiders is also found in the "Historia Insectorum" of Ray.

Lyon.-Lyonnet (Peter), Interpreting Secretary to the United Provinces, born in 1707, died in 1789.

"Traité Anatomique de la Chenille du Saule," 4to. La Haye, 1762, with plates, engraved by the author, a work which is at once the masterpiece of engraving and anatomy.

MACL., or MAC L.-M Mac Leay (W. S.), of the Linnæan Society of London.

"Horæ Entomologicæ," 8vo. vol. 1st, in two parts, with plates. London, $1819,182]$.

"Annulosa Javanica," 4to., with plates. No. 1. London, 1825.

He has also published some general Memoirs on Insects, not referred to, however, in this work.

Maccar,-Maccari (P.), mem- 
ber of the Société de Medicine of Marseilles, \&c.

"Mémoire sur le Scorpion qui se trouve sur la Montaigne de Cette," \&c. 1 vol. 8vo. 1810.

MacQ.-Macquart (J.), member of the Société Royale des Sciences, d'Agriculture et des Arts of Lille.

A series of Memoirs on the "Insectes Diptères du Nord de la France," with plates representing their wings, published among those of the above Society, which form 4 vols. 8vo. with plates, Lille, 1826-1829.

Macr I (Zaverio), a Neapolitan naturalist.

" New Observations on the Pnlmo Marinus of the Ancients," in Italian, 1 vol. 8vo. Naples, 1778.

Mann.-Mannerheim (C. G.), counsellor to the Emperor of Russia.

"Eucnemis Insectorum genus," with two plates, 1 vol. 8vo. Petrop., 1823.

"Observations on the genus Megalopus," in the tenth volume of the Memoirs of the Imperial Academy of Sciences of St. Petersburg, 1824.

"Description of forty new species of Scarabæides from Brazil," with plates, 4 to.

Mantell (G.), member of the College of Surgeons of London resident at Lewes.

"Illustrations of the Geology of the County of Sussex," 2 vols. 4to. London, 1822, 1827.

MarcGr.-MarcGrav de Leibstadt (George), of Meissen in Saxony, a traveller in Brazil; born 1610 , died in Guinea, 1644 .

" Historiæ Rerum Naturalium Brasilæ," lib. 8, in fol. Leyden and Amsterdam, 1648. An excellent work for the times, full of cxact descriptions, and recognizable though rude figures of all kinds of animals.

Marsh.-Marsham, an English naturalist, Treasurer of the Linnæan Society, \&c.
"Entomologia Britannica, sistens Insecta Britanniæ Indigena, secundum methodum Linnæanum disposita," tom. I., Coleoptera. London, 1802.

"A monograph of the genus Notoclea" (Paropsis, Olivier), with plates, published in the ninth volume of the Transactions of the Linnæan Society.

Martens (Frederick), a surgeon at Hambourg.

" A Voyage to Spitzberg," in German, 1 vol. 4to. Hambourg, 1675.

It is useful with respect to animals of the Arctic Ocean.

Martens (George de), Secretary of the Supreme Court of Wirtemberg.

"A Voyage to Venice," 2 vols. 8vo. Ulm, 1824. It contains a Catalogue of the Fishes of that port.

Martini (F. H. G.), a physician of Berlin, born 1729, died 1778 . He commenced the great conchyliological work entitled the

"Systematic Cabinet of Shells," 10 vols. 4to. and 1 of Suppl., with coloured plates. Nuremberg.

The first three volumes, 1769-1777, are from his pen, the other from that of Chemnitz.

Mathiole (P. A.), of Sœna, born 1500, died 1577 .

In his Commentary on Dioscorides, he enters into details of various animals.

Maud.-Mauduit (R. J. E.), a physician at Paris, who died in 1792.

Author of the "Dictionnaire des Oiseaux" of the Encyclopédie Méthodique.

Maupert.-Maupertius (P. L. M. de), member of the Académie des Sciences, president of that of Berlin, \&c. born 1678, died 1759. An astronomer and geometrician, also author of certain Memoirs on Natural History.

"Expéricnces sur les Scorpions," in the Memoires de l'Acad. des Sciences, 1731 .

Maurice de Nassau (Prince), 
or rather Count John Maurice de Nassau-Siegen, born 1604, the Dutch governor of Brazil from 1637 to 1644 .

He encouraged the labours of Marcgrave in that country, and drew several fishes which have been engraved and published in the Ichthyology of Block. He died in the service of Brandebourg in 1679 .

Meckel (J. F.), Professor at Halle. We quote his

"Materials for Comparative Anatomy" (in German,) 8vo. Leipzic, 1808.

"A treatise on the Ornithorhynchus," folio. Leipzic, 1826.

\section{Meg.-Megerle de Muhl- FIELD (J. C.)}

Author of "A Classification of Bivalve Shells," inserted in the Magazine of the Society of the Friends of Nature of Berlin.

\section{Mehlis (Edward).}

"De Distomate Hepatico et Lanceolato," folio. Gottingen, 1825.

\section{Meig.-Meigen (J. G.), a Ger- man naturalist.}

This author has published (in German), a work on the "Diptera of Europe," now forming 5 vols. 8vo. accompanied with plates representing at least one species of each genus, with the details of their characters.

M. Baumauer published an extract from the same work, under the title of a " Nouvelle Classification des Mouches à deux ailes," 8vo. Paris, 1800.

Mem. de la Soc. D'Hist. Nat.

"Mémoires de la Société d'Histoire Naturelle de Paris," l vol. 8vo, 1799 , the only one that appeared.

There is another work with a similar title, in 3 vols. 4 to. 1823 , et seq.

Merian (M. S.), a German lady established in Holland, born 1647, died 1717. She has left us two posthumous works, remarkable for the beauty of the drawings:

"De Generatione et Metamorphosibus Insectorum Surinamensis," 1 vol. folio. The Hague, 1726.

" Histoire des Insectes d'Europe," trans- lated into French by Mairet, 1 vol. folio. Amsterdam, 1730.

Merr.-Merrem (Blaise), born at Bremen, Professor of Natural History at Marburg.

"Avium Rariorum et minus Cognitarum Icones et Descript.," four Nos. 4to. Leipzic, 1786.

"Materials for the Natural History of Reptiles" (in German), 2 Nos. 4to. Duisbourg and Lemgo, 1790. All that it contains relates to Serpents.

"Tentamen Systematis Amphibiorum," in Latin and German, 1 vol. 8vo. Marburg, 1820.

Mesnard. - Mesnard de la Groye, a naturalist of Angers, and my adjunct in the Collége de France, died in 1827.

Author of various Memoirs in the Annales du Museum, Journal de Physique, \&c.

\section{Meyer and Wolf.}

"Taschenbuch," \&c. or Almanack of the Birds of Germany, 2 vols. 8vo. Franckfort, 1810. The first volume contains the terrestrial birds by Wolf; the secoud the water-birds by Meyer. This work is filled with excellent observations.

Mig.-Miger (Felix), a naturalist at Paris.

"Mémoire sur les Larves des Insectes Coléoptères Aquatiques," inserted in the fourteenth volume of the Annales du Museum

Mik.-MikaU (I. C.), a Bohemian naturalist.

"Monographia Bombyliorum Bohemix," with plates, 8vo. Prague, 1796.

Miluer (J.S.), an English naturalist.

"Natural History of the Crinoides," and a "Memoir on the Belemnites," 4to. Bristol, 1821. In the Transactions of the Geological Society of London, second series, vol. II. part I.

Mitchell, an American naturalist and physician.

1 chiefly quote his work on the "Fishes of New York," in the Trans. of the Literary and Philosophical Society of New York. He has also published 
other Memoirs in the Annals of the NewYork Lyceum, and in the Journ. of the Academy of Natural Sciences of Philadelphia.

Moehr.-Moehring (P. H.G.), a physician at Jever.

"Avium Genera," 8vo. Aurich, 1752.

Molin.--Mourna (the Abbé J. I.), an ecclesiastic of Chili, resident in Italy.

"Essai sur 1'Histoire Naturelle du Chili," in Latin, and translated into French by Gruvel, 1 vol. 8vo. Paris, 1789. This work was written in Italy from memory, and contains many doubtful passages.

\section{Montag.-Montagu (George),} an English naturalist.

Author of descriptions of various species of Birds, Fishes, Mollusea and Crustacea, in the Transactions of the Linnæan and Wernerian Societies of London.

Montegre a physician of Paris, who died in the colonies.

I quote his "Mémoire sur les Vers de Terre," published in the Mémoires du Muséum.

Montf.-Montfort (Denis de), a singular man, who styled himself an ancient naturalist of the King of Holland; he perished through want in the streets of Paris in 1820 or 1821. I principally quote his

"Conchyliologie Systématique," a sort of Genera Conchyliorum, where the genera are extremely numerous, and represented by wood cuts, executed by the author, in as exact a manner as can be done by that species of engraving.

There are but two volumes 8vo. which contain the Univalves only. Paris, 1808,1810 .

He is also the author of the first four volumes of the "Histoire Naturelle des Mollusques," that form a sequcl to Sonnini's Buffon, Paris, 1802, in which he has inserted apocryphal figures. They merely contain the generalia and the Cephalopoda.

Moq. TAnd.-Moquin-TAn Don (A.), a physician of Montpellier, Professor at Marseilles.
"Monographie de la famille des Hirudinées," 4to. Montpellier, 1826.

Moreau de Jonnes, corresponding member of the Institute.

Quoted as author of several Memoirs on the animals of the Antilles.

Morren (C. F. A.), a naturalist of Belgium.

"De Lumbrici Terrestris Historia Naturali nec non Anatomia," 4to. Brussels, 1829.

MoufF. - Mouffet (Thomas), an English naturalist, died about 1600 .

"Insectorum sive Minimorum Animalium Theatrum," 1 vol. folio, with five hundred wood cuts. London, 1634.

It was published by Theodore de Mayerno, a Frenchman and physician to James I. It is the first special work on Insects.

Stat. Mull.-Muller (Philip Louis Statius), Professor at Erlang, born in 1725 , died 1776 .

Author of a bad translation, into German, of the Systema Naturæ of Linnæuls, from the Dutch translation of Houttuyn, 9 vols. 8vo. Nuremb. $1773-1776$, containing the animals only.

Mull.-Muller (O. F.), a Dane, Counsellor of State, and one of the most laborious observers of the eighteenth century, born 1730 , died 1784. I quote his

"Von Würmern der Süssen und Salzigen Wassers," 1 vol. 4to. or fresh and salt water worms.

"Vermium Terrestrium et Fluviatilium Historia," 2 vols. 4 to.

"Zoologia Danica," folio, with coloured plates. The first three numbers, Copenhagen, 1788, 1789, are from his pen; the fourth from Abildgaardt, Vahl, \&c.

"Zoologia Darica, Prodromus," 1 vol. 8vo. Hanix, 1776.

"Entomostraca seu Insecta Testacen," 1 vol. 4to. with plates. Lips. and Havniæ, 1785.

"Hydrachnæ," 1 vol. 4to. with coloured plates. Lipsiæ, 1781.

"Animalcula Infusoria," 1 vol. 4to. 
Naccari (L. F.), librarian of the seminary of Chioggia.

"Ittiologia Adriatica," published in the Physical Journal of Pavia, vol. V. Dec. 11, 1822.

Nardo (Domenico), an Italian naturalist established at Chioggia.

He made some additions to the work of Naccari in the Physical Journal of Pavia, XVII.

Natter.-Natterer, an Austrian naturalist, who travelled in Brazil.

Author of various interesting observations on the animals of Germany.

\section{Naturf.-Naturforscher.}

"Der Naturforscher, or the Naturalist." The title of a German Journal on Natural History, of which twentyseven numbers were published at Halle, from 1774 to 1793 . It abounds in important observations and good figures.

Naum. - Nauman (J.A. and J.F.), father and son.

" Natural History of the Birds of Germany." An excellent work, the plates of which, though small, are perfect. The second edition, 8vo. Lips., 1820, et seq.-whieh we chiefly quote-is not yet terminated.

Nees d'Esenb. See GravenHORST.

Nicols.-Nicolson, an Irish Dominican, missionary to St. Domingo.

"Essai sur l'Histoire Naturelle de St. Domingue," 8vo. with plates. Paris, 1776.

Nieremb.-Nieremberg(J.E.), a Jesuit, Professor at Madrid.

"Historia Naturalis maxime peregrina, libris XVI. distincta," folio, Antwerp, a compilation of but little value.

Nils.-Nilson (S. V.), Curator of the Lund Museum.

"Ornithologia Suecica," 2 vols. 8vo. Copenhagen, 1817, 1821.

Nitzch (C. L.), Professor at Halle.

Cuv. Table of Authors.
Author of various Memoirs on the osteology of Birds and the Invertebrata, published among those of Halle, Bonn, \&c.

"Spiropteræ Stramosæ Descr.," 4to. Halle, 1829.

"Materials for a History of the Infusoria, or a description of the Cercariæ and Bacillariæ," 8vo. in German. Halle, 1817.

Nosem.-Noseman (N.), died 1786.

In conjunction with the engraver, Christian Sepp, author of a "History of the Birds of the Netherlands" (in Dutch), folio, with remarkably beautiful plates. The last numbers are by Houttuyn. Amsterdam, 1770, et seq.

Ochsenh. - Ochsenheimer (Ferdinand).

His work, written in German, on the "Lepidoptera of Europe," is the best that has been published with respect to critical accuracy, and the descriptions of the species. The first volume appeared at Leipzic in 1806. The one he is about to publish will contain the Noctuæ.

\section{Odier (Auguste).}

"Mémoire sur la Composition Chimique des parties cornées des Insectes," inserted in the first volume of the "Mémoires de la Soc. d'Hist. Nat.," 4 to. 1823.

Oken, a German naturalist of Fribourg in Brisgau, established at Jena.

"Philosophy of Nature," 3 vols. 8vo. Jena, 1809 .

"A Treatise on Natural History," of which the Zoology forms the third part, in two vols. 8vo. with an Atlas. Jena, 1816.

" A Natural History for Schools," 1 vol. Jena, 1821.

"Esquisse de Système d'Anatomie, de Physiologie, et d'Histoire Naturelle," 8vo. Paris, 1821.

He is the principal editor of the Isis, a journal which abounds in important articles relative to natural history.

Olafsen (Eggert), or Erard Olavius, a naturalist of Iceland, born 1726, died 1768 .

Jointly with Biorn Polvelsen, of PAULI, the first physician of that S s 
island, who died in 1778, author of a "Journey in Iceland," printed in 1772. I quote the French translation, 5 vols. 8vo. with an atlas. Paris, 1802.

Orivi (The Abbé Joseph).

"Zoologia Adriatica," 1 vol. 8vo. with plates. Bassano, 1792.

It contains excellent observations on the Mollusca and Crustacea.

Oliv.-Olivier (Antoine-Guillaume), member of the Académie des Sciences, Professor of Zoology to the Ecole Véterinaire of Alfort, \&c., born at Draguignan 1756, died 1814.

"Entomologie, ou Histoire Naturelle des Insectes" (Coleoptera), 5 vols. folio, with coloured plates. Paris, $1789-1808$.

Insects of the Encyclopédie Méthodique, from the fourth volume of the Natural History to the eighth inclusively.

"Voyage dans l'Empire Ottoman, l'Egypte et la Perse," 3 vols. 4to. with plates. Paris, 1807. It contains interesting species of several classes of animals.

Omalius de Halloy, governor of the province of Namur, and a learned geologist.

Oppel (Michael), a Bavarian naturalist, who died in 18-.

"Sur la Classification des Reptiles." The first Memoir is on the Ophidia, the second on the Batrachia, published in the Annales du Muséum.

"The Orders, Families and Genera of Reptiles" (in German), 4to. Munich, 1811.

I also quote his Memoir on the Tanypus, inserted in the Memoirs of the Academy of Munich, 1812.

In conjunction with Messrs. Tiedeman and Liboschitz, he commenced a work on Reptiles, with numerous plates, of which the Crocodiles only were published. Heidelberg, folio, 1817.

Osbeck (Peter), a pupil of Linnæus, and chaplain of a Swedish vessel that went to China in $\mathbf{1 7 5 0 .}$

His narrative was printed in the Swedish language in 8vo. Stockholm, 1757, and translated into German by G. Rostock, 8vo. 1765.
Отто (A. W.), a German naturalist, Professor at Breslau.

Author of several Memoirs among those of the Academy of Sc., of Nature, and other collections.

"Conspectus Animalium quorundam," \&c. Breslau, 1821.

"De Stermaspide Thalassemoides et Siphostomate Diplochaito," 4to. Breslau, 1820.

Palis. de Beauv.-Palisot, Baron de Beauvois (A. M. F. J.), member of the Académie des Sciences, born 1755, died 1820.

"Insectes recuellis en Afrique et en Amérique," \&c., folio, with coloured plates. Paris, 1805 et seq.

Pall.-Pallas (P. S.), one of the greatZoologists of modern times, born at Berlin 1741, died 1812. I quote his

GLIR.

"Novæ Species Quadrupedum e Glirium Ordine," 4 to. with thirty-nine coloured plates. Erlang, 1778.

SPIC. or SPIC. ZooL.

"Spicilegia Zoologica," fourteen numbers, 4to. Berlin, 1767-1780.

MisceL.

"Miscellanea Zoologica," 1 No. 4to. Haga, 1766.

Voy.

"Voyage dans plusieurs provinces de l'Empire de Russie," French Tr., 8vo. with an atlas. Paris.

NORD. BEYTR.

"Neue Nordische Beytræge," \&c. (or New Materials from the North for Geography, \&c.), 7 vols. 8vo. Petersburg and Leipzic, 1781-1796.

"Zoographia Russo-Adriatica," 3 vols. 4to. Some of the plates of this work having been mislaid, it has not yet been published, though the Academy of St. Petersburg have granted the use of the MSS. to certain naturalists.

Several of his Memoirs inserted among those of the Academy last mentioned.

Panz.-Panzer (G. W. F.), a physician of Nuremberg, born in 1755.

"Faunæ Insectorum Germanicæ initia, or Deutschlands Insecten," one hundred and nine numbers, $12 \mathrm{mo}$. each consisting of twenty-four coloured plates. Nuremberg, 1796, et seq. One of the most useful entomological works we possess, on account of the accuracy of the figures. 
"Entomologischer Versuch uber die Jurineschen Gattungen der Linneischen Hymenoptern, 1 vol. $12 \mathrm{mo}$. Nuremberg, 1806.

"Index Entomologicus, pars prima, Eleutherata," I vol. ]2mo. Nurembergæ, 1813.

He has also published several other works on Insects, which I have not had occasion to quote.

Park.-Parkinson (James), an English naturalist.

"Outlines of Oryctology," 1 vol. 8vo. with plates.

" Organic Remains of a Former World," 3 vols. 4 to. London. 1811.

Parra (Don Antonio), an American naturalist.

Author of a "Description of various portions of Natural History," and chiefly of marine productions, written in Spanish, 4to. Havanna, 1784.

In this work the author describes and figures many fishes and Crustacea.

Passer.-Passerini (Charles).

"Observations on the sound produced by the Sphinx Atropos," in Italian, from which M. Dufronches has given an extract.

Paykull (Gustavus), Counsellor to the King of Sweden, and member of the Academy of Stockholm.

"Fauna Suecica" (Insecta), 3 vols. 8vo. Upsal, 1800

These three volumes refer exclusively to the Coleoptera; his descriptions are carefully and completely given.

He has also published good Monographs of the genera Carabus, Curculio and Staphylinus, but they are incorporated with the Fauna.

"Monographia Histeroideum," with plates of all the species, 1 vol. 8vo. Upsal, 181l. This Monograph is superior to the preceding ones, and is indispensably requisite for the study of these Insects.

$\mathrm{He}$ has published certain Memoirs on Birds.

Peck (William), Professor of Botany at the university of Harvard.

Author of a Memoir inserted in the fourth volume of the Agricultural Journal of Massachusetts, relative to a species of Rhynchænus, that attacks the Pine.
Penn. Pennt.-Pennant (Thomas), a Welchman, born in 1726 , died in 1798. A laborious naturalist. The works we chiefly quote are his

"History of Quadrupeds," 2 vols. 4to.

"British Zoology," 1 vol. folio.

"British Zoology," 4to. and 8vo. 4 vols.

"Arctic Zoology," 2 vols. 4to.

"Indian Zoology," 1 vol. 4to.

Pernetry, a Benedictine who accompanied Bougainville to the Falkland Islands; he was afterwards librarian to Frederick II. of Prussia.

"Voyage aux Iles Malouines," 2 vols. 8vo. Paris, 1770. It contains some valuable details on Natural History, and useful figures.

Per.-Peron (François), born at Cerilly in 1775 , died in 1810 , a zealous traveller, prematurely snatched from the sciences, and one of those who have most contributed to enrich the Museum of Paris.

" He edited the first volume of the "Voyage de découverte aux 'Terres Australes en 1800-1804," 1 vol. 4to, with an atlas. Paris, 1807.

He was also the author of various $\mathrm{Me}$ moirs published in the Annales du Muséum.

Perrault, (Claude), a naturalist, architect of the Louvre and Observatory of Paris, born 1613, died 1688.

$\mathrm{He}$ published, from the dissections of Duverney, the "Mémoires pour servir à l'Histoire Naturelle des Animaux," which form the third volume of the Mém. de l'Acad. des Sciences, previous to 1699 .

Petag.-Petagna, (V.), of Naples.

"Specimen Insectorum Ulterioris Calabriæ," 4to. with one plate. Francofurti, 1787.

"Elements of Entomology," 2 vols. 8vo.

Petersf. or Petrop. Mem., or Comment., or Nov. Comment., or Act., or Nov. Аст.

$$
\text { s } s
$$


Such are the various titles of the Memoirs of the Imperial Academy of Sciences of St. Petersburg.

The "Commentarii," 14 vols. 4to. from 1726 to 1746 .

The "Novi Commentarii," 20 vols. from 1749 to 1775 .

The "Acta," 7 vols. from 1777 to 1782 .

The "Nova Acta," 15 vols. from 1783 to 1802 .

The "Memoirs," from 1809

\section{Phelsum (Murck Van), a Dutch naturalist.}

Quoted for his "Letter to C. Noseman on the Echini," 8vo. Rotterdam, 1774 .

Phillip (Arthur), a German, and Governor of Botany Bay, in the English Service.

"The Voyage of Governor Phillip to Botany Bay," \&c., with fifty-five coloured plates, London, 1789. An anonymous work, the part relative to natural history by Latham. There is a French translation of it without plates, in 1 vol. 8vo. Paris, 1791.

Phipps (C. J.), the celebrated English navigator, subsequently Lord Mulgrave; born 1746, died 1792.

"Voyage to the North Pole in 1773," translated into French by Desmeuniers, 1 vol. 4to. Paris, 1775.

Planc.-Plancus (Janus) or J. Branchi, a physician of Rimini, born in 1693 , died in 1775 .

"De Conchis minus notis," 1 vol. 4to. with plates. Venice, 1739. The second edition greatly enlarged. Rome, 1760 .

\section{Pl. Col.-Planches Colo- RIEES.}

"Planches Coloriées des Oiseaux, par MM. Temminck and Laugier," 4to. and folio, a great work, which forms a sequel to the Planches Enluminées, \&c. of Buffon.

\section{Pl. Enl.-Planches Enlumi-} NEES.

The coloured plates of Birds, published for Buffon's Natural History, by Dubenton, Jun., amounting to one thousand and eight, but arranged without order. It is beyond all doubt the richest collection of that class that has ever appeared. Most of the figures are good.

Plum. - Plumier (Charles), who travelled for a long time in the service of Louis the Fourteenth ; he was a great naturalist in all the branches of the science, although several of his works have remained unpublished.

I have had occasion to quote his Observations on Fishes and Reptiles, part of which are at Paris and part at Berlin, all in MS., with numerous drawings; a portion of them has been published by Bloch and Lacépède.

PoLI, a naturalist and anatomist at Naples, author of the magnificent work, entitled

"Testacea utriusque Siciliæ eorumque Historia et Anatome," 2 vols. folio. Parma, 1791 and 1795. A third volume has been lately published。

Prev.-Prevost (Benedict).

"Mémoire sur le Chirocéphale," published at the end of the Histoire des Monocles of Jurine. See JuRINE.

Preys.-Preysler (J. D.)

"Werzeichniss Bochmischer Insecten," 1 vol. 4to. Prague, 1790.

Pr.Max.-MaximilianPrince, de Wied-Neuwied.

His "Voyage to Brazil," 2 vols. 4to. with an atlas, Franckf., 1820 and 1821, his "Natural History of Brazil," of which two vols. 8vo. were published at Weimar, 1826, and several numbers of coloured plates, in folio. These are among the number of those productions of modern times which are richest in novelties.

Prun.-Prunner (Leonard de).

"Lepidoptera Pedemontana," 1 vol. 8vo. Turin, 1798

Q. and G. or Quoy and Gaym., or Gaim.-Quoy and Gaymard, fellow travellers who have already made two great voyages.

They have published the "Zoologie du Voyage de l'Uranie," 1 vol. Paris, 1824 , with one volume folio of plates. They are at present occupied with 
that of the "Voyage de l'Astrolabe," of which several numbers have already appeared.

Raffles (Sir Stamford), an English general and Governor of Sumatra, who has greatly contributed to our knowledge of the productions of that island.

I quote his paper on this subject in the thirteenth volume of the Linnæan Transactions.

Raf.-Rafinesque Schmaltz. (C. S.), naturalist, long a resident in Sicily, and at present established in the United States.

Author of numerous little works on new species, genera and systems.

"Caratteri di alcuni nuovi Generi et nuove Specie di Animali e Piante della Sicilia," 8vo. Palermo, 1810.

"Indice d'Ittiologia Siciliana," 8vo. Palermo, 1810

"Principes Fondamentaux de Sémiologie." Palermo, 1814.

"Analyse de l'Univers, ou Tableau de la Nature," 8vo. Paris, 1815.

"Ichthyologia Ohiensis, or Natural History of the Fishes inhabiting the river Ohio," \&c. 8vo. Lexington, Kentucky, 1820 .

$\mathrm{R}_{\mathrm{AY}}$ (John), an English theologian, born 1628, died 1704; the first true methodiser of the animal kingdom, and the principal guide of Linnæus in that department of the natural sciences.

"Synopsis Methodica Animalium Quadrupedum et Serpentum," 8vo. London, 1683.

"Synopsis Methodica Avium et Piscium," 8vo. London, 1783.

"Historia Insectorum," 4to. London, 1710 .

\section{Randohr (C. A.), a German} naturalist.

Anthor of a treatise "On the Digestive Organs of Insects," in the German language, 4to. Halle, 1811; and of "Materials for the History of certain German Monoculi," 4to. Ibid. 1805.

$R_{A N G}$ (Sander), and officer of the Corps Royal of the French Navy, an able naturalist.

"Manuel de l'Histoire Naturelle des
Mollusques et de leurs Coquilles," 12 mo. Paris, 1829.

" Etablissement de la famille des Béroides," published in the fourth volume of the Mém, de la Soc. d'Hist. Naturelle.

"Histoire Naturelle des Aplysies," 4to. Paris, 1828.

Ranzani (The Abbé Camillo), Professor of Natural History at Bologna, \&c.

"Elements of Zoology" (in Italian). Bol., 1819, et seq., of which thirteen volumes, 8vo. have already appeared, all relating to Quadrupeds and Birds.

"Memoirs on Natural History" (also in Italian), 4to. Bologna, 1820 .

RaPP (William), Professor at Tubingen.

"On the Polypi in general and the Actiniæ in particular," 4to. Weimar, 1829.

Reaum.-Reaumur (R. A. Ferchault de), member of the Académie des Sciences, born 1683, died 1757 ; his labours were directed to all the sciences. We chiefly quote his

"Mémoires pour servir à l'Histoire des Insectes," 6 vols. 4to. with plates. Paris, 1734-1742. The seventh volume remains in MS.; the others were not commenced. An admirable work.

RED.-REDI (F.), a celebrated literary character and physician of Arezzo, born 1626, died 1698.

"Experimenta circa Generationem Insectorum," 3 vols. $12 \mathrm{mo}$. with plates. Amstelodami, 1671, 1686, 1712. L.)

Reich.-Reichenbach (H. T.

"Monographia Pselaphorum," 1 vol. 8vo. with plates. Lipsix, 1816.

Reinw.-Reinwardt, a German naturalist, Professor at Leyden, who travelled through the Archipelago of India, where he made as plendid collection.

RENARD (Louis), editor of a collection of drawings of Fishes and other marine animals executed in 
India by native painters, which, under a barbarous appearance, exhibit interestiug and true species. One vol. folio. Amsterdam, 1754.

ReNieri, an Italian naturalist, Professor at Padua.

Rets.-Retsius, a Swedish naturalist, Professor at Lund.

Author of a greatly enlarged edition of the "Fanna Suecica" of Linnæus, of various theses, \&c.

RICHARDS.-R R ICHARDSON(John) $_{\text {ICH }}$ surgeon to the first expedition under captain Franklin.

Author of the Zoological appendix attached to the account of that voyage. London, 1823, in 4to.

RIss.-RIsso (A.), a naturalist of Nice, and a zealous observer.

"Ichthyologie de Nice," \&c., 1 vol. 8 vo. Paris, 1810, a work of extreme value on account of the number of new species which it contains.

"Histoire Naturelle des Crustacés des environs de Nice," 1 vol. 8vo. with plates. Paris, 1816.

These works have been reproduced in his "Histoire Naturelle de l'Europe Merid.," 5 vols. 8vo. Paris, 1826.

$\mathrm{He}$ has also published a description of some new Crustacea in the Journal de Physique.

Robin.-Robineau Desvoidy, physician at St. Sauveur, department of the Yonne.

"Recherches sur l'Organization Vertebrales des Crustacés, des Arachnides, et des Insectes," I vol. 8vo. Paris, 1828.

"Essai sur la tribu des Culicides," inserted in the second volume of the Mémoires de la Société d'Histoire Naturelle.

A great work on the Muscidæ, which he calls "Myodaires," published in the Mém, des Savants Etrangéres, \&c.

"Observations on the Olfactory Organ of the Crustacea, and on the use of the Halteres of the Diptera."

Rochefort (N.), a protestant minister of Holland.

"Natural and Moral History of the Antilles and America." The first edition is anonymous, and published at
Rotterdam, 1658. The part relative to Natural History is copied from the first edition of Dutertre, 1654

\section{Ram.-Ramer (J. C.)}

"Genera Insectorum Linnæi et Fabricii, Iconibus illustrata," 1 vol. 4to. Vitoduri Helvetion'um, 1789.

His work is merely an edition of that of Sulzer on the same subject; with some new plates.

Røs.-Røsel de Rosenhof (A. J.), a painter of Nuremberg, born 1705 , died 1759 , one the most ingenious observers, and an able painter of subjects of Natural History.

"Historia Naturalis Ranarum nostratium," l vol. folio. Nuremb., 1758.

"Insecten-Belustigungen," with excellent coloured plates, 4 vols. 4 to. $\mathrm{Nu}$ remb., 1746, et seq. See Kleemann.

Rog. - RogER, a naturalist of Bourdeaux.

"Instructions à l'usage des personues qui voudraient s'occuper a recueillir des Insectes pour les Cabinets d'Histoire Naturelle," 8vo. Bourdeaux.

Roiss. - Roissy (Félix de), a naturalist of Paris.

He completed, by the 5 th and 6 th vols. 8vo. the "Histoire des Mollusques," commenced by Denys de Montfort for Sonnini's Buffon.

Rondel. - Rondelet (Guillaume), Professor at Montpelier, born 1507, died 1566 .

"Libri de Piscibus," 1 vol. folio. Lyons, 1554. A work still useful for its numerous wood-cuts.

Ross.-Rossi (Pietro), an Italian naturalist, Professor at Pisa, died in 18-.

" Fauna Etrusca, sistens Insecta quæ in provinciis Florentina et Pisana præsertim collegit Petrus Rossius," 2 vols. 4 to. with coloured plates. Liburni, 1790 .

"Mantissa Insectorum exhibens Species nuper in Etruria collectas, a Petro Rossio," \&c. with coloured plates, 2 vols. 4to. Pisis, 1792-1794.

Roux (Polydore), Curator of the Museum of Marseilles. 
"Ornithologie Provençale," 4to. with beautiful lithographic plates.

"Crustacés de la Mediterranée et de son littoral," 4to. with plates, the three first numbers. Marseilles, 1827 $-1828$.

Roxburgh, an English physician at Bengal.

I quote his paper on the Dolphin of the Ganges.

Rudolphi (C. A.), a German naturalist and anatornist, Professor at Gripswald and now at Berlin. Chiefly quoted for his classical work on the Intestinal Worms.

" Entozoa seu Vernium Intestinalium Historia Naturalis," 2 vols. 8vo. Amsterdam, 1808.

Rumph (G. E.), a German merchant, born at Hanau in 1637 , Intendant at Amboyna in the Dutch service, died in 1706 .

"The Cabinet of Amboyna" (in Dutch), 1 vol. folio. Amsterdam, 1705.

"Theasurus Imaginum," \&c. Haga, 1739 , 1 vol. folio, with the same plates, but a more abridged text.

\section{REPPel (Edward), a naturalist} of Franckfort.

Author of "Travels in Nubia," with excellent lithographic and coloured plates, representing new species of various classes, of which several numbers are already published in 4 to. Franckf. 1826

Russec (P.), formerly a surgeon at Bengal.

"Serpents of the coast of Coromandel," 1 vol. folio, with a supplement and excellent plates. London, 17-.

"Description and figures of two hundred Fishes from the Coast of Coromandel," 2 vols. folio. London, 1803. Two capital works.

Ruysch (Henry), son of the celebrated anatomist; he died before his father. Under the title of

" Theatrum Animalium," 2 vols. folio, Amsterd., 1718, he gave an edition of Johnstone, to which he added a copy of the same plates of fishes employed by Renard and Valentin.
SABine, an English naturalist.

Author of the Appendix to Captain Parry's first voyage, and of various papers in the Transactions of the Linnæan Society.

SAGE (B. G.), Chemist of the Academy of Sciences, died 1824.

"Mémoire sur les Belemnites," published in the Journal de Physique.

Sahl.-Sahleerg (C. R.).

"Dissertatio Entomologica Insecta Fennica enumerans." Præs. C. R. Sahlberg, 8vo. Aboæ, 1717, 1823.

"Periculi Entomographici," l vol. 8vo. with plates. Aboæ, 1823.

Salerne, a physician of Orleans.

Author of a translation of the "Synopsis Avium " of Ray, under the title of " l'Histoire Naturelle éclaircie dans une de ses principales parties, l'Ornithologie," \&c. 4to. Paris, 1767.

The drawings are by the same hand that furnished those of Brisson and of the Planches Enluminées, and are frequently taken from the same specimens.

Salt, English consul in Egypt.

"Travels in Abyssinia." They contain some observations relative to natural history.

Salv.-Salviani (Ippolito), of Citta di Castello, a physician at Rome, born 1513, died 1572 .

"Aquatilium Animalium Historiæ," 1 vol. folio, with numerous and excellent copperplate engravings of Fishes. Romæ, 1554 .

Sav., or Savign.-Savigny (J. C.), member of the Académie des Sciences.

"Histoire Naturelle et Mythologique de l'Ibis," l vol. 8ve. Paris, 1805.

"Mémoires sur les Oiseaux de l'Egypte," in the great work on Egypt.

"Mémoires sur les Animaux sans Vertèbres," part first, No. 1, 8vo. Paris, 1816.

"Système des Annelides," published in the great work on Egypt, as well as his "Tableau Systématique des Ascidies."

Savi (Paulo), a young natu- 
ralist of Tuscany and Professor at Pisa.

Author of various good observations on the animals of that country, published in the Giornale dei Letterati. He has given in Italian two Memoirs on a species of Iulus, which have lately been reproduced with others of the same savant, in a work entitled "Memorie Scientifiche di Paolo Savi, decade prima con sette tavole," 1 vol. 8vo. Pisa, 1828.

$\mathrm{S}_{\mathrm{AY}}$ (Thomas), a French naturalist, established in the United States.

Author of various papers in the Journal of the Academy of Natural Sciences of Philadelphia, and the Annals of the New York Lyceum.

Scheff.-Schefrer (J. C.), a clergyman at Ratisbon, born in 1718, died in 1799 .

"Elementa Entomologica," with coloured plates, 1 vol. 4to. Ratisbonne, 1769.

"Icones Insectorum circa Ratisbon Indigenorum," 3 vols. 4to. Ratisbon, 1769 .

"Apus pisciformis Insecti Aquatici Species noviter detecta," 4to. with plates. Ratisbonne, 1757. This Crustaceous animal is the Cancer Stagnalis of Linnæus. See Branchipus.

"Abhandlungen von Insecten." Regensburg, 1764-1779.

Schellenb. - Schellenberg, (J. R.), painter and engraver at Zurich.

" Cimicum in Helvetiæ Aquis et Terris degens Genus," with plates, 1 vol. 8vo. Turici, 1800.

"Genres des Mouches Diptéres," in French and German, with coloured plates. Zurich, 1803. The text is by two anonymous writers.

Sch., or Scheuchz.-ScheuchZER (J. J.), a physician of Zurich.

"Physique Sacrée," 4 vols. folio. Amsterdam, 1732. It contains numerous figures of Serpents.

Schrntz, Secretary of the Society of Natural History at Zurich, the translator into German of the Régne Animal.

Author of the "History of the Eggs and Nests of Birds."

\section{Schloss.-Schlosser, a physi-} cian at Amsterdam.

Author of certain "Memoirs on Fishes," jointly with Boddaert, published in the Philosophical Transactions.

Schn.-Schnerder (J. G.), the celebrated hellenist and natural. ist, Professor at Frarckfort-on-theOder, now at Breslau.

"Amphibiorum Physiologiæ Specim." 4to. Fascic. I et 1I. Zullichow, 1797.

" Historix Amphibiorum Naturalis et Litterariæ," 8vo. Fascic. I et II. Jena, 1799, 1801.

"The Natural History of Tortoises in general," (in German), 1 vol. 8vo. Leipzic, 1783.

I frequently quote under his name his edition of the "Systema Ichthyologiæ" of Bloch, 8vo. with one hundred and ten plates. Berlin, 1801.

Schorp (J. D.), a physician at Anspach, born 1752.

"Historia Testudinum Iconibus Illustrata," 4to. with coloured plates. Erlang, 1792, et seq.

Schonefeld (E.de), a physician of Hamburg.

"Ichthyologia, \&c. ducatum Slesvigi et Holsatix," 4to. Hamburg, 1824.

Schon.-or Schønh.-Schønherr (C. J.), a Swede.

"Synonymia Insectorum," 2 vols. 8vo. with plates. Stockholm, 1806-1808.

"Curculionidum Dispositio Methodica," 1 vol. 8vo. Leipzic, 1826.

Schrank (F. de P.), a Bavarian naturalist, Professor at Ingolstadt, born in 1747 .

"Enumeratio Insectorum Austriæ Indigenorum," 1 vol. 8vo. with plates. Augustæ Vindelicorum, 1781.

"Fauna Boica," 6 vols. 8vo. Nuremberg and Ingolstadt, 1798, et seq.

Schreb.-Schreber (J. C. de), Professor at Erlang, born in 1739.

We chiefly quote his "History of the Mammalia" (in German), with coloured plates, 4to. Erlang, 1775, et seq.

There are also some French copies of the first parts. The greater part of 
the plates are copied from Buffon, and coloured from the descriptions, although some of them are original and good.

Schreib.-Schreibers (Charles de), Director of the Imperial $\mathrm{Mu}$ seum of Vienna.

The description of various unpublished or but little known Coleoptera, with plates, inserted in the sixth volume of the Transactions of the Linnæan Society.

A Memoir on the Proteus in the Philosophical Transactions.

Schroet. - Schroeter, (J. S.), Lutheran superintendent at Buttstedt in the Duchy of Weimar, born in 1735. Author of numerous works on Conchyliology ; we quote his

"History of Fresh-water Shells" (in German), 4to. Halle, 1779.

Schweig.-Schweigger(A.F.), a Prussian naturalist who was assassinated by his guide during a journey in the interior of Sicily.

"Prodromus Monographiæ Cheloniorum," in which he particularly describes the new species in the Museum of Paris. It is published in the "Archives of Kœnigsberg," for 1812. He has also given us

"Observations during his Travels," in which he treats of the Corallines and yellow Amber, 4to. Berlin, 1819.

"A Mannal of the Invertebrate and Inarticulated Animals," l vol. 8vo. Leipzic, 1820.

\section{Scilla (Agostino), a Sicilian} painter.

"La Vana Speculatione disingannata dal Senso," 1 vol. 4to. Naples, 1670.

The first exact comparison of fossils with analogous recent bodies that was instituted. There is a Latin translation of this work in 4to. Rome, 1752.

Scop.-Scopoli (J. A.). Professor of Botany and Chemistry at Pa. via, born in 1723 , died in 1788 .

"Entomologia Carniolica," 1 vol. 8vo. Vindebonæ, 1763.

"Deliciæ Floræ et Faunæ Insubricæ" with plates, 4 vols. folio. Ticini, 1786 $-1788$

Cuv. Table of Authors.
"Introductio ad Historiam Naturalem," 1 vol. 8vo. Pragæ, 1777.

"Anni Historici-Naturales V." Lipsæ, $1768-1772$, united in 1 vol. 8vo.

$\mathrm{He}$ also published some plates which are but little known, forming a sequel to his "Entomologia Carniolica."

Scoresby, an English navigator, who re-discovered Oriental Greenland, and author of

"Arctic Regions," \&c, 1 vol. London, 1816 , which contains many valuable observations on the Cetacea.

Seb.-Seba (Albert), a druggist of Amsterdam, born in 1665, died in 1736. Celebrated for his

"Locupletissimi Rerum Naturalium Thesauri Accurata Descriptio," 4 vols. folio. Amsterdam, 1734, 1765.

A work that I have frequently quoted, because it is enriched with numerous and excellent plates; the text, however, is of no authority whatever, being written without accuracy or judgment.

\section{Selby (P. J.)}

Author of "Illustrations of British Ornithology," 8vo. Edinburgh, 1825, with a very large atlas, the most magnificent work on Ornithology that exists.

He also published various papers in the Zoological Journal, \&c.

Senguerd.-Senguerdius(Wolferd.)

"Tractatus Physicus de Tarantula," 1 vol. 12mo. Lugduni Batavorum, 1668.

Serres (Marcel de), Professor of Mineralogy to the Faculté des Sciences of Montpellier. Author of

"Mémoire sur les yeux composés, et les yeux lisses des Insectes," with plates, 1 vol. 8vo. Montpellier, 1813.

Several Memoirs on the Anatomy of Insects, published in the Annales du Muséum.

Serv. - Serville, one of the writers for the Entomological Department of the Faune Française, and of the Encyclopédie Méthodique.

He has also published the last number 
of the work of the late Palisot de Beauvois on the Insects collected by him in Africa and America; as well as extracts from various works on Insects, in the "Bulletin Universel" of Baron Férussac.

Sнаw (Thomas), a theologian of Oxford, who travelled in Africa and the Levant.

His work, published in English at $\mathrm{Ox}$ ford, in folio, 1738, has been translated into French under the title of "Voyage dans plusieurs parties de la Barbarie et du Levant," 2 vols. 4 to. La Haye, 1743.

Sh. or Shaw.-Shaw (George), Adjunct Librarian of the British Museum, a laborious compiler and describer, died in 1815.

"The Naturalist's Miscellany," 8vo. London, 1789, et seq.; a numerous collection of coloured plates, mostly copies, with some that are original.

"General Zoology," London, 1800, et seq., several volumes, 8vo. with plates, most of them copies.

"Zoology of New Holland," a few number's, 8vo. London, 1794 et seq. The work remains unfinished.

Sloane (Hans), a former President of the Royal Society, born in 1660 , died in 1753 .

"Voyage to the Islands of Madeira, Barbadoes, Nevis, St. Christopher and Jamaica," with 274 indifferent or bad plates, 2 vols. folio. London, 1707 , 1727.

\section{Smeath.-Smeathman (Hen.)}

His History of the Termites, published in the seventy-first volume of the Philosophical Transactions, has been translated into French by Dr. Rigaud of Montpellier, and inserted in the French translation of Sparrman's Voyage.

Sмiтн (Hamilton), an officer in the English service and a learned naturalist.

Author of a great portion of the additions to the English translation of the Règne Animal, and particularly of the Synopsis Mammalium, which terminates the third volume.

Soc. NAt. BerL., or BerL. Mem., or Nat. of Berl., or BerL. NAT.
The Memoirs of this Society have appeared successively under four different titles, in German.

1. "Beschæftigungen," (Occupations), 4 vols. 8vo. 1775-1779.

2. "Schriften," (Writings), 11 vols. 8vo. $1780-1794$, the five last of which are also styled "Beobachtungen and Entdeckungen," (Observations and Discoveries).

3. "Neue Schriften," (New Writings), 4to. 1795-17

4. "Magazin," \&c. (The Magazine of New Discoveries in Natural History), quarterly from 3807 .

Sold. - Soldani (Ambrosio), General of the Camaldolites, subsequently Professor at Siena, author of various works on Microscopic Testacea, both fossil and recent.

"Saggio Oritografico Ovvero Osservationi sopra le Terre Nautilitiche," \&c. 1 vol. 4 to. Siena, 1780 .

"Testaceographia ac Zoophytographia Parva et Microscopica," 3 vols. folio. Siena, 1789-1798.

Sonner. - Sonnerat, born at Lyons, died in Paris, 1814, an indefatigable collector.

"Voyage à la Nouvelle-Guinée," with one hundred and twenty plates, 4to. Paris, 1776. His first voyage.

"Voyage anx Indes Orientales et à la Chine," from 1774 to 1781,2 vols. 4 to. with one hundred and forty plates. Paris, 1782. His second voyage.

Sonnini de Manoncourt(C.S.) engineer, born at Lorraine, died in Wallachia in 1814. I quote his

"Voyage dans la Haute et Basse Egypte," with an atlas of forty plates, 3 vols. 8vo. Paris, 1799.

And sometimes his edition of Buffon, 8vo. Paris, Dufart, 1798.

Sowerb.-Sowerby (Jas.), and Sowerby (G. B.), his son, English naturalists and artists.

"The Genera of recent and fossil Shells," thirty numbers, 8vo.

"Fossil Conchology,"

Various papers in the Zoological Journal.

Spai.L.-Spallanzani(Lazzaro), the celebrated observer, Professor at Reggio, then at Modena, and finally at Pavia, born in $\mathbf{1 7 2 9}$, died 
in 1799. Of his numerous works we have only had occasion to quote the

"Opuscoli di Fisica Animale e Vegetabile," 1776 .

They have been translated into French by Sennebier, 3 vols. 8vo. Geneva, 1787.

Sparm.-Sparmann (Andrew), born in 1748, a pupil of Linnæus. He visited the Cape of Good Hope and China, and was subsequently a Professor at Upsal.

Voy.

"Voyage au Cap de Bonne-Espérance," a French translation, 3 vols. 8vo. Paris, 1787.

Mus. Carls.

"Museum Carlsonianum," four small folio numbers. Stock., 1786, et seq. It contains figures of Birds, of which certain varieties are converted into species.

\section{Spence (William), an English naturalist.}

"A Monograph of the Cholevæ," that are found in England, published in the Transactions of the Linnæan Society.

Spengl.-Spengler (L.), $\mathrm{Cu}$ rator of the Cabinet of the King of Denmark, born in 1720 .

Quoted for certain Memoirs in the Naturforscher, \&c.

Spin.-Spinola (Maximilian), a Genoese noble, and a learned naturalist.

"Insectorum Liguriæ Species Novæ aut Rariores," with plates, 2 vols. 4to. Genuæ, 1806-1808.

"Mémoire sur les Poissons de Ligurie ;" one on the "Cératine Albilabre?" and the "Essai d'une Nouvelle Classification Générale des Diplolépaires," in the Annales du Muséum.

SpIx (John), a naturalist of Bavaria and member of the Academy of Munich.

Quoted for his Memoirs in the Annales du Muséum, and for his great works on the Zoology of Brazil, where he travelled with $\mathbf{M}$. de Martius by order of the King of Bavaria.

"The Natural Hist. of New Species of
Monkeys and Bats" (in Lat. and Fr.), 1 vol. folio. Munich, 1823.

" New Species of Birds" (in Latin), with one hundred and nine coloured plates, 1 vol. 4to. Munich, 1824.

"New Species of Tortoises and Frogs" (in Latiu), 4to. Munich, 1824.

"Nat. Hist. of New Species of Serpents," from the notes of the traveller, by John Wagler (Latin and French), 4to. Munich, 1824.

"Selected Generaand Species of Fishes," described by L. Agassiz, 4to. Munich, 1829.

Staab.-Slabeer (M.), a Dutch naturalist.

"Natural Amusements, containing Microscopical Observations," \&c. (in Dutch), 1 vol, 4to. Haarlem, 1778.

$\mathrm{He}$ is also the author of certain Memoirs, published among those of the Academy of Haarlem.

Stev.-Steven (C.) Director of the Imperial Botanical Garden of Odessa.

"Description of certain Insects of Caucasus and of Southern Russia," a Memorial in 4to. printed among those of the Imperial Socicty of Naturalists of Moscow, Vol. II.

\section{Stock. Mem.}

"Memoirs of the Academy of Sciences of Sweden," of which 1 vol. 8vo. (in the Swedish language) has annually appeared since the year 1739 . The first forty reach to 1779 . Since 1780 they have been published under the title of the "New Memoirs," \&c.

Stoll.-Stoll (Casper), a Dutch physician.

Supplement to the work entitled "Les Papillons Exotiques des trois parties du Monde" (in Dutch and French), 1 vol. 4to. Amsterdam, 1790, et seq.

"Représentation exactement coloriée d'après Nature, des Spectres, des Mantes, des Saterelles," \&c. (in Dutch and French), 8 Nos. 4to. Amsterdam, 1780, et seq.

"Représentation exactement coloriée d'après Nature des Cigales et des Punaises" (in Dutch and French), 10 Nos. 4to. Amsterdam, 1780, et seq.

Storr (T. C. C.), Professor at Tubingen. 
His thesis entitled "Prodromus Methodi Mammalium," Tub., 1780, and republished in the "Delectus Opusculorum ad Sc. Nat. Spect. de Ludwig," l vol. 8vo. Leipzic, 1790, has been of great use to us.

(H.)

Straus.-Straus Durckheim

"Considérations Générales sur l'Anatomie Comparée des Animaux Articulés, auxquelles on a jointl'Anatomie Descriptive du Hanneton," with plates, 1 vol. 4to. Paris, 1828.

The only work that can be compared to that of Lyonnet alrearly mentioned.

He has read to the Acad. des Sciences, a "Mémoire sur le Système tégumentaire et musculaire de l'Araignée aviculaire," Mygale of Le Blond, Lat.

Stroem (John), a pastor in Norway, born in 1726 .

Author of several Memoirs inserted among those of Drontheim, Copenhagen, \&c. and of a description of the district of Sondmer.

STuRm (J.), a German naturalist and painter.

"Deutschland Fauna," with excellent plates, 2 vols. 8vo. Nuremberg, 1807.

\section{Sulz.-Sulzer (J. H.)}

"Die Kennzeichen der Insecten," with plates, 1 vol. 4to. Zurich, 1761 .

Surrir.--Surriray, a physician at Havre.

" Observations sur le foetus d'une espèce de Calige," in the third volume of the Annales Générales des Sciences Physiques.

Swains.-Swainson, an English naturalist.

Author of various papers on Birds, published in the Linnæan Transactions and in the Zoological Journal; also of

"Zoological Illustrations," a work which forms a sequel to the Zoological Miscellany of Leach, and to the Naturalist's Miscellany of Shaw.

In conjunction with Dr. Horsefield he has published a Memoir on the Birds of New Holland, in the Linnæan Transactions.

Swammerdam, (John), a Dutch physician, born at Amsterdam in 1637 , died in 1680 .
"Biblia Naturæ," 1 vol. folio (Latin and Dutch). Leyden, 1737, 1738. The principal writer on the Anatomy of Insects.

Swed.-Sweder (N. S.), a Swedish naturalist.

Author of a Memoir published among those of Stockholm, 1784.

Temm., and sometimes T.-TemMiNcK (C. J.), formerly Director of the Society of Sciences of Haarlem, and proprietor of a valuable zoological collection, and now Director of the Royal Museum of Leyden.

"Histoire Naturelle Générale des Pigeons et des Gallinacés," 3 vols. 8vo. Amsterdam and Paris, 1813, 1815.

The part containing the Pigeons has also been published in folio, with splendid coloured plates, by Madame Knip.

"Manuel d'Ornithologie ou Tableau Systématique des Oiseaux qui se trouvent en Europe," 1 vol. 8vo. Amsterdam and Paris, 1815.

"Monographies de Mammalogie," 4to. Paris, 1827.

"Planches Coloriées des Oiseaux," 4to. and folio, forming a sequel to the Planches Enluminées of Buffon. This work was published by Temminck jointly with M. Meiffren de Laugier, Baron de Chartrouse, \&c. \&c.

Thien., or Thienem.-Thieneman, Professor and Curator of the Museum of Dresden.

Author of Observations (in German) on the Animals of the North, and chiefly on the Phocæ, 8vo. with an atlas in 4 to.

Thier. - Thiery de Menonville (N. J.), a French physician who visited Mexico for the purpose of carrying off the Cochineal.

"Traité de la culture du Nopal et de l'Education de la Cochinelle," 2 vols. 8vo. with plates. Paris, 1787.

Thomas (P.), a physician of Montpellier.

"Mémoires pour servir à l'Histoire Naturelle des Sang-sues," pamphlet. 8vo. Paris, 1806.

Thompson (John W.), a surgeon of the English army. 
"A Memoir on the Pentacrinus Europæus," 4to. Cork, 1827.

Tномеs.-Tномpson(William), an English physician established at Naples.

Author of a Memoir on a Hippurites which he calls Cornucopia.

Thunb.-Thunberg (C. P.), a pupil of Linnæus, who visited the Cape of Good Hope and Japan, Professor at Upsal, born in 1743.

Quoted for various Memoirs published among those of the Academy of Stockholm.

\section{Tiedeman (Frederick), Professor at Heidelberg.}

"Anatomy of the Holothuria, Asterias, and Echinus," folio, Landshut, 1805: one of our most splendid Monographs of invertebrated animals.

Tiles.-Tinesius (W. G.), a German naturalist who sailed round the world.

Author of several Memoirs presented to the Academy of St. Petersburg, of observations on various new animals in the Voyage of Krusensterm, and previously of an "Annual of Natural History," in the German, 12mo. Leipzic, 1802.

Trans. Lin. See Linn. Trans.

Treits.-Treitschike (Frederick), a German naturalist.

The continuer of Ochsenheimer's work on the Lepidoptera of Europe. The last volume (1829) contains the Pyralides.

Trmbl.-Trembley (Abraham), a native of Geneva, born in 1710 , and died in 1784 ; immortalized by his discovery of the reproductive power of the Polypus.

" Mémoires pour servir à l'Histoire des Polypes d'eau douce à bras en forme de cornes," with fifteen plates, 4to. Leyden, 1774.

Treutl.-Treutler (F. A.), a German physician, author of a thesis entitled

"Observationes Pathologico-anatomicx Auctarium ad Helminthologiam $\mathrm{Hu}$ - mani Corporis Continentes," 4to. Leipzic, 1793.

Trevir.-Treviranus (G. R.), Professor at Bremen.

"On the Internal Organization of the Arachnides" (in German), with plates, 4to. Nuremberg, 1812.

Tuckey (J. K.), a Captain of the British Navy.

"Relation d'une Expédition pour reconnaître le Zaire," the French translation, with an atlas in 4 to. 2 vols. 8vo. Paris, 3818.

VAHL (Martin), a celebrated Danish botanist.

Author of certain Memoirs on Zoology, published among those of the Society of Natural History of Copenhagen.

VAILL., or Le VAILL.-LevaiLLANT (François), a celebrated traveller and collector, born atSurinam. His father was a Frenchman.

Voy. I.

"Voyage dans l'intérieur de l'Afrique par le Cap de Bonne-Espérance," 2 vols. 8vo. Paris, 1790.

Voy. II.

"Seconde Voyage dans l'intérieur de l'Afrique," \&c., 1 vol. 8vo. Paris, 1795.

AFR.

"Histoire Naturelle des Oiseaux d'Afrique," 5 vols. 4to. Paris, 1799, et seq.

Perr.

"Histoire Naturelle des Perroquets," 2 vols. 4to. and folio. Paris, 1801.

OIS DE PAR.

"Histoire Naturelle des Oiseaux de Paradis et des Rolliers, suivie de celle des Toucans et des Barbas," 2 vols. folio. Paris, 1806.

"Histoire Naturelle des Promerops et des Guépiers," folio. Paris, ]807.

Val.-Valenciennes (A.) Adjunct Naturalist to the Museum of Paris, and my fellow labourer in the great work on Fishes.

Author of various Memoirs published among those of the Museum, of the Annales des Sciences Naturelles, and of the Zoological Observations of M. de Humboldt.

Valentyn (F.), a pastor at Amboyna. 
"The East Indies, Ancient and Modern" (in Dutch), 5 vols. folio. Dordrecht and Amsterdam, 17241726.

The third volume contains numerous observations on the Natural History of Amboyna. The plates of the Fishes are identical with those of Renard.

\section{Dijon.}

VALl. - VALLOT, Professor at

Has presented to the Académie des Sciences a Memoir on certain species of Cecidomyiæ, and has also published in the thirteenth volume of the Annales des Sc. Nat. some observations on the habits of the Anthribus Marmoratus, but which were made in Sweden by Dalman.

VANDELLI, an Italian naturalist, Director of the Museum at Lisbon.

Author of certain Memoirs on the Fishes of the river Amazon, published among those of the Academy of Lisbon.

Vander Lin. - Vander LiNDeN, (P. L.), a physician and Professor of Natural History at Brussels.

Has published, in two Memoirs, 4to. a description of the Libellulæ of the territory of Bologna, and also in 1 vol. 8vo. that of all the species of the same family peculiar to Europe.

Also observations on European Hymenoptera of the family of the Fossores.

The first number of a work entitled "Essai sur les Insectes de Java et des Isles Voisines;" a notice of the impression of an Insect enclosed in a piece of schistous limestone from Solenhofen in Bavaria. These three last Memoirs are published in the General Annals of the Physical Sciences. Brussels, 1819, et seq.

Vaucher (J. P. the Reverend), Professor at Geneva.

"Histoire des Conferves d'eau douce," 1 vol. 4to. Geneva, 1803.

Author of some observations on Zoophytes, published in the Bulletin des Sciences.

Vieill. - Virelot (L. P.), a naturalist of Paris, died 1828.

"Histoire Naturelle des plus beaux Oiseaux chanteurs de la zone torride," 1 vol. folio. Paris, 1805 .
"Histoire Naturelle des Oiseaux de l'Amérique Septentrionale," of which but 2 vols. folio have appeared. Paris, 1807.

He also continued the "Oiseaux Dorées" of Audibert, and has given us an " Analyse d'une nouvelle Ornithologie Elémentaire," pamphlet, 8vo. Paris, 1816.

"Galérie des Oiseaux," which is quoted as Vieill. Gal. He assisted in publishing the edition of Buffon's Birds, printed by Dufart, and the "Nouveau Dictionnaire d'Histoire Naturelle," by Deterville.

Vigors, an English naturalist, and principal editor of the Zoological Journal.

Author of various papers in the Linnæan Transactions," \&c。

Vill.-Villers (Charles de), a naturalist of Lyons.

"C. Linnæi Entomologia," 4 vols. 8vo. with tolerably good plates. Lugduni, 1789.

A useful compilation at the time when it was published, and to which the author has added a description of various Insects peculiar to the southern departments of France.

Vill.-Villiers (Adrian P. de)

Has published in the Annales de la Société Linnéenne de Paris, Nov. 1826, a description of three undescribed or but little known Lepidoptera of the south of France, with a plate in which they are figured. He there also rectifies the description previously given of the "Bombyx Milhauseri."

VIQ D'Az.-VIR D'AzYr (Felix), born at Valogne in 1748, died at Paris in 1794; member of the Acad. des Sciences, and perpetual Secretary to the Société Royale de Medecine. I quote his

"Système Anatomique," which forms a portion of the Encyclopédie Méthodique, and of which only the second volume appeared, containing the Quadrumana and the Rodentia, 1 vol. 4to. Paris, 1795 .

Virey (J. J.), a physician, and one of the editors of the "Journal de Pharmacie et des Sciences ac- 
cessoires," in which he has published his

"Histoire Naturelle des Végétanx et des Insectes qui les produisent," as well as "Recherches sur l'Insecte de la Gomme-laque."

Viv.-Viviani (Domenico), Professor of Botany and Natural History at Genoa.

"Phosphorescentia maris quatuordecim lucescentium Animalculorum, Novis Speciebus illustrata," 1 vol. 4to. Genuæ, 1805.

Vosm.-Vosmaer (Arnold), a Dutch naturalist who died in 1799 ; he was Curator of the Museum and Menagerie of the Stadtholder.

Author of numerous Monographs (in Dutch and French) of various animals, with coloured plates, from 1767.

Voy. De Duper.

The Zoological part of the "Voyage de la Coquille," under M. Duperrey. This portion of the work is by Messrs. Lesson and Garnot.

Voy. de Freycin., or Zool. de FreYcin.

The Zoological portion of the "Voyage de l'Uranie," under M. de Freycinet. It is by Messrs. Quoy and Gaimard.

Wagler (John), a German naturalist, author of Ornithological Fragments, entitled

"Systema Avium," editor of the History of Serpents in the Brazilian Zoology of Spix and Martius, and author of Memoirs on Fishes in the Isis.

Walb.-WALbaum (J. J.), a physician of Lubeck, born 1724,

Besides his edition of "Artedi," has given us (in German) a "Chelonographia," or Description of certain Tortoises, 1 vol. 4to. Lubeck and Leipzic, 1782.

Also some Memoirs inserted amongst those of the Naturalists of Berlin.

Walch (J. E. E.), Professor at Jena,born in 1725 , and died in 1778 .

Author of the text of Knorr's "Monuments," \&c. See Knorr.
Walck.-WALCKenaer (C. A.), member of the Académie des Inscriptions et Belles-Lettres.

"Faune Parisienne," 2 vols. 8vo. Paris, 1802.

"Tableau des Araneides," in numbers, like those of Panzer on the Insects of Germany. But five have appeared.

"Araneides de France," a work which forms part of that entitled "La Faune Française," published by MM. de Blainville, Desmarest, Vieillot, \&c.

"Mémoires pour servir à l'Histoire Naturelle des Abeilles Solitaires," 1 vol. 8vo. Paris, 1817.

Web.-Weber (Frederick), a German naturalist, Professor at Kiel.

"Observationes Entomologicæ," I vol. 8vo. Kiel, 1801.

White, Bот. B., or White Voy. -White (John), a surgeon in the English service at Botany-Bay.

"Journal of a Voyage to New South Wales," with sixty-five plates, 1 vol. 4to. London, 1790. The Zoological part of this work, which is enriched with splendid drawings, appears to have been from the pen of John Hunter, the celebrated anatomist. There is a French edition, 1 vol. 8vo. Paris, 1795, in which useless notes are added to the original work, and the natural history and plates are suppressed.

\section{Wiedemann, C. R. G.}

Diptera exotica, 1 vol. 8vo.

Analecta entomologica, 1824, 4to.

Willoughby de Eresby (F.), born in 1635, and died in 1672 , an English gentleman and a zealous naturalist.

"Ornithologix, lib. III." I vol. folio, London, 1676; published by Ray from his posthumous papers. It was translated by Salerne with additions, in 1 vol. 4 to. Paris, 1767.

"Historia Piscium, lib. IV." 2 vols. folio. Oxford, 1685 .

The plates of these two works are mostly copied from other authors.

Wils.-Wirson (Alexander), an American naturalist, born in $\mathbf{1 7 6 6 ,}$ and died in 1813.

"American Ornithology," with coloured 
plates, 9 vols. 4to. Philadelphia, $1808-1814$.

A new edition, 3 vols. 4to. appeared in 1828.

Wolf (J. F.), a German naturalist.

"Icones Cimicum Descriptionibus Illustratæ," 4 Nos. 4to. Erlangæ, 1804.

WoLfF, joint author with MEYeR of the "Almanack of German Birds."

WORM. or MUS. WORM.-WORmius, or Worm. Olaus, Professor at Copenhagen, born in 1588, died in 1654 .

"Museum Wormianum," 1 vol. folio. Leyden, 1650.

YARR.-YARReL, an English naturalist, author of various papers in the Zoological Journal of London, \&c.

ZED.-ZEDER (J. G. H.), a German naturalist.
Author of "First Supplement to the Natural History of Intestinal Worms by Goeze,"I vol. 4to. Leipzic, 1800.

"An Introduction to the Natural History of the Intestinal Worms," l vol. 8vo. Bamberg, 1803.

Zetterst.-Zettersted (J. G.), a Swedish naturalist.

"Orthoptera Sueciæ," I vol. 8vo. Lundæ, 1811.

"Fauna Laponica," part first, 1 vol. 8vo. Hammone, 1828.

ZOOL. JourN.

Published in London by M. Vigors, aided by Messrs. TH. BeLL, E. T Bennet, J. E. Bicheno, W. J. Broderip, J.G. Children, Gen. T. H. Hardwicke, Dr. Horsefield, W. Kirby, the Messrs. Sow and son, and W. YARrell. We have sixteen numbers, from 18- to 1829.

Zorgdr.-ZorgDrager,a Dutchman.

Author of a treatise on the whale fishery.

THE END.

GILBERT \& RIVINGTON, PRINTERS,

St. John's Square, London. 


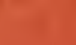

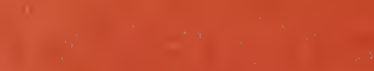

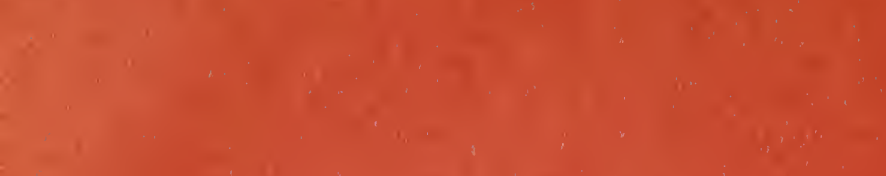

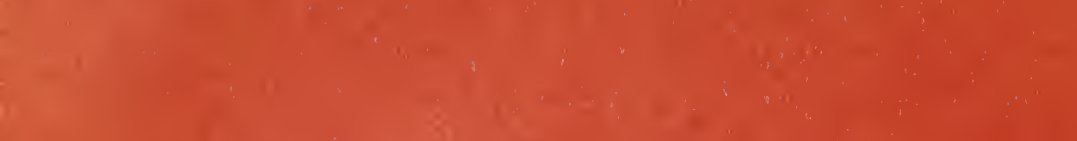

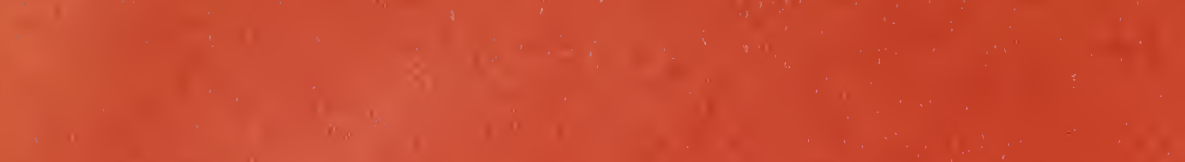

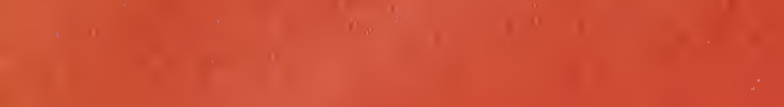

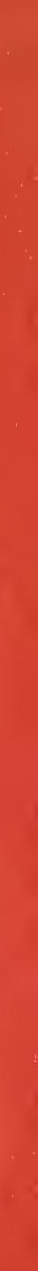

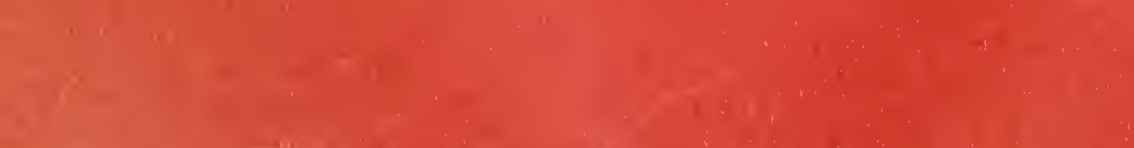

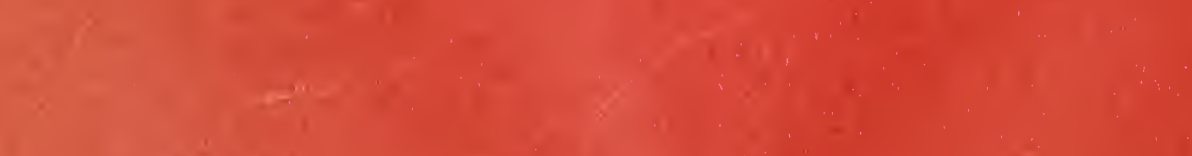

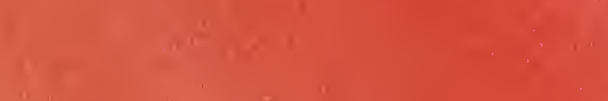

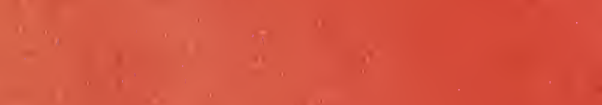
8

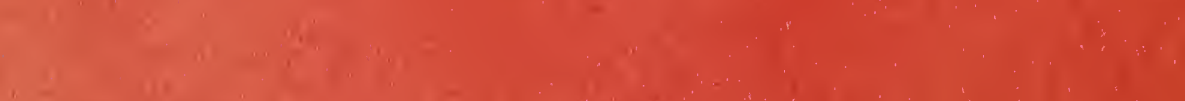

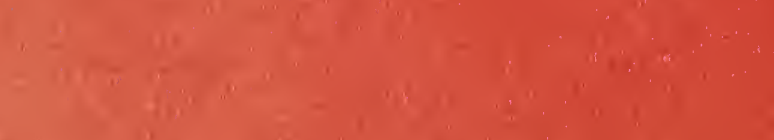

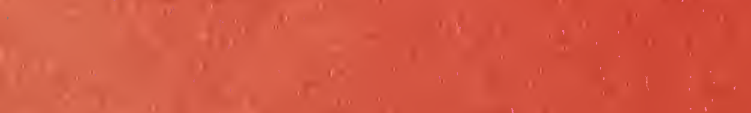
ix: . : 8 
A

\title{
TABULAR VIEW
}

$$
\text { OF THE }
$$

\section{CLASSIFICATION OF ANIMALS}

\author{
ADOPTED \\ BY THE BARON CUVIER;
}

WITH

SPECIFIC EXAMPLES。 



\section{CONSPECTUS}

OF THE

\section{ENTIRE ANIMAL KINGDOM.}

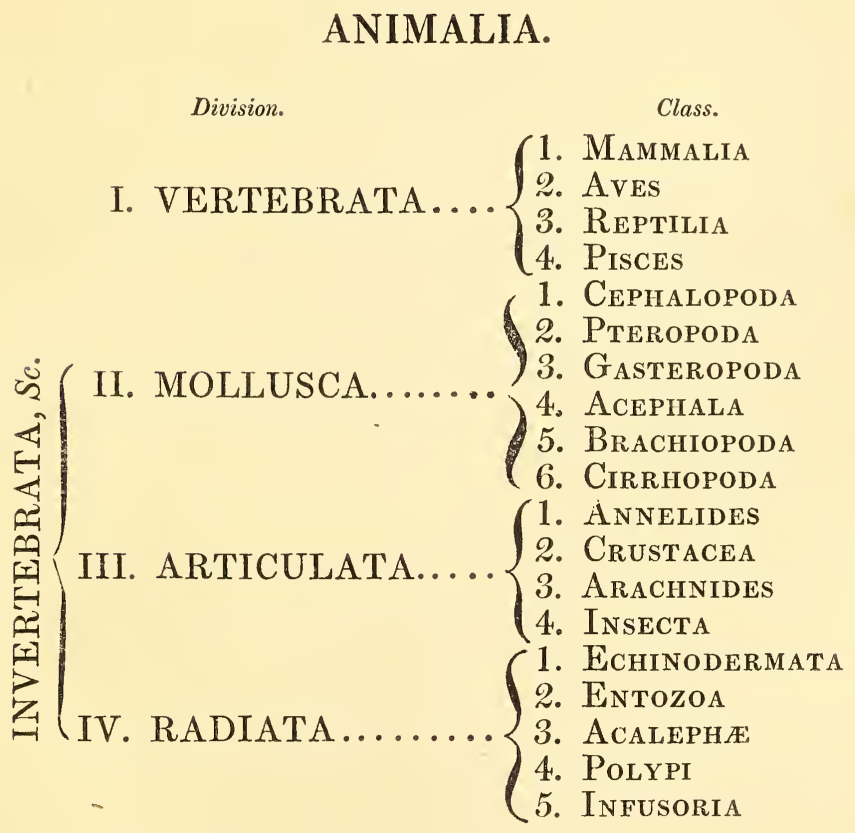

All animals may be reduced to one or other of these classes. It will be observed, that though the term 'Invertebrata' is introduced, it is not employed as significant of any peculiar form. The reason of this is, that it is merely a negative character, and such should never be used where positive can be obtained. Its vagueness is evident from its belonging to three out of four of the great primary divisions of the 'Animal Kingdom.' 


\section{VERTEBRATA.}

\section{CLASS I.-MAMMALIA.}

This class is divided into nine orders.

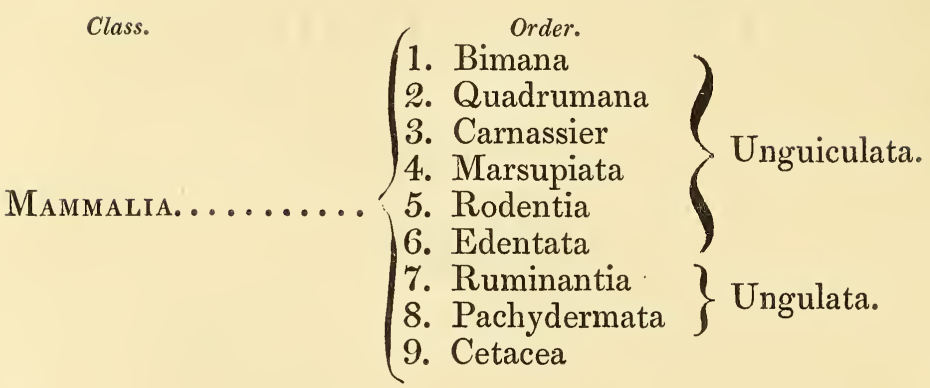

With respect to the term Carnassier, used by Cuvier for his third order, some observations will be found in our second volume, at the commencement of the supplementary matter on the order.

\section{ORDER I.-Bimana.}

This order contains only one genus, and a single species, with its varieties, as here under:

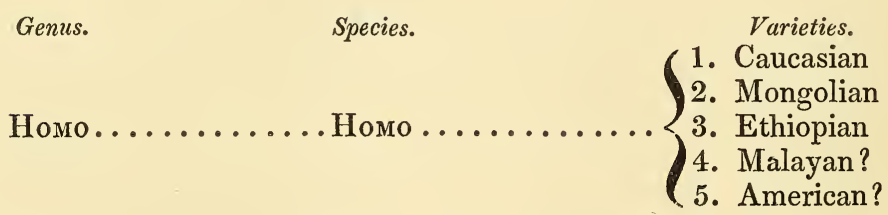

Only the first three varieties are considered by Cuvier as possessing sufficiently distinctive characters to deserve separate description. "The Malays," he says, "pass by imperceptible shades into their neighbours, the Caucasian Hindoos on one side, and the Mongolian Chinese on the other." The Americans also want that precise and constant character necessary to constitute a peculiar race. For these reasons we have marked the two last varieties with a query.

In this order we have given even the varieties; in the others we can go no farther than the subgenera, with one specific example of each : our space compels us to this arrangement. 


\title{
ORDER II.-QuAdRUMANA.
}

Of this order three principal genera are enumerated, viz. I. Simia, Lin., II. Hapale, Illig., and III. Lemur, Lin.; the first and last of these contain so many subgenera and species that they almost deserve to rank as distinct families, for Simia has fifteen subgenera, Lemur five, while Hapale has only two, which however contain more than eight different species.

\author{
Genus. \\ Subgenus. \\ Example of Species. \\ 1. Pithecus, Geoff. ..... Simia satyrus, L. Ourang outang \\ 2. Hylobates, Illig..... S. lar, L. Gibbon \\ 3. Cercopithecus, Erxl. S. Sabæa, L. Green monkey \\ 4. Semnopithecus, F. $\}$ S. Maura, L. Negro monkey \\ 5. Macacus, Lacép. . ...S. Sinica, Gm. Chinese monkey \\ 6. Inuus, Cuv. ......... S. sylvanus, L. Barbary ape \\ 7. Cynocephalus, Cuv. S. cynocephalus, L. Little baboon \\ I. Simia, Lin... $\left\{\begin{array}{l}\text { 8. Papio, Briss. ....... S. maimon, L. Mandrill } \\ \text { 9. Mycetes, Illig. .... S. seniculus, Redhowling monkey }\end{array}\right.$ \\ 10. Ateles, Geoff..... \{ A teles arachnoïdes, Geoff. Spi.- \\ 11. Lagothrix, Geoff. . Lag. Humboldtii, Geoff. Capparo \\ 12. Cebus, Geoff.......... apella, L. Weeper monkey \\ 13. Callithrix, Cuv. ..... S. sciureus, L. Squirrel monkey \\ 14. Pithecia, Desm. .....S. lugens, Humb. Widow monkey \\ 15. Nocthora, F. Cuv. $\left\{\begin{array}{l}\text { Noct. trivirgata, F. Cuv. Dourou- } \\ \text { couli }\end{array}\right.$ \\ II. Hapale, Illig. $\left\{\right.$ 1. Jacchus, Geoff. .. $\left\{\begin{array}{l}\text { S. Jacchus, L. Stunted monkey, } \\ \text { or Jacchus }\end{array}\right.$ \\ 2. Midas, Geoff.......... rosalia, L. Lion monkey \\ III. Lemur, Lin.. $\left\{\begin{array}{l}\text { 1. Lemur, Cuv. ...... Lem. catta, L. Ring-tailed lemur } \\ \text { 2. Lichanotus, Illig. . Lem. indri, Sonn. } \\ \text { 3. Stenops, Illig......... gracilis, Slender loris } \\ \text { 4. Galago, Geoff...... . potto, Gm. the African potto } \\ \text { 5. Tarsius, Storr..... . spectrum, Pall. }\end{array}\right.$
}

This is according to the classification followed by Cuvier in the second edition of his Règne Animal, published in 1829 .

The first eight subgenera of Simice inhabit the old world, the remainder are found in the new, and have received the general appellation of Sapajous (Cebus $E r x l$ ). 


\section{ORDER III.-CARNASSIER.}

This order is divided into three great families, Cheiroptera, Insectivora, and Carnivora, the last of which is subdivided into three tribes, Plantigrada, Digitigrada, and Amphibia. In the first edition of the Règne Animal the Marsupiata were added as a fourth family of this order; they now, with greater propriety, constitute an order by themselves.

Family. Genus. Subgenus. Example of Species.

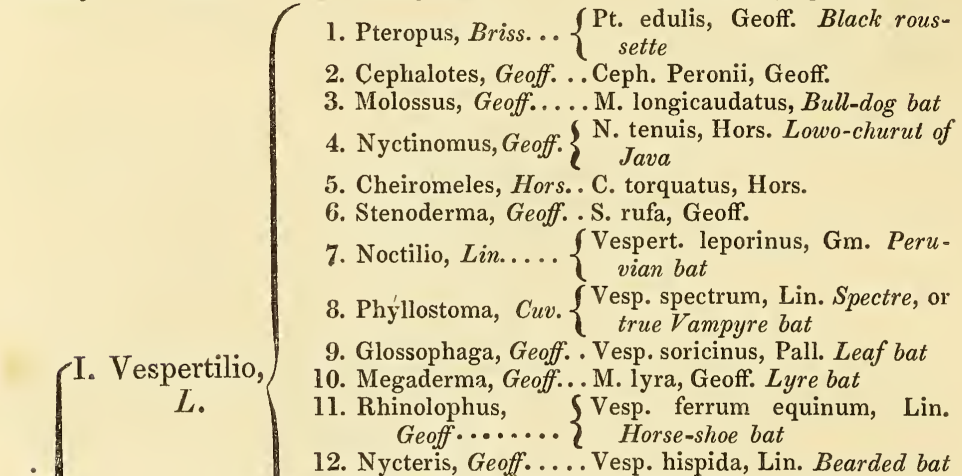

$$
\begin{aligned}
& \text { 13. Rhinopoma, Geoff. . R. microphylla, Desm. } \\
& \text { 14. Taphozous, Geoff. }\left\{\begin{array}{l}
\text { Vesp. lepturus, Schr. Pouched } \\
\text { bat, or Slender-tailed bat }
\end{array}\right. \\
& \text { 15. Mormoops, Leach ... M. Blainvillii, Leach }
\end{aligned}
$$

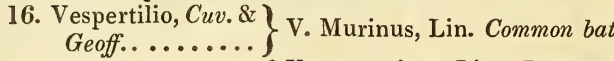

$$
\begin{aligned}
& \text { 17. Plecotus, Geoff... }\left\{\begin{array}{c}
\text { Vesp. auritus, Lin. Long-eared } \\
\text { bat }
\end{array}\right. \\
& \text { 18. Nycticeius, Rafin. }\left\{\begin{array}{c}
\text { Vesp. lasiurus, Schr. Rough- } \\
\text { tailed bat }
\end{array}\right. \\
& \text { II. Galeopithecus, Pall. ...... } \begin{array}{c}
\text { Lemur volans, Lin. Flying ma- } \\
\text { cauco, or Flying colugo }
\end{array} \\
& \text { Family. Genus. Example of Species. }
\end{aligned}
$$

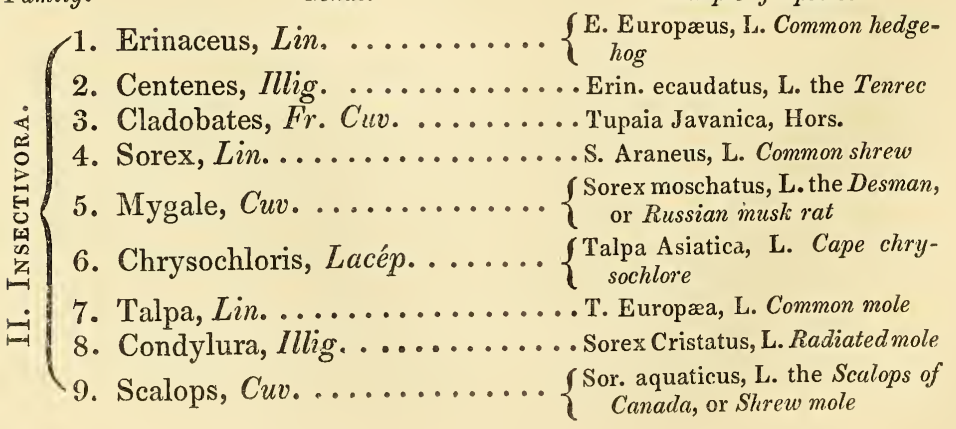


ORDER III.-CARNASSIER (continued).

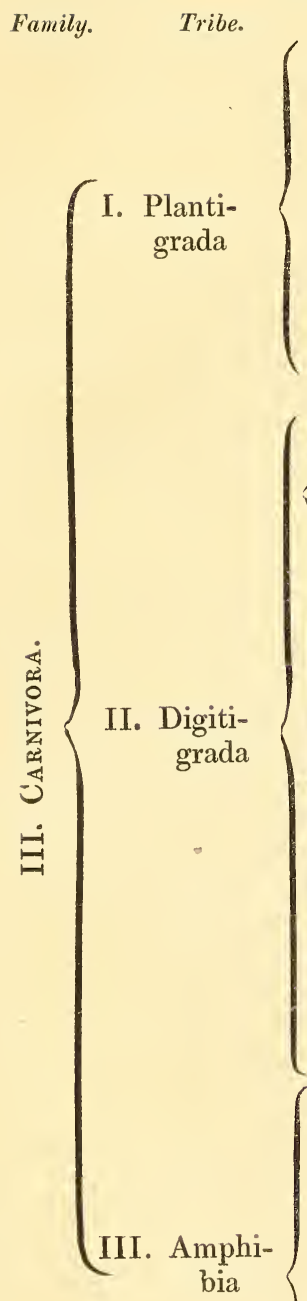

\section{Genus. $\quad$ Example of Species.}

1. Ursus, Lin... . . U. arctos, L. Common brown bear

2. Procyon, Storr. Ursus lota, L. Racoon

3. Ailurus, Fr.Cuv. A. refulgens, Fr. Cuv.

4. Ictides, Valenc. I. albifrons, Fr. Cuv.

5. Nasua, Storr... Viverra nasua, L. Red coati

6. Cercoleptes, Viv. caudivolva, Gm. the AmeIllig. .... $\}$ rican potto

7. Meles, Storr... Ursus meles, L. Common badger

8. Gulo, Storr.... Ursus gulo, L. the Glutton

9. Ratellus, Fr. 2 Cuv...... $\}$ Viv. mellivora, L. Honey ratel

1. Putorius, ZMustela putorius, L. Common Cuv...... $\}$ pole cat

2. Mustela, Cuv. M. martes, L. Common marten

3. Mephitis, Cuv. M. Americana, Desm. Skunk

4. Lutra, Storr. Must. lutra, Common otter

5. Canis, Lin. . . C. familiaris, L. the Dog

6. Vivera, Cuv... V. civetta, L. the Civet

7. Genetta, Cuv. Viverra genetta, L. Common genet

8. Paradoxurus, $\{$ P. typus, Fr. Cuv. Common paFr. Cuv. . \{ $\begin{aligned} & \text { radoxurus, or Palm martin of } \\ & \text { the French }\end{aligned}$

9. Herpestes, YViv. ichneumon, L. the IchneuIllig...... $\}^{\text {mon }}$

10. Ryzæna, Illig. Viv. tetradactyla, L. the Surikate

11. Crossarchus, Fr. Cuv. ... $\}$ C. obscurus, Fr. Cuv.

12. Proteles, Is. $\}$ P. Lalandii, Is. Geoff. Geoff. ..... $\}$ P. Lalandii, Is. Geoff.

\{13. Hyæna, Storr. Canis hyæna, L. Striped hyæna 14. Felis, Lin...... F. leo, L. the Lion

1. Phoca, Cuv. .. P. vitulina, L. Common seal

2. Stenorhyncus, Phoc. leptonyx, Blainv. SmallFr. Cuv. .. $\}$ clawed seal

3. Pelagus, Fr. $\{$ Ph. monachus, Gm. White-belCuv.......\} lied seal

4. Stemmatopus, Ph. cristata, Gm. Hooded seal Fr. Cuv. ...

5. Macrorhyna, $\mathrm{Ph}$. leonima, L. Sea lion, or sea Fr. Cuv. .. elephant

6. Otaria, Peron. Ph. ursina, L. Common sea bear

7. Trichecus, Lin. $\left\{\begin{array}{l}\text { T. rosmarus, L. Morse, sea cow, } \\ \text { sea horse, \&c. }\end{array}\right.$

The tribe Digitigrada contains three subdivisions, distinguished by their mode of dentition, and represented by the weasel, the dog, 
and the cat: these we have endeavoured to indicate above by smaller brackets, within the general bracket including all the genera of the tribe. The Amphibia also formerly made but two genera, the seals (Phoca) and the Morses (Trichecus), the first of which we see Cuvier has here extended to several subdivisions.

\section{ORDER IV.-MARSUPIATA.}

The arrangement of these animals, chiefly inhabitants of the Australian continent, seems by no means decided. In his first edition Cuvier had made of them a family of the order Carnassier; in the second he established them into a separate order; but even then expressed his opinion that they ought rather to be looked on as a class containing several orders running parallel with the orders of ordinary quadrupeds. He finally, however, left them as an order divided into seven genera, three of which are subdivided.

Genus.

1. Didelphis, L. \{

11. Dasyurus, Geoff.....

Subgenus.

Example of Species.

1. Didelphis, prop. $\}$ D. Virginiana, Penn. Virginian Cuv........... opossum

2. Chironectes, Illig. $\{$ Did. palmata, Geoff. Yapock, or 1. Thylacini, Tem.. \{ Dasyurus cynocephalus, Geoff. . Tog-faced dasyurus

2. Phascogali, Tem. . $\left\{\begin{array}{c}\text { Das. minimus, Geoff. Dwarf } d a- \\ \text { syurus }\end{array}\right.$

3. Dasyurus, prop. Cuv. Didelphis ursina, Harr. the Devil

4. Perameles, Geoff. $\{$ P. nasutus, Geoff. the Long-

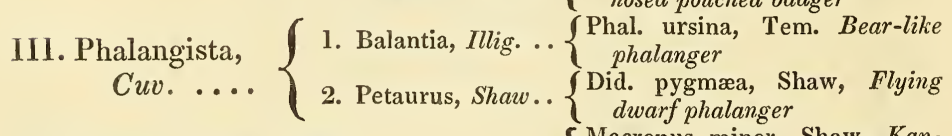

IV. Hypsiprimnus, Illig........ $\begin{gathered}\text { Macropus minor, Shaw, Kan- } \\ \text { garoo rat }\end{gathered}$

V. Macropus, Shaw .......... M. major, Shaw, Gigantic kan-

VI. Lipurus, Goldfuss........... $\left\{\begin{array}{c}\text { L. cinereus, Goldfuss. Koala, or } \\ \text { New Holland sloth }\end{array}\right.$

VII. Phascolomys, Geoff............ Did. ursina, Shaw, the Wombut

\section{ORDER V.-RODENTIA.}

In his first edition Cuvier had divided this order into two sections, the first possessing perfect, the second only rudimentary clavicles : these divisions ran by such gradual shades into each other, that the point of separation was, we may say, almost arbitrary. In his second edition, therefore, he contents himself by arranging his genera and indicating such as possess strong clavicles or the reverse. 


\section{ORDER V.-Rodentia.}

Genus.

Subgenus.

Example of Species.

I. Sciurus, L. $\left\{\begin{array}{l}\text { 1. Sciurus, prop. Cuv. Sc. vulgaris, L. Common squirrel } \\ \text { 2. Pteromys, Cuv... } \begin{array}{l}\text { Sc. volans, L. European fying } \\ \text { squirrel }\end{array} \\ \text { 3. Cheiromys, Cuv.. } \begin{array}{l}\text { Sc. Madagascariensis, Gm. the } \\ \text { Aye-aye }\end{array}\end{array}\right.$

1. Arctomys, Gm... Mus Alpinus, L. Marmot of the

2. Myoxus, Gn.......Mus glis, L. the Dormouse

3. Echimys, Geoff... \{ E. spinosus, Desm. Spiny rat of

4. Hydromys, Geoff. ... H. leucogaster, Geoff.

5. Capromys, Desm... C. Fournieri, Desm.

6. Mus, prop., Cuv. . . M. rattus, L. the Rat

II. Mus, L... 7. Gerbillus, Desm.. $\left\{\begin{array}{c}\text { Dipus tamaricinus, Gm. Tama- } \\ \text { risk rat }\end{array}\right.$

8. Meriones, Fr. Cuv. \{ Mus Labradorius, Sabine, La-

9. Cricetus, Cuv...... Mus cricetus, L. Common hamster

10. Fiber, Cuv...... \{ Mus Zibeticus, Gm. Ondatra, or

10. Fiber, Cuv..... $\begin{array}{r}\text { Musk rat of Canada } \\ \text { Mus }\end{array}$

11. Arvicola, Cuv. ....Mus amphibius, L. Water rat

12. Georychus, Illig. . . Mus lemmus, L. the Lemming

13. Otomys, Fr. Cuv...O. capensis, Fr. Cuv.

14. Dipus, Gm. ......Mus sagitta, L. the Jerboa

III. Helamys, Fr. Cuv............ Dipus Caffer, Gm. Cape jerboa

IV. Spalax, Guldenstedt ........... Mus typhlus, Pall. Blind rat-mole

V. Bathyergus, Illig............. Mus capensis, Gm. Cape mole

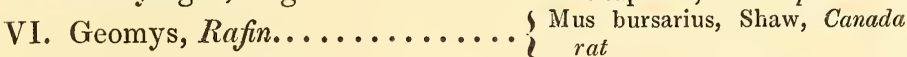

VII. Diplostoma, Rafin. ......... D.

? Rafin. Tailless rat

VIII. Castor, L................ fiber, L. the Beaver

IX. Myopotamus, Comm............Mus coïpus, Molin. the Couïa

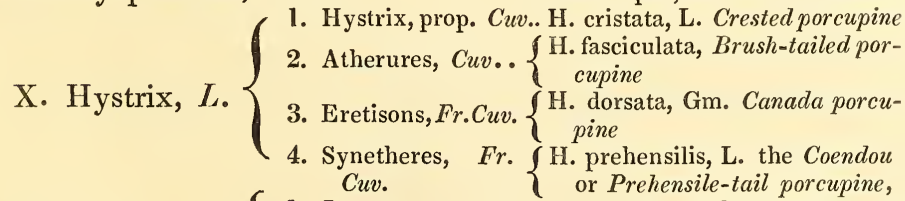

XI. Lepus, L.. $\left\{\begin{array}{l}\text { 1. Lepus, prop. Cuv... L. timidus. L. the Common hare } \\ \text { 2. Lagomys, Cuv. .. }\left\{\begin{array}{c}\text { Lep. pusillus, Pall. the Calling } \\ \text { hare }\end{array}\right.\end{array}\right.$

XII. Hydrochærus, $\operatorname{Erx}$. . . . . . . Cavia capybara, L. the Capybara XIII. Cavia, Illig................... cobaya, Pall. the Guinea-pig XIV. Dasyprocta, Illig......... $\left\{\begin{array}{c}\text { D. acuti, Jll. the Agouti, or Long- } \\ \text { nosed cavy }\end{array}\right.$ XV. Cœlogenys, Fr. Cuv. .......... Cavia paca, L. the Paca

Cuvier suggests that the Chinchilla, the furs of which arrive in such quantities in these countries, should, from its evident connection with the cavies, come at the end of this order. Nothing but want of 
information respecting its dentition prevented him from placing it there. The Marmot Diana, of which we gave a figure in our third volume, p. 172, he says is the same as the Viscache of Azzara, and considers it merely as a larger kind of Chinchilla, with rougher and longer hair.

\section{ORDER VI.-Edentata.}

The Edentata Cuvier divides into three tribes, the Tardigrades, including the first two genera; the common Edentata, including the next four; and the Monotremata, including the remaining two.
Tribe. Genus.
Subgenus.
Example of Species.
1. Acheus, Fr. Cuv. \{ Brad. tridactylus, L. the $A \ddot{i}$, or pus, $L$. \{
2. Bradypus, prop. \{ B. didactylus, L. the Unau, or Fr. Cuv...... two-toed sloth
1
II. Megatherium, Cuv.... \{ $\begin{gathered}\text { Megatherium, C. Great fossil } \\ \text { animal }\end{gathered}$
1. Cachicames, Cuv. $\left\{\begin{array}{c}\text { Das. 9-cinctus, L. Nine-banded } \\ \text { armadillo }\end{array}\right.$
2. Apara, Cuv. ... \{ Das. 3-cinctus, L. Three-banded
III. Dasy-?
S Das. 6-cinctus, L. Six-banded pus, $L$.
3. Encouberts, Cuv. $\left\{\begin{array}{c}\text { Das. 6-cinctus } \\ \text { armadillo }\end{array}\right.$
4. Priodontes, Fr, Cuv. Das gigas, L. Giant armadillo
5. Chlamyphorus, Harl.C. truncatus, Harl.
IV. Orycteropus, Geoff...... 0 . capensis, Illig. Cape ant-
....
V. Myrmecophaga, L.... $\begin{gathered}\text { M. jubata, L. Great or maned } \\ \text { ant-eater, bear ant-eater }\end{gathered}$

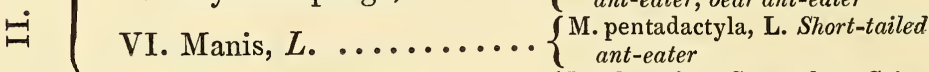

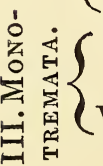
S. hystrix, Cuv. the Spiny $\left\{\begin{array}{l}\text { E. hystrix, Cuv. the Spiny } \\ \text { echidna, or porcupine ornitho- }\end{array}\right.$ rynchus
O. paradoxus, Blain. the Duck-
VIII. Ornithorynchus, Blum... $\begin{array}{r}\text { O. paradoxus, } \\ \text { billed platypus }\end{array}$

\section{ORDER VII.-Pachydermata.}

This order is also divided into three tribes or families, I. the Proboscidiana, or those furnished with a proboscis, including the elephant and some fossil animals; II. the common Pachydermata, including all the rest of the order except the horse, which belongs to family III., Solipeda, or solid-footed.
Family.
Genus.
Example of Species.
I. Proboscidiana $\left\{\begin{array}{l}\text { 1. Elephas, L...... Indicus, Cuv. Indian elephant } \\ \text { 2. Mastodon, Cuv ... Mastodon, Cuv. (fossil) }\end{array}\right.$ 


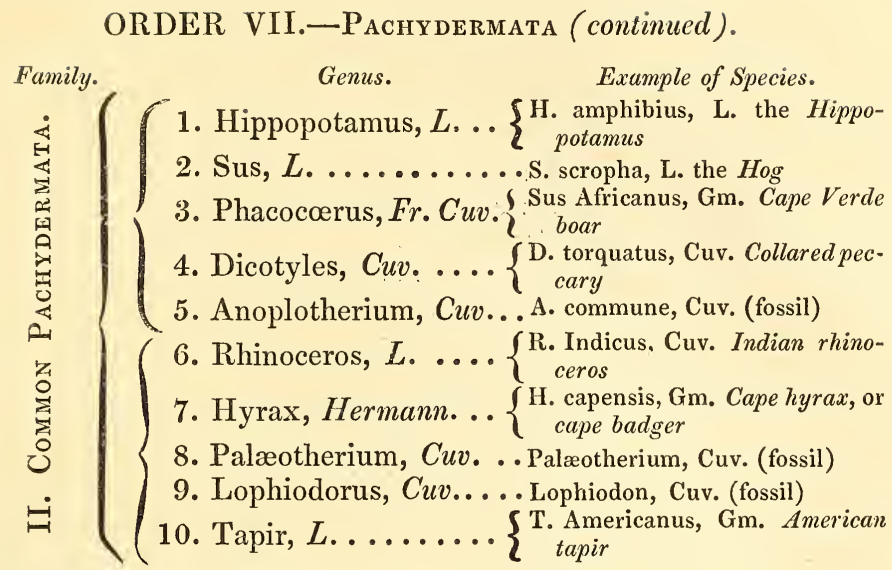

III. Solipeda . . Equus, L........ E. Caballus, L. the Horse

The common Pachydermata we have marked off into two subdivisions, in the first of which the toes are of an even number, four or two, and assume something of a bifurcated appearance, approaching, as do also in some measure their skeletons and stomachs, the forms proper to ruminant animals; in the second subdivision the toes are of an odd number, and want this appearance. Sus, Phacocœrus, and Dicotyles, are merely subgenera of the Linnæan genus Sus, which name we have left to the first of them.

\section{ORDER VIII.-Ruminantia.}

This order is naturally distinguished into two families, those without and those with horns. To the former belong the camel and musk; to the latter all the other genera. Horns are of three kinds, specimens of which may be seen in the deciduous antler of the stag, the permanent invested protuberances of the cameleopard, and the true horn or hollow corneous case of the cow, sheep, antelope, \&c. These differences have been employed to subdivide the second family. Col. Hamilton Smith divides the whole order into five tribes, the types of which are the camel, the stag, the giraffe, the goat, and the ox. For further particulars see our fifth volume, p. 296, et seq.

$$
\text { Family. Genus. Example of Species. }
$$

I. Akeratophora, $\left\{\right.$ 1. Camelus, L... $\begin{array}{l}\text { C. Bactrianus, L. the Bactrian } \\ \text { camel }\end{array}$

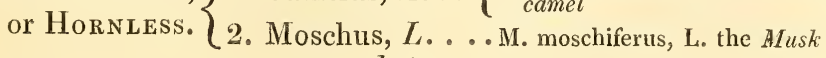


OR DER VIII.-Ruminantia (continued).
Family.
Genus.
Example of Species. 2. Camelopardalis, L.C. girafa, Fr. Cuv. the Giraffe
II. Keratophora, Antelope, L. ....A. dorcas, L. the Gazelle 4. Capra, L. ........ æ. ægagrus, Gm. the Wild goat 5. Ovis, L.......... Ammon, L. the Argali 6. Bos, $L$. . . . . B. taurus, L. the Common ox

\section{ORDER IX.-Cetacea.}

Here also we have two families, the herbivorous cetacea, including the first three genera, and the common cetacea, comprehending all the rest. This latter family is again subdivided, according as the head is of the ordinary proportion to the body, as we see in Delphinus and Monodon, or is disproportionately large, as is the case in Physeter and Balæna.
Genus.
Subgenus.
Example of Species.

I. Manatus, Cuv................ $\begin{gathered}\text { Trichecus manatus, L. the } M a- \\ \text { nati, Sea-cow, or Lamantin }\end{gathered}$

II. Halicore, Illig. ........... . Indicus, Ill. the Dugong

(III. Rytina, Illig. ......... \{ $\begin{gathered}\text { Trich. borealis, Sh. Whate- } \\ \text { tailed manati }\end{gathered}$

IV. Delphinus prop. Cuv. D. delphis, L. Common dolphin

IV. Delphinus, $\left\{\begin{array}{l}\text { 2. Phocæna, Cuv...... Delph. phocæna, L. the Porpoise } \\ \text { 3. Delphinapterus, Lac. D. leucas, Gm. the Beluga } \\ \text { 4. Hyperoodon, Lac. }\end{array}\right.$
V. Monodon, L. $\ldots \ldots \ldots \ldots \ldots \ldots\left\{\begin{array}{c}\text { Balæna rostrata, Klein. Bottle- } \\ \text { nosed whale } \\ \text { or Sea-unicorn }\end{array}\right.$
VI. Physeter, L. the Narwal,

VII. Balæna, L. \{1. Balæna, prop. Lacép. B. mysticetus, L. Common whale

2. Balænoptera, Lacép. B. musculus, L. the Rorqual. 


\section{VERTEBRATA.}

\section{CLASS II.-AVES.}

This class comprises six orders.

Class.

Order.

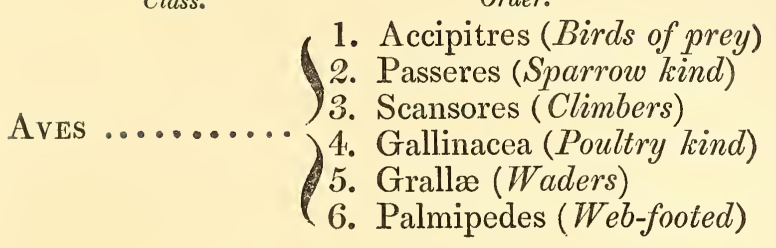

\section{ORDER I.-AccipITRES.}

This order includes birds generally known as birds of prey; and is divided by Cuvier into the two natural families of diurnal and nocturnal; the former including the two great genera of Linnæus, the vultures and falcons; the latter, his genus of owls. These genera Cuvier has subdivided as under.

Genus. Subgenus. Example of Species.

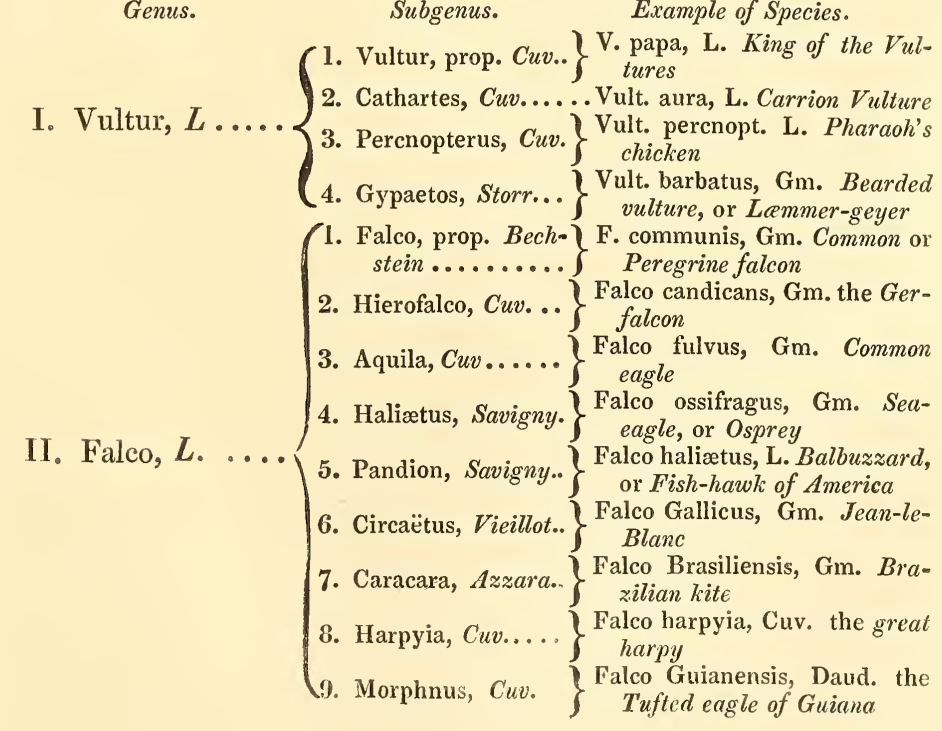




\title{
ORDER I.-Accipitres (continued).
}

\author{
Genus. \\ Subgenus. \\ Example of Species.

\begin{tabular}{|c|c|c|}
\hline Geñus. & Subgenus. & Example of Species. \\
\hline & (10. Cymindis, Cuv... & $\begin{array}{l}\text { Falco Cayennensis, Gm. Small } \\
\text { Cayenne eagle }\end{array}$ \\
\hline & $\begin{array}{l}\text { 11. Astur, Bechstein... } \\
\text { 12. Nisus, Cuv....... } \\
\text { 13. Milvus, Bechstein. }\end{array}$ & $\begin{array}{l}\text { Falco palumbarius, L. Goshawk } \\
\text { Falco nisus, L. Sparrow-hawk } \\
\text { Falco milvus, L. Common kite }\end{array}$ \\
\hline II. Falco, & 14. Pernis, Cuv.... & $\begin{array}{l}\text { Falco apivorus, L. Common } \\
\text { honey-buzzard }\end{array}$ \\
\hline (contint & 15. Buteo, Bec & $\begin{array}{l}\text { Falco buteo, L. Common buz- } \\
\text { zard }\end{array}$ \\
\hline & $\begin{array}{l}\text { 16. Circus, Bechstein. } \\
\text { 17. Serpentarius, Cuv. }\end{array}$ & $\begin{array}{l}\text { Falco cyaneus, Gm. Hen-har- } \\
\text { rier } \\
\text { Falco serpentarius, Gm. Snake- } \\
\quad \text { eater, or Secretary }\end{array}$ \\
\hline & 1. Otus, $C$ & $\begin{array}{l}\text { Str. otus, L. Common long- } \\
\text { eared owl }\end{array}$ \\
\hline & 2. Ulula, Cuv & $\begin{array}{l}\text { Str. laponica, Gm. Great gray } \\
\text { howler }\end{array}$ \\
\hline Strix & $\left.\begin{array}{l}\text { 3. Strix, prop. } S a- \\
\text { vigny .......... }\end{array}\right\}$ & $\begin{array}{l}\text { Str. flammea, L. Common white } \\
\text { or barn owl }\end{array}$ \\
\hline & $\begin{array}{l}\text { 4. Syrnium, Savigny. } \\
\text { 5. Bubo, Cuv ........ } \\
\text { 6. Noctua, Savigny... }\end{array}$ & $\begin{array}{l}\text { Str. stridula, L. the wood-owl } \\
\text { Str. bubo, L. Great horned owl } \\
\text { Str. surnia, Dum. Hawk-owl }\end{array}$ \\
\hline & 7. Scops, Savigny .. & $\begin{array}{l}\text { Str. leucotis, Tem. White-eared } \\
\text { owl }\end{array}$ \\
\hline
\end{tabular}

ORDER II.-Passeres.

This order is not clearly defined, nor does it appear very well divided. As to the former, it seems to contain every bird that does not belong to any other of the orders ; as to the latter, Cuvier appears to have done whatever was possible, under the present state of knowledge, by using for his primary divisions the nature of the feet, and for his secondary, or division into families, the peculiarities of the bill. As respects the feet, they are divided into those ${ }_{j}$ which have the external toe united to the internal only as far as the first or second phalanx, and those called Syndactyles, in which the union extends to all but the last articulation. The first of these divisions is by far the most numerous, and contains the families Dentirostres, Fissirostres, Conirostres, and Tenuirostres, with their several genera and subgenera, as underneath: the second forms but one family, containing six genera. 


\section{ORDER II.-PAsserEs.}

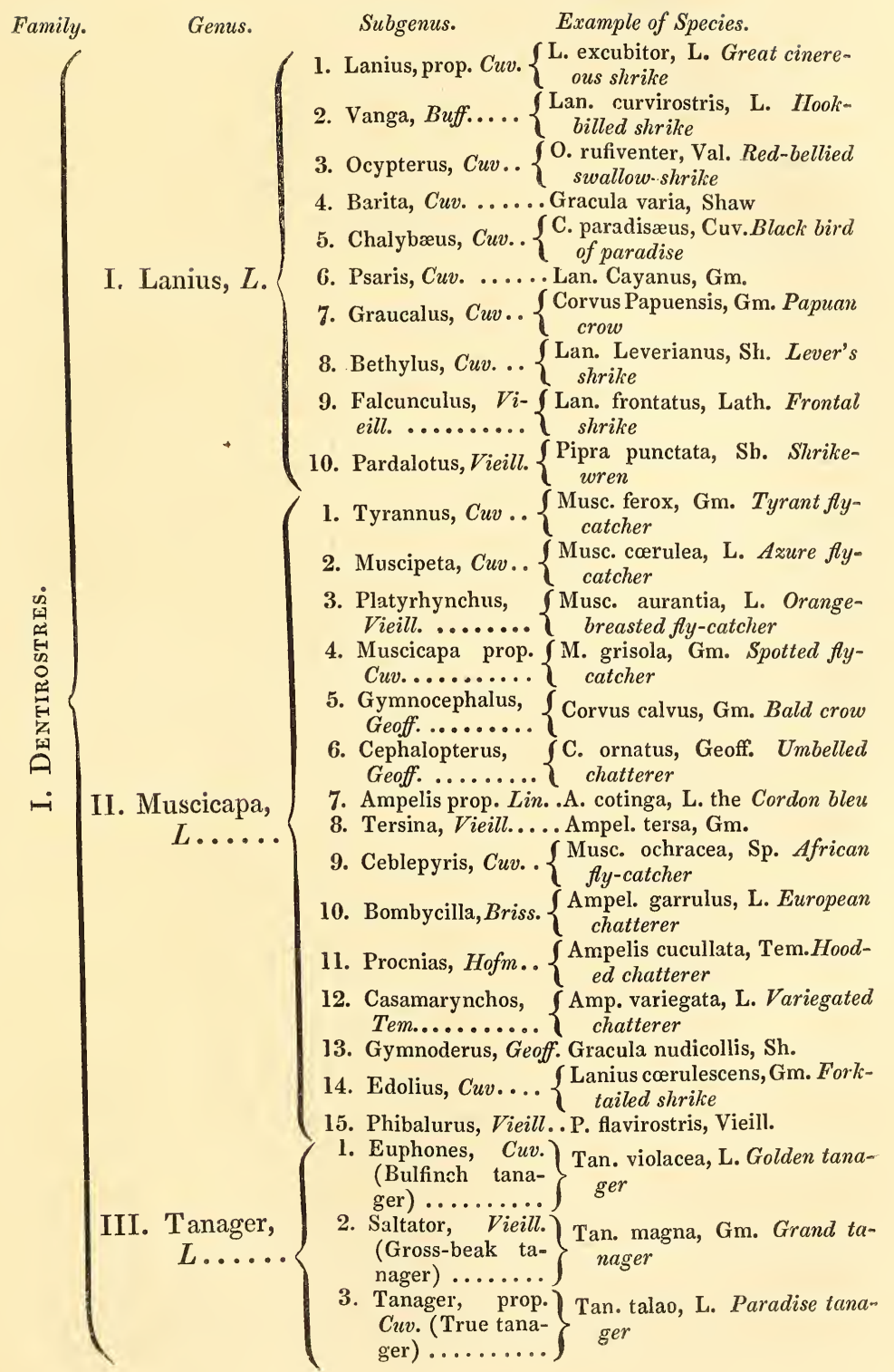


ORDER II.-Passeres (continued).

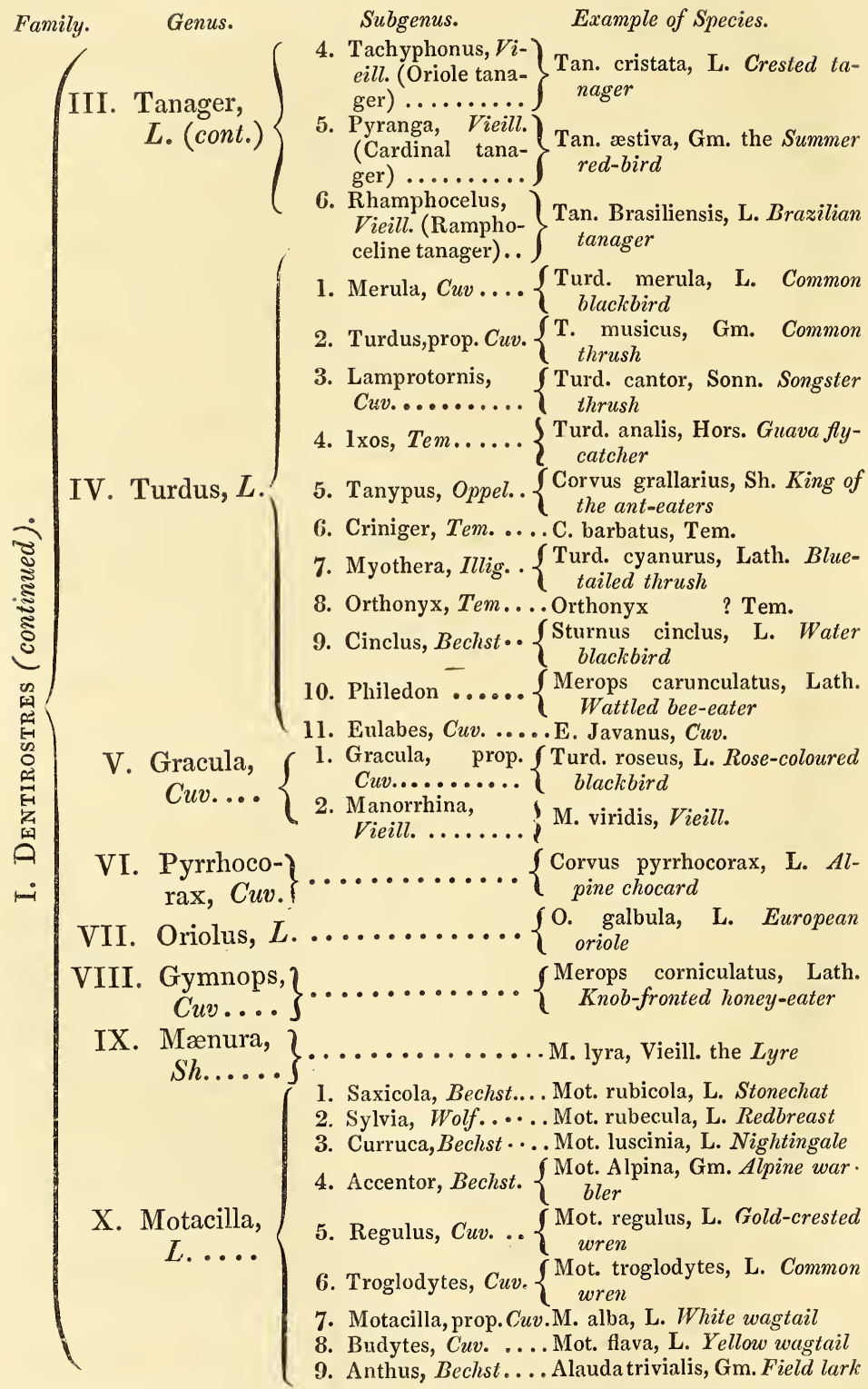




\section{ORDER II.-Passeres (continued).}

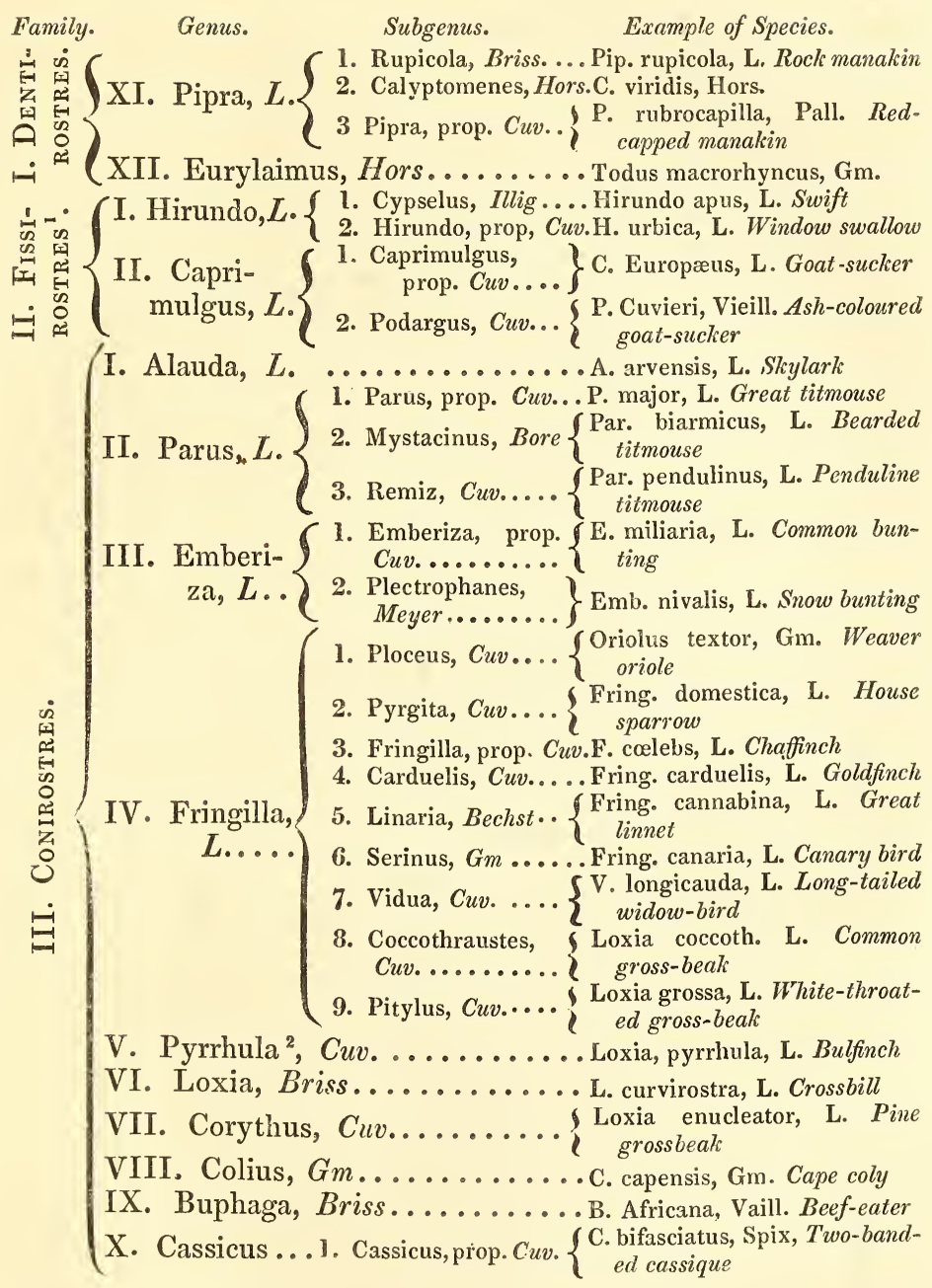

1 The two genera of this family, like the two grand subdivisions of the Accipitres, present a natural distinction of habit, the first being birds that seek their prey by day, the second only by night.

2 It does not seem very evident from the text, whether Pyrrhula should be a genus, or only a subgenus of Fringilla; in truth, the transitions, particularly in this family, are often so gradual as to elude all attempts at definition. 


\section{ORDER II.-PASsERES (continued).}

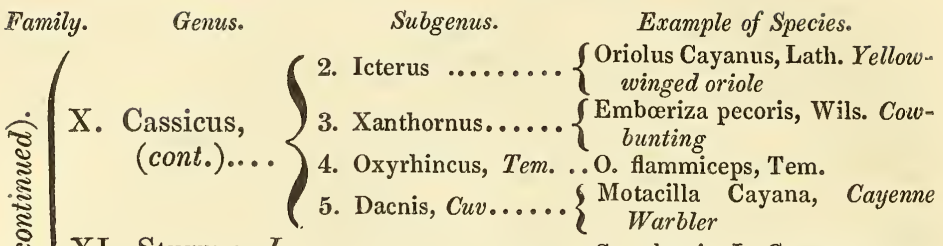

XI. Sturnus, L............... S. vulgaris, L. Common stare

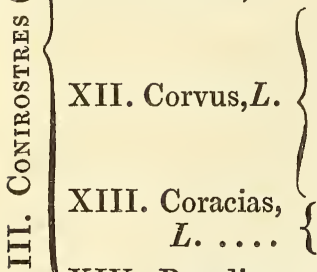

1. Corvus, prop. Cuv...C. frugilegus, L. Rook

2. Pica, Cuv. ......... Corv. pica, L. Magpie

3. Garrulus, Cuv...... Corv. cristatus, L. Blue jay

4. Caryocatactes, Cuv.. Corv. caryocat. L. Nutcracker

5. Temia, Vaill.... $\left\{\begin{array}{l}\text { Corv. varians, Lath. Variable } \\ \text { crow }\end{array}\right.$

6. Glaucopis, Forst....G. cinerea, Lath. Wattle-bird XIII. Coracias, (1. Coracias, prop. Cuv. .C. garrula, L. Garrulous roller

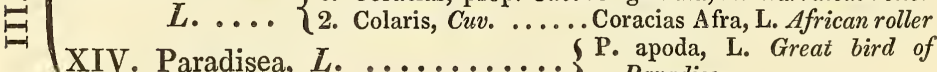

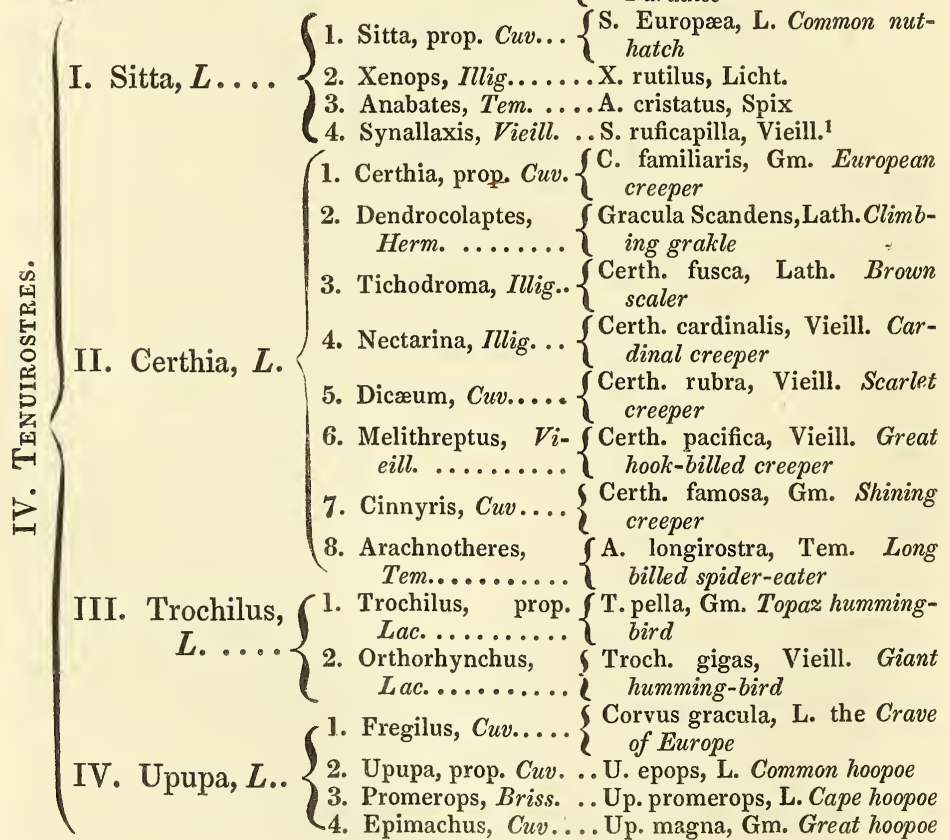

1 These being all South American species, have no English name. 
ORDER II.-PASSERES (continued).

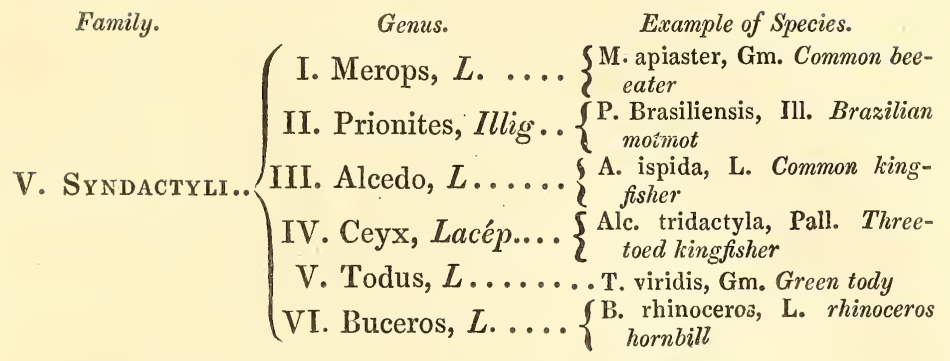

This fifth family is also the second general subdivision of the order distinguished, as we above explained, by the union of its toes, whence the name.

\section{ORDER III.-Scansores.}

Genus.

Subgenus.

Example of Species.

I. Jacamar, Cuv $\{$ 1. Galbula, Briss. .... G. viridis, Lath. Green jacamar

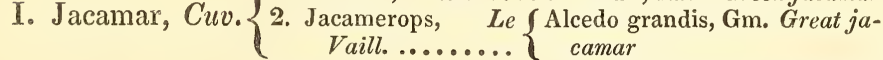

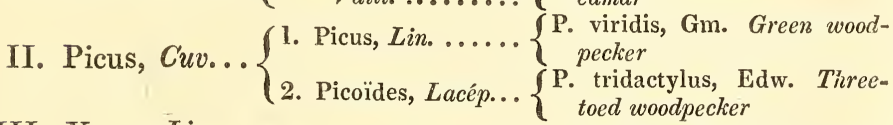
III. Yunx, Lin. ................ torquilla, L. the Wryneck IV. Cuculus, L.. $\begin{aligned} & \text { 1. Cuculus, prop. Cuv.. C. canorus, L. Common cuckoo } \\ & \text { 2. Conas, Vaill..... } \begin{array}{l}\text { Cuc. seniculus, L. Mangrove } \\ \text { cuckoo }\end{array} \\ & \text { 3. Centropus, Illig... } \begin{array}{l}\text { Cuc. Egyptius, L. Egyptian } \\ \text { cuckoo }\end{array} \\ & \text { 4. Leptosomus, Vieill. Cuc. Afer, L. African courol } \\ & \text { 5. Indicator, Vaill... }\left\{\begin{array}{c}\text { Cuc. indicator, Vaill. Honey } \\ \text { cuckoo } \\ \text { 6uc. tranquillus, L. Wax-bill } \\ \text { barbet }\end{array}\right.\end{aligned}$ V. Phœnicophæus, Vieill. .........P. Javanicus, Hors.

VI. Scythrops, Lath............ S. Australasiæ, Sh. Psittaceous S1. Pagonias, Illig. .. \{ Buc. dubius, Gm. Groove-billVII. Bucco, L... $\left\{\begin{array}{l}\text { 1. Pagonias, Illig. .. } \\ \text { 2. Bucco, prop. Cuv. }\end{array}\right.$

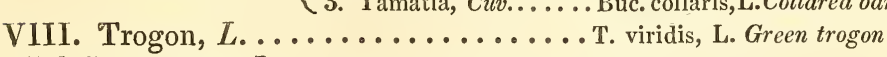

IX.Crotophaga, L............... major, Vieill.

X. Ramphastos, $\left\{\begin{array}{r}\text { 1. Rhamphastos,prop. } \\ \text { Cuv............ toco, L. the Toucan }\end{array}\right\}$ R.

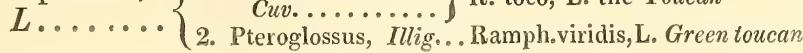




\section{ORDER III.-Scansores (continued).}

Genus.

Subgenus.

Example of Species. $\left\{\begin{array}{l}\text { Ps. Macao, L. Red and blue } \\ \text { maccaw }\end{array}\right.$

2. Conurus Ara, Cuv. $\left\{\begin{array}{c}\text { Ps. vittatus, Vaill. Banded } \\ \text { parrakeet }\end{array}\right.$

3. Palæornis, Vig. \& $\{$ Ps. Alexandri, L. Rose-ringed

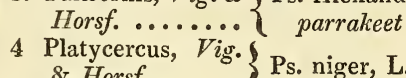

XI. Psittacus, L..\{

5. Plyctolophus, 5ieill. ........ $\left\{\begin{array}{c}\text { Plistatus, } \\ \text { cockatoo }\end{array}\right.$ Ps. niger, L. Black parrakeet

6. Psittacus, prop, Cuv.Ps. erythacus, L. Grey parrot

7. Lory $\quad S h . .$. Ps. lori, L. Black-capped lory

8. Psittaculus, Kuhl..Ps. taïtianus, Gm.Violet parrot

9. Microglossus, Vieill.Ps. gigas, Lath. Black cockatoo 10. Pezoporus, Illig.. $\left\{\begin{array}{c}\text { Ps. formosus, Vaill. Ground } \\ \text { parrot }\end{array}\right.$

XII. Corythaix, Ill............. \{ Cuculus Persa, L. Touraco XIII. Musophaga, Isert. ......... M. violacea, Vieill. Violet

\section{ORDER IV.-GaLLinacea.}

Most of our barn-door fowl, and many excellent game-birds, are to be found in this order, which takes its name from Gallus gallinaceus, the common cock and hen. It is composed in a great measure of one natural family, and is divided into genera, subgenera, and species.
Genus.
Subgenus.
Example of Species.

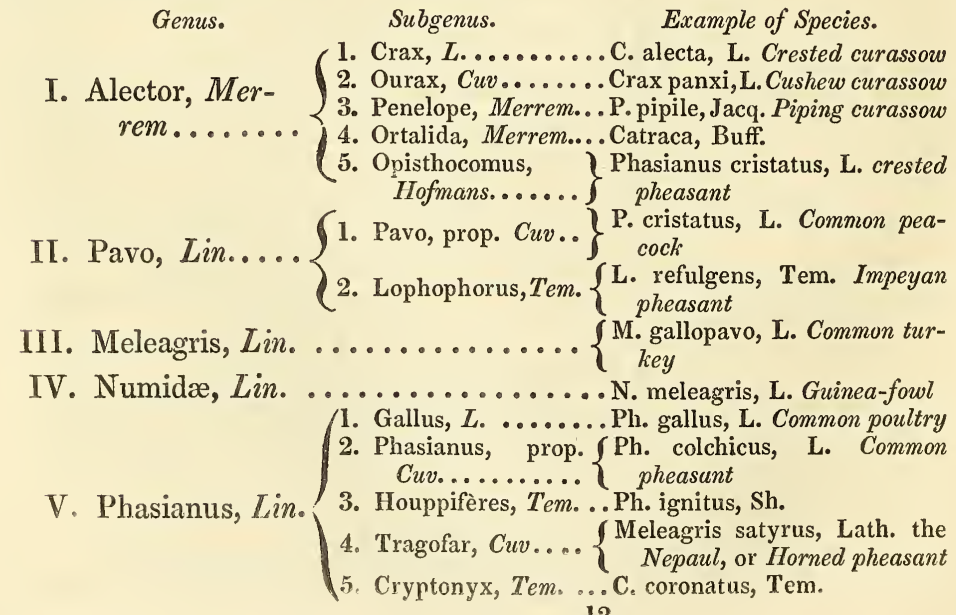


ORDER IV.-Gallinacea (continued).

Genus.

Subgenus.

Example of Species.

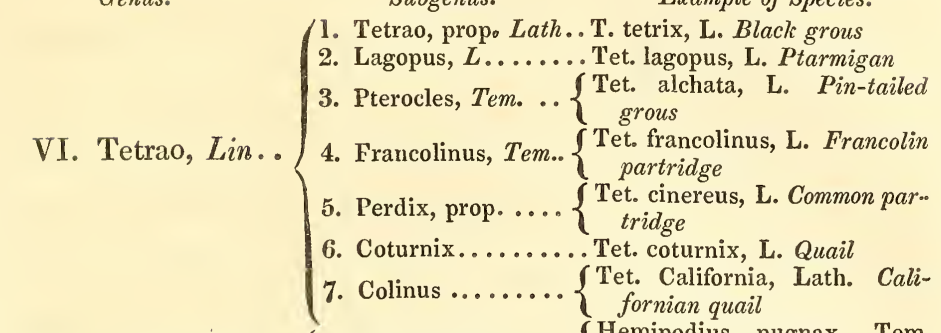

VII. Tridactylus, $\{1$. Ortygis, Illig..... $\{$ Hemipodius pugnax, Tem. Lacép.... 2. Syrrhaptes, Illig.. $\left\{\begin{array}{c}\text { Tet. paradoxurus, Pall. Hete- } \\ \text { roclite grous }\end{array}\right.$

VIII. Tinamus,

1. Pezus, Spix.......Tet. major, Gm.

Lath. .... 2. Tinamus, Spix ..... T. inambui, Azz.

3. Rhyncotus, Spix....T. rufescens, Tem.

$\left\{\begin{array}{l}\text { 1. Columbo-gallina, } \begin{array}{c}\text { C. carunculata, Tem. Carun- } \\ \text { Vaill............ } \\ \text { culated pigeon }\end{array} \\ \text { 2. Columba, prop. }\end{array}\right.$

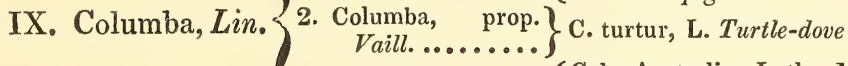

3. Virago, Cuv..... $\left\{\begin{array}{c}\text { Col. Australis, Lath. Mada- } \\ \text { gascar pigeon }\end{array}\right.$

\section{ORDER V.-GRALLE.}

These are generally long-legged wading birds, for the most part living in the vicinity of water, if we except the ostrich and cassowary, which indeed did not originally belong to this order, as established by Linnæus. Cuvier divides the entire order into five families, which we shall indicate by braces, and three distinct genera, which cannot well be reduced to any of the five families, and which he seems to think may almost be looked on as constituting little families in themselves. The families he has named are, 1. Brevipennes, containing genera i. and ii. ; 2. Pressirostres, containing genera iii-viii. ; 3. Cultrirostres, containing genera $\mathrm{ix}-\mathrm{x}$ viii.; 4. Longirostres, containing genera xix. and xx.; and, 5. Macrodactyli, containing genera xxi-xxiv.; after which come the three detached and concluding genera.

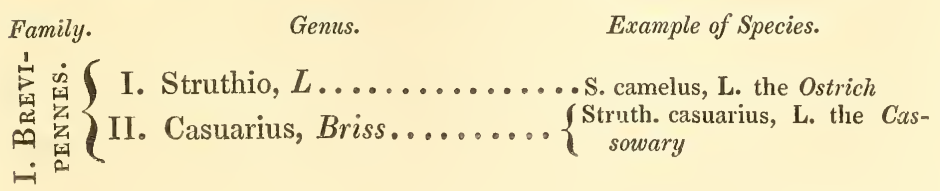




\section{ORDER V.-GRALLE (continued).}

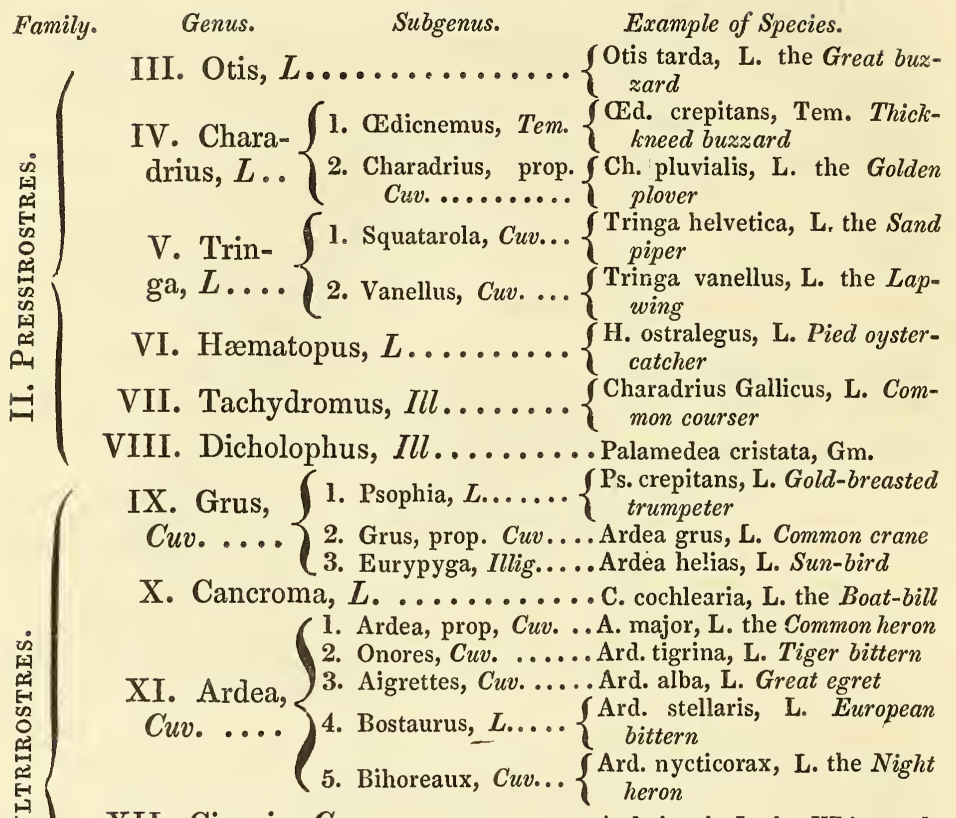

XII. Ciconia, Cuv. ..........Ard.ciconia,L. the White stork XIII. Mycteria, L........ A M. Americana, L. American XIV. Scopus, Briss............. . umbretta, L. Tufted umbre XV. Anastomus, Ill.......... Ard. ponticeriana, Gm. XVI. Dromas, Paykull...... $\begin{gathered}\text { D. ardeola, Payk. Abysinian } \\ \text { erodyla }\end{gathered}$ XVII. Tantalus, L........... T. loculator, L. Wood ibis XVIII. Platalea, L. ......... $\{$ P. leucorodia, Gm. White

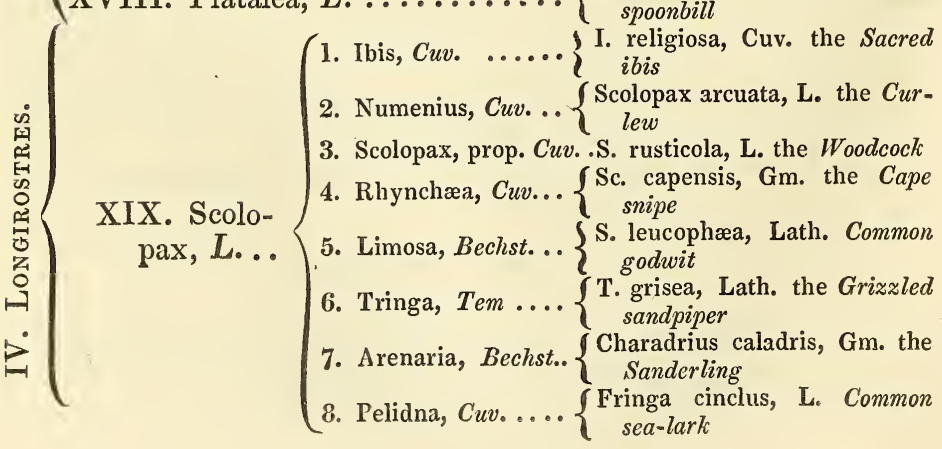




\section{ORDER V.-GRALLE (continued).}

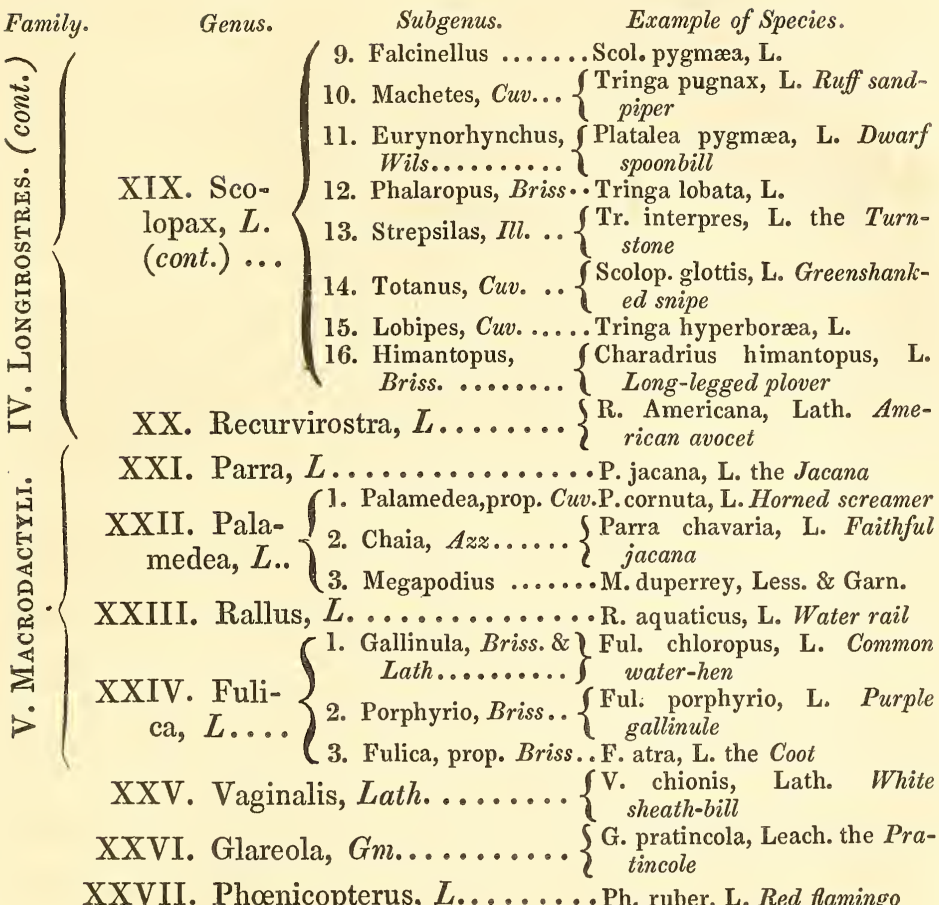

\section{ORDER VI.-PALMipedes.}

This order contains generally such birds as are web-footed, and fitted for an aquatic life. It is divided into four natural families ; 1. the Brachyptera, or Divers ; 2. the Longipennes, or High-flying birds; 3. the Totipalmes, in which the thumb, as well as the other toes, is included in the common web or membrane of the foot; and, 4. the Lamellirostres, which have the bill furnished with rows of laminæ, resembling fine teeth. 


\section{ORDER VI.-Palmipedes.}

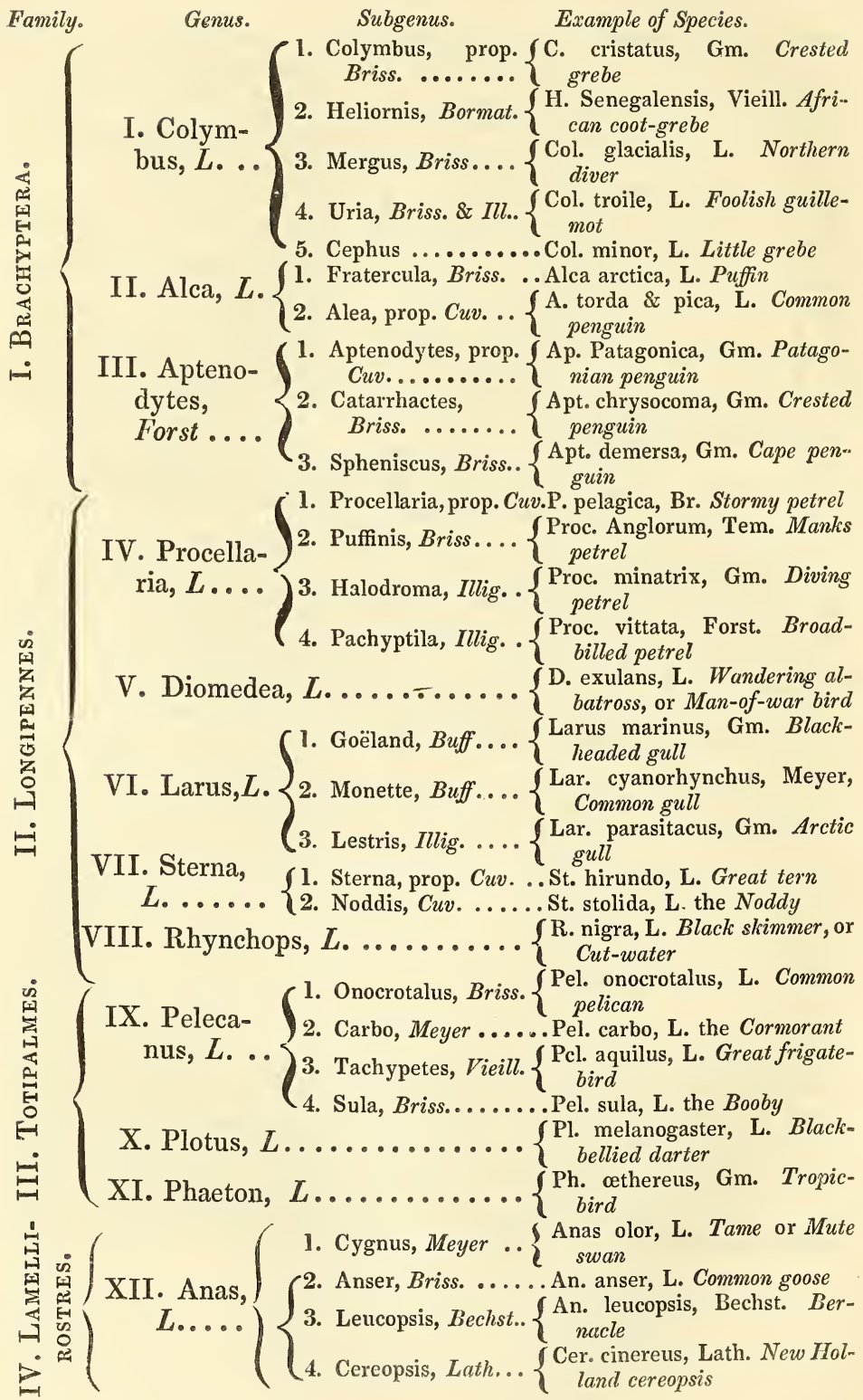




\section{ORDER VI.-PALMipedes (continued).}

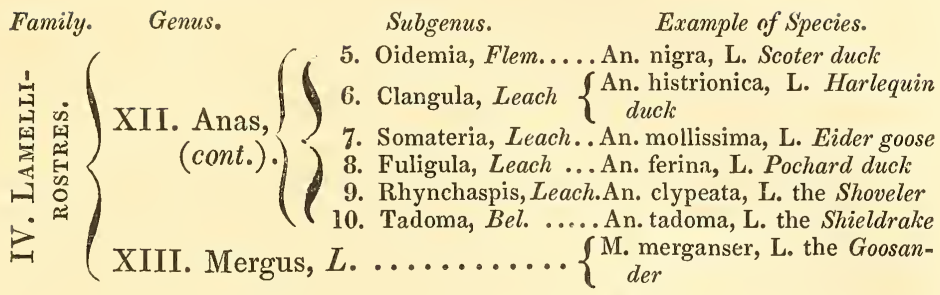

Note.-The great genus Anas is primarily divided into three subgenera: swans, geese, and ducks; which we have indicated by interior braces; the swans forming only one subgenus, but the geese and ducks several. The last subgenus, tadoma, is still further subdivided, and made to contain the Muscovy duck, the mallard, the teal, \&c. ; but our limits prevent us entering into such minute details. 


\section{VERTEBRATA.}

\section{CLASS III.-REPTILIA.}

Reptiles are divided into four sufficiently natural orders; the Chelonian, Saurian, Ophidian, and Batrachian, severally represented by the Tortoise, the Lizard, the Serpent, and the Frog. The last of these is remarkable for presenting in early life a structure different from that which it is to assume when adult: thus the young tadpole, it is well known, breathes by gills, and in some genera of this order the gills are never lost. An easy transition is thus formed from the class Reptiles to the subsequent class Fish.

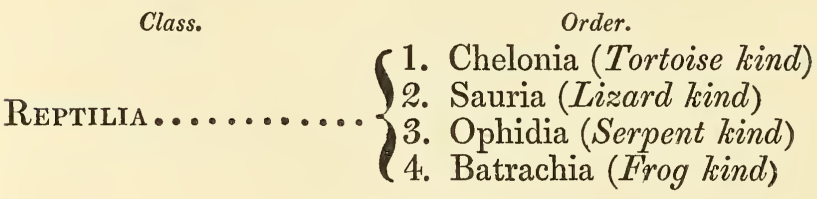

\section{ORDER I.-CheLonia.}

This order contains but one genus, the Linnæan genus Testudo, which has been divided into five subgenera, as follow.

\footnotetext{
Genus. Subgenus. Example of Species.

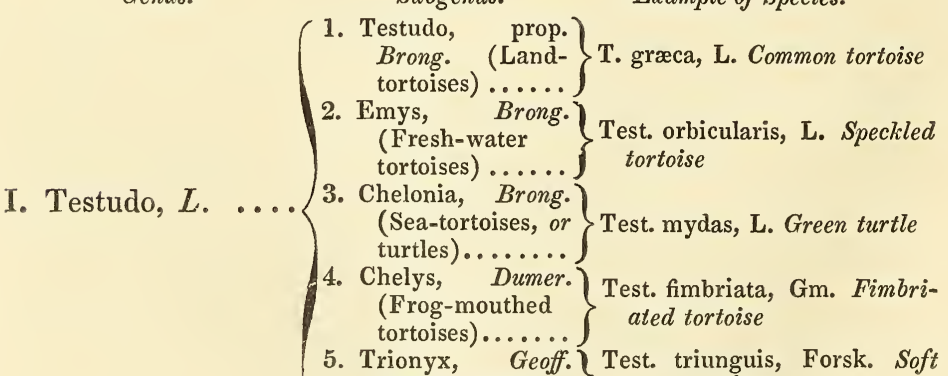
(Soft tortoises)... tortoise of the Nile.
} 


\section{ORDER II.-SAURIA.}

From the considerable differences existing between animals possessing the general characters of this order, it has been found necessary to divide it into six families, represented severally by the Crocodiles, Monitors, Iguanas, Geckos, Cameleons, and Skinks ; of these families, the first and fifth have each only one genus, the second two, and the others have several genera.

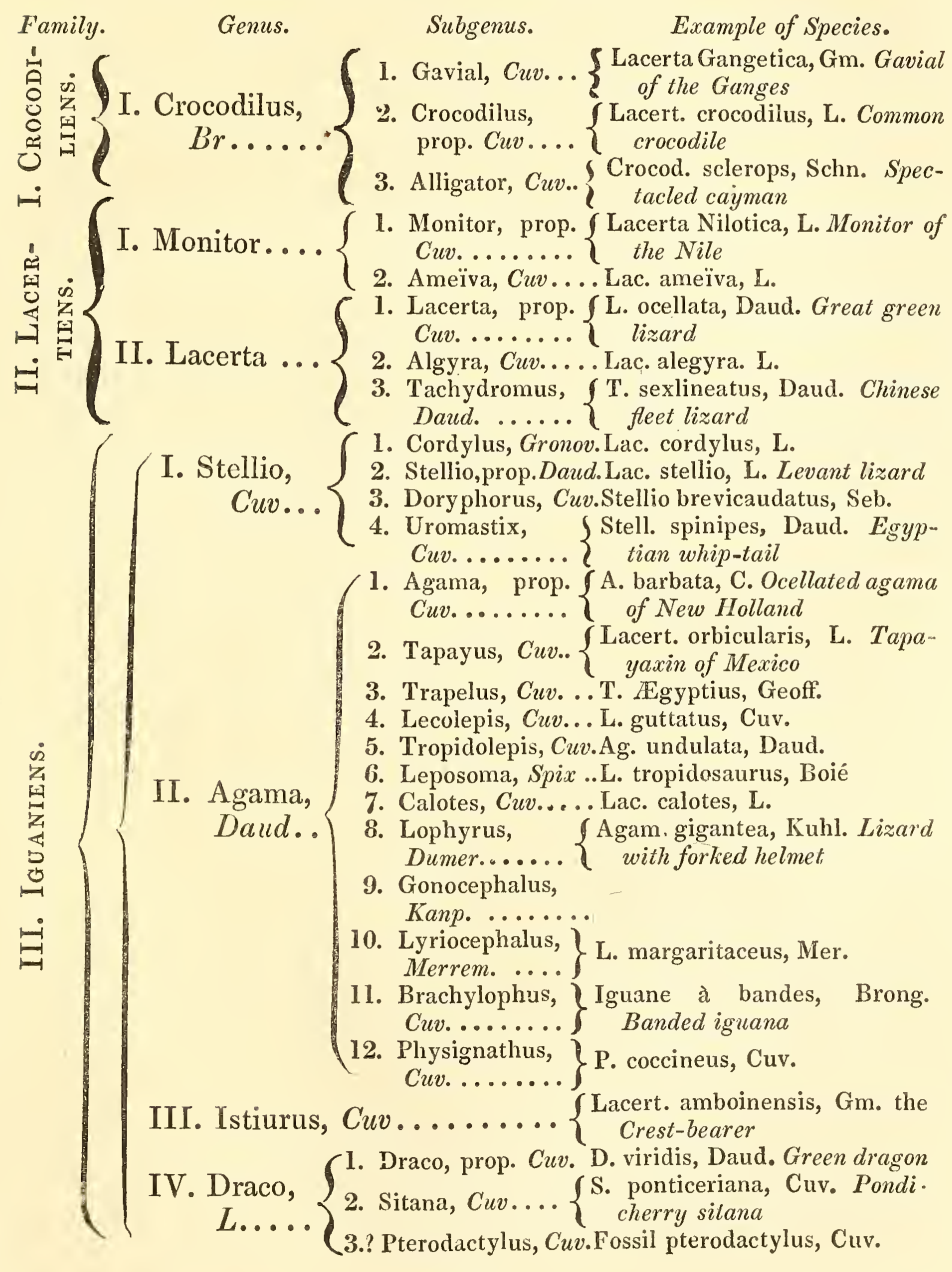


ORDER II.-SAURIA (continued).

\begin{tabular}{|c|c|}
\hline Family. & $\begin{array}{l}\text { le of Species. } \\
\text { a, L. Common } 24\end{array}$ \\
\hline & VI. Opryessa, Boié ...... Lac \\
\hline 国量 & VII. Basiliscus, Daud.... $\begin{array}{c}\text { Lac. basiliscus, L. Guiana } \\
\text { lisk }\end{array}$ \\
\hline 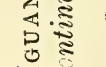 & rus, Cuv. . . . . . Lac. marmora \\
\hline & s, Cuv. Black-co \\
\hline & $\begin{array}{l}\text { X. Uplurus, Cuv. } \\
\text { XI. Anolius, Cuv. . }\end{array}$ \\
\hline
\end{tabular}

Of the Iguaniens, genera $\mathrm{i}$-iv. have no palatine teeth; genera v-xi. have. This gives rise to two sections, indicated by the interior braces. To the last section would belong the fossil animal of Maëstricht called Mososaurus.

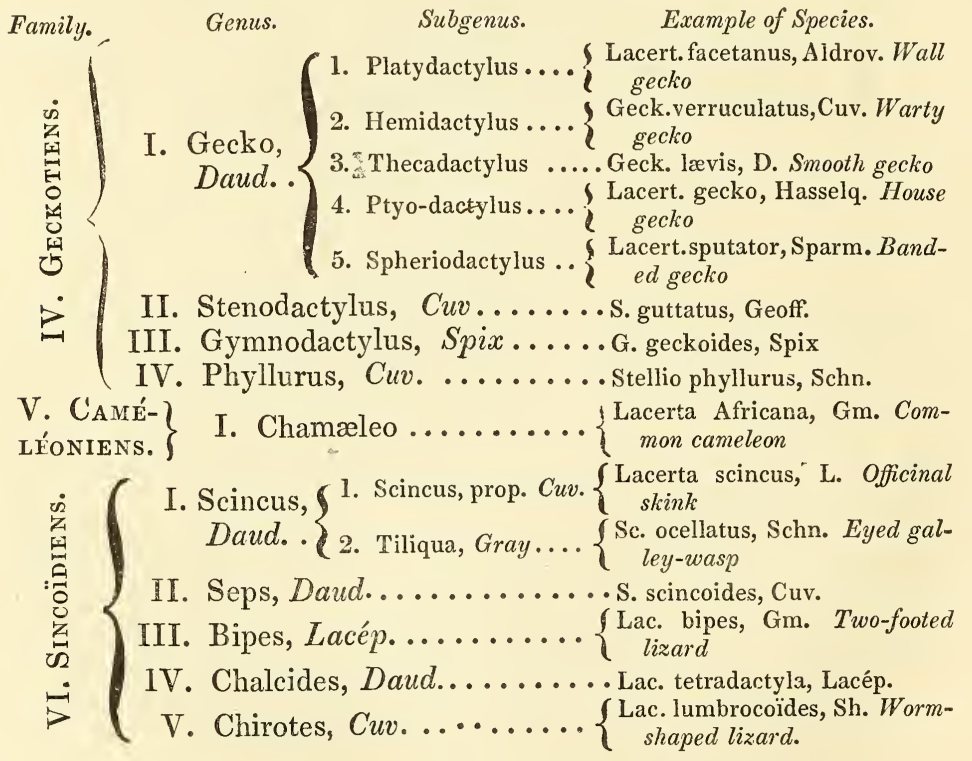

The five subgenera of the genus Gecko all agree in having the toes wide and dilated; the Stenodactyli and Gymnodactyli want this character, and are therefore placed as separate genera. To the preceding family, the Iguaniens, belong the Geosurus, Negulosaurus, Iguanodon, and many other fossil animals. 


\section{ORDER III.-OPHIDIA.}

This order is primarily divided into three families : 1. the Snakes, Anguis; 2. the true Serpents; and, 3. the naked Serpents. Of these, the 1 st and $3 d$ contain each but one genus; the $2 d$ contains all the rest, and, as these differ in several respects, is subdivided, 1st, into two tribes, the Double-marcheurs, that is, those that move with either end foremost, and the Serpents proper, which always advance with the head in front. The Serpents proper are then divided according as they are non-venomous or venomous; and afterwards these latter according as they have isolated fangs, or fangs accompanied by the ordinary jaw teeth. Finally come the division into genera, subgenera, and species; but of all these our plan and limits will only permit us to notice the families, genera, and subgenera, illustrating as usual with a specific example of each.

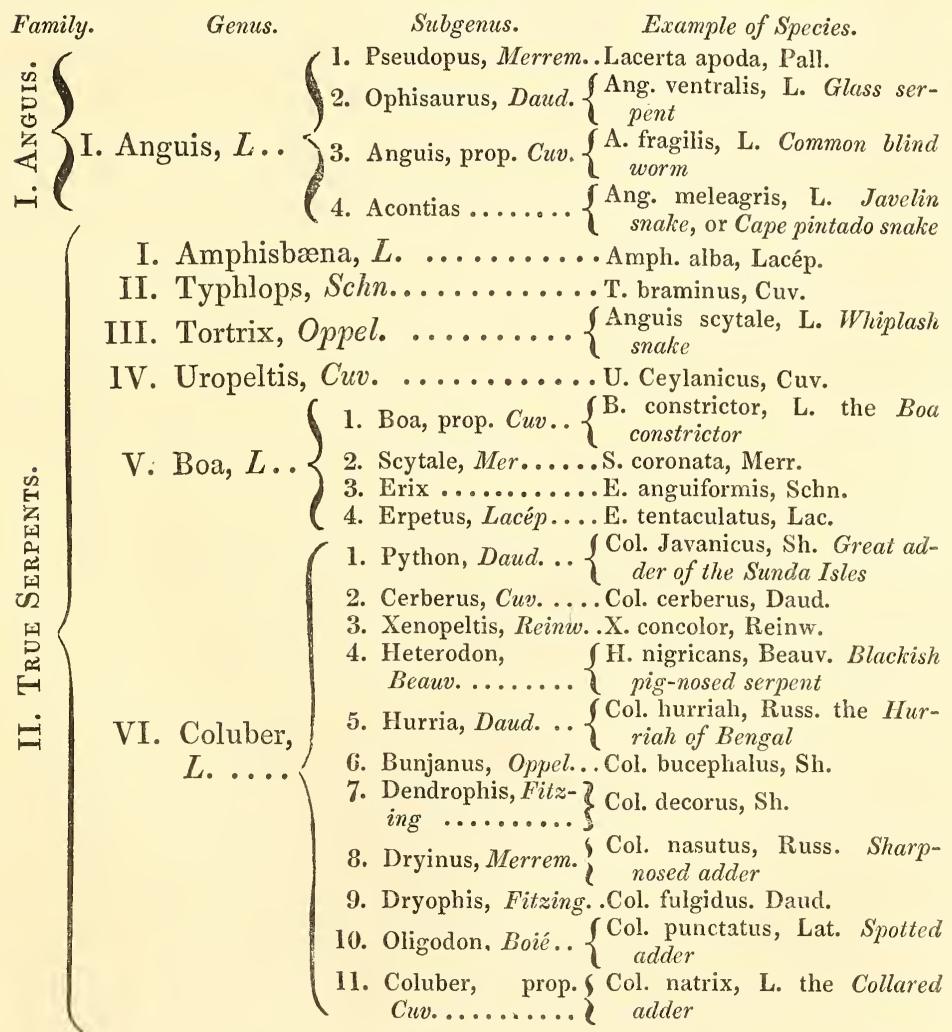




\section{ORDER III.-OPHIDIA (continued).}

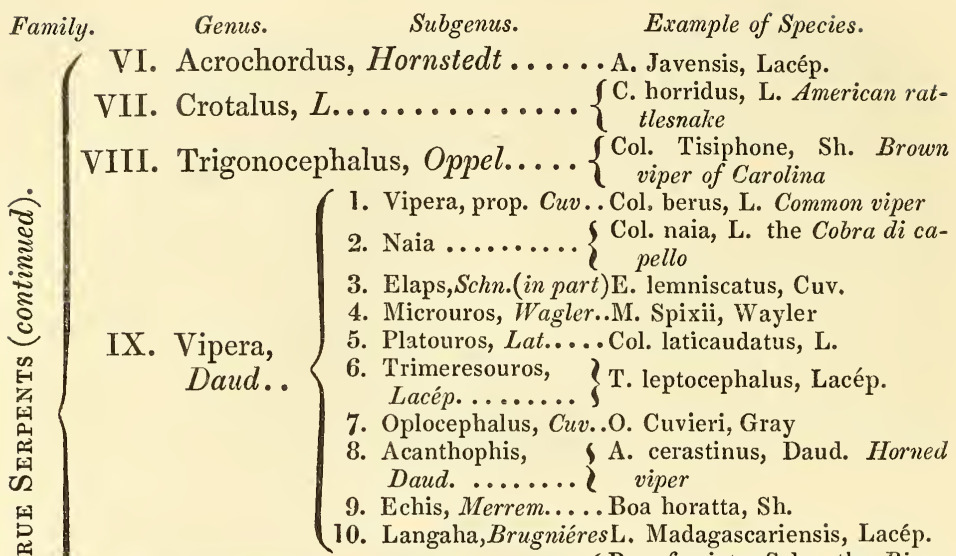

X. Bungarus, Daud. (in part) $\cdots\left\{\begin{array}{c}\text { Boa. fasciata, Schn. the Ring- } \\ \text { ed bungarum-pamma of Ben- } \\ \text { gal }\end{array}\right.$

XI. Hydrus, \{ Schn. (in \{

1. Hydrophis, Daud.. Hydrus curtus, Sh.

2. Pelamides, Daud. $\left\{\begin{array}{r}\text { Hydrus bicolor, } \\ \text { Schn. }\end{array}\right\}$

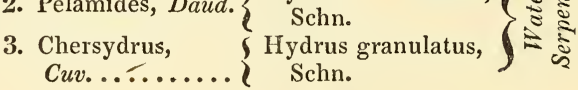

$\left.\begin{array}{r}\text { III. Naked } \\ \text { Serpents }\end{array}\right\} \ldots$. I. Cæcilia, L....... $\begin{gathered}\text { Cæc. annulata, Spix, Annu- } \\ \text { lated cacilia. }\end{gathered}$

\section{ORDER IV.-BATRACHIA.}

This order is divided by Mr. Gray (see Synopsis at the end of our ninth volume) into two very natural orders or families, according as they do or do not undergo metamorphosis. Cuvier has indi. cated the same division, and has then proceeded to subdivide them as follows :-

Genus.

\section{Subgenus.}

Example of Species.

1. Rana, prop. LaurentiR. temporaria, L. Common frog

2. Ceratophris, Boié . . Rana cornuta,Seb. Horned frog

3. Dactylethra, Cuv...Pipa lævis, Merrem.

4. Hyla, Laurenti....Ran. arborea, L. Tree frog

I. Rana, $L$.

5. Bufo, Laurenti .... Ran. bufo, L. Common toad

6. Bombinator, Mer- $\{$ Ran. bombina, Gm. Yellowrem......... \{ bellied toad

7. Oxyrhynchus, $\quad$ Bufo proboscideus, Spix, LongSpix.......... \{ nosed toad 
ORDER IV.-BATRACHIA (continued).
Genus.
Subgenus.
Example of Species.

I. Rana, L. (cont.) $\left\{\begin{array}{l}\text { 8. Otilophus, Cuv... }\left\{\begin{array}{l}\text { Rana margaritifera, L. Pearl- } \\ \text { ed toad }\end{array}\right. \\ \text { 9. Breviceps, Merr. } \text { Bufo gibbosus, Seb. } \\ \text { 10. Pipa, Laurenti .. } \begin{array}{c}\text { Rana pipa, L. the Surinam } \\ \text { toad }\end{array}\end{array}\right.$

II. Salamandra, Brougn....

1. S. prop. Laurenti $\}$ Lacerta salamandra, L. Com-

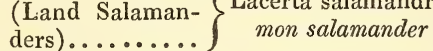

2. Triton, Laurenti

(Water salaman- Salamand. marmorata, L. ders) .......... $\}$ Marbled salamander

Sal. gigantea, Barton, Great

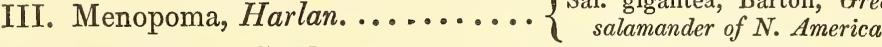

IV. Amphiuma, Garden............ tridactylum, Cuv.

V. Axolotl ............. \{ $\begin{gathered}\text { Siren pisciformis, Sh. the Axo } \\ \text { lotl of Mexico }\end{gathered}$

VI. Menobranchus, Harlan .......... M. lateralis, Harl.

VII. Proteus, Laurenti ........... P. anguinus, Laur. the Proteus

VIII. Siren, L............. \{ S. lacertina, L. the Lacertine

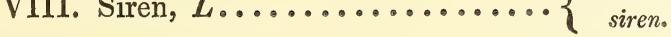




\section{VERTEBRATA.}

\section{CLASS IV.-PISCES.}

This class is divided into two great series, the Ossei and Chondropterygii ; in the former of which the skeleton is formed of true bone, while in the latter it always remains in the state of cartilage or gristle. The former is divided into six, the latter into two orders; the principal characters being derived from the first gills, as the names of the orders will show.

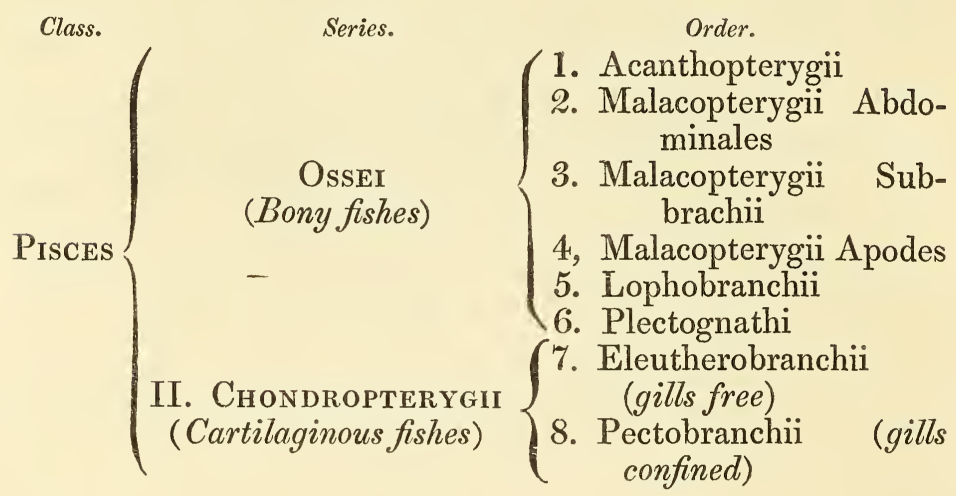

\section{SERIES I.-OSSEI.}

\section{ORDER I.-ACANTHOPTERYGII.}

The Acanthopterygians make three-fourths of all the fishes known, and are the most perfectly wrought type of the class. The families into which they are divided are in general very natural, but present so many varieties with respect to those characters on which it might be supposed they could be grouped into orders or other subdivisions, that it has been found expedient to leave them all together. 


\section{ORDER I.-AcanthopteRYGII.}

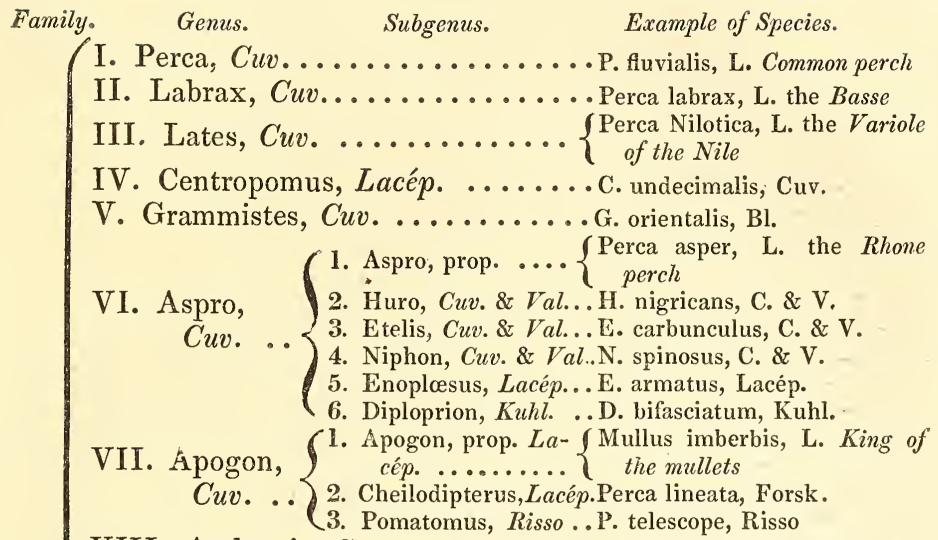

VIII. Ambassis, Commers........... A. Commersonii, C. \& V.

IX. Lucio-perca, Cuv........ $\left\{\begin{array}{c}\text { Perca lucioperca, L. the Pike- } \\ \text { perch }\end{array}\right.$

X. Serranus, $\quad$ 1. Serranus, prop. ....Perca scriba, L. the Sea-perch Cuv..... 2 2. Anthias, Bl. (in part) A. sacer, Bl. the Barber

XI. Plectropoma, Cuv. .........Pl. melanoleucum, Cuv.

XII. Diacope, Cuv............ D. sebæ, Cuv.

XIII. Mesoprion, Cuv. ............ unimaculatus, Russel

XIV. Acerina, Cuv............Perca cernua, L. the Ruffe

XV. Rypticus, Cuv........ Anthias saponaceus, Bl. the

XVI. Polyprion, Cuv ........... cernuum, Valenc.

XVII Centropristis, Cuv. ..... C. nigricans, Cuv. Black basse, XVII. Centropristis, Cuv. $\cdots \cdots\{$ or Black ham

XVIII. Grystes, Cuv....... $\left\{\begin{array}{c}\text { Cichla variabilis, Lesueur, } \\ \text { New York growler }\end{array}\right.$

XIX. Cirrhites, Commers. ........ C. maculatus, Lacép.

XX. Chironemus, Cuv ........... georgianus, Cuv. \& Val.

XXI. Pomotis, Cuv. ............ vulgaris, Cuv. Pond perch

XXII. Centrarchus, Cuv .......... . æneus, Cuv.

XXIII. Priacanthus, Cuv.........Anthias boops, Bloch

XXIV. Dules, Cuv.............

XXV. Therapon, $\{$ l. Therapon, prop...Holocentrus servus, Bl.

Cuv....\{ 2. Datnia, Cuv. \& Val.Coius datnia, Buch.

XXVI. Pelates, Cuv............ 5-linestus, Cuv. \& Val.

XXVII. Helotes, Cuv............ 6-lineatus, Cuv. \& Val.

XXVIII. Trichodon, Steller........ T. stelleri, Cuv.

XXIX. Sillago, Cuv. ....... $\begin{aligned} & \text { S. domina, Cuv. Lady-fish of } \\ & \text { Pondicherry }\end{aligned}$ 


\section{ORDER I.-Acanthopterygir (continued).}

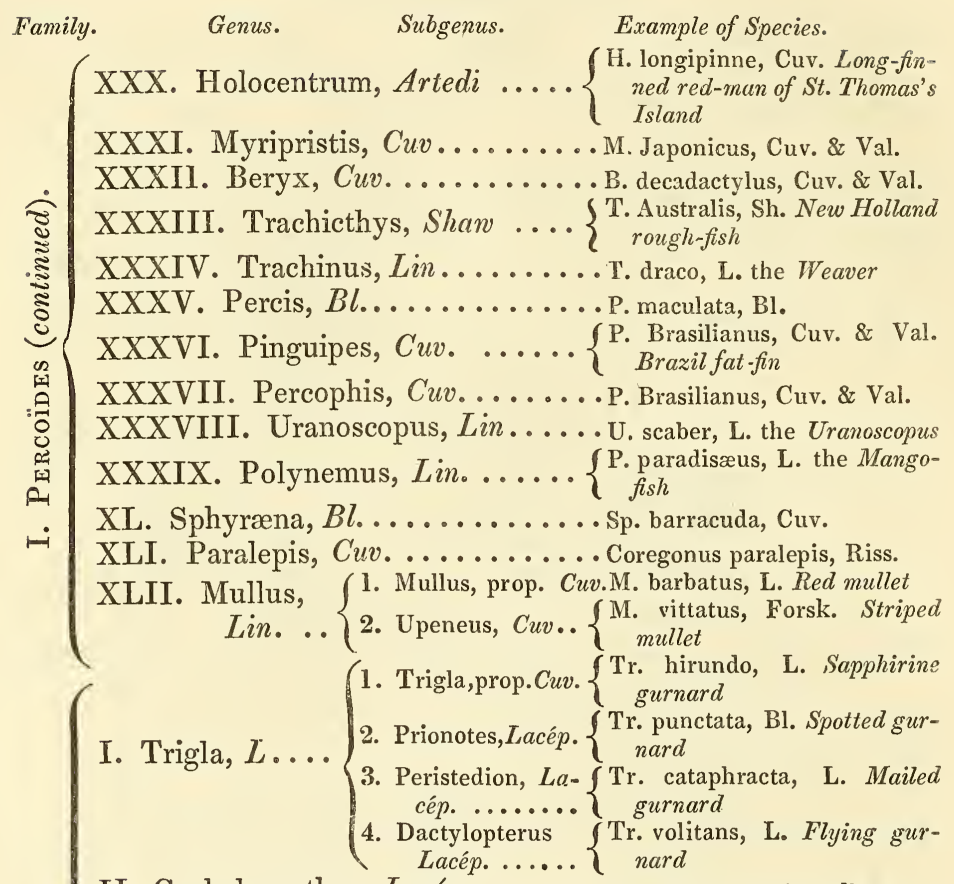

II. Cephalocanthus, Lacép......... Gasterosteus spinarella, L.

III. Cottus, 1. Cottus, prop. Cuv.C. gobio, L. Miller's thumb

2. Aspidophorus, , Cott. cataphractus, L. Armed Lacép.......l bull-head

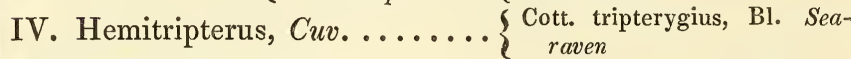

V. Hemilepidotus, Cuv. ........Cott. hemilepidotus, Tiles

VI. Platycephalus, $B l \ldots \ldots \ldots \ldots$ Cott. insidiator, L.

(1. Scorpæna prop.Cuv.S. scrofa, L. Great sea-devil VII. Scorpæna, 2. Sebastes, Cuv...\{ Perca Norvegica, Müll. the

3. Pteroïs, Cuv.... Scorp. volitans, L. Gini-maha,

VIII. Blepsias.............. Blennius villosus, Stell.

IX. Apistes $\ldots \ldots \ldots \ldots \ldots$. . . Ap. alatus, Cuv.

X. Agriopus .............. Blennius torvus, Gronov.

XI. Pelorus . . . . . . . . . . . . . Scorpœna didactyla, Pall.

XII. Synan ceia, $B l \ldots \ldots \ldots$..... Scorp. horrida, Lin.

XIII. Monocentris, $B l$. ........... Japonica, Bl. 


\section{ORDER I.-Acanthopterygir (continued).}

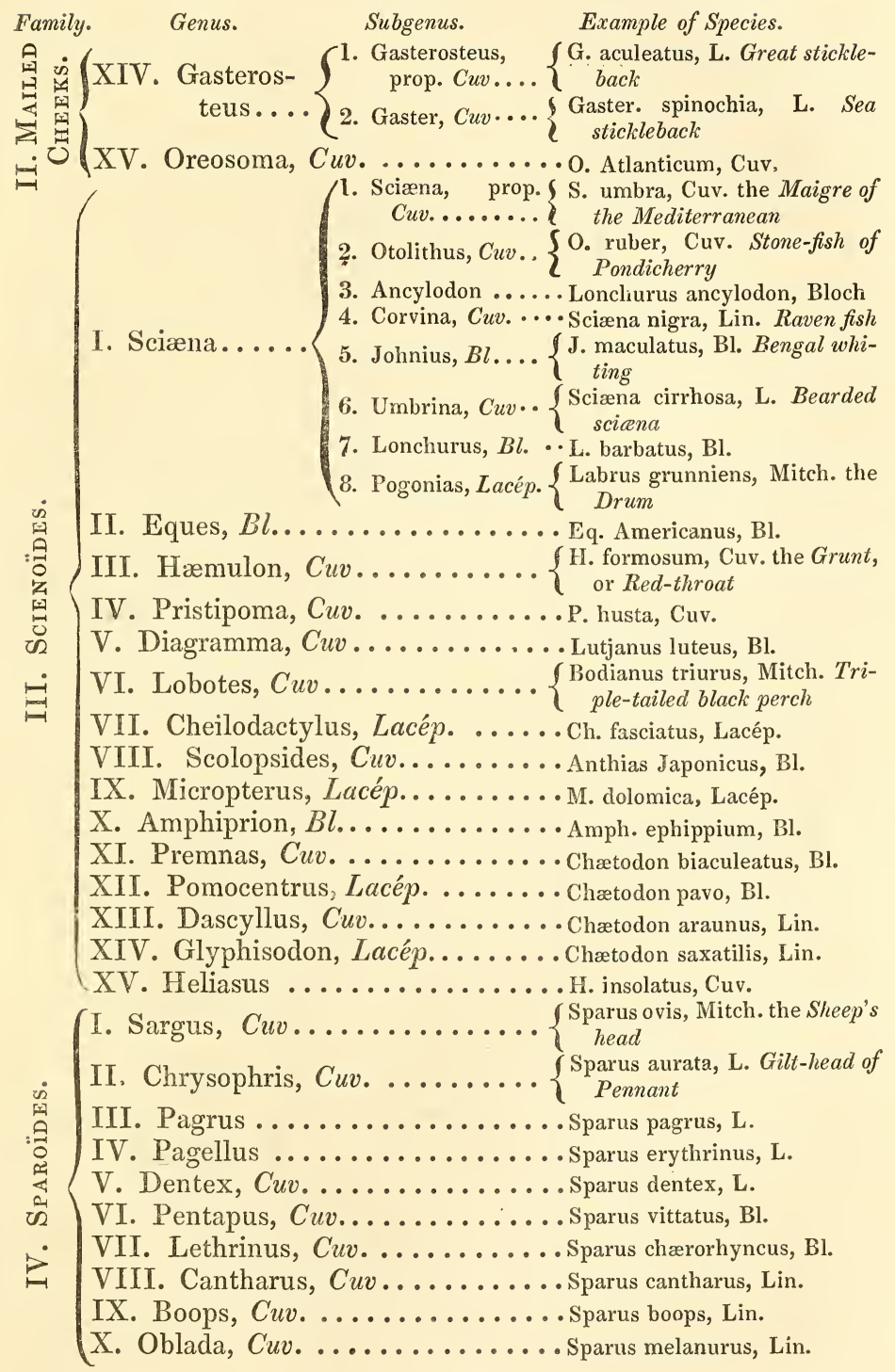




\title{
ORDER I.-AcanthopterygiI (continued).
}

\author{
Family. Genus. Subgenus. Example of Species.
}

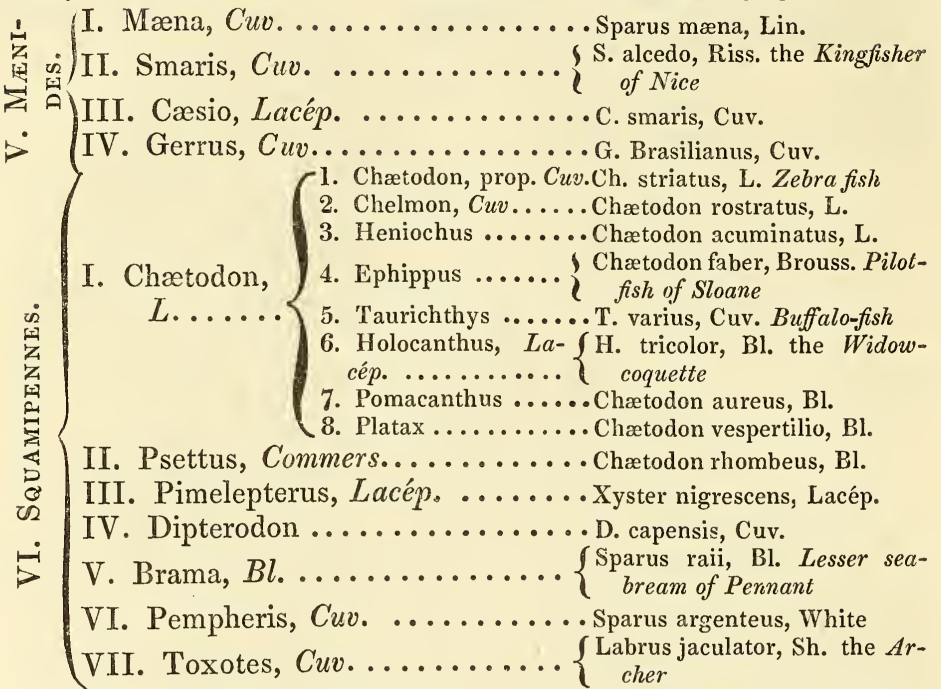

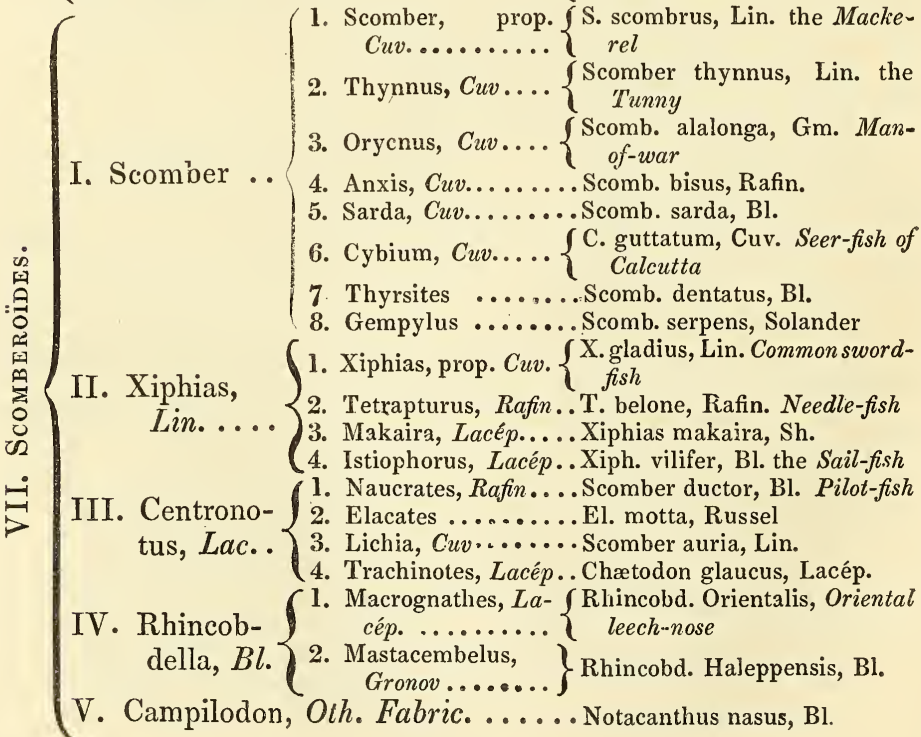




\section{ORDER I.-AcanthopterygiI (continued).}

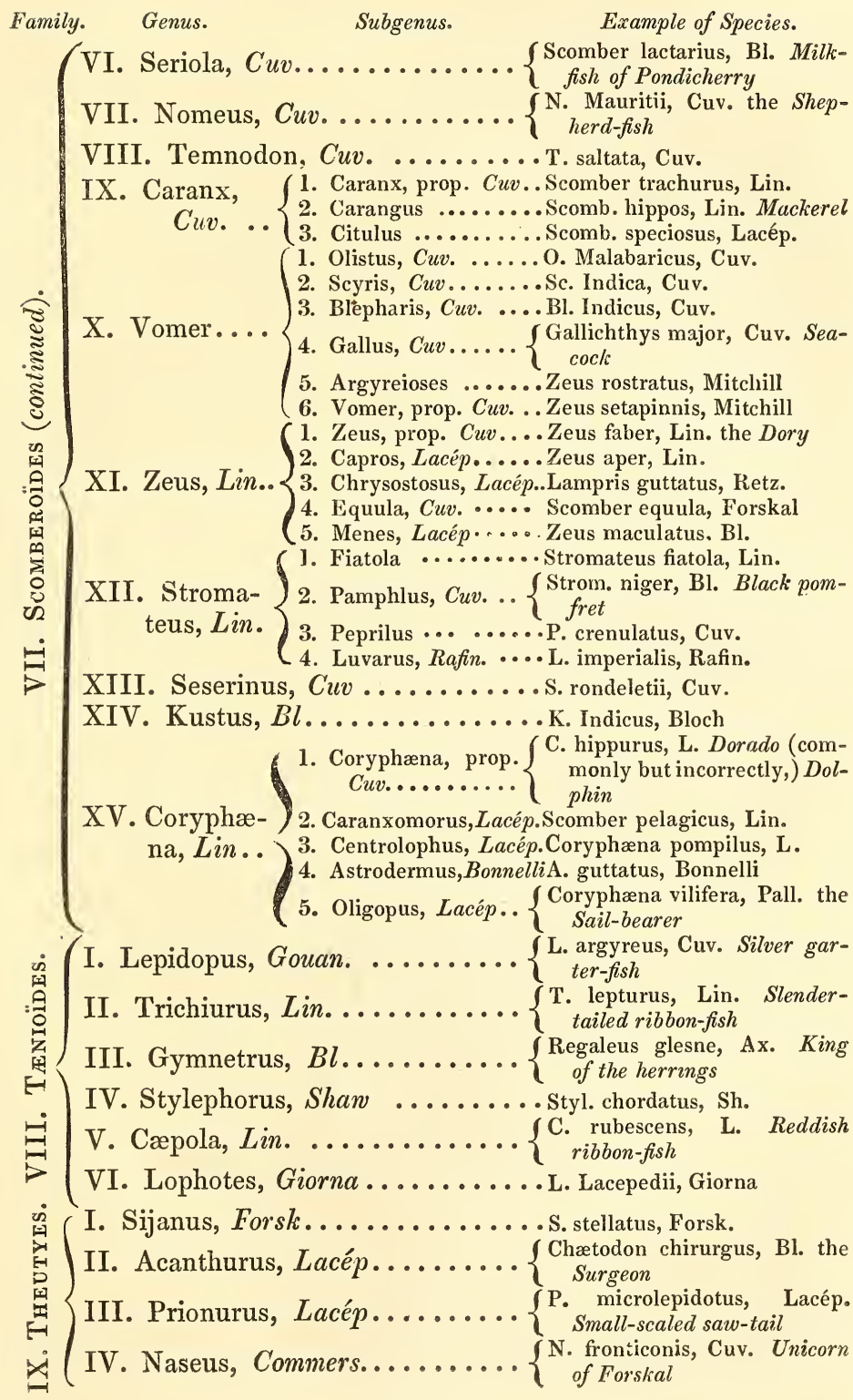




\section{ORDER I.-Acanthopterygil (continued).}

Family. Genus. Subgenus. Example of Species.

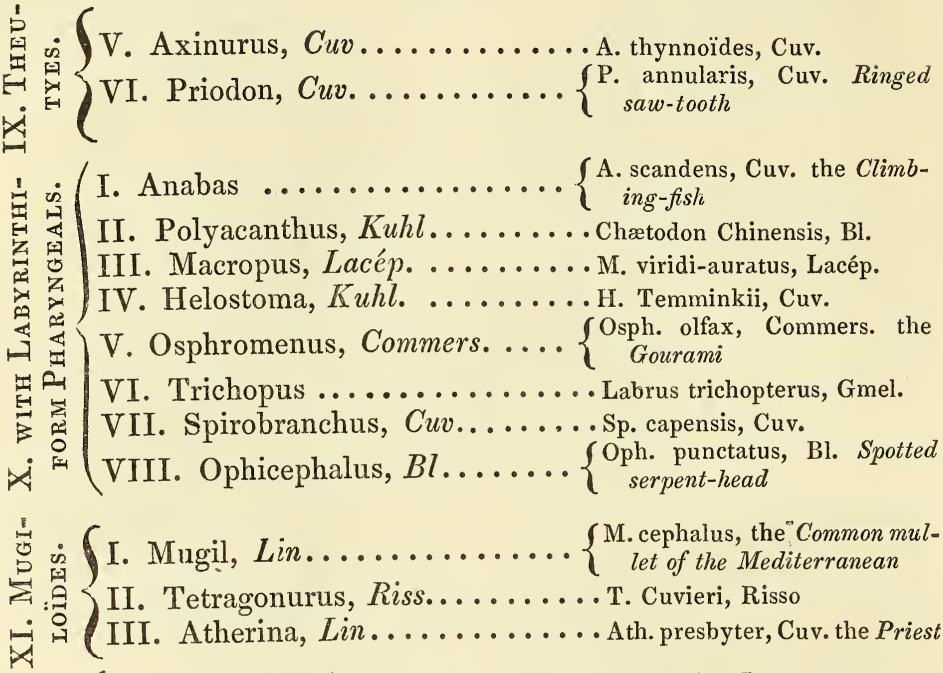

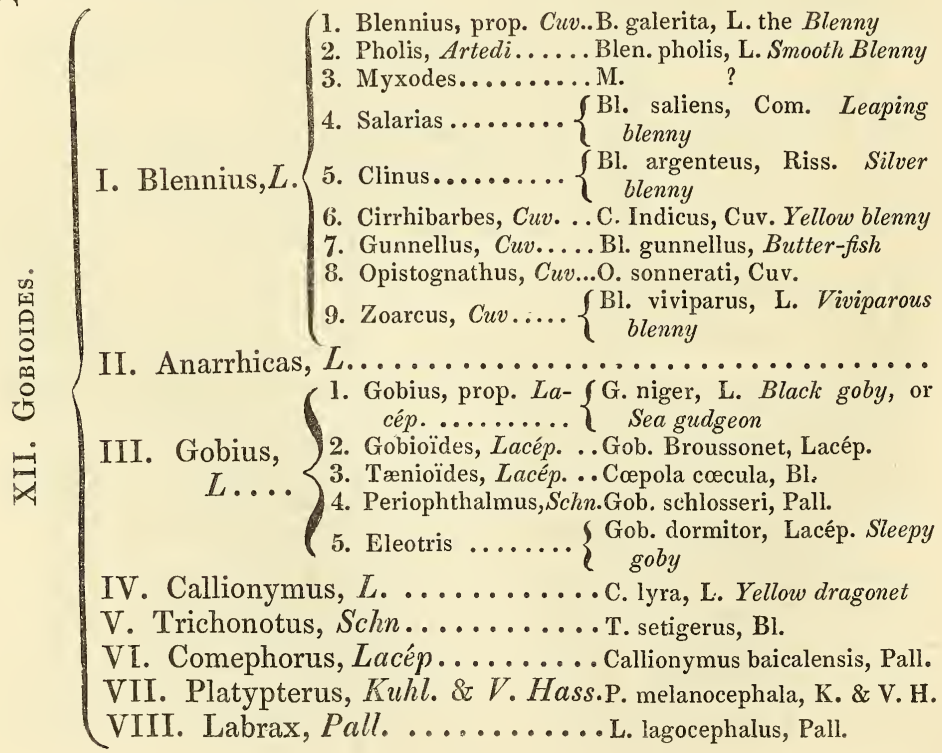


ORDER I.-ACANTHOPTERYGII (continued).

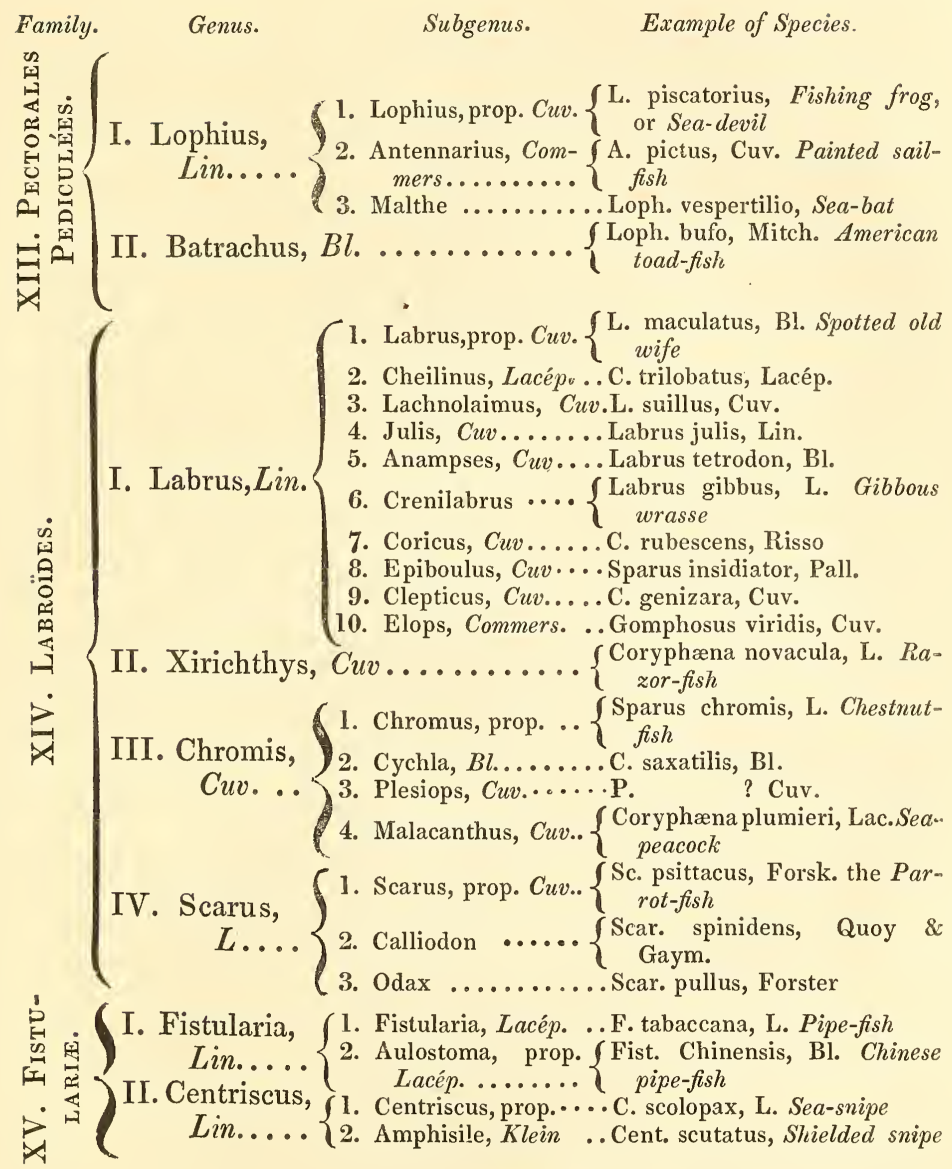

\section{SERIES I.-OSSEI.}

\section{ORDER II.-Malacopterygil Abdominales.}

Ventral fins behind the pectorals; rays soft or articulated. There are five families. 


\section{ORDER II.-Malacopterygit Abdominales.}

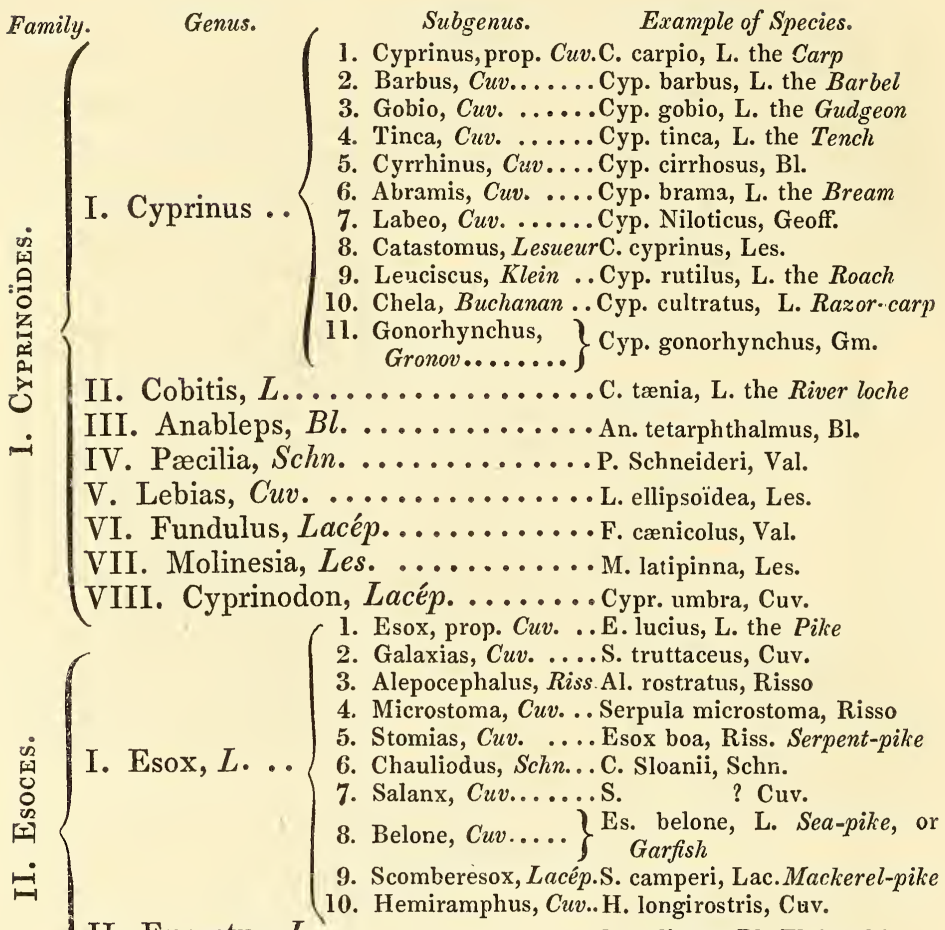

II. Exocetus, L. ........... . volitans, Bl. Flying fish

III. Mormyrus, L. . . . . . . . . . M. Hasselquistii, Geoff.

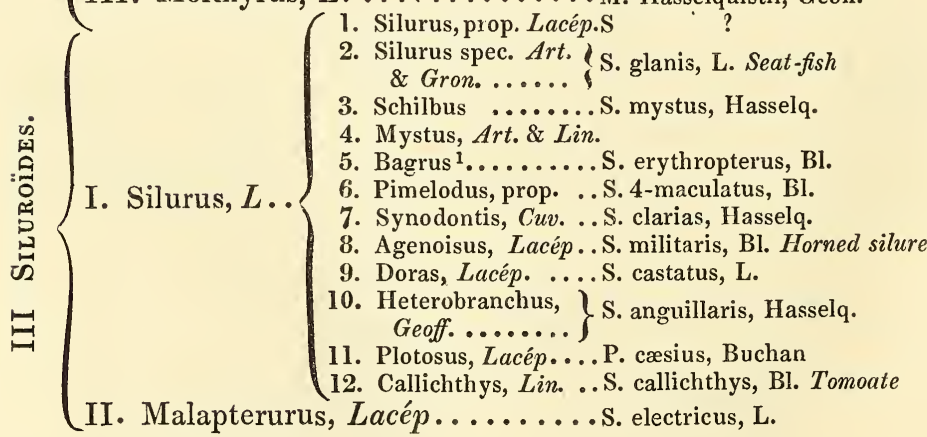

1 Bagrus and Pimelodus, prop. are divisions of the subgenus Pimelodus of Lacépède. 


\section{ORDER II.-Malacopterygir Abdominales (continued).}

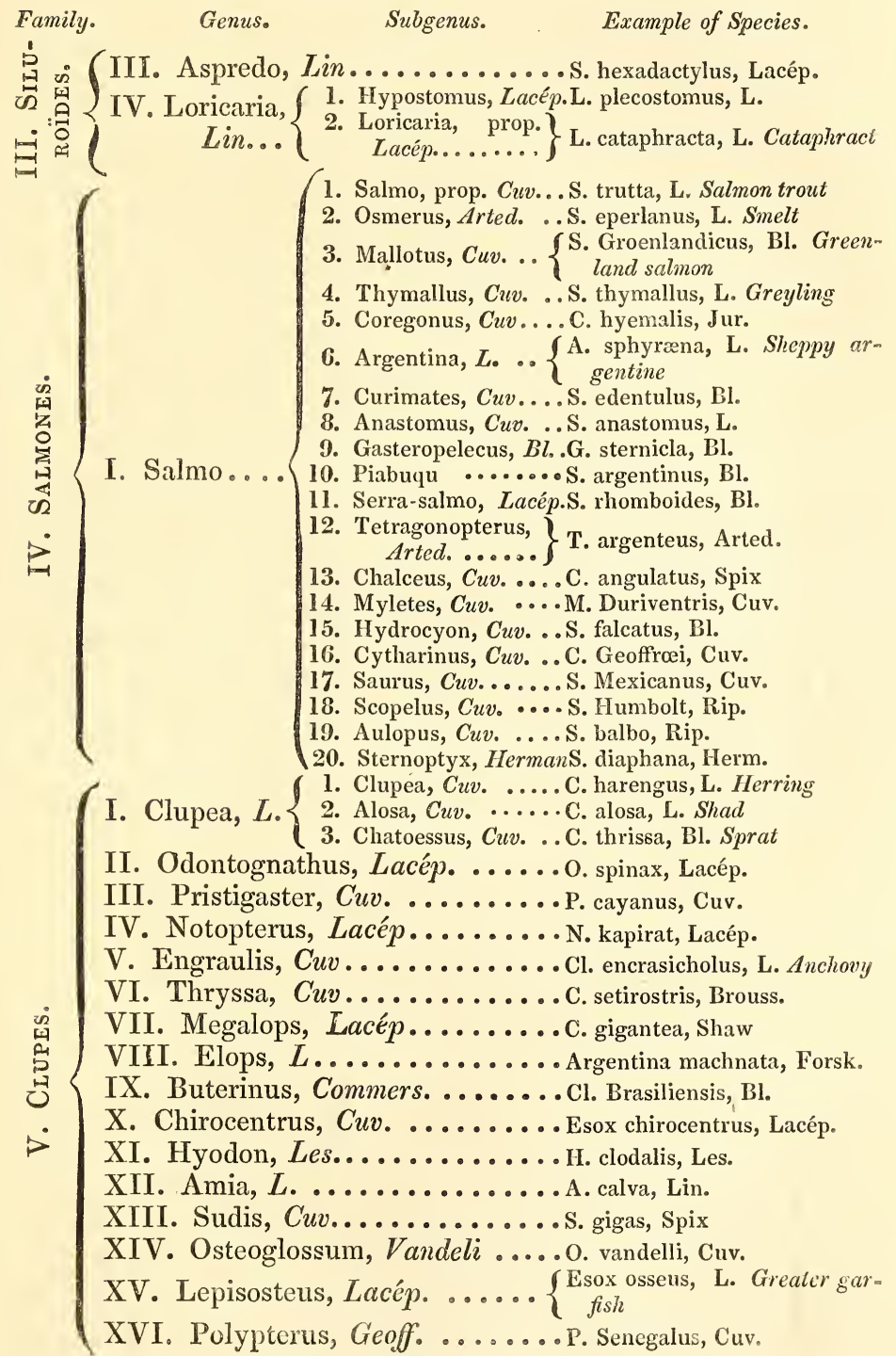




\section{SERIES I.-OSSEI.}

\section{ORDER III.-Malacopterygit Subbrachil.}

Ventral fins under the pectorals.

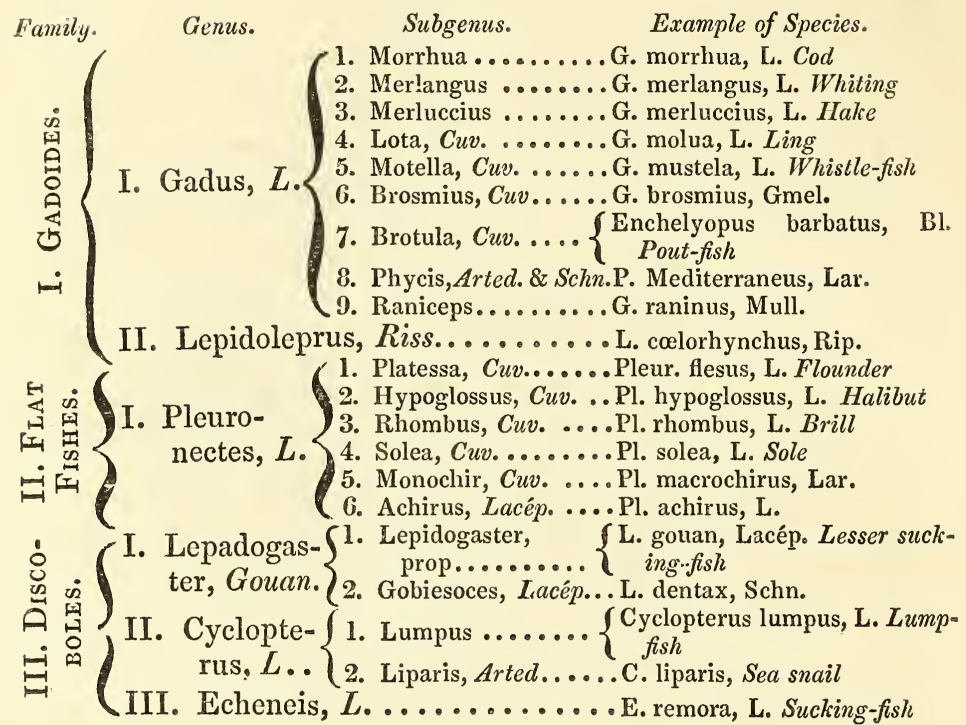

\section{SERIES I.-OSSEI.}

\section{ORDER IV.-Malacopterygit Apodes,}

\section{Constitute one natural family.}

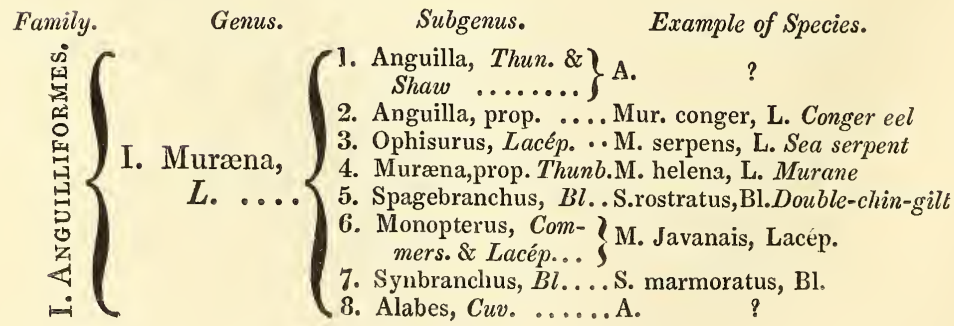




\section{ORDER IV.-Malacopterygir Apodes (continued).}

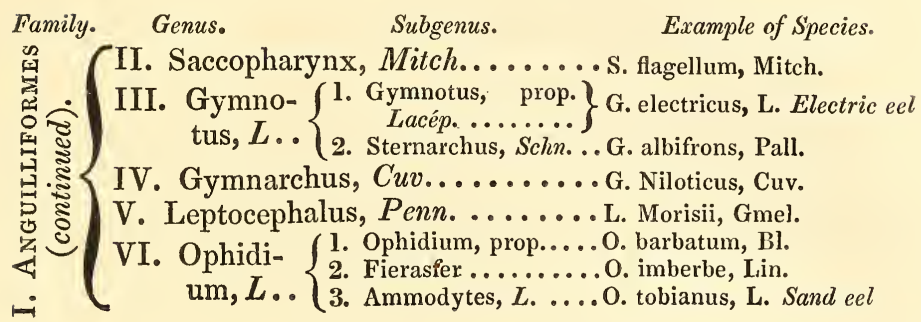

\section{SERIES I.-OSSEI.}

\section{ORDER V.-LOFHOBRANCHII.}

Gills in tufts ; operculum fixed by a membrane which only affords a small aperture for the escape of the water. There are two genera.

Genus.

Subgenus.

Example of Species.

I. Syngnathus, L... $\left\{\begin{array}{l}\text { 1. Syngnathus, prop. .. S. acus, L. Sea adder } \\ \text { 2. Hippocampus, Cuv.. H. brevirostris, Cuv. } \\ \text { 3. Solenostomus, Lacép. Fistularia paradoxa, Pall. }\end{array}\right.$ II. Pegasus, L................... . draco, L. Sea dragon

\section{SERIES I.-OSSEI.}

\section{ORDER VI.-Plectognathi.}

Maxillary fixed to the intermaxillary bones, and the palatal to the cranium. Opercula covered in.

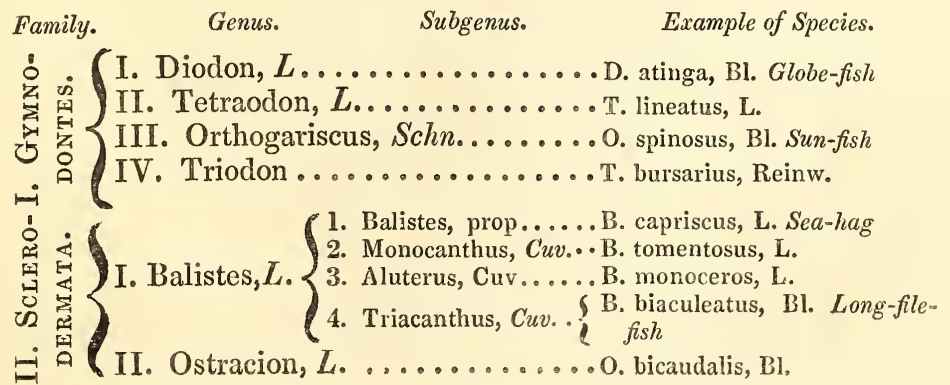




\section{SERIES II.-CHONDROPTERYGII.}

\section{ORDER I.-ELeUtherobranchir.}

Genus.

Subgenus.

Example of Species.

1. Accipenser, L. .................. sturio, L. Common sturgeon

II. Polyodon, Lacép.................... Squalus s patula, Mand.

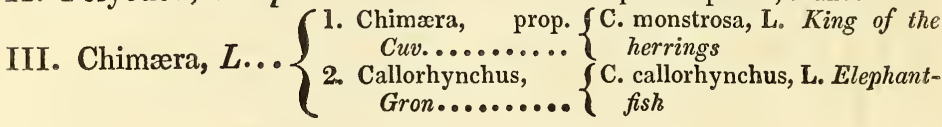

\section{SERIES II.-CHONDROPTERYGII.}

\section{ORDER II.-Pentobranchit.}

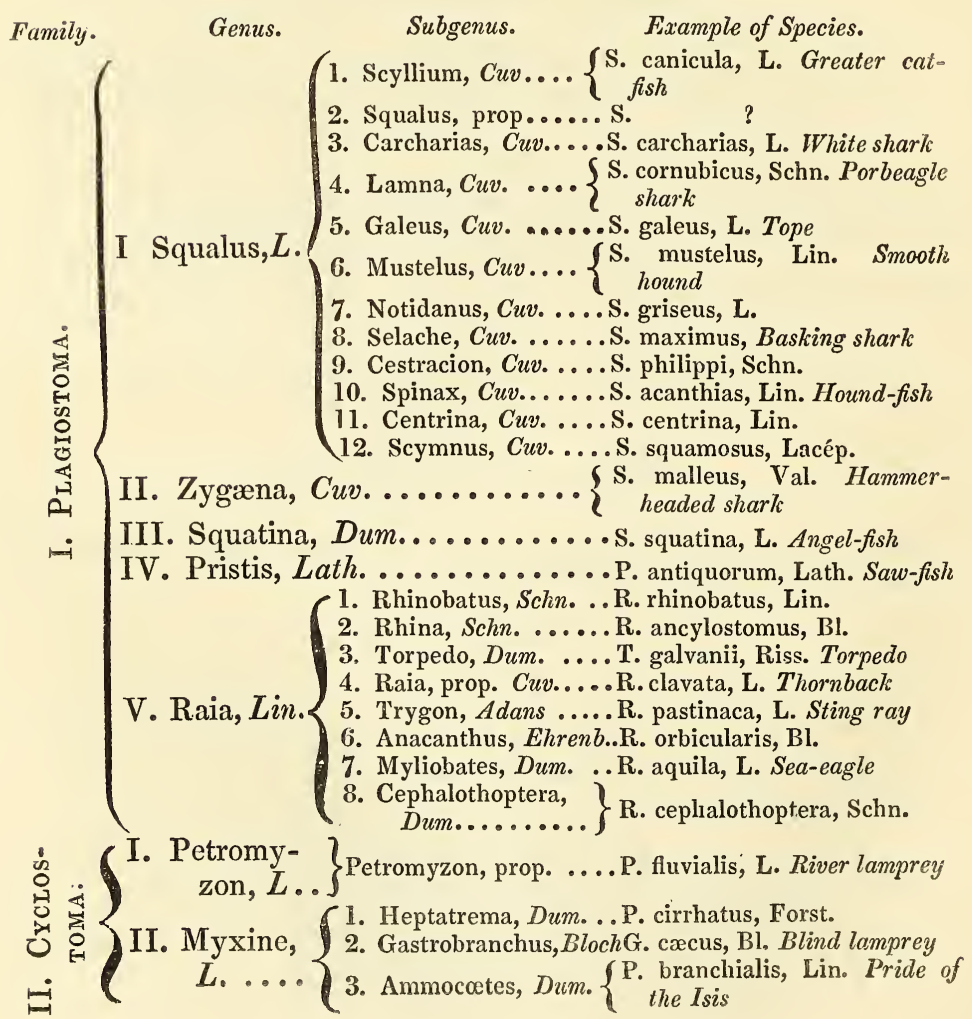




\section{INVERTEBRATA.}

\section{DIVISION II.-MOLLUSCA.}

The animals of this second grand division of the animal kingdom are either naked or testaceous.

They are divided into six classes.

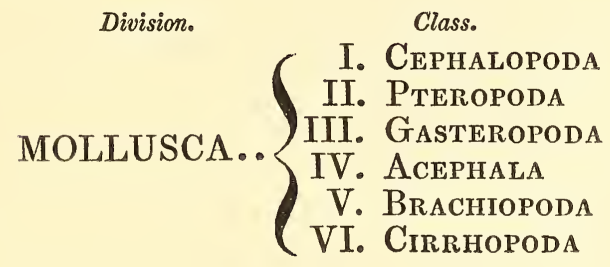

\section{CLASS I.-CEPHALOPODA.}

This class, named from the long prehensile arms which surround the head, includes but one order, designated by the same name, divided into six genera.

\footnotetext{
Genus.

Example of Species.

Subgenus. $\left.\begin{array}{l}\text { Octopus, Lam. Po- } \\ \text { lypus, Aristotle.. }\end{array}\right\}$ Sepia octopodia, L. Eledone, Arist. ..... Octopus moschatus, Lam.

2. Argonauta, Lin... $\begin{gathered}\text { Nautilus \& Pompilus of the } \\ \text { ancients }\end{gathered}$

3. Bellerophon (Fossil).B. vasulites, Montf.

4. Loligo, Lam. ...... Sepia loligo, Lin. Calamary

5. Onykia, Lesueur..... On. caribcea, Lesueur

6. Sepiola, Cuv. ....... Sepia sepiola, Lin. $\left.\begin{array}{c}\text { 7. Chondrosepia,Leu- } \\ \text { kard.......... }\end{array}\right\}$ Chon. loligiformis, Leukard

8. Sepia, prop. Lam. \{ Sepia officinalis, Lin. Common II. Nautilus ..... $\left\{\begin{array}{l}\text { 1. Spirula, Lam...... N. spirula, L. Rams-horn } \\ \text { 2. Nautilus,prop. Cuv. }\left\{\begin{array}{c}\text { N. pompilius, L. Great cham- } \\ \text { bered nautilus }\end{array}\right.\end{array}\right.$
} 


\section{CLASS I.-CEPHALOPODA (continued).}

Genus.

Subgenus.

Example of Species.

III. Belemnites

IV. Actinocamax, Miller

V. Ammonites, Brug. $\left\{\begin{array}{l}\text { 1. Ammonites, prop. } \\ \text { Lam. } \\ \text { 2. Ceratites, Haan } \\ \text { 3. Orbitulites, Lam. } \\ \text { 4. Scaphites, Sowerb. } \\ \text { 5. Baculites, Lam. } \\ \text { 6. Hamites, Sowerb. } \\ \text { 7. Turrilites, Montf. } \\ \text { VI. Camerines, Brug. Siderolithes, Lam. } \\ \text { Nummulites, } \\ \text { Lam. Helicostega } \\ \text { Hel. nautiloida } \\ \text { Hel. turbinoida } \\ \text { Hel. ammonoida } \\ \text { 3. Stygostega } \\ \text { 4. Enallostega } \\ \text { 5. Agathistega }\end{array} \mid \begin{array}{r}\text { These genera, with their } \\ \text { subdivisions, are all fossil. }\end{array}\right.$

\section{CLASS II.-PTEROPODA.}

The feet, so to speak, or rather the organs of locomotion of this class, have the appearance of wings or fins. It constitutes, like the last, but one order.

Genus. Subgenus. Example of Species.

I. Clio, Lin. . ................. Clio borealis, Lin.

II. Cymbulia, Peron

III. Pneumodermon, Cuv...........Pn. Peronii, Cuv.

IV. Limacina, Cuv. ............ Clio helicina, Phips. \& Gm.

V. Hyalea, Lam. ............... Anomia tridentata, Forskall

1. Cleodora, prop.

VI. Cleodora, Peron. $\left\{\begin{array}{l}\text { 2. Creseis } \\ \text { 3. Cuvieries }\end{array}\right.$

4. Psyche

VII. Pyrgo Defrance

5. Eurybia 


\section{CLASS III.-GASTEROPODA.}

Includes the reptilious mollusca. They are naked or testaceous, though all the latter have not a shell large enough to take in the body of the animal. The class is divided into eight orders, viz :-

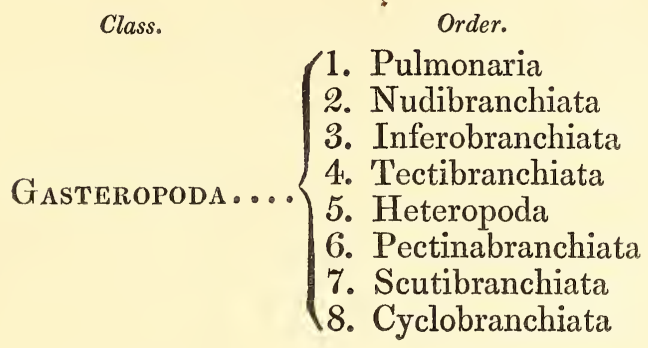

\section{ORDER I. Pulmonaria.}

These mollusca breathe by a sort of internal lungs, instead of gills. It is composed of the following genera.

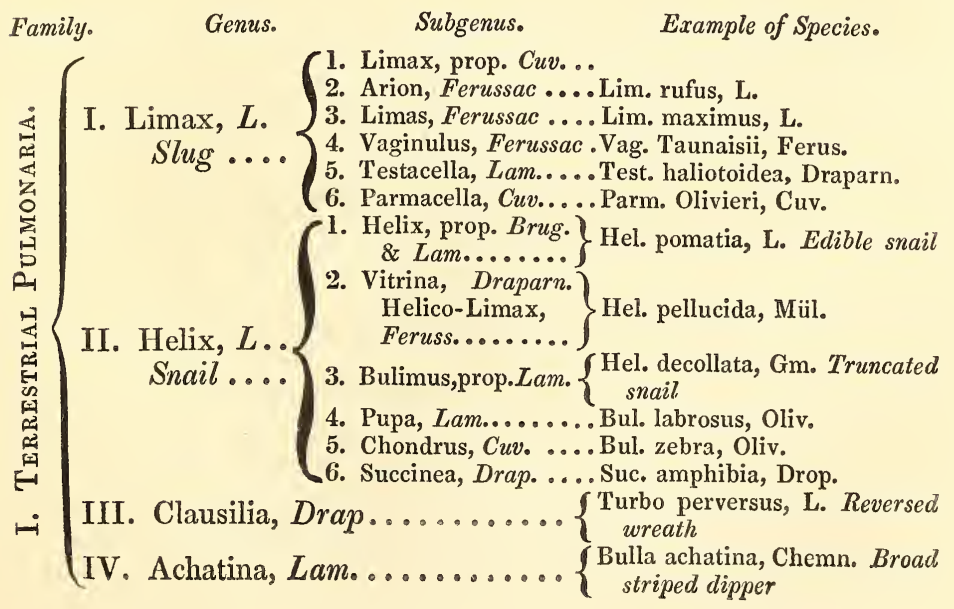




\section{ORDER I.-Pulmonaria (continued).}

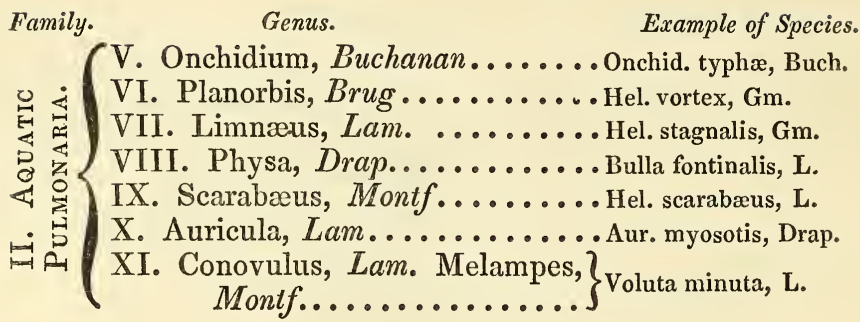

\section{ORDER II.-Nudibranchiata.}

With naked gills on the back. They are divided into the following genera.

Genus.

Example of Species.

I. Doris, Cuv. ................ Dor. verucosa, I.

II. Onchidora, Blainv............. Onch. Leachii, Blain.

III. Plocamoceros, Leuckard .........Ploc. ocellatus, Leach

IV. Polycera, Cuv. ............. Doris quadrilineata, Müll.

V. Tritonia, Cuv................. Trit. Hombergii, Cuv.

VI. Thethys, $\operatorname{Lin} . \ldots \ldots \ldots \ldots \ldots \ldots$........ fimbria, L. Cuv.

VII. Scyllæa, Lin. ................ s. pelagica, L. Cuv.

VIII. Glaucus, Forster ........... Doris radiata, Gm.

IX. Laniogerus, Blain............... Elfortii, Blain.

X. Eolidia, Cuv. ................ Doris papillosa

XI. Cavolina, Brug.............. Doris peregrina, $\mathrm{Gm}$.

XII. Flabellina, Cuv. ........... Doris affinis, $\mathrm{Gm}$.

XIII. Tergipes, Cuv. ............. Limax tergipes, Forskal

XIV. Busiris, Risso............... Bus. griseus, Risso

XV. Placobranchus, Van Hasselt........Plac. Hasseltii, Cuv.

\section{ORDER III.-INFEROBRANCHIATA.}

Gills on the lower sides of the body.

Genus.

Example of Species.

I. Phyllidia, Cuv............... Phil. trilineata, Seba

II. Diphyllidia, Cuv............. Diphyl. Brugmansii, Cuv。 
ORDER IV.-Tectibranchiata-Monopleijobranchiata, Blain.

Gills like leaflets, on the right side, or on the back.
Genus.
Subgenus.
Example of Species.

I. Pleurobranchus, Cuv. ..........Pleur. Peronii, Cuv. II. Pleurobranchæa, Meckel ........ Pleur. Meckellii, Lam. III. Aplysia, Lin............... Apl. fasciata, Poiret IV. Dolabella, Lam.............. Dol. Rumphii, Cuv.

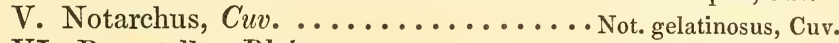
VI. Bursatella, Blain.............. Burs. Leachii, Blain. VII. Akera, Müller $\left\{\begin{array}{l}\text { 1. Bullæa, Lam........ Bull. aperta, Lam. } \\ \text { 2. Bulla, Lam....... Bul. lignaria, Lin. } \\ \text { 3. Akera, Cuv. Dori- }\end{array}\right.$ $\left.\begin{array}{c}\text { dium, Meck. Lo- } \\ \text { baria, Blain..... }\end{array}\right\}$ Bulla carnosa, Cuv.

VIII. Gastropteron, Meckel........... Gast. Meckelii, Kosse IX. Gastroplax, Blain. Umbrella, Lam.

\section{ORDER V.-Heteropoda.}

In this order, the foot is formed into a vertical muscular lamina, and does not form a disk, as in the other orders.

Genus. Subgenus. Example of Species.
1. Carinaria, Lam. .... Car. cymbium, Lam.
2. Atlanta, Lesueur.... At. Peronii, Lesueur
I. Pterotrachea,
Forsk....... $\left\{\begin{array}{r}\text { 3. Timorienna, Quoy. } \\ \text { \& Gaym............. }\end{array}\right\}$
5. Monophora, Peron..

II. Pliylliroe

\section{ORDER VI.-PECTINIBRANCHIATA.}

In this numerous order, the gills are pectinated, and occupy the last whorl of the shell. 


\section{ORDER VI.-PeCtinibRanchiata.}

Family. Genus. Subgenus. Example of Species.

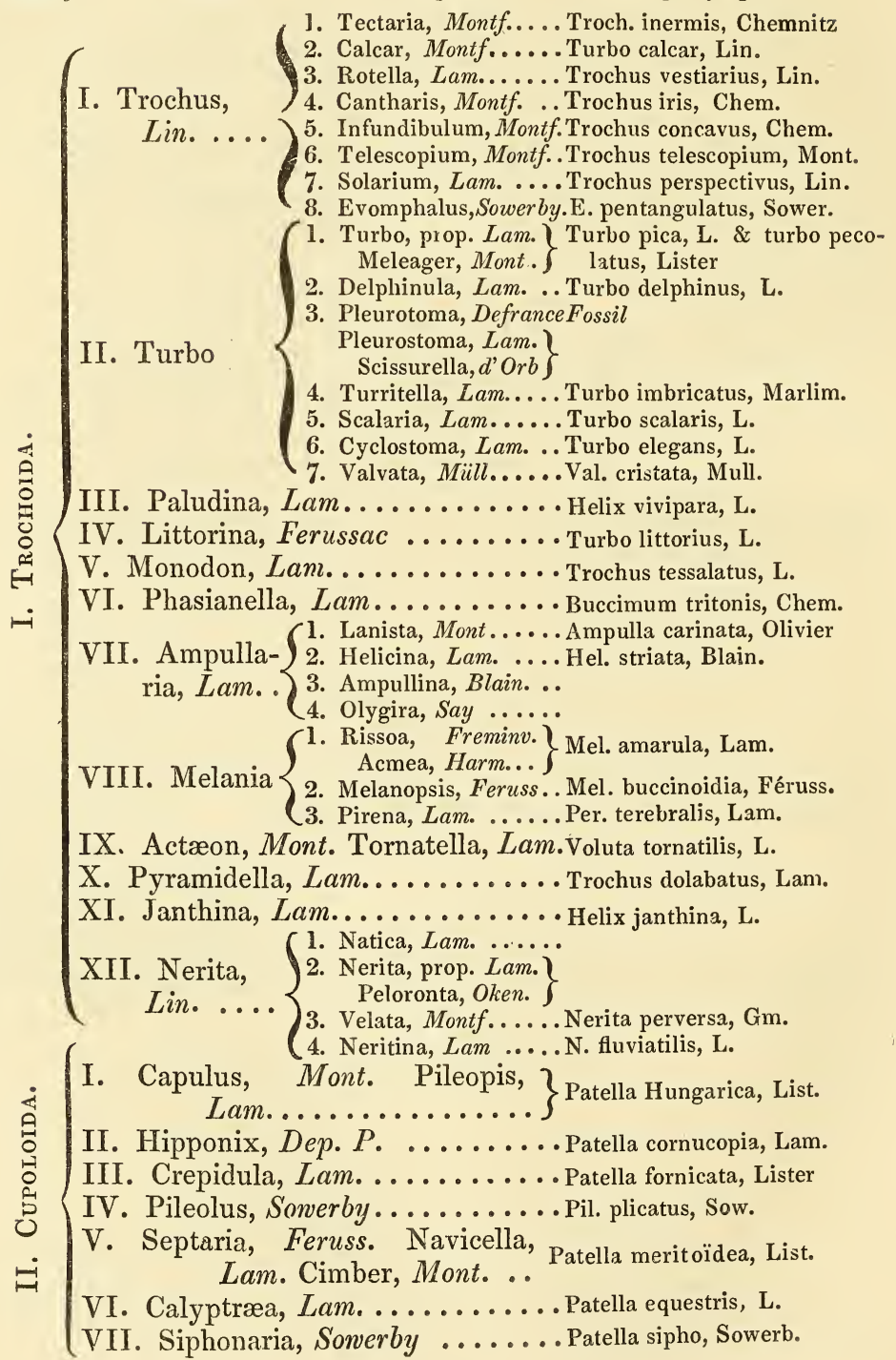


ORDER VI.-Pectinibranchiata (continued).

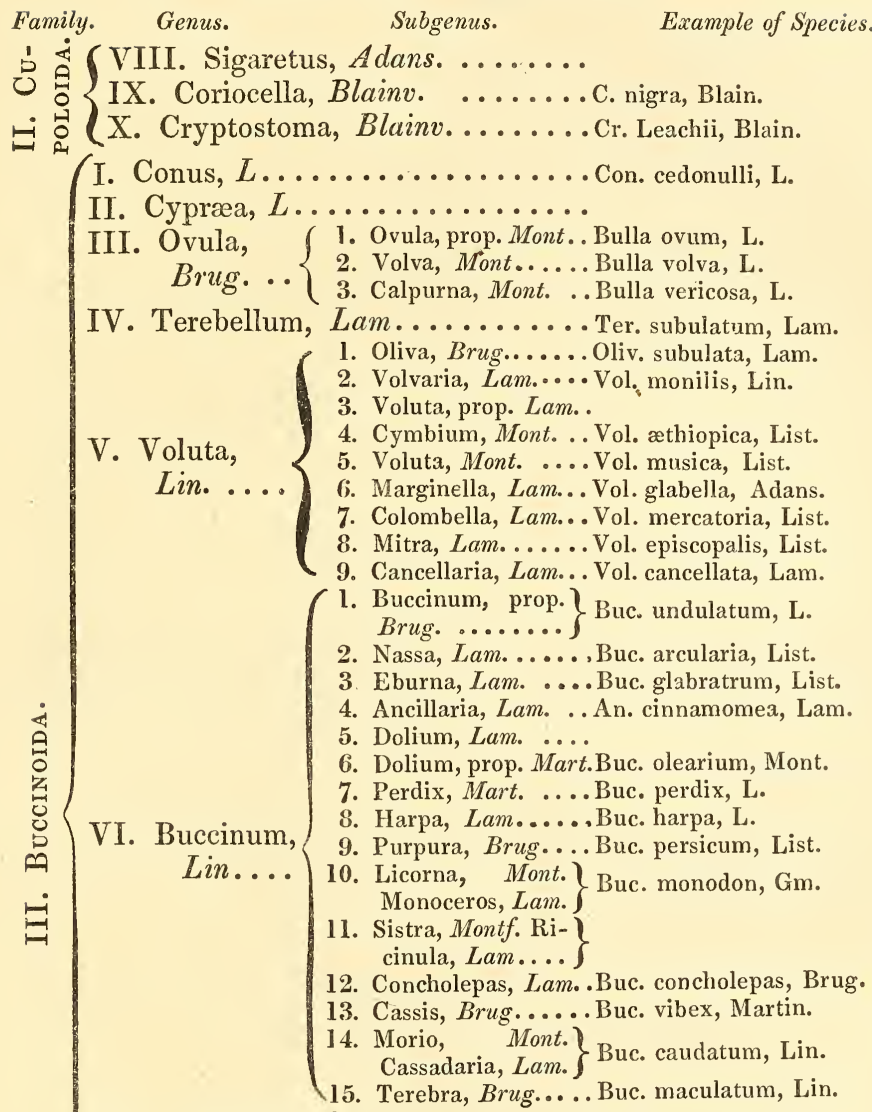

VII. Cerichium. Brug. ......... Murex vertagus, List.

1. Potamida, Brong... Pot. Lamarckii, Brongn.

1. Murex, Brug. $\left.{ }_{\text {Lam. Montf.... }}\right\}$ M. tribulus, Lister

2. Brontis, Montf...... M. haustellum, List.

3. Typhis, Montf......M. tubifer, Roissy

4. Chicoracea, Montf. . M. remosus, Hist.

VIII. Murex,

5. Aquilla, Montf...... M. cutaceus, L.

L. ......

6. Lotorium, Mart. .. M. lotorium, L.

7. Tritonium, Montf... M. tritonis, L.

8. Trophona, Montf... M. Magellanicus, Mart.

9. Ranella, Lam. ..... M. bufo, Mont.

10. Apolles, Montf..... M. gyrinus, Hist.

11. Fusus, Lam. ......

12. Fusus, prop. Montf.M. cochlidium, Seba 


\section{ORDER VI.-Pectinibranchiata (continued).}

\begin{tabular}{|c|c|c|}
\hline & $s$. & \\
\hline 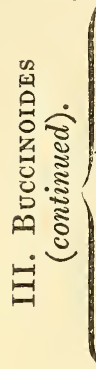 & $\begin{array}{r}\text { VIII. Murex, } \\
\text { L. }(\text { cont }) \cdot\end{array}$ & 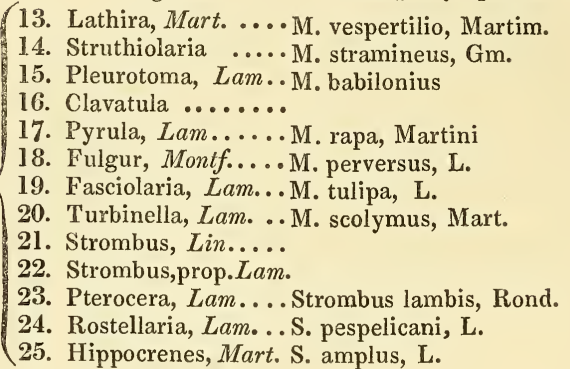 \\
\hline
\end{tabular}

\section{ORDER VII.-Tubulibranchiata.}

Allied in most respects to the previous order, but the shell describes a tube only partially spiral, and is attached to foreign bodies.

Genus. Subgenus. Example of Species.

I. Vermetus, Adans............... Serpula lumbricalis, L. Vermillia, Delamarck ... S. trigusta, Gm.

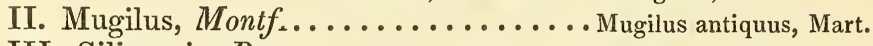
III. Siliquaria, Brug. ............ Serpula anguina

\section{ORDER VIII.-Scutibranchiata,}

In this order, the shell is not turbinated, and is in fact little else than a mere shield to the gills. The animals are true hermaphrodites.

Genus.

$$
\text { Subgenus. }
$$

Example of Species.

I. Halyotis, Lin. $\cdots\left\{\begin{array}{l}\text { 1. Halyotis, prop. Lam.. Halyotis, Gm. } \\ \text { 2. Padolla, Montf. ....Padolla, Montf. }\end{array}\right.$

3. Stromatia, Lam..... Halyotis imperforata, Gm.

II. Fissurella, $L \ldots \ldots \ldots \ldots \ldots \ldots \ldots$. . Patella of Div. $V$. of $\mathrm{Gm}$.

III. Emarginula, $\operatorname{Lam} \ldots \ldots \ldots \ldots$. . . Patella fissura, L.

IV. Parmophorus, Lam. .......... Patella ambigua, Chem. 


\section{ORDER IX.-Cyclobranchiata.}

The gills, in the forms of leaflets, are situated under the edge of the mantle. In other respects the order is assimilated to the last.

$$
\text { Genus. } \quad \text { Example of Species. }
$$

I. Patella, L................P. Patella, Gm.

II. Chiton, L. ................ Chiton, L.

\section{CLASS IV.-ACEPHALA.}

In this class there is no apparent head, the principal ganglion of the brain being over the mouth, which is situated at the bottom, or between the folds of the mantle.

\section{ORDER I.-Acephala Testacea.}

In this order, the animals have four branchial leaflets. All the bivalves, and many multivalves, belong to it.

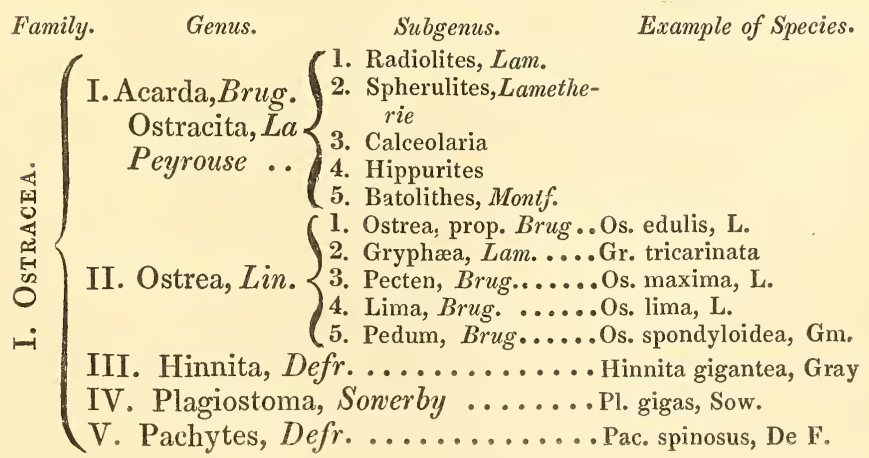




\section{ORDER I.-Acephala Testacea (continued).}

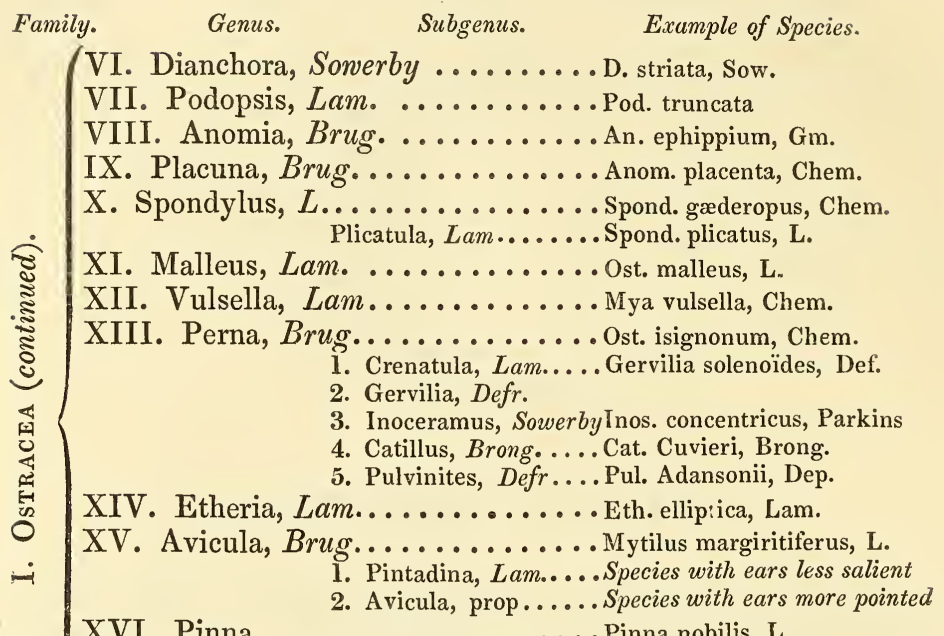

XVI. Pinna ................ Pinna nobilis, L.

XVII. Arca, L. $\left\{\begin{array}{l}\text { 1. Arca, prop. Lam. ..Arca Noæ, Chem. } \\ \text { 2. Cucullæa, DelamarckArca cucullata, Chem. } \\ \text { 3. Pectunculus, Lam... Arca pilosa, L. } \\ \text { 4. Nucula, Lam....... Arca pellucida, Chem. }\end{array}\right.$

XVIII. Trigonia, Brug. ......... La Trigonie nacrée, Lam.

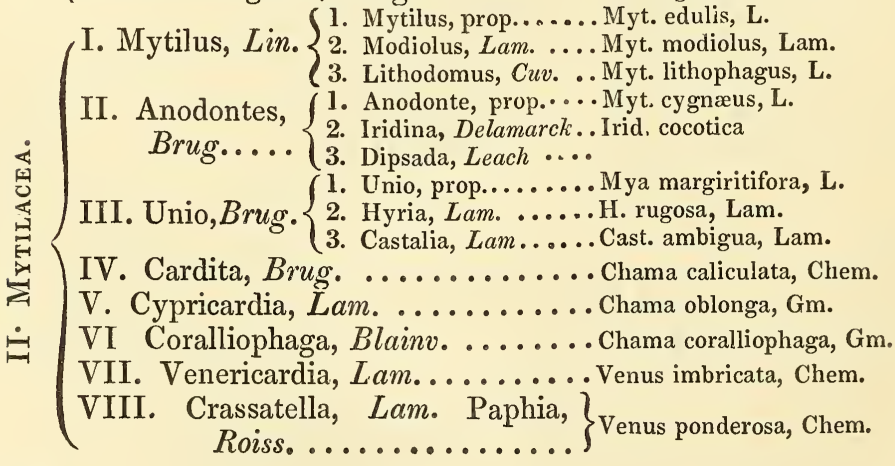

(1. Tridacna, prop. Lam.Chama gigas, L.

2. Hippopus ....... Chama hippopus, $\mathrm{L}$.

I. Chama, Lin. $\{$ 3. Chama, prop. Brug. .Chama Lazarus, Chem.

4. Diceras, Lam...... . Dic. arietina, Lam.

5. Isocardia, Lam. ..... Chama cor, L. 


\section{ORDER I.-Acephala Testacea (continued).}

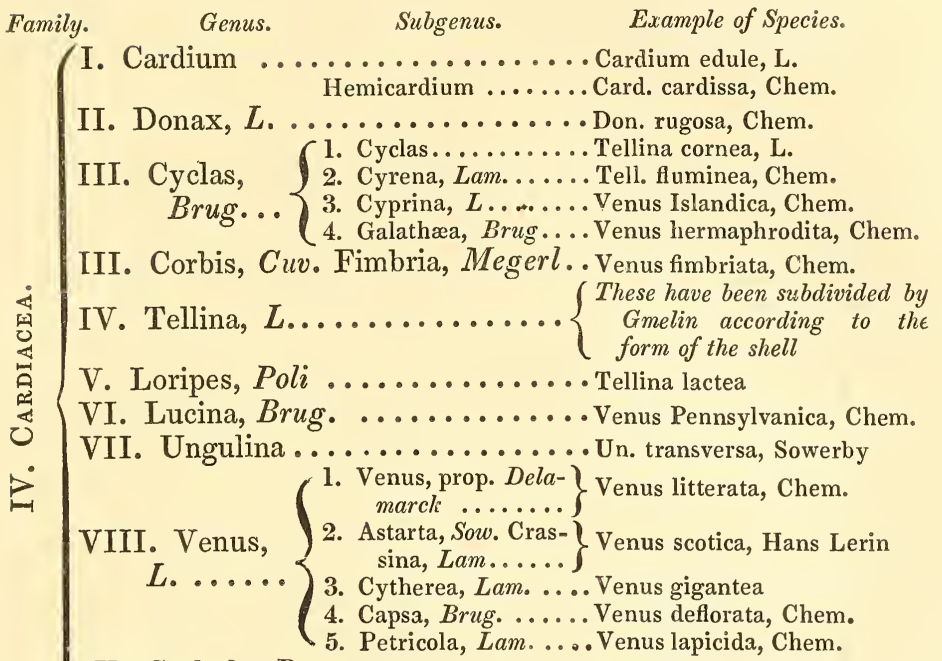

IX. Corbula, Brug. ............. gallica

X. Mactra, L. \{ $\begin{aligned} & \text { 1. Mactra, prop. Lam... Mya australis, Chem. } \\ & \text { 2. Lavignon }\end{aligned}$

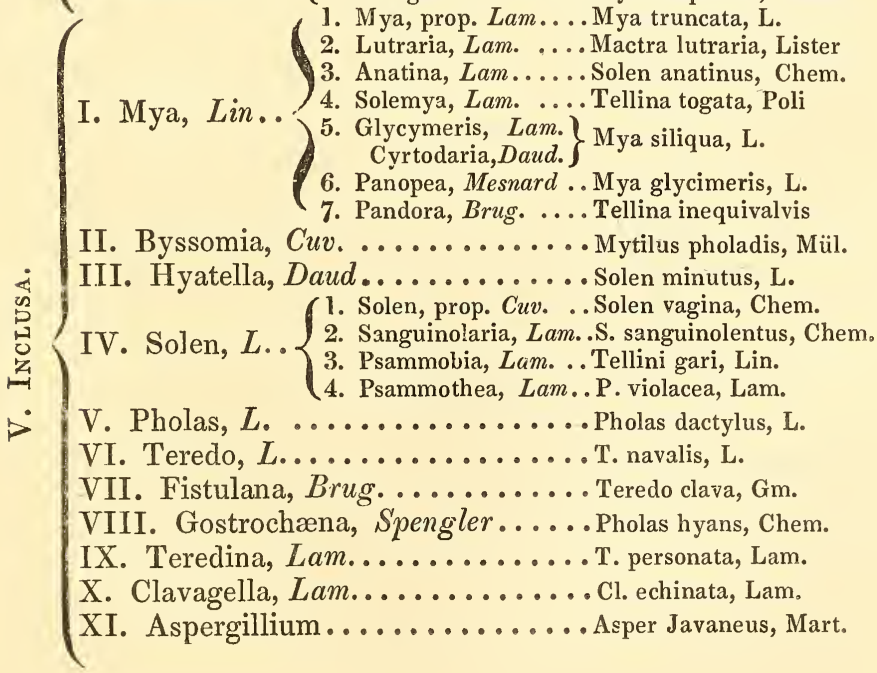




\section{ORDER II.-Acephala Nuda.}

These are without a shell. The gills differ among themselves in form, but never constitute four leaflets, as in the testaceous acephala.

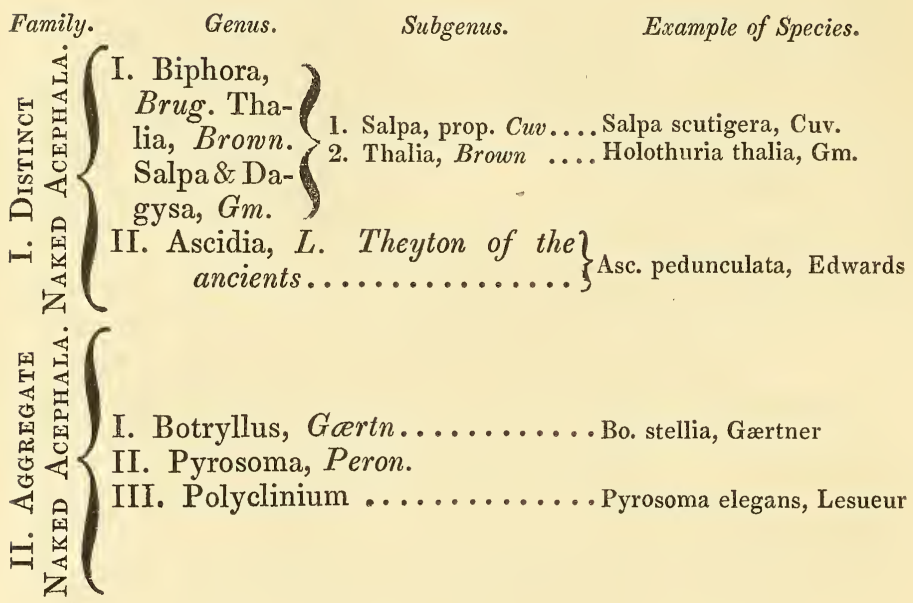

\section{CLASS .V.-BRACHIOPODA.}

In this class, as in the fourth, the mantle has two lobes, and it is always open; but for feet there are fleshy arms, with numerous filaments, which can be withdrawn into the shell. All the genera are bivalves.

$$
\text { Genus. Subgenus. Example of Species. }
$$

I. Lingula, Brug.............. Lin. anatina, Cuv. II. Terebratula,Brug. $\left\{\begin{array}{l}\text { 1. Spirifer, Sowerby ... Anomia scobinata, Gualt. } \\ \text { 2. Thecidia, Def..... The. Mediterranea, Risso }\end{array}\right.$ III. Orbicula, Cuv.............Patella anomala, Müll. Crania, Brug. .........Anomia craniolaris, L. 


\title{
CLASS VI.-CIRRHOPODA.
}

\author{
Lepas and Tritons, Lin.
}

In this class, the animals of which approach the Articulata, the mouth is furnished with jaws, and the abdomen with filaments, or cirrhi, in pairs, like those under the tail of several crustacea.

Genus. Subgenus. Example of Species.
I. Anatifa, Brug. ..
1. Pentalasmis, Leach..Lepas anatifera, L.
2. Pollicipes, Leach ... Lepas pollicipes, L.
3. Cineras, Leach..... . . vittata, Leach
4. Otion, Leach ...... Otion Cuvieri, Leach
5. Tetralasmis, Cuv...T. hirsutus, Cuv.
1. Balanus, prop. ..... Lepas balanus, $\mathbf{L}$.
2. Acasta, Leach ....A. Montagni, Leach
3. Conia, Blainv. .... C. radiata, Blain.
4. Asama, Ranzani .. Lepas porosus, Gm.
II. Balanus, Brug..
5. Pyrgoma, Savig. ..Py. cancellata, Leach
6. Octhosia, Ranzani.. Lepas Stræmii, Müll.
7. Creusia, Leach .... C. spinulosa, Leach
8. Coronula, Lam..... Lepas balænaris, L.
9. Tubicinella, Lam. $\left\{\begin{array}{l}\text { La Tubicinelle, Lam.An. Mus。 } \\ \text { I. xxx. }\end{array}\right.$
10. Diadema, Ranz.... Lepas diademi, Chem. 


\section{DIVISION III.-ARTICULATA.}

In this grand division, the body, and in general the limbs, are surrounded by, or encased in, articulated rings, affording a support for the soft parts, and an attachment for the muscles.

It is divided into four classes.

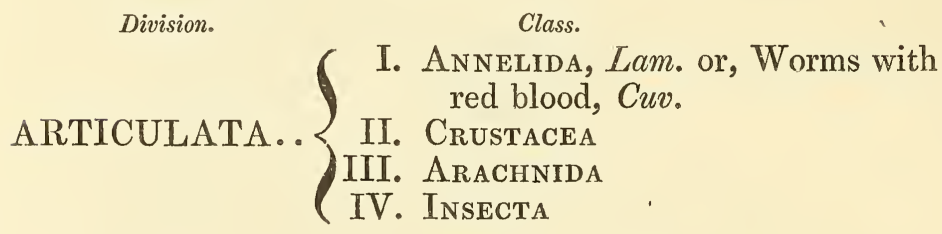

CLASS I.-ANNELIDA, or, Worms with red blood.

Body soft, elongated, articulated or divided into segments or transverse folds.

The Annelida are divided into three orders, viz :-

$$
\begin{gathered}
\text { Class. } \\
\text { ANN ELIDA.. }
\end{gathered} \begin{cases}\text { 1. } & \text { Tubicola } \\
\text { 2. } & \text { Dorsibranchiata } \\
\text { 3. } & \text { Abranchiata }\end{cases}
$$

order I.-Tubicola.

These animals form tubes to live in, either of calcareous matter exuded from their own body, or from foreign substances, to which tubes they are not however attached, like the tubicolous or testaceous mollusca. 


\section{ORDER I.-TuBicola.}

Genus. Subgenus. Example of Species.

I. Serpula, L. .................... Serpula contortuplicata, Ellis

Spirorbis, Lam. $\quad \ldots$... Serpula spirillum, Pallas

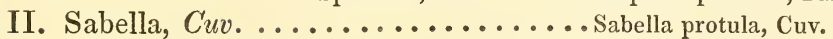

III. Terebella, Cuv.............. Ter. conchilega, Gm.

IV. Amphitrite, Cuv. .................. auricoma Belgica, Gm.

V. Syphostoma, Otto ................. diplocaita, Otto

VI. Dentalium, L. ............. D. elephantinum, Martini

\section{ORDER II.-Dorsibranchiata.}
Genus.
Subgenus.
Example of Species.

I. Arenicola, Lam. Lumbricus marinus, $\mathrm{L}$.
II. Amphinome, Brug...... 3. Euphosine, Sav. ....Ev. laureata, Sav.
1. Chloeia, Sav. ..... Terebella flava, Gm.
2. Pleione, Sav. Am- $\}$ Ter. carunculata, Gm.
4. Hipponoa, Aud. \& $\}$ Hip. Gaudichaudi

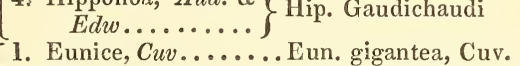

III. Eunice, Cuv..\{ $\begin{aligned} & \text { 2. Marphisa, Sav...... Ner. sanguinea, Montag. } \\ & \text { 3. Lysidica, Sav..... Ly. Valentina, Sav. }\end{aligned}$

4. Aglaura, Sav........ Ag. fulgida
IV. Nereida, Sav. $\left\{\begin{array}{l}\text { 1. Nereis, prop. Cuv. . Phereis versicolor, Gm. } \\ \text { 2. Phyllodoca, Sav. }\end{array}\right.$ Nereis lamifera Atlanti Lycoris, Sav..

V. Alciopa, Aud. \& Edw.............. Reynaudii, Aud. \& Edw.

1. Spio, prop. ......... Spio setticornis, Otto

VI. Spio, Fab. \& 2. Syllis, Sav.......... S. monilaris, Sav. Gmel....... 3. Glycera, Sav. ....... Nereis alba, Müll.

4. Nephthys, Cav. ...... Nephthys Hombergii, Cuv.

VII. Lombrinera, 1 . Lombrinera........ Nereis abranchiata, Pallas Blain....... 2. Aricia, Sav.............. Curvieri, Aud.

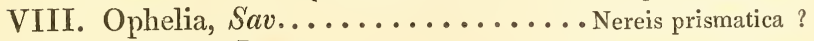

IX. Cirrhatula, Lam. ..................

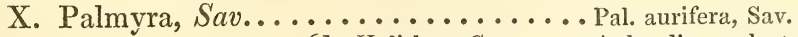

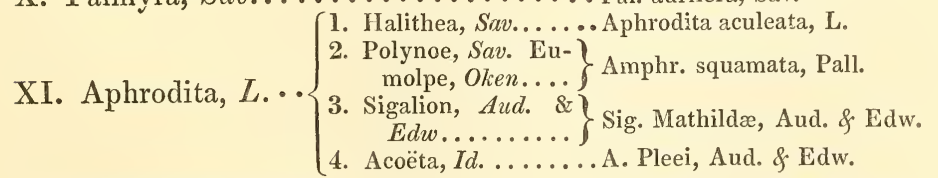

XII. Chætopterus, Cuv. ............... phergamentaceus, Cuv. 


\section{ORDER III.-Abranchiata.}
Family. Genus.
Subgenus.
Example of Species.
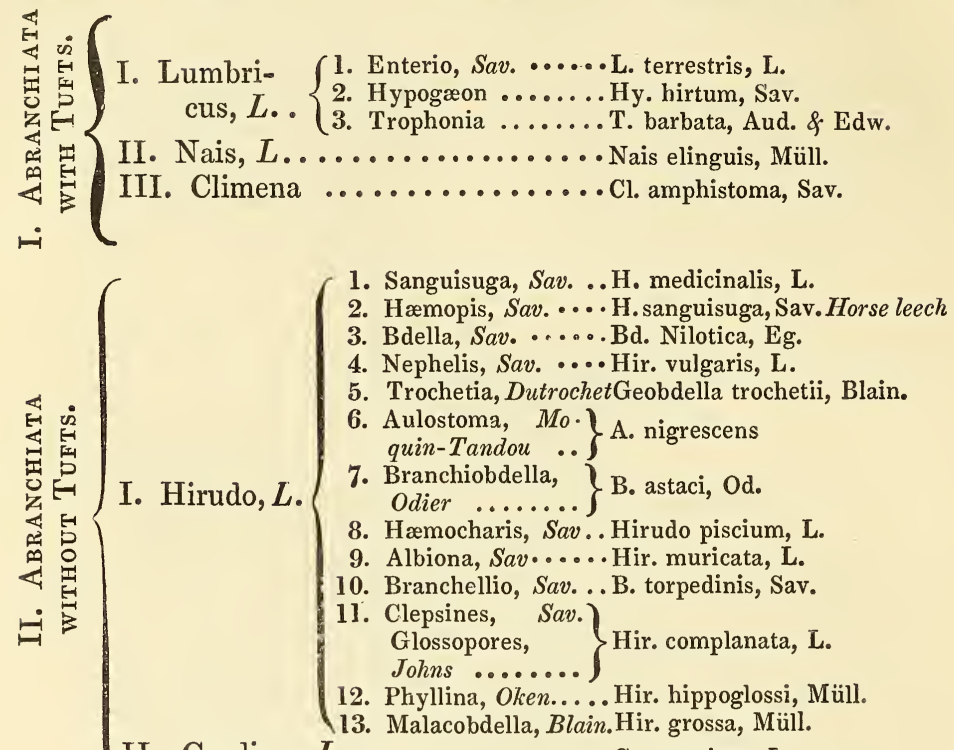

II. Gordius, $L \ldots \ldots \ldots \ldots \ldots$. . . G. aquaticus, L. 


\section{ARTICULATA.}

The following classes of Articulata have articulated feet or legs.

\section{CLASS II._CRUSTACEA.}

This class is divided into two sections.

$$
\begin{gathered}
\text { Class. } \\
\text { CRUSTACEA.. } \\
\begin{array}{c}
\text { Section. } \\
\text { I. Malacostraca }
\end{array} \\
\text { II. Entomostraca }
\end{gathered}
$$

The section Malacostraca is divided into five orders.

$$
\begin{aligned}
& \text { Section. } \\
& \text { Order. } \\
& \text { Malacostraca } . .\left\{\begin{array}{l}
\text { 1. Decapoda } \\
\text { 2. Stomapoda } \\
\text { 3. Amphipoda } \\
\text { 4. Læmodipoda } \\
\text { 5. Isopoda }
\end{array}\right.
\end{aligned}
$$

The first four orders embrace the genus Cancer of Linnæus, and the last that which he names Oniscus.

\section{ORDER I.-DECAPODA.}

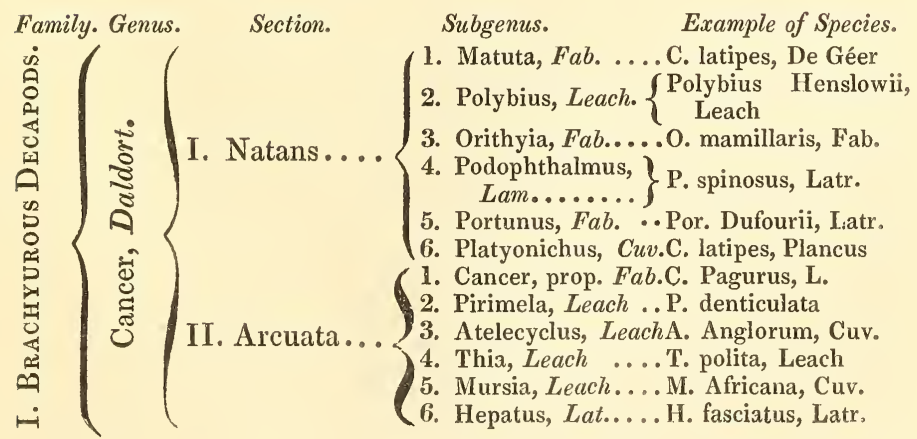




\section{ORDER I.-DEcapoda (continued).}

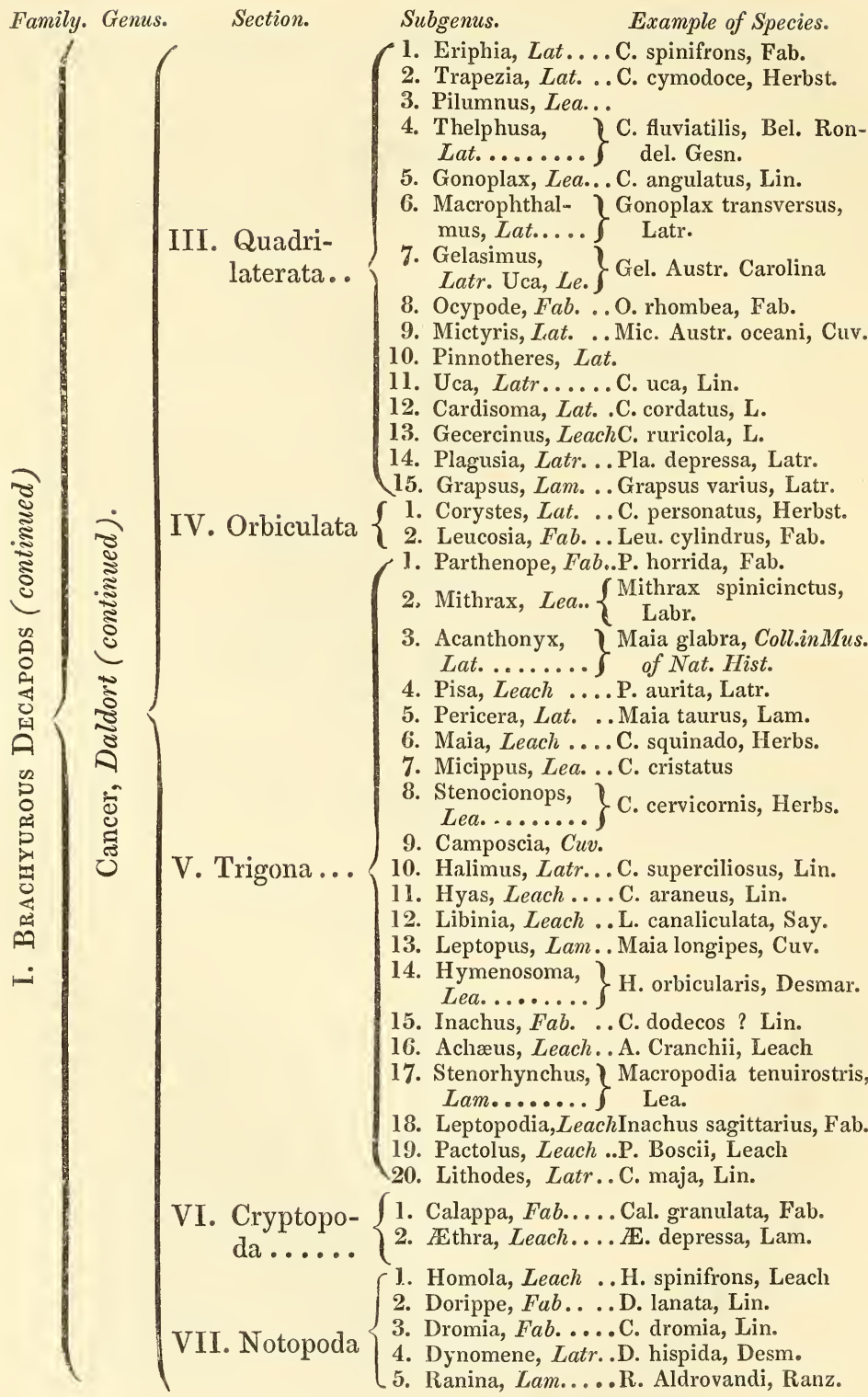


ORDER I.-DECAPoda (continued).

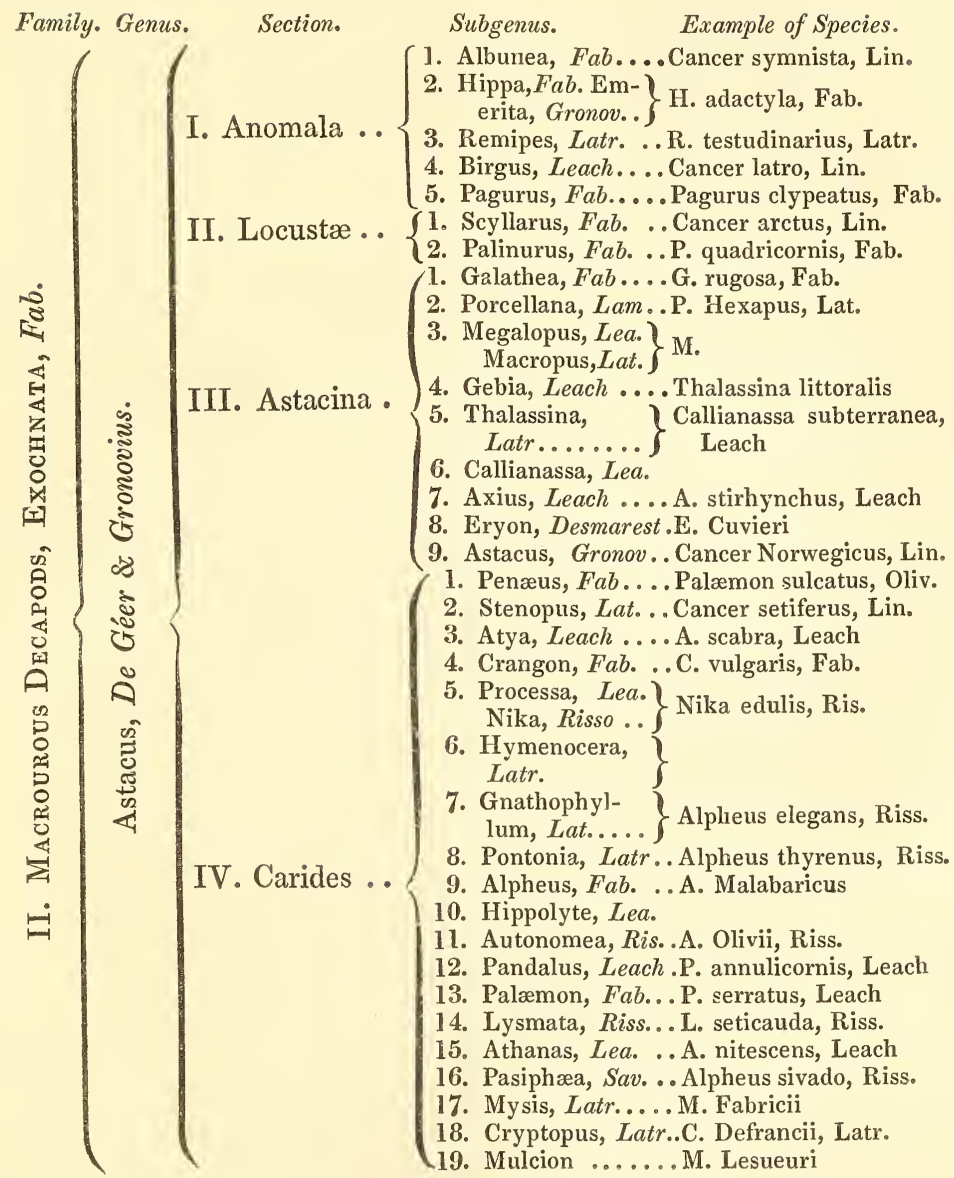




\section{ORDER II.-STOMAPODA.}

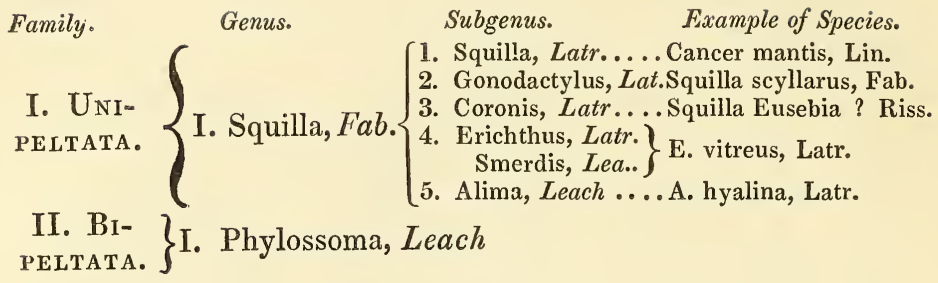

ORDER III.-AMPHIPODA.

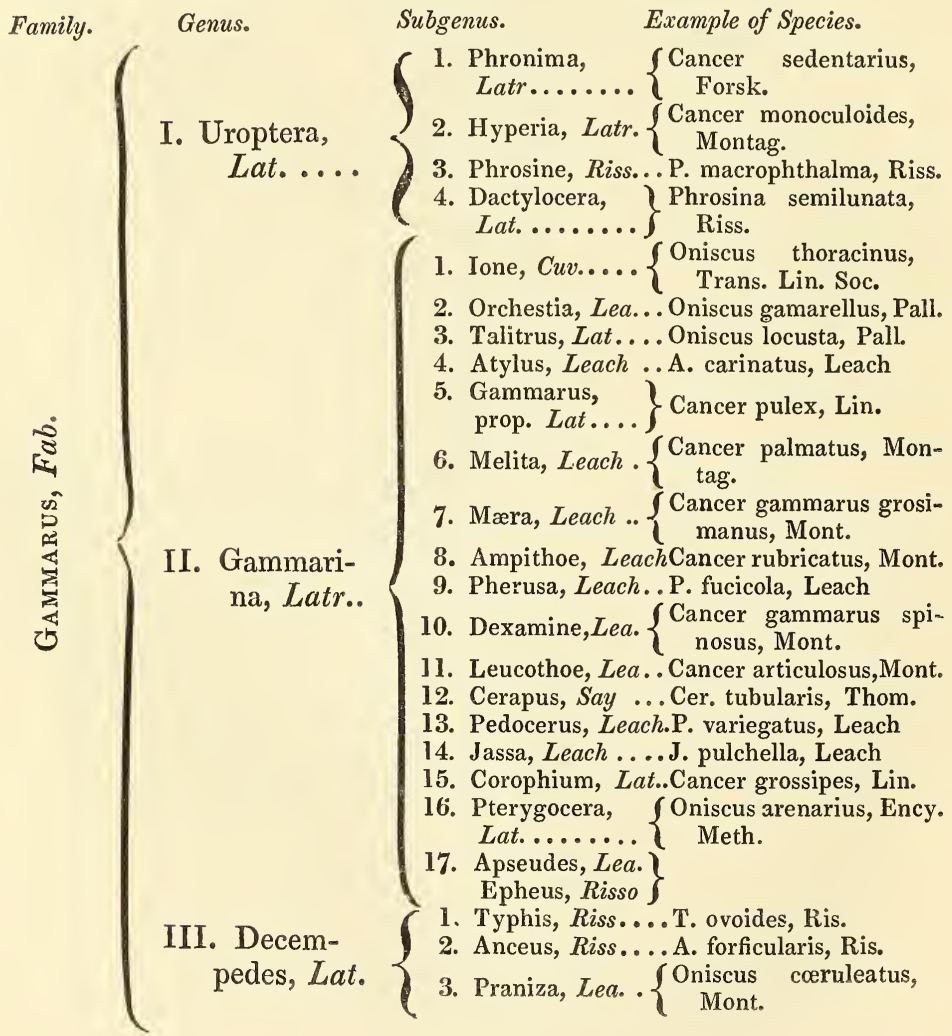




\section{ORDER IV.-LEMODIPODA.}

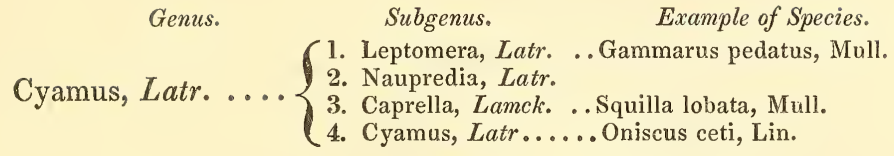

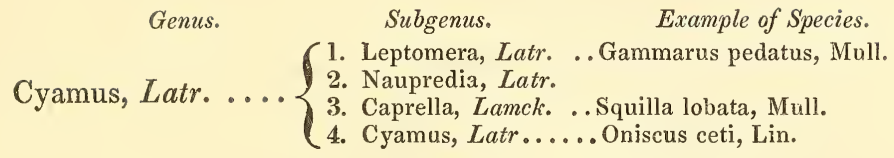

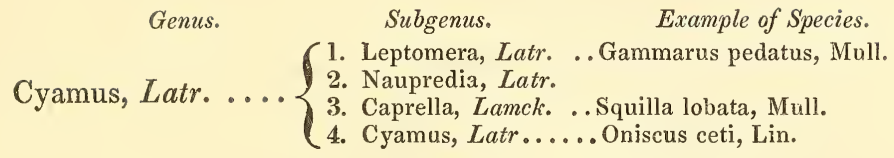

\section{ORDER V.-IsopodA.}

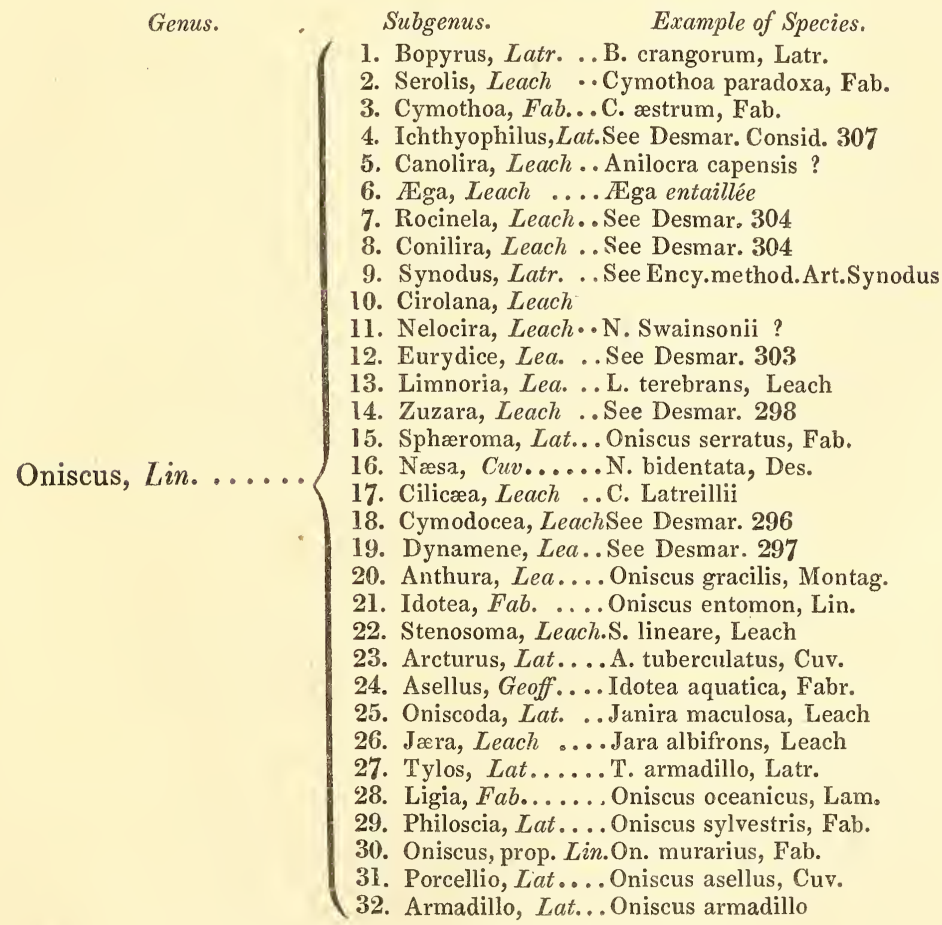




\title{
CRUSTACEA.
}

\section{GENERAL DIVISION II.-ENTOMOSTRACA}

is divided into two orders.

\author{
Division. \\ Order. \\ Entomostraca.. $\left\{\begin{array}{l}\text { 1. Branchiopoda } \\ \text { 2. Pæcilopoda }\end{array}\right.$
}

ORDER I.-BRANCHIOPODA.

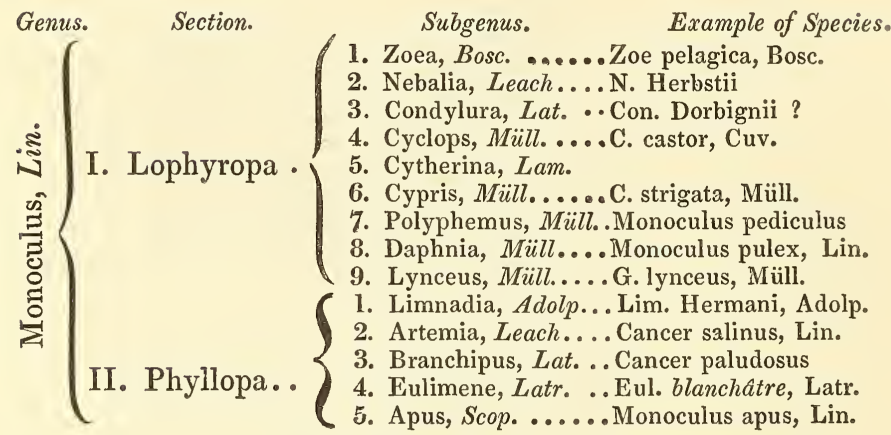

ORDER II.-P屃ILOPODA.

Family.

Genus.

Example of Species.

I. Xiphosura ... $\left\{\begin{array}{l}\text { Limulus, Fab. . . . . L Limula heterodactyla, Cuv. } \\ \text { Tachypleus ..... . Lim. cyclops, Fab. }\end{array}\right.$

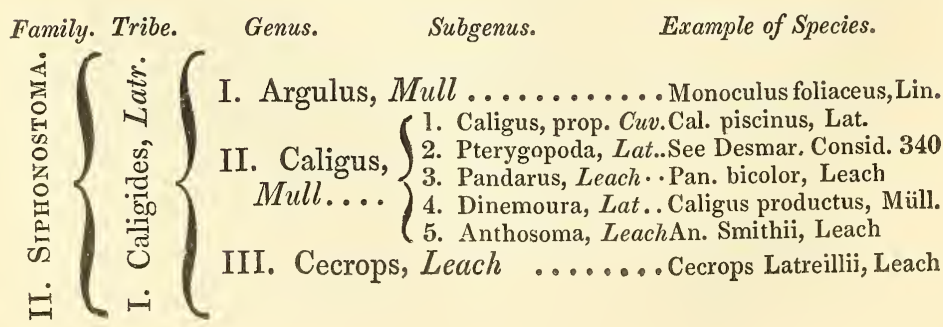




\section{ORDER II._PæcILopoda (continued).}

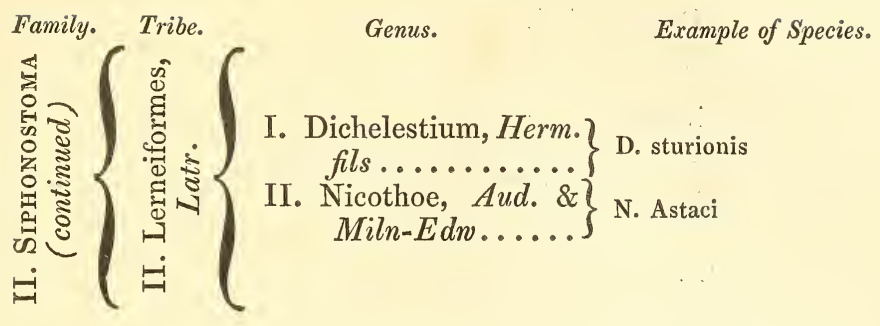

\section{TRILOBITES.}

The singular fossils called Trilobites, are supposed by naturalists to have been crustacea allied to entomostraca.

The principal genera are

1. Agnostus, Brong .

2. Calymene, Brong.

3. Asaphus, Brong.

4. Ogygia, Brong.

5. Parodoxides, Brong. 


\section{CLASS III.-ARACHNIDA.}

The Arachnida are divided into two orders.

Class.

order.

Arachnida .. $\left\{\begin{array}{l}\text { 1. Pulmonariæ } \\ \text { 2. Tracheariæ }\end{array}\right.$

\section{ORDER I.-PULMonaria.}

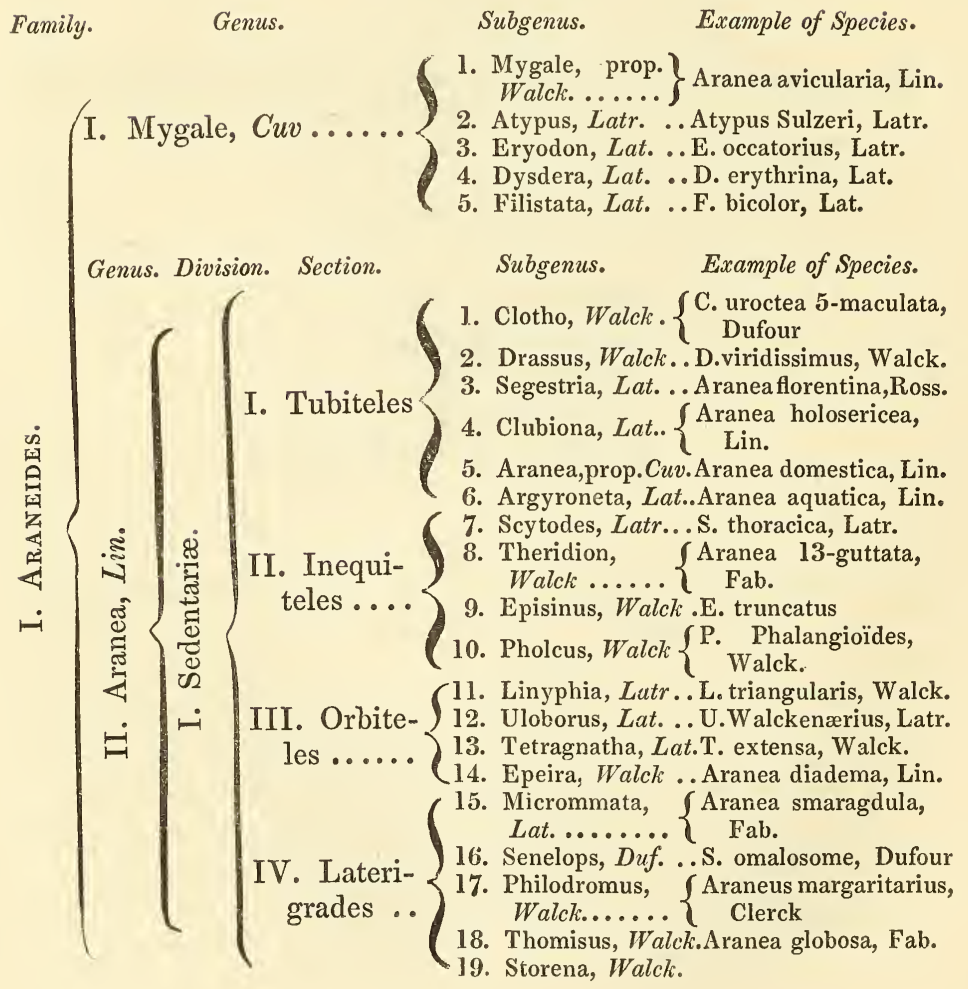




\section{ORDER I.-Pulmonaria (continued).}

Family. Genus. Division. Section. Sibgenus. $\quad$ Example of Species.
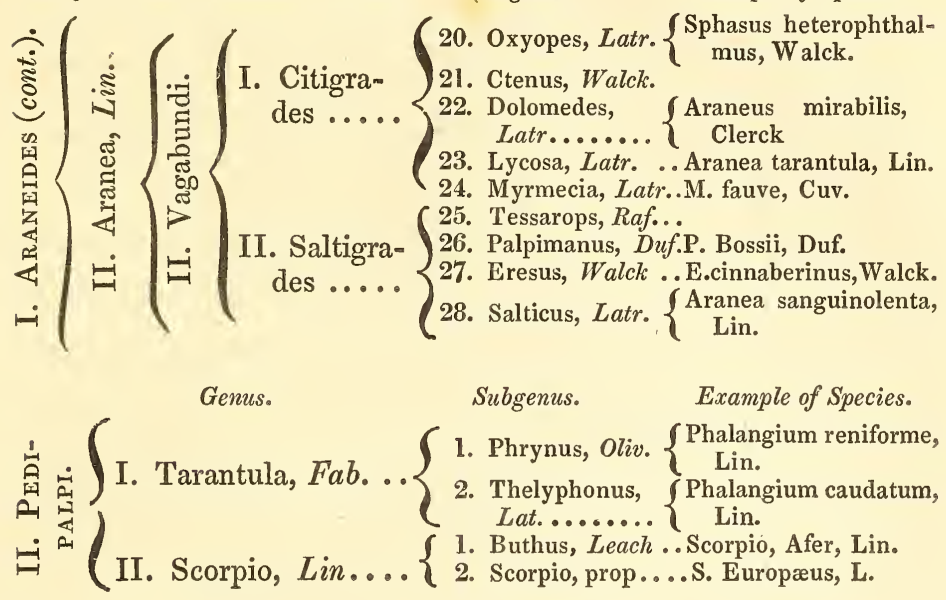

ORDER II.-TracheariE.

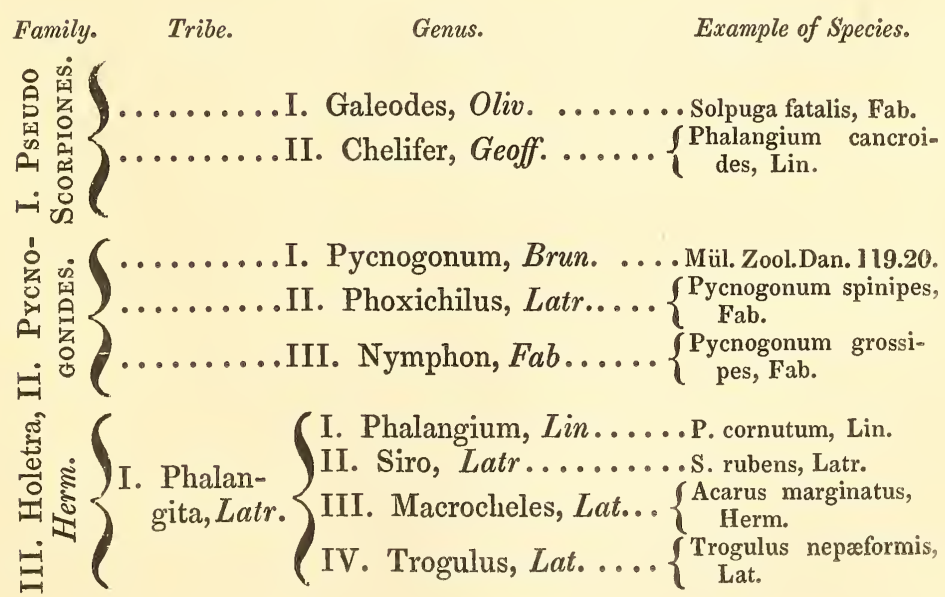




\section{ORDER II.-Trachearie (continued).}

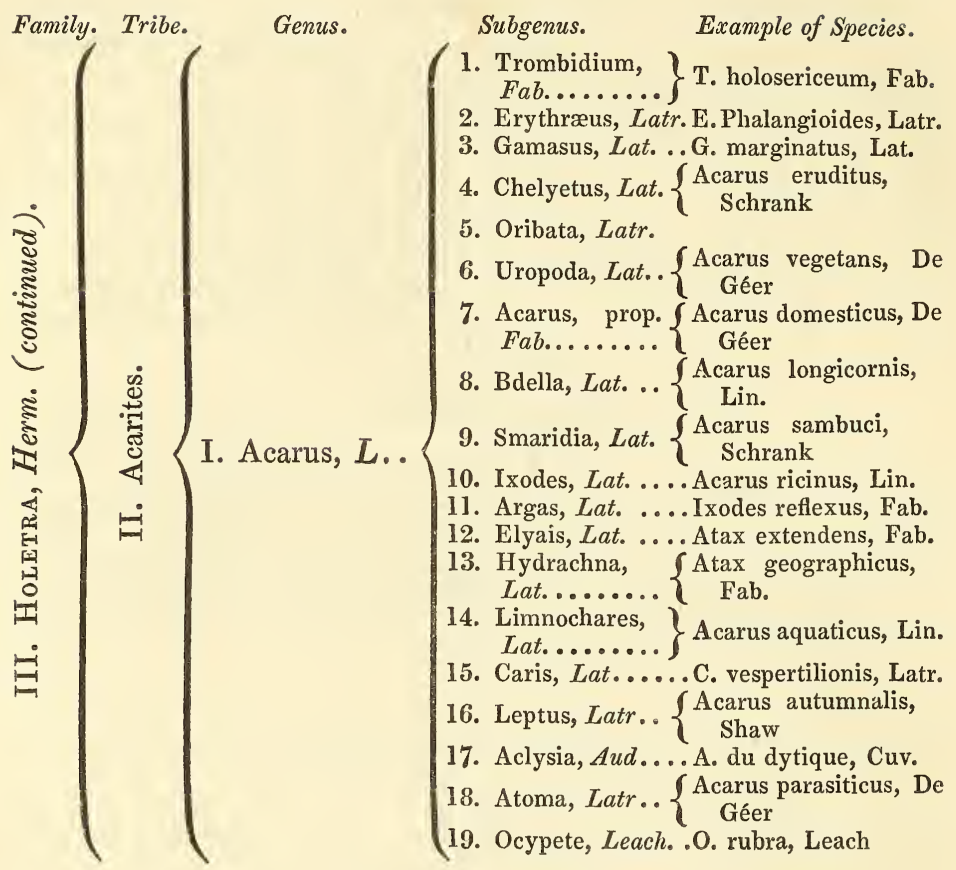




\section{CLASS IV.-INSECTA.}

The Insects are divided into twelve orders.

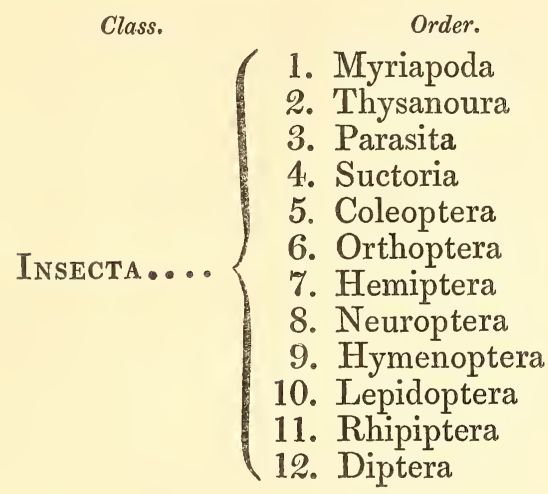

ORDER I.-MYriaPodes.

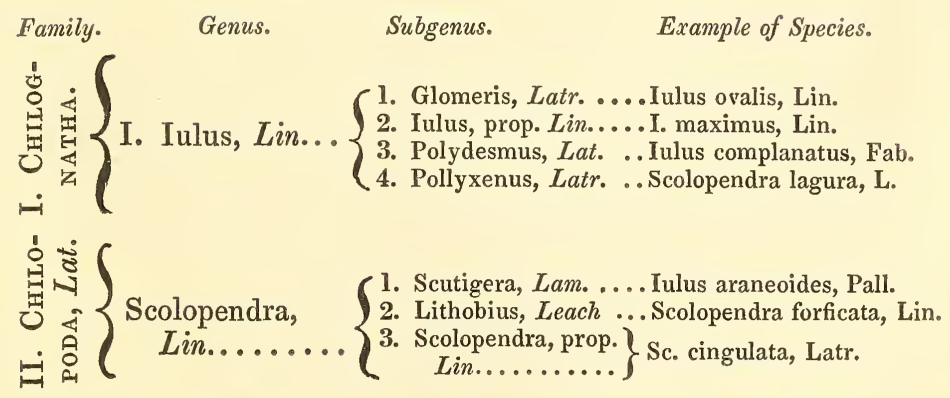

ORDER II.-Thysanoura.

Family. 'Genus. Subgenus. Example of Species. $\left.\begin{array}{c}\text { I. LePIS- } \\ \text { MENE, Lat. }\end{array}\right\}$ Lepisma, L. $\left\{\begin{array}{l}\text { 1. Machilis, Latr. . Leprisma polypoda, Lin. } \\ \text { 2. Lepisma,prop. Lin.L. saccharina, Lin. }\end{array}\right.$ II. PodU- $\}$ Podura, L.. \{1. Podura, Lat. .... P. arborea, Lin.

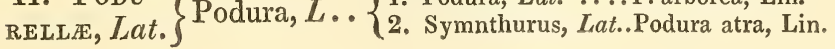




\section{ORDER III.-PARASITA.}
Genus.
Subgenus.
Example of Species.

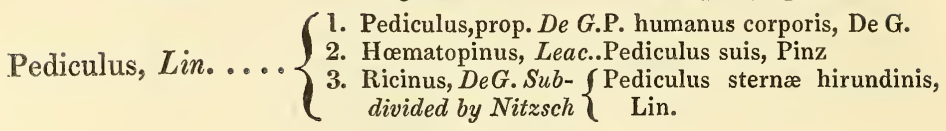

ORDER IV.-SUCtoRIA.

Genus. Example of Species.

Pulex, L. ..................Pulex irritans, L.

\section{ORDER V.-Coleoptera.}

\section{Section I.-Pentamera.}

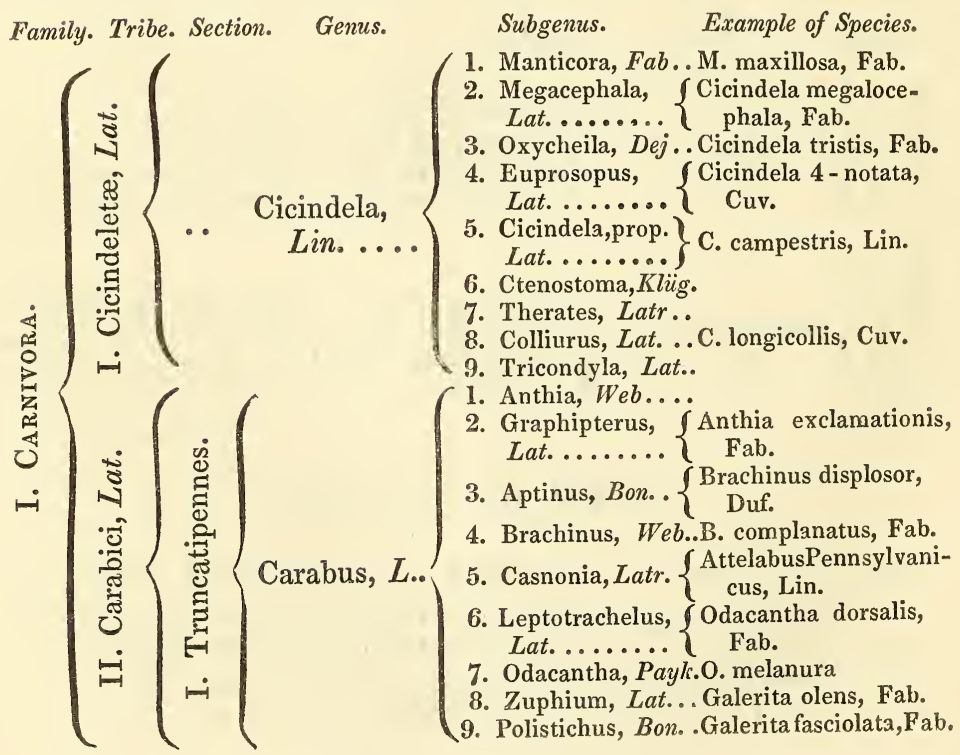


ORDER V.-Coleoptera (continued).

Section I.-Pentamera (continued).

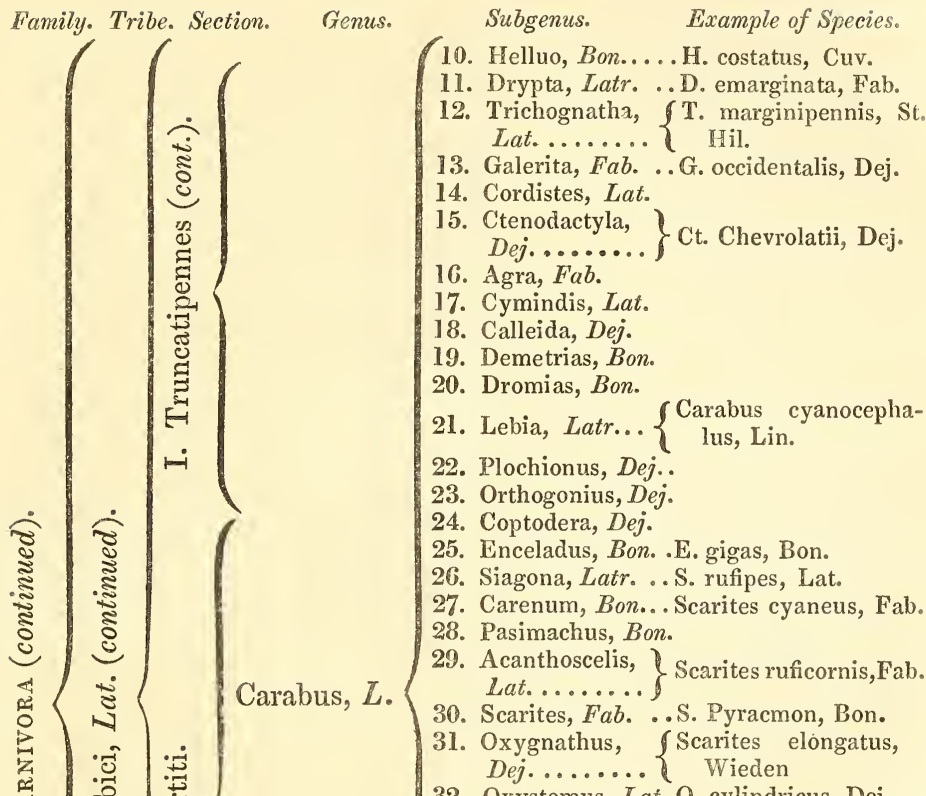

32. Oxystomus, Lat..O. cylindricus, Dej.

33. Camptodontus, $\}$ C. Cayennensis, Dej. Dej. ........

34. Clivina, Lat.... . Tenebrio fossor, Lin.

35. Dyschirius, Bon..D.

36. Morio, Lat. . \{ Harpalus monilicornis,

37. Ozæna, Oliv..... O. dentipes, Oliv.

38. Ditomus, Bon.. $\left\{\begin{array}{c}\text { Carabus Calydonius, } \\ \text { Fab. }\end{array}\right.$

39. Apotomus, Hoff. .Scarites rufus, Oliv.

40. Acinopus, $\{$ Harpalus megacephaZeigl. ..... \{ lus, Lat.

41. Daptus, Fisch. Acinopus maculipen-

42. Harpalus, Dej. . Carabus æneus, Fab.

43. Ophonus, Zeigl..

44. Stenolophus, S. vaporariorum, Dej.

45. Acupalpus, Carabus meridianus, Lat. ....... Lin.

\section{$k$}




\section{ORDER V.-Coleoptera (continued).}

\section{Section I.-Pentamera (continued).}

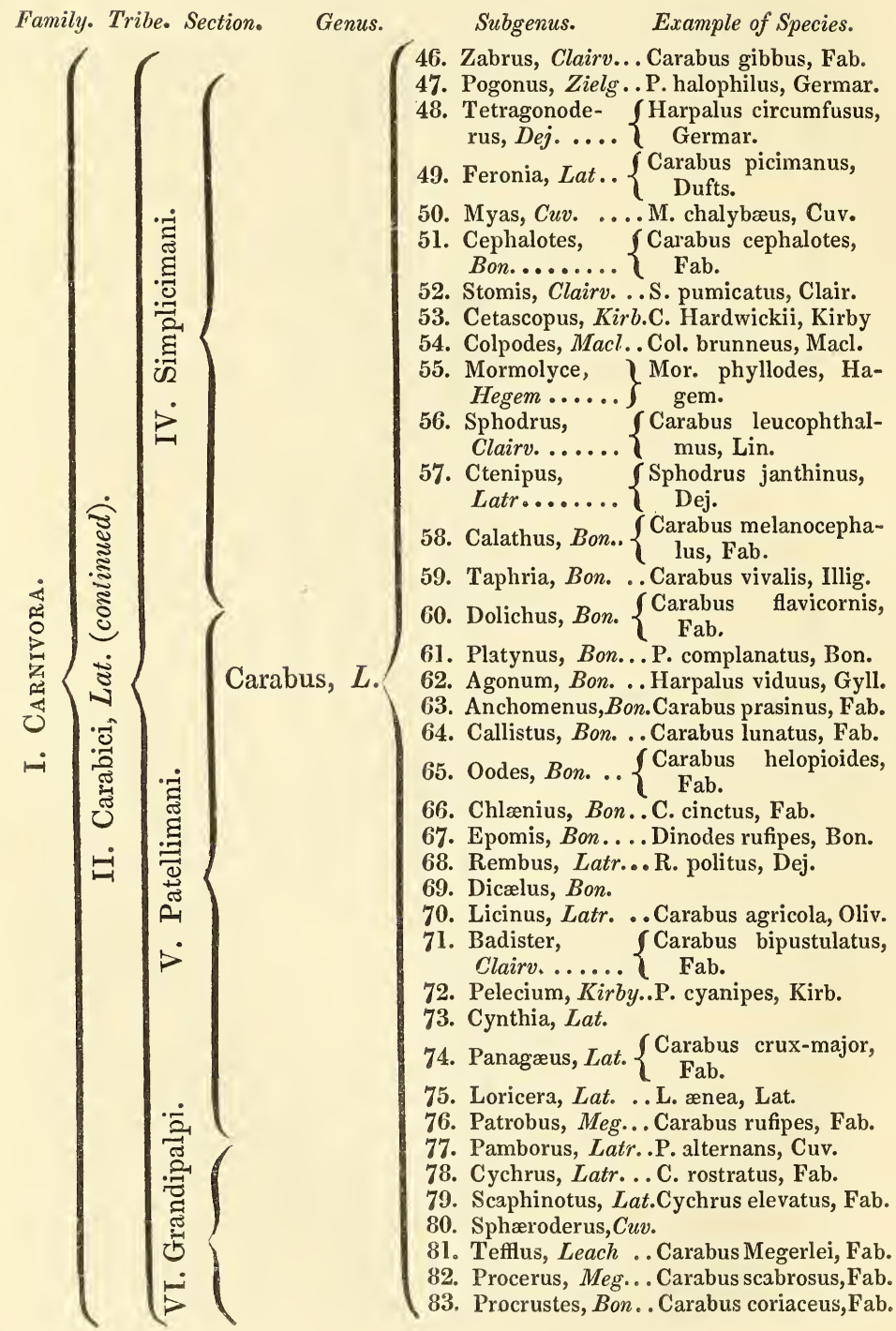




\section{ORDER V.-Coleoptera (continued).}

\section{Section I.-Pentamera (continued).}

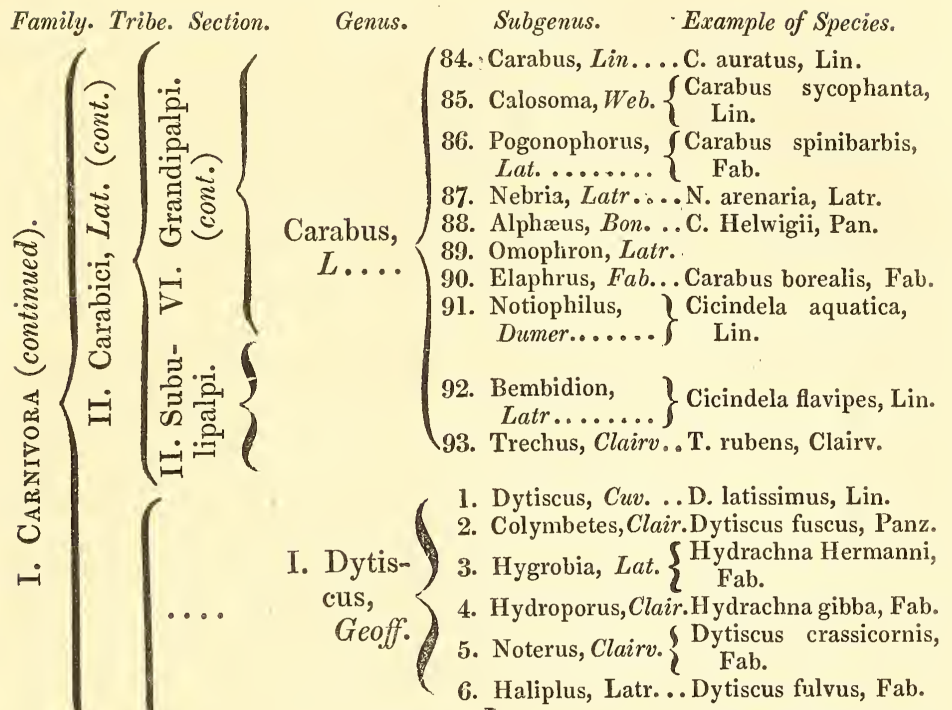




\section{ORDER V.-Coleoptera (continued).}

\section{Section I.-Pentamera (continued).}
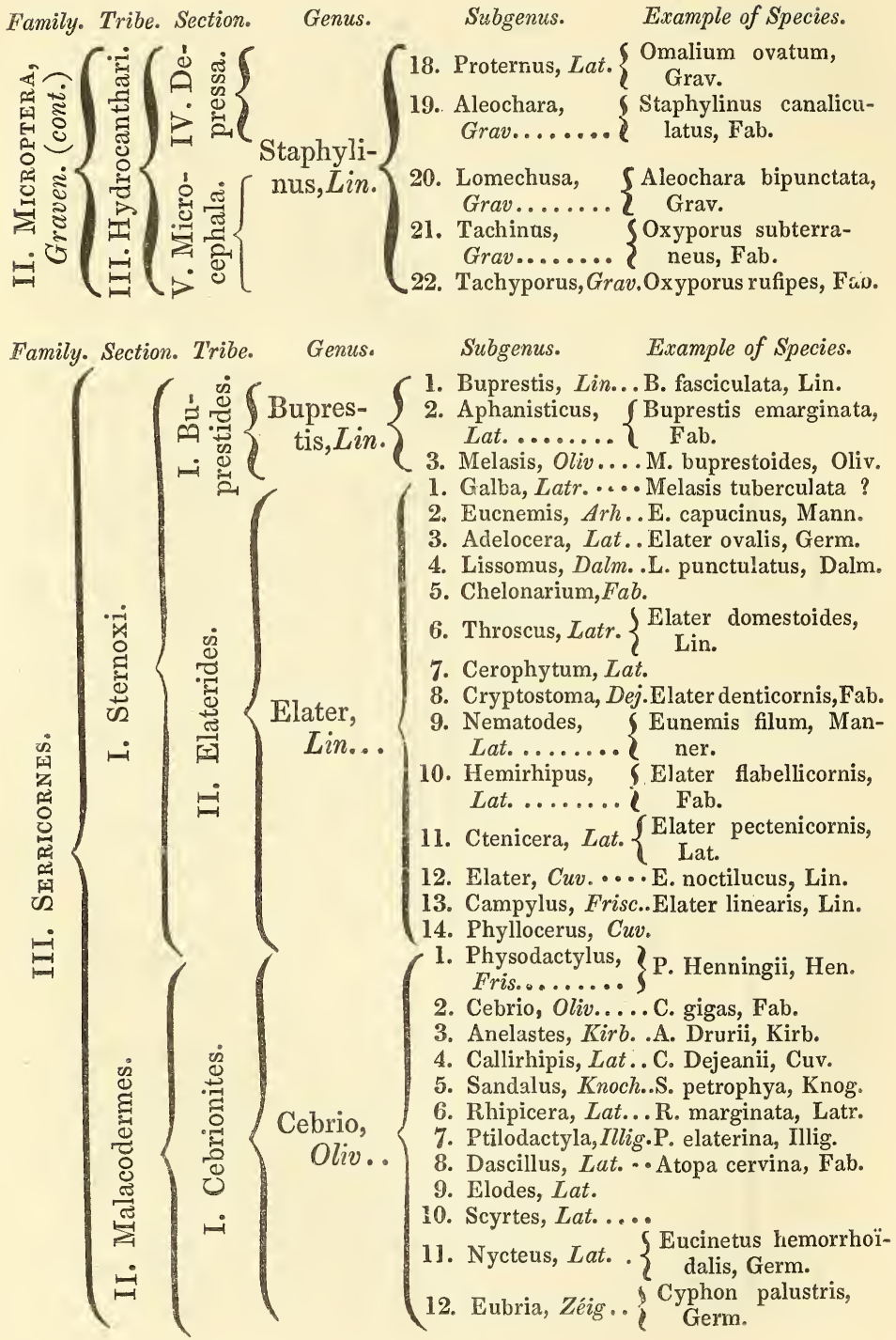


\section{ORDER V.-Coleoptera (continued).}

Section I.-Pentamera (continued),

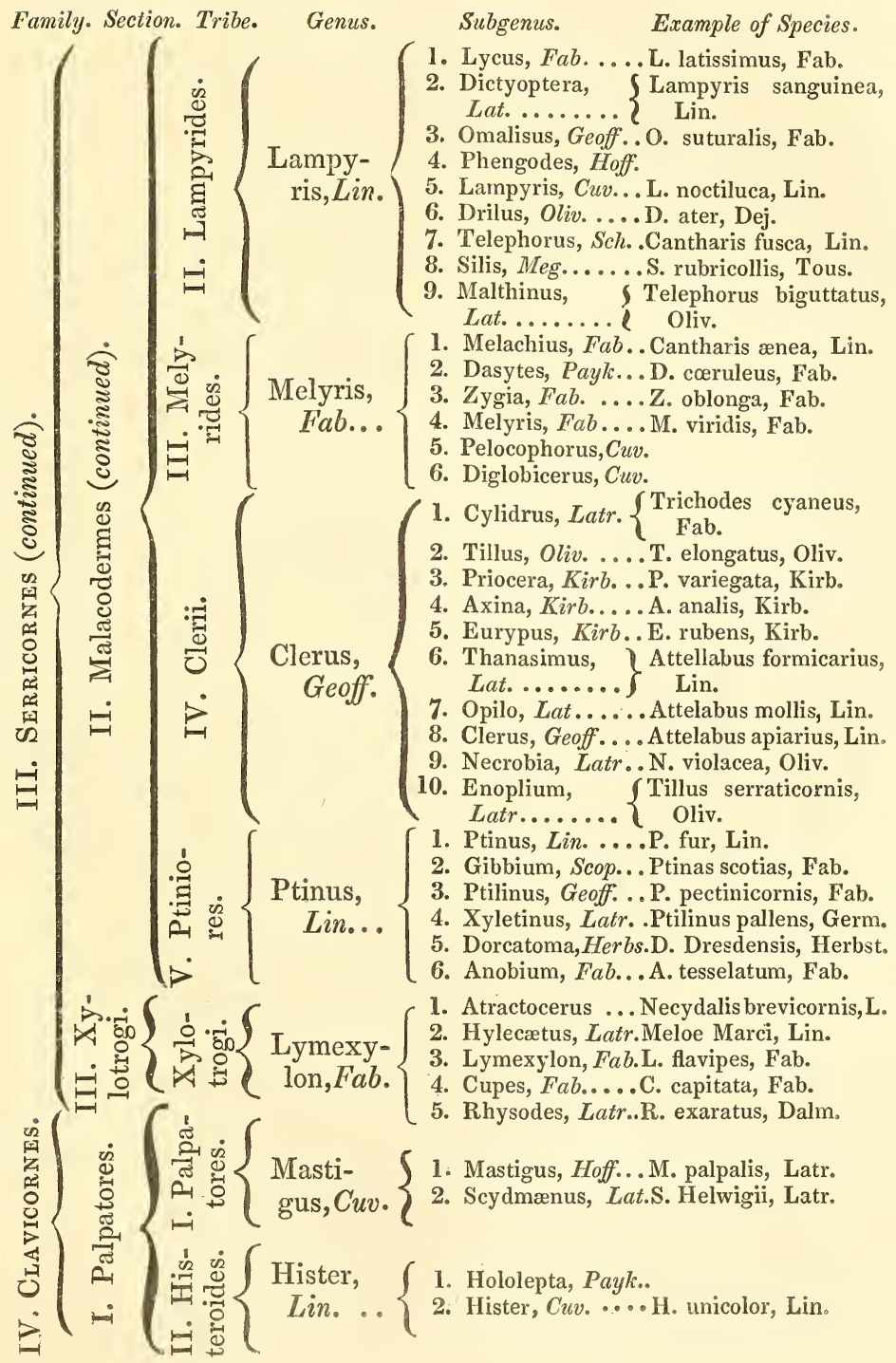




\section{ORDER I.-Coleoptera (continued).}

\section{Section I.-Pentamera (continued).}

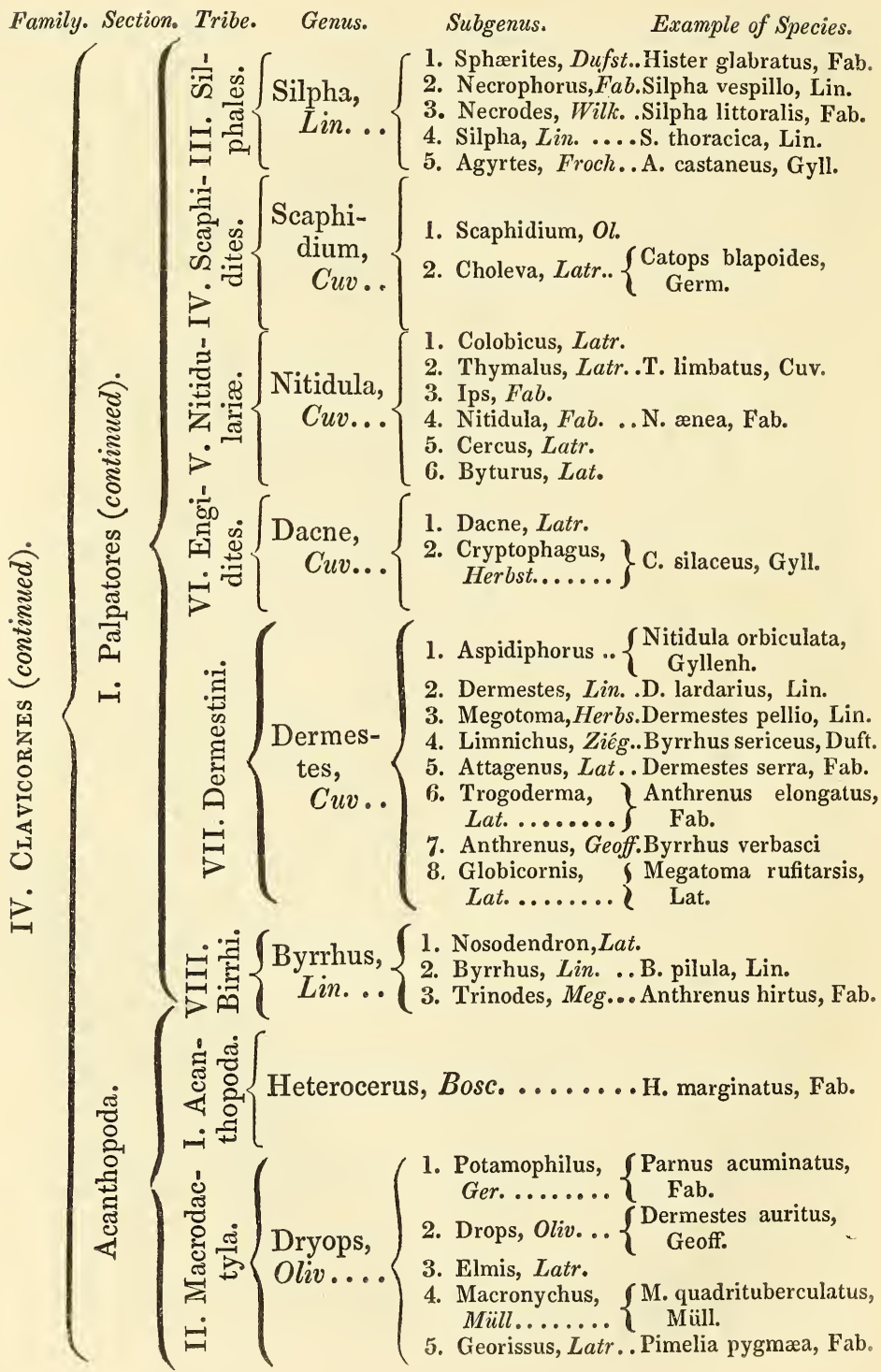




\section{ORDER V.-Coleoptera (contimued).}

\section{Section I.-Pentamera (continued).}

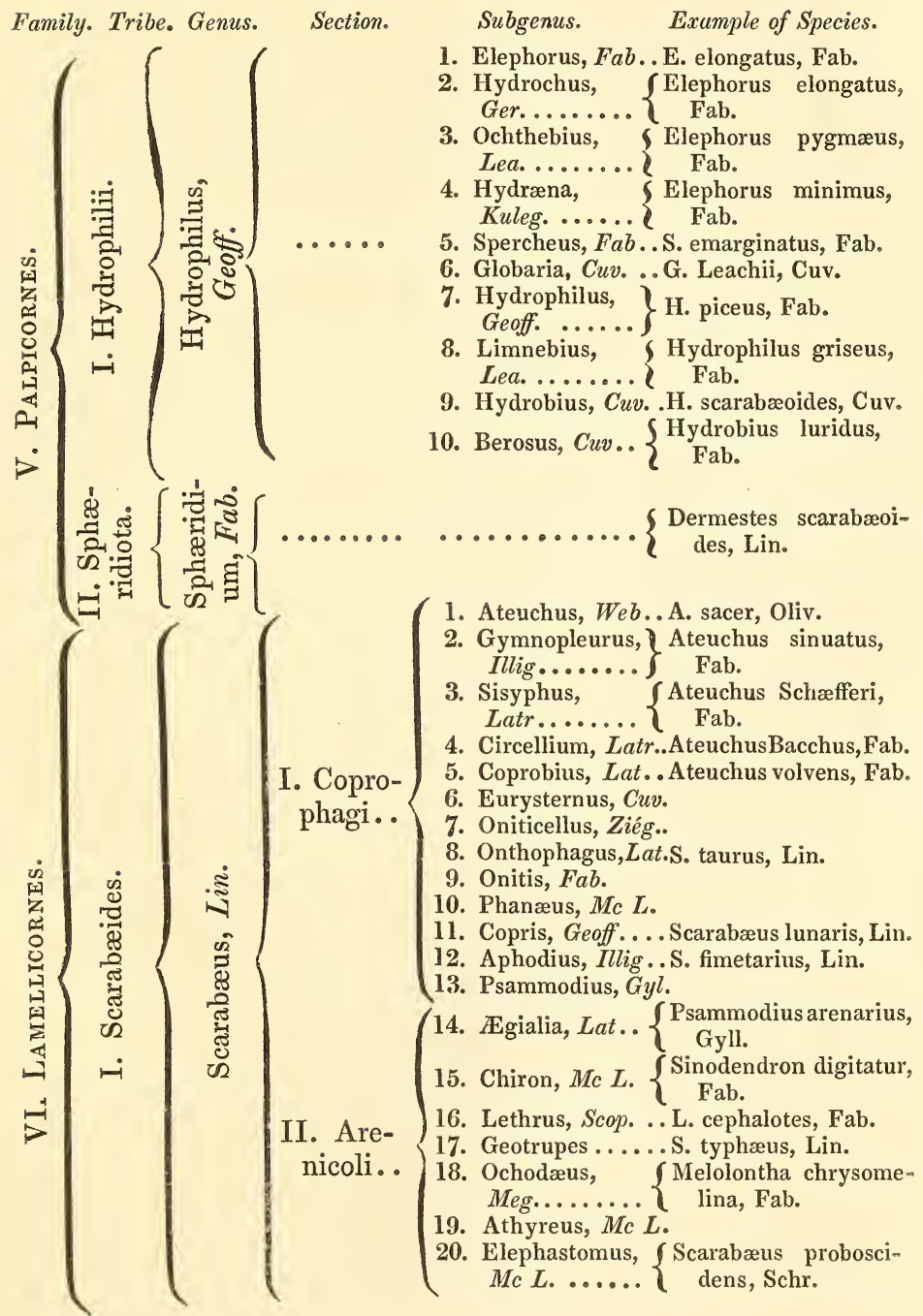




\title{
ORDER V.-Coleoptera (continued).
}

\author{
Section I. - Pentamera (continued).
}

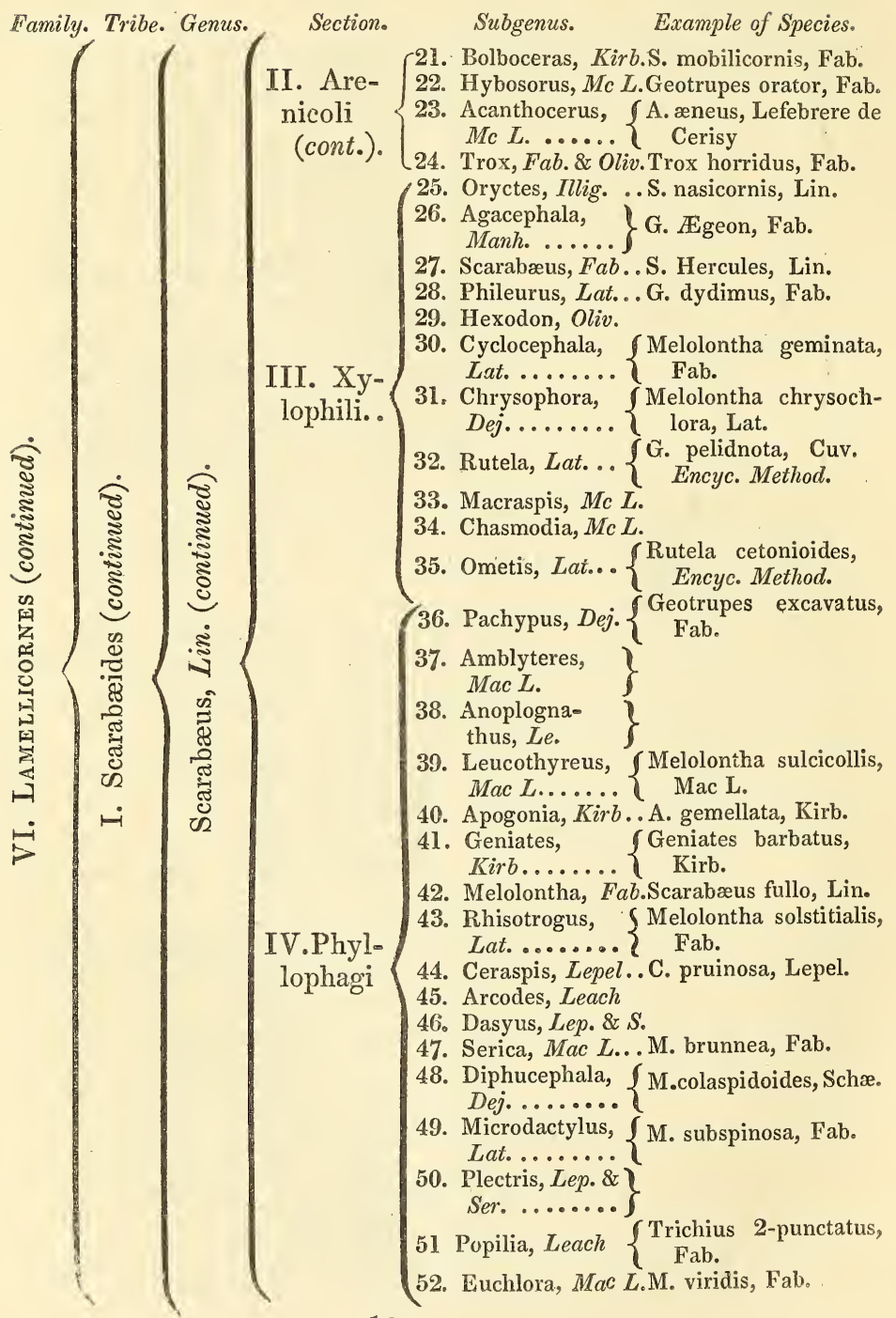


ORDER V.-Coleoptera (continued)。

Section I.-Pentamera (continued).

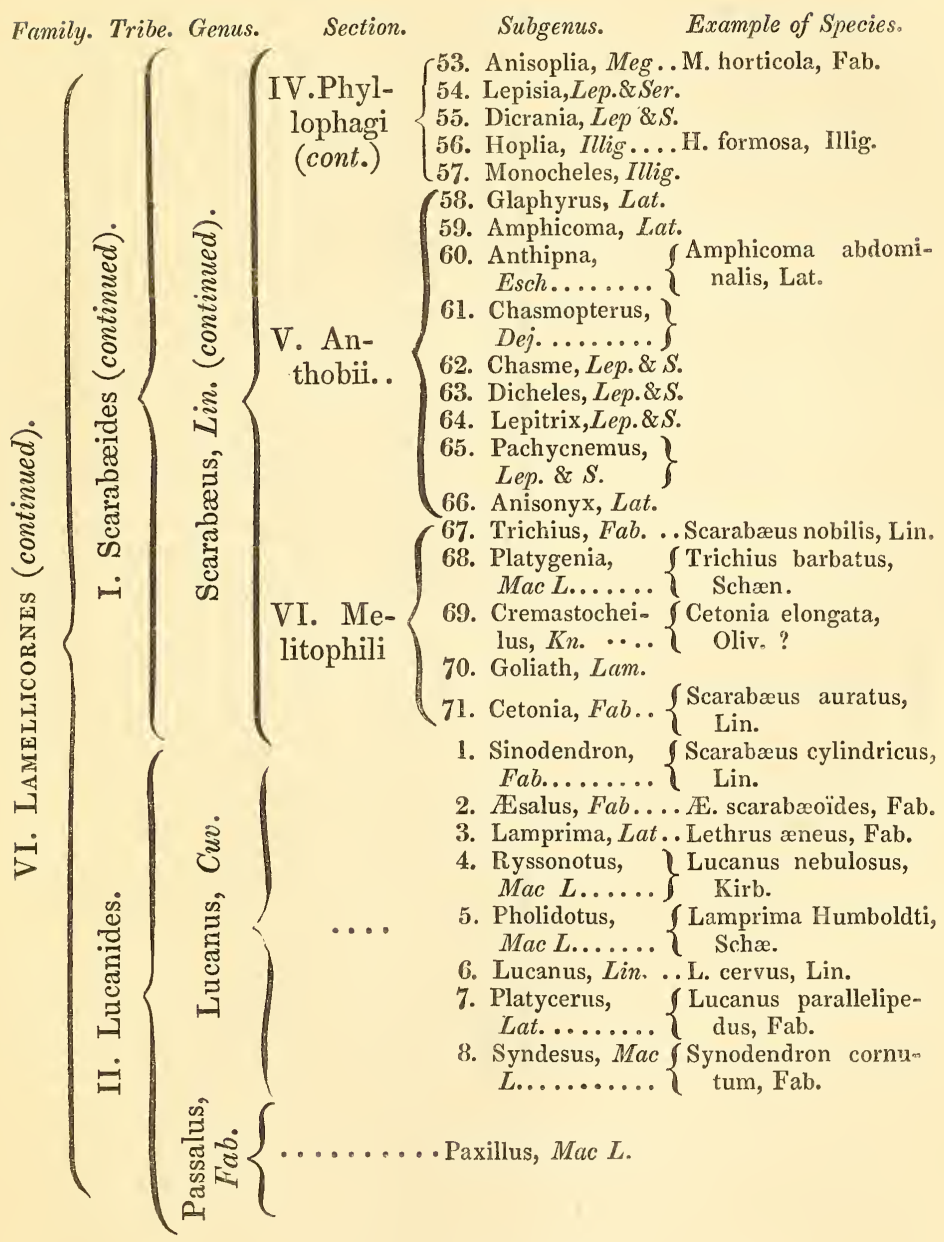




\section{ORDER V.-CoLeoptera (continued).}

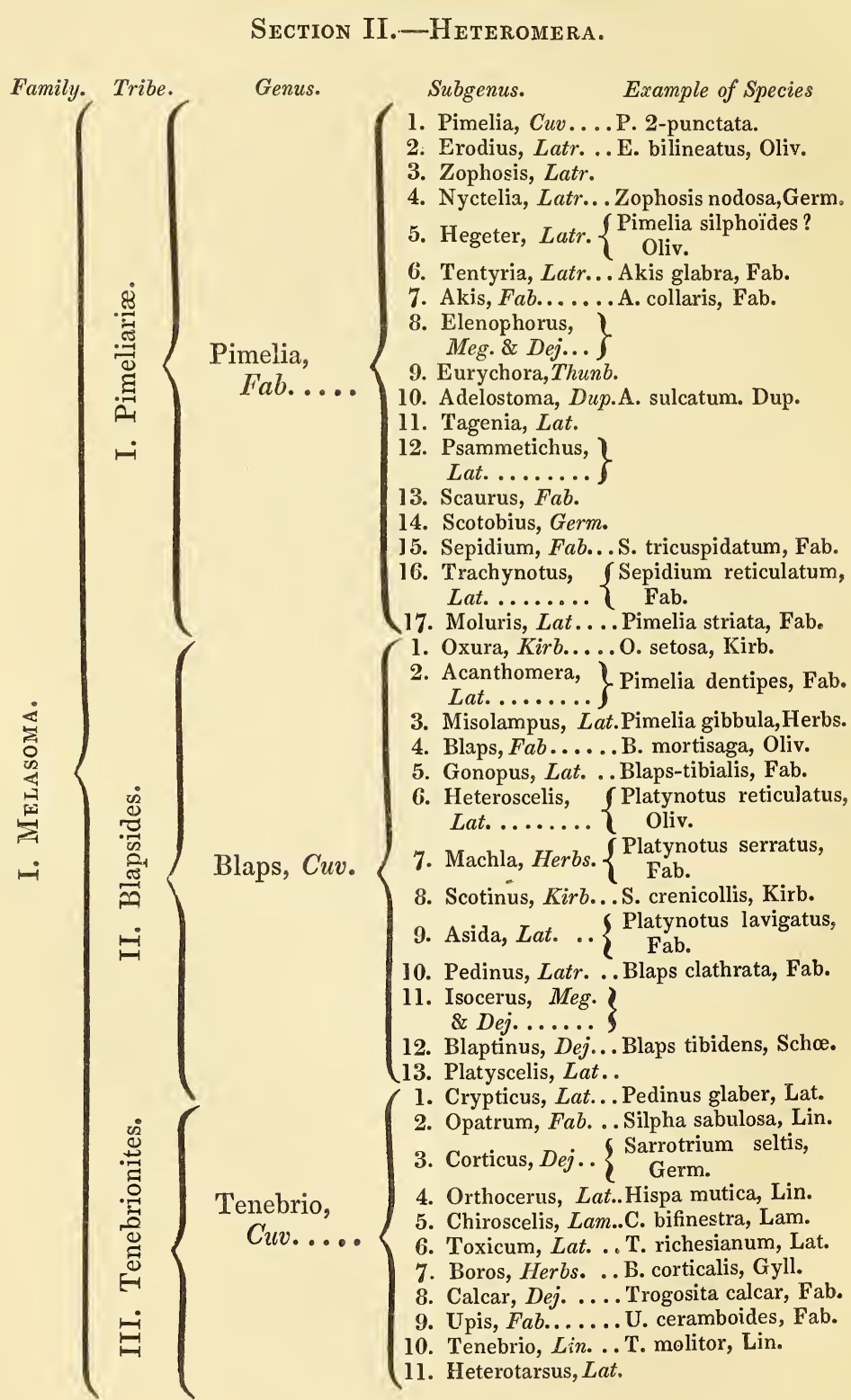


ORDER V.-Coleoptera (continued).

Section II.-Heteromera (continued).

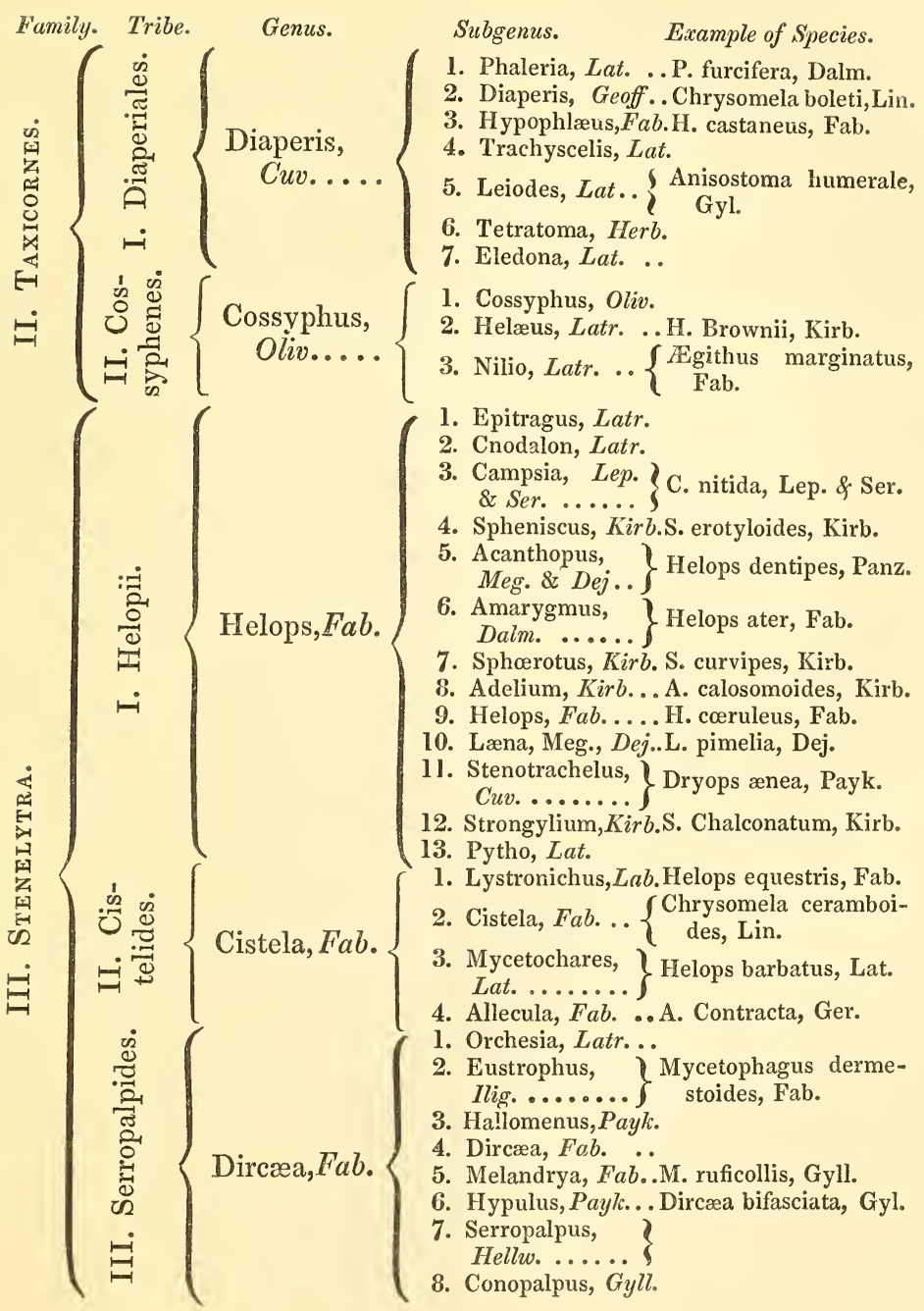




\section{ORDER V.-Coleoptera (continued).}

Section II.-Heteromera (continued).

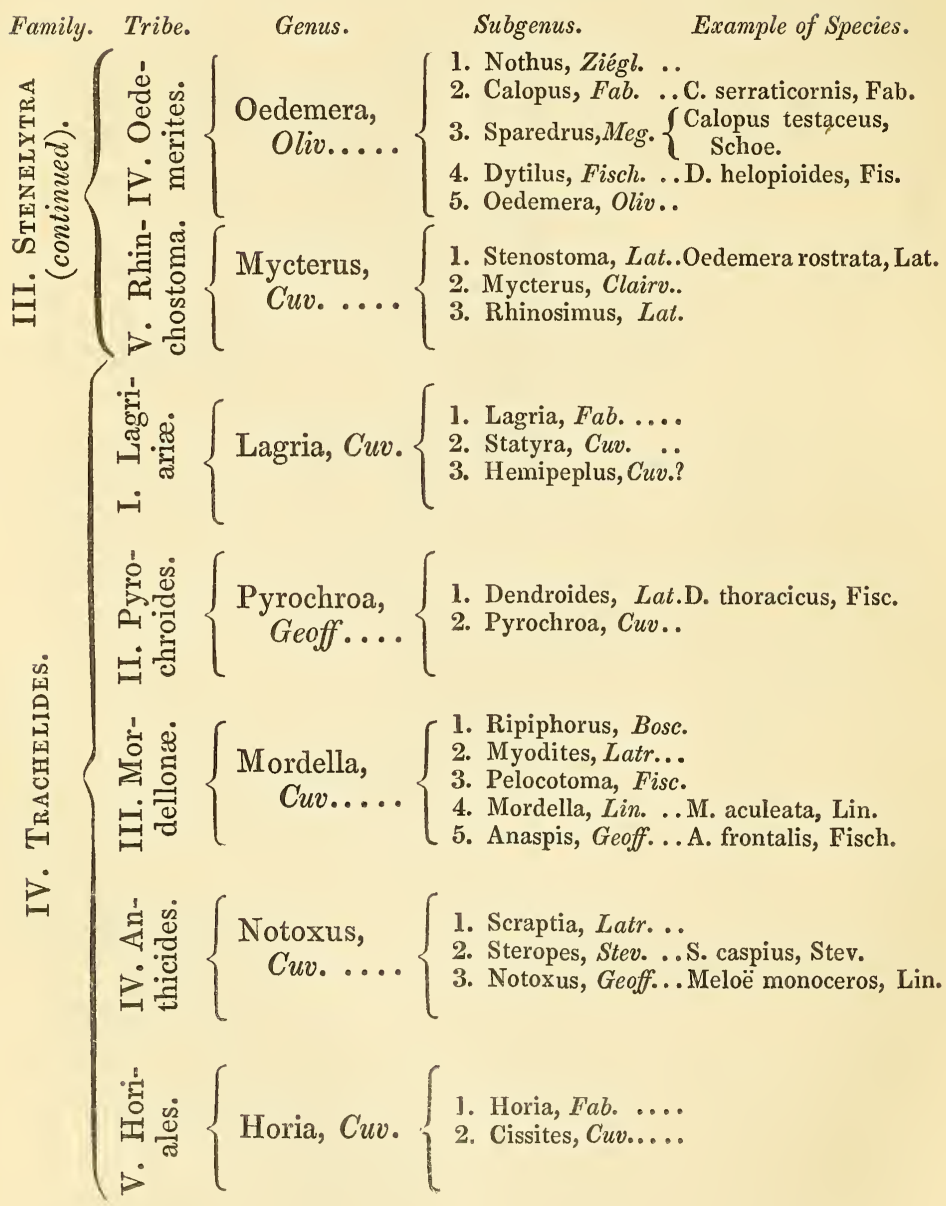




\section{ORDER V.-Coleoptera (continued).}

\section{Section II.-Heteromera (continued).}

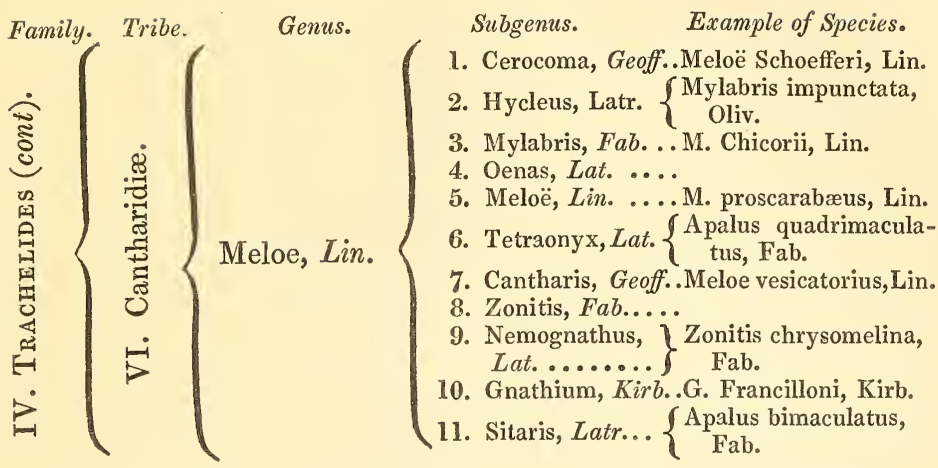

\section{Section III.-Tetramera.}

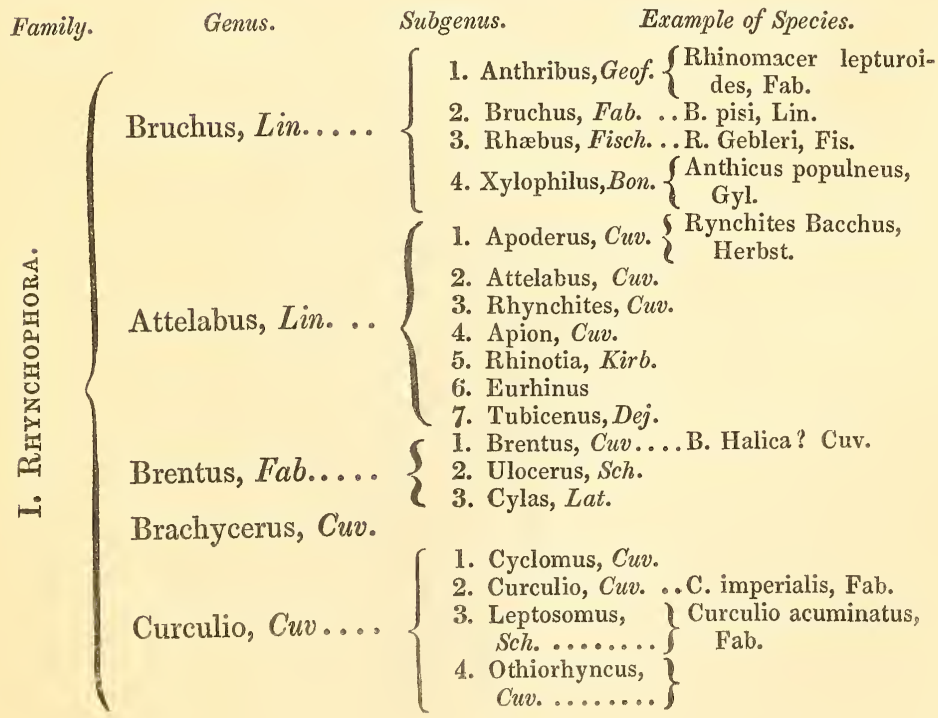


ORDER V.-Coleoptera (continued).

Section III.-Tetramera (continued).

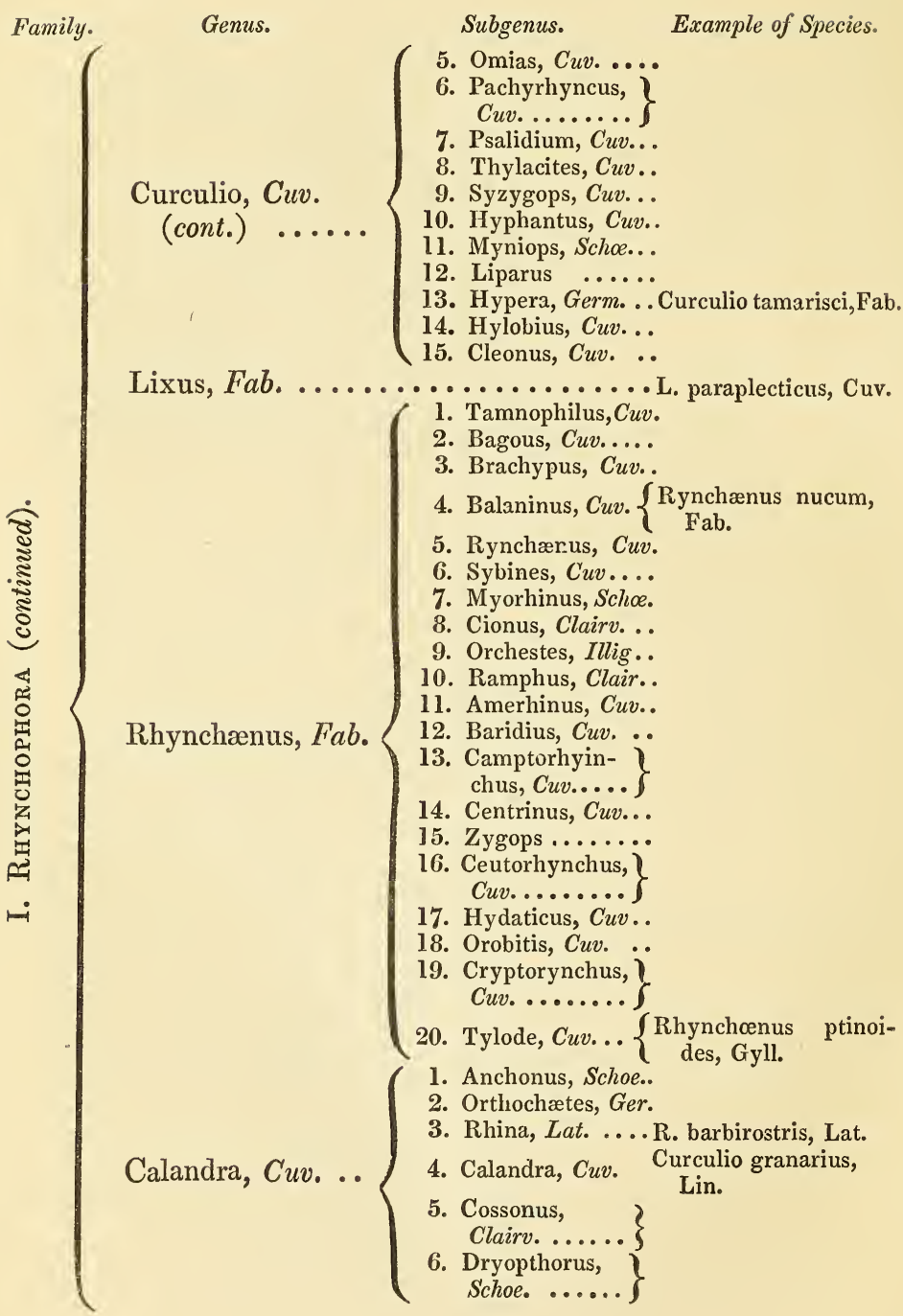


ORDER V.-Coleoptera (continued).

Section III.-Tetramera (continued).

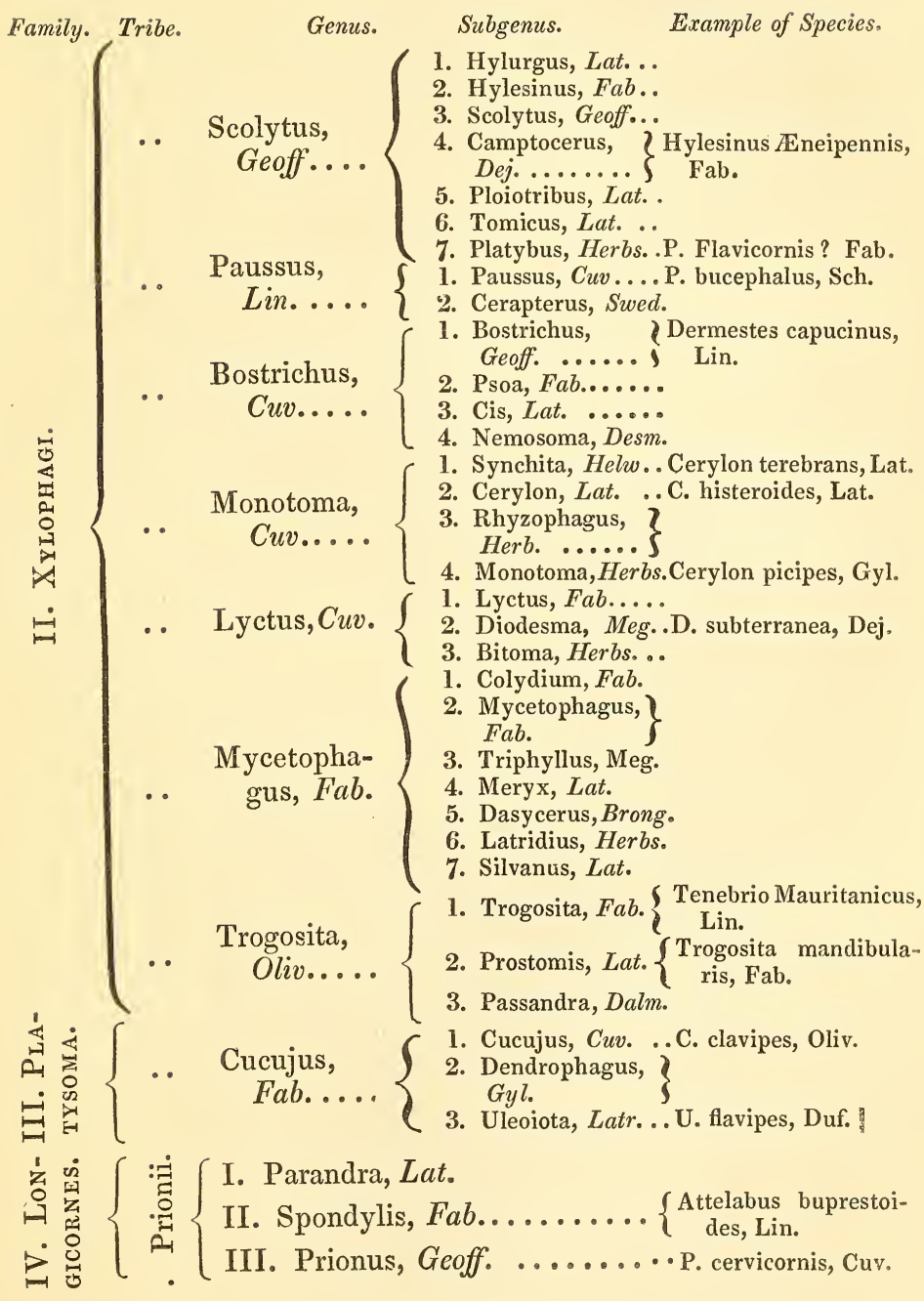


ORDER V.-Coltoptera (continued).

Section III.-Tetramera (continued).

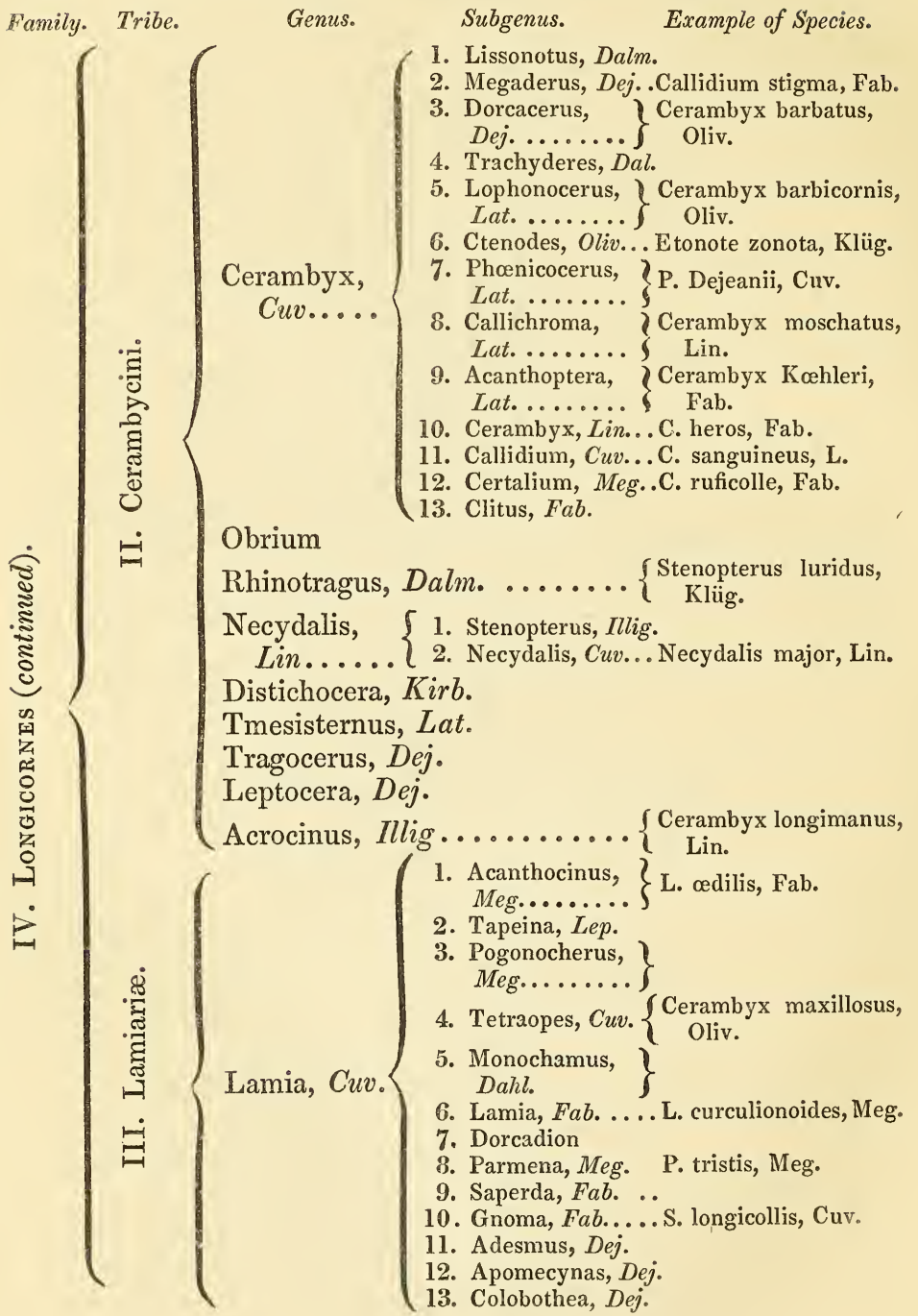




\section{ORDER V.-Coleoptera (continued).}

\section{Section III.-Tetramera (continued).}

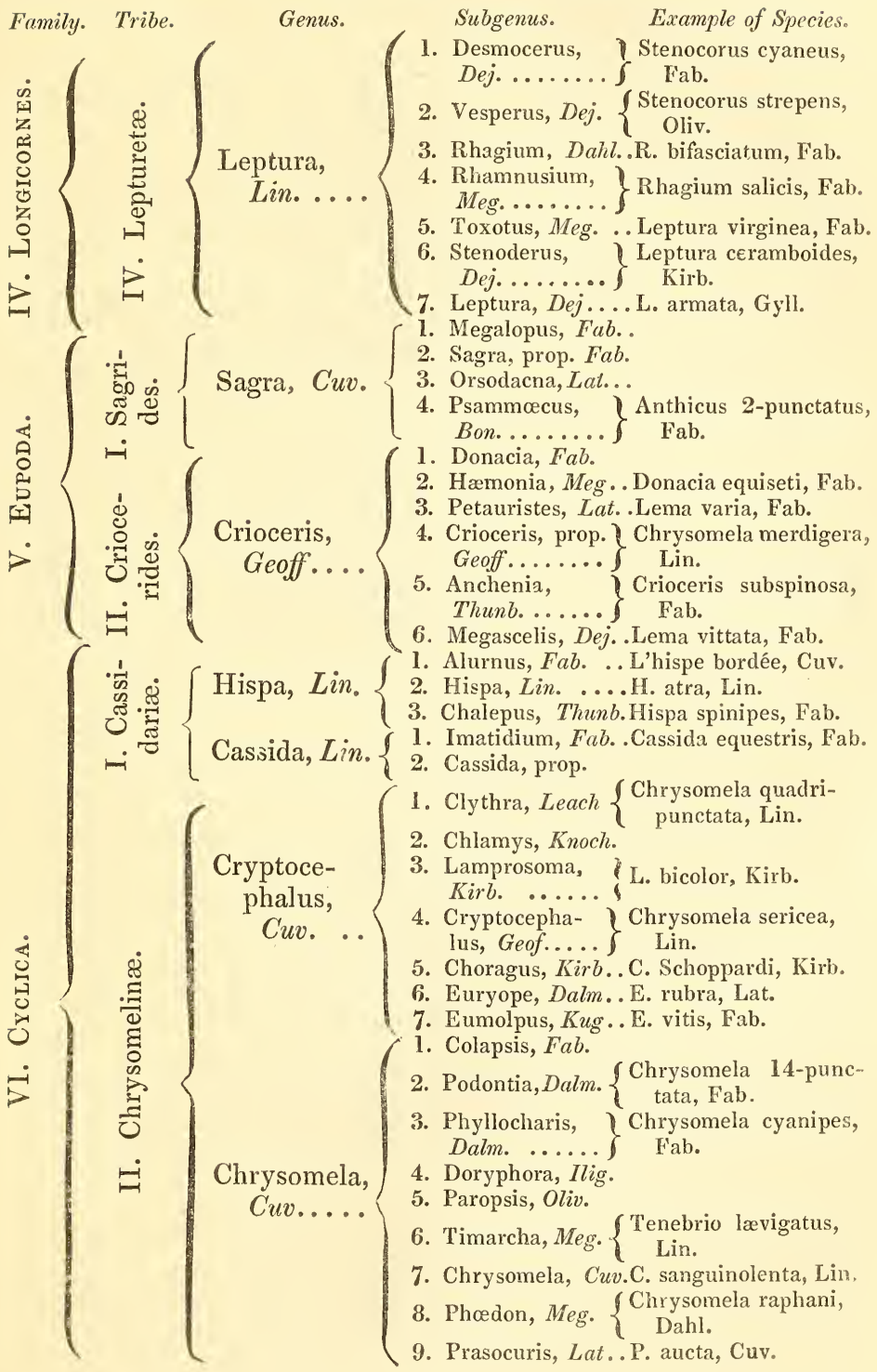




\section{ORDER V.-Coleoptera (continued).}

\section{Section III.-Tetramera (continued).}

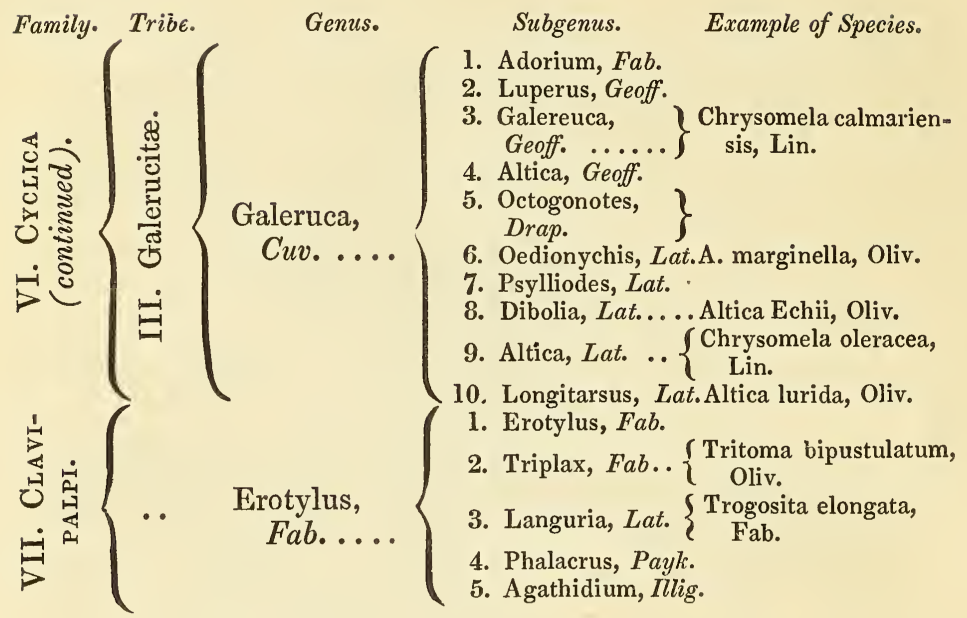

\section{Section IV.-Trimera.}

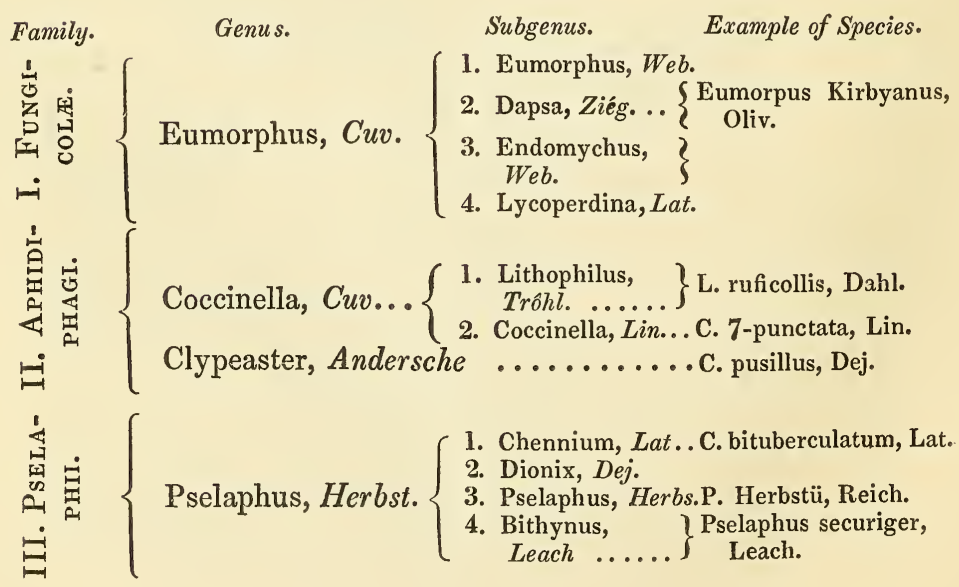




\section{ORDER V.-Coleoptera (continued).}

\section{Section IV.-Trimera (continued).}

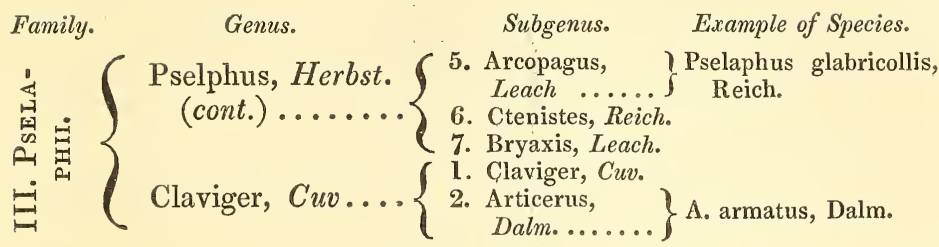

ORDER VI.-ORTHOPtERA.

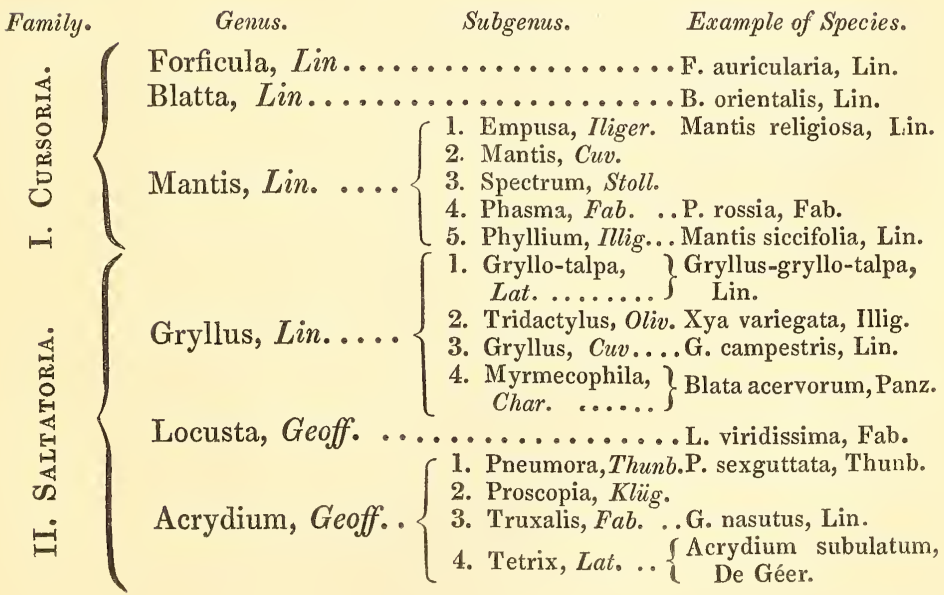




\section{ORDER VII.-Hemiptera.}

\section{Section I.-Heteroptera.}

Family.

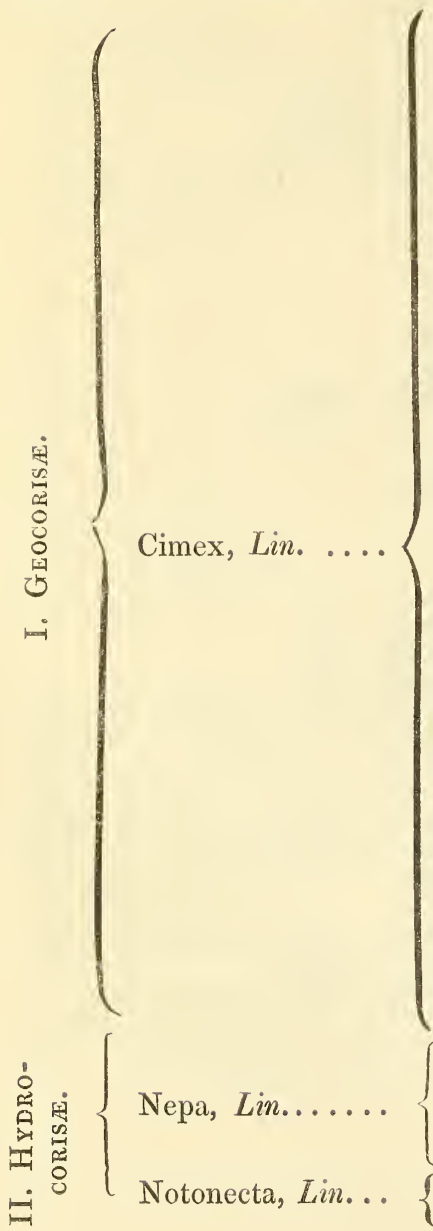

Subgenus. $\quad$ Example of Species.

1. Scutellera, Lam. . Cimex lineatus, Lin.

2. Pentatoma, Oliv. .Cimex ornatus, Lin.

3. Tesseratoma, Pel.Edessa papillosa, Fab.

4. Phlœa,Lep.\& Ser.

5. Coreus, Fab.... Cimex marginatus, Lin.

6. Holhymenia, $\}$ Lygæus biclavatus, Lep. \& Ser. .. S Fab.

7. Pachylis, Lep. ? $\&$ Ser.

8. Anisoscelis, Lat. .L. membranaceus, Fab.

9. Alydus, Fab.

10. Leptocorisa, Lat.

11. Neides, Lat.

12. Lygæus, $F a b$. . . Cimex equestris, Lin.

13. Salda, Fab. .....S. atra, Fab.

14. Myodocha, Latr.

15. Astemma, Cuv. . . Salda pallicornis, Fab.

16. Miris, Fab.

17. Capsus, Fab.

18. Heterotoma, Capsus spissicornis, Lat. ........

19. Acanthia, $F a b$.

20. Syrtis, Fab. ....S. manicata, Fab.

21. Tingis, Fab. .... T. pyri, Fab.

22. Aradus, $\mathrm{Fab}$.

23. Cimex, Latr... . . C. lectularius, Lin.

24. Reduvius, $F a b$.

25. Holoptilus, Lep. \& Ser. ..... s

26. Nabis, Lat. . . . Cimex personatus, Lin. 27. Zelus, $\mathrm{Fab}$.

28. Ploiaria, Scop. $\{$ Gerris vagabundus,

29. Leptopus, Lat. $\cdots$ Salda striata, Lat.

30. Hydrometra, Fab.

31. Gerris, Lat.

32. Velia, Lat.

1. Galgulus, Lat. Naucoris oculata, Fab.

2. Naucoris, Geoff..Nepa cimicoides, Lin.

3. Belostoma, Lat.. Nepa grandis, Fab.

4. Nepa

5. Ranatra, Fab. ... Nepa linearis, Lin.

1. Corixa, Geoff. ... Notonecta striata, Lin.

2. Notonecta, Geoff..N. glauca, Lin. 


\title{
ORDER VII.-Hemiptera (continued)。
}

\author{
Section II.-Homoptera.
}

Family.

Genus.

Subgerus.

Exumple of Species.

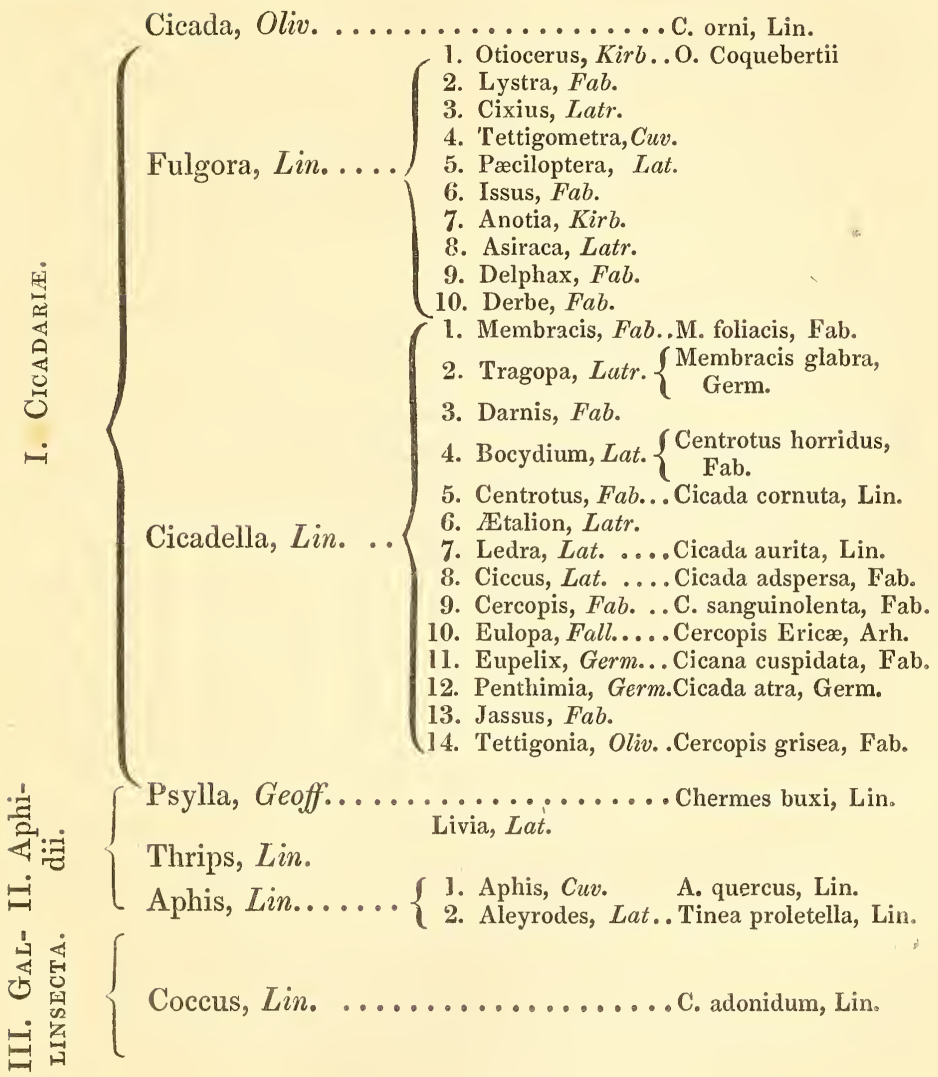




\section{ORDER VIII.-NEUROPTERA.}

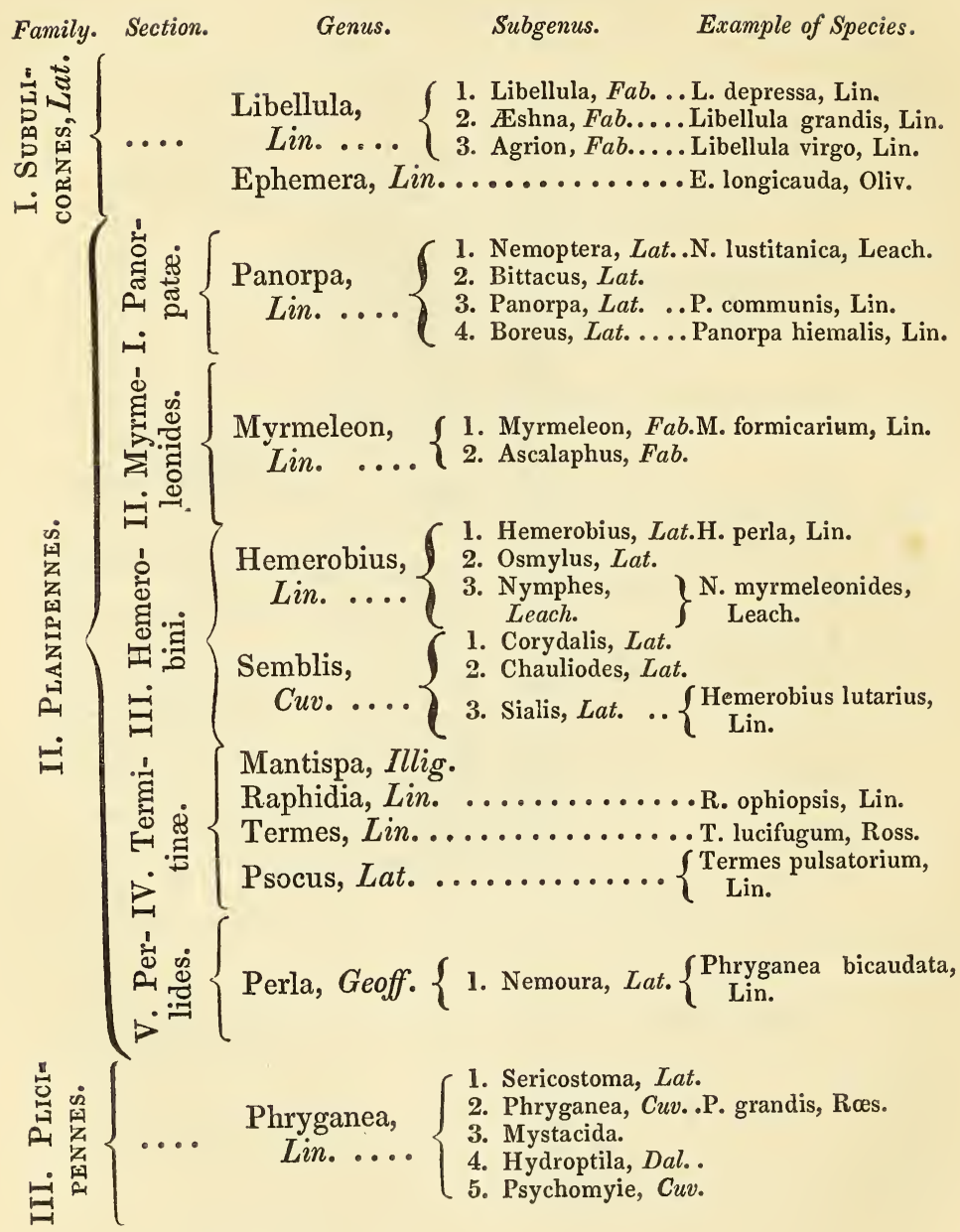




\section{ORDER IX.-Hymenoptera.}

\section{Section I.-Terebrantia.}

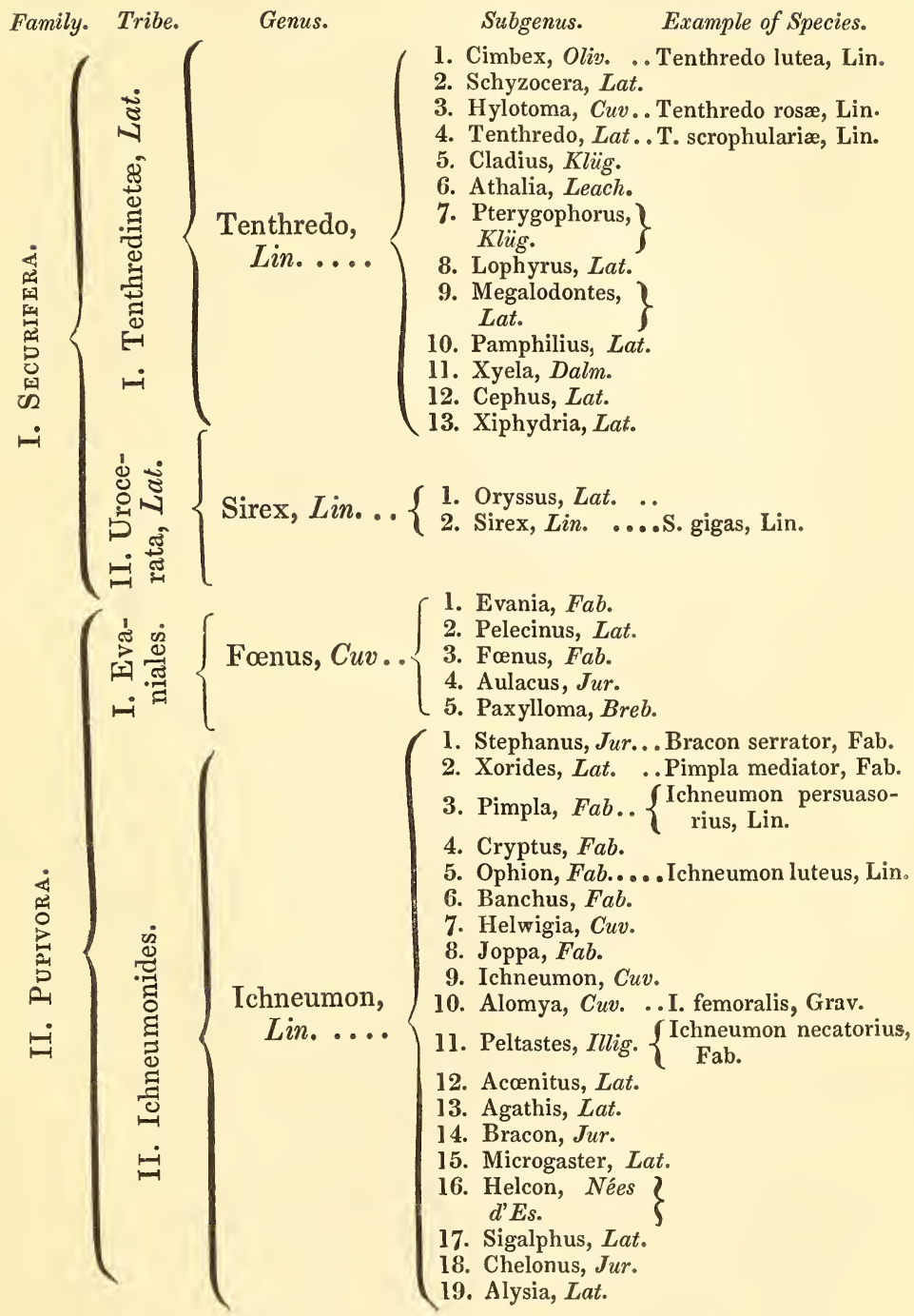


ORDER IX.-Hymenoptera (continued).

\section{Section I.-Terebrantia (continued).}

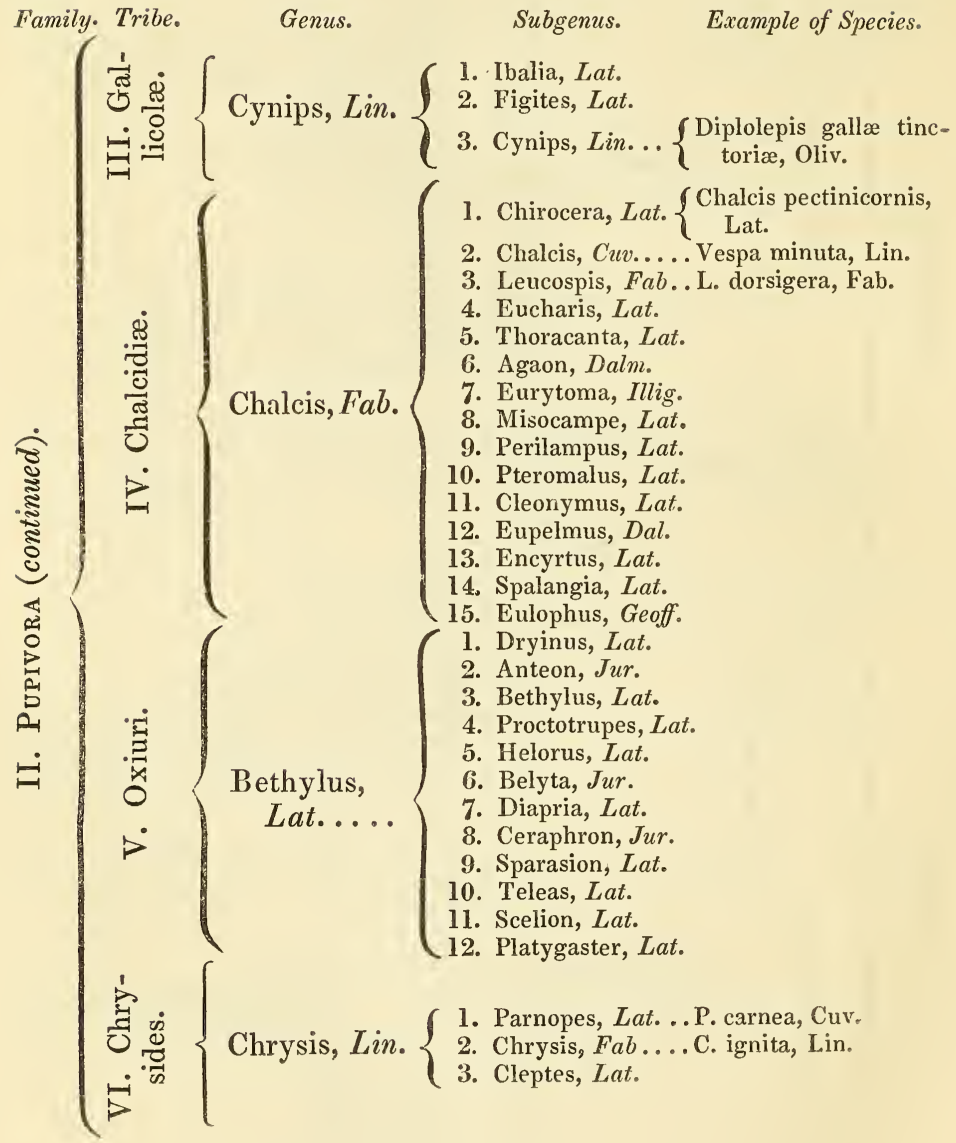


ORDER IX.-Hymenoptera (continued).

\section{Section II.-Aculeata.}

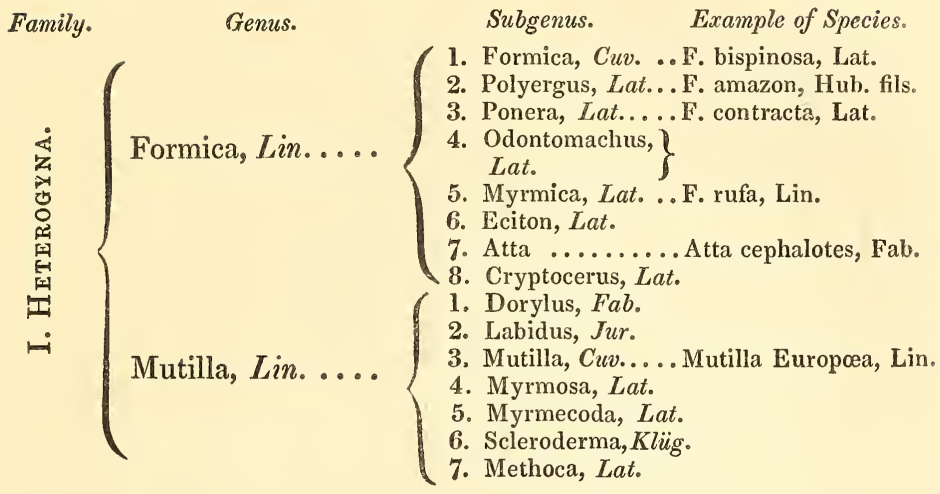

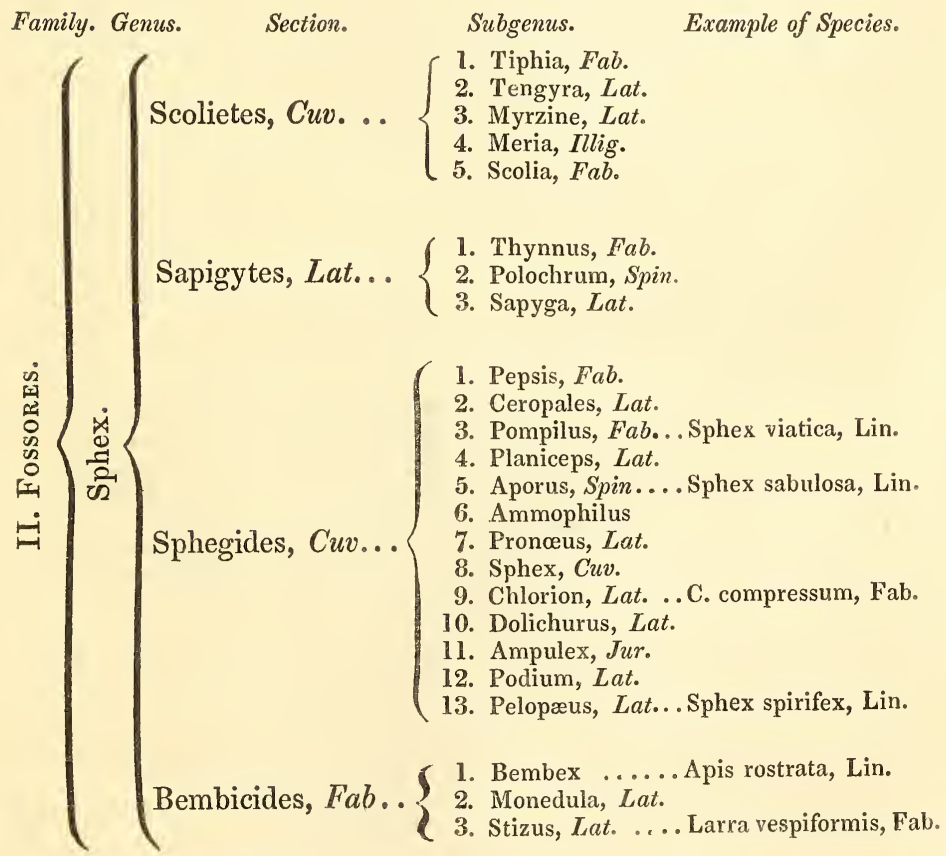




\section{ORDER IX.-Hymenoptera (continued).}

Section II.-Aculeata (continued).

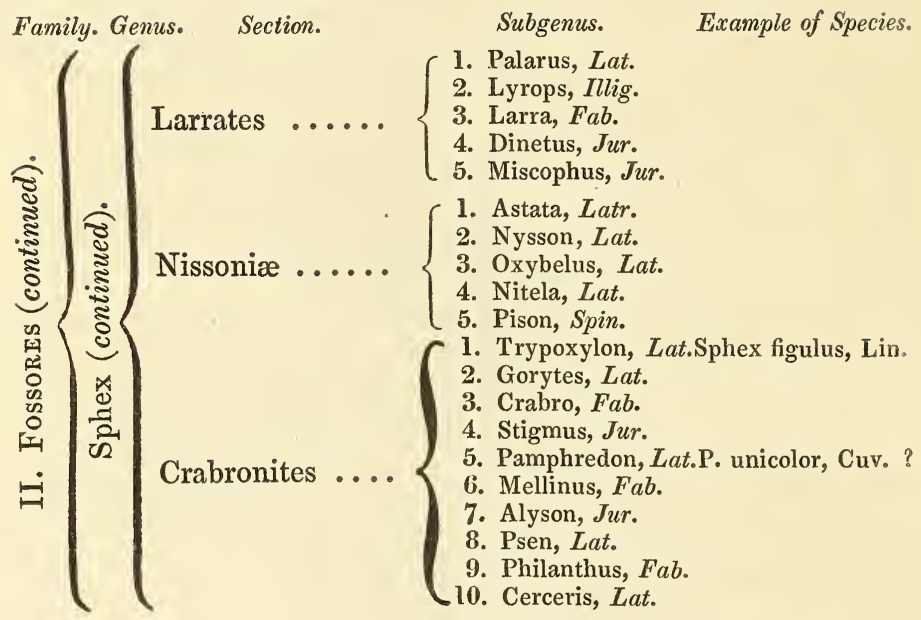

Family. Tribe. Genus. Subgenus. Example of Species.
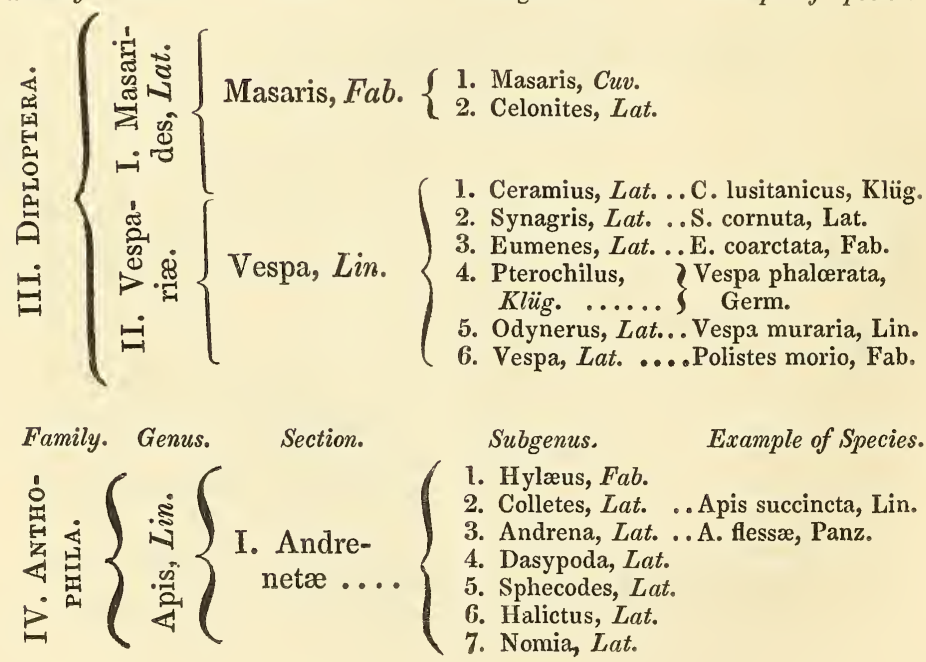


\section{ORDER IX.-Hymenoptera (continued).}

\section{Section II.-Aculeata (continued).}

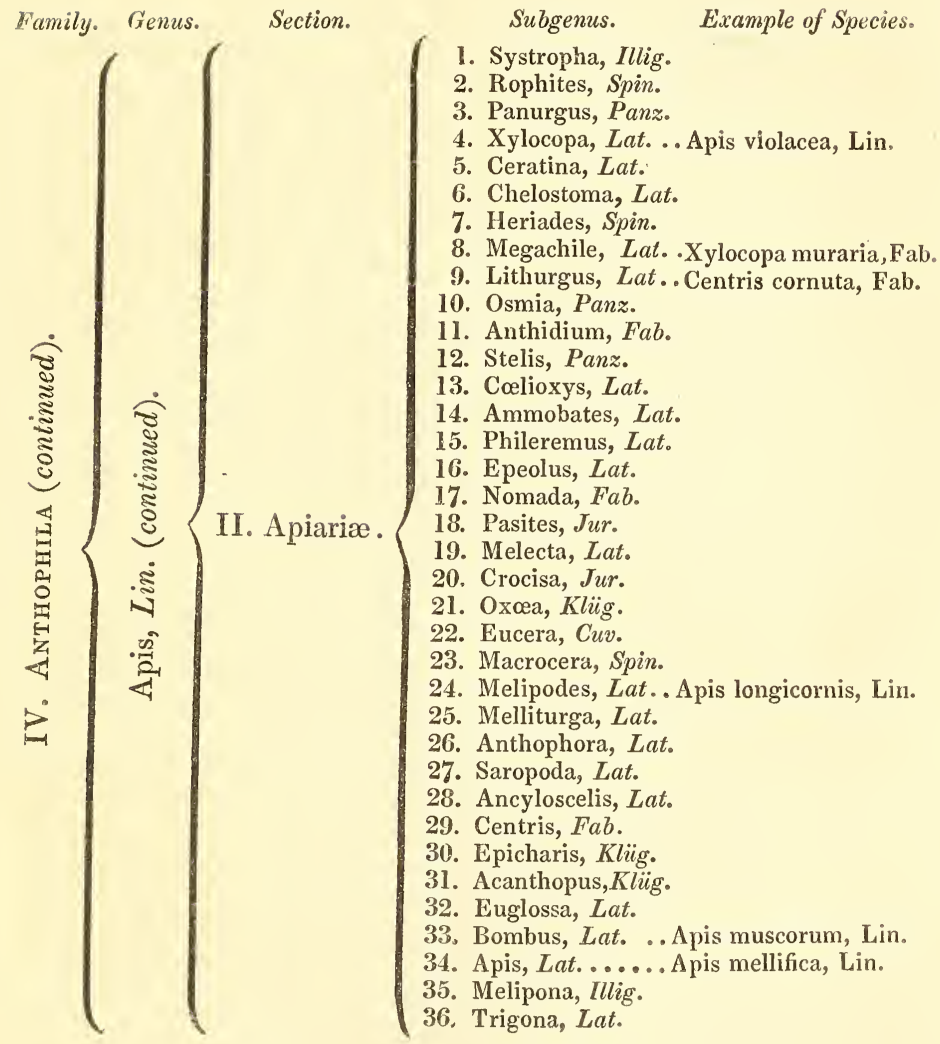




\section{ORDER X.-Lepidoptera.}

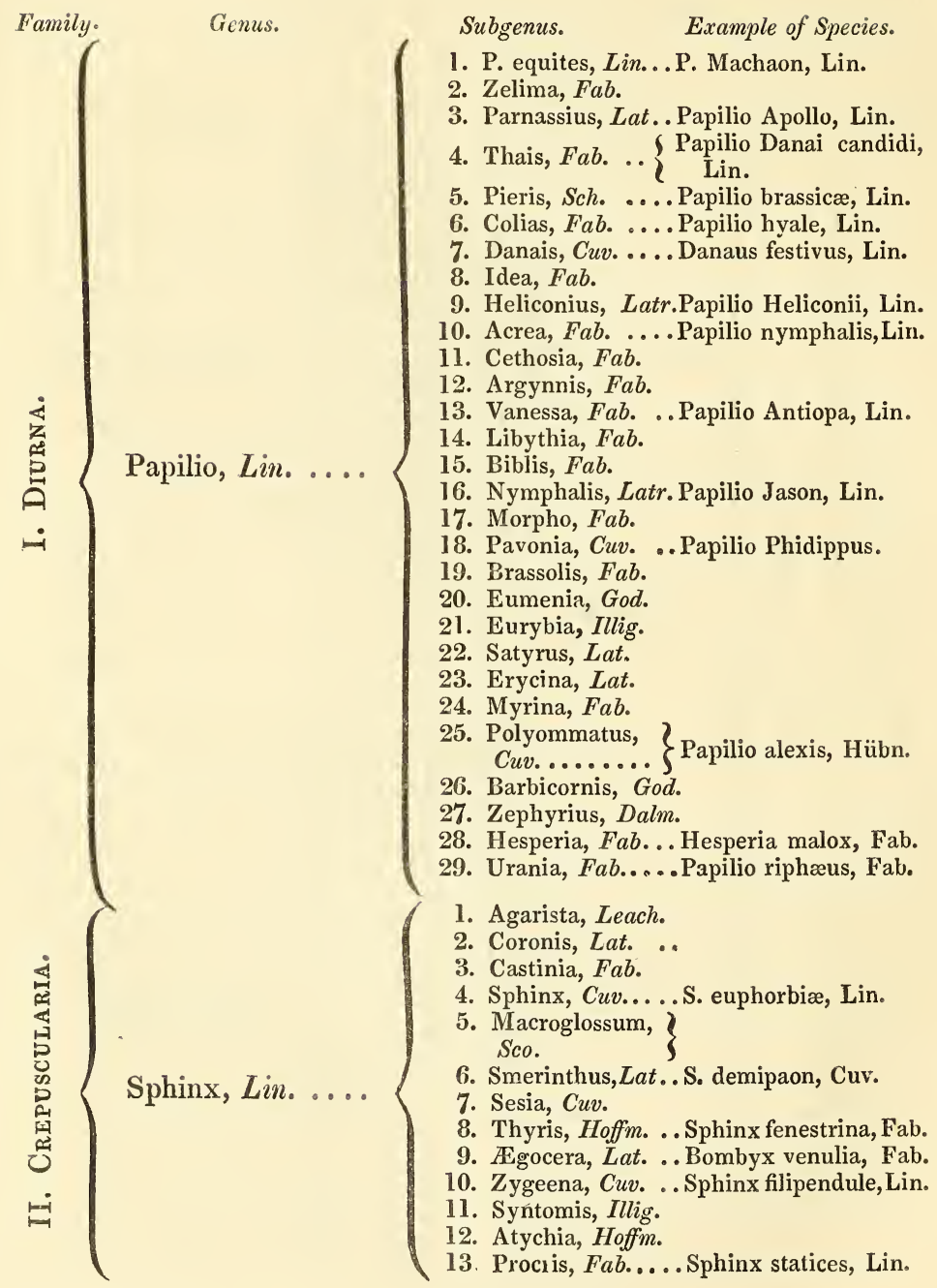




\section{ORDER X.-Lepidoptera (continued).}

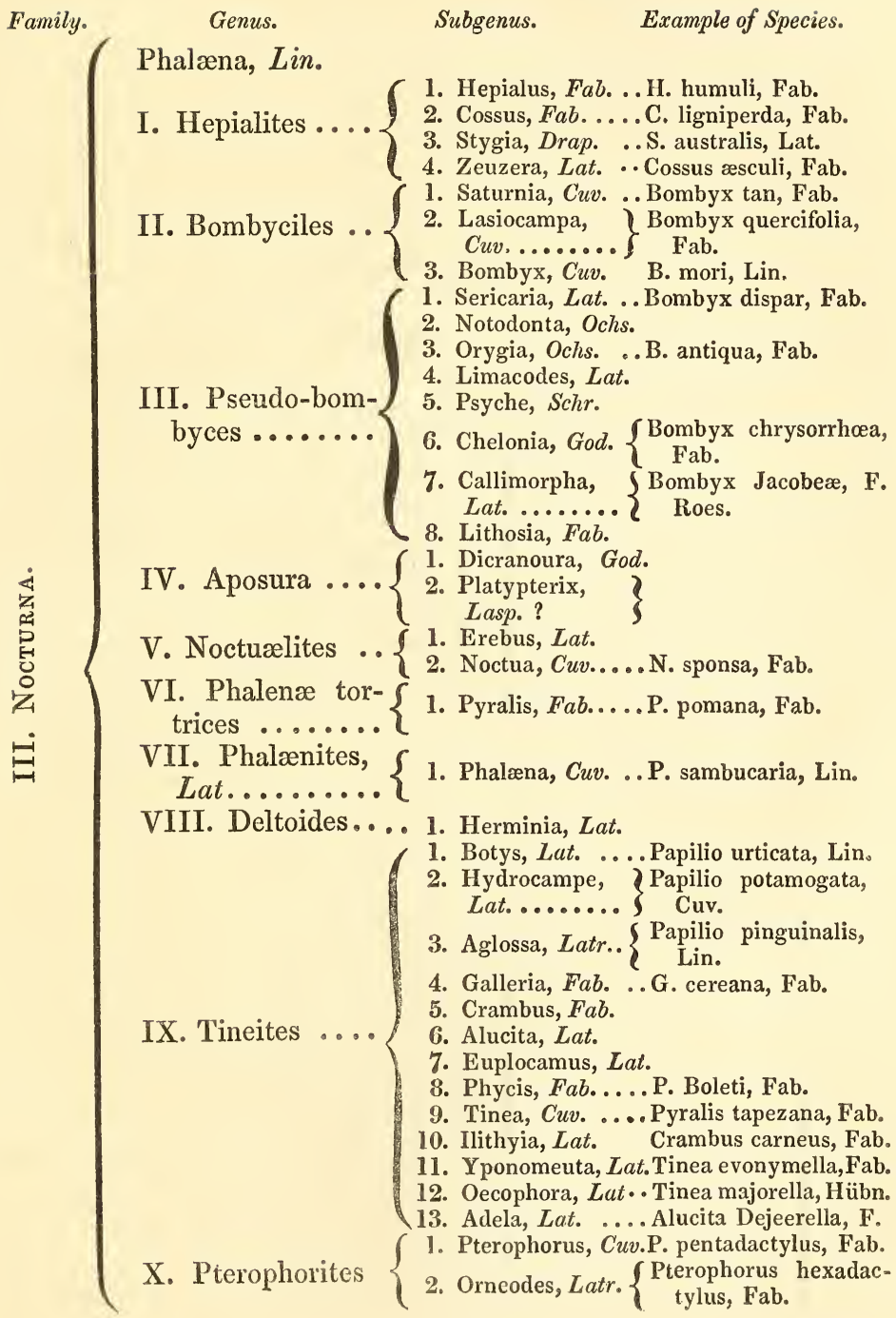




\title{
ORDER XI.-RHIPIPTERA.
}

\author{
Genus.
}

I. Xenos, Ros.

II. Stylops, Kir.

ORDER XII.-DipteRA.

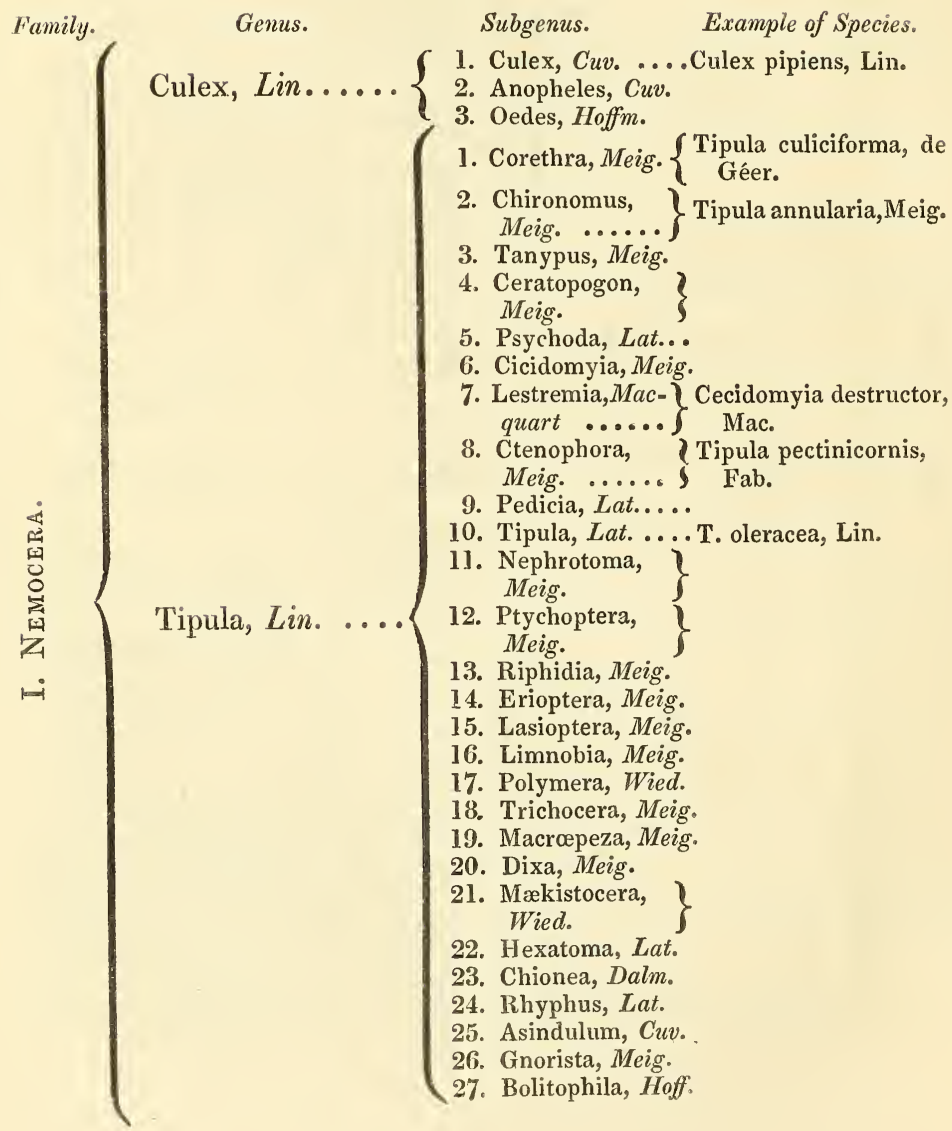


ORDER XII.-Diptera (continued).

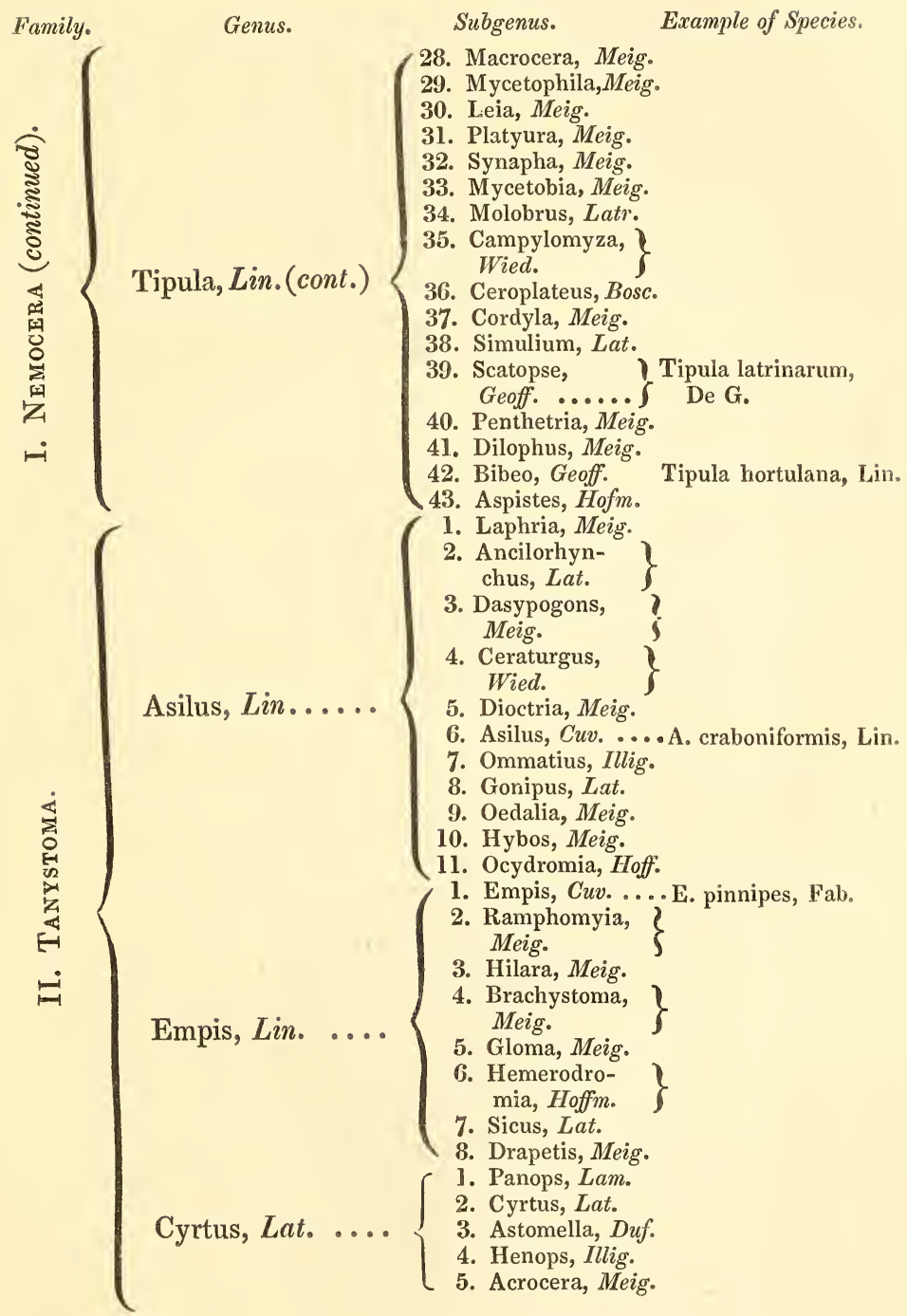




\section{ORDER XII.-Diptera (continued).}

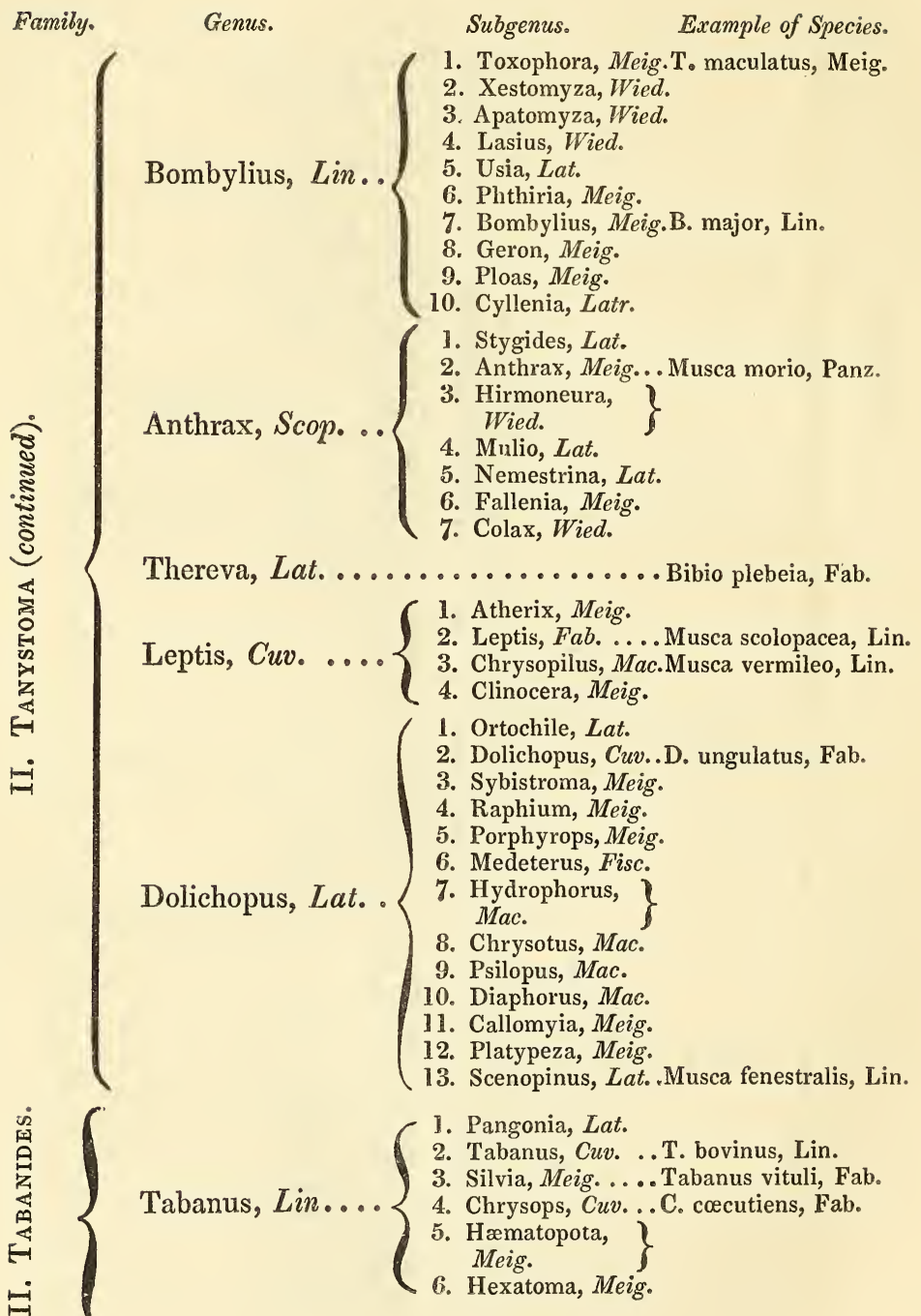




\section{ORDER XII.-DipterA (continued).}

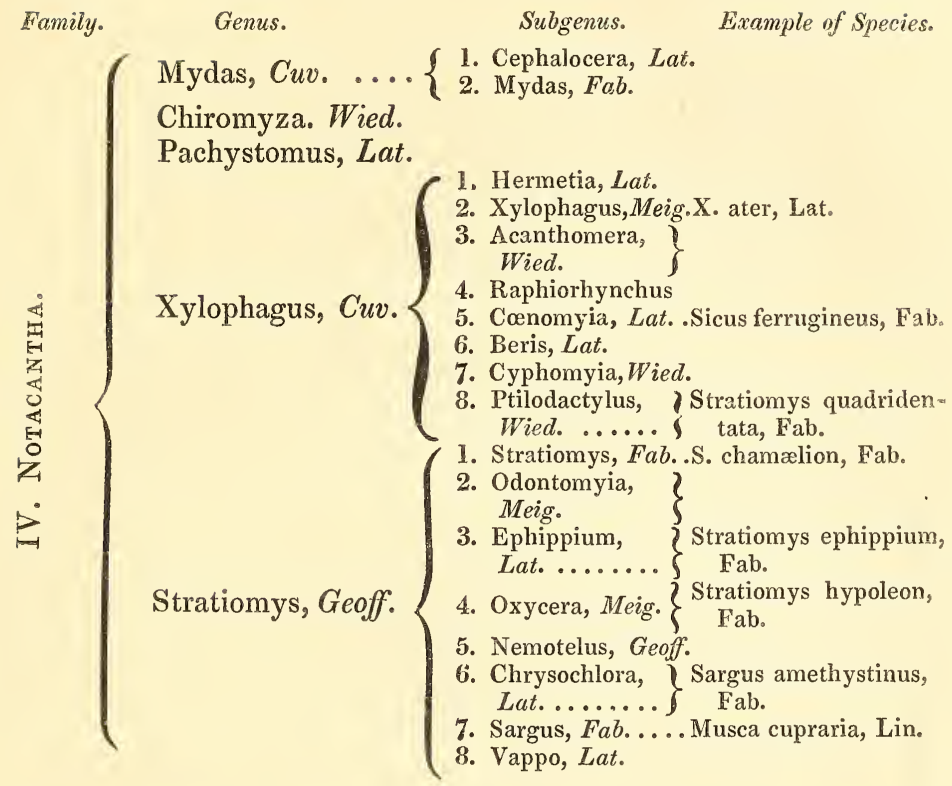

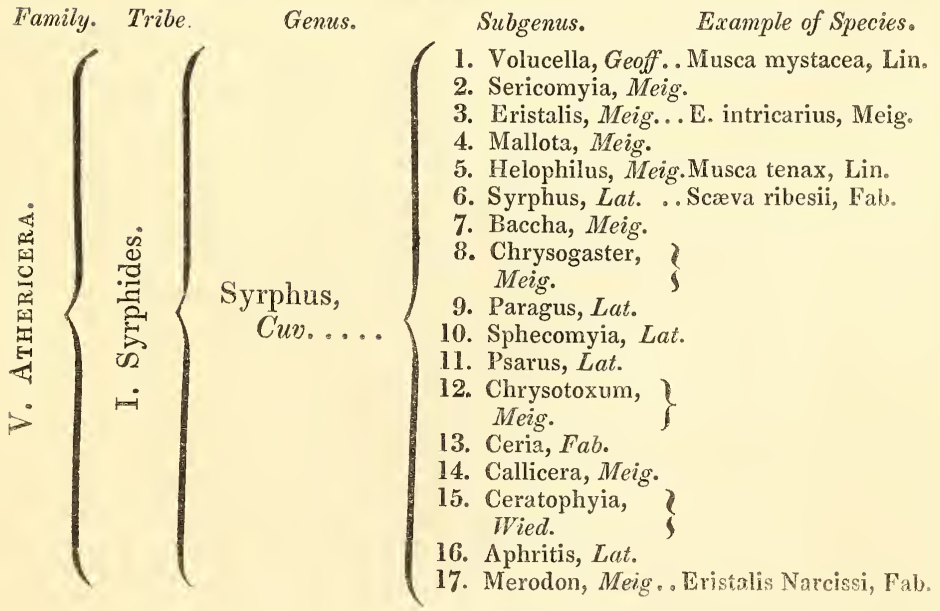




\section{ORDER XII.-Diptera (continued).}

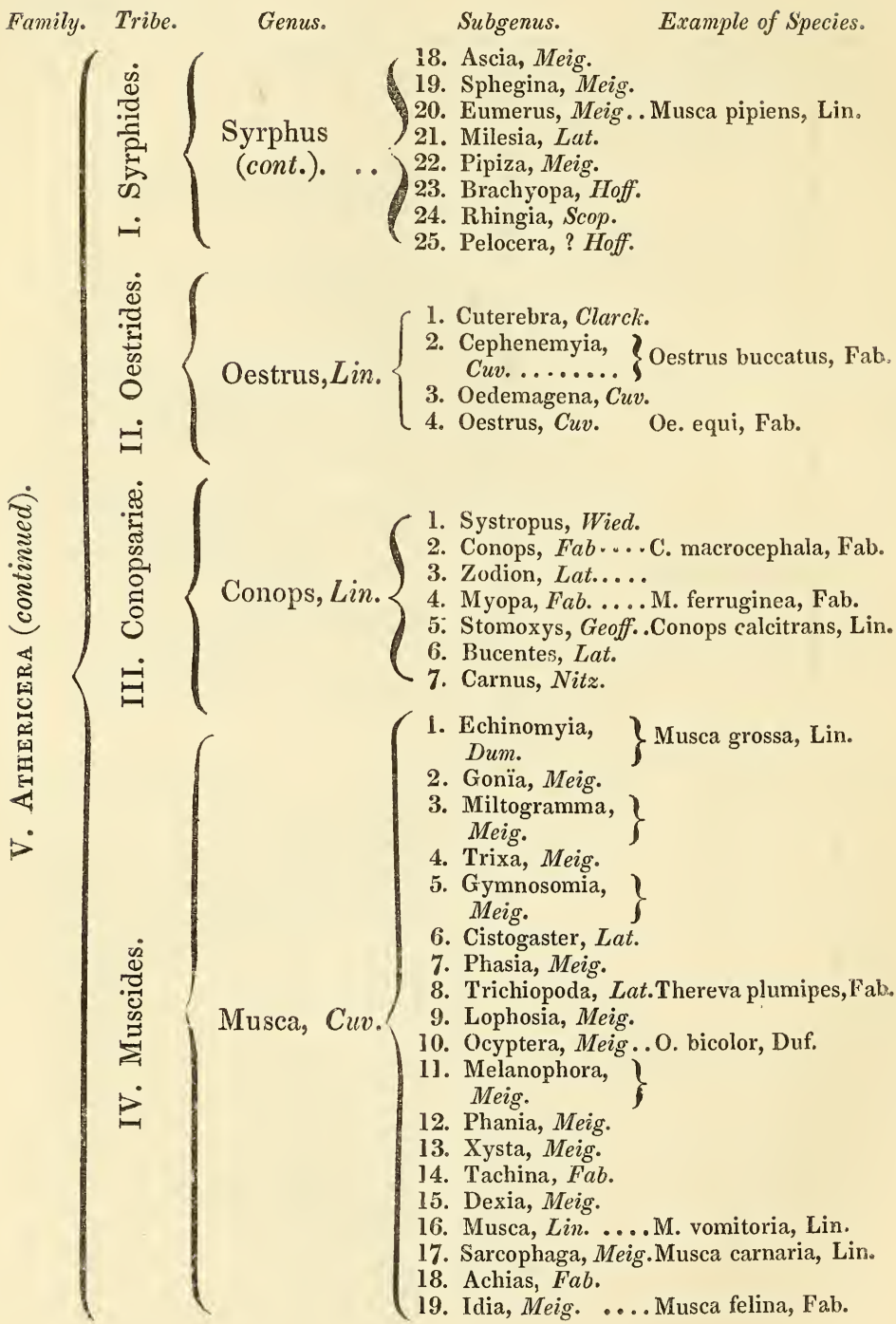




\section{ORDER XII.-Diptera (continued).}

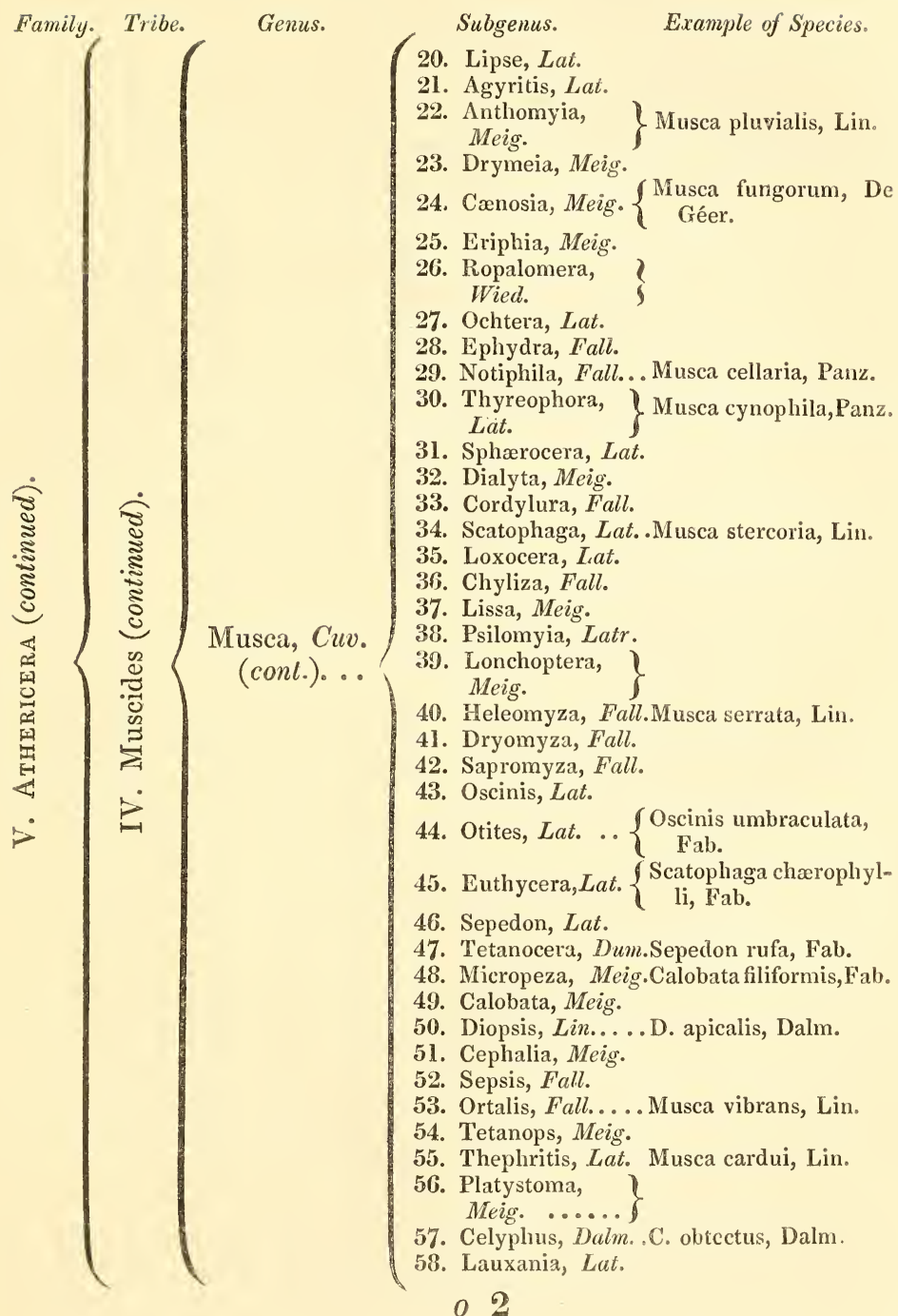




\section{ORDER XII.-Diptera (continued).}

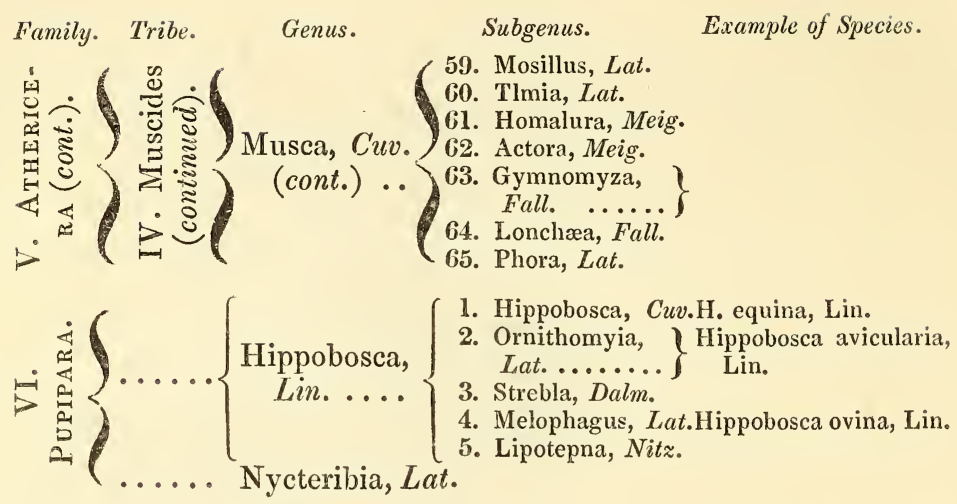




\section{DIVISION IV.-RADIATA, OR ZOOPHYTES.}

THIs division includes the most simple forms of animated nature, and although these forms vary considerably in the several classes, all show traces, more or less distinct, of radiation from a common centre.

It includes five classes.

Division.

Class.

RADIATA.. $\left\{\begin{array}{c}\text { I. Echinodermata } \\ \text { II. Entozoa, or, Intestinal Worms } \\ \text { III. Acalephe } \\ \text { IV. Polypi } \\ \text { V. Infusoria }\end{array}\right.$

\section{CLASS I.-ECHINODERMATA.}

This name is strictly applicable to the genus Echinus, sea-urchins, or hedgehogs, but is applied more generally to the most complicated of the radiated animals. All the genera have a well organized skin, with distinct viscera, and a sort of imperfect vascular system.

The class includes two orders.

$$
\text { Echinodermata.. }\left\{\begin{array}{l}
\text { 1. } \text { Pedicellata } \\
\text { 2. Apedicellata }
\end{array}\right.
$$

\section{ORDER I.-Pedicellata.}

The envelope in this order is pierced with apertures, through which issue numerous membranaceous tentacula, which acting as organs of motion, are called the feet. 


\section{ORDER I.-Pedicellata.}

Genus.

Subgenus.

Example of Species.

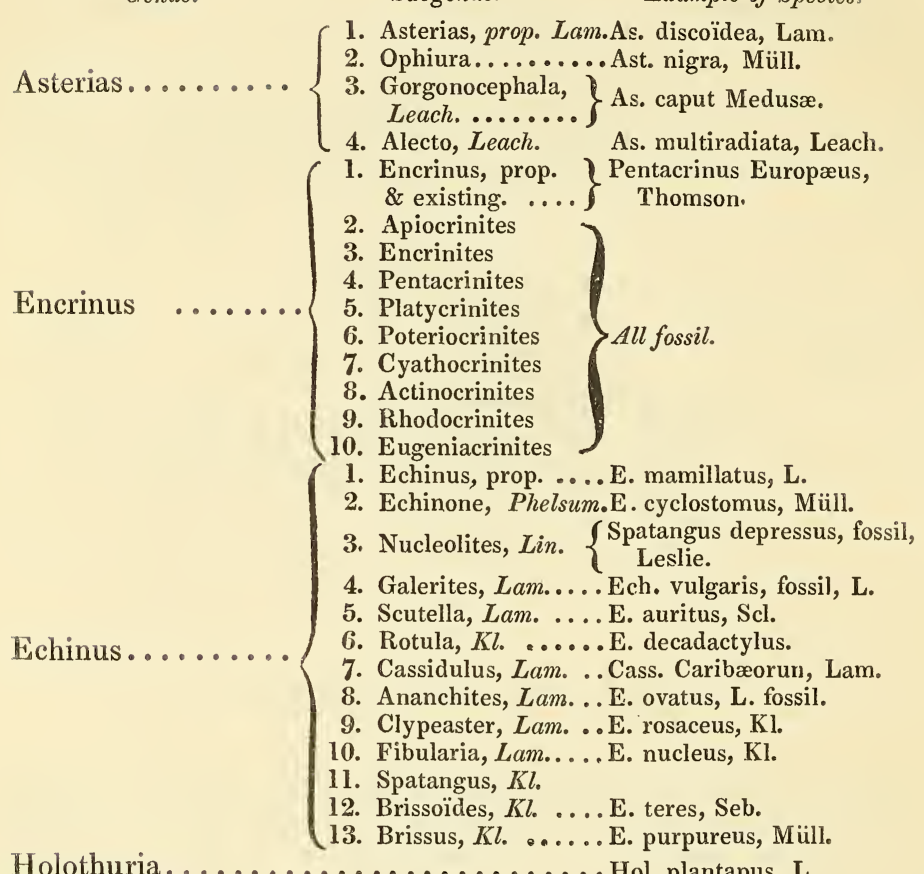

Holothuria................... Hol. plantapus, L.

\section{ORDER II.-APEdicellata.}

Genus. Subgenus. Example of Species.

Molpadia .......................... holothuroïdes, Cuv.

Mynias ....................... cyanea, Cuv.

Priapulus ......................... priapulus, L.

Lithodermes ......................... cuneus, Cuv.

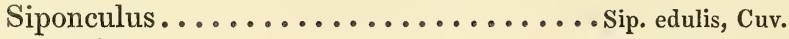

Bonellia.................... Bon. viridis, Rol.

Thalassema $\ldots . .\left\{\begin{array}{l}\text { 1. Thalassema, prop..T. Neptuni, Gertner. } \\ \text { 2. Echiurus. ........ Lumbricus echiurus, Gmel. }\end{array}\right.$

3. Sternapsis, Otto $\because$ Thalassima scutatum, Ranzan. 


\section{CLASS II.-INTESTINA-ENTOZOA, Rudolphi。}

This class, so far as has been observed, is destitute of any true circulation, though there are obscure vestiges of nerves. Most of the genera live only in the interior of other animals.

They are divided into two orders.

Class.

Order.

INTESTINA.. $\left\{\begin{array}{l}\text { 1. Intestina cavitaria } \\ \text { 2. Intestina parenchymata }\end{array}\right.$

ORDER I.-Intestina Cavitaria-Nematoidea, Rudolphi.

Genus. Subgenus. Example of Species.

Filaria, $L . \ldots \ldots \ldots \ldots$...........Fil. Medinensis, Gm.

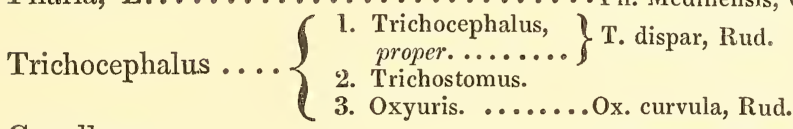

Cucullanus...................... lacustris, Gm.

Ophiostomus ................... cystidicola.

Ascaris ......................... lumbricoïdes, L.

Strongylus................... Stron. equinus, Gm.

Spiroptera ...................... strumosa, Nits.

Physaloptera................... See Rud. Syn. 29.

Scelorostoma, Blain. $\left\{\begin{array}{l}\text { 1. Scelorostoma, Blain. } \\ \text { 2. Liorhynchus, Rud. }\end{array}\right.$

Linguatula

Prionoderma ................. Cucullanus ascarhoides

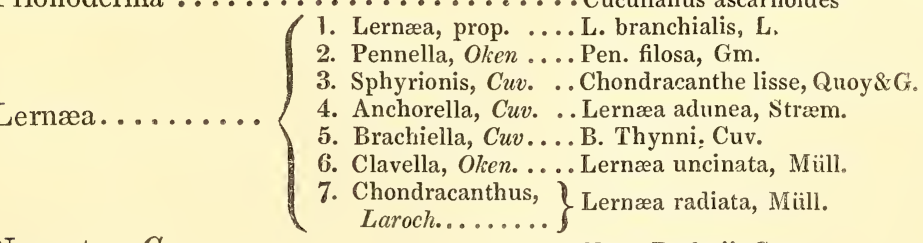

Nemertes, Cuv.................. Nem. Borlasii, Cuv.

Tubularia, Renimeri.

Ophiocephalus, Quoy \& G.

Cerebratula, Renimeri 


\section{ORDER II.-PARENCHYMATA.}

This order is divided into four families.

$$
\text { Prder. } \quad \begin{aligned}
& \text { Family. } \\
& \text { Parenchymata. }
\end{aligned} \quad \begin{aligned}
& \text { Acanthocephala } \\
& \text { Trematodes } \\
& \text { Tenioides } \\
& \text { Cestoides }
\end{aligned}
$$

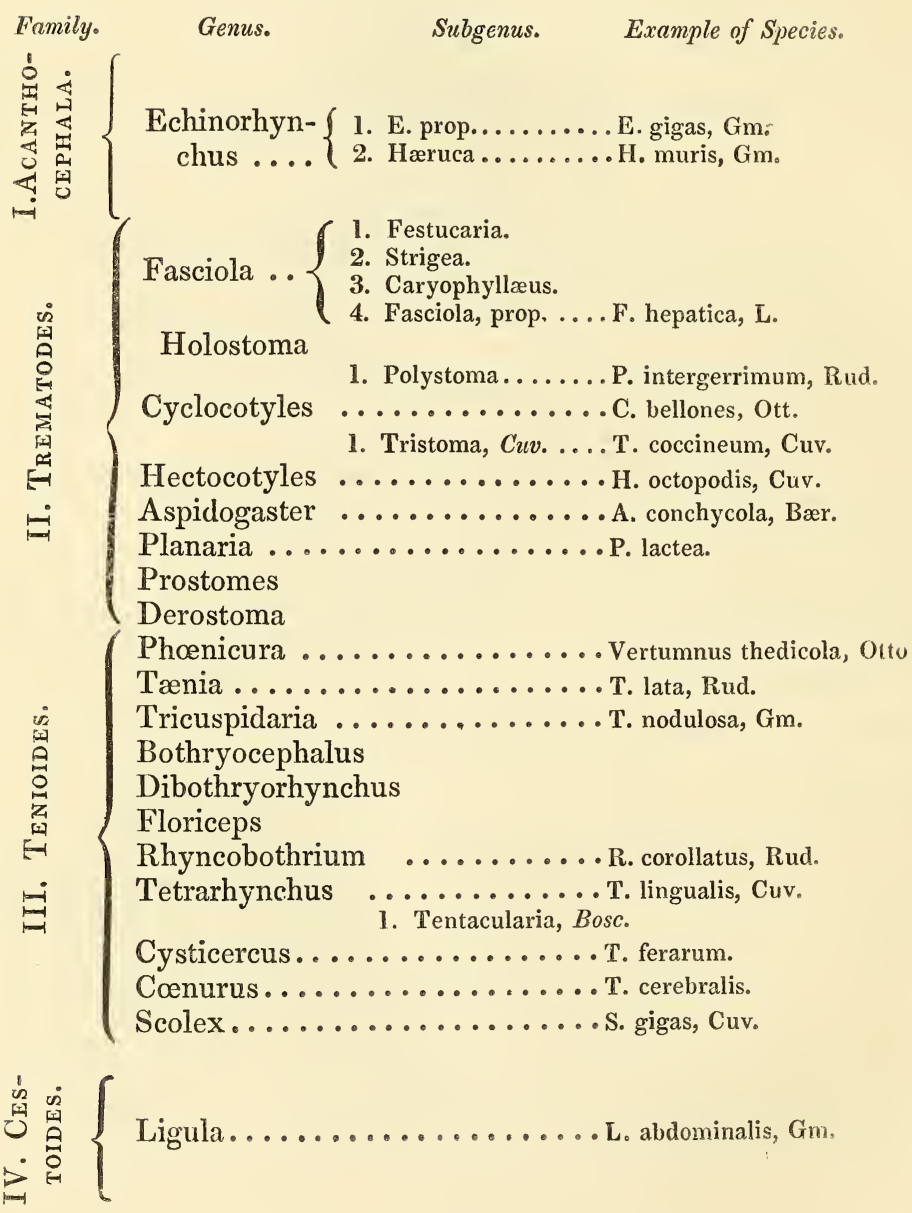




\section{CLASS III.-ACALEPHA.}

Commonly called sea-nettles. These display some appearance of internal vessels, which, however, are mere productions of the intestines in the substance of the body.

They include two orders.

Class.

Order.

Acalepнa $\ldots\left\{\begin{array}{l}\text { Acalephæ simplices. } \\ \text { Acalephæ hydrostaticæ. }\end{array}\right.$

\section{ORDER I.-Acalephe Simplices.}

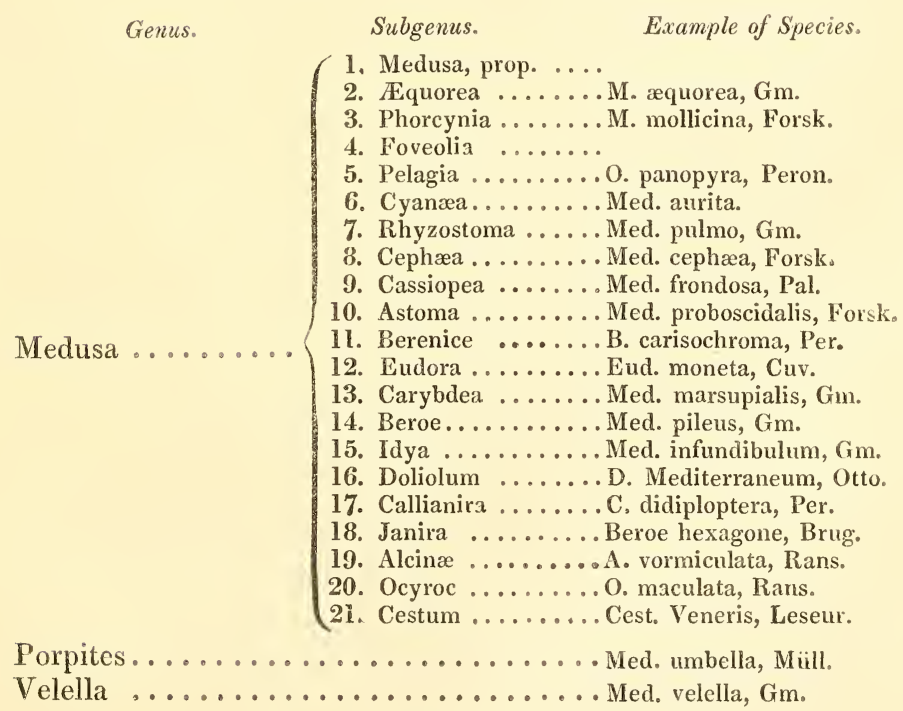




\section{ORDER II.-Hydrostatica.}

This order is distinguished by air vesicles or bladders, by which the animals are usually suspended in the water.

$$
\text { Genus. Subgenus. Example of Species. }
$$

Physalia, Lam. . . . . . . . . . . Holothuria physalis, L.

Physsophora, Forsk. $\left\{\begin{array}{l}\text { 1. Physsophora, prop..P. hydrostatica, Gm. } \\ \text { 2. Hippopus, Quoy \& G. } \\ \text { 3. Cupulites. } \\ \text { 4. Racemides, Cuv. } \\ \text { 5. Rhyzophyza, Per..P. filliformis, Forsk. } \\ \text { 6. Stephanomia....... amphitritis, Per. }\end{array}\right.$

Diphyes, Cuv..... $\left\{\begin{array}{l}\text { 1. Diphyes, prop. } \\ \text { 2. Calpe. } \\ \text { 3. Abyle. } \\ \text { 4. Cuboides. } \\ \text { 5. Navicula. }\end{array}\right\}$ See An. de Sc. X. 


\section{CLASS IV.-POLYPI.}

In this class there are tentacula, which surround the mouth. Most of the genera propagate by off-shoots, as well as by eggrs.

It includes three orders, viz.-

$$
\begin{gathered}
\text { Class. } \\
\text { Pouy } \\
\text { Order. }
\end{gathered}
$$

\section{ORDER I.-Polypi Carnosi.}

Called also fixed sea-nettles. They have a fleshy envelope, and are generally fixed to some submarine object, but occasionally free.

$$
\text { Genus. Subgenus. Example of Species. }
$$
Actinia
1. Actinia, prop. .....Ac. senilis, L.
2. Thalassianthus .... Thal. aster, Ruppel.
3. Discosoma........ D. nummiforme, Id.
4. Zoanthus ........Hydra sociata, Gm.

Lucernaria...................... quadricornis, Müll.

\section{ORDER II.-Polypi Gelatinosi.}

Are without any general envelope, and are gelatinous. Their body represents a mere bag.

$$
\text { Genus. } \quad \text { Example of Species. }
$$

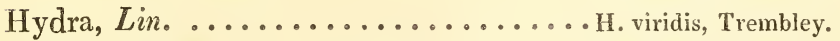

Corine, Gartner. .............. Tabularia coryna, Gm.

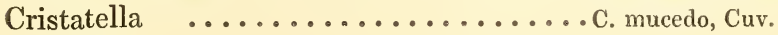

Vorticella

Pedicellaria ............................ Mëller, Zool. Dan. xvi. 


\section{ORDER III.-Polypi Polypiferi.}

In this order the animals are composite, inhabiting, in numbers, a single habitaculum or polyparia, with a community of nutriment and volition.

The order is divided into three families.

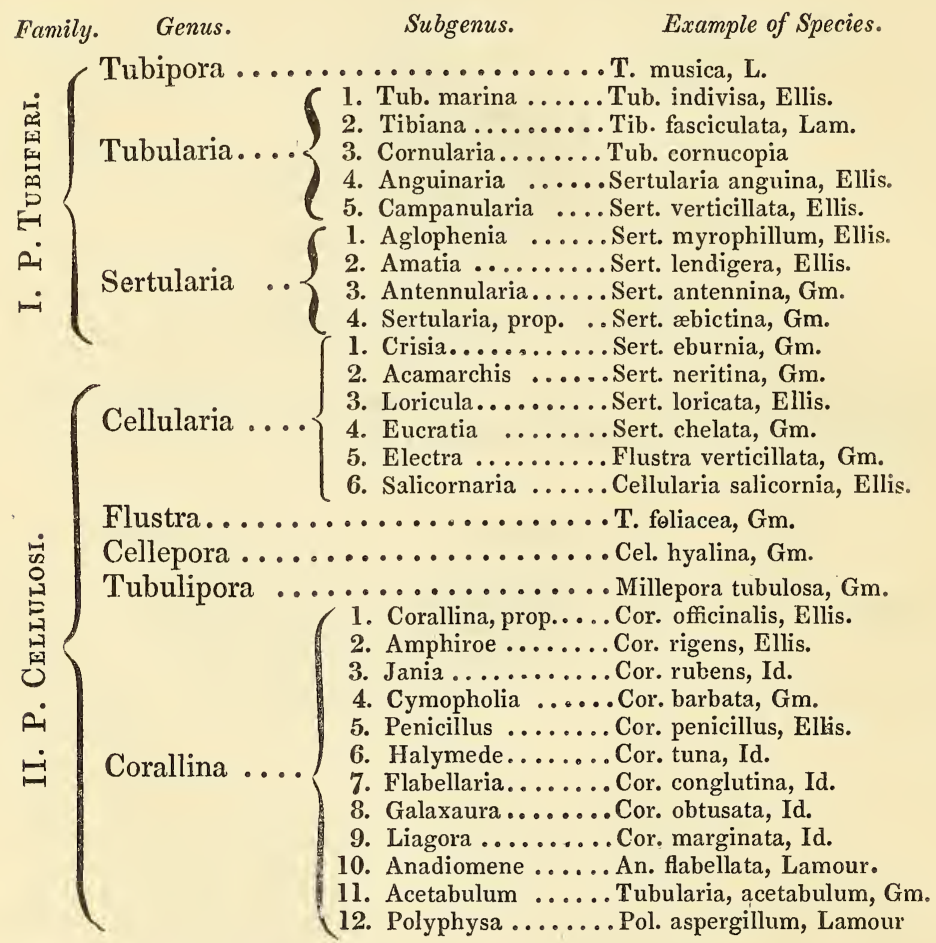

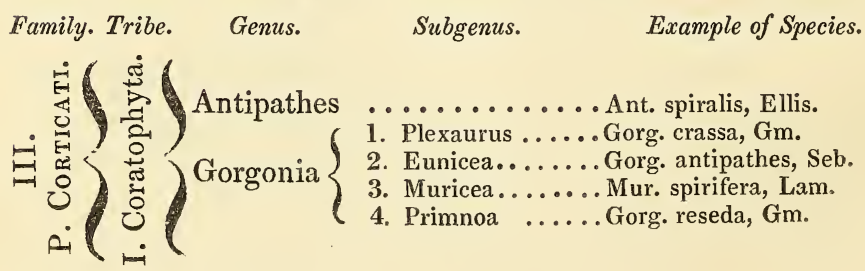




\section{ORDER III.-PoLyPI Polypiferi (continued).}

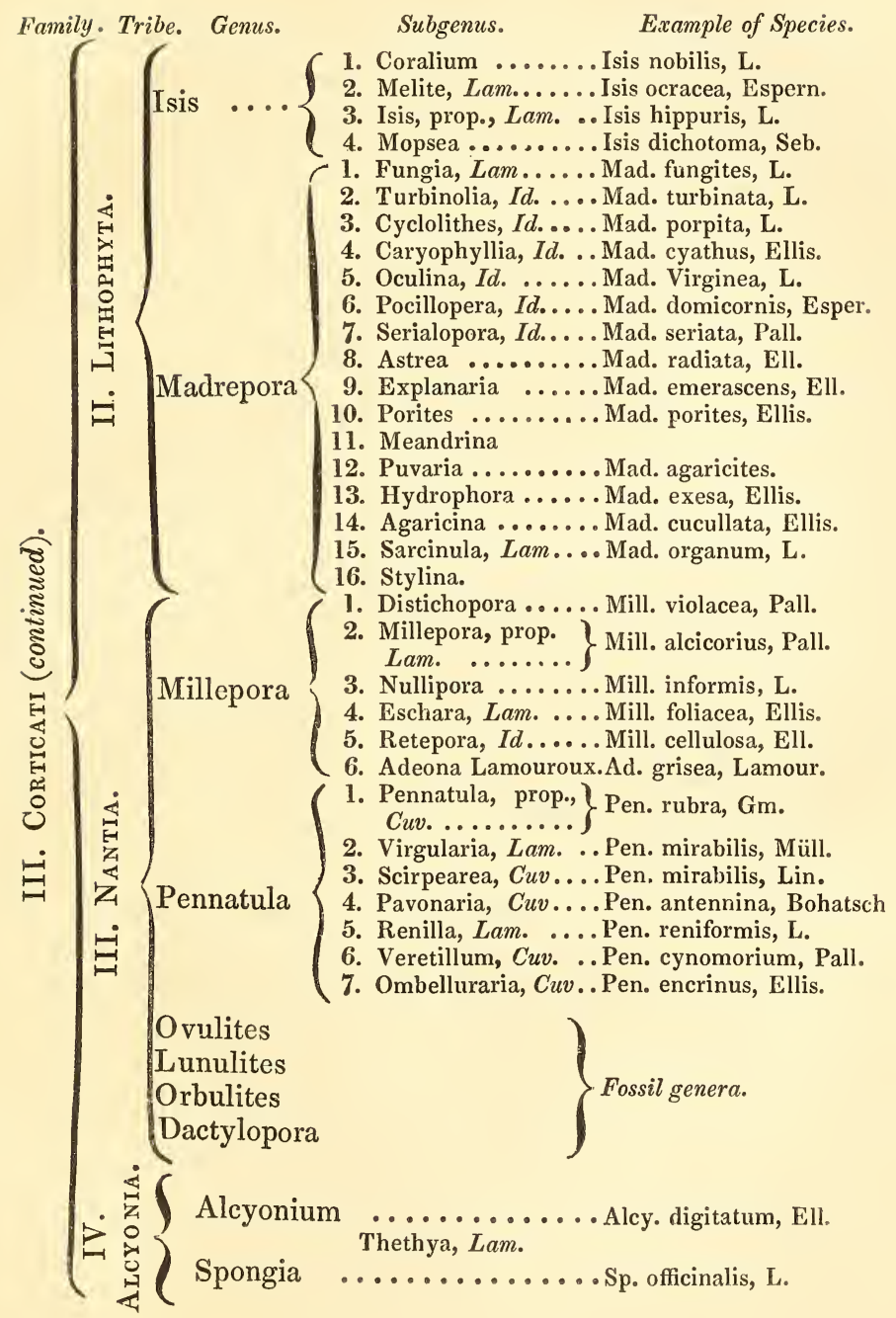




\section{CLASS V.-INFUSORIA.}

Microscopic animals, in general of extreme simplicity of organization. Some of the genera, however, appear to be more complicated, though the details of their organization escape the deepest powers of the microscope.

The class is divided into two orders.

$$
\begin{array}{cl}
\text { Class. } & \begin{array}{l}
\text { Order. } \\
\text { InfUSORIA }
\end{array} \cdot \begin{cases}\text { 1. } & \text { Rotifera. } \\
\text { 2. } & \text { Homogenea. }\end{cases}
\end{array}
$$

\section{ORDER I.-ROTIFERA.}

Body oval and gelatinous; a mouth, stomach, intestines, and arms have been observed. The mouth or head is surrounded by denticulated lobes, which vibrate with great rapidity, and present the appearance under the glass of a revolving wheel.

Genus.

Subgenus.

Example of Species.

Furcularia ...... $\left\{\begin{array}{l}\text { 1. Furcularia, prop. ..F. } \\ \text { 2. Trichocerca, Lam... Tri. paxillum, Müll. } \\ \text { 3. Vaginicola, Lam. . Tri. innata, Müll. }\end{array}\right.$ Tubicolaria, Lam. ............... Vorticella tetrapetala Brachionus, Müll. 


\section{ORDER II.-HoMOgENEA.}

Body gelatinous, but without appearance of stomach, intestines, anus, and sometimes of mouth.

\section{Genus.}

Urceolaria, Lam.

Trichoda

Leucophra

Kerona

Himantopus

Cercaria

Vibrio

Enchelis

Cyclidium

Paramecium

Kolpoda

Gonium

Bursaria

Proteus

Monas

Volvox. 






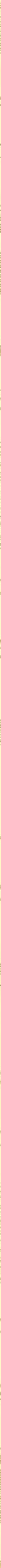


SMITHSONIAN INSTITUTION LIBRARIES

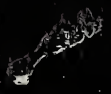

